\section{$\mathrm{Y}-12$}
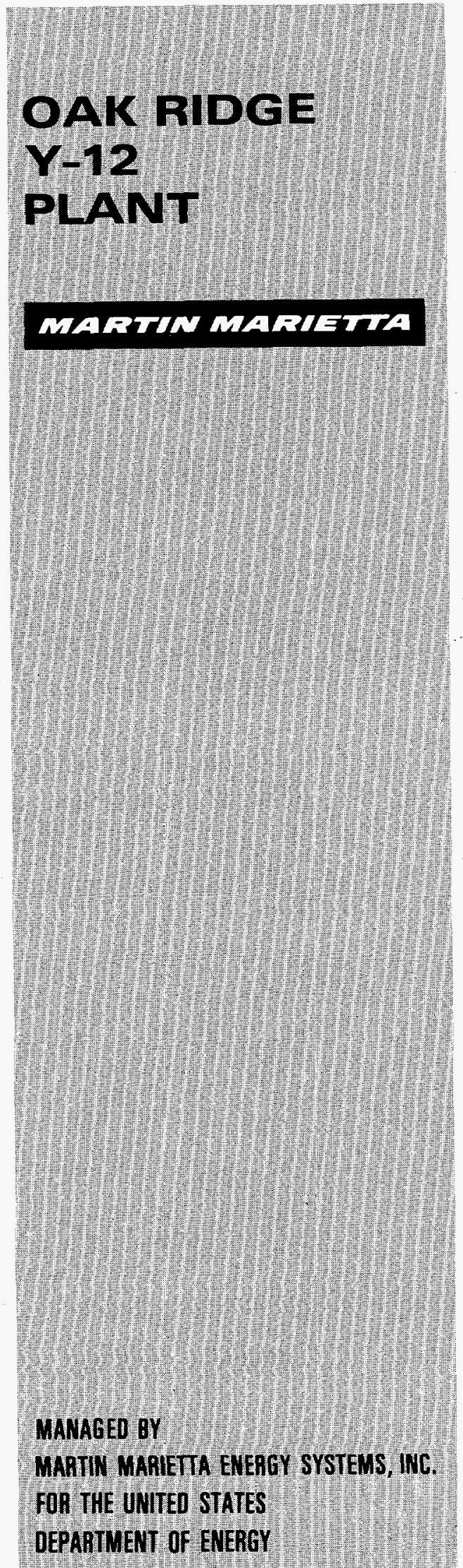

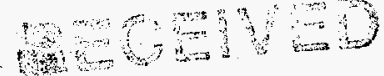

Y/ER-159/V3/PtB

Aบ5 281997

OSTI

\section{Y-12 Plant Decontamination and Decommissioning Technology Logic Diagram for Building 9201-4}

\author{
Volume 3 \\ Technology Evaluation Data Sheets \\ Part B \\ Decontamination . Robotics/Automation \\ Waste Management
}

September 1994

Prepared for the Office of

Environmental Restoration

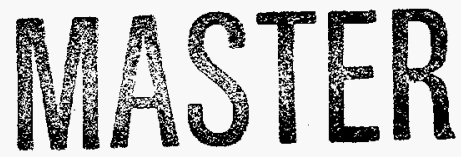

Prepared by

Oak Ridge K-25 Site

Oak Ridge, Tennessee 37831

managed by

MARTIN MARIETTA ENERGY SYSTEMS, INC.

for the

U.S. DEPARTMENT OF ENERGY

under contract DE-AC05-84OR21400 


\section{DISCLAIMER}

This report was prepared as an account of work sponsored by an agency of the United States Government. Neither the United States Government nor any agency thereof, nor any of their employees, makes any warranty, express or implied, or assumes any legal liability of responsibility for the accuracy, completeness, or usefulness of any information, apparatus, product, or process disclosed, or represents that its use would not infringe privately owned rights. Reference herein to any specific commercial product, process, or service by trade name, trademark, manufacturer, or otherwise, does not necessarily constitute or imply its endorsement, recommendation, or favoring by the United States Government or any agency thereof. The views and opinions of authors expressed herein do not necessarily state or reflect those of the United States Government or any agency thereof.

This report has been reproduced directly from the best available copy.

Available to DOE and DOE contractors from the Office of Scientific and Technical Information, P. O. Box 62, Oak Ridge, TN 37831; prices available from 615-576-8401.

Available to the public from the National Technical Information Service, U.S. Department of Commerce, 5285 Port Royal Rd., Springfield, VA 22161. 


\title{
Y-12 Plant Decontamination and Decommissioning Technology Logic Diagram for Building 9201-4
}

\author{
Volume 3 \\ Technolgy Evaluation Data Sheets
}

Part B

Decontamination . Robotics/Automation

Waste Management

Prepared for the

Office of Environmental Restoration

September 1994

DISTRIBUTION OF THIS DOCUMENT IS UNLPRTED

Prepared by

Oak Ridge K-25 Site

Oak Ridge, Tennessee 37831

managed by

MARTIN MARIETTA ENERGY SYSTEMS, INC.

for the

U.S. DEPARTMENT OF ENERGY

under contract DE-AC05-84OR21400 


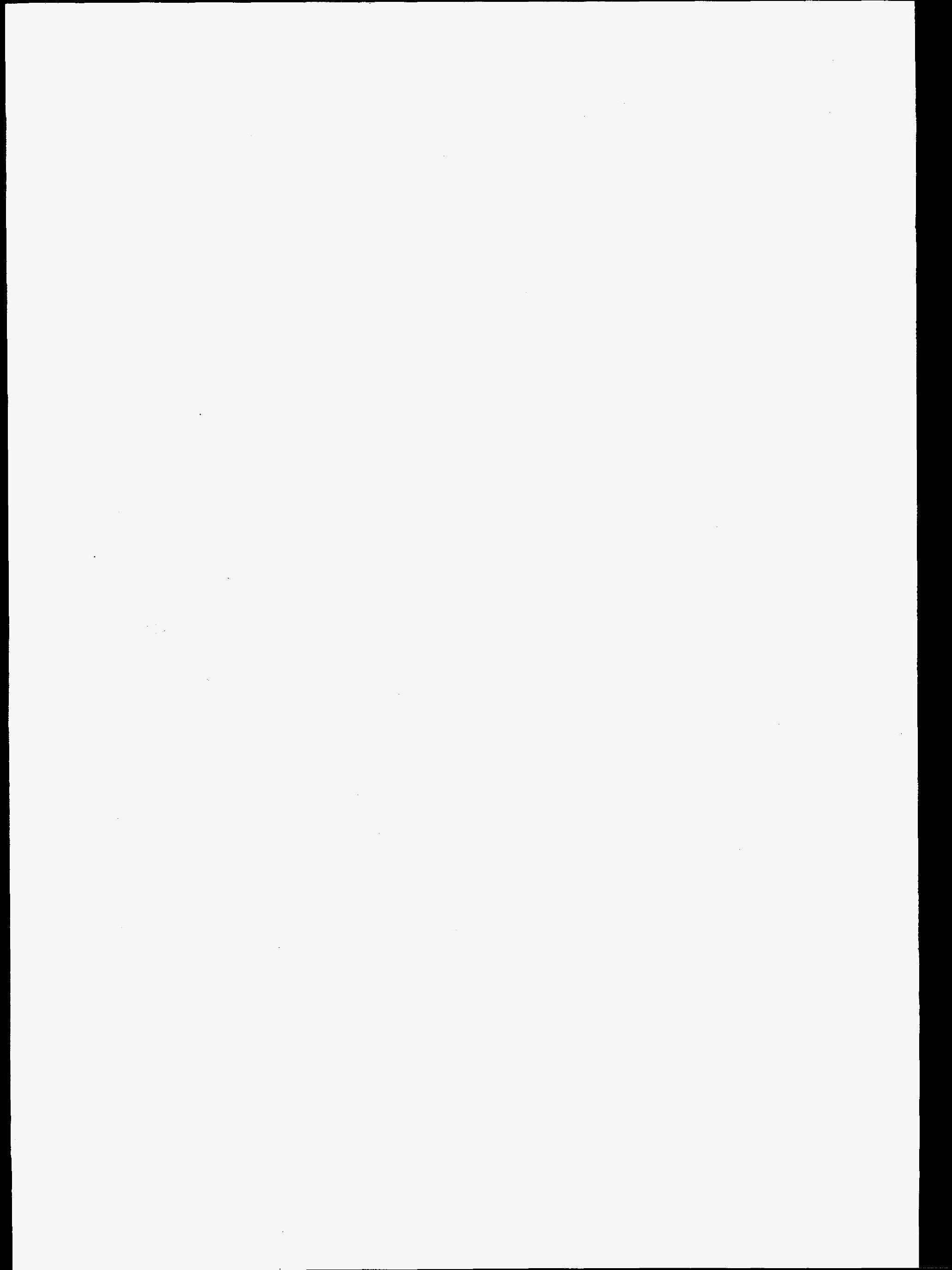




\section{DISCLAMMER}

Portions of this document may be illegible in electronic image products. Images are produced from the best available original document. 


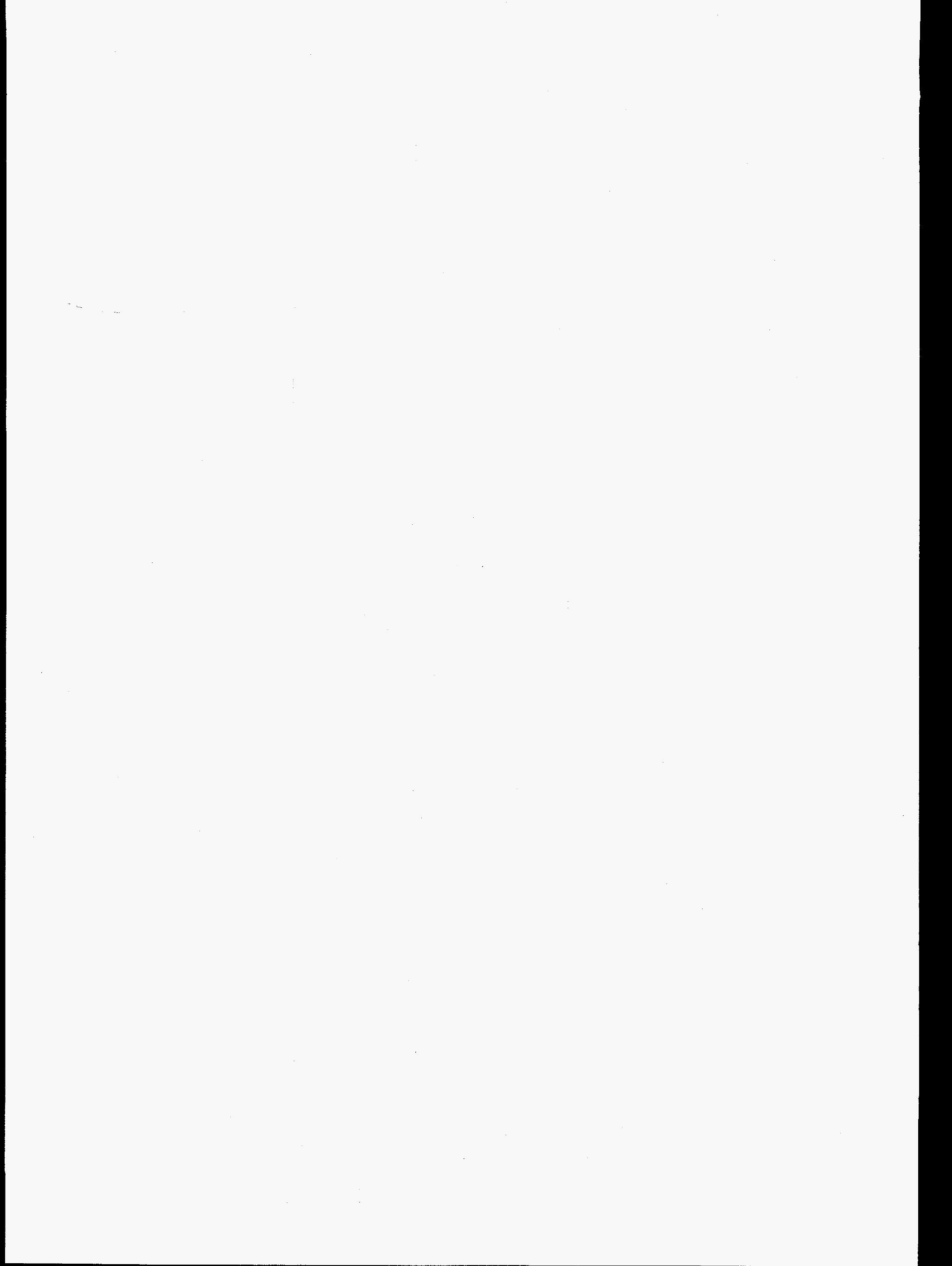


John M. Googin, a member of the Senior Advisory Group, was a significant contributor to this Technology Logic Diagram project before his death this year. We feel it appropriate to express our appreciation of him and our sense of loss. His insightful comments at project meetings never failed to enliven the discussions and guide our thinking.

The authors acknowledge the significant contributions made by the DOE Overview Team for the Technology Logic Diagram Review and Development: Robert Benedetti, Holmer Dugger, Mitchell Erickson, Ned Hutchins, William Schutte, and Randall Snipes.

\section{Project Manager}
R.L. Fellows
K-25 Tech.

\section{Senior Advisory Review}
V. Boston
DOE
R.M. Cannon
Eng.
F.P. DeLozier
ER
J.M. Googin
Y-12 Develop.
J.T. Grumski
Y-12 ER
J.E. Heiskell
WM
R.C. Reipe
Y-12 Develop.

Planning/Oversight/Coordination Review
M.L. Baker
K.L. Brady
Y.C. Childs
S.L. Cross
R.L. Fellows
S.M. Howell
J.M. Kennerly
J.O. Moore
Y-12 Develop.
ER
Y-12 D\&D
MMES
K-25 Tech.
Y-12 D\&D
ER
M.J. Norris
DOE
J.E. Stone
Y-12 ER
WM

Planning \& Integration
C.A. Chandler
Eng.
R.L. Fellows
K-25 Tech.
D.W. Phifer
K-25 Tech.

Team Leaders
G.A. Blankenship
Eng.
R.D. Bundy
K-25 Tech. 
R.L. Fellows

C.G. Jones

S.P.N. Singh

Deputy Team Leader

D.E. Beck

\section{Publications}

J.A. Getsi

G.W. Llanos

M.W. Marsh

G.E. Powell

W.C. Russell

N.C. Smith

R.H. Van Dyke
K-25 Tech.

MMES Env. Compl.

ORNL CTD

\section{Contributors}

R.B. Alderfer

T. Barnes

J.A. Basford

D.E. Beck

G.L. Bell

Z.W. Bell

D.H. Bunch

R.M. Davis

J.R. Devore

E.L. Etnier

R.L. Fellows

A.P. Fleming

C.A. Foster

W.L. Gardner

C.M. Goddard

D.C. Haley

C.E. Hamilton

J.P. Hitch

J.M. Hiller

P. Hoskins

L.L. Houlberg

K.A. Kitzke

K.E. Lott

E.B. Munday

W.D. Nelson

F. Roettger

T. Ross

S.L Schrock

D.D. Smith

C.W. Smith

D.B. Smith
K-25 Tech.

Technical Editor

Electronic Publisher

Technical Editor

Technical Editor

Technical Editor

Electronic Publisher

Technical Editor

K-25 Tech.

Mach. Kinetics Corp.

Y-12 Develop.

K-25 Tech.

ORNL Fusion Energy

Y-12 Develop.

K-25 Tech.

Y-12 Develop.

Eng.

ORNL HSRD

K-25 Tech.

Y-12 EM

ORNL Fusion Energy

ORNL Fusion Energy

Y-12 ER

ORNL Robotics

Eng.

ORNL HSRD

Y-12 Develop.

Eng.

ORNL HSRD

Y-12 Develop.

Eng.

K-25 Tech.

ORNL Fusion Energy

Y-12 Develop.

ORNL CEA

ORNL Robotics

Y-12 Develop.

Y-12 ER

ORNL Eng. Tech. 
C.C. Tsai

R. Whaley

T.L. White

J.H. Wilson

L.M. Woodard
ORNL Fusion Energy

Eng.

ORNL Fusion Energy

ORNL CTD

Y-12 Develop. 

The Y-12 Plant Decontamination and Decommissioning Technology Logic Diagram for Building 9201-4 (TLD) was developed to provide a decision-support tool that relates decontamination and decommissioning (D\&D) problems at Bldg. 9201-4 to potential technologies that can remediate these problems. The TLD uses information from the Strategic Roadmap for the Oak Ridge Reservation, the Oak Ridge K-25 Site Technology Logic Diagram, the Oak Ridge National Laboratory Technology Logic Diagram, and a previous Hanford logic diagram.

This TLD identifies the research, development, demonstration, testing, and evaluation needed for sufficient development of these technologies to allow for technology transfer and application to D\&D and waste management (WM) activities. It is essential that follow-on engineering studies be conducted to build on the output of this project. These studies will begin by selecting the most promising technologies identified in the TLD and by finding an optimum mix of technologies that will provide a socially acceptable balance between cost and risk.

The TLD consists of three fundamentally separate volumes: Vol. 1 (Technology Evaluation), Vol. 2 (Technology Logic Diagram), and Vol. 3 (Technology Evaluation Data Sheets). Volume 1 presents an overview of the TLD, an explanation of the programspecific responsibilities, a review of identified technologies, and the rankings of remedial technologies. Volume 2 contains the logic linkages among environmental management goals, environmental problems, and the various technologies that have the potential to solve these problems. Volume 3 contains the TLD data sheets.

Volume 3 has been divided into five sections comprising two parts. Volume 3, Part A, consists of the characterization and dismantlement data sheets. Volume 3, Part B, consists of the decontamination, robotics/automation, and WM data sheets. Data sheets are arranged alphanumerically.

The technology evaluations contained in these volumes are based on the best information available during compilation of the TLD. New or more accurate information is solicited to improve the TLD data base. Please send comments to R. $L$. Fellows, Editor, Y-12 Plant Decontamination and Decommissioning Technology Logic Diagram for Building 9201-4, Martin Marietta Energy Systems, Inc., P.O. Box 2003, Oak Ridge, TN 37831-7274. FAX 615-576-8558. 

DECONTAMINATION 

EM Problem: Decontamination and Decommissioning

Y-12 Plant Problem: Building 9201-4 (Alpha-4)

Problem Area/Constituents: Insulation and building paper contaminated with $\mathrm{Hg}$, $\mathrm{U}$ (and daughters), and PCBs; wood and sheet rock contaminated with $\mathrm{Hg}, \mathrm{LiOH}, \mathrm{H}_{2} \mathrm{SO}_{4}, \mathrm{HNO}_{3}$, and asbestos

Reference Requirements: Refer to Vol. 1, Chap. 10, for potentially applicable proposed and promulgated environmental laws, signed and pending agreements for the Oak Ridge Reservation, radiation protection standards, DOE orders, and nonregulatory guidance. As site- and waste-specific characteristics are provided for each technology, specific regulatory requirements will be specified.

\section{Subelement: Decontamination}

Alternative: Bulk decontamination methods

Technology: Solvent extraction. Organic contaminants are selectively removed by dissolution in a solvent. This process could be applied as a "factory style" process in either batch or continuous fashion. Contaminated materials are fed into processing equipment and cleaned material exits. This would be applicable to wood, sheet rock, and building paper for example. A countercurrent extractor, such as developed by the ORNL Fuel Recycle Division for fuel dissolution, would appear to be an appropriate contractor and availability of both laband pilot-scale equipment would need to be pursued for Y-12 applications. This process could also be applied as a portable technology; the solvent could be applied to a localized spot with a device patterned after a carpet cleaning machine.

Status: Solvent extraction is a mature technical field, but specific extractants have not been identified for all radionuclides, PCB extraction from insulation, etc. An attractive solvent, triethylamine, has been evaluated for organic extraction from sludge (TTP-OR-373-AH) and found to be a very effective solvent, and one for which industrial processing equipment is available commercially. Solvent recycle equipment and off-gas treatment equipment could be easily adapted for use here.

Efficacy - Limitations; has been demonstrated remotely, but not for Hg-contaminated waste materials.

Waste-Produces a liquid raffinate stream.

Science/Technology Needs: Technology development needs are the following: (1) survey and tests of specific extractants applicable to $\mathrm{Hg}$ - and PCB-contaminated insulation, wood, and sheet rock materials and (2) development or adaption of a portable "spot" remover from commercial devices. Technology demonstration will involve (3) the applicability of rotary 
contractor, solvent recycle, and, if appropriate because of the solvent selection, an off-gas treatment system.

Implementation Needs: Development costs will run less than $\sim 250 \mathrm{~K}, \$ 500 \mathrm{~K}$, and $\$ 1000 \mathrm{~K}$ for 1, 2, and 3, (under Science/Technology Needs), respectively, assuming development of lab-scale and pilot-scale extraction units at Y-12 and industrial participation in demo. Deployment will require no unusual costs. Development (pilot-scale) equipment may be used in production.

Author: K. E. Lott/615-574-6537

References: MMES staff members 
EM Problem: Decontamination and Decommissioning

Y-12 Plant Problem: Building 9201-4 (Alpha-4)

Problem Area/Constituents: Insulation and building paper contaminated with $\mathrm{Hg}$ and $\mathrm{U}$ (and daughters); carbon chunks contaminated with $\mathrm{Hg}, \mathrm{LiOH}, \mathrm{H}_{2} \mathrm{SO}_{4}$, and $\mathrm{HNO}_{3}$

Reference Requirements: Refer to Vol. 1, Chap. 10, for potentially applicable proposed and promulgated environmental laws, signed and pending agreements for the Oak Ridge Reservation, radiation protection standards, DOE orders, and nonregulatory guidance. As site- and waste-specific characteristics are provided for each technology, specific regulatory requirements will be specified.

\section{Subelement: Decontamination}

Alternative: Bulk decontamination methods

Technology: Incineration. Contaminated combustible materials are burned. The contamination is collected in the ash and the off-gas treatment system. The volume of the waste is reduced.

Status: Accepted. The incineration of contaminated combustible building materials is common in the nuclear industry. ${ }^{1}$ The system will have to be designed to prevent mercury emissions.

Efficacy-Limitations: has been demonstrated remotely, but not for Hg-contaminated waste materials.

Waste-Produces a liquid raffinate stream. The amount of waste produced will depend on the design of the incinerator and the ash content of the waste being fed.

Cost-Capital cost is $\$ 26 \mathrm{M}$; operating cost is $\$ 10 / \mathrm{lb}^{2}$.

Science/Technology Needs: None

Implementation Needs: About 8-10 years will be required for writing an environmental impact statement, holding public hearings, and obtaining the necessary permits: TSCA permit, RCRA permit, NESHAP permit, and Clean Air Act permit. ${ }^{3}$ 


\section{Author: D. H. Bunch/615-576-0201}

\section{References:}

1. R. Kohout, "Incineration," in Radioactive Waste Technology, ed. A. Alan Moghissi, et al., American Society of Mechanical Engineers and American Nuclear Society, 1986.

2. T. O. Rogers, telephone conversation with D. H. Bunch, May 28, 1992.

3. L. V. Gibson, Jr., telephone conversation with D. H. Bunch, June 16, 1992. 
EM Problem: Decontamination and Decommissioning

Y-12 Plant Problem: Building 9201-4 (Alpha-4)

Problem Area/Constituents: Steel and nickel process equipment contaminated with $\mathrm{Hg}$, $\mathrm{H}_{2} \mathrm{SO}_{4}, \mathrm{HNO}_{3}, \mathrm{LiOH}$, and asbestos. Insulation and building paper contaminated with $\mathrm{Hg}$ and $\mathrm{U}$.

\section{Reference Requirements:}

- Federal Facility Compliance Agreements (e.g., FFCA for Oak Ridge Site)

- Applicable federal and state laws and regulations (e.g., Clean Air Act and Toxic Substance Control Act)

- DOE orders

\section{Subelement: Decontamination}

Alternative: Bulk decontamination methods

Technology: Dry heat (thermal desorption or roasting). The process involves heating the bulk material to a temperature high enough to volatilize the contaminants. Volatilized vapors are oxidized in a secondary combustion chamber or collected for other treatment. Development efforts have demonstrated its use to remove PCBs, solvents, and mercury. Significant volume reduction for burnable feed materials is not expected. The contaminated feed materials are fed to a rotating metal shell inside a gas fired furnace. A wet scrubber and/or bag house is used for final filtering of the off-gases from the secondary combustion chamber.

Status: Demonstration. To be successful, this technology must be demonstrated as an effective contaminant removal. The contaminants characteristics must be matched to the decontamination advantages of the process.

Efficacy/strengths/weaknesses-Secondary waste streams must be characterized during development activities.

Waste-The contaminants characteristics must be matched to the decontamination advantages of its development need.

Cost-Capital cost will be about $\$ 1$ million; operating cost, typical for this process, $-\$ 12 / 1 b$ of feed.

Science/Technology Needs: The availability of engineered equipment is adequate provided that laboratory studies verify effective contamination removal by heat. Secondary waste treatment would need to be addressed. Engineering data are needed for this process. 
Implementation Needs: Development costs to define and demonstrate effective removal of contaminants and volume reduction are estimated at $\$ 500 \mathrm{~K}$. Establishment of waste criteria for the commercial unit could cost $\$ 300 \mathrm{~K}$. Separation of asbestos would require a special facility; however, the cost cannot be estimated. Compliance is a minor concern for moderate contamination levels.

Author: R. B. Alderfer/615-576-0225

References: MMES staff members

Technical vendor release from IT Corporation, Knoxville, Tenn.

Techical vendor release from Hazen Research, Inc. Golden, Colo. 
EM Problem: Decontamination and Decommissioning

Y-12 Plant Problem: Building 9201-4 (Alpha-4)

\section{Problem Area/Constituents:}

- In situ decontamination of the interior of steel and nickel process equipment contaminated with $\mathrm{Hg}, \mathrm{LiOH}, \mathrm{H}_{2} \mathrm{SO}_{4}$, and $\mathrm{HNO}_{3}$.

- In situ decontamination of the exterior of steel and nickel process equipment contaminated with $\mathrm{Hg}, \mathrm{LiOH}, \mathrm{H}_{2} \mathrm{SO}_{4}, \mathrm{HNO}_{3}$, asbestos, and PCBs.

- Ex situ decontamination of the exterior of steel and nickel process equipment contaminated with $\mathrm{Hg}, \mathrm{LiOH}, \mathrm{H}_{2} \mathrm{SO}_{4}, \mathrm{HNO}_{3}$, asbestos, and PCBs.

- Painted concrete and clay tile contaminated with PCBs, oil, U (and daughters), electrolytes, $\mathrm{Cd}, \mathrm{Pb}$, and $\mathrm{Cr}$.

- Insulation and building paper contaminated with PCBs, oil, U (and daughters), electrolytes, $\mathrm{Cd}, \mathrm{Pb}$, and $\mathrm{Cr}$.

- Packing contaminated with $\mathrm{Hg}, \mathrm{LiOH}, \mathrm{H}_{2} \mathrm{SO}_{4}$, and $\mathrm{HNO}_{3}$.

- Carbon chunks contaminated with $\mathrm{Hg}, \mathrm{LiOH}, \mathrm{H}_{2} \mathrm{SO}_{4}$, and $\mathrm{HNO}_{3}$.

- Wood and sheet rock contaminated with $\mathrm{Hg}, \mathrm{LiOH}, \mathrm{H}_{2} \mathrm{SO}_{4}, \mathrm{HNO}_{3}, \mathrm{U}$ (and daughters), asbestos, and/or PCBs.

Reference Requirements: Refer to the Regulatory Compliance chap. of Vol. 1 for potentially applicable proposed and promulgated environmental laws, signed and pending agreements for the Oak Ridge Reservation, radiation protection standards, DOE orders, and nonregulatory guidance. As site- and waste-specific characteristics are provided for each technology, specific regulatory requirements will be specified.

\section{Subelement: Decontamination}

Alternative: Bulk decontamination methods

Technology: Chemical leaching. This process uses a liquid chemical agent to dissolve soluble contaminants from a porous solid. The technology could be applied as a "factory style" process in either batch or continuous fashion. It could also be applied as a portable technology (e.g., the solvent is applied in some way to a localized spot and vacuumed).

Status: Evolving technology. Although leaching for removal of PCBs with organic solvents has been developed to a considerable extent, the method has not yet been applied to the contaminants at the Y-12 Plant. The technology is probably best described as preconceptual when applied to Y-12 problems. The efficacy of the technology when applied to Y-12 problems cannot be determined until lab studies are concluded. The operating costs are roughly estimated at $<\$ 1 / \mathrm{ft}^{2}$ of surface, assuming the method is effective. Waste solutions would be generated that would contain the extracted contaminants. This solution would require recycle or disposal. The time required to implementation is estimated at 1.5 years. 
Science Technology Needs: Literature studies to select effective agents and laboratory studies are needed. Demonstrations will follow if warranted.

Implementation Needs: Development costs, $\$ 350 \mathrm{~K}$ for a laboratory study to confirm efficacy, $\$ 150 \mathrm{~K}$ for application tools (shop vac or carpet cleaner-style machine, leaching tanks, chemical preparation equipment, and facility modifications to accommodate the project).

Author: R. L. Fellows/615-576-5632. Reviewed by E. B. Munday/615-576-5806

References: MMES staff members 
EM Problem: Decontamination and Decommissioning

Y-12 Plant Problem: Building 9201-4 (Alpha-4)

Problem Area/Constituents:

- Ex situ decontamination of the exterior of steel and nickel process equipment contaminated with $\mathrm{Hg}, \mathrm{LiOH}, \mathrm{H}_{2} \mathrm{SO}_{4}, \mathrm{HNO}_{3}$, asbestos, and PCBs.

- Insulation and building paper contaminated with PCBs, oil, U (and daughters), electrolytes, $\mathrm{Cd}, \mathrm{Pb}$, and $\mathrm{Cr}$.

- Packing contaminated with $\mathrm{Hg}, \mathrm{LiOH}, \mathrm{H}_{2} \mathrm{SO}_{4}$, and $\mathrm{HNO}_{3}$.

- Carbon chunks contaminated with $\mathrm{Hg}, \mathrm{LiOH}, \mathrm{H}_{2} \mathrm{SO}_{4}$, and $\mathrm{HNO}_{3}$.

- Wood and sheet rock contaminated with $\mathrm{Hg}, \mathrm{LiOH}, \mathrm{H}_{2} \mathrm{SO}_{4}, \mathrm{HNO}_{3}, \mathrm{U}$ (and daughters), asbestos, and/or PCBs.

Reference Requirements:

- Federal Facility Compliance Agreements (e.g., FFCA for Oak Ridge Site)

- Applicable federal and state laws and regulations (e.g., Clean Air Act, RCRA, NEPA, and NESHAP)

- DOE orders

Refer to the final chap. of Vol. 1 for potentially applicable proposed and promulgated environmental laws, signed and pending agreements for the Oak Ridge Reservation, radiation protection standards, DOE orders, and nonregulatory guidance. As site- and waste-specific characteristics are provided for each technology, specific regulatory requirements will be specified.

\section{Subelement: Decontamination}

Alternative: Bulk decontamination methods

Technology: Catalytic extraction process (CEP). The catalytic extraction process uses an induction or electric arc furnace to form a molten metal bath. It can use the molten bath not only as a means for metal purification but also as a high-temperature, high-energy density medium for more effectively reducing hazardous waste materials, such as PCBs, hydrocarbons, and cyanide, to nonhazardous materials. The molten metal (about $3,000^{\circ} \mathrm{F}$ ) provides much more effective contact with the material in a smaller volume than is possible with the hot gases in a conventional incinerator. ${ }^{1}$

Status: Predemonstration. CEP was developed by a commercial vendor to use standard "offthe-shelf" steel industry equipment. ${ }^{1}$ The use of a molten metal bath to more effectively dissociate hazardous materials with potential recovery of valuable or usable constituents is unique. CEP reportedly will allow many hazardous wastes to be processed ten times more effectively than an incinerator, because the molten metal bath at $3,000^{\circ} \mathrm{F}$ provides much 
better thermal contact than hot gases. If the wastes being processed require oxygen, it is added as pure oxygen rather than air, and off-gases are 1/5-1/50 the volume of an incinerator for the same amount of material. Capital costs are estimated at one-half that of an incinerator, and operating costs are estimated at one-third that of an incinerator's operating costs. The costs to incinerate mixed wastes in the TSCA incinerator at K-25 is estimated ${ }^{2}$ at $\$ 6 / 1 \mathrm{~b}$ if solids were fed with liquids. Thus the payback potential for CEP is great. This process has not yet been used to incinerate or smelt scrap bearing radioactivity ${ }^{3}$, but the experience from Y-12 and elsewhere for smelting can be used to roughly gauge expected success when applied to melt processing. Experiments at $Y-12$ have shown that mild steel, stainless steel, nickel, copper, and Monel can be purified by smelting to levels of $0.01 \mathrm{ppm}-4.0 \mathrm{ppm}$ residual uranium. Aluminum was more difficult to purify with levels of residual uranium remaining at concentrations from $3 \mathrm{ppm}-100 \mathrm{ppm}$. Technetium reportedly was not removed. ${ }^{4}$ Hesmatpour and Copeland ${ }^{5}$ report better results for aluminum (1 ppm-2 ppm). Based on information contained in a report by Kellogg et al. ${ }^{6}$, a cost of roughly $\$ 0.93 / \mathrm{lb}$ of metal smelted may be estimated, which includes capital and operating costs. Their estimate concludes that if a de minimis standard is obtained, thus allowing the ingots to be sold, the value of the metal could totally pay for the project. Since the Kellogg study, was done in 1982 and since environmental regulations have become more stringent, this conclusion is probably no longer valid. The wastes would be in the form of radioactive slag and perhaps scrubber solution (although wet scrubbers may be avoided in many applications). ${ }^{1}$ Quantities are not available, but Kellogg et al. ${ }^{6}$ estimated that $\sim 50 \mathrm{lb}$ of slag would be generated per ton of scrap metal processed by smelt purification. CEP is likely to be a very satisfactory method for disposal of combustible wastes.

Science/Technology Needs: Technology Development Needs. The use of CEP as a substitute for incineration of mixed waste has not been done on any scale at this point and must be developed. The effectiveness of CEP for the materials and contaminants of interest in Building 9201-4 should be demonstrated.

Implementation Needs: The technology development will require further lab-and pilot-scale evaluation. Detailed requirements for the development of CEP production phase could not be obtained but are assumed to closely resemble those roughly estimated for smelt purification. The costs are briefly enumerated as follows: development costs, $\$ 3 M-\$ 5 M$; capital costs, $1 / 2$ the amount of an incinerator of equal capacity $(\sim 16 \mathrm{M})$; operating costs, $1 / 3$ the amount of a TSCA incinerator $(\$ 2-\$ 3 / \mathrm{lb}$ for nonmetals, $\$ 0.93 / \mathrm{lb}$ for metal smelting).

The following requirements are based on that assumption. The development costs would include a small electric induction furnace, two full-time technical people (one with experience in metallurgy); and analytical, maintenance, and other support personnel. The duration may be 24 months and the development costs are roughly estimated by the investigator at $\$ 2.0 \mathrm{M}-\$ 3.5 \mathrm{M}$. An additional 24 months is roughly estimated as the time required for fall implementation. Kellogg et al. ${ }^{6}$, lists the following as requirements for a smelt facility: scrap handling and preparation, scrap transportation, scrap weighing and drying facility (the scrap must be heated prior to placing in the smelter to drive off oil and water), 8-ton capacity electric induction furnace with two crucibles, mold preparation and cooling, slag process

September 1994

Decontamination and Decommissioning 
refractory repair, flux storage and preparation, ingot dumping and storage, offices, laboratory and control room, maintenance areas, and personnel convenience stations. Capital and operating costs were not indicated, but as mentioned above, an overall cost of roughly $\$ 0.93 / \mathrm{lb}$ can be estimated based on their conclusions that all costs could be recovered from the sale of 90,000 tons of metal presently in the DOE inventory (valued at $\$ 102$ million in 1982 dollars) assuming a de minimis is established. (About 74\% of the metal value is nickel, which amounts to only $11 \%$ by weight of the metals in the Kellogg study.)

Money likely no existing facility at Y-12 will satisfy the operational requirements of this facility (the Kellogg study assumes new facilities are constructed). The design and construction phase will require the support of many engineering disciplines: structural and site engineering, engineering mechanics, process design, electrical engineering, instrument engineering, safety engineering, and metallurgy. Following are the numerous support services that will be required: health physics, industrial hygiene, health, safety, environmental, and analytical. Following are the numerous construction and maintenance craftsmen that will be required: welders, carpenters, electricians, mechanics, ETI personnel, and supervisors. These requirements cannot be quantified at present. The establishment of a de minimis standard is very critical for the success of this project because the resulting ingots cannot be sold without this legislation.

Author: E. B. Munday/615-576-5806

\section{References:}

1. J. D. Smith, "Molten Metal Technology," EI Digest, Industrial and Hazardous Waste Management Environmental Information Ltd., July 1991.

2. Tom Rodgers, Martin Marietta Energy Systems, Inc., personal communication with E. B. Munday, June 1992.

3. Victor Gatto, Molten Metal Technology, Inc., personal communication with E. B. Munday, June 1992.

4. Y-12 Plant data.

5. B. Hesmatpour and G. L. Copeland, The Effects of Slag Composition and Process Variable on Decontamination of Metallic Wastes by Melt Refining, ORNL/TM-7501, Union Carbide Corporation, January 1981.

6. D. R. Kellogg et al., Metal-Smelting Facility, CONF-820418-13, DE-82 013539, Union Carbide Corporation Nuclear Division, 1982. 


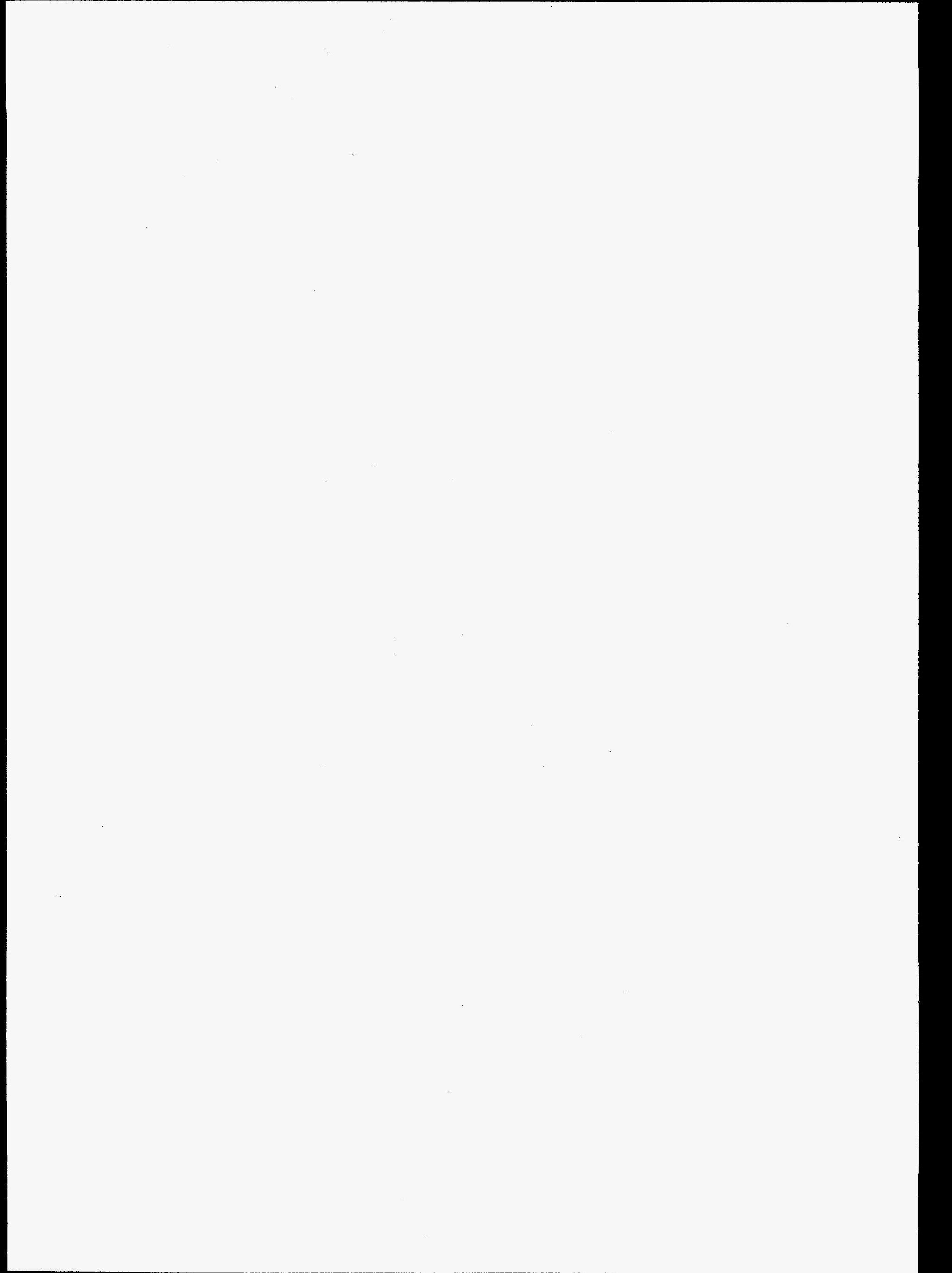


EM Problem: Decontamination and Decommissioning

Y-12 Plant Problem: Building 9201-4 (Alpha-4)

Problem Area/Constituents: Steel and nickel process equipment contaminated with Hg, $\mathrm{H}_{2} \mathrm{SO}_{4}, \mathrm{HNO}_{3}, \mathrm{LiOH}$, and asbestos; insulation and building paper contaminated with $\mathrm{U}$ and $\mathrm{Hg}$.

\section{Reference Requirements:}

- Federal Facility Compliance Agreements (FFCAs) (e.g., FFCA for Oak Ridge Site)

- Applicable Federal and State laws and regulations (e.g., Clean Air Act and Toxic Substance Control Act)

- DOE orders

Refer to the Regulatory Compliance chapter of Vol. 1 for potentially applicable proposed and promulgated environmental laws, signed and pending agreements for the Oak Ridge Reservation, radiation protection standards, DOE orders, and nonregulatory guidance. As site- and waste-specific characteristics are provided for each technology, specific regulatory requirements will be identified.

Subelement: Decontamination

Alternative: Bulk decontamination methods

Technology: Vacuum (low pressure with heat)

Status: Conceptual. The technology exists but requires quantitative verification of effectiveness and subsequent demonstration. Operating costs could be roughly $\$ 2 / 1 b$.

Science Technology Needs: Development-The characteristics of the specific contaminants must be matched to the advantages of decontamination by volatilization under vacuum conditions with the appropriate heat available.

Implementation Needs: The specific areas must be characterized and evaluated for feasibility for heating and applying low pressure conditions for optimum removal rate. Capital costs are $\$ 1 \mathrm{M}$. Development costs will be about $\$ 500 \mathrm{~K}$.

Author: R. B. Alderfer/615-576-0225

Reference: MMES staff members 


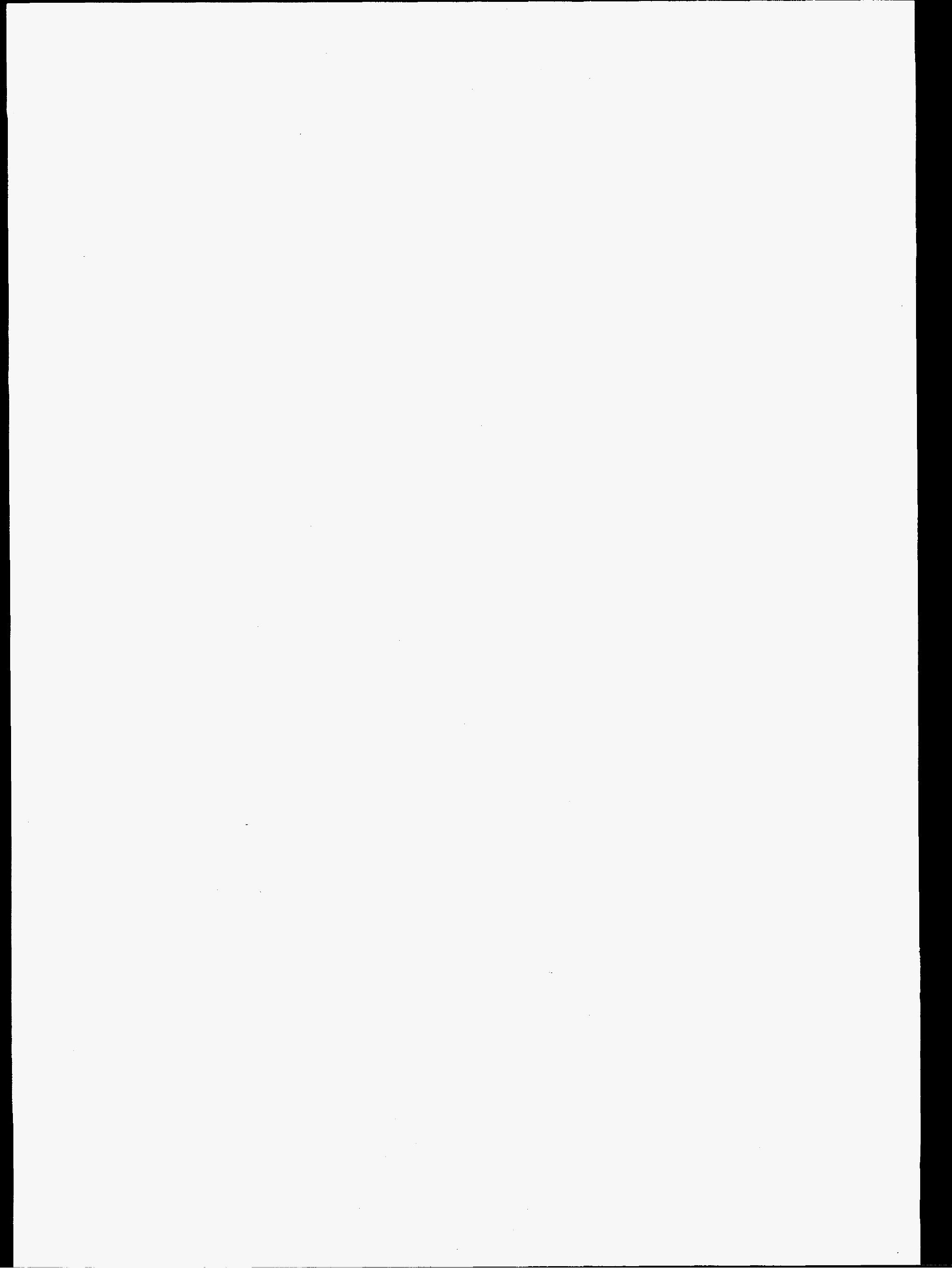


EM Problem: Decontamination and Decommissioning

Y-12 Plant Problem: Building 9201-4 (Alpha-4)

Problem Area/Constituents: Used for in situ decontamination of vertical surfaces, large components, and internal surfaces of nickel-plated steel equipment or steel pipes. Contamination (smearable) can be uranium or other contaminants as dictated by foam constituents.

Reference Requirements: Refer to the Regulatory Compliance chap. of Vol. 1 for potentially applicable proposed and promulgated environmental laws, signed and pending agreements for the Oak Ridge Reservation, radiation protection standards, DOE orders, and nonregulatory guidance. As site- and waste-specific characteristics are provided for each technology, specific regulatory requirements will be identified.

\section{Subelement: Decontamination}

Alternative: Chemical surface cleaning (fixed and transferable contamination) methods

Technology: Chemical foams. Foams are used to remove smearable contamination from contacted surfaces.

Status: Accepted. Widely used throughout the nuclear industry, foam serves as a carrier of chemical decontamination agents (in the early 1980s, detergents and wetting agents), not as the agent itself. It is sprayed onto component walls, or the component is filled with the foam. For vertical surfaces, decontamination agents are suspended in a thick dry foam that is applied to the surface to be cleaned. Ideally, a light, even coating (1-2 in. thick) is formed, with a resident time on vertical surfaces of at least several minutes. It can be sprayed on and wiped, rinsed, or vacuumed off. Repeated applications can give "several orders of magnitude reduction in surface contamination."1 More recently, gels have been preferred for equipment exteriors, and foams are used for circulating in pipes and systems in situ. ${ }^{2}$

Efficacy/strengths/weaknesses

Advantages: This is a good method for large components with complex shapes; for in situ internal decontamination to eliminate smearable contamination prior to dismantlement; and low final waste volume. Remote application is easily done. ${ }^{2}$

Disadvantages: Fully controlled foaming times are needed. It is difficult to keep foam circulating while integrally filling large cavities. It is difficult to obtain a good decontamination factor (DF) by one-time spraying (batch process). Foam is not appropriate for use on cracked surfaces or where there are deep or convoluted crevices.

Effectiveness: In one study ${ }^{1}$, decontamination effectiveness using Turco was $63-92 \%$, with a DF of 2.7 to 12.5 on painted surfaces inside a hot cell. On unpainted carbon steel, it was 
75-90\% effective. Turco does not remove oxides, but it does remove lightly bonded contaminants. The contact time is too short to obtain DFs currently desired in a batch process (spraying or filling). One approach is to fill the component and then circulate the foam for as long as necessary. ${ }^{2}$ A spray system developed by TURCO is in use at Rockwell International. DFs are not applicable to mercury-contaminated surfaces.

Waste-The technology will produce small amounts of contaminated sulfonated detergents, synthetic wetting agents, coupling agents, rinse water, and drying cloth.

Cost-The technology itself is inexpensive, but development and scale-up costs will probably require significant financial support.

Science/Technology Needs: Full control over the mean bubble dimensions and the volume swell factor are needed, indicating that some basic research will be necessary.

Implementation Needs: Scale-up and development of the process to a size appropriate for $\mathrm{Y}-12$ Plant use is necessary. Following are estimated costs: development costs, $\$ 1 \mathrm{M}-4 \mathrm{M}$; capital costs, $<\$ 50 \mathrm{~K}$; operating costs, $\$ 0.50-\$ 2 / \mathrm{ft}^{2}$.

Author: D. E. Beck/615-574-0706. Reviewed by: K. E. Lott/615-574-6537

\section{References:}

1. J. M. Harris, J. R. Miller, R S. Freezer, J. H. Walter, "Foam Process for Application of Decontaminating Agents," from the International Conference on Decontamination of Nuclear Facilities, Niagara Falls, Canada, September 19, 1982.

2. J. R. Costes, J. P. Gouchon, M. Montjoie (Commissariat à l'Energie Atomique, France) "Recent Developments in Gel and Foam Decontamination Processes Applied to Decommissioning," Proceedings of the Nuclear and Hazardous Waste Management International Topical Meeting (Spectrum '88), pp. 24-33, Pasco, Wash., Sept. 11-15, 1988, ANS, LaGrange Park, Ill., September 1988.

September 1994

Decontamination and Decommissioning 
EM Problem: Decontamination and Decommissioning

Y-12 Plant Problem: Building 9201-4 (Alpha-4)

Problem Area/Constituents: Nickel and steel process equipment contaminated with mercury, lithium hydroxide, sulfuric acid, asbestos, and PCBs. Painted concrete and clay tile contaminated with $\mathrm{PCBs}$, oil, $\mathrm{U}$ (and daughters), electrolytes, $\mathrm{Cd}, \mathrm{Pb}$, and $\mathrm{Cr}$

Reference Requirements: Refer to Vol. 1, Chap. 10, for potentially applicable proposed and promulgated environmental laws, signed, and pending agreements for the Oak Ridge Reservation, radiation protection standards, DOE orders, and nonregulatory guidance. As site- and waste-specific characteristics are provided for each technology, specific regulatory requirements will be identified.

\section{Subelement: Decontamination}

Alternative: Chemical surface cleaning methods

Technology: Chemical gels. Used to remove smearable contamination from contacted surfaces

\section{Status: Demonstration.}

Efficacy/strengths/weaknesses-Chemical gels are most suited to in situ decontamination of large surfaces. ${ }^{2}$ Gel is used as a carrier of chemical decontamination agents, not as the agent itself. It is sprayed onto component walls; allowed to work; and then scrubbed, wiped, rinsed, or peeled off. Solutions with viscosities of $300-600 \mathrm{cps}$ form a reasonably stable film on the contaminated surface. An airless compressor can be used for spraying the gel and, with a change in heads, for rinsing. Typical reagent combinations are a nitric-hydrofluoric-oxalic acid mixture and a nonionic detergent mixed with a carboxymethylcellulose gelling agent, with aluminum nitrate used as a fluoride chelating agent. Steps include scraping and vacuuming of solid waste material, preliminary hot water rinsing, and gel spraying throughout the cell.

Advantages: Gel spraying is a good method for large component in situ removal of smearable contamination. It creates minimum secondary waste volumes, ${ }^{2}$ and remote application is readily done. Disadvantages: Gel spraying is a complex system that requires laboratory optimization for each change in variables. It is costly and time-consuming for decommissioning but possibly worthwhile for maintenance and cleaning operations. Reagent action is limited by the solution viscosity, which reduces the ion diffusion rate at the gel/surface interface. Amount of active reagents in gel film must be kept low $\left(<10 \mathrm{~g} / \mathrm{m}^{2}\right)$.

Effectiveness: The decontamination factor can be as high as $100 .^{1}$ In some cases, as many as three gel applications have been required (e.g., two acid and one base application). 
Waste-After spraying and rinsing the gel film two or three times, the volume of waste to be neutralized was four or five times less than that for decontamination of the same item by chemical solutions such as nitric acid. Treatment of the acidic and basic wastes can be by phosphate precipitation, sulfate precipitation, simple neutralization, or neutralization and addition of preformed nickel ferrocyanide precipitate. ${ }^{1}$

Cost—capital cost, $<\$ 50,000$; operating cost, $\$ 0.50-\$ 2.00 / \mathrm{ft}^{2}$

Science/Technology Needs: Complex gel formulation with a number of compounds may be required, depending on the objectives. With any change in variables, laboratory optimization will be necessary. Development costs may be $\$ 1 \mathrm{M}-\$ 4 \mathrm{M}$.

Implementation Needs: N/A

Author: D. E. Beck/615-574-0706. Reviewed by K. E. Lott/615-574-6537.

\section{References:}

1. J. M. Harris, J. R. Miller, R S. Frazier, and J. H. Walter, "Foam Process for Application of Decontaminating Agents," from the International Conference on Decontamination of Nuclear Facilities, Niagara Falls, Canada, September 19, 1982.

2. J. R. Costes, J. P. Gouchon, and M. Montjoie (Commissariat à l'Energie Atomique, France) "Recent Developments in Gel and Foam Decontamination Processes Applied to Decommissioning," Proceedings of the Nuclear and Hazardous Waste Management International Topical Meeting (Spectrum '88), pp. 24-29, Pasco, Wash., Sept. 11-15, 1988, ANS, LaGrange Park, Ill., September 1988.

September 1994

Decontamination and Decommissioning 
EM Problem: Decontamination and Decommissioning

Y-12 Plant Problem: Building 9201-4 (Alpha-4)

Problem Area/Constituents: Painted concrete and clay tile contaminated with PCBs, oil, $\mathrm{U}$ (and daughters), electrolytes, $\mathrm{Cd}, \mathrm{Pb}$, and $\mathrm{Cr}$

Reference Requirements: Refer to Vol. 1, Chap. 10, for potentially applicable proposed and promulgated environmental laws, signed and pending agreements for the Oak Ridge Reservation, radiation protection standards, DOE orders, and nonregulatory guidance. As site- and waste-specific characteristics are provided for each technology, specific regulatory requirements will be identified.

\section{Subelement: Decontamination}

Alternative: Chemical surface cleaning (fixed and transferable contamination) methods

Technology: Organic acid treatments

Status: Demonstrated. The technology is currently in use by BNFL at Capenhurst Gaseous Diffusion Plant. BNFL uses citric and sulfuric acids in sequence for removal of uranium (U) and technetium (TC), respectively, from metal surfaces (mostly wrought aluminum). Citric acid portion is dilute ("soft"), with correspondingly low corrosion. The carboxylate ion is strongly complexing. The ability to remove other radionuclides has not been demonstrated.

Efficacy/strengths/weaknesses

Advantages: Demonstrated effectiveness in decontamination of GDP facility. BNFL is willing to market the technology. Limitations: If uranium recovery is required, citric acid may be inappropriate. Citric tends to prevent $U$ precipitation. Although the BNFL process works well on wrought aluminum, it may have only limited success with cast $\mathrm{Al} .^{{ }^{1}}$ No data on radionuclides other than that of $\mathrm{U}$ or $\mathrm{TC}$.

Effectiveness: BNFL process achieved surface decontamination factor of 300 for alpha and 40 for Tc beta. ${ }^{2}$ Decontaminated to below British standards for release as scrap: $11.1 \mathrm{~Bq} / \mathrm{g}$ alpha and $0.4 \mathrm{~Bq} / \mathrm{g}$ beta. The high enrichment portion of Capenhurst decontamination was completed in 1985. Recycled to metals market were 1000 tons Al, 1700 tons steel, and 350 tons other metals. ${ }^{3}$ The low-enrichment portion of the facility is now in the process of decontamination.

Waste-Secondary wastes are (1) citric acid containing metal ions, especially $U$, and (2) sulfuric acid containing metal ions. Citric acid is relatively benign to the environment, biodegradable, and treatable by ion exchange removal of radionuclides and other metal ions. Remaining effluent may be treatable at sewage treatment plant. Sulfuric acid is also treatable by ion exchange with possible recycle of cleaned acid. Tertiary wastes are depleted ion 
exchange resins. NOTE: SEG and possibly other companies compact and solidify ion exchange resin wastes on contract, or they will provide complete ion exchange services.

Cost-Total net costs for the Capenhurst decontamination project are expected to be around one hundred million pounds. Four years ago, BNFL's operating cost was approximately $\$ 300 /$ metric ton. Ours would rate higher because of more sophisticated effluent treatment needs. Capital costs will include spray booth and tank installation with fluid recirculation systems, but costs will not be significantly higher for the BNFL process than for any other proven acid dissolution decontamination system.

Science/Technology Needs: Technology development needs: Pilot plant development for waste treatments (ion exchange columns) to verify ability to meet standards and regulations. More fundamental studies are needed in secondary waste disposal. ${ }^{4}$

Implementation Needs: Development. The financial resources necessary for purchase of technology, regulatory compliance, pilot-scale decontamination, and waste treatment demonstrations. For personnel, a Ph.D. and/or an engineer to direct development. Cost $\$ 400 \mathrm{~K}-\$ 1000 \mathrm{~K}$. Implementation: The financial resources necessary for construction of facility, environmental compliance, and procedural requirements. Hardware: No present Y-12 facility could be adapted for citric acid cleaning. BNFL's expertise may reduce the amount of original research, paperwork, etc., necessary to begin implementation. ${ }^{2}$ In general, decontamination with BNFL's citric acid process should pose no undue difficulties. It may well be less costly to implement than the more familiar nitric acid process would be due to the relative nontoxicity and ease of treating secondary waste stream. Capital costs would be $\$ 4 \mathrm{M}-\$ 10 \mathrm{M}$ if new equipment is required; operating costs would be $\$ 300 / 2200 \mathrm{lb}$ (BNFL estimatel.

Author: D. E. Beck/615-574-0706. Reviewed by: K. E. Lott/615-574-6537

\section{References:}

1. John Barber, John Kennerly, and others of Martin Marietta Energy Systems, personal communication to D. E. Beck, May-June 1992.

2. BNFL, personal communication to D. E. Beck, July 20, 1992.

3. D. W. Clements and G. H. C. Begg, "Decommissioning Britain's Gaseous Diffusion Plant-With Special Reference to Volume Reduction Techniques," Proc. Inst. Mech. Eng., IMechE Conference, pp. 179-88 (1992).

4. G. D. Del Cul, P. E. Osborne, and D. E. Beck, Evaluation of Alternatives for Best Available Technology Treatment and Retreatment of Uranium-Contaminated Wastewater, K/QT-394, Part 1, January 1991.

September 1994

Decontamination and Decommissioning 


\section{EM Problem: Decontamination and Decommissioning}

\section{Y-12 Plant Problem: Building 9201-4 (Alpha-4)}

Problem Area/Constituents: Nickel and steel process equipment contaminated with mercury, lithium hydroxide, sulfuric acid, asbestos, and PCBs.

Reference Requirements: Refer to Vol. 1, Chap. 10, for potentially applicable proposed and promulgated environmental laws, signed and pending agreements for the Oak Ridge Reservation, radiation protection standards, DOE orders, and nonregulatory guidance. As site- and waste-specific characteristics are provided for each technology, specific regulatory requirements will be identified.

\section{Subelement: Decontamination}

Alternative: Chemical surface cleaning (fixed and transferable contamination)

Technology: Fluoboric acid treatment. Decontamination and decommissioning (D\&D) using fluoboric acid $\left(\mathrm{HBF}_{4}\right)$ in dipping baths, loop systems, or by spraying

Status: Demonstration. Technology was designed specifically for D\&D. ${ }^{1}$ Attacks nearly every metal surface and metallic oxide. Removes oxide and contaminated outer layer in controllable, uniform, and efficient manner. Unrestricted use of materials should be possible after decontamination because of a high decontamination factor (DF); however, corrosion is high (DF is a function of the depth of metal removed).

Efficacy/strengths/weaknesses-The reaction is heterogeneous and proceeds as follows:

1. $\mathrm{nHBF}_{4}+\mathrm{Me}=\mathrm{Me}\left(\mathrm{BF}_{4}\right) \mathrm{n}+\mathrm{n} / 2 \mathrm{H}_{2}$

2. $\mathrm{nHBF}_{4}+$ metallic oxide $=\mathrm{Me}\left(\mathrm{BF}_{4}\right) \mathrm{n}+$ water

Fluoboric (or fluosilicic) acid can also scabble and clean cement and brickwork. If it is contaminated to a depth of only a few $\mathrm{mm}$, concrete flooring and structures can be returned to unrestricted use. ${ }^{1}$ Fluoboric acid exhibits low-vapor pressure and requires only the standard precautions for acid or base work.

Waste-The fluoboric acid technology generates metal waste as a result of surface removal. About $1 \mathrm{~g}$ of this waste is generated per meter ${ }^{2}$ per micron depth of bare metal removal. The acid can be electrolytically regenerated and recycled, with the radioactive waste plated out at the cathode and solidified in cement. Final quantity of cement-solidified waste is 20-50 $\mathrm{g} / \mathrm{m}^{2}$ decontaminated metal. Other waste treatment options are neutralization and precipitation with solidification in cement $\left(200-500 \mathrm{~g} / \mathrm{m}^{2}\right.$ or treatment by ion exchange followed by solidification of the resin in cement $\left(400-700 \mathrm{~g} / \mathrm{m}^{2}\right)$. The optimum material for solidification of this waste needs to be determined.

Cost-Capital costs in 1990 to set up a plant for decontaminating 5 tons/d of steel was $\$ 1.3 \mathrm{M}$ (\$774K excluding development, planning and buildings). ${ }^{2}$ Operating costs will be 
relatively low; this is a very simple process. The plant can be manufactured in modular form ${ }^{2}$ and installed in existing buildings.

Science/Technology Needs: Depends to a certain extent on similarities between $\mathrm{Y}-12$ and Chernobyl operations. ${ }^{2}$ Results of the technology usage at Chernobyl may answer many questions for us.

Implementation Needs: Hardware (equipment): fabrication of all key plant parts from polypropylene or from Halar-coated metal. Development cost will be about \$1M.

Author: D. E. Beck/615-574-0706. Reviewed by K. E. Lott/615-574-6537.

\section{References:}

1. J. D. Hanulik and W. T. Rippin, "Chemical Decontamination for Decommissioning with Application of DECOHA Technology on Metal, Concrete, and Brickwork," Proc. Ins. Mech. Eng. Nucl. Decom '92, Decommissioning of Radioactive Facilities International Conference, London, England, February 17-19, 1992.

2. H. W. Beaujean, J. Fiala-Goldiger, and J. Hanulik, "DECOHA at Chernobyl," Nuclear Engineering International, April 30, 1991. 


\section{EM Problem: Decontamination and Decommissioning}

Y-12 Plant Problem: Building 9201-4 (Alpha-4)

Problem Area/Constituents: Nickel and steel process equipment contaminated with mercury, lithium hydroxide, sulfuric acid, nitric acid asbestos, and PCBs. Painted concrete and clay tile contaminated with PCBs, oil, $\mathrm{U}$ (and daughters), electrolytes, $\mathrm{Cd}, \mathrm{Pb}$, and $\mathrm{Cr}$.

Reference Requirements: Refer to Vol. 1, Chap. 10, for potentially applicable proposed and promulgated environmental laws, signed and pending agreements for the Oak Ridge Reservation, radiation protection standards, DOE orders, and nonregulatory guidance. As site- and waste-specific characteristics are provided for each technology, specific regulatory requirements will be identified.

\section{Subelement: Decontamination}

Alternative: Chemical surface cleaning (fixed and transferable contamination)

Technology: Inorganic acid treatments. The removal of tightly adhered contamination using nitric acid solutions in spray booth or dipping decontamination processes. Use of other inorganic acids.

Status: Accepted. Nitric acid decontamination has been the preferred method at Y-12 for cleaning items in various facility operations since the 1940 s. In addition, the technique has been widely used at building K-1420 at the K-25 Site and at Portsmouth's X-705 facility. Generally, all DOE sites decontaminate for maintenance but not for release. Concentrated $(\mathrm{pH}<2)$ solution with concurrent corrosion; however, additives ( $1 \%$ urea) and modifications can greatly reduce corrosion. Decontamination factor (DF) for concentrated acid treatment is in the 100 range. ${ }^{2}$

Efficacy/strengths/weaknesses-Advantages/effectiveness: Excellent DFs, but secondary waste requires treatment and disposal as an RCRA waste.

Waste-Large quantities of corrosive nitrates are produced. Difficult and expensive to treat by today's standards; however, Portsmouth has built a biodenitrification facility which meets National Pollutant Discharge Elimination System outfall standards. Prior to biodenitrification, waste treatment would likely involve removal of radionuclides by neutralization/precipitation. Waste is presently evaporated and the concentrate stored, with the distillate treated at the West End Treatment Facility and Central Pollution Control Facility prior to discharge.

Cost-Nitric acid decontamination was used extensively at most DOE plants, but data is not avallable regarding costs. Operating costs should be less than $\$ 1 / \mathrm{ft}^{2}$, excluding waste treatment costs. Currently, Portsmouth plant is developing a data base to track costs, ${ }^{3}$ but it will not contain historical data. 
Although nitric acid is by far the most common, sulfuric acid has been used in decontamination, notably to dissolve nickel-plating prior to removal of technetium at the nickel-steel interface. ${ }^{5}$ Other inorganic acids, such as hydrochloric, are rarely mentioned in the literature. The exception is fluoboric acid, for which a separate input sheet is written.

Science/Technology Needs: Adaptations of modifications to system (e.g., scrubbers, filters, treatment for nitrates and heavy metals) are needed to meet regulatory requirements. ${ }^{6}$ Possible development of nitric acid/hydrogen fluoride decontamination methods with or without ultrasonic agitation. ${ }^{7,8}$ Continuing research and development, waste treatment, and volume reduction are needed. Possible research and development in control of base metal dissolution.

Implementation Needs: Nitrate treatment facility either on site or accessible to decontamination facility. Support personnel to sample, analyze, develop treatments, and ensure compliance with RCRA regulations will be necessary; thus, operating costs may be relatively high. If a waste treatment plant were required solely for this process, capital costs could be as high as $\$ 4 \mathrm{M}-\$ 10 \mathrm{M}$.

Author: D. E. Beck/615-574-0706. Reviewed by: K. E. Lott/615-574-6537

\section{References:}

1. J. C. Bailey, J. Dykstra, and R. A. Joseph, Review and Appraisal of K-1420 Decontamination Uranium Recovery (U), K/D-5819, Martin Marietta Energy Systems, Inc., Engineering Division, Oak Ridge Gaseous Diffusion Plant, April 1989.

2. X. H. Miyamaru, H. Mizuno, and Y. Imal, Cost Benefit Study of Chemical Decontamination presented at Decontamination of Nuclear Facilities, International Joint Topical Meeting, ANS-CNA, Niagara Falls, Canada. Sept. 19-22, 1982.

3. A. J. Saraceno, Portsmouth Gaseous Diffusion Plant. personal communication to D. E. Beck, April 29, 1992.

4. G. D. Del Cul, P. E. Osborne, and D. E. Beck, Evaluation of Alternatives for Best Available Technology and Treatment of Uranium Contaminated Solutions, K/QR-394, Oak Ridge K-25 Site. April 1991.

5. G. H. Goodall and B. E. Gillespie, "Decontamination of Surfaces", (BNFL) Patent GB 2191329 A, December 1987.

6. Personal opinion, based on J. C. Bailey et al., Review and Appraisal of K-1420 Decontamination - Uranium Recovery (U), K/D-5819, Martin Marietta Energy Systems, Inc., Engineering Division, Oak Ridge Gaseous Diffusion Plant. April 1989.

7. S. Buck and A. Colquhoun, "Decommissioning of a Mixed Oxide Fuel Fabrication Facility," (BNFL), eds., Pflugrad et al., International Conference on Decommissioning of Nuclear Installations, (Brussels, Oct. 24-27, 1989), CONF-891077-EUR-12690, Barking (UK) Elsevier Applied Science, 1990.

8. Allen, Schneider, Konzek, and Smith, "Decontamination Technology Assessment," Proceedings of the International Topical Meeting for Nuclear and Hazardous Waste Management Spectrum '88, pp. 511-513, ANS, La Grange Park, Ill., September 1988. 
EM Problem: Decommissioning

Y-12 Problem: Building 9201-4 (Alpha-4)

Problem Area/Constituents: Nickel and steel process equipment contaminated with mercury, lithium hydroxide, sulfuric acid, nitric acid, asbestos, and PCBs. Painted concrete and clay tile contaminated with $\mathrm{PCBs}$, oil, $\mathrm{U}$ (and daughters), electrolytes, $\mathrm{Cd}, \mathrm{Pb}$, and $\mathrm{Cr}$.

Reference Requirements: Refer to Vol. 1, Chap. 10, for potentially applicable proposed and promulgated environmental laws, signed and pending agreements for the Oak Ridge Reservation, radiation protection standards, DOE orders, and nonregulatory guidance. As site- and waste-specific characteristics are provided for each technology, specific regulatory requirements will be specified.

\section{Subelement: Decontamination}

Alternative: Chemical Surface Cleaning (fixed and transferable contamination)

Technology: The use of alkaline sodium carbonate, ammonium carbonate, sodium EDTA, sodium citrate, trisodium phosphate other sequestering agents, and detergents for surface washing and removal of particulates; the use of alkaline permanganate to oxidize chromium in high-Cr oxides (preconditioning the oxide for further treatment).

Status: Accepted/demonstrated. Surface smearable decontamination with caustic chemicals (essentially soap and water scrubbing) is accepted technology at Y-12 and other DOE sites. ${ }^{2}$ Using alkaline potassium permanganate to dissolve high-chromium oxides by oxidizing the chrome content in Pressurized Water Reactors (PWRs) has been demonstrated. ${ }^{3}$ The latter may or may not be relevant to ORNL needs; the need for dissolution of high-Cr oxides appears unique to PWRs in the commercial sector. The caustic hand-scrubbing process was applied to cleaning small items.

Efficacy/strengths/weaknesses-Effectiveness: Decontamination factors (DFs) for removal of smearable contaminants are also not available; however.

Waste-Moderate quantities of contaminated caustic solutions are produced. Simple neutralization and precipitation has been the traditional treatment. The sludge produced could be solidified and the effluent sent to the Central Pollution Control Facility.

Cost-Caustic decontamination is used extensively at $\mathrm{Y}-12$, in conjunction with nitric acid, but, at this time cost information is not available. Capital costs could be minimal, but scrubbing with caustic solutions is labor intensive.

Science/Technology Needs: None for removal of smearable contamination with carbonates, soaps, etc. ${ }^{2}$ 
Implementation Needs: This is an established process that could be implemented at Y-12 with no major changes. Capital costs $\sim<\$ 10 \mathrm{~K}$; operating costs $\sim>\$ 1 / \mathrm{ft}$ unless a riding scrubber is used.

Author: K. E. Lott/615-574-6537

\section{References:}

1. G. D. Conner, the K-25 facility, June 16, 1992, and J. M. Urik the X-705 facility (Portsmouth), June 16, 1992, personal communication to D. E. Beck.

2. SOP 1410.20, "Large Parts Decontamination ('C' Floor Plan)," July 1989, and SOP 1410.21, "Small Parts Decontamination ('A' Floor Plan)," July 1989, Martin Marietta Energy Systems, Inc., Oak Ridge Gaseous Diffusion Plant Standard Operating Procedures.

3. T. Swan, M. G. Segal, W. J. Williams, M. E. Pick, LOMI Decontamination Reagents and Related Preoxidation Processes, EPRI-NP-5522M, Research Project 1329-1. Prepared by Central Electricity Generating Board, Berkeley Nuclear Laboratories, UK, for the Electric Power Research Institute, Palo Alto, December 1987. 


\section{EM Problem: Decontamination and Decommissioning}

\section{Y-12 Plant Problem: Building 9201-4 (Alpha-4)}

Problem Area/Constituents: Nickel and steel process equipment contaminated with mercury, lithium hydroxide, sulfuric acid, nitric acid, asbestos, and PCBs

Reference Requirements: Refer to Vol. 1, Chap. 10, for potentially applicable proposed and promulgated environmental laws, signed and pending agreements for the Oak Ridge Reservation, radiation protection standards, DOE orders, and nonregulatory guidance. As site- and waste-specific characteristics are provided for each technology, specific regulatory requirements will be specified.

\section{Subelement: Decontamination}

Alternative: Chemical surface cleaning (fixed and transferable contamination)

Technology: Redox treatments. Various "designer" combinations, which include the following: (1) AP-Citrox-A two-step process using an alkaline permanganate oxidizing step followed by a citric acid-oxalic acid reducing step. (2) LOMI (low-oxidation-state metal ion)a one-step process that uses vanadous picolinate solution to reduce ferric ion to ferrous (using vanadium ion) and subsequent chelation with picolinate. (3) Ceric acid-solutions of cerium IV in inorganic acids (nitric, sulfuric, sulfamic, etc.); several different formulations, but all appear to work by oxidization with Ce followed by complex formation with na inorganic acid to keep metals in solution.

Status: Demonstrated. Nuclear power plant decontamination. Predemonstration, gaseous diffusion plant and fluoride film decontamination.

Efficacy/strengths/weaknesses-Citrox: A mixture of oxalic and citric acids (Turco 4521 is a Citrox reagent) has been used since the early 1980 s to decontaminate boiling water reactors (BWRs). Its decontamination factors (DFs) ranged from 3 to 17 in a 1984 series of tests. ${ }^{1}$ P-Citrox (Citrox with preoxidation by alkaline permanganate) was developed for Boiling and Pressurized Water Reactors (BWRs and PWRs), oxides of which have a high percentage of chrome. The purpose of the AP step is to oxidize and remove chrome from the high-chromium $(>20 \%)$ oxide film prior to completion by citric and oxalic acids. Addition of preoxidation step to Citrox process increases the DF by a factor of 10 to 100 , for high-chrome oxides.

Disadvantage: There can be intergranular attack and stress corrosion cracking on steel when oxalic acid is present in the decontamination solution.

LOMI: This process (for low oxidation-state metal ion) is a noncorrosive process using a vanadous picolinate solution to rapidly reduce iron-based oxides. LOMI was developed in response to the oxalic acid corrosion problems mentioned above and has been used for decontamination of BWRs and PWRs since the mid-1980s. It is often combined with a 
preoxidation step, making it a process of two or more steps using acidic or alkaline permanganate or both. Multistep processes may be NP-LOMI (nitric acid/permanganate), AP-LOMI (alkaline permanganate), or AP/NP/LOMI, depending on the substrate. Other LOMI combinations can be tailored, such as LOMI-NP-LOMI, etc., to obtain the optimum decontamination. ${ }^{2}$

Advantage: An advantage to LOMI is that it has proven to be unaffected by the incorporation of nickel in the oxide lattice, which can make dissolution of iron-based oxides by simple complexing acids difficult. ${ }^{3}$

Disadvantages: Possible storage problems; both permanganate and LOMI solutions are thermodynamically unstable when exposed to water. Tailoring is essential for efficient decontamination; not only from system to system, but sometimes within the system (e.g., chromium content in the oxide can vary from location to location within a piece of equipment).

Effectiveness: The overall decontamination factor (DF) at Monticello recirculation system was 22.9 (removal of $96 \%$ of the contamination), using LOMI. On high-chrome equipment, the DF was 3.6 (removal of $72 \%$ ). LOMI was less effective than a Citrox process in decontaminating a TMI-2 reactor cooling system. ${ }^{4}$.

Cerium solutions in acid: These solutions (e.g., cerium nitrate) are the most recent developments in REDOX decontamination. They produce a high DF, are excellent for use on complex configurations, and produce a resolution rate higher than electropolishing. ${ }^{8} \mathrm{Cerium}$ oxidation is also called promising for TRU decontamination. ${ }^{7}$ At least one of the ceric acid methods, using tetrasulfato ceric acid, is patented. ${ }^{8}$

Advantage: Ceric acid solutions eliminate the need for separate oxidizing and decontaminating solutions. Wide applicability to complex configurations. Dilute (soft) processing and temperatures as low as $70^{\circ} \mathrm{C}$ help keep corrosion rates low.

Disadvantage: New technology, requiring more development. Must be tailored to substrate and oxide film.

Effectiveness: A solution of ceric acid and inorganic acid was found to remove more than $97 \%$ of contamination in a reactor cooling system. ${ }^{9}$ For greater effectiveness, ceric acid cleaning may be followed by conventional decontamination (mixture of a chelate and an organic acid) and a final rinse with deionized water.

Waste-Citrox produces largest quantity ion-exchange resin waste of the three technologies discussed. LOMI-Volume of secondary waste generated is about one-half that of the Citrox process. It does contain relatively high metal concentrations which could be precipitated out before passing the remaining waste through ion-exchange columns. A cation resin from DOW and an anion resin from Rohm \& Haas are effective in cleaning the waste stream. "Cleanup by ion-exchange is straightforward and very efficient." However, disposal of ion-exchange resins is becoming a problem. Cerium solutions in acid-The spent ceric acid solution can 
be cleaned with a mixed anion-cation exchange resin and reused, or neutralized, evaporated, and disposed of as solid waste. In order to recycle, the cell can be oxidized back to CeIV by oxidizing with ozone or peroxide. Subsequent passage through ion exchange columns will remove most radioactive metal ions. Uranyl and plutonyl will pass through and can then be recovered by solvent extraction or more ion exchange. Disposal of ion-exchange resins or solidified wastes may present a problem. Another option is for electrolytic regeneration of the spent ceric acid solution.

Cost-Citrox: Operating costs are relatively high, especially for a two-step process.

LOMI: No hard cost data have been collected, but both capital and operating costs are probably higher than for Citrox. Cerium solutions in acid: Cerium solutions are nearly as effective as permanganate solutions for oxidation. However, cerium solutions are more expensive. Research and development are necessary for application of the process to Y-12 equipment.

Science/Technology Needs: All REDOX techniques will require demonstration to determine their usefulness in decontaminating facilities in Building 9201-4 and applicability to mercury. It is estimated that development costs would be about $\$ 1 \mathrm{M}$ to bring a REDOX decontamination development through bench-scale and demonstration phases.

Implementation Needs: Capital cost $>\$ 1 \mathrm{M}$; operating cost $>\$ / \mathrm{ft}^{2}$.

Author: D. E. Beck/615-574-0706. Reviewed by K. E. Lott/615-574-6537.

\section{References:}

1. R. Sjoblom, P. M. Olson, J. M. Parke, and D. Schneidmiller, Postaccident Chernobyl Decontamination: Method Development, DPRI-NP-4999, Research Project 2012-8, prepared by Pacific Nuclear Services for the Electric Power Research Institute, Palo Alto, Calif., January 1987.

2. T. Swan, M. G. Segal, W. J. Williams, and M. E. Pick, LOMI Decontamination Reagents and Related Preoxidation Processes, EPRI-NP-5522M, Research Project 1329-1, prepared by Central Electricity Generating Board, Berkeley Nuclear Laboratories, UK, for the Electric Power Research Institute, Palo Alto, Calif., December 1987.

3. C. J. Wood, "Chemical Decontamination Techniques," eds., A. A. Moghissi, H. W. Godbee, and S. A. Hobart, Radioactive Waste Technology, ASME, New York, NY.

4. R. Sjoblom et al., Ibid.

7. A. P. Murray, C. G. Slater, and R. W. White, Ceric Acid Decontamination of Nuclear Reactors, U. S. Patent 4,880,559. November 1989.

8. W. S. Bennett and A.L. Taboas, "Decontamination of Transuranically Contaminated Metallic Waste," in Proceedings of the 2nd U.S. Department of Energy Environmental Control Symposium, Vol. 2, Nuclear Energy, Conservation, and Solar Energy. Argonne National Laboratory, Ill., June 1980. 
9. A. P. Murray et al., "Ceric Acid Decontamination of Nuclear Reactors," U. S. Patent 4,880,559, Nov. 14, 1989. 
EM Problem: Decontamination and Decommissioning

Y-12 Plant Problem: Building 9201-4 (Alpha-4)

\section{Problem Area/Constituents:}

- In situ decontamination of the interior of steel and nickel process equipment contaminated with $\mathrm{Hg}, \mathrm{LiOH}, \mathrm{H}_{2} \mathrm{SO}_{4}$, and $\mathrm{HNO}_{3}$.

- In situ decontamination of the exterior of steel and nickel process equipment contaminated with $\mathrm{Hg}, \mathrm{LiOH}, \mathrm{H}_{2} \mathrm{SO}_{4}, \mathrm{HNO}_{3}$, asbestos, and PCBs.

- Ex situ decontamination of the exterior of steel and nickel process equipment contaminated with $\mathrm{Hg}, \mathrm{LiOh}, \mathrm{H}_{2} \mathrm{SO}_{4}, \mathrm{HNO}_{3}$, asbestos, and PCBs.

- Painted concrete and clay tile contaminated with PCBs, oil, U (and daughters, electrolytes, $\mathrm{Cd}, \mathrm{Pb}$, and $\mathrm{Cr}$.

- Air contaminated with Hg.

- Packing contaminated with $\mathrm{Hg}, \mathrm{LiOH}, \mathrm{H}_{2} \mathrm{SO}_{4}, \mathrm{HNO}_{3}$, and asbestos.

Reference Requirements: Refer to the Regulatory Compliance chapter of Vol. 1 for potentially applicable proposed and promulgated environmental laws, signed and pending agreements for the Oak Ridge Reservation, radiation protection standards, DOE orders, and nonregulatory guidance. As site- and waste-specific characteristics are provided for each technology, specific regulatory requirements will be identified.

\section{Subelement: Decontamination}

Alternative: Chemical surface cleaning methods

Technology: Electropolishing. A surface removal process that is traditionally applied to conductive metallic substrates. The electropolished area is defined by the path of the electric current (the path of lowest resistance) from electrode to substrate, hence a rough surface is smoothed because the "high" spots are closer to the electrode than the low spots. Cracks or crevices will not be electropolished until the surrounding "high" areas are removed. Traditionally applied as a portable or "localized" technology on small areas, the solvent and electricity are applied in some way to a localized spot. Could be applied as a "factory style" process in batch fashion to contaminated parts. Contaminated materials are fed into processing equipment, and cleaned material exits.

Status: Demonstration. Direct applicability must be demonstrated.

Efficacy/strengths/weaknesses-The amount of surface removed is proportional to factors such as current, time, and voltage. These factors can be increased so that the entire surface is removed. For smooth contaminated materials, the level of decontamination is dependent on transport of electropolishing products away from the cleaned surface, and in practice levels of zero added contamination can be achieved. 
Limitations: Electropolishing is essentially a "line-of-sight" process, so cracks, crevices, areas out of sight of, or shadowed by, the electrode will not be effectively decontaminated. Contaminated electrolytes must be removed for complete decontamination to be achieved. Electropolishing is difficult to apply to in situ equipment.

Waste-A contaminated electrolyte solution is a product of this process.

Science/Technology Needs: Primary and secondary waste treatment and solution recycle need to be developed. Cleanup principles (e.g., ion exchange and filtering) are well established, so that only design and demonstration are needed.

Implementation Needs: Development costs, $\sim \$ 1 \mathrm{M}$; operating costs, $>\$ 2.00 / \mathrm{ft}^{2}$, with capital costs of $\$ 1 M$.

Author: R. L. Fellows/615-576-5632. Reviewed by R. B. Alderfer/615-576-0225

References: MMES staff members 
EM Problem: Decontamination and Decommissioning

Y-12 Problem: Building 9201-4 (Alpha-4)

\section{Problem Area/Constituents:}

- In situ decontamination of the interior of steel and nickel process equipment contaminated with $\mathrm{Hg}, \mathrm{LiOH}, \mathrm{H}_{2} \mathrm{SO}_{4}$, and $\mathrm{HNO}_{3}$.

- In situ decontamination of the exterior of steel and nickel process equipment contaminated with $\mathrm{Hg}, \mathrm{LiOH}, \mathrm{H}_{2} \mathrm{SO}_{4}, \mathrm{HNO}_{3}$, asbestos, and PCBs.

- Ex situ decontamination of steel and nickel process equipment contaminated with $\mathrm{Hg}$, $\mathrm{LiOH}, \mathrm{H}_{2} \mathrm{SO}_{4}, \mathrm{HNO}_{3}$, asbestos and PCBs.

- Painted concrete and clay tile contaminated with PCBs, oil, U (and daughters), electrolytes, $\mathrm{Cd}, \mathrm{Pb}$, and $\mathrm{Cr}$.

- Air contaminated with $\mathrm{Hg}$.

- Packing contaminated with $\mathrm{Hg}, \mathrm{LiOH}, \mathrm{H}_{2} \mathrm{SO}_{4}$, and $\mathrm{HNO}_{3}$.

- Carbon chunks contaminated with $\mathrm{Hg}, \mathrm{LiOH}, \mathrm{H}_{2} \mathrm{SO}_{4}$, and $\mathrm{HNO}_{3}$.

\section{Reference Requirements:}

\section{Subelement: Decontamination}

Alternative: Chemical surface cleaning methods

Technology: Amalgamation. Mercury readily forms stable alloys (amalgams) with numerous metals, but copper and zinc are perhaps the most practical metal candidates for forming amalgams on a large scale. This technology has been used to immobilize spills of elemental mercury by sprinkling powdered zinc over the mercury. The resulting zinc-mercury amalgam is solid and has low solubility. The solid amalgam can often be recovered more readily than the heavy liquid mercury metal that may run and seep deeper into cracks or porous substrates during attempts to remove it

Status: Predemonstration. Although amalgamation of mercury with the metals of interest is well known, application to the problem areas in the Alpha-4 building have not been demonstrated. This technology does not of itself remove the mercury but transforms elemental mercury to a form that is easier to recover by vacuuming or by other mechanical recovery methods. Since the amalgam is a much less pure form of mercury, this method may actually be undesirable in applications in which the mercury can be conveniently recovered for resell. (Presently, mercury once contaminated with radioactive elements can not be sold in the United States regardless of its purity.) The amalgam itself is the chief waste formed by the process. The amalgam may be suitable for ultimate disposal, but this assumption must be demonstrated especially in the case of a zinc-mercury amalgam, because zinc is a reactive metal. About 57 wt\% mercury is the maximum amount of mercury that would be suitable in a mercury-zinc amalgam. The maximum amount of mercury suitable for a mercury-copper 
amalgam is $<76 \mathrm{wt} \%$ mercury. ${ }^{1}$ This method is not expected to aid in recovery of mercury that is not in elemental form. This method therefore has limited applicability.

Science/Technology Needs: Technology Development Needs. An assessment must be conducted to determine the suitability of the process to the decontamination problems of interest and to determine the suitability of the amalgams as an ultimate disposal form for the mercury.

Implementation Needs: The technology development will require further lab-and pilot-scale evaluation. The hardware and facility needs are expected to be simple assuming that mercury can be handled in an existing laboratory. Further literature reviews, lab studies, and benchscale evaluations, if warranted, are roughly estimated at $\$ 500 \mathrm{~K}$, which includes equipment and personnel costs. The development project is expected to last about 8 months. An additional 4 months is roughly estimated for implementation of the process. The equipment needed for full-scale application is expected to be very similar to that used during the demonstration and is not expected to exceed $\$ 100 \mathrm{~K}$ in additional costs. These comparatively low costs are based on the assumption that the method simply consists of contacting the mercury with powdered metal followed by vacuuming the residual.

Author: E. B. Munday/615-576-5806

References:

1. A. H. Gorin and J. H. Leckey, "Find Disposal Options for Mercury/Uranium Mixed Wastes from Building 9201-4," Draft, Martin Marietta Energy Systems, Oak Ridge K-25 Site, October 1993. 
EM Problem: Decontamination and Decommissioning

Y-12 Plant Problem: Building 9201-4 (Alpha-4)

\section{Problem Area/Constituents:}

- In situ decontamination of the interior of steel and nickel process equipment contaminated with $\mathrm{Hg}, \mathrm{LiOH}, \mathrm{H}_{2} \mathrm{SO}_{4}$, and $\mathrm{HNO}_{3}$.

- In situ decontamination of the exterior of steel and nickel process equipment contaminated with $\mathrm{Hg}, \mathrm{LiOH}, \mathrm{H}_{2} \mathrm{SO}_{4}, \mathrm{HNO}_{3}$, asbestos, and PCBs.

- Ex situ decontamination of the exterior of steel and nickel process equipment contaminated with $\mathrm{Hg}$, $\mathrm{LiOh}, \mathrm{H}_{2} \mathrm{SO}_{4}, \mathrm{HNO}_{3}$, asbestos, and PCBs.

- Painted concrete and clay tile contaminated with PCBs, oil, U (and daughters), electrolytes, $\mathrm{Cd}, \mathrm{Pb}$, and $\mathrm{Cr}$.

- Packing contaminated with $\mathrm{Hg}, \mathrm{LiOH}, \mathrm{H}_{2} \mathrm{SO}_{4}$, and $\mathrm{HNO}_{3}$.

\section{Reference Requirements:}

- Federal Facility Compliance Agreement for Oak Ridge Site

- Federal Facility Compliance Agreement for PCBs

- Clean Air Act

- Toxic Substances Control Act

- DOE orders

Refer to the Regulatory Compliance chapter of Vol. 1 for potentially applicable proposed and promulgated environmental laws, signed and pending agreements for the Oak Ridge Reservation, radiation protection standards, DOE orders, and nonregulatory guidance. As site- and waste-specific characteristics are provided for each technology, specific regulatory requirements will be specified.

\section{Subelement: Decontamination}

Alternative: Chemical surface cleaning methods

Technology: Biological surface cleaning. Living organisms are used to clean or mobilize surface contamination.

Status: Evolving technology (preconceptual). A knowledge base exists for biological treatment of mercury and other contaminants; however, there is no database for application of the technology to surface decontamination. The technology is likely to work if methods can be developed to apply a layer of microbes to surfaces to be decontaminated, supply needed nutrients to the microbes, and finally remove the microbe layer from the decontaminated surface. The technology may possibly be applied in situations where other technologies cannot be used (e.g., microbes could be transported by water to contaminated internal surfaces of equipment that are inaccessible to other technologies). At this time, an estimate 
of the expected decontamination efficiency cannot be made. The waste generated would be the contaminated layer of microbes removed from the treated surface. An estimate of the quantity of waste cannot be made at this time.

Science/Technology Needs: A research project that investigates the feasibility of using biological treatment for decontamination of mercury from surfaces is needed.

Implementation Needs: Because of its preconceptual status, a reliable estimate of the capital and operating costs of the technology cannot be made at this time. The capital cost might be around $\$ 200 \mathrm{~K}$. The cost of a research and development project on surface decontamination by biological treatment would most likely range from $\$ 300 \mathrm{~K}-\$ 600 \mathrm{~K} / \mathrm{year}$ for at least two years.

Author: J. H. Wilson/615-576-4413. Reviewed by: R. D. Bundy/615-576-0192

References: MMES staff members. 
EM Problem: Decontamination and Decommissioning

Y-12 Plant Problem: Building 9201-4 (Alpha-4)

\section{Problem Area/Constituents:}

- In situ decontamination of the interior of steel and nickel process equipment contaminated with $\mathrm{Hg}, \mathrm{LiOH}, \mathrm{H}_{2} \mathrm{SO}_{4}$, and $\mathrm{HNO}_{3}$.

- In situ decontamination of the exterior of steel and nickel process equipment contaminated with $\mathrm{Hg}, \mathrm{LiOH}, \mathrm{H}_{2} \mathrm{SO}_{4}, \mathrm{HNO}_{3}$, asbestos, and PCBs.

- Ex situ decontamination of steel and nickel process equipment contaminated with $\mathrm{Hg}$, LiOH, $\mathrm{H}_{2} \mathrm{SO}_{4}, \mathrm{HNO}_{3}$, asbestos, and PCBs.

- Painted surfaces, $\mathrm{PCB}$, and oil on various substrates.

\section{Reference Requirements:}

- Federal Facility Compliance Agreement (e.g., FFCA for Oak Ridge Site)

- applicable federal and state laws and regulations (e.g., Clean Water Act and Toxic Substance Control Act)

- DOE orders

Refer to Vol. 1, Chap. 10, for potentially applicable proposed and promulgated environmental laws, signed and pending agreements for the Oak Ridge Reservation, radiation protection standards, DOE orders, and nonregulatory guidance. As site- and waste-specific characteristics are provided for each technology, specific regulatory requirements will be specified.

\section{Subelement: Decontamination}

Alternative: Chemical surface cleaning methods

Technology: Ultraviolet light (UV)/ozone. Oxygen molecules absorb UV to form ozone that dissociates to form atomic oxygen. Furthermore, the contaminant molecules are excited and/or dissociated by the absorption of UV. The excited contaminant molecules and the free radicals produced by dissociation of contaminant molecules react with atomic oxygen to form simpler, volatile molecules, such as carbon dioxide, water, and $\mathrm{N}_{2}$. UV highly activates mercury. This process could be applied either in a closed glovebox-like environment or could use ambient oxygen in air to produce ozone. In the latter case, a waste collection filtering system using HEPA filters would be implemented.

Status: Predemonstration. In the semiconductor industry UV/ozone surface-cleaning is an effective method of removing a variety of contaminants from surfaces. Representative contaminants including various oils such as human skin oils, cutting oil, and vacuum pump oil; solder fluxes; greases; and cleaning solvent residues such as acetone, ethanol, methanol, isopropyl alcohol, trichloroethane, and trichlorotrifluoroethane. In most instances UV/ozone 
surface-cleaning is a simple-to-use process that is inexpensive to set up and operate. Cleaning systems are available commercially primarily for the semiconductor industry. The method can produce clean surfaces in air or in a controlled atmosphere, at ambient to slightly elevated temperatures.

However, there are limitations to this cleaning method. Only those contaminants that will dissociate and react with atomic oxygen to form simpler, volatile molecules, such as carbon dioxide, water, and $\mathrm{N}_{2}$, are effectively removed. The distance of the surface from the UV source has to be minimized for best cleaning results. Inorganic contaminants such as dust, salts, and solid oxides cannot be removed by this method.

The specific application to decontamination is in the problem definition stage since UV/ozone cleaning technology exists and may have some applicability to cleaning tasks. The efficacy of this technology to the particular target areas/problem constituents is unknown at this time.

Waste-The UV/ozone cleaner operates with $\mathrm{O}_{2}$ and produces active ozone gas. No significant addition to the waste stream is expected because gas flow would be of the order of $10 \mathrm{ft}^{3} / \mathrm{min}$ or less. The existing waste will be converted to a different form (gaseous) and will be collected in a standard HEPA system. The operating gas (ozone) will most likely combine with the waste, forming some new materials. These new materials will also be in gaseous form and will also be handled with standard systems.

Cost-An operating UV/ozone system should have a capital cost of less than $\$ 100 \mathrm{~K}$, including exhaust gas handling. The equipment could be handled by a single person at less than $\$ 100 / \mathrm{h}$. The gas consumption would be around $10 \mathrm{ft}^{3} / \mathrm{min}$ with a cost of about $\$ 0.10 / \mathrm{ft}^{3}$. The electrical cost will be less than $\$ 60 / \mathrm{h}$, based on $100 \mathrm{~kW} @ \$ 0.06 \mathrm{~kW} / \mathrm{h}$. The cleaning ratio, which will be demonstrated in a proof-of-principle experiment, is unknown and depends heavily on the waste. The cost leading to an operating commercially produced system is expected to be about $\$ 1$ million.

Science/Technology Needs: The potential of the UV/ozone surface cleaning method to reduce organic contaminants to rather innocuous byproducts warrants further development. Especially, development of a safe, portable, efficient system is desirable. Techniques of combining this method and other efficient methods for removing contaminant metals, axides, etc., would be particularly advantageous and justifies further investigation. However, its efficacy in removing the specific organic and radioactive contaminants is not demonstrated. Using a commercial UV/ozone hardware, laboratory-scale experiments could be conducted to establish a data base of cleaning rates on removing organic contaminants from different substrates and to develop necessary techniques to handle the waste generated. Subsequently, the equipment would be incorporated with robotic, remote, and automatic operations with computer control.

Implementation Needs: Development activities: to address science needs, the approximate cost of equipment and staff time is $\$ 1 \mathrm{M}$ (capital equipment, $\$ 100 \mathrm{~K}$ ). For the above activities,

September 1994

Decontamination and Decommissioning 
the staff are required to be highly trained in the fields of plasma technology and photochemistry: one-half would be scientists at the M.S. and/or Ph.D. level, and one-half would be highly trained technicians at the B.S. level. A rough estimate of the operating cost is $\$ 3 / \mathrm{ft}^{2}$.

Author: W. L. Gardner/615-574-1221 and W. D. Nelson/615-574-0966

References: J. R. Vig, "UV/Ozone Cleaning of Surfaces, Semiconductor Cleaning Technology/1989," Electrochem. Soc. Proc., 90-9, 105-1 13 (1990). 


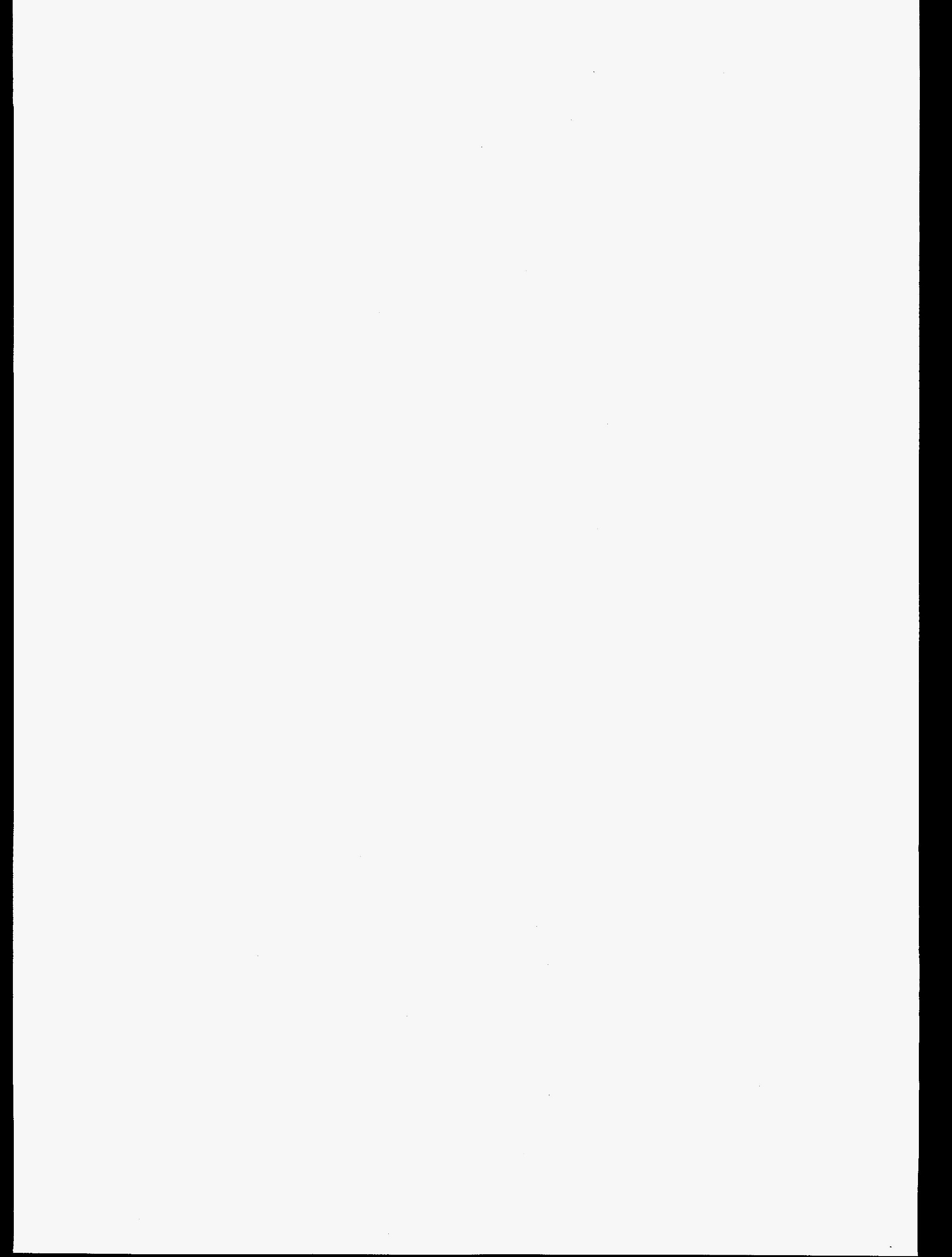




\section{EM Problem: Decontamination and Decommissioning}

\section{Y-12 Plant Problem: Building 9201-4 (Alpha-4)}

Problem Area/Constituents: Nickel and steel process equipment contaminated with mercury, lithium hydroxide, sulfuric acid, nutric acid, asbestos, and PCBs. Painted concrete and clay tile contaminated with PCBs, oil, $\mathrm{U}$ (and daughters), electrolytes, $\mathrm{Cd}, \mathrm{Pb}$, and $\mathrm{Cr}$. Packing contaminated with $\mathrm{Hg}, \mathrm{LiOH}, \mathrm{H}_{2} \mathrm{SO}_{4}$, and $\mathrm{HNO}_{3}$.

Reference Requirements: Refer to Vol. 1, Chap. 10, for potentially applicable proposed and promulgated environmental laws, signed and pending agreements for the Oak Ridge Reservation, radiation protection standards, DOE orders, and nonregulatory guidance. As site- and waste-specific characteristics are provided for each technology, specific regulatory requirements will be specified.

\section{Subelement: Decontamination}

\section{Alternative: Chelation treatment}

Technology: Chelation treatment. Selective removal of organic and inorganic compounds by chemical dissolution with organic chelating agents, such as carboxylic acids. The agent breaks the chemical bond between the contaminant ion and the substance it is attached to and forms a soluble complex. Additional reagents, such as surfactants and emulsifiers, can be added depending on the situation. The technology differs from solvent extraction in that an acid solution is not required (acts at or near pH-neutral). Both loose and fixed contamination can be removed using these agents with either batch or continuous process (with optional ultrasonic agitation), a spray booth, or through in situ application by wet-vac cleaning machine. Chelating agents can be destroyed in solution, producing a filterable sludge-containing contaminant. Waste products from chelate solution are nonhazardous, nonfuming, have no gas evolution, and are biodegradable.

Status: The technology has been employed at various facility operations and nuclear power plant sites, achieving acceptable decontamination levels resulting in unconditional release.

Waste produced-The technology minimizes waste through oxidative destruction of chelate agents, partitioning of organics (including $\mathrm{PCBS}$ ), and concentration of radionuclides precipitated out in the flocculent sludge, which can be further dewatered. Oxidation of chelating agents removes any potential for mixed classification from that source.

Overall cost- $<\$ 1 / \mathrm{ft}^{2}$ for large areas

Efficacy/strengths/weaknesses-Excellent decontamination factors. Easy to apply in situ, since the agents act at neutral pH and are nonhazardous, nonfuming, have no gas evolution, and are biodegradable. Worker safety is positively affected versus acids, solvents, and heavy equipment used for bulk extraction. Simple waste minimizing treatment and disposal as a non-RCRA waste. 
Science/Technology Needs: Current validation of updated chelating agents is underway at ORNL to confirm total efficacy and economic advantages. The development cost should be about $\$ 1.5 \mathrm{M}$.

Implementation Needs: Application equipment for in situ decontamination. Support personnel and equipment to sample, analyze, develop appropriate concentrations, ensure oxidation of organics, and/or complete partitioning will be needed. Spray booth or dipping tank facilities with heating capabilities are desirable on-site or accessible to the decontamination facility. Capital costs should be about $\$ 1.5$ million.

Author: J. R. DeVore/615-574-7092. Reviewed by K. E. Lott/615-574-6537

\section{References:}

1. Chelant Mixture Regeneration with Strong Acid Cation and Weak Base Anion Resin Mixtures, Nutek Canada Limited and Prime Energy Systems, Ltd., Downsview Ontario, 1977.

2. "Chemical Decontamination of Large Metal Objects," Bouchard and D'Muhala, September 1993 paper to be delivered, American Nuclear Society Global, Seattle.

3. "Ultrasonic Cleaning," Decontamination of Nuclear Reactors and Equipment, Ronald Press, ed., A. J. Ayers, Hanford Operations, USAEC, 1970.

4. "Ultrasonic Decontamination of Control Rod Drive Mechanisms," General Dynamics Corp., Electric Boat Division, 1963. 
EM Problem: Decontamination and Decommissioning

Y-12 Plant Problem: Building 9201-4 (Alpha-4)

Problem Area/Constituents:

- In situ decontamination of the interior of steel and nickel process equipment contaminated with $\mathrm{Hg}, \mathrm{LiOH}, \mathrm{H}_{2} \mathrm{SO}_{4}$, and $\mathrm{HNO}_{3}$.

- In situ decontamination of the exterior of steel and nickel process equipment contaminated with $\mathrm{Hg}, \mathrm{LiOH}, \mathrm{H}_{2} \mathrm{SO}_{4}, \mathrm{HNO}_{3}$, asbestos, and PCBs.

- Ex situ decontamination of steel and nickel process equipment contaminated with $\mathrm{Hg}$, $\mathrm{LiOH}, \mathrm{H}_{2} \mathrm{SO}_{4}, \mathrm{HNO}_{3}$, asbestos, and PCBs.

- Painted concrete and clay tile contaminated with PCBs, oil, U (and daughters), electrolytes, $\mathrm{Cd}, \mathrm{Pb}$, and $\mathrm{Cr}$.

- Air contaminated with $\mathrm{Hg}$.

- Packing contaminated with $\mathrm{Hg}, \mathrm{LiOH}, \mathrm{H}_{2} \mathrm{SO}_{4}$, and $\mathrm{HNO}_{3}$.

- Carbon chunks contaminated with $\mathrm{Hg}, \mathrm{LiOH}, \mathrm{H}_{2} \mathrm{SO}_{4}, \mathrm{HNO}_{3}, \mathrm{U}$ (and daughters), asbestos, and/or PCBs.

\section{Reference Requirements:}

- Federal Facility Compliance Agreements (e.g., FFCA for Oak Ridge Site and FFCAs for PCBs)

- Applicable federal and state laws and regulations (e.g., Clean Air Act and Toxic Substances Control Act)

- DOE orders

\section{Subelement: Decontamination}

Alternative: Chemical surface cleaning methods

Technology: Sulfide conversion. The concept is to contact elemental mercury with sulfur at room temperature to form mercury sulfide (HgS). The red hexagonal form of HgS is the most important mercury mineral found in the earth's crust from which mercury is recovered. ${ }^{1} \mathrm{HgS}$ is a desirable form because mercury will not leach from it and because it is a solid rather than a liquid. The solid compound can often be recovered more readily than the heavy liquid mercury metal that may run and seep deeper into cracks or porous substrates during attempts to remove it. The sulfur could be applied as a powder directly to the mercury; or alternately, the sulfur could be used to form a colloidal suspension in an aqueous solution that can be used to flush a contaminated object.

Status: Evolving Technology. The direct reaction of elemental mercury and elemental sulfur is thermodynamically favorable at room temperature, but experiments are needed to determine if the kinetics are suitable. ${ }^{1}$ This technology does not of itself remove the mercury contamination but transforms elemental mercury to a form that is easier to recover by 
vacuuming or by other mechanical recovery methods. Because the HgS is a much less pure form of mercury, this method may actually be undesirable in applications in which the mercury can be conveniently recovered for resell. (Presently, mercury once contaminated with radioactive elements can not be sold in the United States regardless of its purity.) The HgS may be suitable for ultimate disposal, because mercury is mixed from the earth in the hexagonal form of this compound. HgS is also a desirable form for fixing the mercury, since $84 \mathrm{wt} \%$ of the compound is mercury. This method is very similar in application and limitation to the amalgamation technology. This method has a limited application to elemental mercury.

Science/Technology Needs: Science and Technology Development Needs. Perhaps additional literature reviews will answer. The questions concerning the room temperature reaction of mercury and sulfur; otherwise, experiments must be conducted to evaluate the kinetics of the reaction. An assessment must be performed to determine the suitability of this process to the decontamination problems of interest and to determine the suitability of the HgS formed as an ultimate disposal matrix for the mercury.

Implementation Needs: The technology will require further lab-scale and bench-scale evaluation. The hardware and facility needs are expected to be single. Further literature reviews, lab studies and bench-scale evaluations, if warranted, one roughly estimated at $\$ 500 \mathrm{~K}$. The development project is expected to last about 8 months. An additional 4 months is roughly estimated for implementation of the process. The equipment needed for full-scale application is expected to be very similar to that used during the demonstration. This equipment is expected to cost an additional \$100K.

Author: E. B. Munday/615-576-5806

\section{Reference:}

1. A. H. Gorin and J. H. Leckey, "Final Disposal Options for Mercury/Uranium Mixed Wastes from Building 9201-4," Draft, Martin Marietta Energy Systems, Oak Ridge K-25 Site, October 1993.

September 1994

Decontamination and Decommissioning 
EM Problem: Decontamination and Decommissioning

Y-12 Plant Problem: Building 9201-4 (Alpha-4)

\section{Problem Area/Constituents:}

- Ex situ decontamination of steel and nickel process equipment contaminated with $\mathrm{Hg}$, $\mathrm{LiOH}, \mathrm{H}_{2} \mathrm{SO}_{4}, \mathrm{HNO}_{3}$, asbestos, and PCBs.

- Painted concrete and clay tile contaminated with PCBs, oil, U (and daughters), electrolytes, $\mathrm{Cd}, \mathrm{Pb}$, and $\mathrm{Cr}$.

\section{Reference Requirements:}

- Federal Facility Compliance Agreements (e.g., FFCA for Oak Ridge Site and FFCAs for PCBs)

- Applicable federal and state laws and regulations (e.g., Clean Air Act and Toxic Substances Control Act)

- DOE orders

Subelement: Decontamination

Alternative: Surface cleaning

Technology: Solutions of chemical bases and alkaline salts are used to clean the surface of contaminated items.

Status: Accepted. Cleaning with caustic solutions has long been used at $\mathrm{K}-25^{1}$ and Portsmouth. ${ }^{2}$ Operating cost, $\sim \$ 1 / \mathrm{ft}^{2}$.

Science/Technology Needs: None

Implementation Needs: A waste treatment system would be needed to treat or recycle spent decontamination solutions from this technology. Capital costs should be less than $\$ 100 \mathrm{~K}$.

Author: D. H. Bunch/615-576-0201

\section{References:}

1. Personal communication, G. D. Conner, K-25 Site, to D. E. Beck, June 16, 1992.

2. Personal communication, J. M. Urik, X705 Facility, Portsmouth Gaseous Diffusion Plant, to D. E. Beck, June 16, 1992. 


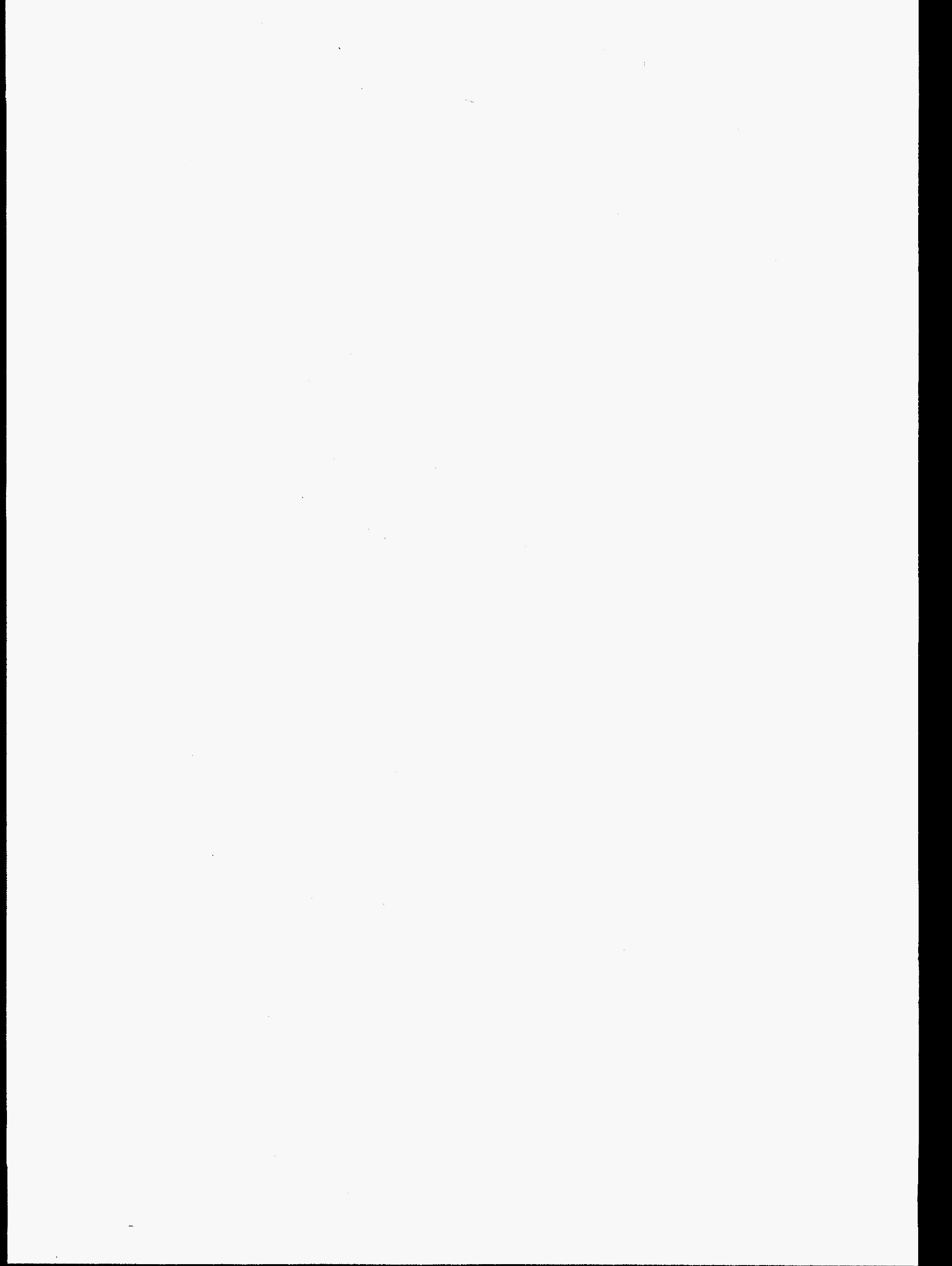




\section{EM Problem: Decontamination and Decommissioning}

\section{Y-12 Plant Problem: Building 9201-4 (Alpha-4)}

Problem Area/Constituents: Nickel and steel process equipment contaminated with mercury, lithium hydroxide, sulfuric acid, nitric acid, asbestos, and PCBs. Packing contaminated with $\mathrm{Hg}, \mathrm{LiOH}, \mathrm{H}_{2} \mathrm{SO}_{4}$, and $\mathrm{HNO}_{3}$.

Reference Requirements: Refer to Vol. 1, Chap. 10, for potentially applicable proposed and promulgated environmental laws, signed and pending agreements for the Oak Ridge Reservation, radiation protection standards, DOE orders, and nonregulatory guidance. As site- and waste-specific characteristics are provided for each technology, specific regulatory requirements will be specified.

\section{Subelement: Decontamination}

Alternative: Metal refining

Technology: Smelt purification. Purifies metals by adding suitable fluxes that will react with the impurities when the metal is melted. The impurities are then removed from the slag that separates from the molten metal and floats to the top. The items requiring smelting must be cut to suitable size for addition to the smelter.

Status: Demonstration. Smelt purification of radioactive metals has been performed by a large number of investigators on a lab-scale and by some investigators on a large-scale. The metals include mild steel, stainless steel, nickel, copper, monel, aluminum, and others. Metal purification has been demonstrated to levels of $0.01-4.0 \mathrm{ppm}$ residual uranium. Aluminum smelting was not as successful (3-100 ppm). Heshmatpour and Copeland ${ }^{1}$ report better results for aluminum (1-2 ppm). Technetium reportedly is not removed by smelt purification.

Waste produced-It has been estimated that about $50 \mathrm{lb}$ of slag would be generated per ton of metal smelted. No figures for off-gas treatment are available.

Cost-Scientific Ecology Group, Inc., personnel estimate ${ }^{2}$ the costs (operating and capital combined) of metal smelting at $\$ 0.65-\$ 2.00 / \mathrm{lb}$ depending on the type and configuration of the metal. Steel will probably cost $\$ 0.65-\$ 0.95 / 1 \mathrm{~b}$ to process. Kellogg et al. estimated that the capital and operating costs of smelting the 90,000 tons of DOE scrap metal presently on hand at the K-25 Site could be recovered through sale of the metal if a de minimis were established. Based on their conclusion, a cost of roughly $\$ 0.93 / 1 \mathrm{~b}$ of metal can be estimated in 1992 dollars. They assumed construction of a new facility with an 8-ton capacity, electric induction furnace capable of handling $\sim 6$ tons $/ \mathrm{h}$ ferrous or 1.6 tons/h nonferrous metals.

Science/Technology Needs: Investigation of fluxing agents and conditions that would permit purification of the metals used in process equipment in Building 9201-4 from the 
contaminants of interest should be investigated first in the laboratory and then on a larger scale. Development costs should be roughly $\$ 3.5 \mathrm{M}$.

Implementation Needs: The technology development needs will require further lab- and pilot-scale evaluation. The development costs will include a small electric induction furnace; two full-time technical people (one with experience in metallurgy); and analytical, maintenance, and other support personnel. The duration may be 24 months and the development costs are roughly estimated by the investigator at $\$ 3 \mathrm{M}-\$ 5 \mathrm{M}$.

The following requirements for a smelt facility are listed by Kellogg et al.: scrap handling and preparation; scrap transportation; scrap weighing and drying facility (the scrap must be heated prior to placing in the smelter to drive off oil and water); eight ton capacity electric induction furnace with two crucibles, mold preparation, and cooling; slag processing, refractory repair, flux storage and preparation; ingot dumping and storage; offices, laboratory, and control room; maintenance areas; and personnel convenience stations. Capital and operating costs were not indicated, but as mentioned above, an overall cost of roughly $\$ 0.93 / \mathrm{h}$ can be estimated based on their conclusions that all costs could be recovered from the sale of 90,000 tons of metal presently in the DOE inventory (valued at $\$ 102 \mathrm{M}$ in 1982 dollars) assuming a de minimis is established. About $74 \%$ of the metal value is the nickel, which amounts to only $11 \%$ by weight of the metals in the Kellogg study. Most likely no existing facility will satisfy the operational requirements of this facility (the Kellogg study assumes new facilities are constructed). The design and construction phase will require the support of numerous engineering disciplines: structural, site, electrical, instrument, and safety engineering, as well as engineering mechanics, process design, and metallurgy. Numerous support services will be required: health physics, industrial hygiene, health, safety, environmental, and analytical services. Numerous construction and maintenance craftsmen will be required: welders, carpenters, electricians, mechanics, ETI personneL and supervisors. These requirements cannot be quantified at present. The establishment of a de minimis standard is very critical for the success of this project since the resulting ingots cannot be sold without this legislation.

Author: E. B. Munday/615-576-5806. Reviewed by K. E. Lott/615-574-6537

\section{References:}

1. B. Heshmatpour and G. L. Copeland, The Effects of Slag Composition and Process Variables on Decontamination of Metallic Wastes by Melt Refining, Union Carbide Corporation, January 1981.

2. Scientific Ecology Group, Inc., personal communication to E. B. Munday, Martin Marietta Energy Systems, Inc., June 1992.

3. D. R Kellogg et al., Metal-Smelting Facility, CONF-82041-13, DE 82013539 , Union Carbide Corporation Nuclear Division, 1982.

September 1994

Decontamination and Decommissioning 
EM Problem: Decontamination and Decommissioning

Y-12 Plant Problem: Building 9201-4 (Alpha-4)

Problem Area/Constituents: Nickel and steel process equipment contaminated with mercury, lithium hydroxide, sulfuric acid, nitric acid, asbestos, and PCBs.

Reference Requirements: Refer to Vol. 1, Chap. 10, for potentially applicable proposed and promulgated environmental laws, signed and pending agreements for the Oak Ridge Reservation, radiation protection standards, DOE orders, and nonregulatory guidance. As site- and waste-specific characteristics are provided for each technology, specific regulatory requirements will be specified.

\section{Subelement: Decontamination}

\section{Alternative: Metal refining}

Technology: Electrorefining. As a nickel purification method, it uses an electrolysis cell that contains a flat anode made from the impure nickel and a flat cathode made from pure nickel. The pure nickel used for the cathode is stripped off of a stainless steel blank, which was briefly used as a cathode. An electrolytic solution provides a conductive medium for the voltage applied between the anode and cathode that causes the impure nickel to ionize into positive nickel ions in the solution and nickel ions at the cathode to plate out. The anode and cathode compartment are separated by a porous diaphragm. Impurities in the nickel anode that are less than nickel in the electromotive series will usually drop out as a sludge beneath the anode. Impurities greater than nickel in the electromotive series will travel with the nickel ions. Therefore, the anode solution is continuously circulated by pump from the anode compartment to a purification train and cathode compartment where the pure nickel is plated out on the cathode. The direction of the solution flow through the diaphragm is from cathode to anode. Electrorefining would require that the scrap nickel be smelted and poured into suitable anode molds first. ${ }^{1}$ Leach/electrowinning technology (DCON-34-OL) combines leaching with electrorefining and avoids the need to smelt the scrap nickel into anodes by substituting a process that leaches the nickel into an electrolytic solution.

Status: Predemonstration. Electrorefining is a well-established commercial technology. However, this technology cannot be considered mature concerning its use for decontaminating radioactive metal because this application is much less established. Uranium is easier to remove than technetium, which means this method will likely allow favorable decontamination factors. The method has not been evaluated for treatment of metals other than nickel. An evaluation to determine the cost effectiveness of recovering other metals besides nickel would be required. At Y-12, nickel is not used extensively.

Waste-Recycle of the electrolytic solutions is assumed with resulting mixed solid waste bearing nickel uranium, technetium, and other compounds amounting to roughly $2 \%$ by weight of the nickel processed. ${ }^{2}$ 
Cost-The cost of an operating facility with a 10 million lb/year capacity is estimated at $\$ 2 \mathrm{M}$ capital and $\$ 6 / \mathrm{lb}$ operating costs. The operating costs exceed the present value of nickel $(\$ 3.60 / \mathrm{lb})$ because commercial nickel companies operate on a much larger scale. ${ }^{3}$ The development costs are estimated at \$3M.

Science/Technology Needs: Pilot-scale testing of this method is needed with special attention to decontamination efficiency and recycle of the electrolytic solutions.

Implementation Needs: An electrorefining plant used for recovery of nickel would also require a smelting and anode forming facility to form the impure nickel into anodes of proper configuration. A tank house containing the electrolytic cells and an electrical capacity of roughly $1 \mathrm{kWh} / \mathrm{lb}$ nickel refined are required. The capital costs altogether are estimated at $\$ 200 \mathrm{M}$, and the operating costs are estimated at $\$ 6 / \mathrm{lb}$ of nickel. ${ }^{3}$ The development costs are roughly estimated at $\$ 3 \mathrm{M}$. Estimates of the number of personnel are not yet available. The design will require the support of virtually all engineering disciplines. Facility operation will require process and electrical engineers, supervisors with at least two years technical background, chemical operators, electricians, mechanics, and general shop crafts. Metallurgical and analytical chemistry support is also required. To allow sale of the resulting purified nickel, either a de minimis level must be established in the United States or permission must be obtained to sell the metal in Europe where a de minimis level exists.

Author: E. B. Munday/615-576-5806. Reviewed by K. E. Lott/615-574-6537

\section{References:}

1. J. R. Boldt, Jr., The Winning of Nickel, D. Van Nostrand Company, Inc., Princeton, N.J., 1967.

2. Rough estimate by E. B. Munday, Martin Marietta Energy Systems, Inc., July 1992.

3. L. G. Guglielmin, INCO Exploration and Technical Services, Inc., personal communication to E. B. Munday, Martin Marietta Energy Systems, Inc., July 1992.

September 1994

Decontamination and Decommissioning 


\section{EM Problem: Decontamination and Decommissioning}

\section{Y-12 Plant Problem: Building 9201-4 (Alpha-4)}

Problem Area/Constituents: Nickel and steel process equipment contaminated with mercury, lithium hydroxide, sulfuric acid, nitric acid, asbestos, and PCBs.

Reference Requirements: Refer to Vol. 1, Chap. 10, for potentially applicable proposed and promulgated environmental laws, signed and pending agreements for the Oak Ridge Reservation, radiation protection standards, DOE orders, and nonregulatory guidance. As site- and waste-specific characteristics are provided for each technology, specific regulatory requirements will be specified.

\section{Subelement: Decontamination}

\section{Alternative: Metal refining}

Technology: Leach/electrowinning. This process would begin by pulverizing the impure metal and then leaching it (dissolve the metal into an electrolytic solution). At Y-12, International Nickel Company (INCO) has proposed that contaminated nickel could be leached into a solution containing dissolved chorine gas and copper ions. The copper acts as a chlorine exchange agent for the nickel to form soluble nickel chloride from the nickel metal. The required chlorine gas is generated as a by-product during the electrowinning of the nickel from the solution. Except for small amounts, the copper is recycled. Uranium in the leach solution is removed by solvent extraction, stripped with weak aqueous chlorine solution, and recovered by precipitation with sodium hydroxide as sodium diuranate. The electrolytic solution containing the nickel is plated onto a cathode. ${ }^{1}$ Leach/electrowinning avoids smelting anodes as required in the electrorefining method, but requires a leaching step that was not needed in the electrorefining method.

Status: Predemonstration. Leach/electrowinning is a well-established commercial method for producing nickel from nickel ore, but is not used commercially to refine impure nickel. Scientific Ecology Group, Inc. (SEG), has conducted bench-scale evaluations that purified nickel contaminated with technetium to less than $1 \mathrm{bq} / \mathrm{g}$ (the European release standard). The method used to get the nickel into solution and the purification train are proprietary information of SEG. ${ }^{2}$ Uranium is easier to remove than technetium, which means this method will likely allow favorable decontamination factors. Personnel at INCO prefer leach/electrowinning to electrorefining because it is believed that leach/electrowinning will give a purer product and leaching will have operational advantages to smelting anodes. ${ }^{1}$ The method has not been evaluated for treatment of metals other than nickel. An evaluation to determine the cost effectiveness of recovery of other metals besides nickel would be required. At $\mathrm{Y}-12$, nickel is not used extensively. 
Waste-Recycle of the electrolytic solutions is assumed with resulting mixed solid waste bearing nickel, uranium, technetium. and other compounds amounting to roughly $2 \%$ by weight of the nickel processed. ${ }^{3}$

Cost-The costs of an operating facility with a 10 million $\mathrm{lb} /$ year capacity are estimated by INCO at $\$ 200 \mathrm{M}$ capital and $\$ 3.80 / \mathrm{lb}$ operating costs. The operating costs exceed the present value of nickel $(\$ 3.60 / \mathrm{lb})$ because commercial nickel companies operate on a much larger scale. The development costs are estimated by INCO at $\sim \$ 440 \mathrm{~K}$ if the work were done at their facilities. These cost figures compare closely to the electrorefining process because of the similarity of the processes and because both estimates are very approximate.

Science/Technology Needs: Pilot-scale testing of this method is needed with special attention to the leaching process, decontamination efficiency, and recycle of the electrolytic solutions. The development work by SEC may satisfy some of this requirement.

Implementation Needs: A leach/electrowinning plant would require a size-reduction facility, a leaching facility, and a tankhouse for the electrolytic cells. Electrical capacity to supply roughly $1 \mathrm{kWh} / \mathrm{lb}$ nickel refined is required. ${ }^{3}$ The capital costs altogether are estimated at $\$ 200$ million, and the operating costs are estimated at $\$ 3.80 / \mathrm{lb}$ of nickel. ${ }^{1}$ The development costs are estimated by INCO in two phases: Phase 1 would be a bench-scale, batch operation requiring $\$ 170 \mathrm{~K}$ and Phase 2 would be a continuous small-scale process with recycle of solutions and would cost an additional $\$ 270 \mathrm{~K} .^{1}$ These estimates assume the development activities are done at INCO's facility, appear low, and could be higher if done at a DOE facility. Estimates of the numbers of personnel are not yet available. The design will require the support of virtually all engineering disciplines. Facility operation will require process engineers, electrical engineers, supervisors with at least 2 years technical background, chemical operators, electricians, mechanics, and general shop crafts. Metallurgical and analytical chemistry support is also required. To allow sale of the resulting purified nickel, either a de minimis must be established for the United States or permission must be obtained to sell the metal In Europe where a de minimis standard exists.

Author: E. B. Munday/615-576-5806. Reviewed by K. E. Lott/615-574-6537

\section{References:}

1. INCO Exploration and Technical Services, Inc., personal communication to E. B. Munday, Martin Marietta Energy Systems, Inc., July 1992.

2. Scientific Ecology Group, Inc., personal communication to E. B. Munday, Martin Marietta Energy Systems, Inc., July 1992.

3. Rough estimate by E. B. Munday, Martin Marietta Energy Systems, Inc., July 1992.

September 1994

Decontamination and Decommissioning 


\section{EM Problem: Decontamination and Decommissioning}

\section{Y-12 Plant Problem: Building 9201-4 (Alpha-4)}

Problem Area/Constituents: Nickel and steel process equipment contaminated with mercury, lithium hydroxide, sulfuric acid, nitric acid, asbestos, and PCBs. Painted concrete and clay tile contaminated with $\mathrm{PCBs}$, oil, $\mathrm{U}$ (and daughters), electrolytes, $\mathrm{Cd}, \mathrm{Pb}$, and $\mathrm{Cr}$.

Reference Requirements: Refer to Vol. 1, Chap. 10, for potentially applicable proposed and promulgated environmental laws, signed and pending agreements for the Oak Ridge Reservation, radiation protection standards, DOE orders, and nonregulatory guidance. As site- and waste-specific characteristics are provided for each technology, specific regulatory requirements will be specified.

\section{Subelement: Decontamination}

\section{Alternative: Mechanical abrasive methods}

Technology: Ultrahigh-pressure water (UHPW). An ultrahigh-pressure intensifier pump pressurizes water up to $55,000 \mathrm{psi}$ and forces it through small-diameter nozzles, generating high velocity waterjets at speeds up to $3,000 \mathrm{ft} / \mathrm{s}$. The nozzles may be mounted in various types of cleaning heads for different contaminated surfaces. The waterjets thoroughly penetrate and remove surface contaminants. Care must be taken so as to not damage the substrate. The UHPW cleaning head, attached to a lance, may be manually moved about on the surfaces being decontaminated. The decontamination efficiency depends on the applicator translation speed. Remote operation of the UHPW cleaning head would be desirable. Also, in some applications (such as decontamination of hot cells), remote operation will be required. Consequently, the adaptation of the equipment to a robotics control system would be necessary.

Status: Accepted by industry. UHPW decontamination technology is available and has been used by industry. The addition of abrasives is also available for industrial cutting and milling. Before acceptance at $\mathrm{Y}-12$ and other DOE sites, the issues of criticality, wastewater treatment, and prevention of spread of contamination must be addressed. The technology can produce high levels of decontamination. Removal of a galvanized layer from sheet metal has been demonstrated. Waste generated is contaminated water from the cleaning operation. In cleaning concrete, for example, a typical flow rate for one cleaning head would be 3-5 gal $/ \mathrm{min}$ at a surface treatment rate of about $1 \mathrm{ft}^{2} / \mathrm{min}$.

Science/Technology Needs: Existing vacuum systems, which recover water from the cleaning (or power) head of the unit, need to be developed further. To minimize waste generation, a water treatment system is needed for decontamination of the wastewater so that the water can be recycled and reused in the UHPW cleaning operation. The system must be capable of treating contaminants specific to the site and reducing their concentrations to allowable levels for water recycle. Remote operation will necessitate the adaptation of the 
ultrahigh-pressure cleaning heads and the vacuum collection systems to a robotic control system. A data base on UHPW cleanup experience is desirable. This could be developed through communication with the Water Jet Technology Association. The data would be useful in optimization of cleaning parameters in future UHPW decontamination activities. Nozzle configuration, water pressure and flow rate, distance of the cleaning head to the substrate, and the geometric complexities of the substrate are all parameters that needed to be evaluated.

\section{Implementation Needs:}

Development costs: For development and demonstration of a water recycle system, in combination with UHPW decontamination, an estimate of funding requirements is $\$ 1.2 \mathrm{M}$. Personnel requirements are estimated to be $\$ 1.7 \mathrm{M}$ (approximately $50 \%$ engineer and $50 \%$ technician time) and 4.1 FTEs (approximately $10 \%$ P\&E, 45\% engineer, and $45 \%$ technician time) for FY 93 and FY 94, respectively. The cost of a robotic control system is estimated at $\$ 250 \mathrm{~K}$.

Deployment costs: FLOW International supplies ultrahigh-pressure power units, with intensifier pumps rated at Pressures of 40,000 to 55,000 psi, are supplied at costs up to $\$ 200 \mathrm{~K}$. Several ultrahigh-pressure tools are available for use with the power unit. Operating costs for a UHPW unit would primarily involve labor costs. Assuming a minimum two-person operating costs would be approximately $\$ 350 /$ day. These costs do not include either the disposal cost of the wastewater that is generated or the capital and operating costs of a system to treat the wastewater and recycle it to the UHPW unit. Development of an acceptable water recycle system is needed to establish estimates for the latter costs. Personnel training for supervisors and for operators will be needed for operation of the UHPW unit and recycle system. Facilities for performing prototype cleaning operations for each decontamination project would be beneficial. Capital costs: UHPW system, $-\$ 500 \mathrm{~K}$ with vacuum system; glove box, $>\$ 500 \mathrm{~K}$; and work room, $-\$ 250 \mathrm{~K}$. Operating costs are estimated at $>\$ 1 / \mathrm{ft}^{2}$.

Author: K. E. Lott/615-574-6537

References: MMES staff members, especially C. E. Benson and J. H. Wilson

September 1994

Decontamination and Decommissioning 


\section{EM Problem: Decontamination and Decommissioning}

\section{Y-12 Plant Problem: Building 9201-4 (Alpha-4)}

\section{Problem Area/Constituents:}

- In situ decontamination of the exterior of steel and nickel process equipment contaminated with $\mathrm{Hg}, \mathrm{LiOH}, \mathrm{H}_{2} \mathrm{SO}_{4}, \mathrm{HNO}_{3}$, asbestos, and PCBs.

- Ex situ decontamination of steel and nickel process equipment contaminated with $\mathrm{Hg}$, $\mathrm{LiOH}, \mathrm{H}_{2} \mathrm{SO}_{4}, \mathrm{HNO}_{3}$, asbestos, and PCBs.

- Painted concrete and clay tile contaminated with PCBs, oil, U (and daughters), electrolytes, $\mathrm{Cd}, \mathrm{Pb}$, and $\mathrm{Cr}$.

\section{Reference Requirements:}

- Federal Facility Compliance Agreements (e.g., FFCA for Oak Ridge Site)

- Applicable Federal and State laws and regulations (e.g., Clean Water Act \& Toxic Substance Control Act)

- DOE orders

Refer to Vol. 1, Chap. 10, for potentially applicable proposed and promulgated environmental laws, signed and pending agreements for the Oak Ridge Reservation, radiation protection standards, DOE orders, and nonregulatory guidance. As site- and waste-specific characteristics are provided for each technology, specific regulatory requirements will be specified.

\section{Subelement: Decontamination}

Alternative: Mechanical surface substrate removal methods

Technology: Shot blasting. Uses mechanically accelerated iron shot (pellets). After the shot hits the surface to be cleaned, it is recovered from the contaminated dust by a magnetic system and reused.

Status: Accepted. Commercial units are available ${ }^{1}$ that have been used in one step to prepare large areas of concrete floors for painting, cleaning rust and marine growth from ship hulls, and cleaning structural steel elements. A Wheelbrator Blastrac is being used to decontaminate floors at the K-25 Site. Cleaning rates for floors are quoted as being faster than acid etching, sandblasting, and scarifying by factors of 1.7,1.3, and 2.0, respectively. ${ }^{2}$ Portable machines with design rates of $2,500 \mathrm{ft}^{2} / \mathrm{h}$ are available. ${ }^{3}$ The method is considered advantageous because it is commercially available and because there is considerable cleaning experience on various surfaces. ${ }^{4}$ Shot blasting differs from sand blasting in that it can be controlled to give more accurate results. ${ }^{5}$ Decontamination factors on the order to $10-100$ and costs on the order of $\$ 10 / \mathrm{m}^{2}$ are reported for similar technologies. ${ }^{4}$ Shot is recycled many 
times during cleaning, but ultimately erodes and becomes part of the waste stream at the rate of approximately $0.1 \mathrm{lb} / \mathrm{m}^{2}$.

\section{Science/Technology Needs: None}

Implementation Needs: A collection system with adequate filtration and sorption would be needed to use this technology. Such systems are an integral part of many commercial shot blasters. However, these collection systems will require the addition of sorption cannisters to remove mercury vapor.

Author: H. H. Haselton/615-574-1115. Reviewed by E. B. Munday/615-576-5806

\section{References:}

1. Blastrac Division the Wheelbrator Corp., Shenandoah, Ga. 30265.

2. "The Original Blastrac Portable Shot Blast Cleaning Systems the Preferred Way to Prepare Floor Surfaces," Wheelbrator B218.00A.

3. "The Original Blastrac Portable Shot Blast Cleaning Systems Specifications," Wheelbrator B219.00B CC/2-90.

4. "Cleanup of Large Areas Contaminated as a Result of a Nuclear Accident," IAEA Technical Report 300, 1989.

5. Metals Handbook, Vol 5, Surface Cleaning, Finishing and Coating, Ninth Edition, 1978. 
EM Problem: Decontamination and Decommissioning

Y-12 Plant Problem: Building 9201-4 (Alpha-4)

Problem Area/Constituents: Painted concrete and clay tile contaminated with PCBs, oil, $\mathrm{U}$ (and daughters), electrolytes, $\mathrm{Cd}, \mathrm{Pb}$, and $\mathrm{Cr}$.

\section{Reference Requirements:}

- Federal Facility Compliance Agreement for Oak Ridge Site

- Federal Facility Compliance Agreement for PCBs

- Clean Air Act and Toxic Substance Control Act

- DOE orders

Refer to Vol. 1, Chap. 10, for potentially applicable proposed and promulgated environmental laws, signed and pending agreements for the Oak Ridge Reservation, radiation protection standards, DOE orders, and nonregulatory guidance. As site- and waste-specific characteristics are provided for each technology, specific regulatory requirements will be specified.

\section{Subelement: Decontamination}

Alternative: Mechanical surface removal methods

Technology: Scabblers/scarifiers. This technology decontaminates a concrete substrate by using mechanical impact methods to remove the contaminated surface. Many vendors market units that use high-speed reciprocating tungsten-carbide tipped pistons to pulverize protective coatings and concrete substrate in a single-step process. Other types of units use a shrouded needle scaler to remove concrete from outside edges and inside corners, as well as from wall surfaces. The solid debris produced by mechanical scabbling is removed and collected by a vacuum system. Mechanical scabblers are usually operated manually. If desired, in order to generate more uniform removal rates, or required (i.e., high levels of radioactivity), the units could be adapted for remote-controlled operation).

Status: Accepted. The technology has been used for decontamination purposes in numerous applications involving hazardous and/or radioactive contaminants. Because the technology involves removal of contaminated surfaces, the decontamination efficiency should be $95 \%$ or higher. The waste generated is the pulverized surface layer that is collected by a vacuum system. The amount of waste generated depends upon the depth of the surface layer that needs to be removed to achieve decontamination. For example, two different commercial units provide removal of concrete at rates of $3-4.5 \mathrm{in}^{3} / \mathrm{min}(8-12 \mathrm{lb} / \mathrm{h})$ and $60 \mathrm{in} .{ }^{3} / \mathrm{min}(160 \mathrm{lb} / \mathrm{h})$, respectively, at a removal depth of $1 / 16$ in. under optimum conditions in a non-contaminated environment, sustained operating rates in a contaminated environment are normally substantially smaller. 
Science/Technology Needs: Remote operation will require the adaptation of the scabbler to a robotic control system.

Implementation Needs: Improvement costs: Robotic control system, $\sim \$ 250 \mathrm{~K}$. Deployment costs: The capital costs of the two different commercial units mentioned above are $\$ 10 \mathrm{~K}$ and $\$ 110 \mathrm{~K}$, respectively. The largest size commercial scabber costs about $\$ 500 \mathrm{~K}$. Operating costs for a scabbler would primarily involve labor costs. Assuming a minimum two-person operation (at a labor rate of $\$ 40 \mathrm{~K} / \mathrm{year} /$ person) of a scabbler during the day shift, operating costs would be approximately $\$ 350 /$ day. Total operating costs normally exceed $\$ 1 / \mathrm{ft}^{2}$. Training of personnel to operate mechanical scabblers will be needed.

Author: J. H. Wilson/615-576-4413. Reviewed by R. D. Bundy

\section{References:}

1. MMES staff members.

2. Dust/Contaminate Free Surface Preparation, Desco Mfg. Co., Inc., Long Beach, Calif. 


\section{EM Problem: Decontamination and Decommissioning}

Y-12 Plant Problem: Building 9201-4 (Alpha-4)

\section{Problem Area/Constituents:}

- In situ decontamination of the exterior of steel and nickel process equipment contaminated with $\mathrm{Hg}, \mathrm{LiOH}, \mathrm{H}_{2} \mathrm{SO}_{4}, \mathrm{HNO}_{3}$, asbestos, and PCBs.

- Ex situ decontamination of steel and nickel process equipment contaminated with $\mathrm{Hg}$, $\mathrm{LiOH}, \mathrm{H}_{2} \mathrm{SO}_{4}, \mathrm{HNO}_{3}$, asbestos, and PCBs.

\section{Reference Requirements:}

- Federal Facility Compliance Agreements (e.g., FFCA for Oak Ridge Site)

- Applicable Federal and State laws and regulations (e.g., Clean Water Act and Toxic Substance Control Act)

- DOE orders

Refer to Vol. 1, Chap. 10, for potentially applicable proposed and promulgated environmental laws, signed and pending agreements for the Oak Ridge Reservation, radiation protection standards, DOE orders, and nonregulatory guidance. As site- and waste-specific characteristics are provided for each technology, specific regulatory requirements will be specified.

\section{Subelement: Decontamination}

Alternative: Mechanical surface substrate removal methods

Technology: Grit blasting, Commonly known as sand blasting, this pneumatic cleaning process can use a wide variety of grits.

Status: Accepted by industry. Grit blasting has been used many times in the nuclear industry with applications ranging from heavily contaminated pipework, contamination fixed in the oxide, to lightly contaminated surfaces. Commercial units are readily available. ${ }^{1}$ Typical abrasives include sand, glass beads, metallic beads, and soft materials such as nut shells and rice hulls. Grit blasting has been shown to be an efficient cleaning method, with unlimited decontamination factors being obtained. ${ }^{2}$ Grit blasting is widely used in industry for removing all classes of scale and rust from a wide range of metal products. ${ }^{3}$ Waste production rates depend on the media/surface combination. Highly abrasive grits will clean more effectively and faster than soft grits but will ultimately become part of the waste stream. Waste production rates, including grit plus filters, could range from 0.005 to $0.1 \mathrm{lb} / \mathrm{ft}^{2}$. The cost of the largest size grit blaster is about $\$ 500 \mathrm{~K}$.

Science/Technology Needs: Technology improvement is needed in waste minimization related to blast media erosion and disposal; containment of waste and vacuum systems; and 
demonstration of specific blast-media substrate/contaminant capabilities, including determination of decontamination factors and process automation. The cost of developing these improvements is estimated to be $\$ 0.25 \mathrm{M}$.

Implementation Needs: This technology, while developed and commercially available, will require a system for processing waste. For instance, use of the device will generate contaminated dust, gases, and/or fluids. This will require a system to separate and package the contaminants. Total operating costs in a contaminated environment are estimated at about $\$ 2.50 / \mathrm{ft}^{2}$.

Authors: J. H. Wilson/615-576-4413

\section{References:}

1. Progressive Blasting Systems, Grand Rapids, MI 49508.

2. Metals Handbook, 9th Ed., Vol. 5, "Surface Cleaning, Finishing and Coating," 1978.

3. Cleanup of Large Areas Contaminated as a Result of a Nuclear Accident, IAEA Technical Report 300, 1989. 
EM Problem: Decontamination and Decommissioning

Y-12 Plant Problem: Building 9201-4 (Alpha-4)

\section{Problem Area/Constituents:}

- In situ decontamination of the interior of steel and nickel process equipment contaminated with $\mathrm{Hg}, \mathrm{LiOH}, \mathrm{H}_{2} \mathrm{SO}_{4}$, and $\mathrm{HNO}_{3}$.

- In situ decontamination of the exterior of steel and nickel process equipment contaminated with $\mathrm{Hg}, \mathrm{LiOH}, \mathrm{H}_{2} \mathrm{SO}_{4}, \mathrm{HNO}_{3}$, asbestos, and PCBs.

- Ex situ decontamination of steel and nickel process equipment contaminated with $\mathrm{Hg}$, $\mathrm{LiOH}, \mathrm{H}_{2} \mathrm{SO}_{4}, \mathrm{HNO}_{3}$, asbestos, and PCBs.

\section{Reference Requirements:}

- Federal Facility Compliance Agreements (e.g., FFCA for Oak Ridge Site)

- Applicable Federal and State laws and regulations (e.g., Clean Water Act and Toxic Substance Control Act)

- DOE orders

Refer to Vol. 1, Chap. 10, for potentially applicable proposed and promulgated environmental laws, signed and pending agreements for the Oak Ridge Reservation, radiation protection standards, DOE orders, and nonregulatory guidance. As site- and waste-specific characteristics are provided for each technology, specific regulatory requirements will be specified.

\section{Subelement: Decontamination}

Alternative: Mechanical surface substrate removal

Technology: Centrifuge cryogenic $\mathrm{CO}_{2}$ pellet blasting uses a high-speed rotating wheel to accelerate $\mathrm{CO}_{2}$ pellets. The centrifuge system is 25 times more efficient than commercial compressed air $\mathrm{CO}_{2}$ systems and can attain much higher pellet speeds. For example, the centrifuge with a $15 \mathrm{hp}$ electric motor replaces a $150 \mathrm{hp}$ air compressor system used in the commercial system and can strip paint at a rate five times that of the commercial system. This increased capability will allow cleaning and etching at greatly increased rates. In comparison to the compressed air technology, the centrifuge system can achieve much higher and more controllable pellet speeds. The centrifuge $\mathrm{CO}_{2}$ system can also produce the same or more aggressive etching than the ultrahigh velocity water blasting system, with comparable or increased efficiencies, and comparable capital equipment costs. The most important difference is that the $\mathrm{CO}_{2}$ method is dry. This also makes $\mathrm{CO}_{2}$ blasting technology usable in enriched uranium decontamination areas and eliminates the need for downstream water decontamination system. 
The high-speed $\mathrm{CO}_{2}$-pellet centrifugal-accelerator blaster unit applies directly to numerous decontamination problems, for instance, such substrates and coatings as concrete, painted concrete, concrete block, painted tiles, stainless steel sheet, structural steel, steel plate, galvanized metals, and heavily oxidized metals can be cleaned, stripped, or etched. The system can be used in situ for cleaning and etching the inside of large pipes and tanks. It can be used to clean the outside of in-place process equipment, and if the equipment has been dismantled it will be effective on all surfaces. The high-speed $\mathrm{CO}_{2}$ pellets are expected to be effective against $\mathrm{Hg}$, amalgams of $\mathrm{Hg}, \mathrm{LiOH}, \mathrm{PCB}, \mathrm{oil}, \mathrm{U}, \mathrm{Cd}, \mathrm{Pb}$, and $\mathrm{Cr}$ and other contaminants.

Status: Predemonstration. $\mathrm{CO}_{2}$ pellet blasting, using compressed air to accelerate the pellets, is a demonstrated technology for decontaminating equipment at nuclear reactor sites without producing secondary wastes. ${ }^{1.2}$ The use of centrifuge pellet acceleration has been established in the DOE Fusion Energy Program to form and accelerate pellets of frozen deuterium and tritium for fueling fusion reactors. ${ }^{3}$ Accelerating pellets of argon and $\mathrm{CO}_{2}$ for the purpose of cleaning heavy oxidation deposits from uranium surfaces has been demonstrated as part of the DOE Y-12 waste minimization program. ${ }^{4}$ With the higher speeds available, the centrifuge technology can remove hard oxide layers from steel, zinc coatings from galvanized steel/sheet metal, and nickel plating from brass screws. Cutting has been demonstrated at higher pellet speeds for aluminum and copper, and during experimental evaluation sufficient damage has been observed to steel and ceramics to include them in the potential cutting list. For very hard materials, a pellet gun can be developed to cut or destroy any construction material. A brief program with the Air Force Warner Robins Air Logistics Center demonstrated the removal of the urethane and epoxy paint surfaces from $F-15$ aircraft at a rate of $120 \mathrm{ft}^{2} / \mathrm{h}$ for a 15- $\mathrm{HP}$ accelerator. Because the pellets evaporate to gaseous $\mathrm{CO}_{2}$ upon impact, the secondary waste stream is limited to the materials removed from the surface. A vacuum shroud surrounding the blast nozzle and covering the impact area will be used to produce a negative pressure and collect the airborne contaminants in a conventional HEPA filtering system. The secondary waste stream would thereby be limited to the HEPA filters.

Efficacy/strengths/weaknesses-The $\mathrm{CO}_{2}$ centrifugal system is very efficient and is usable for a wide variety of contaminates and substrates.

Waste-No additional waste is produced by the system. Existing waste is converted into a more acceptable and easier to handle form.

Cost-Development costs, $\$ 750 \mathrm{~K}$; the operating cost is stated above.

The capital cost of a centrifuge blaster capable of accelerating 1 ton/h of $\mathrm{CO}_{2}$ at speeds up to $400 \mathrm{~m} / \mathrm{s}$ would be $\$ 100 \mathrm{~K}-\$ 200 \mathrm{~K}$. The unit would be remotely manipulated by an operator and would be capable of cleaning rates of $200-2000 \mathrm{ft}^{2} / \mathrm{h}$ depending on the nature of the surface to be cleaned.

$\mathrm{CO}_{2}$ costs- $\$ 50 / \mathrm{h}$

Electricity costs $30 \mathrm{~kW} @ \$ .06 / \mathrm{h}-\$ 1.80 / \mathrm{h}$

September 1994

Decontamination and Decommissioning 
Labor; one person @ $\$ 100 / \mathrm{h}-\$ 100 / \mathrm{h}$

Total cost- $\$ 151.80 / \mathrm{h}$

Cost $/ \mathrm{ft}^{2}-\$ 0.075 / \mathrm{ft}^{2} @ 2000 \mathrm{ft}^{2} / \mathrm{h}$ to $\$ 0.75 / \mathrm{ft}^{2} @ 200 \mathrm{ft}^{2} / \mathrm{h}$

Because the removal or etching rate of the surface varies dramatically with the specific application, data on specific decontamination efficiencies needs to be determined to obtain accurate cost estimates. However, decontamination factors for compressed air systems range from 3 to $>5000$ for various materials. ${ }^{5}$ Due to the higher speed capability and the increased throughput capabilities, the costs will be one-fifth or less than that of present compressed air systems.

Science/Technology Needs: The fundamental concepts of centrifugal pellet accelerator are well proven and have been demonstrated. The only science and technology needs are those associated with tailoring the accelerator to the particular contamination problem. In most cases this will simply be adjusting the pellet speed and determining the specific decontamination rate. In some contaminated environments, it may be desirable to detect the degree of contamination and develop feedback control that will speed the overall cleaning rate and thus help reduce operating cost. Since the $\mathrm{CO}_{2}$ is in a gaseous state after impact, they can be collected in normal gas collection systems. The blasted off materials will require only established collection technology. There will be no additions and surprises in the waste stream problem.

Implementation Needs: An existing accelerator developed for the Y-12 waste minimization program should be upgraded to operate in a laboratory for scoping studies. Additions to the system would be a high throughout pellet maker (up to a ton per hour) and a particulate collection system. This unit would be operated to collect decontamination data (and rates). If the application chosen is one with extreme contamination, a robot system would be designed along with the application specific accelerator. The resulting unit would be field tested, demonstrated, and put to use.

The development cost of a concrete cleaning $\mathrm{CO}_{2}$ blaster unit would be about $\$ 750 \mathrm{~K}$. Additional applications would require further equipment modifications and cost. The pellet accelerator capital cost is estimated at $\$ 100 \mathrm{~K}$. Additional capital costs would be required for the waste collection system. The operating cost is indicated above.

Authors: C. A. Foster/615-574-1128 and W. D. Nelson/615-574-0966

\section{References:}

1. Alpheus Cleaning Technologies, Rancho Cucamonga, Calif. 91730.

2. Cold Jet, Loveland, Ohio 45140.

3. C. A. Foster, "Solid Deuterium Centrifuge Pellet Injector," J. Vac. Sci Tech Al(2), 952-958 (1983).

4. C. A. Foster, "Surface Impact Cleaning by High-Speed Cryogenic Pellets," Second International Workshop on Solvent Substitution, Phoenix, Ariz., December 10-13, 1991. 
5. R. J. Dabolt, Evaluation of Pelletized Carbon Dioxide as a Fluidized Abrasive Agent for Removal of Radioactive Contamination, Document No. ER-89-002 (1989), Chem-Nuclear Systems, Columbia, S.C. 29210. 


\section{EM Problem: Decontamination and Decommissioning}

T-12 Plant Problem: Building 9201-4 (Alpha-4)

\section{Problem Area/Constituents:}

- In situ decontamination of the interior of steel and nickel process equipment contaminated with $\mathrm{Hg}, \mathrm{LiOH}, \mathrm{H}_{2} \mathrm{SO}_{4}$, and $\mathrm{HNO}_{3}$.

- In situ decontamination of the exterior of steel and nickel process equipment contaminated with $\mathrm{Hg}, \mathrm{LiOH}, \mathrm{H}_{2} \mathrm{SO}_{4}, \mathrm{HNO}_{3}$, asbestos, and PCBs.

- Ex situ decontamination of steel and nickel process equipment contaminated with $\mathrm{Hg}$, $\mathrm{LiOH}, \mathrm{H}_{2} \mathrm{SO}_{4}, \mathrm{HNO}_{3}$, asbestos, and PCBs.

\section{Reference Requirements:}

- Federal Facility Compliance Agreement for Oak Ridge Site

- Federal Facility Compliance Agreement for PCBs

- Clean Air Act

- Clean Water Act

- Toxic Substance Control Act

- DOE orders

Refer to Vol. 1, Chap. 10, for potentially applicable proposed and promulgated environmental laws, signed and pending agreements for the Oak Ridge Reservation, radiation protection standards, DOE orders, and nonregulatory guidance. As site- and waste-specific characteristics are provided for each technology, specific regulatory requirements will be specified.

\section{Subelement: Decontamination}

\section{Alternative: Mechanical surface removal}

Technology: Ice blasting. It is similar to other decontamination technologies that direct a high velocity stream of fine particles, such as shot, grit, cryogenic pellets, or plastic pellets, onto a surface to remove contamination. The contaminated wastewater generated by the melting ice particles is the controlling medium for the displaced contamination. The water must then be treated for discharge by processing in commercially available evaporators. The cleaning head may be manually moved about on the surfaces being decontaminated. Decontamination efficiency depends on the applicator translation speed, cleaning head distance from substrate, operating pressure, and geometric complexities of the substrate. The velocities of the ice particles will be similar to those achieved by the "sand blasting" type $\mathrm{CO}_{2}-$ pellet blasters and thus its applications will be limited to cleaning and removing relatively soft paints and other coatings. For in situ inside cleaning, this technique would apply for large pipes and empty tanks. For outside in situ cleaning, it could apply to most surfaces. 
Status: Accepted. Ice blasting technology has been used recently for decontamination at the Oconee Nuclear Power Plant in Seneca, South Carolina. Decontamination testing was performed at ORNL.

Ice blasting will remove coatings and some fixed surface contamination, but it will not take off concrete to a significant depth. The waste produced is contaminated wastewater. The amount of wastewater generated depends upon the ice blasting rate. For example, a unit recently demonstrated at ORNL operated at $11 \mathrm{gal} / \mathrm{h}$.

Science/Technology Needs: Remote-controlled operation of the cleaning head would be desirable. The cost of a robotics control system is estimated at $\$ 250 \mathrm{~K}$ (personal communication with C. E. Benson). The efficacy of commercial systems needs demonstration. Total development costs are estimated to be $\$ 0.75 \mathrm{M}$.

Implementation Needs: The capital cost of an ice blasting machine such as that used at a nuclear power plant, as mentioned above, would be $\$ 155 \mathrm{~K}$. A commercially available evaporator will be required. Because of the low volume of water generated during operation, evaporation-and not recycle-will likely be more cost-effective. Capital costs, $\$ 60 \mathrm{~K}-\$ 90 \mathrm{~K}$ for an evaporator. Remote operation will require the adaptation of the ice blasting and water collection systems to a robotics control system. Total capital costs could range from $\$ 100 \mathrm{~K}$ to $\$ 1 \mathrm{M}$ depending on the application.

Operating costs for an ice blasting unit would primarily involve labor costs. Assuming a minimum two-person operation (at a labor rate of $\$ 40 \mathrm{~K} /$ year/person) of an ice blasting unit during the day shift, operating costs would be $\sim \$ 350 /$ day. Training of personnel to operate the ice blasting equipment and the wastewater treatment system will be needed. Total operating costs in a Hg-contaminated environment are expected to be about $\$ 2 / \mathrm{ft}^{2}$.

Author: J. H. Wilson/615-576-4413

References: Personal communication between C. E. Benson and the author.1 
EM Problem: Decontamination and Decommissioning

T-12 Plant Problem: Building 9201-4 (Alpha-4)

\section{Problem Area/Constituents:}

- In situ decontamination of the interior of steel and nickel process equipment contaminated with $\mathrm{Hg}, \mathrm{LiOH}, \mathrm{H}_{2} \mathrm{SO}_{4}$, and $\mathrm{HNO}_{3}$.

- In situ decontamination of the exterior of steel and nickel process equipment contaminated with $\mathrm{Hg}, \mathrm{LiOH}, \mathrm{H}_{2} \mathrm{SO}_{4}, \mathrm{HNO}_{3}$, asbestos.

- Ex situ decontamination of steel and nickel process equipment contaminated with $\mathrm{Hg}$, $\mathrm{LiOH}, \mathrm{H}_{2} \mathrm{SO}_{4}, \mathrm{HNO}_{3}$, asbestos.

- Painted concrete and clay tile contaminated with PCBs, oil, U (and daughters), electrolytes, $\mathrm{Cd}, \mathrm{Pb}$, and $\mathrm{Cr}$.

\section{Reference Requirements:}

- Federal Facility Compliance Agreement for Oak Ridge Site

- Clean Air Act

- Toxic Substance Control Act

- DOE orders

Refer to Vol. 1, Chap. 10, for potentially applicable proposed and promulgated environmental laws, signed and pending agreements for the Oak Ridge Reservation, radiation protection standards, DOE orders, and nonregulatory guidance. As site- and waste-specific characteristics are provided for each technology, specific regulatory requirements will be specified.

\section{Subelement: Decontamination}

Alternative: Mechanical surface removal methods

Technology: Supercritical $\mathrm{CO}_{2}$ blasting. Supercritical $\mathrm{CO}_{2}$ (above its critical temperature of $87.8^{\circ} \mathrm{F}$ and at high pressure) is pressurized by an ultrahigh-pressure intensifier pump up to $55,000 \mathrm{psi}$ and forced through nozzles, generating high velocity $\mathrm{CO}_{2}$ jets at speeds up to $3,000 \mathrm{ft} / \mathrm{s}$. The nozzles may be mounted in different cleaning heads for various contaminated surfaces. The $\mathrm{CO}_{2}$ jets thoroughly penetrate and remove surface contaminants without damaging the healthy substrate. The removed contaminants, any of the substrate surface layer that may be removed, and the $\mathrm{CO}_{2}$ are captured by a vacuum recovery system. In the recovery system, the substrate surface layer, if removed, and the contaminants are collected by a cyclone separator and a HEPA filter. The $\mathrm{CO}_{2}$, now in the gaseous state, is discharged to the atmosphere or recovered and recycled to the supercritical cleaning step.

Status: Predemonstration. This technology is being developed by a private company. Similar to the ultrahigh-pressure water process, which has been demonstrated to remove the 
galvanized layer from sheet metal, the supercritical $\mathrm{CO}_{2}$ technology should produce high levels of decontamination. The waste generated is the removed contaminants and the substrate surface layer collected in the vacuum recovery system. The $\mathrm{CO}_{2}$ is either discharged to the atmosphere or recycled to the supercritical cleaning operation.

Science/Technology Needs: Evaluation of the effect of operating parameters (e.g., pressure, distance between nozzle and substrate, traversing speed of cleaning head) on the removal of various contaminants from different substrates is needed.

Implementation Needs: Development costs: The cost of a development program for the supercritical $\mathrm{CO}_{2}$ blasting technology is estimated to be from $\$ 250 \mathrm{~K}-\$ 1 \mathrm{M}$. Deployment costs: The capital cost of a commercial supercritical $\mathrm{CO}_{2}$ cleaning unit is estimated to be $\$ 150 \mathrm{~K}$. With vacuum handling and gas treatment systems, the total capital cost for this technology could easily be $\$ 250 \mathrm{~K}$ to $\$ 1 \mathrm{M}$, depending upon the application. Operating costs for a supercritical $\mathrm{CO}_{2}$ unit would primarily involve labor costs and cost of $\mathrm{CO}_{2}$. Assuming a minimum two-person operation (at a labor rate of $\$ 50 \mathrm{~K} /$ year/person) of a $\mathrm{CO}_{2}$ blasting unit during the day shift, labor costs would be approximately $\$ 400 /$ day. Total operating costs in a contaminated environment are expected to be over $\$ 1 / \mathrm{ft}^{2}$. The cost of $\mathrm{CO}_{2}$ will depend upon the blasting rate, which would be determined during the development phase of the technology. Other implementation needs include personnel training for operation of the supercritical $\mathrm{CO}_{2}$ decontamination unit and vacuum recovery system and facilities for performing prototype cleaning operations for each decontamination project.

Author: J. H. Wilson/615-574-4413. Reviewed by R. B. Alderfer/615-576-0225

\section{References:}

1. MMES staff members, especially C. E. Benson.

2. FLOW International Corporation, Seattle, Wash.

September 1994

Decontamination and Decommissioning 
EM Problem: Decontamination and Decommissioning

Y-12 Plant Problem: Building 9201-4 (Alpha-4)

\section{Problem Area/Constituents:}

- In situ decontamination of the exterior of steel and nickel process equipment contaminated with $\mathrm{Hg}, \mathrm{LiOH}, \mathrm{H}_{2} \mathrm{SO}_{4}, \mathrm{HNO}_{3}$, asbestos, and PCBs.

- Ex situ decontamination of steel and nickel process equipment contaminated with $\mathrm{Hg}$, $\mathrm{LiOH}, \mathrm{H}_{2} \mathrm{SO}_{4}, \mathrm{HNO}_{3}$, asbestos, and PCBs.

- Painted concrete and clay tile contaminated with PCBs, oil, U (and daughters), electrolytes, $\mathrm{Cd}, \mathrm{Pb}$, and $\mathrm{Cr}$.

\section{Reference Requirements:}

- Federal Facility Compliance Agreements (e.g., FFCA for Oak Ridge Site)

- Applicable Federal and State laws and regulations (e.g., Clean Water Act and Toxic Substance Control Act)

- DOE orders

Refer to the Regulatory Compliance chapter of Vol. 1 (Pt. A, B, or C as appropriate) for potentially applicable proposed and promulgated environmental laws, signed and pending agreements for the Oak Ridge Reservation, radiation protection standards, DOE orders, and nonregulatory guidance. As site- and waste-specific characteristics are provided for each technology, specific regulatory requirements will be specified.

Subelement: Decontamination

Alternative: Mechanical surface substrate removal

Technology: Plastic pellet blasting. A cleaning process similar to sand blasting that uses less abrasive plastic pellets.

Status: Demonstrated. Plastic pellet blasting is an alternative to sand blasting for applications in which it is desirable to remove surface contamination or contaminated coatings while imparting minimal damage to the substrate. ${ }^{1,2}$ Cost of the media is high $(\$ 1-\$ 2 / l b)$ and pellet erosion can also be a factor, but cyclone systems are capable of recycling pellets up to thirty times for reuse. Cleaning rates of up to $4 \mathrm{ft}^{2} / \mathrm{min}$ are quoted for a $1 / 2$ in. nozzle at $30 \mathrm{psi}$ with operating costs in the range of $\$ 1.75 / \mathrm{ft}^{2}$ or $\$ 45-\$ 65 / \mathrm{h}$ using skilled labor. ${ }^{3}$ Plastic media has some advantages over grits, like sand and metal shot, in that it can more easily be separated from eroded metallic substrate and it can be disposed by incineration, thus giving it the potential to significantly reduce waste disposal costs.

Science/Technology Needs: Technology improvement is needed in waste minimization related to blast media erosion and disposal; containment of waste; and demonstration of 
specific blast-media substrate/contaminant capabilities, including determination of decontamination factors and process automation.

Implementation Needs: This technology, while developed and commercially available, will require a system for processing the waste. The device will generate contaminated dust, gases, and/or fluids. This will require a system to separate and to package the contaminants. These developments should cost less than $\$ 200 \mathrm{~K}$. Capital cost-\$100K; operating cost $>\$ 1 / \mathrm{ft}^{2}$.

Author: J. H. Wilson/615-574-4413. Reviewed by R. B. Alderfer/615-576-0225

\section{References:}

1. Stripping Technologies, Tucson, Ariz. 85706.

2. Progressive Blasting Systems, Grand Rapids, Mich. 49508.

3. K. E. Abott, Plastic Media Blasting-The Maturing of the Technology, 27th Annual Aerospace/Airline Plating \& Metals Finishing Forum \& Exposition, San Antonio, Tex., March 26-28, 1991.

4. J. H. Wilson and other MMES staff members. 
EM Problem: Decontamination and Decommissioning

Y-12 Plant Problem: Building 9201-4 (Alpha-4)

\section{Problem Area/Constituents:}

- In situ decontamination of the exterior of steel and nickel process equipment contaminated with $\mathrm{Hg}, \mathrm{LiOH}, \mathrm{H}_{2} \mathrm{SO}_{4}, \mathrm{HNO}_{3}$, and asbestos.

- Ex situ decontamination of steel and nickel process equipment contaminated with $\mathrm{Hg}$, $\mathrm{LiOH}, \mathrm{H}_{2} \mathrm{SO}_{4}, \mathrm{HNO}_{3}$, and asbestos.

\section{Reference Requirements:}

- Federal Facility Compliance Agreements (e.g., FFCA for Oak Ridge Site and FFCAs for PCBs)

- Applicable federal and state laws and regulations (e.g., Clean Air Act and Toxic Substances Control Act)

- DOE orders

\section{Subelement: Decontamination}

Alternative: Mechanical surface removal methods

Technology: Hand grinding, honing, and scraping. Hand-held, power-driven grinding equipment is used to remove the surface from the contaminated object.

Status: Accepted. Grinding with hand-held power grinders has been successfully used for small-scale decontamination. ${ }^{1}$ The head generated by the grinding process will vaporize mercury.

Efficacy/strengths/weaknesses-Grinding is effective only on surface contamination.

Waste-Surface area of item being decontaminated, spent grinding media (such as emery paper), and the adsorption canisters and HEPA filters on the vacuum system.

Cost-Operating costs, $\$ 1 / \mathrm{ft}^{2}$; capital costs, $\$ 150 \mathrm{~K}$ ( $\$ 10 \mathrm{~K}$ with vacuum system and $\$ 50-500 \mathrm{~K}$ with remote operation).

Science/Technology Needs: If possible, a remotely operated system should be developed to reduce workers' radiation exposure.

Implementation Needs: A system will be needed to remove dust and mercury vaporized by the heat generated during grinding. 
Author: D. H. Bunch/615-576-0201

Reference:

1. D. H. Bunch, June 16, 1992. 
EM Problem: Decontamination and Decommissioning

Y-12 Plant Problem: Building 9201-4 (Alpha-4)

\section{Problem Area/Constituents:}

- In situ decontamination of the exterior of steel and nickel process equipment contaminated with $\mathrm{Hg}, \mathrm{LiOH}, \mathrm{H}_{2} \mathrm{SO}_{4}, \mathrm{HNO}_{3}$, and asbestos.

- Ex situ decontamination of steel and nickel process equipment contaminated with $\mathrm{Hg}$, $\mathrm{LiOH}, \mathrm{H}_{2} \mathrm{SO}_{4}, \mathrm{HNO}_{3}$, and asbestos.

\section{Reference Requirements:}

- Federal Facility Compliance Agreements (e.g., FFCA for Oak Ridge Site and FFCAs for PCBs)

- Applicable federal and state laws and regulations (e.g., Clean Air Act and Toxic Substances Control Act)

- DOE orders

\section{Subelement: Decontamination}

Alternative: Mechanical surface removal methods

Technology: Automated grinding. Remotely operated, power-driven grinding equipment with a manipulator for turning and moving the contaminated item is used to remove the top surface layer of the contaminated object.

Status: Conceptual. Grinding has been successfully used for small-scale decontamination. This grinding was done with hand-held power grinders. ${ }^{1}$ Remotely operated grinding equipment is available, ${ }^{2}$ but no references to its use for decontamination have been found. The heat generated by the grinding process will vaporize mercury.

Efficacy/strengths/weaknesses-Grinding is effective only on surface contamination.

Waste-The surface layer of the item being decontaminated, spent grinding media (such as emergy paper), and the adsorption canisters and HEPA filters on. the vacuum system.

Cost-Capital costs, $\$ 250 \mathrm{~K}$; operating costs, $\$ 1 / \mathrm{ft}^{2}$.

Science/Technology Needs: The applicability of this technology to items with rad contamination should be demonstrated. A system to contain and treat the off-gas needs to be developed.

Implementation Needs: Development cost- $\$ 1 \mathrm{M}$. A collection system for the dust and vaporized mercury will be needed to implement this technology. 
Author: D. H. Bunch/615-576-0201

\section{References:}

1. D. H. Bunch, June 16, 1992.

2. Harry G. Movat Company, telephone conversation with D. H. Bunch, June 15, 1992. 
EM Problem: Decontamination and Decommissioning

Y-12 Plant Problem: Building 9201-4 (Alpha-4)

Problem Area/Constituents: Ex situ decontamination of steel and nickel process equipment contaminated with $\mathrm{Hg}, \mathrm{LiOH}, \mathrm{H}_{2} \mathrm{SO}_{4}, \mathrm{HNO}_{3}$, and asbestos.

Reference Requirements: Refer to Vol. 1, Chap. 10, for potentially applicable proposed and promulgated environmental laws, signed and pending agreements for the Oak Ridge Reservation, radiation protection standards, DOE orders, and nonregulatory guidance. As site- and waste-specific characteristics are provided for each technology, specific regulatory requirements will be specified.

\section{Subelement: Decontamination}

Alternative: Mechanical surface removal methods

Technology: Metal milling. A machine shaves off a layer of metal.

Status: Accepted. Milling has been used to decontaminate metal items. ${ }^{1}$ This method is most suitable when there are many similar items to be decontaminated because there is a $1 / 2$ to $3 / 4 \mathrm{~h}$ setup time required between differently shaped items. ${ }^{2}$ The heat generated by the milling process causes organic compounds on the surface of the item being decontaminated to decompose. ${ }^{3}$ Milling has been accepted in the past because the generation of these vapors was overlooked. The technology needed to control these vapors has not been identified.

Efficacy/strengths/ weaknesses-This method can remove only surface contamination.

Waste-The top layer (up to $1 / 8$ in.) of the metal would be chipped off. ${ }^{1}$

Cost-Capital costs, $\sim \$ 150 \mathrm{~K}$; operating costs, $\sim \$ 20 / \mathrm{ft}^{2}$.

Science/Technology Needs: Improvement-An off-gas containment and treatment system needs to be developed.

Implementation Needs: The contaminated equipment must be removed and disassembled before this technology can be used. Development cost (Improvement)-Off-gas containment and treatment, \$1M.

Author: D. H. Bunch/615-576-0201 


\section{References:}

1. M. A. Trisel, telephone conversation with D. H. Bunch, June 5, 1992.

2. N. F. Knueman and M. A. Trisel, personal communication to D. H. Bunch, June 12, 1992.

3. John Googin, written comments on draft of $K-25$ Site Technology Logic Diagram, November 1992. 
EM Problem: Decontamination and Decommissioning

Y-12 Plant Problem: Building 9201-4 (Alpha-4)

Problem Area/Constituents: See decontamination section Vol. 1.

\section{Reference Requirements:}

- Federal Facility Compliance Agreements (e.g. FFCA for Oak Ridge Site and FFCAs for PCBs)

- Applicable federal and state laws and regulations (e.g. Clean Air Act and Toxic Substances Control Act)

- DOE orders

\section{Subelement: Decontamination}

Alternative: Mechanical Surface Removal Methods

Technology: Electro-Hydraulic Scabbling. The process involves aggressive scabbling of contaminated concrete surfaces by an extension of the EHS system in use for over 20 years. The initial application of EHS was for pulsed lasers and currently uses electro-hydraulic forces to grind minerals and pump fluids. The scabbling of concrete is considered an extension of these technologies and of pulsed systems. In this process, shock waves and cavitating bubbles are generated in water by electronic pulses. The pulse energy and frequency controls the depth of the scabbling action.

Status: Demonstration. To be successful, the established technology must be demonstrated for effective scabbling action on concrete surfaces.

Efficacy/strengths, weaknesses - The secondary waste stream will include a large amount of concrete fines and rubble that must be processed to remove the aggregate contaminants or otherwise safely stored. Thus waste characterization is an important prerequisite. The advantages include fast, aggressive surface removal with an operation that lends itself to remove robotic operations. There are no airborne dust formation concerns and a moderate equipment cost.

Waste-Scabbled concrete and decontamination materials.

Cost-Operating cost is estimated at $\$ 2.00 / \mathrm{ft}^{2}$ of surface.

Science/Technology Needs: The availability of engineered equipment is adequate provided that demonstrations verify effective surface removal rates. Secondary waste treatment effectiveness needs to be addressed. Effective integration with robotic control should be determined for special control areas. 
Implementation Needs: Demonstration costs are estimated at $\$ 750 \mathrm{~K}-\$ 1000 \mathrm{~K}$ including $\$ 250$ K for equipment procurement.

Author: R. B. Alderfer/615-576-0225

\section{References:}

1. Public information release from Textron Defense Systems (TDS) Everett, Mass.

2. MMES staff members. 
EM Problem: Decontamination and Decommissioning

Y-12 Plant Problem: Building 9201-4 (Alpha-4)

Problem Area/Constituents: Painted concrete and clay tile contaminated with PCBs, oil, $\mathrm{U}$ (and daughters), electrolytes, $\mathrm{Cd}, \mathrm{Pb}$, and $\mathrm{Cr}$.

\section{Reference Requirements:}

- Federal Facility Compliance Agreements (e.g., FFCA for Oak Ridge Site and FFCAs for PCBs)

- Applicable federal and state laws and regulations (e.g., Clean Air Act and Toxic Substances Control Act)

- DOE orders

Subelement: Decontamination

Alternative: Mechanical surface removal methods

Technology: Concrete milling. A machine shaves away the top layer of the concrete. For large-area horizontal surfaces the large milling vehicles used by paving contractors would be employed. Smaller equipment is available for milling smaller areas.

Status: Conceptual. The equipment is commercially available, ${ }^{1}$ but no reference to its use for decontamination has been found. ${ }^{2}$

Efficacy/strengths/weaknesses-This technology is only suited for horizontal surfaces.

Waste-The top 6-25 $\mathrm{mm}$ of the concrete is removed. ${ }^{1}$

Cost-For large areas: Capital costs, $\$ 50 \mathrm{~K}-\$ 363 \mathrm{~K}$ (this does not include the cost of trucks to haul away the debris); operating costs, $\$ 0.5 \mathrm{M}-\$ 1.6 \mathrm{M} / \mathrm{sq}$. mile. ${ }^{2}$ For small areas: Capital costs, $\$ 11 \mathrm{~K}_{;}^{3}$ operating costs, $\$ 2 / \mathrm{ft}^{2}$.

Science/Technology Needs: The technology to operate this equipment remotely needs to be developed and demonstrated.

Implementation Needs: Development costs, $\$ 3 \mathrm{M}$ for automation

Author: D. H. Bunch/615-576-0201 


\section{References:}

1. M. M. Barbier and C. V. Chester, "Decontamination of Large Horizontal Concrete Surfaces Outdoors," Proc. of the Concrete Decontamination Workshop, Richland, Wash., September 1980.

2. C. V. Chester, telephone conversation with D. H. Bunch, June 4, 1992.

3. John Summer, ASPC, Bellevue, Tenn., facsimile to D. H. Bunch, March 29, 1993. 
EM Problem: Decontamination and Decommissioning

X-12 Plant Problem: Building 9201-4 (Alpha-4)

Problem Area/Constituents: Painted concrete and clay tile contaminated with PCBs, oil, $\mathrm{U}$ (and daughters), electrolytes, $\mathrm{Cd}, \mathrm{Pb}$, and $\mathrm{Cr}$.

\section{Reference Requirements:}

- Federal Facility Compliance Agreements (e.g., FFCA for Oak Ridge Site and FFCAs for PCBs)

- Applicable federal and state laws and regulations (e.g., Clean Air Act and Toxic Substances Control Act)

- DOE orders

Subelement: Decontamination

Alternative: Mechanical surface removal methods

Technology: Explosive. The top 3-4 in. of concrete are removed by detonating carefully placed and timed explosive charges.

Status: Accepted. Blasting has been used to decontaminate nuclear reactor shielding. ${ }^{1}$ Demonstration may be needed for other applications.

Cost-Capital costs, $<\$ 50 \mathrm{~K}$; operating costs, $\$ 50 / \mathrm{ft}^{2}$

Science/Technology Needs: Safety concerns need to be resolved; care must be taken to contain the dust and to prevent structural damage to the building being decontaminated and the surrounding structures. ${ }^{1}$ Improvements in the methods of applying explosives and in the uniformity of the detonation are needed. ${ }^{2}$ The explosion will generate toxic organic vapors and mercury vapors. ${ }^{3}$ The technology to control these vapors must be developed and demonstrated.

Implementation Needs: A certified blasting technician will be needed. ${ }^{2}$ Development costs: up to $\$ 4 \mathrm{M}$

Author: D. H. Bunch/615-576-0201 


\section{References:}

1. T. S. Laguardia, "Concrete Decontamination and Demolition Methods," Nuclear Energy Services, Inc. Danbury, Conn., Proceedings of the Concrete Decontamination Workshop, Pacific Northwest Laboratory, September 1980.

2. Decontamination and Decommissioning Integrated Demonstration Strategy (Draft for Review), November 1991, page 2-38. 


\section{EM Problem: Decontamination and Decommissioning}

\section{Y-12 Plant Problem: Bldg. 9201-4 (Alpha-4)}

Problem Area/Constituents: Painted concrete and clay tile contaminated with PCBs, oil, $\mathrm{U}$ (and daughters), electrolytes, $\mathrm{C}_{\mathrm{d}}, \mathrm{P}_{\mathrm{b}}, \mathrm{C}_{\mathrm{r}}$ and $\mathrm{H}_{\mathrm{g}}$

\section{Reference Requirements:}

- Federal Facility Compliance Agreements (e.g., FFCA for Oak Ridge Site and FFCAs for PCBs)

- Applicable federal and state laws and regulations (e.g., Clean Air Act and Toxic Substances Control Act)

- DOE orders

\section{Subelement: Decontamination}

\section{Alternative: Surface Cleaning Methods}

Technology: Concrete chipping. Paving breakers and chipping hammers remove concrete (and asphalt) by mechanically fracturing localized sections of the surface. Fracturing is caused by the impact of a hardened tool steel bit of either a chisel or moil point shape. The bit is driven in a reciprocating motion by either a compressed air or hydraulic fluid pressure source. Removed material is collected, treated, and disposed of.

Paving breakers (i.e., jackhammers pneumatic drills) weight approximately 35-100 lb and are intended for use on floors. Paving breakers deliver about 1500 blows per minute at up to $95 \mathrm{ft}-\mathrm{lb}$ of energy per blow, depending on the size of the unit. The chipping hammer is similar in concept to the paving breaker but is light enough (15-35 lb) to be hand held for use on walls or ceilings. Chipping hammers deliver about 2000 blows per minute.

Paving breakers are recommended for use on floors to remove small areas that are inaccessible for other heavy equipment. They may also be used to expose reinforcing rods after controlled blasting to permit cutting of the rods. The chisel point may be used to scarify surface areas of concrete floors where contamination may have penetrated several inches deep in localized areas. Contamination control may be accomplished using water or fog sprays. Chipping hammers are recommended for use on walls to scarify small areas where contamination may have penetrated several inches deep over localized areas. However, the limited removal capacity and significant weight (up to $35 \mathrm{lb}$ ) make it impractical for use on large areas.

Paving breakers and chipping hammers leave the treated surface very rough and resurfacing is required if future use of the structure is applicable. 
Status: Accepted. This technology has been used successfully for many applications in the nuclear industry. Technology is generally effective. Waste is up to $20 \mathrm{yd}^{3}$ removed concrete per day for breaker, and $27 \mathrm{ft}^{3} /$ day for a chipper containing removed contaminants plus worn hammers and chippers.

Science/Technology Needs: Improvement-Automation, especially for walls and ceilings, including collection system for chipped, contaminated concrete.

Implementation Needs: A dust collection system is needed for the chipped concrete. Development Cost: None; Capital cost: $<\$ 50 \mathrm{~K}$; Operating cost: $\$ 5 / \mathrm{ft}^{2}$

Author: W. D. Nelson/615-574-0966 
EM Problem: Decontamination and Decommissioning

Y-12 Plant Problem: Building 9201-4 (Alpha-4)

Problem Area/Constituents: Painted concrete and clay tile contaminated with PCBs, oil, $\mathrm{U}$ (and daughters), electrolytes, $\mathrm{C}_{\mathrm{d}}, \mathrm{P}_{\mathrm{b}}, \mathrm{C}_{r}$, and $\mathrm{H}_{\mathrm{g}}$

\section{Reference Requirements:}

- Federal Facility Compliance Agreements (e.g., FFCA for Oak Ridge Site and FFCAs for PCBs)

- Applicable federal and state laws and regulations (e.g., Clean Air Act and Toxic Substances Control Act)

- DOE orders

\section{Subelement: Decontamination}

Alternative: Mechanical Surface Removal Methods

Technology: Slurry Blasting. The process uses a combination of grit and high-pressure water to provide an intermediate aggressive decontamination process. Its aggressiveness is between water blasting and grit blasting. Engineered systems are available for a variety of decontamination applications including in situ and ex situ installations. The process includes on-line recycling with special separators and filters used as required. Pressures up to 3000 psi have been used with water temperatures up to $180 \mathrm{deg} F$. Both smearable and fixed radiological contamination have been removed successfully. Normally the grit is aluminum oxide, however, glass beads have been used successfully when protection of the substrate is important. About 500 gal of water are used with 75 lbs of grit for a typical application. Depths of several mils have been demonstrated in varying applications. It is claimed that paint and corrosion products are removed quite easily.

Status: Demonstration. To be successful, the established technology should efficiently decontaminate tile, concrete, and a variety of metal process equipment configurations.

Efficacy/strengths/weaknesses-The secondary waste stream will include the spent grit in an aqueous matrix along with the contaminants. The separation methods will depend on the properties an volumes of the overall contaminant mixture. The advantages include a moderately aggressive process compared to high pressure water alone and no significant dusting problems compared with grit blasting alone. Equipment costs should be $<\$ 0.5 \mathrm{M}$ due to the variety of existing engineering designs currently in operation. The wastes will include the abraded material surface and the contaminate materials. The overall cost is estimated at $\$ 1.75 / \mathrm{ft}^{2}$ of surface. 
Science/Technology Needs: The availability of engineered equipment should be adequate provided that demonstrations verify effective removal rates on the substrates and contaminants in question. The on-line recycle of the decontaminating materials needs to be verified under operating conditions.

Implementation Needs: Demonstration costs are estimated at $\$ 500 \mathrm{~K}$. The possible need to evaluate more than one configuration to cover both in situ and ex situ applications may boost this figure by $75 \%$.

Author: R. B. Alderfer/615-576-0225

\section{References:}

1. Public information release from Kleiber\&Schulz, Inc., Farmingdale, N.Y. 11735.

2. Jim Turley/615-574-2492.

3. MMES staff members. 
EM Problem: Decontamination and Decommissioning

Y-12 Plant Problem: Bldg. 9201-4 (Alpha-4) decontamination and decommissioning

\section{Problem Area/Constituents:}

- In situ decontamination of the interior of steel and nickel process equipment contaminated with $\mathrm{Hg}, \mathrm{LiOH}, \mathrm{H}_{2} \mathrm{SO}_{4}$, and $\mathrm{HNO}_{3}$.

- In situ decontamination of the exterior of steel and nickel process equipment contaminated with $\mathrm{Hg}, \mathrm{LiOH}, \mathrm{H}_{2} \mathrm{SO}_{4}$, and $\mathrm{HNO}_{3}$ asbestos, and PCBs.

- Ex situ decontamination of steel and nickel process equipment contaminated with $\mathrm{Hg}$, $\mathrm{LiOH}, \mathrm{H}_{2} \mathrm{SO}_{4}$, and $\mathrm{HNO}_{3}$, asbestos, and PCBs.

\section{Reference Requirements:}

- Federal Facility Compliance Agreements (e.g., FFCA for the Oak Ridge Site)

- Applicable federal and state laws and regulations

- Clean Water Acts and Toxic Substances Control Act

- DOE orders.

Refer to the Regulatory Compliance chapter of Vol. 1 for potentially applicable proposed and promulgated environmental laws, signed and pending agreements for the Oak Ridge Reservation, radiation protection standards, DOE orders, and nonregulatory guidance. As site- and waste-specific characteristics are provided for each technology, specific regulatory requirements will be specified.

\section{Subelement: Decontamination}

Alternative: Surface cleaning (transferable contamination) methods

Technology: Compressed-air cryogenic $\mathrm{CO}_{2}$ pellet blasting. Similar to traditional sandblasting except that pellets made of solid $\mathrm{CO}_{2}$ (dry ice) are used instead of sand. Depending on the pellet impact velocity and the substrate material, the pellets clean by removing surface contamination or removing/etching the substrate. As in sandblasting, the surfaces to be decontaminated must be accessible to the blast nozzle.

Status: Demonstration. This technology is commercially available. ${ }^{1.2}$ It has been used at nuclear reactor sites to decontaminate hand tools and some equipment..$^{3-6}$ With a person operating the "gun," it requires a few minutes to decontaminate a hand tool. The operation of the device is tiring to the operator so that either a remote manipulator or frequent rest periods would be required for large tasks or continuous use.

Because the dry ice pellets evaporate on contact, the secondary waste stream is minimized. The contamination "blasted" from the surface is collected via vacuum/HEPA filtration system, which is either a closely coupled shroud over the surface or a specially designed room air 
filtration system. The secondary wastes generated would be limited to the HEPA and rough filters. The technology is known to be effective at removing smearable contamination. The removal of fixed contamination depends on the nature of the bonding. The technology, for example, can remove paint and loose rust, but it is not aggressive enough to etch steel or remove black oxide from steel. Tests using a $\mathrm{CO}_{2}$ system for decontamination are limited, but a fixed contamination reduction ratio of $3: 1$ was reported for concrete at a rate of $90 \mathrm{ft}^{2} / \mathrm{h}$ and 5000:1 for angle iron at $10 \mathrm{ft}^{2} / \mathrm{h}^{3}$

Waste-This system uses large volumes of compressed air to accelerate $\mathrm{CO}_{2}$ pellets. This air will need to be processed to extract primary waste components once the contaminating waste is mobilized.

Science/Technology Needs: Because the cleaning rate is a dominant factor in the cost, and it is highly variable, depending on the nature of the specific contaminant and surface material, more extensive tests need to be done to produce accurate cost estimates. Automation should be developed for cleaning high walls and ceilings. Improved waste handling is needed.

Implementation Needs: Waste handling, design, and construction of equipment to handle the waste stream is needed. Application of this technology requires staff with technician training. The operating cost of a typical unit is approximately $\$ 160 \mathrm{~K}$. For a $100-\mathrm{kW}$ unit at $\$ .06 \mathrm{kWh}$, energy costs would be $\$ 6 / \mathrm{h}$. Labor costs for a manually operated system would require two operators, alternating, at $\$ 200 / \mathrm{h}$. A remotely manipulated/robotics system could reduce labor costs by allowing multiple guns to be operated by one person. Manual operation would be $\$ 216 / \mathrm{h}$ (G) $100 \mathrm{ft}^{2} / \mathrm{h}=\$ 2.0 / \mathrm{ft}^{2} ; 10 \mathrm{ft}^{2} / \mathrm{h}=\$ 20 / \mathrm{ft}^{2}$. A remote manipulator system with one operator and four guns would be $\$ 164 / \mathrm{h} @ 400 \mathrm{ft}^{2} / \mathrm{h}=\$ 0.41 / \mathrm{ft}^{2}$; (a) $40 \mathrm{ft}^{2} / \mathrm{h}=\$ 4.10 / \mathrm{ft}^{2}$; capital costs, $\sim \$ 200 \mathrm{~K}$; development cost, $-\$ 0.25 \mathrm{M}$.

Authors: C. A. Foster/615-574-1128 and W. D. Nelson/615-574-0966

\section{References:}

1. Alpheus Cleaning Technologies, Rancho Cucamonga, Calif. 91730.

2. Cold Jet, Loveland, Ohio 45140.

3. Chem Nuclear Systems, Evaluation of Pelletized Carbon Dioxides a Fluidized Abrasive Agent for Removal of Radioactive Contamination, April 1989.

4. Power Reactor and Nuclear Fuel Development Corporation,"Overview and Strategy for Managing TRU Waste," PNC/DOE/PNC Specialist Meeting, October 17-18, 1989.

5. Environmental Alternatives: Summary and Analysis for a Carbon Dioxide Blasting Demonstration at Georgia Power Company, Hatch Station.

6. TTI Engineering, "Report on Nondestructive Cleaning Decontamination Facility at Surry Power Station," November 1990, Seminar viewgraphs from seminar held March 22, 1991.

September 1994

Decontamination and Decommissioning 


\section{EM Problem: Decontamination and Decommissioning}

\section{Y-12 Plant Problem: Bldg. 9201-4 (Alpha-4)}

Problem Area/Constituents: Nickel and steel process equipment contaminated with mercury, lithium hydroxide, sulfuric acid, nitric acid, asbestos, and PCBs. Painted concrete and clay tile contaminated with $\mathrm{PCBs}$, ofl, $\mathrm{U}$ (and daughters), $\mathrm{Cd}, \mathrm{Pb}$, and $\mathrm{Cr}$.

Reference Requirements: Refer Vol. 1, Chap. 10, for potentially applicable proposed and promulgated environmental laws, signed and pending agreements for the Oak Ridge Reservation, radiation protection standards, DOE orders, and nonregulatory guidance. As site- and waste-specific characteristics are provided for each technology, specific regulatory requirements will be specified.

\section{Subelement: Decontamination}

Alternative: Surface cleaning (transferable contamination) methods

Technology: High-pressure water (5,000-20,000 psig). Blasting of surfaces with high pressure water will remove deposits that are tightly adhered to substrate materials. Water pressure at the nozzle of commercially available high-pressure blasting systems is typically $5,000-20,000 \mathrm{psig}$. Flow rates vary from 4 to $100 \mathrm{gpm}$. When used with chemical additives, this technique can be effective for decontaminating piping trays, overhead surfaces, upper regions of walls, gratings, pumps, and similar items. However, splashing from blast operations can be extensive and result in recontamination if precautions are not taken. In the use of this decontamination technology, the high-pressure water cleaning head may be manually moved on the surfaces being decontaminated. Because the decontamination efficiency depends on the applicator translation speed, as well as on other operating parameters, remote operation of the high-pressure-water cleaning head would be desirable.

Status: Accepted by industry. High-pressure water blasting has been used very successfully to decontaminate various large and complex surfaces at nuclear power plants. These include coolant pump impellers and cavities, fuel storage racks and handling equipment, floor drains and sumps, reactor cavity walls, pipes, and valves. When used as a preliminary step to wiping or scrubbing activities, decontamination factors (DFs) in the range of 50 are commonly achieved for loosely deposited and loosely adherent contamination. Experience has demonstrated that blasting effectiveness becomes negligible when the cleaning nozzle tip is more than 10-12 in. away from the surface being cleaned. However, before acceptance at ORNL and other DOE sites, the issues of wastewater treatment and prevention of spread of contamination must be addressed. The waste generated is the contaminated water from the cleaning operation. The waste production rate is essentially the same as the water rate in the cleaning step, which can range from 4 to $>100 \mathrm{gpm}$.

Science/Technology Needs: To minimize waste generation, a water treatment system is needed to decontaminate the wastewater so that the water can be recycled and reused in the 
high-pressure water cleaning operation. Remote operation will necessitate the adaptation of the mobile high-pressure water and vacuum collection systems to a robotics control system.

Implementation Needs: Development costs: For development and demonstration of a water recycle system, in combination with high-pressure water decontamination, an estimate of funding requirements is $\$ 1.2 \mathrm{M}$. Personnel requirements are estimated to be 4.1 FTEs (approximately 10\% P\&E, 45\% engineer, and 45\% technician time) for FY 93 and FY 94, respectively. The cost of a robotics control system is estimated at $\$ 250 \mathrm{~K}$. This is based on the cost of a system that is available from the Remotek Company. Estimated cost of a glove box is $<\$ 50 \mathrm{~K}$; work room, $>\$ 250 \mathrm{~K}$. Operating cost will be $<\$ 1 / \mathrm{ft}^{2}$.

Deployment costs: The capital cost of a high-pressure water cleaning unit will be in the $\$ 50 \mathrm{~K}-\$ 175 \mathrm{~K}$ range. Operating costs for a high-pressure water cleaning unit would primarily involve labor costs. Assuming a minimum two-person operation (at a labor rate of $\$ 40 \mathrm{~K} /$ year/person) during the day shift, operating costs would be approximately $\$ 350 /$ day. Personnel training for operation of a high-pressure water unit and a water treatment and recycle system would be required.

Author: K. E. Lott/615-574-6537

References: MMES staff members. 
EM Problem: Decontamination and Decommissioning

Y-12 Plant Problem: Bldg. 9201-4 (Alpha-4)

Problem Area/Constituents: Nickel and steel process equipment contaminated with mercury, lithium hydroxide, sulfuric acid, nitric acid, asbestos, and PCBs. Painted concrete and clay tile contaminated with PCBs, oil, $\mathrm{U}$ (and daughters), $\mathrm{Cd}, \mathrm{Pb}$, and $\mathrm{Cr}$.

Reference Requirements: Refer Vol. 1, Chap. 10, for potentially applicable proposed and promulgated environmental laws, signed and pending agreements for the Oak Ridge Reservation, radiation protection standards, DOE orders, and nonregulatory guidance. As site- and waste-specific characteristics are provided for each technology, specific regulatory requirements will be specified.

\section{Subelement: Decontamination}

Alternative: Surface cleaning (transferable contamination)

Technology: Superheated water (e.g., at conditions of $300 \mathrm{psi}$ and $300^{\circ} \mathrm{F}$ ). Directed as a high velocity stream on a surface to remove contamination. The contaminated wastewater that is must be treated for discharge or for recycle back to the operation. The superheated water cleaning head may be manually moved about on the surfaces being decontaminated. Also, a hand-held wand may be more conveniently used for vertical surfaces, equipment surfaces, etc. Because the decontamination efficiency depends on the applicator translation speed, as well as on other operating parameters, remote operation of the superheated water cleaning head would be desirable. Also, in some applications (such as decontamination of hot cells), remote operation will be required. Consequently, the adaptation of the equipment to a robotic control system would be necessary.

Status: Accepted in industry. Technology is available and has been used by industry. However, before acceptance at ORNL and other DOE sites, the issues of wastewater treatment and prevention of spread of contamination must be addressed. Because of the relatively low operating pressures (as compared to ultrahigh-pressure water at 30,000-50,000 psi), superheated water will only remove surface contamination that is soluble or loosely bound to the surface. In these cases, the decontamination efficiency should be high. However, for tightly bound or imbedded surface contamination, removal may be insignificant. The waste generated is the contaminated water from the cleaning operation. The wastewater generation rate for a typical commercial unit ranges from $0.4-2 \mathrm{gpm}$.

Science/Technology Needs: To minimize waste generation, a water treatment system is needed to decontaminate the wastewater so that the water can be recycled and reused in the superheated water cleaning operation. Remote operation will necessitate the adaptation of the mobile cleaning and vacuum collection system to a robotic control system. 
Implementation Needs: Development costs: For development and demonstration of a water recycle system, in combination with high-pressure water decontamination, an estimate of funding requirements is $\$ 1.2 \mathrm{M}$. Personnel requirements are estimated to be 4.1 FTEs (approximately 10\% P\&E, 45\% engineer, and 45\% technician time) for FY 93 and FY 94, respectively. The cost of a robotics control system is estimated at $\$ 250 \mathrm{~K}$. This is based on the cost of a system that is available from the Remotek Company.

Deployment costs: The capital cost of a basic unit (spray-vacuum cleaning system) is $\$ 50 \mathrm{~K}-\$ 175 \mathrm{~K}$. Various attachments are available for specialty cleaning. Operating costs for a superheated water cleaning unit would primarily involve labor costs. Assuming a minimum two-person operation (at a labor rate of $\$ 40 \mathrm{~K} / \mathrm{year} /$ person) of a cleaning unit during the day shift, operating costs would be approximately $\$ 350 /$ day. Training of personnel for operation of the superheated water cleaning unit and the wastewater treatment system will be needed. Other capital costs include $<\$ 50 \mathrm{~K}$ for a glove box and $>\$ 250 \mathrm{~K}$ for a work room. Operating costs will be $-\$ 2 / \mathrm{ft}^{2}$.

Author: K. E. Lott/615-574-6537

References: MMES staff members, especially J. H. Wilson.

September 1994

Decontamination and Decommissioning 
EM Problem: Decontamination and Decommissioning

Y-12 Plant Problem: Bldg. 9201-4 (Alpha-4)

Problem Area/Constituents: Nickel and steel process equipment contaminated with mercury, lithium hydroxide, sulfuric acid, nitric acid, asbestos, and PCBs. Painted concrete and clay tile contaminated with $\mathrm{PCBs}$, oil, $\mathrm{U}$ (and daughters), electrolytes, $\mathrm{Cd}, \mathrm{Pb}$, and $\mathrm{Cr}$.

Reference Requirements: Refer to Vol. 1, Chap. 10, for potentially applicable proposed and promulgated environmental laws, signed and pending agreements for the Oak Ridge Reservation, radiation protection standards, DOE orders, and nonregulatory guidance. As site- and waste-specific characteristics are provided for each technology, specific regulatory requirements will be specified.

\section{Subelement: Decontamination}

Alternative: Surface cleaning methods

Technology: Water flushing. Removal of soluble or loosely adherent contamination using water wash.

Status: Accepted. Water flushing is a very simple process which is a widely used pretreatment to most of the wet-process decontamination technologies.

Efficacy/strengths/weaknesses-Decontamination factors depend on the nature of contaminant and quantity of water used. Secondary waste requires treatment and disposal.

Cost-Water flush decontamination is used extensively at most DOE plants, but data are not available regarding costs. Costs at ORNL for processing low level liquid waste and process water are $\$ 8 / \mathrm{gal}$ and $5 \$ / \mathrm{gal}$ respectively. Rough cost estimates are development cost, -\$1.2M; capital cost, <\$50K; operating cost, $<\$ 1 / \mathrm{ft}^{2}$.

Science/Technology Needs: A water treatment system is needed to minimize liquid wastes from this technology.

Implementation Needs: None

Authors: K. E. Lott/615-574-6537

\section{Reference:}

1. J. R. DeVore, Martin Marietta Energy Systems, personal communication to K. E. Lott, November 1993. 


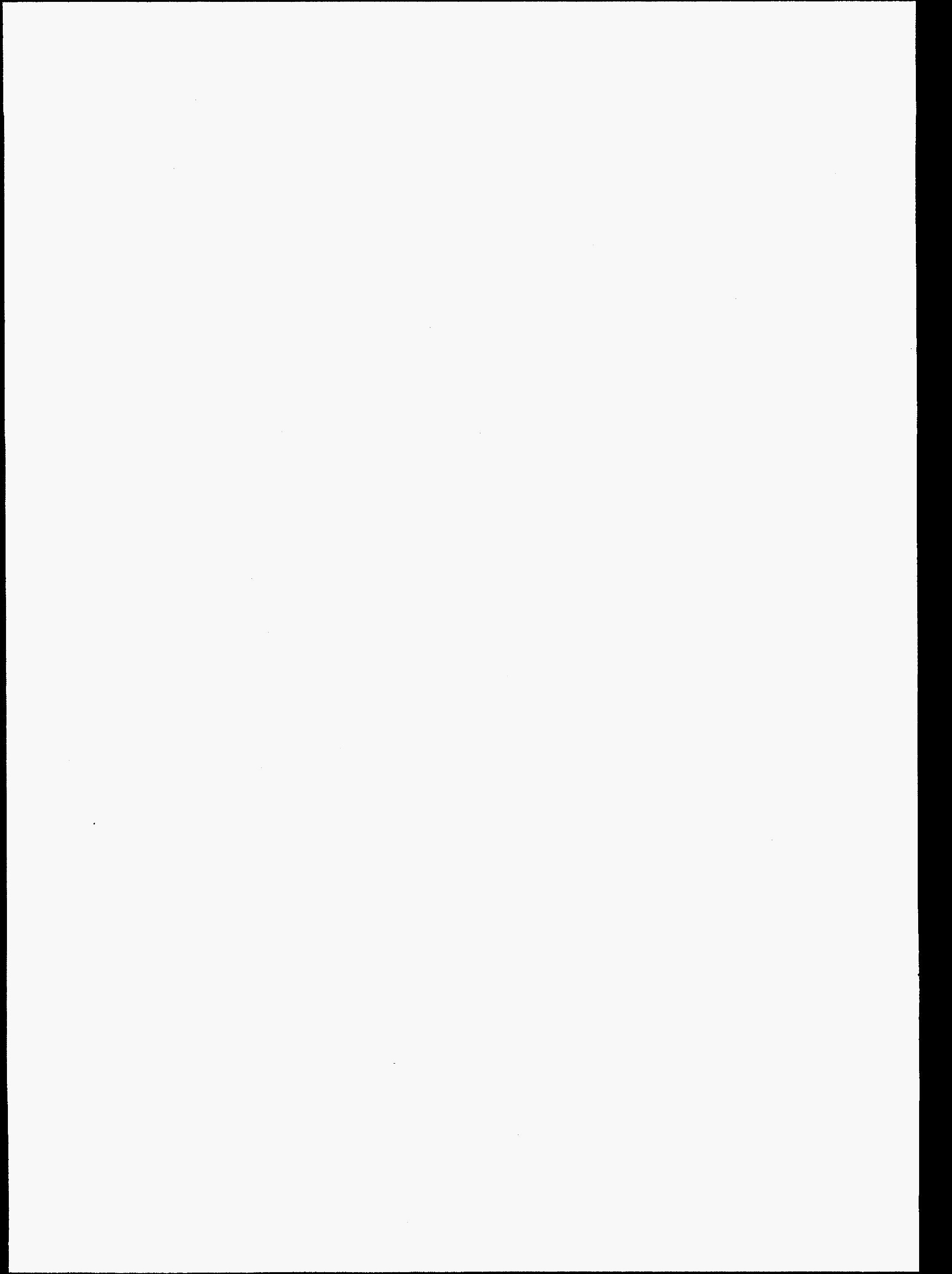


EM Problem: Decontamination and Decommissioning

Y-12 Plant Problem: Building 9201-4 (Alpha-4)

Problem Area/Constituents: Nickel and steel process equipment contaminated with mercury, lithium hydroxide, sulfuric acid, nitric acid, asbestos, and PCBs. Painted concrete and clay tile contaminated with $\mathrm{PCBs}$, oil, $\mathrm{U}$ (and daughters), electrolytes, $\mathrm{Cd}, \mathrm{Pb}$, and $\mathrm{Cr}$. Packing wood, and sheet rock contaminated with $\mathrm{Hg}, \mathrm{LiOH}, \mathrm{H}_{2} \mathrm{SO}_{4}$, and $\mathrm{HNO}_{3}$.

Reference Requirements: Refer to Vol. 1, Chap. 10, for potentially applicable proposed and promulgated environmental laws, signed and pending agreements for the Oak Ridge Reservation, radiation protection standards, DOE orders, and nonregulatory guidance. As site- and waste-specific characteristics are provided for each technology, specific regulatory requirements will be specified.

\section{Subelement: Decontamination}

Alternative: Surface Cleaning (transferable contamination)

Technology: Steam cleaning. Combines the solvent action of water with the kinetic energy effect of blasting. At relatively high temperatures, the solvent action is increased and the water volume requirements are reduced compared to water blasting. In the use of this decontamination technology, the stream cleaning head may be manually moved about on the surfaces being decontaminated. Also, a hand-held wand may be more conveniently used for vertical surfaces, equipment surfaces, etc. Because the decontamination efficiency depends on the applicator translation speed, as well as on other operating parameters, remote operation of the steam cleaning head would be desirable. Some applications (such as decontamination of hot cells) will require remote operation.

Status: Accepted. The technique has proven useful, especially on complex shapes and large surfaces. Good results can be obtained for surface decontamination with minimum rad waste volumes. Steam cleaning is quite effective for encrusted salt formations where leakage of chemically treated water has occurred. However, before acceptance at Y-12 and other DOE sites, the issues of wastewater treatment and prevention of the spread of contamination must be addressed. The waste generated is the contaminated condensed steam from the cleaning operation.

Efficacy/strengths/weaknesses-DFs, depend on the nature of contaminant. Not expected to be effective for removing fixed contamination of equipment interiors. Effectiveness for transferable contamination must be demonstrated.

Science/Technology Needs: To minimize waste generation, a water treatment system is needed for decontamination of the wastewater so that the water can be recycled and reused in the steam cleaning operation. 
Implementation Needs: Development costs-For development and demonstration of a water recycle system, in combination with steam decontamination, an estimate of funding requirements is $\$ 1.2 \mathrm{M}$. Personnel requirements are estimated to be 4.1 FTEs (approximately 10\% P\&E, 45\% engineer, and 45\% technician time) for FY 1993 and FY 1994, respectively.

Deployment costs-The capital cost of a steam cleaning unit will be in the $\$ 50 \mathrm{~K}-\$ 75 \mathrm{~K}$ range. Operating costs for a steam cleaning unit would primarily involve labor costs. Assuming a minimum two-person operation (at a labor rate of $\$ 40 \mathrm{~K} /$ year/person) of a steam cleaning unit during the day shift, operating costs would be approximately $\$ 350 /$ day or $\$ 1-\$ 2 / \mathrm{ft}^{2}$. Personnel training for operation of a steam cleaning unit and water recycle system will be needed. Capital costs, $<\$ 50 \mathrm{~K}$ for glove box.

Author: K. E. Lott/615-574-6537

\section{Reference:}

1. J. H. Wilson and C. E. Benson, Martin Marietta Energy Systems. 
EM Problem: Decontamination and Decommissioning

Y-12 Plant Problem: Building 9201-4 (Alpha-4)

Problem Area/Constituents:

- In situ decontamination of the exterior of steel and nickel process equipment contaminated with $\mathrm{Hg}, \mathrm{LiOH}, \mathrm{H}_{2} \mathrm{SO}_{4}, \mathrm{HNO}_{3}$, and asbestos.

- Ex situ decontamination of steel and nickel process equipment contaminated with $\mathrm{Hg}$, $\mathrm{LiOH}, \mathrm{H}_{2} \mathrm{SO}_{4}, \mathrm{HNO}_{3}$, and asbestos.

\section{Reference Requirements:}

- Federal Facility Compliance Agreements (e.g., FFCA for Oak Ridge Site and FFCAs for PCBs)

- Applicable federal and state laws and regulations (e.g., Clean Air Act and Toxic Substances Control Act)

- DOE orders

Subelement: Decontamination

Alternative: Surface cleaning methods

Technology: Hand brushing used to remove surface contamination.

Status: Accepted. Brushing is a common decontamination technique. ${ }^{1}$

Efficacy/strengths/weaknesses-Brushing is effective for smearable contamination and less effective for fixed contamination. ${ }^{2}$

Waste-HEPA filters and adsorption canisters on the vacuum cleaner used to pick up the particles generated by brushing and the worn brushes.

Cost-Capital costs, negligible; operating costs, $\$ 1 / \mathrm{ft}^{2}$.

Science/Technology Needs: None

Implementation Needs: None

Author: D. H. Bunch/615-576-0201 


\section{References:}

1. Paul Osbourne, telephone conversation with D. H. Bunch, August 10, 1992 .

2. R. P. Allen, "Overview of Nonchemical Decontamination Techniques," American Nuclear Society Executive Conference on Decontamination Techniques, CONF-840956, September 1984. 
EM Problem: Decontamination and Decommissioning

Y-12 Plant Problem: Building 9201-4 (Alpha-4)

Problem Area/Constituents: In situ decontamination of the interior of steel and nickel process equipment contaminated with $\mathrm{Hg}, \mathrm{LiOH}, \mathrm{H}_{2} \mathrm{SO}_{4}, \mathrm{HNO}_{3}$, and asbestos.

\section{Reference Requirements:}

- Federal Facility Compliance Agreements (e.g., FFCA for Oak Ridge Site and FFCAs for PCBs)

- Applicable federal and state laws and regulations (e.g., Clean Air Act and Toxic Substances Control Act)

- DOE orders

Subelement: Decontamination

Alternative: Surface cleaning methods

Technology: Automated brushing. Power-driven brushes are used to remove smearable contamination inside equipment such as pipes.

Status: Demonstration. This technology has been used at Rocky Flats to remove plutonium contamination from the inside of pipes. ${ }^{1}$ Tests would be needed to demonstrate its effectiveness for other contaminants on other substrates.

Efficacy/strengths/weaknesses-Brushing is effective for smearable contamination and less effective for fixed contamination. The interior of the pipes at Rocky flats was cleaned to shiny metal. ${ }^{1}$

Waste-The HEPA filters and adsorption canisters on the vacuums used to pick up the particles of contamination and the worn brushes.

Cost-Capital costs, $\$ 250 \mathrm{~K}$ (remotely operated system); operating costs, $\$ 300 /$ linear foot of pipe. ${ }^{1}$

Science/Technology Needs: Test to demonstrate that this technology is effective for the problems at Alpha 4

Implementation Needs: Development costs, $\$ 1 \mathrm{M}$

Author: D. H. Bunch/615-576-0201 


\section{Reference:}

1. T. Karas, Rocky Flats, telephone conversation with D. H. Bunch, May 13, 1993. 
EM Problem: Decontamination and Decommissioning

Y-12 Plant Problem: Building 9201-4 (Alpha-4)

\section{Problem Area/Constituents:}

- In situ decontamination of the exterior of steel and nickel process equipment contaminated with $\mathrm{Hg}, \mathrm{LiOH}, \mathrm{H}_{2} \mathrm{SO}_{4}, \mathrm{HNO}_{3}$, asbestos, and PCBs.

- Ex situ decontamination of steel and nickel process equipment contaminated with $\mathrm{Hg}$, $\mathrm{LiOH}, \mathrm{H}_{2} \mathrm{SO}_{4}, \mathrm{HNO}_{3}$, asbestos, and PCBs.

- Painted concrete and clay tile contaminated with PCBs, oil, U (and daughters), electrolytes, $\mathrm{Cd}, \mathrm{Pb}$, and $\mathrm{Cr}$.

\section{Reference Requirements:}

- Federal Facility Compliance Agreement for Oak Ridge Site

- Federal Facility Compliance Agreement for PCBs

- Clean Air Act

- Clean Water Act

- Toxic Substance Control Act

- DOE orders

Refer to the Regulatory Compliance chapter of Vol. 1 (Pt. A, B, or C as appropriate) for potentially applicable proposed and promulgated environmental laws, signed and pending agreements for the Oak Ridge Reservation, radiation protection standards, DOE orders, and nonregulatory guidance. As site- and waste-specific characteristics are provided for each technology, specific regulatory requirements will be specified.

\section{Subelement: Decontamination}

Alternative: Surface cleaning (transferable contamination) methods

Technology: Sponge blasting. Using air under 110 psig pressure as the propellant, the Sponge-Jet system decontaminates by blasting surfaces with various grades of patented water-based urethane foam cleaning media. The foam may be used dry or wetted for a variety of surface contaminants such as oils, greases, lead compounds, chemicals, and radionuclides. The following two types of foam cleaning media are used: (1) a nonaggressive grade that is used for surface cleaning of sensitive or otherwise critical surfaces, and (2) aggressive grades that are impregnated with abrasives that can remove tough materials such as paints, protective coatings, and rust (it can also roughen concrete and metallic surfaces if desired). The cleaning heads of the Sponge-Jet unit are similar to those of other blasting technologies and could be readily adapted to a robotic control system. Robotic control is required in applications where workers could be exposed to unacceptable levels of contamination. 
Status: Accepted. The technology is relatively new. It is currently being used by a company in Tennessee that produces projectiles containing uranium and by a nuclear power plant in Pennsylvania. Extensive data on decontamination factors are not available. Foam blasting media are recyclable in a closed-cycle wash unit that centrifugally launders the cleaning media. The media typically can be recycled eight to ten times. On the first time through, the sponge blasting unit uses $6-8 \mathrm{ft}^{3}$ of media per hour at a surface cleaning rate of about $1 \mathrm{ft}^{2} / \mathrm{min}$. Thus, the solid waste produced (foam media with the absorbed contaminants) is approximately $0.01 \mathrm{ft}^{3} / \mathrm{ft}^{2}$ of surface cleaned. For waste minimization purposes, the contaminated washwater from the wash unit can be treated and recycled.

Science/Technology Needs: To minimize waste generation, a water treatment system is needed to decontaminate the wastewater so that the water can be recycled and reused in the sponge blasting operation. The system must be capable of treating the contaminants specific to the site and reducing their concentrations to allowable levels for water recycle. Remote operation will require the adaptation of the cleaning heads to a robotic control system.

Implementation Needs: Development costs-The cost of a robotic control system is estimated at $\$ 250 \mathrm{~K}$ by Remotek Company (personal communication with C. E. Benson). For development and demonstration of a water recycle system in combination with sponge blasting, an estimate of funding requirements is $\$ 1.2 \mathrm{M}$.

Deployment costs-Operating costs for a sponge blasting unit would primarily involve labor and material costs. Assuming a minimum two-person operation (at a labor rate of $\$ 40 \mathrm{~K} / \mathrm{year} /$ person) of a blasting unit during the day shift, labor costs would be approximately $\$ 350 /$ day. A conservative estimate of blasting media material costs is $\$ 70 / \mathrm{h}$. Based on an eight hour day with about $75 \%$ operating time, operating costs would be approximately $\$ 2 / \mathrm{ft}^{2}$ of surface cleaned. The capital cost of a standard sponge blasting unit is $\$ 13 \mathrm{~K}$. A sifter unit, used to separate the used blasting media from debris such as paint flakes, rust particles, etc., costs $\$ 6 \mathrm{~K}$. The blasting media wash unit is not standard and is designed for each specific application. The time required for development and implementation is roughly estimated at 1.5 years.

Author: J. H. Wilson \615-574-4413; edited by E. B. Munday/615-576-5806

Reference: Sponge-Jet, Inc., "C" Dow Highway, Eliot, ME 03903.

September 1994

Decontamination and Decommissioning 
EM Problem: Decontamination and Decommissioning

Y-12 Plant Problem: Building 9201-4 (Alpha-4)

\section{Problem Area/Constituents:}

- In situ decontamination of the interior of steel and nickel process equipment contaminated with $\mathrm{Hg}$, $\mathrm{LiOH}, \mathrm{H}_{2} \mathrm{SO}_{4}$, and $\mathrm{HNO}_{3}$.

- In situ decontamination of the exterior of steel and nickel process equipment contaminated with $\mathrm{Hg}, \mathrm{LiOH}, \mathrm{H}_{2} \mathrm{SO}_{4}, \mathrm{HNO}_{3}$, and asbestos.

- Ex situ decontamination of steel and nickel process equipment contaminated with $\mathrm{Hg}$, $\mathrm{LiOH}, \mathrm{H}_{2} \mathrm{SO}_{4}, \mathrm{HNO}_{3}$, and asbestos.

- Painted concrete and clay tile contaminated with PCBs, oil, U (and daughters), electrolytes, $\mathrm{Cd}, \mathrm{Pb}$, and $\mathrm{Cr}$.

- Insulation and building paper contaminated with $\mathrm{Hg}$ and $\mathrm{U}$ (and daughters).

- Packing contaminated with $\mathrm{Hg}, \mathrm{LiOH}, \mathrm{H}_{2} \mathrm{SO}_{4}$, and $\mathrm{HNO}_{3}$.

\section{Reference Requirements:}

- Federal Facility Compliance Agreements (e.g., FFCA for Oak Ridge Site)

- Applicable federal and state laws and regulations (e.g., Clean Air Act and Toxic Substances Control Act)

- DOE orders

Refer to the Regulatory Compliance chapter of Vol. 1 for potentially applicable proposed and promulgated environmental laws, signed and pending agreements for the Oak Ridge Reservation, radiation protection standards, DOE orders, and nonregulatory guidance. As site- and waste-specific characteristics are provided for each technology, specific regulatory requirements will be specified.

\section{Subelement: Decontamination}

Alternatives: Surface cleaning methods

Technology: Hot air stripping. The basic air-stripping technology is well known for volatiles in liquids, and many commercial designs are readily available if secondary treatment or effluent is required. Any released contaminants would likely stay with the off-gas.

Status: Demonstration. What the technology needs in specific applications needs to be determined.

Efficacy/strengths/weaknesses-See below.

Waste-Since no significant substrate will be removed, there is the potential to minimize waste volume. 
Cost-Dependent on the decontamination rate and amount of decontaminant to be removed. Secondary separation and disposal costs will largely determine overall costs.

Science/Technology Needs: Investigation of the conditions and removal efficiency for the removal of mercury and other volative contaminants by hot air stripping is required. Development of a hot air cleanup system is needed. Specific needs depend on specific secondary treatment requirements.

Implementation Needs: An air cleanup system is needed to use this technology. Development cost, $-\$ 2 \mathrm{M}$; capital cost, $\$ 250 \mathrm{~K}-500 \mathrm{~K}$; operating cost, $\$ 3 / \mathrm{ft}^{2}$.

Author: R. B. Alderfer/615-576-0225

References: MMES staff members. 
EM Problem: Decontamination and Decommissioning

Y-12 Plant Problem: Building 9201-4 (Alpha-4)

\section{Problem Area/Constituents:}

- Ex situ decontamination of steel and nickel process equipment contaminated with $\mathrm{Hg}$, $\mathrm{LiOH}, \mathrm{H}_{2} \mathrm{SO}_{4}, \mathrm{HNO}_{3}$, asbestos, and PCBs.

- Painted concrete and clay tile contaminated with PCBs, oil, U (and daughters), electrolytes, $\mathrm{Cd}, \mathrm{Pb}$, and $\mathrm{Cr}$.

- Insulation and building paper contaminated with $\mathrm{Hg}$ and $\mathrm{U}$ (and daughters).

- Packing contaminated with $\mathrm{Hg}, \mathrm{LiOH}, \mathrm{H}_{2} \mathrm{SO}_{4}$, and $\mathrm{HNO}_{3}$.

Reference Requirements:

- Federal Facility Compliance Agreements (e.g., FFCA for Oak Ridge Site)

- Applicable federal and state laws and regulations (e.g., Clean Air Act and Toxic Substance Control Act)

- DOE orders

Refer to the Regulatory Compliance chapter of Vol. 1 for potentially applicable proposed and promulgated environmental laws, signed and pending agreements for the Oak Ridge Reservation, radiation protection standards, DOE orders, and nonregulatory guidance. As site- and waste-specific characteristics are provided for each technology, specific regulatory requirements will be specified.

Subelement: Decontamination

Alternative: Surface decontamination methods

Technology: Dry heat. The technology is simple in concept. Its application has not been demonstrated for these problem areas.

Status: Conceptual-The effective application of dry heat to the above problem areas is uncertain and untried. A significant number of bench-scale laboratory studies would be needed to define applicability of the process to the problem areas discussed above.

Science/Technology Needs: Technology improvement needs would include demonstrations on specific problems needing treatment and comparison to other alternatives.

Implementation Needs: An air cleanup system is needed to use this technology. Development cost, \$1M; capital cost, $\$ 250 \mathrm{~K}-500 \mathrm{~K}$; operating cost, $>\$ 1 / \mathrm{ft}^{2}$.

Author: R. B. Alderfer/615-576-0225

References: MMES staff members. 


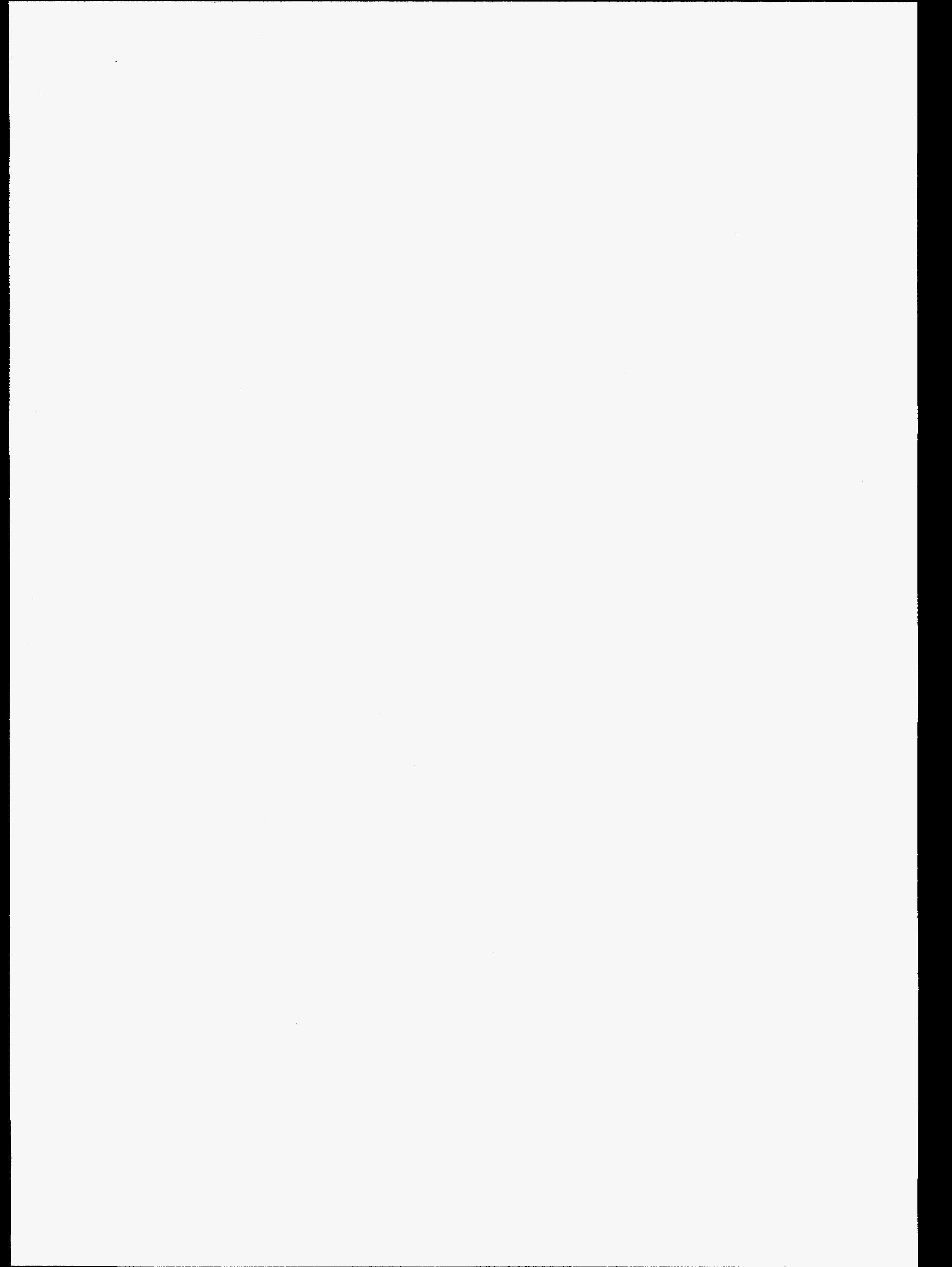


EM Problem: Decontamination and Decommissioning

Y-12 Plant Problem: Building 9201-4 (Alpha-4)

Problem Area/Constituents: Nickel and steel process equipment contaminated with mercury, lithium hydroxide, sulfuric acid, nitric acid, asbestos, and PCBs. Painted concrete and clay tile contaminated with $\mathrm{PCBs}$, oil, $\mathrm{U}$ (and daughters), electrolytes, $\mathrm{Cd}, \mathrm{Pb}$, and $\mathrm{Cr}$.

Reference Requirements: Refer to Vol. 1, Chap. 10, for potentially applicable proposed and promulgated environmental laws, signed and pending agreements for the Oak Ridge Reservation, radiation protection standards, DOE orders, and nonregulatory guidance. As site- and waste-specific characteristics are provided for each technology, specific regulatory requirements will be specified.

\section{Subelement: Decontamination}

Alternative: Chemical surface cleaning methods

Technology: In one technology, contaminated items are washed in solvent and an automated system is used to spray and recover the solvent.

A new proprietary process has been developed by EET, Inc., for removing PCBs, heavy metals, radionuclides, and other hazardous substances from solid materials such as concrete, steel and metals using a chemical-based extraction technology. This process is also referred to as the Enclean process. ${ }^{1}$ Due to the proprietary nature of the technology, the chemical constituents are not available.

The extraction technology is a multistep process in which proprietary chemicals are applied to the contaminated surface and then removed. The specific chemicals and cleaning techniques required are custom engineered for the specific application. Removal of any debris from the surface is required initially. Then a custom-blended liquid is applied as a surface preparation step. A second chemical blend (the extraction step) is applied to extract the contaminants from the surface and substrate. The surface is again rinsed and the liquids removed. The cycle may be repeated several times to achieve the desired levels of decontamination.

\section{Status: Conceptual}

Efficacy/strengths/weaknesses-For the EnClean process as it pertains to PCBs, beginning levels of $82,000 \mu \mathrm{g} / 100 \mathrm{~cm}^{2}$ have been reduced to nondetectable levels. ${ }^{3}$ Combinations of PCBs, heavy metals, and radionuclides in high concentration have been brought down to nondetectable levels as well. Reductions for radionuclides have been successful for both surface contamination (through "smear" testing with beginning levels at 50,000 dpm reduced 
to nondetectable levels) and fixed radiation (subsurface through geiger testing with beginning levels of $800,000 \mathrm{dpm}$ reduced to less than $2,000 \mathrm{dpm}$ ).

Waste-Only the surface material removed. ${ }^{2}$ One typical EnClean process application generated $4 \mathrm{gal} / 100 \mathrm{ft}^{2}$ of liquid waste. ${ }^{3}$

Cost-\$250-500K; labor cost-an average of $7.5 \mathrm{~min} /$ item was required when decontaminating small items such as pipe wrenches, vise grips, and lard cans. ${ }^{4}$

Demonstration costs $-\$ 1 \mathrm{M}$ to identify and demonstrate the replacement solvent. Plutoniumcontaminated items at Richland, Washington, were rinsed with Freon. ${ }^{5}$ Solvents that are less damaging to the environment need to be identified and demonstrated. ${ }^{6}$ The vendor who supplied the equipment for Richland ten years ago no longer sells it. ${ }^{7}$ In EET's experience, the cost range of extraction projects has been from $\$ 6$ to $\$ 50 / \mathrm{ft}^{2}$ cleaned, with relatively small additional expenditures required for transportation and disposal. ${ }^{3}$ Cost ranges reflect many factors, including the size of the area to be cleaned, level of contamination, required level of decontamination, and types of contaminants.

Science/Technology Needs: Solvents on mercury-contaminated surfaces need to be identified, researched, and demonstrated. ${ }^{8}$

Implementation Needs: Solvent off-gas collection and treatment would be needed.

Author: K. E. Lott/615-574-6537

\section{References:}

1. Personal communication, EET, Inc., November 30, 1993.

2. Richard P. Allen, H. W. Arrowsmith, and M. W. McCoy, "New Decontamination Technologies for Environmental Applications," Environmental Decontamination, Proceedings of the workshop, December 4-5, 1979, Oak Ridge, Tenn., CONF-791234, February 1981.

3. Ronald E. Borah, M. W. Bonem, and S. E. Rathke, "Extraction of Contaminants from Porous Surfaces Case History," presentation at the ER ' 93 Department of Environmental Remediation Conference, Augusta, Georgia, October 24-28, 1993.

4. T. Shapiro et al., Building Characterization Report, ORGDP Diffusion Facilities Permanent Shutdown, Building K-25, KD-5748, March 10, 1988, p. 64.

5. Telephone conversation with D. H. Bunch, June 17, 1992.

6. Decontamination and Decommissioning Integrated Demonstration Strategy (Draft for Review), November 1991.

7. Telephone conversation with D. H. Bunch, June 5, 1992.

8. J. M. Chiang et al., Building Characterization Report, ORGDP Diffusion Facilities Permanent Report, Building K-33, KD-5752, April 6, 1988, p. 14. 
EM Problem: Decontamination and Decommissioning

Y-12 Plant Problem: Building 9201-4 (Alpha-4)

Problem Area/Constituents: Nickel and steel process equipment contaminated with mercury, lithium hydroxide, sulfuric acid, nitric acid, asbestos, and PCBs. Painted concrete and clay tile contaminated with $\mathrm{PCBs,}$ oll, $\mathrm{U}$ ( and daughters), electrolytes, $\mathrm{Cd}, \mathrm{Pb}$, and $\mathrm{Cr}$.

Reference Requirements: Refer to Vol. 1, Chap. 10, for potentially applicable proposed and promulgated environmental laws, signed and pending agreements for the Oak Ridge Reservation, radiation protection standards, DOE orders, and nonregulatory guidance. As site- and waste-specific characteristics are provided for each technology, specific regulatory requirements will be specified.

Subelement: Decontamination

Alternative: Chemical surface cleaning methods

Technology: Solvent washing to remove PCBs

Status: Conceptual

Efficacy/strengths/weaknesses-This equipment is produced by a number of manufacturers ${ }^{1}$ and its use for removing organic surface contamination is common in a variety of industries. ${ }^{2}$

Waste-Solvent degreasing was used successfully, but its use was stopped to avoid exposing the workers and the environment to the hazardous solvents. Other solvents might be available, but their effectiveness would have to be demonstrated. ${ }^{2}$ Maintenance mechanics operated degreasing equipment at the K-25 Site.

Cost-Capital cost, $\$ 400 \mathrm{~K}$ (1992 dollars) for a 4000-lb capacity unit. Labor costs-One operator for one hour to load, clean, and unload $4000-1 \mathrm{~b}$ of metal. ${ }^{3}$ Total operating cost, $>\$ 1 / \mathrm{ft}^{2}$.

Science/Technology Needs: Solvents that are less damaging to the environment need to be identified and demonstrated. Development cost, $<\$ 1 M$.

Implementation Needs: Solvent off-gas collection and treatment would be needed.

Author: K. E. Lott/615-574-6537

\section{References:}

1. Thomas Register of American Manufacturers, Thomas Publishing Company, New York, 1992, pp. 7651-7663.

2. Personal communication to D. H. Bunch, June 5, 1992.

3. Durr Corp., phone conversation with D. H. Bunch, June 8, 1992. 


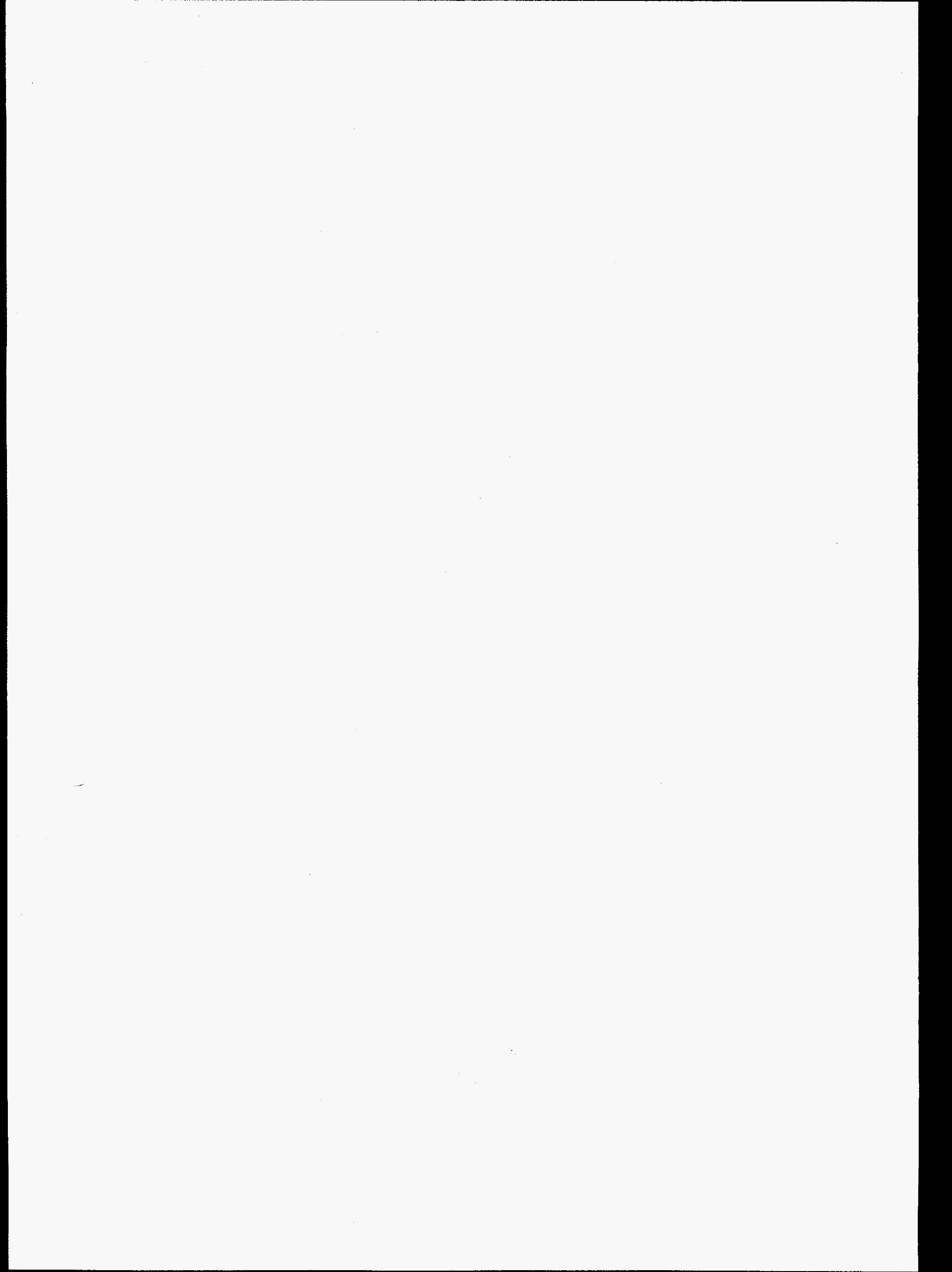




\section{EM Problem: Decontamination and Decommissioning}

Y-12 Plant Problem: Building 9201-4 (Alpha-4)

\section{Problem Area/Constituents:}

- In situ decontamination of the exterior of steel and nickel process equipment contaminated with $\mathrm{Hg}, \mathrm{LiOH}, \mathrm{H}_{2} \mathrm{SO}_{4}, \mathrm{HNO}_{3}$, and asbestos.

- Ex situ decontamination of steel and nickel process equipment contaminated with $\mathrm{Hg}$, $\mathrm{LiOH}, \mathrm{H}_{2} \mathrm{SO}_{4}, \mathrm{HNO}_{3}$, and asbestos.

- Concrete and clay tile contaminated with $\mathrm{Hg}, \mathrm{LiOH}, \mathrm{H}_{2} \mathrm{SO}_{4}, \mathrm{HNO}_{3}$, and $\mathrm{U}$ (and daughters)

\section{Reference Requirements:}

- Federal Facility Compliance Agreement for Oak Ridge Site

- Clean Air Act

- Toxic Substances Control Act

- DOE orders

Refer to the Regulatory Compliance chapter of Vol. 1 for potentially applicable proposed and promulgated environmental laws, signed and pending agreements for the Oak Ridge Reservation, radiation protection standards, DOE orders, and nonregulatory guidance. As site- and waste-specific characteristics are provided for each technology, specific regulatory requirements will be specified.

\section{Subelement: Decontamination}

Alternative: Surface cleaning (transferable contamination) methods

Technology: Strippable coatings. A coating, such as a water-based organic polymer, is applied to a contaminated surface by methods such as spraying, brushing, and rolling (as may be used for paint). When the coating dries, it is either manually stripped from the surface or, in the case of "auto-release" coatings, it is collected by vacuuming. The surface contamination is removed with the coating, producing a dry, hard, nonairborne waste product. Water-based strippable coatings are intended for use in decontaminating smooth and semirough porous surfaces, including steel, concrete, aluminum, wood, and painted surfaces.

Status: Accepted. The technology has been used for decontamination purposes in applications involving hazardous and/or radioactive contaminants. The waste product is the solid polymer that contains the removed contaminants. Typical coverages would be $50 \mathrm{ft}^{2} / \mathrm{gal}$ of polymer. Strippable coatings are very effective. However, the application and removal times are relatively long in some instances, the cost of materials is high, and the radioactive waste aspects for disposal must be carefully considered. Most commercial strippable coatings may be incinerated. 
Science/Technology Needs: A potential area of improvement for strippable coatings is the reduction of material costs.

Implementation Needs: Normal implementation needs.

Deployment costs-The cost of a spray unit for applying strippable coatings is $\$ 20 \mathrm{~K}$. The cost of the polymer is $\$ 50-\$ 75 / \mathrm{gal}$. At the coverage stated above, the cost of materials per unit area of contaminated surface is $>\$ 1.50 / \mathrm{ft}^{2}$.

Author: J. H. Wilson/615-574-4413. Reviewed by: R. B. Alderfer/615-576-0225

References: MMES staff members. 
EM Problem: Decontamination and Decommissioning

Y-12 Plant Problem: Building 9201-4 (Alpha-4)

\section{Problem Area/Constituents:}

- In situ decontamination of the exterior of steel and nickel process equipment contaminated with $\mathrm{Hg}, \mathrm{LiOH}, \mathrm{H}_{2} \mathrm{SO}_{4}, \mathrm{HNO}_{3}$, and asbestos.

- Ex situ decontamination of steel and nickel process equipment contaminated with $\mathrm{Hg}$, $\mathrm{LiOH}, \mathrm{H}_{2} \mathrm{SO}_{4}, \mathrm{HNO}_{3}$, and asbestos.

\section{Reference Requirements:}

- Federal Facility Compliance Agreements (e.g., FFCA for Oak Ridge Site)

- Applicable federal and state laws and regulations (e.g., Clean Air Act and Toxic Substances Control Act)

- DOE orders

Refer to the Regulatory Compliance chapter of Vol. 1 for potentially applicable proposed and promulgated environmental laws, signed and pending agreements for the Oak Ridge Reservation, radiation protection standards, DOE orders, and nonregulatory guidance. As site- and waste-specific characteristics are provided for each technology, specific regulatory requirements will be specified.

Subelement: Decontamination

Alternative: Surface decontamination methods

Technology: Vacuum cleaning. Use of a vacuum cleaner to remove, by air entrainment, loosely adhering contamination

Status: It is widely accepted by industry for removing loose solid contaminants. When solids are present in significant amounts but are not loose, they may be broken free by hand scraping or more automated means and then vacuumed by a HEPA filtration vacuum systems. Dust-laden areas are also good candidates where the possibility of contamination exists.

Science/Technology Needs: Improvement-Cleanable, reusable prefilters and HEPA filters should be developed to minimize wastes.

Implementation Needs: Disassembly of contaminated equipment and possible prior loosening of the deposits using another technology are needed to use this technology.

Capital cost, $<\$ 25 \mathrm{~K}$; operating cost, $\$ 0.75 / \mathrm{ft}^{2}$ 
Author: R. B. Alderfer/615-576-0225

References: MMES staff members. 


\section{EM Problem: Decontamination and Decommissioning}

Y-12 Plant Problem: Building 9201-4 (Alpha-4)

\section{Problem Area/Constituents:}

- Ex situ decontamination of steel and nickel process equipment contaminated with $\mathrm{Hg}$, $\mathrm{LiOH}, \mathrm{H}_{2} \mathrm{SO}_{4}, \mathrm{HNO}_{3}$, asbestos, and $\mathrm{PCBs}$.

- Packing contaminated with $\mathrm{Hg}, \mathrm{LiOH}, \mathrm{H}_{2} \mathrm{SO}_{4}$, and $\mathrm{HNO}_{3}$.

\section{Reference Requirements:}

- FFCA for K-25 Site

- RCRA and TSCA regulations as applied to solvents used in washing and organic contaminants on the metal surfaces (oils, PCB, etc.)

- NRC NUREG 1.86 as applied to release standards for radioactive surface contamination

Refer to the Regulatory Compliance chapter of Vol. 1 for potentially applicable proposed and promulgated environmental laws, signed and pending agreements for the Oak Ridge Reservation, radiation protection standards, DOE orders, and nonregulatory guidance. As site- and waste-specific characteristics are provided for each technology, specific regulatory requirements will be specified.

\section{Subelement: Decontamination}

Alternative: Surface cleaning (transferable contamination) methods

Technology: Ultrasonic cleaning. It uses the scrubbing action of liquid excited by ultrasonic frequencies to remove surface deposits of oils, organics, and loosely bound solids from metals, plastic, glass, and other solids. It produces no abrasion, distortion, or changes in most solids and is ideally suited for delicate or valuable parts and materials that are to be reused.

Status: Accepted. The operating cost is high compared to that of other decontamination methods. The cost is greater than $\$ 5 / \mathrm{ft}^{2}$ and it may exceed $\$ 10 / \mathrm{ft}^{2}$.

Ultrasonic cleaning has been used for many years in the private sector and government installations for removing surface contamination from relatively small metal parts that can fit into an ultrasonic bath. This technology has been demonstrated on a plant scale at Y-12 for the past 4 years as a substitute for solvent washing. Initial funding was provided by HAZWRAP and DOE-Albuquerque Operations Office. Ultrasonic cleaning is not used at ORNL on a large scale. The technology will work well for loosely bound surface deposits such as dust, dirt, organic, oils, PCBs, etc., on metal equipment, sheet metal, and solid depleted uranium. The technique is not practical for large items that would require size reduction. It is ineffective for tightly bound materials such as paints, varnishes, and other materials that are difficult to remove. The technique may generate secondary wastes that are expensive to 
manage (such as solvent and detergent solutions requiring processing). The technique is intended for valuable parts that could be recycled "as is" after cleaning. The technique is also good for removing deposits in difficult-to-access places, and it may be useful for electronic parts and electric motors.

Science/Technology Needs: Technology development needs to include definition of acceptable cleaning liquids that (1) are not hazardous, (2) can be separated from the contaminants, and (3) can be reused to minimize secondary wastes. Technology improvements should include more aggressive cleaning action.

Implementation Needs: The technology is sufficiently mature to be used in most decontamination and decommissioning (D\&D) applications without the need for additional personnel and educational investments. Investment in means to produce a more aggressive cleaning action may be cost effective and could likely be accomplished on a pilot scale for about $\$ 0.4 \mathrm{M}$. If the needs for more aggressive cleaning within the DOE D\&D were great enough, private suppliers of ultrasonic equipment would likely bear the cost of its development. The time required before implementation is roughly estimated at one year. Capital costs, $\$ 10 \mathrm{~K}-\$ 100 \mathrm{~K}$; operating costs, $\$ 5-\$ 10 / \mathrm{ft}^{2}$ or more.

Author: J. M. Kennerly/615-574-9935. Reviewed by: E. B. Munday/615-576-5806

\section{References:}

1. L. M. Thompson, R. F. Samandl, and H. L. Richards, "Solutions for the Chlorinated Solvent Debacle," YDB 904, Rev. 1.

2. L. M. Thompson and R. F. Samandl, "Ultrasonic Aqueous Cleaning as a Replacement for Chlorinated Solvent Cleaning," report in preparation.

September 1994

Decontamination and Decommissioning 


\section{EM Problem: Decontamination and Decommissioning}

Y-12 Plant Problem: Building 9201-4 (Alpha-4)

\section{Problem Area/Constituents:}

- Ex situ decontamination of steel and nickel process equipment contaminated with $\mathrm{Hg}$, $\mathrm{LiOH}, \mathrm{H}_{2} \mathrm{SO}_{4}, \mathrm{HNO}_{3}$, asbestos, and PCBs.

- Packing contaminated with $\mathrm{Hg}, \mathrm{LiOH}, \mathrm{H}_{2} \mathrm{SO}_{4}$, and $\mathrm{HNO}_{3}$.

\section{Reference Requirements:}

- Federal Facility Compliance Agreements (e.g., FFCA for Oak Ridge Site and FFCAs for PCBs)

- Applicable federal and state laws and regulations (e.g., Clean Air Act and Toxic Substances Control Act)

- DOE orders

\section{Subelement: Decontamination}

Alternative: Surface cleaning methods

Technology: Vibratory cleaning. Vibratory cleaning has been used commercially for many years for a large variety of applications. A vibrator causes vibrations throughout a bowl containing pellets and the items to be cleaned or finished. The vibrations usually cause the pellets to flow in circular fashion within the bowl. Pellets of different sizes, shapes, and materials may be used. For rough abrasive action, ceramic pellets may be used, and for less abrasive action, plastic pellets may be used. Solutions and detergents may also be used. A large variety of vibratory cleaning equipment is available from numerous vendors, which offer units that vary in capacity from several cubic feet to over $30 \mathrm{ft}^{3}$.

Status: Demonstration. Vibratory cleaning is likely to be affective for decontaminating a variety of Y-12 problems, but waste will be generated from the cleaning pellet medium and cleaning solutions used. The problems of cross-contamination within the vibratory cleaner pellet medium and how best to recycle the pellets and solutions remain. The general cost effectiveness of the process compared to other methods has not been evaluated. The quantity of the waste generated cannot be reliably estimated at this time.

Science/Technology Needs: Technology development needs. The problems identified in the status section above must be evaluated during a bench-scale demonstration.

Implementation Needs: A bench-scale demonstration could be done in about nine months at a cost roughly estimated at $\$ 500 \mathrm{~K}$. Of this amount, about $\$ 60 \mathrm{~K}$ is needed for equipment and installation costs. These estimates assume that an existing facility is available for use during the project. Although operating rate and costs cannot be accurately determined until 
after the demonstration, these costs are roughly estimated at $\$ 6 / \mathrm{ft}^{2}$. Because equipment sizing is a function of operating rate and amount of material to be processed, the capital costs can not be fully estimated, However, a large operating unit can be installed in an existing facility for a cost roughly estimated at $\$ 60 \mathrm{~K}$. The time required to implement the project is roughly estimated at one year.

Author: E. B. Munday/615-576-5806 
EM Problem: Decontamination and Decommissioning

Y-12 Plant Problem: Building 9201-4 (Alpha-4)

\section{Problem Area/Constituents:}

- Painted concrete and clay tile contaminated with PCB, oil, U (and daughters), electrolytes, $\mathrm{Cd}, \mathrm{Pb}, \mathrm{Cr}$, and $\mathrm{Hg}$

- Insulation and building paper contaminated with $\mathrm{Hg}$ and $\mathrm{U}$ (and daughters).

- Packing contaminated with $\mathrm{Hg}, \mathrm{LiOH}, \mathrm{H}_{2} \mathrm{SO}_{4}$, and $\mathrm{HNO}_{3}$.

- Carbon chunks contaminated with $\mathrm{Hg}, \mathrm{LiOH}, \mathrm{H}_{2} \mathrm{SO}_{4}$, and $\mathrm{HNO}_{3}$.

- Wood and sheet rock contaminated with $\mathrm{Hg}, \mathrm{LiOH}, \mathrm{H}_{2} \mathrm{SO}_{4}$, and $\mathrm{HNO}_{3}, \mathrm{U}$ (and daughters), asbestos, and/or PCBs.

\section{Reference Requirements:}

- Federal Facility Compliance Agreements (e.g., FFCA for Oak Ridge Site and FFCAs for PCBs)

- Applicable federal and state laws and regulations (e.g., Clean Air Act and Toxic Substances Control Act)

- DOE orders

Subelement: Decontamination

Alternative: Surface Cleaning Methods

Technology: Microwave vaporization-Microwave energy may be used to remove mercury adsorbed by concrete floors and walls. Microwave energy deposition interior to the surface can establish a temperature gradient that can drive mercury to the surface where it can be collected by an integrated vacuum system. Directing the air stream over cooled panels would condense and collect the mercury prior to passing through HEPA filters. The mechanical structure of the floor or wall is preserved and no resurfacing is required. For other problems indicated above, the microwave system would have a different configuration but would still be used for in-depth heating of the contaminated material. The principal would still be to mobilize the mercury for extraction/collection.

Status: Predemonstration. ORNL has designed, fabriacted and tested a mobile system for microwave removal of concrete surfaces. ${ }^{1}$ In contrast to the concrete removal system where the application of microwave power to the concrete results in violent steam explosions which explosively fracture the surface, the microwave vaporization technique relies on gentle heating of the concrete to establish a warm zone in the center of the wall or floor. The microwave vaporization technique is an extension of an ongoing microwave concrete removal program. The specific application to remove $\mathrm{Hg}$ is in the early investigation stage.

This technique has the potential to decrease $\mathrm{Hg}$ content in surface layers to manageable levels. 
The amount of waste produced is minimal compared to other techniques. The method has the potential to produce no dust or debris and would not require the use of solvents, water or additional items that require handling and disposal. It would not generate contaminated dust or fluids and would be a quiet operation.

Microwave vaporization avoids costs, hazards, and paperwork associated with the largevolume waste stream generated by chipping/blasting techniques. This technique avoids the cost and handling required for thermal desorption and collection of the Hg from rubble.

Unit Operating Cost-An operating microwave vaporization system would have a capital cost of less than $\$ 130 \mathrm{~K}$, not including the $\mathrm{Hg}$ recovery system. The equipment would require an operator at about $\$ 100 / \mathrm{hr}$. The electrical cost would be less than $\$ 8.60 / \mathrm{hr}$ $(110 \mathrm{kWh} \times \$ 0.08 / \mathrm{kWh})$.

The surface processing rate would be about $25 \mathrm{ft}^{2} / \mathrm{hr}$. The system development cost is estimated to be $\$ 1.4 \mathrm{M}$.

Science/Technology Needs: The use of microwave power to drive adsorbed mercury from a concrete surface requires a proof-of-principle experiment using laboratory-scale equipment. The steps needed to assess the principle and determine feasibility for application to Bldg. 9201-4 surfaces for the purpose of $\mathrm{Hg}$ removal are (1) establish a laboratory setup and test removal of a nontoxic simulant from a concrete surface; (2) test an $\mathrm{Hg}$-contaminated surface, with characterization of the $\mathrm{Hg}$ concentration profile before and after application of microwave energy; and (3) follow the laboratory-scale demo test with an investigative program to develop the technology for a prototype system. The investigative program would demonstrate field use of the Phase II lessons learned and characterize in-field performance. Other questions addressed are the effects of real-world surfaces and structural supports (rebar, support columns, cross members, etc.).

Implementation Needs: Development Cost-The total cost of the proof-of-principle testing and additional development along with system optimization and delivery of a functioning fixed-frequency unit is estimated at about $\$ 1.4 \mathrm{M}$.

Capital Cost-For an operating unit involving a microwave generator, power supplies, data acquisition, electrical monitoring and control, vacuum system and materials the cost would be approximately $\$ 130 \mathrm{~K}$.

Operating Cost-The operating cost, including labor, amortized capital cost, electricity, filters and other supplies, is estimated at about $\$ 130 / \mathrm{h}$, or $\$ 3 / \mathrm{ft}^{2}$.

The cleaning rate is highly speculative at this time and depends on the diffusion rate of the mercury to the surface. The illuminated surface will be approximately $25 \mathrm{ft}^{2}$ (adjustable) with an assumed diffusion time estimate of an hour, the cleaning cost would be about $\$ 5 \mathrm{ft}^{2}$.

Author: Gary L. Bell/615-241-4400 and Terry L. White/615-574-0983

September 1994

Decontamination and Decommissioning 
EM Problem: Decontamination and Decommissioning

Y-12 Plant Problem: Building 9201-4 (Alpha-4)

Problem Area/Constituents: Painted concrete and clay tile contaminated with PCBs, oil, $\mathrm{U}$ (and daughters), electrolytes, $\mathrm{Cd}, \mathrm{Pb}$, and $\mathrm{Cr}$.

\section{Reference Requirements:}

- Federal Facility Compliance Agreement for Oak Ridge Site

- Federal Facility Compliance Agreement for PCBs

- Clean Air Act

- Toxic Substances Control Act

- DOE orders

Refer to the Regulatory Compliance chapter of Vol. 1 for potentially applicable proposed and promulgated environmental laws, signed and pending agreements for the Oak Ridge Reservation, radiation protection standards, DOE orders, and nonregulatory guidance. As site- and waste-specific characteristics are provided for each technology, specific regulatory requirements will be specified.

\section{Subelement: Decontamination}

\section{Alternatives: Thermal surface removal methods}

Technology: Microwave scabbling. This technology directs microwave energy at a concrete surface, using a specialized waveguide applicator, and heats the concrete and free water present in the concrete matrix. Continued heating produces thermal- and steam-pressureinduced mechanical stresses that cause the concrete surface to burst. The concrete particles from this steam explosion are small enough to be removed by a vacuum system, yet less than $1 \%$ of the debris is small enough to pose an airborne contamination hazard. The process is fast, dry, generates little dust, and avoids mechanical impacts. The microwave applicator head may be manually moved about on the concrete surfaces being decontaminated. Because the rate and depth of surface removal depend on the applicator translation speed, remote operation of the mobile microwave equipment would be desirable. Also, in some applications (such as decontamination of hot cells), remote operation will be required. Consequently, the adaptation of the equipment to a robotics control system would be necessary.

Status: Predemonstration. In FY 1991, ORNL demonstrated ${ }^{1}$ reliable removal of noncontaminated concrete surfaces using microwave energy. At microwave frequencies of $2.45 \mathrm{GHz}$ and $10.6 \mathrm{GHz}$, continuous concrete removal rates of $1.1 \mathrm{~cm}^{3} / \mathrm{s}$ at $5.2 \mathrm{~kW}$ and $2.1 \mathrm{~cm}^{3} / \mathrm{s}$ at $3.6 \mathrm{~kW}$, respectively, were obtained. Removal rate and removal depth are controlled by choosing the proper frequency and varying the power and translation speed of the applicator on the concrete surface. Higher frequencies preferentially remove surface contamination. A mobile prototype microwave concrete removal machine is being built at 
ORNL. A rugged, compact, and electrically efficient microwave applicator that is better integrated with the required concrete debris collection system is being developed. ${ }^{2}$ In 1987 , a group from Japan reported on a mobile microwave decontaminator that could perform removal at a rate of $11 \mathrm{~cm}^{3} / \mathrm{s}^{3}$ This removal rate was equal to that of the fastest commercial mechanical concrete breaking machines. It is anticipated that, with an upgrade of power and improvements to the applicator design to spread out the microwave power in a larger area, concrete removal rates comparable to or exceeding those obtained with conventional concrete removal equipment can be achieved. ${ }^{4}$ The microwave technique is a dry process that generates little dust and avoids the need to work when the concrete surfaces are wet. Because microwave scabbling removes the contaminated concrete surface, the decontamination efficiency should be $100 \%$ if there is no recontamination from the removed scabble. The waste generated is concrete rubble that has particle diameters lying primarily in the range of 1-10 mm. The quantity of waste generated depends upon the translation speed of the microwave applicator and the depth of the concrete surface layer that is removed. For example, for a removal depth of $10 \mathrm{~mm}$ and a surface area treatment rate of $1 \mathrm{ft}^{2} / \mathrm{min}, 150 \mathrm{lb} / \mathrm{h}$ of concrete waste would be generated.

Science/Technology Needs: The development and testing of a mobile prototype microwave concrete removal machine needs to be completed (TTP No. OR-3DAA; DOE No. OR-1012-04). Significant improvements in concrete removal rates and efficiencies can be achieved by modeling the microwave scabbling process as an unsteady, three-dimensional, finite-element, nonlinear, thermal and mechanical stress problem. Because the microwave absorption properties of concrete are critically dependent on temperature and frequency, the frequency and temperature dependent dielectric properties of concrete are being measured and the data will be used in a one-dimensional model developed at Florida International University to characterize the stresses generated in the concrete under the conditions of rapid microwave heating. ${ }^{5,6}$ Extensions of this work to three dimensions will follow. Remote operation will necessitate the adaptation of the mobile microwave and vacuum collection systems to a robotics control system. In addition, the development of real-time monitoring sensors for measuring the progress of the decontamination process is needed so that only the contaminated portion of the concrete is removed, thus minimizing the amount of waste produced.

Implementation Needs: Development costs-The cost for development and testing of a mobile prototype microwave concrete removal machine is estimated to be $\$ 2.5 \mathrm{M}$ for FY 1993-1995. ${ }^{2}$ The personnel requirements are 2.5 FTEs/year (50\% technical, 50\% support personnel). The cost of a robotics control system is estimated at $\$ 250 \mathrm{~K}$. This is based on the cost of a system that is available from the Remotek Company (personal communication with C. E. Benson).

Deployment costs: Roughly $\$ 250 \mathrm{~K}-\mathrm{A}$ credible estimate of capital cost for a microwave scabbler unit cannot be made until a prototype unit has been developed and demonstrated. Operating costs for a scabbler would primarily include power and labor costs. Assuming a two-person (at a labor rate of $\$ 40 \mathrm{~K} /$ year/person) operation of a $100-\mathrm{kW}$ unit at $75 \%$ usage

September 1994

Decontamination and Decommissioning 
during the day shift, operating costs would be approximately $\$ 350 / d$, or somewhat greater than $\$ 1 / \mathrm{ft}^{2}$. Training personnel to operate the microwave scabbler at DOE sites will be needed. Also, this training will need to be included in any technology transfer activities.

Author: J. H. Wilson/615-576-4413 and R. D. Bundy/615-576-0192

\section{References:}

1. T. L. White, R. G. Grubb, L. P. Pugh, D. Foster, Jr., and W. D. Box, "Removal of Contaminated Concrete Surfaces by Microwave Heating-Phase I Results," published in the proceedings of the 18th American Nuclear Society Symposium on Waste Management, Waste Management '92, Tucson, Ariz., March 1-5, 1992.

2. TTP No. OR-3DAA (DOE No. OR-1012-04).

3. H. Yasunaka et al., "Microwave Decontaminator for Concrete Surface Decontamination in JPDR," Proc. Int. Decommissioning Symp., 109-1 15, October 4-8, 1987.

4. T. L. White, private communication with J. H. Wilson.

5. W. Li et al., "Heat Transfer with a Radiation Contaminated Slab Applying a Microwave Heating Technique," in press, ASME Trans., J. of Heat Transfer, ASME National Heat Transfer Conference, San Diego, Calif., August 8-11, 1992.

6. W. Li et al., "Heat and Mass Transfer in a Contaminated, Porous Concrete Slab Subjected to Microwave Heating," 1992 Annual Winter Meeting of ASME, Anaheim, Calif., November 25-December 2, 1992. 


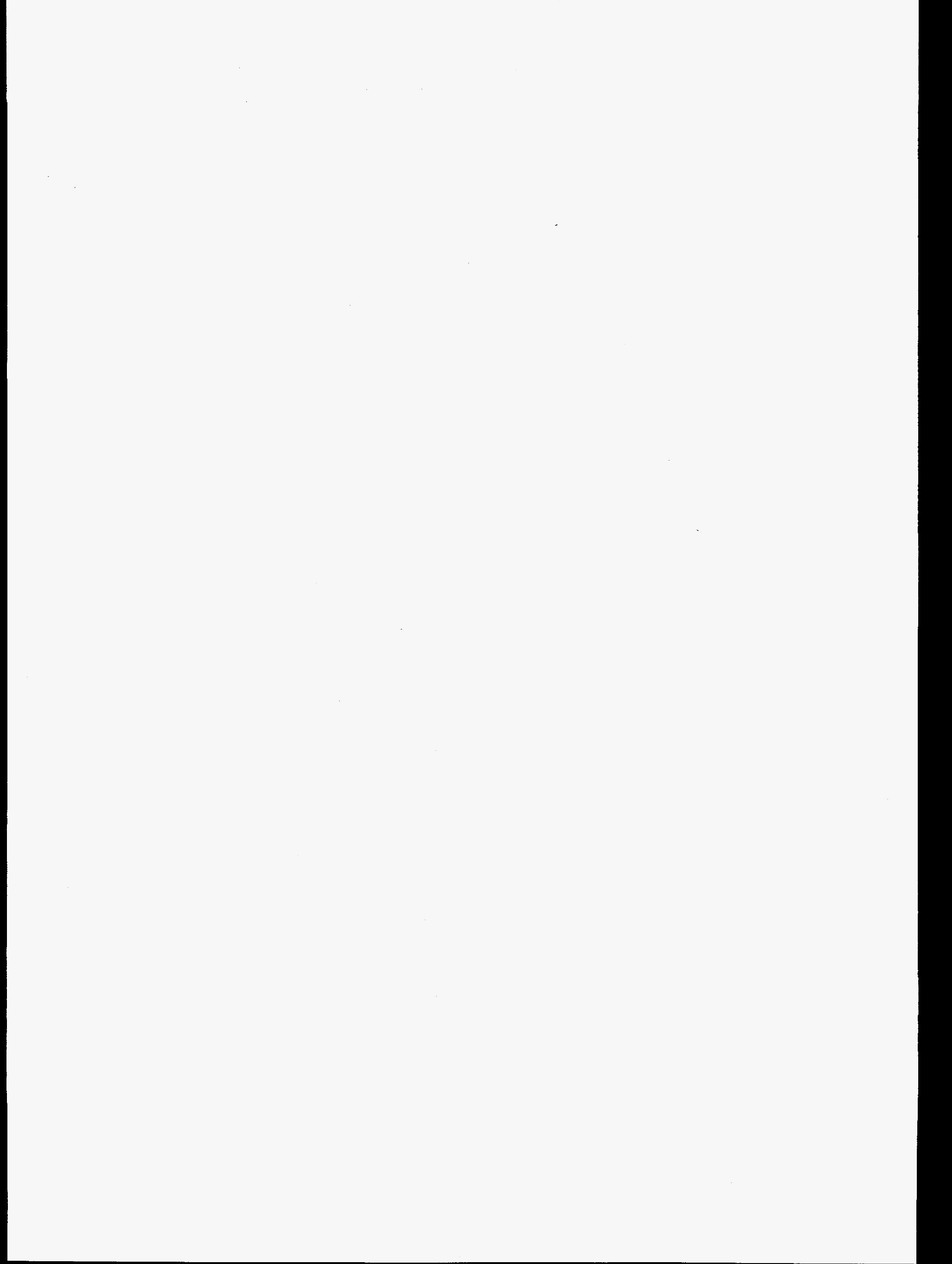


EM Problem: Decontamination and Decommissioning

Y-12 Plant Problem: Building 9201-4 (Alpha-4)

\section{Problem Area/Constituents:}

- In situ decontamination of the interior of steel and nickel process equipment contaminated with $\mathrm{Hg}, \mathrm{LiOH}, \mathrm{H}_{2} \mathrm{SO}_{4}$, and $\mathrm{HNO}_{3}$.

- In situ decontamination of the exterior of steel and nickel process equipment contaminated with $\mathrm{Hg}, \mathrm{LiOH}, \mathrm{H}_{2} \mathrm{SO}_{4}, \mathrm{HNO}_{3}$, asbestos, and PCBS.

- Ex situ decontamination of steel and nickel process equipment contaminated with $\mathrm{Hg}$, $\mathrm{LiOH}, \mathrm{H}_{2} \mathrm{SO}_{4}, \mathrm{HNO}_{3}$, asbestos, and PCBs.

\section{Reference Requirements:}

- Federal Facility Compliance Agreements (e.g., FFCA for Oak Ridge Site)

- Applicable federal and state laws and regulations (e.g., Clean Water Act and Toxic Substances Control Act)

- DOE orders

Refer to the Regulatory Compliance chapter of Vol. 1 for potentially applicable proposed and promulgated environmental laws, signed and pending agreements for the Oak Ridge Reservation, radiation protection standards, DOE orders, and nonregulatory guidance. As site- and waste-specific characteristics are provided for each technology, specific regulatory requirements will be specified.

\section{Subelement: Decontamination}

Alternatives: Thermal surface and substrate removal methods

Technology: Plasma torch. Uses an inert gas passing through a high-power DC or RF arc discharge to produce a very high temperature gas stream that is capable of melting nearly all uncooled materials. Such torches are used in plasma synthesis and decomposition of materials.

Status: Conceptual-Potential uses in decontamination of materials include evaporating mercury, breaking down oils and PCBs into less harmful or harmless substances, rapid spalling of concrete, and using the difference in coefficient of thermal expansion to delaminate contaminants from underlying substrates. Because of its very high gas temperature it should also be effective in removing other coatings on substrates such as $\mathrm{Hg}$, amalgam, L, OH, rust, etc. A data base needs to be established for this process. The torch's inert gas is recyclable and creates no additional waste stream of its own, as compared to the carbon dioxide and $\mathrm{NO}_{\mathrm{x}}$ product created by a combustion torch. Plasma torches exist commercially to weld and cut materials that either have a very high melting temperature or require an inert atmosphere to prevent oxidation. 
Waste-The plasma torch can operate with either an inert gas like argon or an active gas. In the inert mode, no significant addition to the waste stream is expected because gas flow from the torch would only be of the order of $10 \mathrm{ft}^{3} / \mathrm{min}$. A "vacuum" shroud will need to surround the plasma torch nozzle to collect waste gases (including mercury vapor). The existing waste will be converted to a different form (gaseous) and will be collected in a standard HEPA system. In the active gas mode the operating gas will most likely combine with the waste, forming new material. This new material will also be in gaseous form and will also be handled with standard systems.

Cost-An operating plasma torch system should have a capital cost of about $\$ 200 \mathrm{~K}$, including exhaust gas handling. The equipment could be handled by a single person at less than $\$ 100 / \mathrm{h}$. The gas consumption would be around $10 \mathrm{ft}^{3} / \mathrm{min}$, with a cost of about $\$ 0.10 / \mathrm{ft}^{3}$. The electrical cost will be less than $\$ 60 / \mathrm{h}$, based on $100 \mathrm{~kW} @ \$ 0.06 \mathrm{~kW} / \mathrm{h}$. The cleaning ratio is unknown and depends heavily on the waste. Operating costs should be about $\$ 2 / \mathrm{ft}^{2}$. The cost leading to an operating, commercially produced system is expected to be less than $\$ 500 \mathrm{~K}$.

Science Technology Needs: While plasma torch technology exists, its efficacy in removing various mercury, organic, and radioactive contaminants has not been demonstrated, along with $\mathrm{Hg}$ vapor collecting and handling equipment. Elevation to the predemonstration stage involves laboratory-scale experiments, which will be designed using experimental results obtained from on-going development activities associated with plasma cleaning and etching technologies. The laboratory equipment will be constructed and operated for generating hightemperature thermal plasmas to destroy organic contaminants and to vaporize and encapsulate heavy metals. A data base of decontamination rates by plasma torch techniques needs to be established. The equipment required for plasma torch cleaning of large surface areas that are contaminated will be developed to accommodate robotic, remote, and automatic operations with computer control. Also, computer modeling of plasma-surface interactions will be used to provide significant improvements in the removal rate and efficiencies of plasma torch methods. Standard filters can be used to clean the working gas.

Implementation Needs: Development costs-To address science needs, the approximate cost of equipment and staff time is about $\$ 500 \mathrm{~K}$ (capital equipment, $\$ 200 \mathrm{~K}$ ). For these activities, the personnel must be highly trained in the fields of plasma technology and chemistry. One-half are scientists with masters or doctoral degrees, and one-half are highly trained technicians with bachelor of science degrees.

Author: W. L. Gardner/615-574-1121 and W. D. Nelson/615-574-0966

\section{Reference:}

1. J. R. Hollis, Environ Progress 2,7 (1983).

September 1994

Decontamination and Decommissioning 
EM Problem: Decontamination and Decommissioning

Y-12 Plant Problem: Building 9201-4 (Alpha-4)

Problem Area/Constituents: In situ decontamination of the interior of steel and nickel process equipment contaminated with $\mathrm{Hg}, \mathrm{LiOH}, \mathrm{H}_{2} \mathrm{SO}_{4}$, and $\mathrm{HNO}_{3}$.

\section{Reference Requirements:}

- Federal Facility Compliance Agreements (e.g., FFCA for Oak Ridge Site and FFCAs for PCBs)

- Applicable federal and state laws and regulations (e.g., Clean Air Act and Toxic Substances Control Act)

- DOE orders

\section{Subelement: Decontamination}

\section{Alternative: Thermal surface removal methods}

Technology: Laser heating. Laser-based photothermal heating is a method for removing contaminants at a surface or at very shallow depths below the surface. Energy from a laser is absorbed at a contaminated surface, the absorbed energy produces a rapid temperature rise in the subsurface, and the material evaporates or decomposes to gaseous and solid residues.

Status: Laser-based photothermal heating is currently being developed and commercialized for coating removal and concrete etching. Several commercial organizations are marketing Nd:YAG and $\mathrm{CO}_{2}$ laser systems that efficiently and safely remove organic coatings (e.g., polyurethane paint) from metal and composite surfaces. The removal rates for these systems are reported to be several hundred square feet per hour. Hanford-Westinghouse Laboratory is developing, testing and using Nd:YAG laser-based decontamination on metal and concrete surfaces. Their process has been effective at removing a variety of organic compounds, inorganic acids such as $\mathrm{HNO}_{3}, \mathrm{PCBs}$ and heavy metals such as cadmium, lead, and chromium.

The primary application for decontamination with laser-based photothermal heating is when large surface areas need a high degree of decontamination with the absolute minimum amount of waste generation. The technology produces as waste only the material that is vaporized from the surface (all the vaporized material is collected in a filtration system) or left as a loose, powdery residue. The technology removes surface contamination without harming the underlying surface. The technology lends itself to a high degree of automation.

Science/Technology Needs: The commercial and prototype laser-based photothermal decontamination systems can be evaluated for possible use on Alpha-4 decontamination problem areas. The cost of this demonstration should be about $\$ 0.5 \mathrm{M}$. 
Implementation Needs: The successful development of this technology will require that the existing laser systems be adapted and optimized to meet the requirements of specific problem areas. Ultimately, commercially available systems should be capable of turn-key operation by trained technicians. Commercial paint stripping systems have capital costs of about $\$ 500 \mathrm{~K}$. Operating costs should be about $\$ 1 / \mathrm{ft}^{2}$.

Author: D. B. Smith/615-574-2196

\section{References:}

1. N. S. Cannon and D. J. Flesher, "Lasers for the Radioactive Decontamination of Concrete," presented at the Sixteenth International Conference on Lasers and Applications, Lake Taho, Nev., Dec. 6-10, 1993; Report WHC-SA-2116-FP, Westinghouse Hanford Company, Richland, Wash., October 1993.

2. Dann J. Flesher, Westinghouse-Hanford Company, Richland, Wash., personal communications to D. B. Smith, Oak Ridge National Laboratory, November 30, 1993.

3. J. S. Foley, "Laser Paint Stripping: An Automated Solution," Industrial Laser Review, August 1991, p. 4-9; J. D. Head, "New $\mathrm{CO}_{2}$ Laser Cuts Paint-stripping Damage," Industrial Laser Review, August 1991, p. 11-14.

4. J. J. Wong and D. J. Flesher, Light Aided (Laser) Decontamination. Report WHC-SD-WMTI-518, Westinghouse Hanford Company, Richland, Wash., July 1992.

5. "Lasers Can Cost-effectively Erase Graffiti, Study Shows," $R$ \& D Magazine, November 1993, p. 10.

September 1994

Decontamination and Decommissioning 
EM Problem: Decontamination and Decommissioning

Y-12 Plant Problem: Building 9201-4 (Alpha-4)

Problem Area/Constituents: In situ decontamination of the interior of steel and nickel process equipment contaminated with $\mathrm{Hg}, \mathrm{LiOH}, \mathrm{H}_{2} \mathrm{SO}_{4}$, and $\mathrm{HNO}_{3}$.

\section{Reference Requirements:}

- Federal Facility Compliance Agreements (e.g., FFCA for Oak Ridge Site and FFCAs for PCBs)

- Applicable federal and state laws and regulations (e.g., Clean Air Act and Toxic Substances Control Act)

- DOE orders

\section{Subelement: Decontamination}

Alternative: Thermal surface removal methods

Technology: Laser ablation. Laser ablation is a method for removing contaminants at the surface or at very shallow depths below the surface. Laser ablation can be used to remove contaminants from a surface or from very shallow depths below the surface. Albation occurs when a tightly focused beam from a pulsed laser is incident on a surface and the fluence (energy per unit area) of the beam is sufficient to initiate a rapid ejection of material from the surface. Ablation is a complex combination of photochemical and photothermal effects. The rate of ablative removal can in most instances be highly controlled by adjusting parameters such as laser wavelength, pulse duration, pulse repetition rate, and fluence.

Status: Demonstration-Decontamination using laser ablation is being demonstrated and evaluated at Ames Laboratory in Ames, Iowa, at Westinghouse Idaho Nuclear Company in Idaho Falls, Idaho, and at Hanford-Westinghouse Laboratory in Hanford, Washington. Some work is also being done at Penn State University and Oak Ridge National Laboratory in Oak Ridge, Tennessee. The technology has been used to remove radiological and organic contaminants from a variety of surface types. For example, at the Ames Laboratory, laser ablation has been used to remove cesium from contaminated stainless steel and to remove contaminants from aluminum ductwork. In addition, laser ablation can easily remove paint or oll from surfaces by decomposing the organic film and reducing it to carbon dioxide and carbon ash.

Capital cost, $\$ 0.5-\$ 1 \mathrm{M} /$ machine; operating cost, $>\$ 1 / \mathrm{ft}^{2}$.

Waste produced-This technology produces a minimal amount of waste. The primary waste produced is the thin layer of material that is ablated from the surface. All material is collected in an off-gas filtration system. Laser ablation is a radiative, noncontact process. 
Science/Technology Needs: Laser ablation for decontamination is between the laboratory demonstration and prototype stage. The versatility and effectiveness of the technique has been demonstrated, and the hardware and other materials required for implementing the technique (e.g., lasers, optics, vacuum and filtration systems) already exist. What is needed is a concerted effort to bring all of the components together in a prototype turn-key system. The development of the technique will need to be coordinated with these requirements. This effort should cost about \$1M.

Implementation Needs: The successful development of laser ablation decontamination will require a team of researchers directed toward its development. There are several laboratories and many qualified researchers available. A best guess of the human resources required is that 5 person-years of effort over a period of one to two years will be sufficient to develop and demonstrate a prototype system. Most of the hardware needed will consist of off-the-shelf equipment such as laboratory glove boxes, laser systems, and data acquisition systems. A rough estimate of the financial investment required for development and demonstration is \$2 over a 3-year period.

The successful deployment of the technology will require a transfer of the technology from laboratory researchers to a commercial manufacturer. The prototype system would serve as the basis for large-scale or mass production system. In concept, a laser-based decontamination system should lend itself to a high degree of automation, and a commercially available system should be capable of turn-key operation. One technician would be capable of overseeing the operation of multiple systems.

Author: D. B. Smith/615-574-2196

\section{References:}

1. M. C. Edelson, Ames Laboratory, Ames, Iowa, personal communication to D. B. Smith, Oak Ridge National Laboratory, March 26, 1993.

2. H. M. Pang et al., "Laser Decontamination: A New Strategy for Facility Decommissioning," p. 1335 in Proceedings of the International Topical Meeting on Nuclear and Waste Management (SPECTRUM '92), Boise, Idaho, August 23-27, 1992.

3. H. M. Pang, D. Baldwin, and M. C. Edelson, "Macroscopic Surface Cleaning Using a High Repetition Rate Ultraviolet Laser," p. 150 in Proceeings of the IEEE Lasers and Electro-Optics Society (LEOS '92), Hynes Convention Center, Boston, November 16-19, 1992.

4. H. M. Pang, M. C. Edelson, and R. Demmer, "Metal Decontamination Using High Power Lasers," presented at ER '93, Radisson Riverfront Hotel, Augusta, Georgia, October 24-28, 1993.

September 1994

Decontamination and Decommissioning 
EM Problem: Decontamination and Decommissioning

Y-12 Plant Problem: Building 9201-4 (Alpha-4)

\section{Problem Area/Constituents:}

- In situ decontamination of the interior of steel and nickel process equipment contaminated with $\mathrm{Hg}$, $\mathrm{LiOH}, \mathrm{H}_{2} \mathrm{SO}_{4}$, and $\mathrm{HNO}_{3}$.

- Ex situ decontamination of steel and nickel process equipment contaminated with $\mathrm{Hg}$, $\mathrm{LiOH}, \mathrm{H}_{2} \mathrm{SO}_{4}, \mathrm{HNO}_{3}$, asbestos, and PCBs.

- Packing contaminated with $\mathrm{Hg}, \mathrm{LiOH}, \mathrm{H}_{2} \mathrm{SO}_{4}$, and $\mathrm{HNO}_{3}$.

- Metal equipment and sheet metal contaminated with uranium, oil, and polychlorinated biphenyls.

\section{Reference Requirements:}

- Federal Facility Compliance Agreements (e.g., FFCA for Oak Ridge Site and FFCAs for PCBs)

- Applicable federal and state laws and regulations (e.g., Clean Air Act and Toxic Substances Control Act)

- DOE orders

Refer to the Regulatory Compliance chapter of Vol. 1 for potentially applicable proposed and promulgated environmental laws, signed and pending agreements for the Oak Ridge Reservation, radiation protection standards, DOE orders, and nonregulatory guidance. As site- and waste-specific characteristics are provided for each technology, specific regulatory requirements will be specified.

\section{Subelement: Decontamination}

Alternatives: Thermal surface and substrate removal methods

Technology: Plasma surface cleaning. With a reactive plasma, chemically reactive species clean contaminated surfaces by chemisorption, reaction and desorption, or gasification. For example, in oxygen plasma cleaning, oxygen atoms, ozone, and UV photons (reactive species) convert hydrocarbon contaminants into volatile products. This plasma cleaning technology is being developed for the DOE's waste minimization program. The plasma cleaning technology can be developed for decontamination for $\mathrm{Hg}-$, oil-, and PCB-contaminated metal process equipment. This technology can be used for in situ cleaning of the inside of process equipment. For inside application, a partial vacuum will be required and a plasma produced: then it would apply to pipes and large tanks. For ex situ cleaning, various parts and pieces of equipment can be processed in a chamber. Metal packing could also be processed in a chamber. 
Status: Predemonstration. Plasma surface cleaning methods by glow discharge are commonly and effectively used for cleaning high-bonding-energy contaminants from surfaces of metals, metal oxides, and glasses. ' Plasma cleaning is done in vacuum chambers for accelerators and magnetic fusion devices. ${ }^{2}$ Recently tritium contaminated hardware has been plasma cleaned with a decontamination factor of $1000 .^{3}$ Plasma processes of etching and deposition are also used in material processing and microelectronic manufacturing in industry. ${ }^{4-7}$ Extrapolating these plasma cleaning techniques for decomposing and destroying oil and PCB contaminants and for recovering Hg (using Ar or He plasmas) in metal process equipment is feasible. Based on known plasma assisted etching rates, decontamination rates by reactive plasmas are expected to be higher than that by gas phase decontamination methods. Moreover, the plasma cleaning process provides a means of separating and recovering mercury from the mixed contaminants, recycling the process equipment, reducing the volume of generated secondary wastes, and helping to minimize the final waste deposition cost. Though additional radio frequency or microwave power is required, the plasma cleaning techniques will be approximately a factor of ten cheaper and faster than the gas cleaning techniques. Together with a scrubber, the supporting equipment used for a gas phase decontamination system, including thermal management system, vacuum system, computer control and monitors can be used for the waste generated in plasma cleaning methods. It is the author's opinion that a savings of a factor of ten could be realized in a production environment.

Efficacy/strengths/weaknesses:

Efficacy-The plasma cleaning process is an excellent technique to remove contaminants without creating additional secondary waste. It is capable of cleaning interior surfaces as well as exterior surfaces. It could be used to clean contaminated equipment and hardware, which could be recycled for other industrial and governmental applications.

Strengths-Plasma processing devices have been used in manufacturing microelectronics, which require the process equipment to be operated in an ultraclean environment. Of course, this cleaning device can be designed and operated to meet environmental regulations. It always operates in a chamber at sub atmospheric pressures, and no effluent gaseous products or $\mathrm{Hg}$ vapor will leak out to the atmosphere without being exhausted through a filter system.

Weaknesses-It needs additional development to demonstrate large surface area cleaning on real contaminated equipment, to maximize cleaning rate, and to minimize secondary waste production.

Waste-Plasma cleaning is effective for cleaning surface contaminants. It can be operated to remove the contaminated surface layer only. Thus, the amount of waste produced will be minimal. In fact, plasma physical processes of sputtering and vaporizing can be employed to recover mercury without producing secondary wastes. A Hg vapor handling device needs to be implemented. Because plasma cleaning is a unique cleaning process for achieving atomiclevel cleaning, valuable metals or hardware can be recycled for other applications.

September 1994

Decontamination and Decommissioning 
Cost-Plasmas of argon/oxygen have been used to clean oil contaminants on a metal surface at a rate of $2.7 \mu \mathrm{m} / \mathrm{min}$. For a contaminated substrate with a dirty surface layer of $20 \mu \mathrm{m}$, with a plasma apparatus capable of creating uniform plasma over $5 \mathrm{ft}^{2}$ and having an average plasma cleaning rate of $3 \mu \mathrm{m} / \mathrm{min}$, the estimated overall cleaning rate is about $45 \mathrm{ft}^{2} / \mathrm{h}$. Such a plasma cleaning machine could require $50 \mathrm{~kW}$ to operate (@ $\$ 0.06 \mathrm{~kW} / \mathrm{h}$ ) and cost about $\$ 3 / \mathrm{h}$. Using $50 \mathrm{~atm} / \mathrm{L} / \mathrm{h}$ for operating the plasma device might cost $\$ 0.50 / \mathrm{h}$. Using computer control, a skillful operator ( $\$ 100 / \mathrm{h}$ ) may be able to oversee the operation of two plasma cleaning devices. Each plasma cleaning device will clean $45 \mathrm{ft}^{2} / \mathrm{h}$ @ $<\$ 53.5 / \mathrm{h}$ (or $\left.>\$ 1 / \mathrm{ft}^{2}\right)$. If an enhanced plasma cleaning technique can be developed to achieve a rate of $6 \mu \mathrm{m} / \mathrm{min}$, then the area cleaning rate of each machine can be increased to $90 \mathrm{ft}^{2} / \mathrm{h}$, and the operating cost can be lowered to $<\$ 0.60 / \mathrm{ft}^{2}$.

Science Technology Needs: A plasma decontamination method should be developed to fulfill certain science and technology needs, even though plasma discharges have been used in laboratories and industry for cleaning and etching of many materials. Prototype equipment needs to be constructed and operated for generating reactive plasmas to clean and recover mercury. A database of decontamination rate on mercury needs to be established. The capability of plasma generation and cleaning on internal surfaces of contaminated equipment that have large surface areas needs to be established. Also, computer modeling of plasmasurface interactions will be used for providing significant improvement in the removal rate and efficiencies of plasma methods. The modeling will provide guidance on key technical issues associated with geometrical configuration, size, time dependent, and nonlinear plasma chemical reactions. Collection systems for vaporized contaminants should be demonstrated.

Implementation Needs: An experimental facility has been built and used to demonstrate cleaning capability for some Y-12 Plant materials. This facility needs to be upgraded to handle the specific contaminated materials that may be present in some parts of the Y-12 Alpha-4 facility. With the upgraded facility, cleaning rates and decontamination rates would be established. Next, a prototype would be designed, constructed, and demonstrated. This would be followed by operational equipment supplied by the industry.

The overall development cost (to show proof of principle) for the total of these items is estimated at $\$ 1 \mathrm{M}$. The plasma cleaning equipment capital cost cannot be accurately estimated at this time for the range of applications indicated, but it is expected to be in the $\$ 0.5 \mathrm{M}-\$ 1 \mathrm{M}$ range. Operational cost is expected to be very small because of the very low power requirements and limited labor requirements (for maintenance and handling of parts to be cleaned and for operation of the system).

Author: C. C. Tsai/615-574-1124 and W. D. Nelson/615-574-0966

\section{References:}

1. K. L. Mittal, "Surface Contamination: An Overview," Proc. Symp. on Surface Contamination: Its Genesis, Detection and Control, pp. 1-45, Washington, D.C., September 13-14, 1978. 
2. H. F. Dylla, "Glow Discharge Techniques for Conditioning High-Vacuum Systems," J. Vac. Sci Technol. A6 (3), May-June 1988.

3. W. T. Shmayda et al., "Plasma-Driven Decontamination Technique," Canadian Fusion Fuels Technology Report, 8(3), May 1992.

4. J. W. Coburn, "Surface Processing With Partially Ionized Plasmas," IEEE Trans. on Plasma Science, 19(6), December 1991.

5. D. L. Flamm, "Plasma Chemistry in Etching," Proc. of the NATO Advanced Study Institute on Plasma-Surface Interactions and Processing of Materials, pp. 35-55, Alicante, Spain, September 4-6, 1988.

6. H. W. Lehmann, "Applications of Plasma Etching," ibid., 1988, pp. 345-375.

7. In the draft document of the DOE Environmental Management D\&D Workshop, Knoxville, Tenn., August 12-16, 1991. 
EM Problem: Decontamination and Decommissioning

Y-12 Plant Problem: Building 9201-4 (Alpha-4)

Problem Area/Constituents:

- In situ decontamination of the interior of steel and nickel process equipment contaminated with $\mathrm{Hg}, \mathrm{LiOH}, \mathrm{H}_{2} \mathrm{SO}_{4}$, and $\mathrm{HNO}_{3}$.

- Ex situ decontamination of steel and nickel process equipment contaminated with $\mathrm{Hg}$, $\mathrm{LiOH}, \mathrm{H}_{2} \mathrm{SO}_{4}, \mathrm{HNO}_{3}$, asbestos, and PCBs.

- Carbon chunks contaminated with $\mathrm{Hg}, \mathrm{LiOH}, \mathrm{H}_{2} \mathrm{SO}_{4}$, and $\mathrm{HNO}_{3}$.

- Metal equipment contaminated with mercury, $\mathrm{LiOH}, \mathrm{H}_{2} \mathrm{SO}_{4}, \mathrm{HNO}_{3}$, asbestos, uranium, oil, PCBs, etc.

Reference Requirements: Refer to the Regulatory Compliance chapter of Vol. 1 for potentially applicable proposed and promulgated environmental laws, signed and pending agreements for the Oak Ridge Reservation, radiation protection standards, DOE orders, and nonregulatory guidance. As site- and waste-specific characteristics are provided for each technology, specific regulatory requirements will be specified.

\section{Subelement: Decontamination}

Alternative: Thermal surface removal methods

Technology: Plasma etching. Using a hydrogen plasma discharge, volatile $\mathrm{HgH}$ is produced that promotes rapid etching. The contaminated metal surfaces are exposed to energetic ions, electrons, and photons. The decontamination rate is greatly enhanced. The plasma etching technology can be developed for decontamination of DOE process equipment.

Status: Predemonstration. Plasma surface cleaning methods by glow discharges are commonly and effectively used for cleaning high-bonding energy contaminants from surfaces of metals, metal oxides, and glasses. Plasma cleaning is done in vacuum chambers for accelerators and magnetic fusion devices. Recently, tritium-contaminated hardware has been plasma cleaned with a decontamination factor of 1000 . Plasma processes of etching and deposition are also used in material processing and microelectronic manufacturing in industry. The plasma cleaning and etching is a predemonstration technology. Extrapolating these plasma cleaning techniques for gasifying and recovering mercury deposits in process equipment is feasible. Based on plasma-assisted etching rates, decontamination rates by reactive plasmas will be higher than that by gas phase decontamination methods. Moreover, the plasma cleaning process provides a means of separating and recovering mercury from the mixed contaminants, recycling the process equipment, reducing the volume of generated secondary wastes, and helping to minimize the final waste deposition cost.

Although requiring additional radio frequency or microwave power, the plasma cleaning and fluorination techniques could be cheaper and faster than the gas cleaning techniques. The 
supporting equipment used for the gas phase decontamination system, including a gas handling system for trapping $\mathrm{Hg}$ vapor and $\mathrm{HgH}$ gas, thermal management system, vacuum system, computer control, and monitors, can be used for the waste generated in plasma cleaning and etching methods. Since the plasma surface cleaning methods are expected to be much faster than the gas phase decontamination method, it will potentially save (in conjunction with the gas phase system) tens of millions of dollars for the decontamination and decommissioning (D\&D). About $\$ 2 M$ for development costs and $\$ 1 M$ capital equipment costs will be required.

If successfully developed, the prototype hardware could decontaminate a $10-\mathrm{m}^{3} \operatorname{tank}$ in 1 month and cost $\$ 0.25 \mathrm{M}$ (or $\$ 0.75 \mathrm{M}$ per stage). It is the author's opinion that a savings of a factor of ten could be realized in a production environment. Wastes would be vaporized contaminants plus filters and HEPA filters from the collection system.

Efficacy-The plasma etching process is an excellent technique to remove mercury contaminants without creating additional secondary waste. It is capable of etching interior surfaces. It could be used to etch mercury and produce $\mathrm{Hg}$ vapor and gaseous $\mathrm{HgH}$, which could be collected for recovering valuable mercury for other applications. Ex situ, the parts and pieces of equipment and metal packing can be processed with this technology.

Strengths-Plasma-enhanced decontamination may have two separate applications: etching and inert gas cleaning. As a first step, as outlined in the proposal, it is appropriate that this work be carried out in a nonradiological environment, with limited application of hazardous gases. This technology may be used as an alternative to gas phase decontamination. Plasma processing devices have been used in manufacturing microelectronics. It is a useful tool for the electronics industry because plasmas are very reactive in gasifying materials. The etching device can be designed and operated to meet OSHA regulations. It always operates in a chamber at sub atmospheric pressures, and no effluent gaseous products, including $\mathrm{Hg}$ vapor, will leak out to the atmosphere without being exhausted through a filter system.

Weaknesses-It needs scientific data on etching rates for decontaminating real substrate contaminated with mercury and its compounds. It also needs further development for demonstrating large-surface-area etching on real contaminated equipment, maximizing etching rate, and performing both the collection of gaseous $\mathrm{HgH}$ and $\mathrm{Hg}$ vapor and the recovery of mercury.

Waste-The plasma etching technology would not produce additional secondary waste. It will gasify mercury into $\mathrm{Hg}$ vapor and $\mathrm{HgH}$, which can be recovered by collection on a cold trap and reprocessed for other applications.

Cost-At higher etching rates, the plasma etching technology should cost less than the gas phase decontamination. Given an average plasma etching rate of $1 \mu \mathrm{m} / \mathrm{min}$, the overall area etching rate of a 5- $\mathrm{ft}^{2}$ plasma etching machine is estimated to be about $15 \mathrm{ft}^{2} / \mathrm{h}$ for etching a 20- $\mu \mathrm{m}$ thick surface layer. It could take $50 \mathrm{~kW}$ (@ $\$ 0.06 \mathrm{~kW} / \mathrm{h}$ ) to operate such a plasma cleaning device and cost about $\$ 3 / \mathrm{h}$. Using gas at $5 \mathrm{~atm} / \mathrm{L} / \mathrm{h}$ for operating the plasma device

September 1994

Decontamination and Decommissioning 
may cost $\$ 0.50 / \mathrm{h}$. Using computer control, a skillful operator (@ $\$ 100 / \mathrm{h}$ ) could clean $15 \mathrm{ft}^{2}$ (1) $<\$ 103.5 / \mathrm{h}$ (or $<\$ 7 / \mathrm{ft}^{2}$ ). If an enhanced plasma cleaning technique can be developed to achieve a rate of $10 \mu \mathrm{m} / \mathrm{min}$, then the area cleaning rate can be increased to $150 \mathrm{ft}^{2} / \mathrm{h}$, and the operating cost can be lowered to $<\$ 0.7 / \mathrm{ft}^{2}$. A reasonable expectation for the operating cost is $\$ 3 / \mathrm{ft}^{2}$.

Science/Technology Needs: Data on cleaning rates for contaminants and substrates of interest are needed. The capability of plasma etching on deposits in complex equipment with large surface areas needs to be established.

Implementation Needs: An experimental facility has been built and used to demonstrate cleaning capability for some Y-12 Plant materials. This facility needs to be upgraded to handle the specific contaminated materials that may be present in some parts of the Y-12 Alpha-4 facility. With this upgraded facility, cleaning rates and decontamination rates would be established. Next, a prototype would be designed, constructed, and demonstrated. This would be followed by operational equipment supplied by industry. Finally, a collection system with appropriate filters for the vaporized deposits would be needed to use this technology.

The plasma surface cleaning technology project directly applies to numerous decontamination problems. For instance, in addition to the removal of mercury deposits, it will be useful for etching and cleaning stainless steel or nickel processing equipment and packing equipment. The overall development cost ( to show proof of principle) for the total of these items is estimated in a technical task plan at $\$ 1.3 \mathrm{M}$. Thus, the prorated development cost for the machine parts cleaning area would be about $\$ 250 \mathrm{~K}$. The plasma etch equipment capital cost cannot be accurately estimated at this time for the range of applications indicated, but it is expected to be in the $\$ 1 \mathrm{M}$ range. Operational cost is expected to be very small because of the very low power requirements and limited labor requirements (for maintenance and handling of parts to be cleaned and for operation of the system).

Author: C. C. Tsai/615-574-1124 and W. D. Nelson/615-574-0966

References: MMES staff members. 


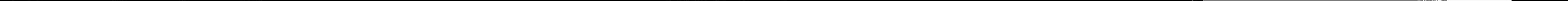


EM Problem: Decontamination and Decommissioning

Y-12 Plant Problem: Building 9201-4 (Alpha-4)

\section{Problem Area/Constituents:}

- In situ decontamination of the interior of steel and nickel process equipment contaminated with $\mathrm{Hg}, \mathrm{LiOH}, \mathrm{H}_{2} \mathrm{SO}_{4}$, and $\mathrm{HNO}_{3}$.

- In situ decontamination of the exterior of steel and nickel process equipment contaminated with $\mathrm{Hg}, \mathrm{LiOH}, \mathrm{H}_{2} \mathrm{SO}_{4}, \mathrm{HNO}_{3}$, asbestos, and PCBs.

- Ex situ decontamination of steel and nickel process equipment contaminated with $\mathrm{Hg}$, $\mathrm{LiOH}, \mathrm{H}_{2} \mathrm{SO}_{4}, \mathrm{HNO}_{3}$, asbestos, and PCBs.

- Packing contaminated with $\mathrm{Hg}, \mathrm{LiOH}, \mathrm{H}_{2} \mathrm{SO}_{4}$, and $\mathrm{HNO}_{3}$.

Reference Requirements: Refer to the Regulatory Compliance chapter of Vol. 1 for potentially applicable proposed and promulgated environmental laws, signed and pending agreements for the Oak Ridge Reservation, radiation protection standards, DOE orders, and nonregulatory guidance. As site- and waste-specific characteristics are provided for each technology, specific regulatory requirements will be specified.

\section{Subelement: Decontamination}

Alternative: Thermal surface removal methods

Technology: Flashlamp cleaning. A method for removing contaminants at the surface or at very shallow depths below the surface. Energy from a high-energy xenon flashlamp is absorbed at the surface, and the ensuing rapid temperature rise causes material to evaporate or decompose to a particulate residue. Flashlamp systems are being evaluated for radionuclide decontamination applications at various $D O E$ sites.

Status: Radiological decontamination using high-energy xenon flashlamps is in the demonstration phase. Hanford-Westinghouse is conducting laboratory tests of xenon flashlamp systems for removing radionuclide contamination from the surfaces inside metal storage vessels.

The primary application for flashlamp cleaning is large surface areas that need a high degree of decontamination with the absolute minimum amount of waste generation. The technology produces as waste only the material that is removed from the surface (all the vaporized material is collected in a filtration system).

Science/Technology Needs: The commercially available flashlamp systems and prototype decontamination systems should be evaluated for possible use on contaminated areas. In particular, a mercury vapor handling equipment needs to be incorporated into flashlamp system for collecting and processing Hg vapors produced. This development may cost about $\$ 2 \mathrm{M}$. 
Implementation Needs: The successful development of this technology will require that commercial vendors of xenon flashlamp systems be encouraged to adapt their systems to meet the requirements of specific problem areas. Commercially available systems should be capable of turn-key operation by trained technicians. Currently available systems cost $\$ 50 \mathrm{~K}-\$ 100 \mathrm{~K}$. Operating costs should be about $\$ 3 / \mathrm{ft}^{2}$.

Author: D. B. Smith/615-574-2196

\section{References:}

1. Dann J. Flesher, Westinghouse-Hanford Laboratory, Hanford, Wash., personal communications to D. B. Smith, Oak Ridge National Laboratory, July 22, 1992, and May 14, 1993.

2. David Van Alstyne, "The Polygon Paint Removal System," Reducing Risk in Paint Stripping, Proceedings of an International Conference sponsored by Office of Toxic Substances, U.S. Environmental Protection Agency, Washington, D.C., February 12-13, 1991.

3. Karl Bremer, "New Paint Strippers Keep Earth Clean and Save You Some Green," Commuter Air International, pp. 8-11, July 1992.

September 1994

Decontamination and Decommissioning 
EM Problem: Decommissioning

ORNL Problem: See Appendix B, lists $\mathrm{C}$ and $\mathrm{H}$

Problem Area/Constituents: See Appendix B, lists $\mathrm{C}$ and $\mathrm{H}$

Reference Requirements: Refer to the Regulatory Compliance chapter of Vol. 1 (Pt. A, B, or $\mathrm{C}$ as appropriate) for potentially applicable proposed and promulgated environmental laws, signed and pending agreements for the Oak Ridge Reservation, radiation protection standards, DOE orders, and nonregulatory guidance. As site- and waste-specific characteristics are provided for each technology, specific regulatory requirements will be specified.

\section{Subelement: Decontamination}

Alternative: Bulk decontamination methods

Technology: Transmutation. Long-lived radioactive isotopes are transformed into radioactive isotopes with shorter half lives through the use of neutron capture, proton capture, or fission. To avoid activation of the stable isotopes, which the radioisotope to be transmuted is mixed with, the stable isotopes must be separated. The radioactive species is then exposed to the neutron or proton flux for a sufficient time to completely transmute the long-lived isotope into stable or shorter-lived isotopes. A particle accelerator would be used to produce protons (and neutrons with a spallation source and moderator), or a fission reactor would be used.

Status: Preconceptual. Although this idea has been advanced for several years, until the advent of Strategic Defense Initiative particle accelerators, sufficiently powerful accelerators were not available.

Waste produced-Potential waste reduction by decreasing the half-life of the irradiated radioisotope.

Overall cost-No cost data is available, but the development cost of technologies like this is extremely expensive.

Efficacy/strengths/weaknesses-Advantages: conversion of long-lived waste into shorterlived waste (converts TRU into short-lived fission products). The only method applicable to activation product wastes. Disadvantages: Applicability to activation product wastes would depend upon the availability of a suitable isotope separation technique so that the bulk of unactivated material is preserved.

Science/Technology Needs: Development of a suitably intense neutron or proton source to reduce reaction times is needed. Tests of the suitability of direct waste irradiation is needed. Development of isotope separation techniques for long-lived activation products is needed.

Implementation Needs: A long-term development program is needed. 
Authors: J. R. DeVore and H. H. Haselton/615-574-7092

References: R\&D Magazine 43 (1993). 
EM Problem: Decontamination and decommissioning

Y-12 Plant Problem: Building 9201-4 (Alpha-4)

Problem Area/Constituents: Mercury and other metals

Reference Requirements: Refer to Volume 1, Chapter 8, for potentially applicable proposed and promulgated environmental laws, signed and pending agreements for the Oak Ridge Reservation, radiation protection standards, DOE orders, and nonregulatory guidance. As site- and waste-specific characteristics are provided for each technology, regulatory requirements will be specified.

To be eligible for exemption from EPA regulation, the maximum concentration of mercury must be less than $50 \mathrm{pg} / \mathrm{m}^{3}$ at the plant property line.

Personnel exposure limits to mercury in air

\begin{tabular}{llllll}
\hline \multirow{2}{*}{ Contaminant } & \multicolumn{2}{c}{ Time-weighted average } & & \multicolumn{2}{c}{ Short-term exposure limit } \\
\cline { 2 - 3 } \cline { 5 - 6 } & \multicolumn{1}{c}{ ICGIH } & Y-12 & & ICGIH & Y-12 \\
\hline Mercury alkyls & $0.01 \mathrm{mg} / \mathrm{m}^{3}$ & $0.005 \mathrm{mg} / \mathrm{m}^{3}$ & & $0.03 \mathrm{mg} / \mathrm{m}^{3}$ & $0.015 \mathrm{mg} / \mathrm{m}^{3}$ \\
All mercury except alkyls & 0.05 & 0.025 & & $\mathrm{NA}$ & $\mathrm{NA}$ \\
Aryl and inorganic mercury & 0.1 & 0.05 & & $\mathrm{NA}$ & $\mathrm{NA}$ \\
The OSHA air contaminant limit for mercury vapor is $0.05 \mathrm{mg} / \mathrm{m}^{3}$. &
\end{tabular}

Subelement: Decontamination

Alternative: Air cleaning methods

Technology: Adsorption. Mercury vapors in the air are collected on an adsorbent such as activated charcoal

Status: Accepted. This is the accepted method for controlling mercury emissions.

Efficacy-The adsorption canister on the bulb crusher at the K-25 Site operates at over $99.5 \%$ efficiency.

Waste- $150 \mathrm{ft}^{3} / \mathrm{h}$.

Cost-Capital cost, $\sim \$ 20 \mathrm{~K}$; operating cost, $\sim \$ 200 \mathrm{~K} /$ year.

Science/Technology Needs: Improvement-System to recover mercury and spent adsorbent. Improvement cost-\$1.3M. 
Implementation Needs: None. This is readily available technology.

Author: D. H. Bunch/615-576-0201

Reference:

1. H. B. McElhoe, personal conversation with D. H. Bunch, $8 / 27 / 93$. 
EM Problem: Decontamination and decommissioning

Y-12 Plant Problem: Building 9201-4 (Alpha-4)

Problem Area/Constituents: Mercury and other metals

Reference Requirements: Refer to Volume 1, Chapter 8, for potentially applicable proposed and promulgated environmental laws, signed and pending agreements for the Oak Ridge Reservation, radiation protection standards, DOE orders, and nonregulatory guidance. As site- and waste-specific characteristics are provided for each technology, regulatory requirements will be specified.

To be eligible for exemption from EPA regulation, the maximum concentration of mercury must be less than $50 \mathrm{pg} / \mathrm{m}^{3}$ at the plant property line.

Personnel exposure limits to mercury in air

\begin{tabular}{llllll}
\hline \multirow{2}{*}{ Contaminant } & \multicolumn{2}{c}{ Time-weighted average } & & \multicolumn{2}{c}{ Short-term exposure limit } \\
\cline { 2 - 3 } \cline { 5 - 6 } & \multicolumn{1}{c}{ ICGIH } & Y-12 & & ICGIH & Y-12 \\
\hline Mercury alkyls & $0.01 \mathrm{mg} / \mathrm{m}^{3}$ & $0.005 \mathrm{mg} / \mathrm{m}^{3}$ & & $0.03 \mathrm{mg} / \mathrm{m}^{3}$ & $0.015 \mathrm{mg} / \mathrm{m}^{3}$ \\
All mercury except alkyls & 0.05 & 0.025 & & $\mathrm{NA}$ & $\mathrm{NA}$ \\
Aryl and inorganic mercury & 0.1 & 0.05 & $\mathrm{NA}$ & $\mathrm{NA}$ \\
\multicolumn{2}{l}{ The OSHA air contaminant limit for mercury vapor is $0.05 \mathrm{mg} / \mathrm{m}^{3}$. }
\end{tabular}

Subelement: Decontamination

Alternative: Air cleaning methods

Technology: Condensation. Mercury vapors in the air are condensed. To reduce the mercury concentration below the time-weighted average (TWA) the condenser would operate at $-31^{\circ} \mathrm{C}$, just above the melting point $\left(38^{\circ} \mathrm{C}\right)$. This technology has been common practice in laboratories, but no references to its use on a large scale for mercury was found.

Status: Conceptual. Waste produced-The mercury itself. Overall cost-Capital cost, $\sim \$ 50 \mathrm{~K}$; operating cost, $\sim \$ 100 \mathrm{~K} /$ year.

Science/Technology Needs: Demonstration on a large scale. Development cost $\$ 1.3 \mathrm{M}$.

Implementation Needs: Distillation may be required to purify the mercury.

Author: D. H. Bunch/615-576-0201 


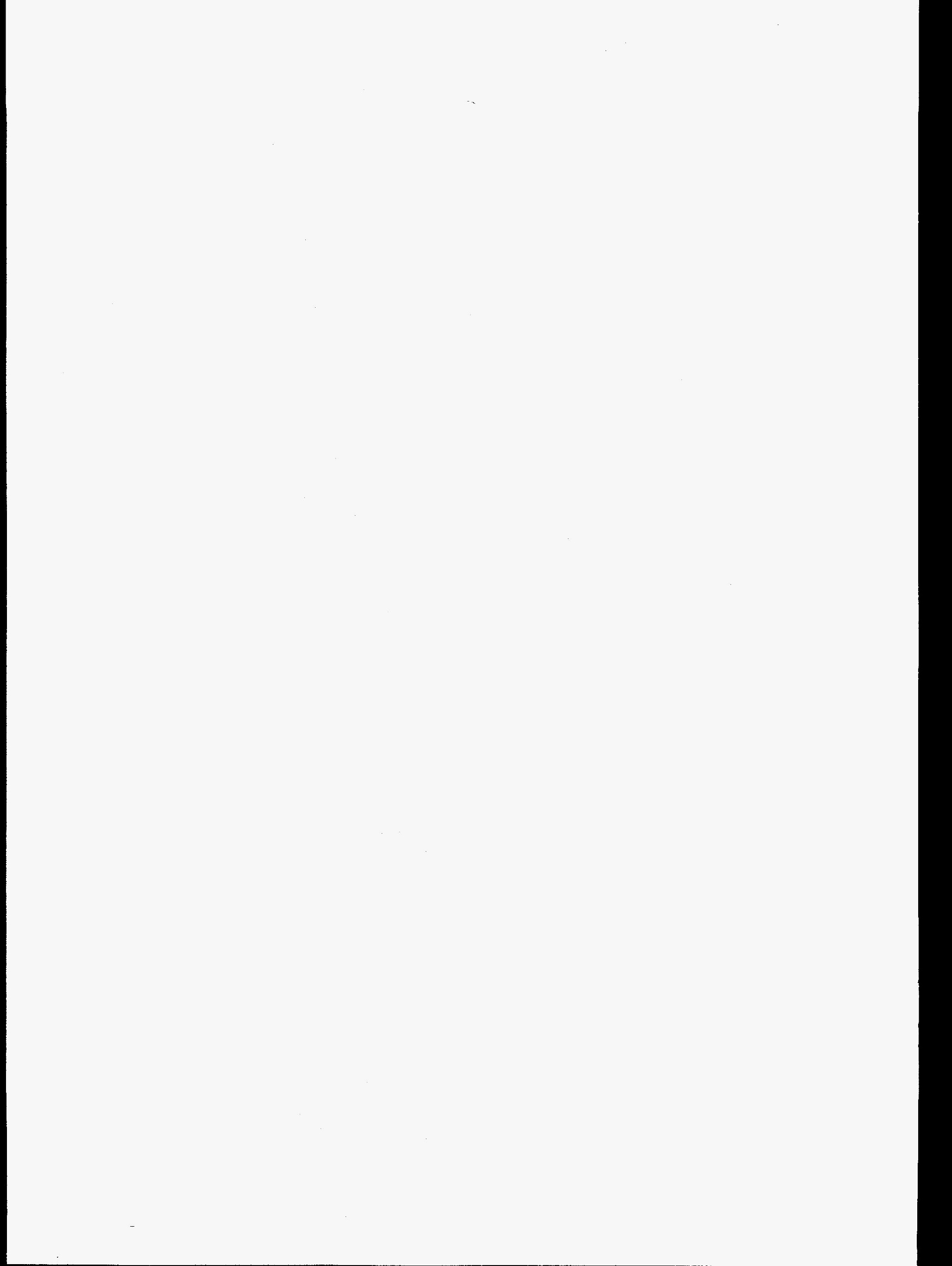


EM Problem: Decontamination and decommissioning

Y-12 Plant Problem: Building 9201-4 (Alpha-4)

Problem Area/Constituents: Mercury and other metals

Reference Requirements: Refer to Volume 1, Chapter 8, for potentially applicable proposed and promulgated environmental laws, signed and pending agreements for the Oak Ridge Reservation, radiation protection standards, DOE orders, and nonregulatory guidance. As site- and waste-specific characteristics are provided for each technology, regulatory requirements will be specified.

To be eligible for exemption from EPA regulation, the maximum concentration of mercury must be less than $50 \mathrm{pg} / \mathrm{m}^{3}$ at the plant property line.

Personnel exposure limits to mercury in air

\begin{tabular}{llllll}
\hline \multirow{2}{*}{ Contaminant } & \multicolumn{2}{c}{ Time-weighted average } & & \multicolumn{2}{c}{ Short-term exposure limit } \\
\cline { 2 - 3 } \cline { 5 - 6 } & \multicolumn{1}{c}{ ICGIH } & Y-12 & & ICGIH & Y-12 \\
\hline Mercury alkyls & $0.01 \mathrm{mg} / \mathrm{m}^{3}$ & $0.005 \mathrm{mg} / \mathrm{m}^{3}$ & & $0.03 \mathrm{mg} / \mathrm{m}^{3}$ & $0.015 \mathrm{mg} / \mathrm{m}^{3}$ \\
All mercury except alkyls & 0.05 & 0.025 & $\mathrm{NA}$ & $\mathrm{NA}$ \\
Aryl and inorganic mercury & 0.1 & 0.05 & & $\mathrm{NA}$ & $\mathrm{NA}$ \\
\multicolumn{2}{l}{ The OSHA air contaminant limit for mercury vapor is $0.05 \mathrm{mg} / \mathrm{m}^{3}}$. &
\end{tabular}

Subelement: Decontamination

Alternative: Air cleaning methods

Technology: Electrostatic precipitation. Particles of the contaminant become charged as they pass through an electrical field. They are then collected on electrodes.

Status: Preconceptual. No references to the use of electrostatic precipitation to removal of mercury to low levels was found.

Efficacy-Very poor. Mercury is very volatile, and electrostatic precipitation is only effective on contaminants in condensed forms.

Waste-The mercury itself.

Cost-Capital cost, <\$1M; operating cost, \$500K/year. 
Science/Technology Needs: Science: Tests to demonstrate the use of electrostatic precipitation to remove mercury from air. Development: Design and testing of a full-scale system.

Implementation Needs: Development costs, $\$ 5 \mathrm{M}$.

Author: D. H. Bunch/615-576-0201

\section{Reference:}

1. R. W. Scheck et al., Economics of Fabric Filters and Electrostatic Precipitators - 1984, Stearns Catalytic Corporation, for Electric Power and Research Institute, 1985. 
EM Problem: Decontamination and decommissioning

Y-12 Plant Problem: Building 9201-4 (Alpha-4)

Problem Area/Constituents: Mercury and other metals

Reference Requirements: Refer to Volume 1, Chapter 8, for potentially applicable proposed and promulgated environmental laws, signed and pending agreements for the Oak Ridge Reservation, radiation protection standards, DOE orders, and nonregulatory guidance. As site- and waste-specific characteristics are provided for each technology, regulatory requirements will be specified.

To be eligible for exemption from EPA regulation, the maximum concentration of mercury must be less than $50 \mathrm{pg} / \mathrm{m}^{3}$ at the plant property line.

Personnel exposure limits to mercury in air

\begin{tabular}{llllll}
\hline \multirow{2}{*}{ Contaminant } & \multicolumn{2}{c}{ Time-weighted average } & & \multicolumn{2}{c}{ Short-term exposure limit } \\
\cline { 2 - 3 } \cline { 5 - 6 } & \multicolumn{1}{c}{ ICGIH } & Y-12 & & ICGIH & Y-12 \\
\hline Mercury alkyls & $0.01 \mathrm{mg} / \mathrm{m}^{3}$ & $0.005 \mathrm{mg} / \mathrm{m}^{3}$ & & $0.03 \mathrm{mg} / \mathrm{m}^{3}$ & $0.015 \mathrm{mg} / \mathrm{m}^{3}$ \\
All mercury except alkyls & 0.05 & 0.025 & & $\mathrm{NA}$ & $\mathrm{NA}$ \\
Aryl and inorganic mercury & 0.1 & 0.05 & $\mathrm{NA}$ & $\mathrm{NA}$ \\
\multicolumn{2}{l}{ The OSHA air contaminant limit for mercury vapor is $0.05 \mathrm{mg} / \mathrm{m}^{3}}$. &
\end{tabular}

Subelement: Decontamination

Alternative: Air cleaning methods

Technology: Scrubbing. Mercury is absorbed by a solution

Status: Accepted. This technology has been used by a glass manufacturer for seven years.

Efficacy-90\%.

Waste- $1 \mathrm{gal} / \mathrm{lb}$ mercury.

Cost-Capital cost, $\$ 200-\$ 300 \mathrm{~K}$; operating cost, $\$ 10-\$ 20 / \mathrm{ft}^{3}$.

Science/Technology Needs: None

Implementation Needs: A system for treating the scrubber solution for rebase is needed to use this technology. 
Author: D. H. Bunch/615-576-0201

Reference:

1. Beco Engineering Company, Oakmont, Pennsylvania, 8/24/93 
EM Problem: Decontamination and decommissioning

Y-12 Plant Problem: Building 9201-4 (Alpha-4)

Problem Area/Constituents: Mercury and other metals

Reference Requirements: Refer to Volume 1, Chapter 8, for potentially applicable proposed and promulgated environmental laws, signed and pending agreements for the Oak Ridge Reservation, radiation protection standards, DOE orders, and nonregulatory guidance. As site- and waste-specific characteristics are provided for each technology, regulatory requirements will be specified.

To be eligible for exemption from EPA regulation, the maximum concentration of mercury must be less than $50 \mathrm{pg} / \mathrm{m}^{3}$ at the plant property line.

Personnel exposure limits to mercury in air

\begin{tabular}{llllll}
\hline \multirow{2}{*}{ Contaminant } & \multicolumn{2}{c}{ Time-weighted average } & & \multicolumn{2}{c}{ Short-term exposure limit } \\
\cline { 2 - 3 } \cline { 5 - 6 } & \multicolumn{1}{c}{ ICGIH } & Y-12 & & ICGIH & Y-12 \\
\hline Mercury alkyls & $0.01 \mathrm{mg} / \mathrm{m}^{3}$ & $0.005 \mathrm{mg} / \mathrm{m}^{3}$ & & $0.03 \mathrm{mg} / \mathrm{m}^{3}$ & $0.015 \mathrm{mg} / \mathrm{m}^{3}$ \\
All mercury except alkyls & 0.05 & 0.025 & & $\mathrm{NA}$ & $\mathrm{NA}$ \\
Aryl and inorganic mercury & 0.1 & 0.05 & & $\mathrm{NA}$ & $\mathrm{NA}$ \\
The OSHA air contaminant limit for mercury vapor is $0.05 \mathrm{mg} / \mathrm{m}^{3}$. &
\end{tabular}

Subelement: Decontamination

Alternative: Air cleaning methods

Technology: Filtration. A high-efficiency particulate air (HEPA) filter usually having sufficiently small pores to remove virtually all particles and droplets down to 0.5 micron is used. Prefilters are often used to remove the bulk of larger particles to extend the life of the HEPA filter. Usually a filter removes mercury aerosol droplets or other contaminants from the air. Filters, except for mersorb and similar filters, which are actually sorbents, cannot remove mercury vapor from air. Mercury that is sorbed on droplets or particles resulting from decontamination can be removed.

Status: Accepted.

Efficacy-Conventional HEPA filters remove only aerosols not gaseous contaminants. Because mercury has a high-vapor pressure at ambient temperatures, HEPA filtration at ambient temperature can only reduce the concentration of mercury to about $1.5 \mathrm{mg} / \mathrm{m}^{3}$. This is above the $\operatorname{TLV}\left(0.5 \mathrm{mg} / \mathrm{m}^{3}\right)$. 
The use of a HEPA filter upstream of an adsorbent trap might be economical. The HEPA filter would reduce the amount of spent sorbent that would be disposed of, and the adsorbent would reduce the mercury concentration to the desired value.

Waste- $150 \mathrm{ft}^{3} /$ year

Cost-Capital cost, $\sim \$ 20 \mathrm{~K}$; operating cost, $\sim \$ 12 \mathrm{~K} /$ year.

Science/Technology Needs: None

Implementation Needs: No unusual implementation needs. This is a readily available technology.

Author: D. H. Bunch/615-576-0201

References: (AUTHOR: Please complete.) 


\section{EM Problem: Decontamination and decommissioning}

\section{Y-12 Plant Problem: Building 9201-4 (Alpha-4)}

Problem Area/Constituents: Nickel and steel process equipment contaminated with mercury, concrete and clay tile contaminated with $\mathrm{Hg}$, carbon chunks contaminated with $\mathrm{Hg}$, packing contaminated with $\mathrm{Hg}$.

Reference Requirements: Refer to the Regulatory Compliance chapter of Vol. 1 for potentially applicable proposed and promulgated environmental laws, signed and pending agreements for the Oak Ridge Reservation, radiation protection standards, DOE orders, and nonregulatory guidance. As site- and waste-specific characteristics are provided for each technology, specific regulatory requirements will be specified.

\section{Subelement: Decontamination}

Alternative: Chemical surface cleaning methods

Technology: Hypochlorite oxidation of $\mathrm{Hg}$. Mercury (metallic or ionic) is mobilized for removal by oxidization with a common oxidizing agent, sodium hypochlorite (NaOCl) in water solution. The mercury is oxidized to $\mathrm{Hg}^{2+}$ which is soluble in water. The mercury-laden water is collected and flushed through an ion exchange resin with thiol (-SH) functional groups which preferentially retains $\mathrm{Hg}^{2+}$. The $\mathrm{Hg}$-loaded resin is regenerated with acid and the $\mathrm{Hg}$ recovered as a metal by reduction with $\mathrm{Zn}$ metal, for example, and amalgamation with $\mathrm{Zn}$, $\mathrm{Cu}, \mathrm{Au}$ or other metal.

Status: Accepted: The hypochlorite oxidation aspects of the process have been common practice in Alpha-4 to clean surfaces and reduce the mercury vapor concentration in ambient air. The core process is commercially practiced on an industrial scale at chlor-alkali plants as the Akzo Process. Specific application to Alpha-4 would additionally require surface cleaning equipment, solution recovery equipment, and procedures to use $\mathrm{NaOCl}$ solutions on floor, wall, and machinery surfaces. The equipment pieces could also be cleaned using an immersion procedure. Equipment in place could be cleaned internally by in situ flushing.

Efficacy: Useful for $\mathrm{Hg}, \mathrm{Hg}^{1+}, \mathrm{Hg}^{2+}$. Mercury $<2 \mathrm{ppb}$ (detection limit of analytical procedure) left in processed liquid effluent. Efficacy would depend on the solution recovery effectiveness which is undemonstrated, or the solution contact if a tank dipping procedure is used.

Weakness: Use in the open air requires personnel protection from fumes from $\mathrm{NaOCl}$.

Waste produced- Debris from the hypochlorite washing process must be filtered out. Reductant ( $\mathrm{NaHSO}_{3}, \mathrm{Na}_{2} \mathrm{SO}_{3}$ or $\mathrm{SO}_{2}$ to remove excess $\left.\mathrm{NaOCl}\right)$ and spent reductant $\left(\mathrm{Na}_{2} \mathrm{SO}_{4}\right)$ are left in effluent. Spent resin is left at the conclusion of its useful life. Spent activated carbon is left from a process cleanup step.

Science/Technology Needs: Technology needs are minimal. While improvements can be made, in general, the core technology is ready to use. 
Implementation Needs: Technology integration of application, washing or dipping and solution recovery systems with Akzo process will be necessary.

Operating cost: $>\$ 1 / \mathrm{ft}^{2}$.

Capital cost: $>\$ 300 \mathrm{k}$

Author: R. L. Fellows/615-576-5632

\section{References:}

1. Akzo Zout Chemie, The Netherlands: Akzo Process.

2. Rohm and Haas Company, Philadelphia, PA, Duolite GT-73 thiol resin.

3. C. Calmon, Ion Exchange and Pollution Control, p 201 (1979)

4. M. D. Rosenweig, Chemical Engineering, 82, 60 (1975) 
ROBOTICS/AUTOMATION 


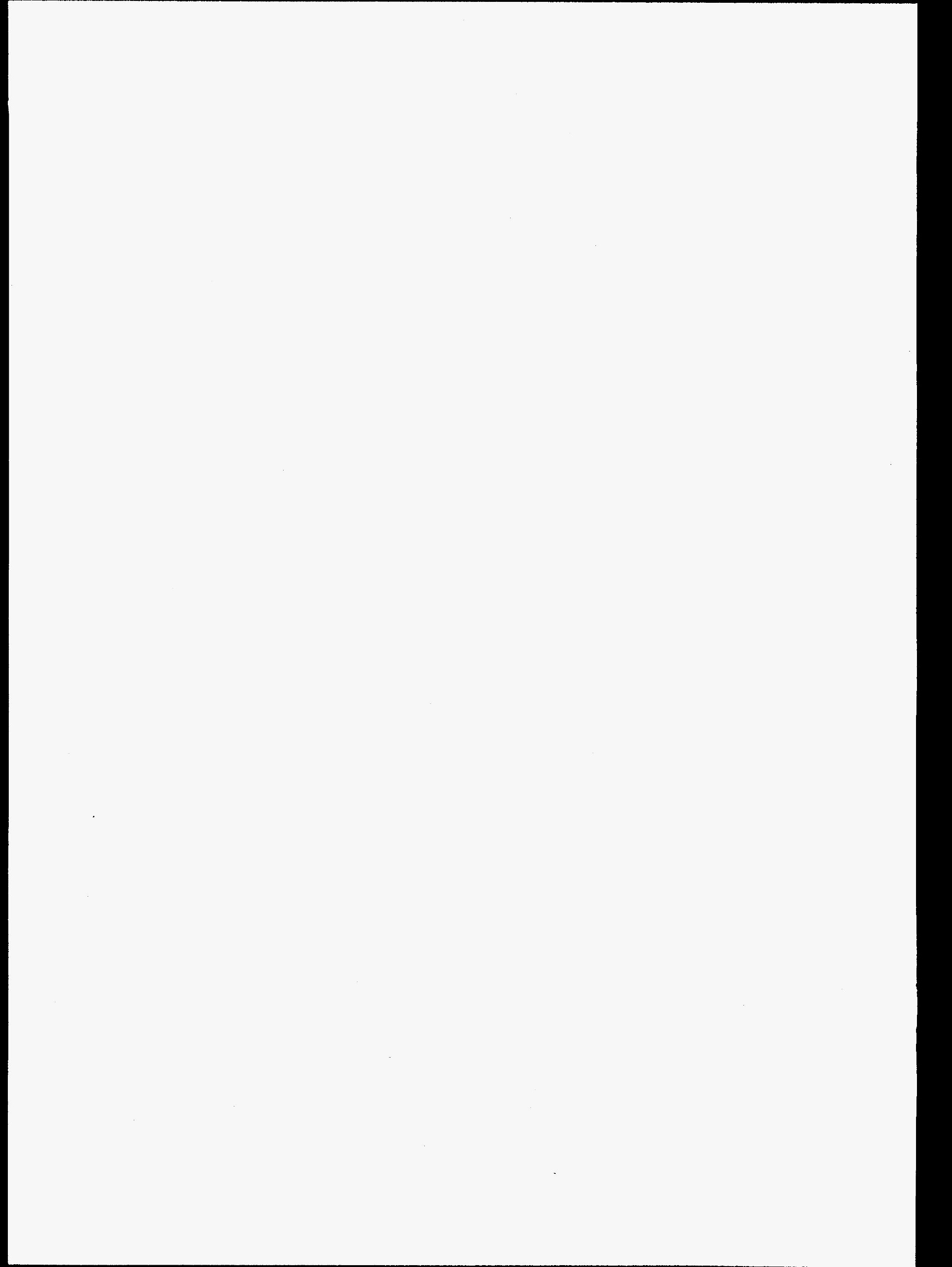


EM Problem: Decontamination and decommissioning (D\&D); remedial action; waste management

Y-12 Plant Problem: Characterization, D\&D of buildings and equipment, characterization surveys and retrieval of buried waste materials, and removal of contaminated soils. Building 9201-4 (Alpha-4)

Problem Area/Constituents: Radioactive and hazardous materials encountered in characterization, decontamination, dismantlement, and retrieval operations

Reference Requirements: Refer to the Regulatory Compliance chapter of Vol. 1 for potentially applicable proposed and promulgated environmental laws, signed and pending agreements for the Oak Ridge Reservation, radiation protection standards, DOE orders, and nonregulatory guidance. As site- and waste-specific characteristics are provided for each technology, specific regulatory requirements will be specified.

Subelement: Robotics/automation

Alternatives: Mechanical; mobility

Technology: Wheeled/tracked vehicles. Robotically or teleoperated vehicles provide methods for deploying sensors or tools required for characterization, decontamination, dismantlement, and retrieval activities. Applications can range from light duty (100-1000 lb) to heavy duty $(>1,000 \mathrm{lb})$. Tracked vehicles would be used primarily on external missions and wheeled vehicles for inside of buildings or structures.

Status: Generally an accepted technology. Many examples of both wheeled and tracked vehicles exist, and they are commercially available. These generally require a dedicated operator for each vehicle. Autonomous controls/capabilities generally are not available.

Efficacy-Very high. Vehicles will be the primary method of deploying tools and sensors required for environmental management problems.

Waste-Emissions for internal combustion power sources. Possible lubrication or hydraulic oil leaks. Decontamination of system after use.

Science/Technology Needs: The primary needs for this technology are not related to its mechanical characteristics, but rather to improvements in the remote control station to allow more autonomy of operation and in the decontamination features to permit faster and more efficient cleaning of the system after use.

Implementation Needs: There are very few implementation needs for this technology. Some training of operators would be required particularly if advanced control stations are developed. Development costs are estimated to range between $\$ 2 \mathrm{M}$ and $\$ 3 \mathrm{M}$, primarily to provide the enhanced control stations. Capital costs, which will depend on the size and characteristics of the vehicle, could range from $\$ 100 \mathrm{~K}$ to $\$ 300 \mathrm{~K} /$ vehicle. 
Author: B. H. Singletary/615-574-6394

References: Personal experience with such systems 
EM Problem: Decontamination and decommissioning (D\&D)

Y-12 Plant Problem: Buildings and equipment. Building 9201-4 (Alpha-4)

Problem Area/Constituents: Pipe/duct crawlers for characterization and decontamination of the inside surfaces of pipes and ducts

Reference Requirements: Refer to the Regulatory Compliance chapter of Vol. 1 for potentially applicable proposed and promulgated environmental laws, signed and pending agreements for the Oak Ridge Reservation, radiation protection standards, DOE orders, and nonregulatory guidance. As site- and waste-specific characteristics are provided for each technology, specific regulatory requirements will be specified.

Subelement: Robotics/automation

Alternatives: Mechanical; mobility

Technology: Pipe/duct crawler (internal). A remote deployment system is required for deployment of sensors for characterization and the deployment of process methods for decontamination within pipes and ductwork. Technology exists to deploy remote devices within pipes and ductwork; however, the capability of those devices to do useful work is limited. Work to date has been limited to primarily video and simple sensing.

A major driver for pursuing internal pipe or ductwork systems is to help establish the exact extent of contamination within these areas. Without internal characterization, all of these extensive systems must be considered contaminated and treated as such in dismantlement and waste handling. Internal characterization and possibly decontamination could potentially limit the extent of work required and waste generated from the $D \& D$ of these systems.

\section{Status: Predemonstration}

Efficacy-The ability for internal pipe and duct crawlers to provide adequate mobility has been demonstrated in the absence of obstacles.

Waste-Secondary waste may be generated during decontamination of the system after use.

Science/Technology Needs: Technology development is required in the following areas:

- Miniaturization of sensors and tooling. Considerable effort is required to develop the small scale sensors, manipulation and/or decontamination process systems needed within this highly constrained environment.

- Obstruction removal or bypass. A major impediment to extensive deployment of internal pipe and ductwork systems is the frequent existence of obstructions such as valves, flow vanes, etc. 
- Development of control and data collection autonomy for these systems is required to allow systems to traverse longer runs with little or no direct operator supervision.

Implementation Needs: No development or demonstration program in this area is currently under way. A viable demonstration project would require hardware and software development, integration, and testing. A rough order of magnitude estimate for such a feasibility demonstration program would take from 1 to 1.5 years and would cost a total of from $\$ 1 M$ to $\$ 2 M$.

Current technology exists to deploy remote video within pipes and ductwork. Current internal pipe and ductwork systems are primarily deployed with tethers for communications, power, and retrieval. An area of concern is cable management for tethered systems. Engineering modifications of development systems, as well as integration and test for specific applications will be required for implementation. Mobility systems with simple manipulation, but excluding decon or sensing systems, are estimated to cost under $\$ 1 \mathrm{M} /$ vehicle.

Author: D. C. Haley/615-576-4388

References: Personal communication and vendor literature 
EM Problem: Decontamination and decommissioning

Y-12 Plant Problem: Buildings and equipment. Building 9201-4 (Alpha-4)

Problem Area/Constituents: Support of characterization, dismantlement, and decontamination. Requires robotic device capable of negotiating the external surface of a pipe or duct. Asbestos removal and packaging is one particularly large application.

Reference Requirements: Refer to the Regulatory Compliance chapter of Vol. 1 for potentially applicable proposed and promulgated environmental laws, signed and pending agreements for the Oak Ridge Reservation, radiation protection standards, DOE orders, and nonregulatory guidance. As site- and waste-specific characteristics are provided for each technology, specific regulatory requirements will be specified.

\section{Subelement: Robotics/automation}

Alternatives: Mechanical; mobility

Technology: Pipe/duct crawler (external). A remote deployment system is required for deployment of sensors for characterization and the deployment of process methods for decontamination on external pipes and ductwork. Similar crawler/climber systems are required for other complex access or complex geometry areas. Technology exists to deploy remote devices on external pipes and ductwork; however, the capability is primarily in laboratory development stages.

Status: Predemonstration. Current commercial costs for asbestos removal from linear pipe runs is approximately $\$ 35 / \mathrm{ft}$ and is expected to more than double when low-level radiation contamination is present. An effective remote system would provide cost benefits, particularly considering applications at other sites and possible commercial potential. Primary considerations for robotic deployment include reduced secondary waste generation from protective clothing and barriers, current low worker productivity, and reduced worker exposure to asbestos.

Efficacy-The ability of an external crawler to provide mobility for sensors or tooling is high in the absence of obstacles in the path.

Waste-Secondary waste may be generated during decontamination of the system after use.

Science/Technology Needs: Technology development is required in the following areas:

- Robotic compatibility of tooling. Current manual methods for removing asbestos must be modified for robotic deployment or new robotic methods developed.

- Obstruction bypass. A major impediment to extensive deployment of external pipe crawling systems is the frequent encounter with obstructions such as valves and pipe hangers. 
Implementation Needs: An Office of Technology Development Robotics Technology Development Program development project for a robotic demonstration system for asbestos removal and bagging from straight pipe runs was planned and estimated. Demonstration on straight runs with no obstacles was estimated at approximately $\$ 1 \mathrm{M}$ for 1 year. Development for other applications would require similar funding levels.

Engineering modifications of development systems, as well as integration and tests for specific applications, will be required for implementation. Depending on the complexity of the crawler and tooling, system cost of as much as \$1M could be expected.

Author: D. C. Haley/615-576-4388

References: MMES staff members' personal experiences/opinions; vendor literature 
EM Problem: Decontamination and decommissioning (D\&D); remedial action (RA)

Y-12 Plant Problem: Buildings, equipment, and impoundments. Building 9201-4 (Alpha-4)

Problem Area/Constituents: Support of characterization, decontamination, dismantlement, remediation, packaging, handling, and inspection. Specialized robotic deployment platforms are required to deploy tools and sensors.

Reference Requirements: Refer to the Regulatory Compliance chapter of Vol. 1 for potentially applicable proposed and promulgated environmental laws, signed and pending agreements for the Oak Ridge Reservation, radiation protection standards, DOE orders, and nonregulatory guidance. As site- and waste-specific characteristics are provided for each technology, specific regulatory requirements will be specified.

Subelement: Robotics/automation

Alternatives: Mechanical; mobility

Technology: Specialized robotic deployment platforms. This category includes all specialized robotic deployment systems that might be advantageous for deployment of tools, sensors, or processes in support of D\&D and RA tasks. Such specialized systems would include climbing robots, submersibles, variable geometry truss structures, etc. Any robotic system specially designed to meet unique deployment requirements could fall under this category.

\section{Status: Demonstration}

Efficacy/strengths/weaknesses-Climbing robots exist for maintenance of high-voltage power line towers. Submersibles are available and well tested, although visibility in fresh-water impoundments is a significant problem. Variable geometry truss structures and specific designs to meet specific constraints are less well documented as to potential effectiveness. Waste-Possible hydraulic fluid during operation and secondary waste generated during decontamination after use.

Overall cost-Because such systems are specialized in nature (i.e., their use is restricted to special cases), estimates for costs are difficult to make. A rough order of magnitude for development costs is $\$ 5 \mathrm{M}$.

Science/Technology Needs: In general, specialized systems require specialized control systems and possibly specialized sensor systems. Also, in general, vision systems for use with specialized platforms are ineffective, particularly for constrained areas and for submersibles.

Implementation Needs: Specialized systems development would only be undertaken as a direct result of a specific requirement that could not be met with existing or more conventional systems. If such a system were expected to have widespread use, it might be a likely candidate for research and development activity. Such determination would have to be made on a case-by-case basis, as development costs could be high. At the very least, cold 
testbeds and operator training would be required to ensure the effectiveness of the specialized platform.

Author: D. C. Haley/615-576-4388

References: MMES staff members' personal experiences/opinions; personal communication; and vendor literature 
EM Problem: Decontamination and decommissioning; remedial action

Y-12 Plant Problem: Buildings and equipment. Building 9201-4 (Alpha-4)

Problem Area/Constituents: Support of characterization, dismantlement, and decontamination. Long-reach arms with high payloads and a reasonable level of dexterity are required as the mobility system to deploy more dexterous robotic systems.

Reference Requirements: Refer to the Regulatory Compliance chapter of Vol. 1 for potentially applicable proposed and promulgated environmental laws, signed and pending agreements for the Oak Ridge Reservation, radiation protection standards, DOE orders, and nonregulatory guidance. As site- and waste-specific characteristics are provided for each technology, specific regulatory requirements will be specified.

Subelement: Robotics/automation

Alternatives: Mechanical; mobility

Technology: Long-arm deployed robotic platforms. A heavy-duty, long-reach manipulator system is required to deploy more dexterous robotic systems throughout large-volume areas. Payload capacity in excess of $1,000 \mathrm{lb}$ will be required. Long-arm platforms will be required for highly contaminated buildings where extended reach is necessary to access heavy equipment items.

Status: Predemonstration

Efficacy-Long-reach manipulators exhibit structural and joint flexibility that may limit positioning accuracy. Control system development or accurate positioning mechanisms at the end of the manipulators may solve this concern.

Waste-Hydraulic fluid during operation and secondary waste during decontamination after use.

Science/Technology Needs: Technology development is required in the following areas:

- Flexible body control. As weight-to-payload ratios are reduced, flexibility characteristics of long manipulators becomes a major control issue and must be addressed.

- End-point sensing. With flexible members, external sensing is required to correctly determine the end-point position of the manipulator for proper position control.

- Collision avoidance. The size of the manipulator and the large volumes swept during motion make collision avoidance capabilities essential.

- Weight-to-payload ratio reduction. Currently available commercial systems are extremely heavy, limiting deployment (mobility) options. 
Implementation Needs: Office of Technology Development (OTD) Robotics Technology Development Program (RTDP) demonstration project. A development project for a heavy-duty, long-reach system is under way within the OTD RTDP. This project is a multimillion dollar development effort. Engineering modifications of development systems, as well as integration and testing for specific applications, will be required for implementation. Low-volume production or unique hardware requirements could drive system cost from $\$ 1 \mathrm{M}$ to $\$ 5 \mathrm{M}$ or higher.

Author: D. C. Haley/615-576-4388

References: Personal communication and vendor literature 
EM Problem: Decontamination and decommissioning; remedial action; waste manage-ment

Y-12 Plant Problem: Buildings, equipment, buried waste, contaminated soils, and sediments. Building 9201-4 (Alpha-4)

Problem Area/Constituents: Support of characterization, dismantlement, and decontamination. Methods of providing power/energy to mobile platform, manipulator arms, sensors, and tools in the remote environment must be developed. Generic methods identified include batteries, fuel cells, internal combustion engines, and umbilical cords.

Reference Requirements: Refer to the Regulatory Compliance chapter of Vol. 1 for potentially applicable proposed and promulgated environmental laws, signed and pending agreements for the Oak Ridge Reservation, radiation protection standards, DOE orders, and nonregulatory guidance. As site- and waste-specific characteristics are provided for each technology, specific regulatory requirements will be specified.

Subelements: Robotics/automation

Alternatives: Mechanical; mobility

Technology: Power sources for mobile platforms. Mobile robotic platforms are required for deployment of characterization, dismantlement, and decommissioning sensors and tooling. All mobile platforms require power sources for platform mobility, manipulation, sensors, and/or controls. Batteries, internal combustion engines, and umbilical cords are accepted technologies and have been widely used on mobile robotic platforms. Each has limiting features requiring science/technology investments or implementation investments. Fuel cells are at the demonstration level for this application and require science/technology investment.

Status: Accepted-batteries, internal combustion engines, umbilicals. Predemonstrationfuel cells.

Efficacy — High for internal combustion engines and umbilicals, medium for batteries and fuel cells.

Waste-Potential chemical waste from batteries and fuel cells, emissions for IC engines, and decontamination wastes for umbilicals.

Science/Technology Needs: Battery power for mobile robotic platforms is severely limited by the energy storage capacity on a weight basis. Lead acid batteries as well as other newer materials remain much too heavy for practical use in operations other than characterization. In addition, the charge/discharge characteristics limit system availability. Fuel cells are limited in current capacity and discharge rate and are not demonstrated for this application to date. Internal combustion engines for power generation require emission control development to be suitable for applications within buildings. To successfully implement hardwired umbilical power supplies in remote robotic applications, clear and unobstructed movement passageways must be allowed to minimize the likelihood of snagging or cutting the 
trailing cable. The link must use either a retrievable or sacrificial approach. Costs will be prohibitive for sacrificial power cables, and the safety issues would likely preclude this use. Impediments to maintaining continuity (i.e., closing doors, multitraverse operation, etc.) must be eliminated. These requirements can make trailing cable applications undesirable and/or uneconomical for many applications where task area is complex and movement restrictions are undesired. The other approach would be for the design to allow the mobile platform to maintain the ability to plug its power cord into available power supplies. This solution would require the demonstration of a hybrid battery/hard-wire power system.

Implementation Needs: Normal deployment needs. Development costs for umbilical cords are negligible. For other power sources, the estimates costs are as follows:

- batteries-improved capacity and duty cycles (\$1M-\$2M),

- fuel cells-improved capacity and discharge work (\$2M-\$4M), and

- internal combustion engines-improved emission controls (\$1M-\$2M).

Author: J.N. Herndon/615-574-7121

References: MMES staff members' personal experiences/opinions 
EM Problem: Decontamination and decommissioning; remedial action; waste management

Y-12 Plant Problem: Buildings, equipment, buried waste, storage tanks, and contaminated soils. Building 9201-4 (Alpha-4)

Problem Area/Constituents: Radioactive and hazardous materials encountered in characterization, decontamination, dismantlement, and retrieval operations.

Reference Requirements: Refer to the Regulatory Compliance chapter of Vol. 1 for potentially applicable proposed and promulgated environmental laws, signed and pending agreements for the Oak Ridge Reservation, radiation protection standards, DOE orders, and nonregulatory guidance. As site- and waste-specific characteristics are provided for each technology, specific regulatory requirements will be specified.

Subelement: Robotics/automation

Alternatives: Mechanical; mobility

Technology: Failure recovery systems for mobile platforms. Methods of retrieving a robotic device-such as tethers, redundant drives, or recovery vehicles-are required if the device fails while performing a remote task in a hazardous environment. Recovery should occur in such a way as to not damage the robotic device or the sensors or tools it may be carrying.

Status: Accepted. The type of failure recovery system selected must be based on the task being performed. For example, tethers may not be suitable for the interior of cluttered buildings whereas they would be the preferred method for vehicles deployed on waste fields.

Efficacy-High. The alternative is to abandon the vehicle in the environment, an option which may not be acceptable for high cost systems.

Waste-None

Development costs-Less than $\$ 0.5 \mathrm{M}$

Science/Technology Needs: No science/technology needs can be identified. Failure recovery systems are either available on commercially procured devices or easy to install on the devices.

Implementation Needs: Some minimal level testing to verify the suitability of the failure recovery system may be required.

Author: J. N. Herndon/615-574-7121

References: Personal experience with such systems 
1 
EM Problem: Decontamination and decommissioning; remedial action (RA); waste management activities

Y-12 Plant Problem: Buildings, equipment, buried waste, buried pipes, and contaminated soils. Building 9201-4 (Alpha-4)

Problem Area/Constituents: Radioactive and hazardous materials encountered in characterization, decontamination, dismantlement, and retrieval operations

Reference Requirements: Refer to the Regulatory Compliance chapter of Vol. 1 for potentially applicable proposed and promulgated environmental laws, signed and pending agreements for the Oak Ridge Reservation, radiation protection standards, DOE orders, and nonregulatory guidance. As site- and waste-specific characteristics are provided for each technology, specific regulatory requirements will be specified.

Subelement: Robotics/automation

Alternatives: Mechanical; mobility

Technology: Transportation methods for mobile platforms. Methods of transporting mobile platforms are needed to eliminate the necessity for decontaminating the device after each application. These methods would reduce the quantity of decontamination fluids and improve the efficiency of the system. This would be particularly useful for RA tasks where frequent movement between waste sites could be anticipated.

Status: Demonstration-small devices; Predemonstration-large mobile platforms. Transportation containers are commercially available for small mobile platforms. Such containers are not available for medium- or heavy-duty mobile platforms.

Efficacy-Medium. Alternatives (i.e., decontamination) are available that may be more cost effective.

Waste-Minimal. The purpose of the technology is to minimize the quantity of decontamination waste.

Development costs-Estimated at $\$ 0.5 \mathrm{M}$

Science/Technology Needs: Technology development of light-weight but durable containers, which themselves can be periodically decontaminated, is needed for medium- and heavy-duty mobile platforms.

Implementation Needs: None

Author: J. N. Herndon/615-574-7121

References: Personal experience and vendor contacts 


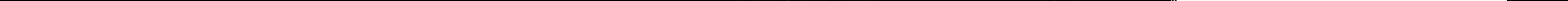


EM Problem: Decontamination and decommissioning; remedial action; waste management

Y-12 Plant Problem: Buildings, equipment, buried waste, and contaminated soils. Building 9201-4 (Alpha-4)

Problem Area/Constituents: Radioactive and hazardous materials encountered in characterization, decontamination, dismantlement, and retrieval operations

Reference Requirements: Refer to the Regulatory Compliance chapter of Vol. 1 for potentially applicable proposed and promulgated environmental laws, signed and pending agreements for the Oak Ridge Reservation, radiation protection standards, DOE orders, and nonregulatory guidance. As site- and waste-specific characteristics are provided for each technology, specific regulatory requirements will be specified.

Subelement: Robotics/automation

Alternatives: Mechanical; mobility

Technology: Data/signal transmission. Methods of transmitting data and control signals from the robotic device to the control station are required for all remote operations. Various options available to the designer include umbilical cords, radio frequency, microwave, and laser-based systems. The option chosen will depend on the particular application and the transmission rate required.

Status: Accepted. Radio frequency is limited in transmission rate. Lasers and, to a lesser extent, microwaves depend on line-of-sight between the transmitter and the receiver, and umbilical cords lead to potential snagging problems.

Efficacy-High. Data/signal transmission system are required for all robotic applications. Waste-None

Overall Cost-Development costs will range from $\$ 0 \mathrm{~K}$ for umbilical cords to $\$ 200 \mathrm{~K}-\$ 500 \mathrm{~K}$ for advanced microwave systems.

Science/Technology Needs: None. The necessary technology currently exists.

Implementation Needs: Integration of existing technology into robotic systems and testing of representative environments to ensure functionality are needed.

Author: J. N. Herndon/615-574-7121

References: Personal experience and discussions with other MMES personnel 


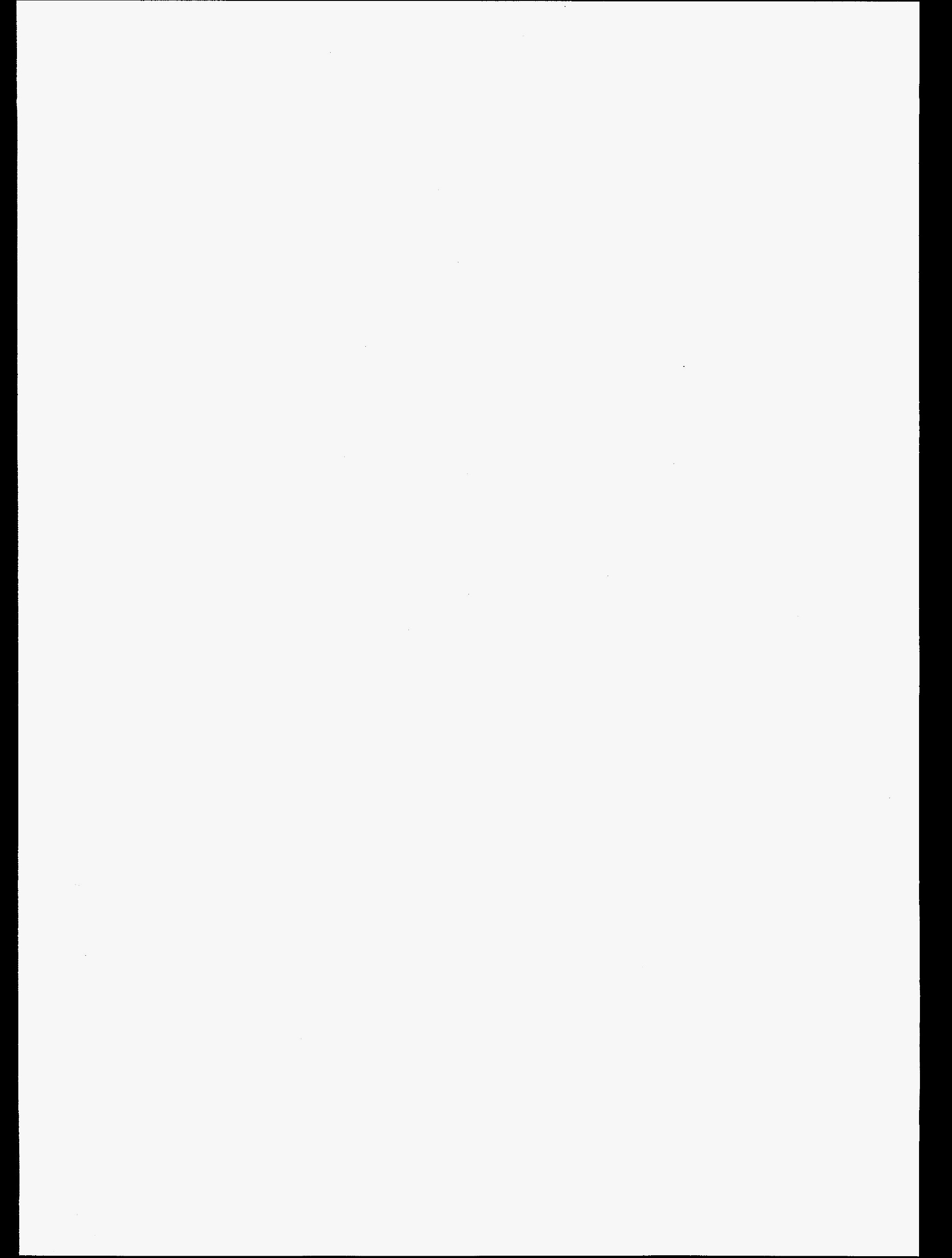


EM Problem: Decontamination and decommissioning

Y-12 Plant Problem: ORNL buildings and equipment. Building 9201-4 (Alpha-4)

Problem Area/Constituents: Support of characterization. Long-reach arms with a reasonable level of dexterity are required to deploy tools and sensors.

Reference Requirements: Refer to the Regulatory Compliance chapter of Vol. 1 for potentially applicable proposed and promulgated environmental laws, signed and pending agreements for the Oak Ridge Reservation, radiation protection standards, DOE orders, and nonregulatory guidance. As site- and waste-specific characteristics are provided for each technology, specific regulatory requirements will be specified.

Subelement: Robotics/automation

Alternatives: Mechanical; manipulation

Technology: Long-reach manipulator (light-duty). A light-duty (50-lb payload), long-reach (2- to $30-\mathrm{m})$ manipulator system is required to deploy sensor systems throughout largevolume areas during characterization.

Status: Predemonstration

Efficacy-Long-reach manipulators exhibit structural and joint flexibility which may limit positioning accuracy. Control system development or accurate positioning mechanisms at the end of the manipulators may solve this concern.

Waste-Hydraulic fluid during operation and secondary waste generated during decontamination after use

Science/Technology Needs: Technology development is required in the following areas:

- Flexible body control. Flexibility characteristics of long manipulators is a major control issue.

- End-point sensing. With flexible members, external sensing is required to correctly determine the end-point position of the manipulator for proper position control.

- Collision avoidance. The size of the manipulator and the large volumes swept during motion make collision avoidance capabilities essential.

- Light-weight, small cross-section design. Light-weight, long-reach systems are required for deployment on mobile systems for large-area characterization.

Implementation Needs: Office of Technology Development (OTD) demonstration project. A development project for a light-duty, long-reach manipulator system is under way within the OTD. This multimillion dollar development effort is targeted at underground storage tank remediation. 
Engineering modifications of development systems, as well as integration and tests for specific applications, will be required for implementation. Low-volume production or unique hardware requirements would dictate system cost of $\$ 1 \mathrm{M}-\$ 2 \mathrm{M}$.

Author: D. C. Haley/615-576-4388

References: MMES staff members' personal experiences/opinion; personal communication; and vendor literature 
EM Problem: Decontamination and decommissioning

Y-12 Plant Problem: Buildings and equipment. Building 9201-4 (Alpha-4)

Problem Area/Constituents: Support of characterization, dismantlement, and decontamination. Long-reach arms with a reasonable level of dexterity are required to deploy tools and sensors.

Reference Requirements: Refer to the Regulatory Compliance chapter of Vol. 1 for potentially applicable proposed and promulgated environmental laws, signed and pending agreements for the Oak Ridge Reservation, radiation protection standards, DOE orders, and nonregulatory guidance. As site- and waste-specific characteristics are provided for each technology, specific regulatory requirements will be speciffed.

Subelement: Robotics/automation

Alternatives: Mechanical; manipulation

Technology: Long-reach manipulator (heavy duty). A heavy-duty ( $>50-1 \mathrm{~b}$ payload), long-reach (2- to 30-m) manipulator system is required to deploy tooling systems throughout largevolume areas during decontamination and dismantling. Payload capacity in excess of $50 \mathrm{lb}$ will be required, with payloads of 1000-2000 lb likely.

Status: Predemonstration

Efficacy-Long-reach manipulators exhibit structural and joint flexibility which may limit positioning accuracy. Control system development or accurate positioning mechanisms at the end of the manipulators may solve this concern.

Waste-Hydraulic fluid during operation and secondary waste during decontamination after use may be generated.

Science/Technology Needs: Technology development is required in the following areas:

- Flexible body control. As weight-to-payload ratios are reduced, flexibility characteristics of long manipulators becomes a major control issue and must be addressed.

- End-point sensing. With flexible members, external sensing is required to correctly determine the end-point position of the manipulator for proper position control.

- Collision avoidance. The size of the manipulator and the large volumes swept during motion make collision avoidance capabilities essential.

- Weight-to-payload ratio reduction. Currently available commercial systems are extremely heavy, limiting deployment (mobility) options.

Implementation Needs: Office of Technology Development (OTD) Robotics Technology Development Program (RTDP) demonstration project. A development project for a heavy-duty, long-reach manipulator system is under way within the OTD RTDP. This project is a 
multimillion dollar development effort. Engineering modifications of development systems, as well as integration and tests for specific applications, will be required for implementation. Low-volume production or unique hardware requirements could drive system cost to $\$ 5 \mathrm{M}$ or higher.

Author: D. C. Haley/615-576-4388

References: Personal communication and vendor literature 
EM Problem: Decontamination and decommissioning (D\&D); remedial action; and waste management

Y-12 Plant Problem: Activities in which constrained access or precise motions are required. Building 9201-4 (Alpha-4)

Problem Area/Constituents: Support of characterization, dismantlement, and decontamination. For some of the activities, robotic arms with a high level of dexterity and redundant kinematics will be required.

Reference Requirements: Refer to the Regulatory Compliance chapter of Vol. 1 for potentially applicable proposed and promulgated environmental laws, signed and pending agreements for the Oak Ridge Reservation, radiation protection standards, DOE orders, and nonregulatory guidance. As site- and waste-specific characteristics are provided for each technology, specific regulatory requirements will be specified.

Subelement: Robotics/automation

Alternatives: Mechanical; manipulation

Technology: Dexterous manipulators. Arms with $\geq 6$ degrees of freedom (DOF). To provide manipulation for the variety of characterization, remediation, waste management, decontamination, and dismantlement tasks, dexterous manipulators with a minimum of $6 \mathrm{DOF}$ are required. Kinematically redundant manipulators ( $>6 \mathrm{DOF}$ ) may be required for access in cluttered environments. Force reflection and tactile capabilities may be required when the operator must be aware of the forces being executed by or on the manipulator. Configuration consisting of a single manipulator arm is required for many tasks, while other tasks require deployment of dual arm manipulation capabilities. In general, currently available 6-DOF manipulators are adequate for many tasks. The areas of concern relative to technology are >6-DOF systems, modular systems, and systems with tactile capabilities. Modular systems are desirable both for reconfiguration to more directly address unique tasks and for ease of maintenance.

\section{Status: Predemonstration}

Efficacy-High. Dexterous manipulators will be required for most remotely performed tasks. Kinematically redundant manipulations have been shown to greatly increase dexterity, whereas systems with force reflection/tactile capabilities will be necessary for sensor deployment, sampling, sample management, etc.

Waste-Hydraulic fluid may be generated during operation and secondary waste during decontamination after use. 
Science/Technology Needs: Technology development is required in the following areas:

- Redundant manipulator planning and control. For >6-DOF arms, reliable kinematically redundant manipulator control algorithms are required.

- Modular manipulator design. Modular manipulator designs must be developed and verified as providing high payback due to standardization and ease of maintenance.

- Nonreplica master/slave operation. Teleoperation of redundant manipulators with a replica master controller requires excessive operator mental and physical agility to control the physical redundance of the mechanical manipulator. Use of nonreplica masters requires the redundant manipulator planning and control capability stated earlier.

- Cooperating and coordinated dual arm control. Dual arm manipulation requires task planning for safe and effective multiarm activities. Coordinated dual arm control requires specialized algorithms for two manipulators in contact with a single object.

Implementation Needs: Development of redundant control technologies is an active area of university research. This activity is predominantly a software development in algorithm design, implementation, and verification. This area generally requires advanced-degree-level personnel in servo-control systems (generally an electrical engineering discipline). Fairly robust capability should be available for demonstration and evaluation within 2 to 5 years. Modular robotic systems are also under study within the university community. Modular mechanical and actuator design, fabrication, integration, and testing are required to evaluate these concepts. This area generally requires advanced-degree-level personnel in mechanical design (mechanical engineering). Engineering development units at the component level exist today. Total system implementations for demonstration and evaluation should be available in 2 to 5 years.

Currently available 6-DOF manipulators with force-reflecting capabilities are adequate for many tasks. A basic commercially available hydraulic, force-reflecting, master/slave 6-DOF manipulator system can be purchased for approximately $\$ 300 \mathrm{~K}$. Deployment of redundant manipulators will increase the applicability of robotics to more complex access and geometry areas. Robotic systems for D\&D applications are under development within the OTD RTDP and will be available within the 2- to 5-year time frame for feasibility demonstrations and benefit evaluations. Efficient teleoperation of redundant systems requires the implementation of compact nonreplica Cartesian master controllers with redundance control provided via software. Development costs for >6-DOF systems or systems with unique capabilities are estimated to be $\$ 1 \mathrm{M}$.

Author: D. C. Haley/615-576-4388

References: MMES staff members' personal experiences/opinions; CMU modular system research

September 1994

Decontamination and Decommissioning 
EM Problem: Decontamination and decommissioning; remedial action; and waste management activities

Y-12 Plant Problem: Buildings, equipment, buried waste, and contaminated soils. Building 9201-4 (Alpha-4)

Problem Area/Constituents: Radioactive and hazardous materials encountered in characterization, decontamination, dismantlement, and retrieval operations

Reference Requirements: Refer to the Regulatory Compliance chapter of Vol. 1 for potentially applicable proposed and promulgated environmental laws, signed and pending agreements for the Oak Ridge Reservation, radiation protection standards, DOE orders, and nonregulatory guidance. As site- and waste-specific characteristics are provided for each technology specific regulatory requirements will be specified.

Subelement: Robotics/automation

Alternatives: Mechanical; manipulation

Technology: Compact, high-capacity, manipulators. For many robotic tasks, particularly those requiring the deployment of decontamination or dismantlement tools, the lifting capacity of the robotic arms may be the constraining feature. Although hydraulic arms have greater lifting/manipulating capacity, those may not be suited for many tasks where hydraulic lines or on-board hydraulic units are not feasible.

Status: Evolving technology; conceptual. For constrained areas, small robotic arms with lifting capabilities sufficient to carry decontamination or dismantlement tools, will be required. Although, hydraulic units exist, there are no equivalent, electrically driven arms.

Efficacy-High. There are many tasks, particularly in D\&D, that require this technology. Waste-Minimal. Only decontamination fluid after use Overall Cost-Development costs would be in the range of $\$ 1 \mathrm{M}-\$ 2 \mathrm{M}$.

Science/Technology Needs: Compact, high-output, electrical motors. Two potential approaches are $\mathrm{AC}$ induction motors or superconducting motors. Neither of these possibilities, however, has been demonstrated for this type of application.

Implementation Needs: Survey of existing commercial motors, followed by an analysis and conceptual design of a compact robotic arm. The survey may indicate that existing motors with the necessary characteristics do not exist and that new motors may have to be developed.

Author: J. N. Herndon/615-574-7121

References: Personal experience and discussions with other MMES personnel 


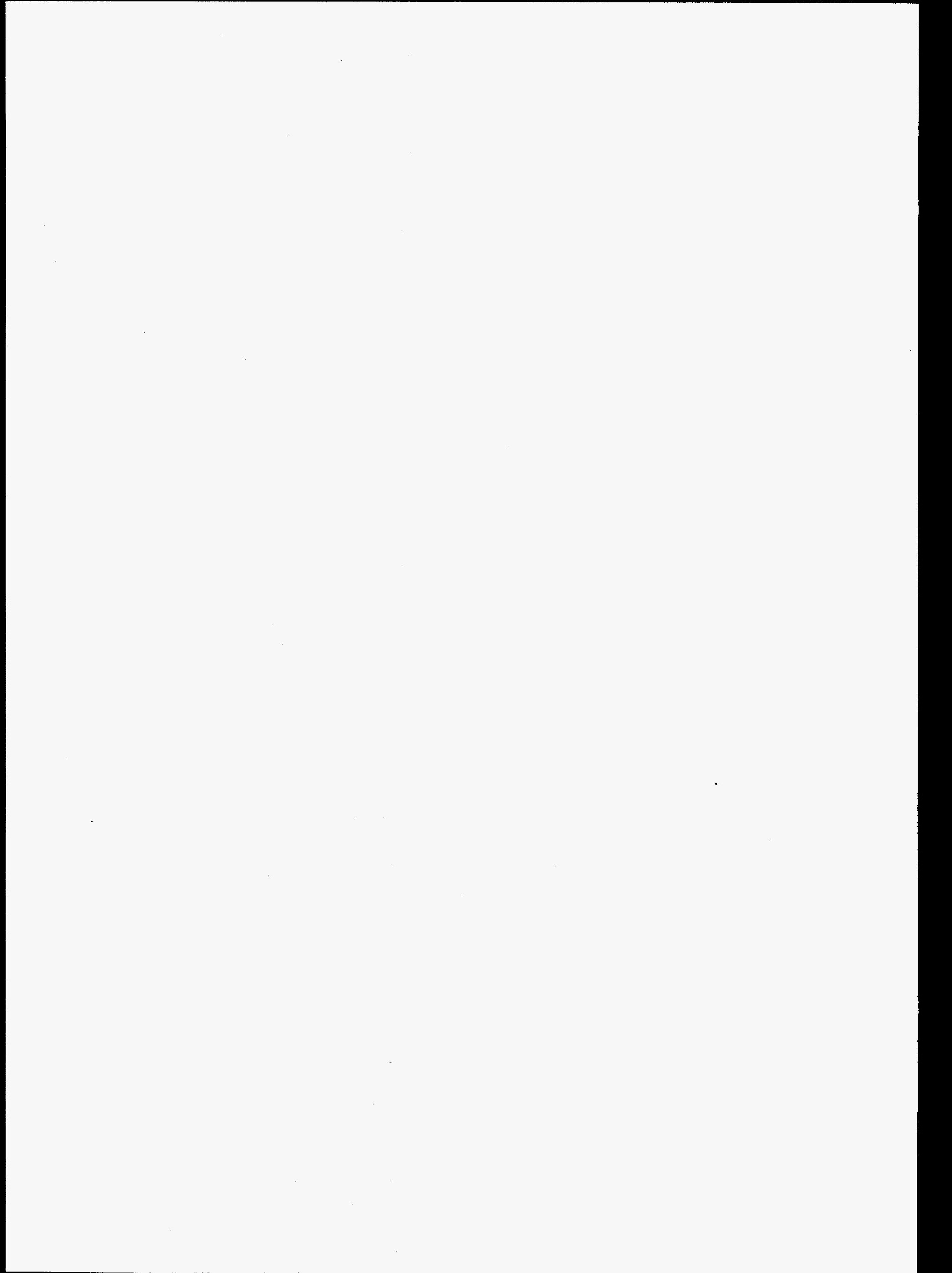


EM Problem: Decontamination and decommissioning; remedial action; waste management

Y-12 Plant Problem: Buildings and equipment. Building 9201-4 (Alpha-4)

Problem Area/Constituents: Support of characterization. Precise programmable mechanisms are required to automate many functions in laboratory processing of characterization samples.

Reference Requirements: Refer to the Regulatory Compliance chapter of Vol. 1 for potentially applicable proposed and promulgated environmental laws, signed and pending agreements for the Oak Ridge Reservation, radiation protection standards, DOE orders, and nonregulatory guidance. As site- and waste-specific characteristics are provided for each technology, specific regulatory requirements will be specified.

Subelement: Robotics/automation

Alternatives: Mechanical; mechanisms

Technology: Precise programmable motions. Precise programmable mechanisms will be required to completely automate laboratory functions in support of characterization activities. These mechanisms will be used to handle and transport samples and chemicals within automated laboratory modules.

\section{Status: Demonstration}

Efficacy-Mechanical and electronic components required to implement precision mechanisms exist and operate within the performance ranges needed. Previous efforts at implementation of such systems have been successful.

Waste-None

Science/Technology Needs: Considerable technology exists currently for automated laboratory processing. Development is required in sample handling, sample preparation, and data interpretation areas (see ROBA-55, -57, and -59). Technology improvement is needed in the mechanism area to standardize mechanical interfaces to allow integration of multiple standard laboratory modules (SLMs) into larger systems and provide mechanical handling of samples and chemicals within SLMs and throughout the entire system.

Implementation Needs: OTD RTDP demonstration project. A development project for laboratory automation is underway within the Office of Technology Development OTD) Robotics Technology Development Program (RTDP). Development and demonstration of a number of standard laboratory modules (SLMs) is expected in FY 1993 and beyond. This multimillion dollar development effort is targeted at providing SLMs that can be plugged together to automate some of the EPA protocols for high-volume sample processing. Engineering modifications of existing laboratory automation hardware and development 
systems, as well as integration and testing for specific applications, will be required for implementation. Development costs for this particular capability are expected to be minimal and are estimated to be $\$ 0.5 \mathrm{M}$.

Author: D. C . Haley/615-576-4388

References: MMES staff members' personal experiences/opinions; personal communication; and vendor literature 
EM Problem: Decontamination and decommissioning (D\&D); remedial action; waste management

Y-12 Plant Problem: Buildings and equipment. Building 9201-4 (Alpha-4)

Problem Area/Constituents: Support of remote operations required for characterization, decontamination, dismantlement, and retrieval

Reference Requirements: Refer to the Regulatory Compliance chapter of Vol. 1 for potentially applicable proposed and promulgated environmental laws, signed and pending agreements for the Oak Ridge Reservation, radiation protection standards, DOE orders, and nonregulatory guidance. As site- and waste-specific characteristics are provided for each technology, specific regulatory requirements will be specified.

Subelement: Robotics/automation

Alternatives: Mechanical; mechanism

Technology: Tool caddies. To allow a single robotics system to perform a variety of tasks at a single work site a number of different hand and power tools must be utilized. The tools will be transported and stored in tool caddies. The tool caddie will hold and position the tools to allow easy access to the robotics end-effector. The caddie should present the tools to the end effector such that a minimum of intelligence is required to access a tool. Unique designs will be required for special purpose tools.

\section{Status: Demonstration}

Efficacy-Tool holders/positioners are used in commercial robotics industry, but most often with non-mobile robots. Use of existing technology with adaption for mobility and custom designs for special purpose tools/sensors will be required for use in $\mathrm{D} \& \mathrm{D}$.

Waste-Secondary waste may be generated during decontamination after use.

Science/Technology Needs: None

Implementation Needs: Adaptation of existing technologies, as well as integration and testing with specific robotics systems, will be required for implementation. Total development costs are estimated at $\$ 0.5 \mathrm{M}$.

Author: R. E. Eversole/615-574-7483

References: MMES staff members' personal experiences/opinions 


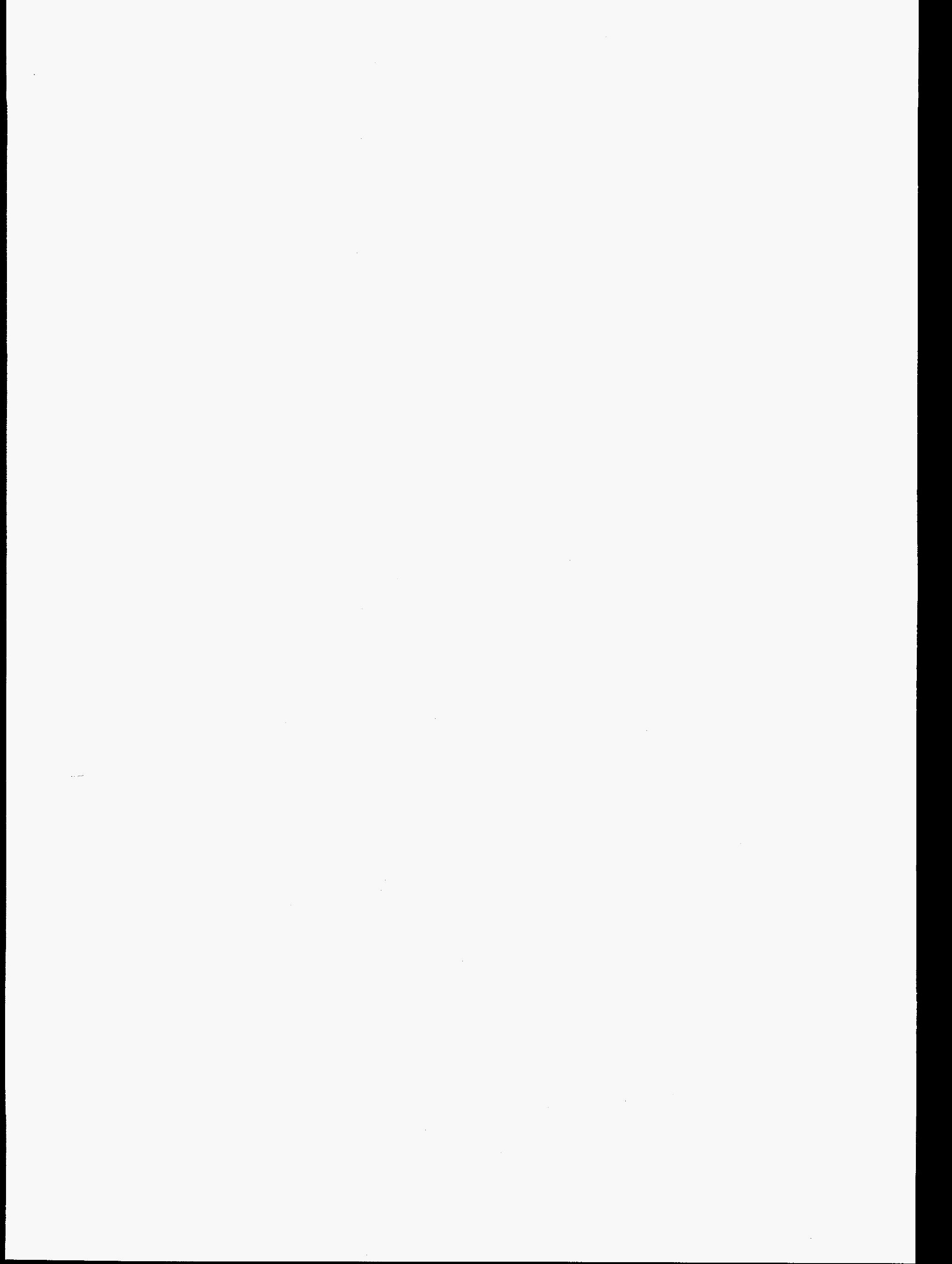


EM Problem: Decontamination and decommissioning (D\&D), remedial action (RA), and waste management (WM)

Y-12 Plant Problem: Buildings and equipment. Building 9201-4 (Alpha-4)

Problem Area/Constituents: Support of remote operations required for characterization, decontamination, and dismantlement

Reference Requirements: Refer to the Regulatory Compliance chapter of Vol. 1 for potentially applicable proposed and promulgated environmental laws, signed and pending agreements for the Oak Ridge Reservation, radiation protection standards, DOE orders, and nonregulatory guidance. As site- and waste-specific characteristics are provided for each technology, specific regulatory requirements will be speciffed.

Subelement: Robotics/automation

Alternatives: Mechanical; mechanisms

Technology: Stabilization/support mechanisms or booms. Many applications of robotics will require the robotics device to perform tasks at distances from a stable area from which the robot is deployed. Optimum performance of robotic devices requires that the operational loads reacted with little motion or movement. Primary options are to develop long reach booms with adequate stiffness or to design stabilization mechanisms that can be deployed near the area of operation.

\section{Status: Demonstration}

Efficacy-The technology to develop stabilization/support mechanisms or booms is available, but such devices are typically a custom design for a given application. Unique custom designs are anticipated for use in D\&D, RA, and WM.

Waste-Secondary waste may be generated during decontamination after use.

Science/Technology Needs: While adequate technology exists, design challenges could be simplified by advanced materials. Advanced materials with higher strength-to-weight ratios could be effectively utilized.

Implementation Needs: Adaptation of existing technologies, as well as integration and testing with specific robotics systems, will be required for implementation. Total development costs are estimated at $\$ 0.5 \mathrm{M}$.

Author: R. E. Eversole/615-574-7483

References: MMES staff members' personal experiences/opinions 


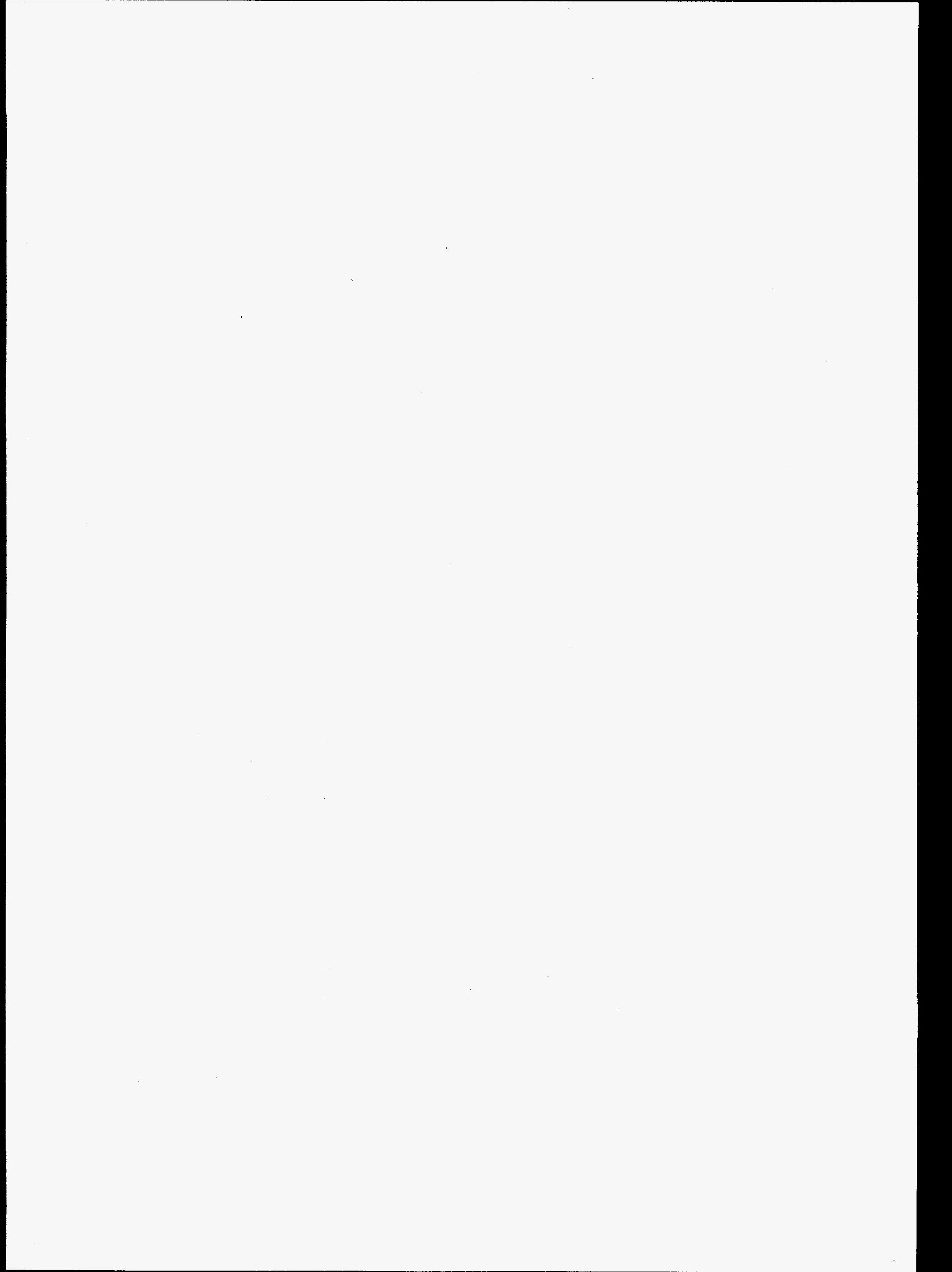


EM Problem: Decontamination and decommissioning (D\&D); remedial action (RA); waste management (WM)

Y-12 Plant Problem: Buildings and equipment. Building 9201-4 (Alpha-4)

Problem Area/Constituents: Support of characterization, decontamination, dismantlement, remediation, packaging, handling, and inspection. End effectors, or alternatives such as special purpose tooling, are required for manipulator systems to be able to perform work.

Reference Requirements: Refer to the Regulatory Compliance chapter of Vol. 1 for potentially applicable proposed and promulgated environmental laws, signed and pending agreements for the Oak Ridge Reservation, radiation protection standards, DOE orders, and nonregulatory guidance. As site- and waste-specific characteristics are provided for each technology, specific regulatory requirements will be specified.

Subelement: Robotics/automation

Alternatives: Mechanical; end-of-arm tooling

Technology: End effectors/alternatives. End effectors or tooling allow manipulators to grasp or manipulate objects. End effectors can range from parallel jaw grippers to complex multifingered, handlike mechanisms. An alternative to the use of grasping end effectors is to directly mount the required tooling on the end of the manipulator to eliminate the need to pick up the tool and operate it via an end effector. The use of changeout mechanisms at the manipulator tool plate (see ROBA-24) allows a selection of special purpose tools and end effectors to be attached, as needed, to the manipulator.

Status: Demonstration for many end effectors and robotically compatible tools. Predemonstration for multifingered grippers

Efficacy-Mechanically, end effectors and robotically deployed tools have proven very effective. The effectiveness of using end effectors to grasp tools or other devices is greatly enhanced by the use of standard mechanical interfaces (see ROBA-25). Complexity and reliability may reduce the effectiveness of multifingered grippers.

Waste-None

Science/Technology Needs: Technology development and testing is required for multifingered grippers.

Technology improvement is needed for robotic compatibility of the tooling required for D\&D, RA, and WM. 
Implementation Needs: Engineering modifications of development systems, as well as integration and testing for specific applications, will be required for implementation. Total development costs of $\$ 1 \mathrm{M}$ are estimated.

Author: D. C. Haley/615-574-4388

References: MMES staff members' personal experiences/opinions; personal communication; and vendor literature 
EM Problem: Decontamination and decommissioning (D\&D); remedial action (RA); waste management (WM)

Y-12 Plant Problem: Buildings, equipment, buried waste, and waste processing. Building 9201-4 (Alpha-4)

Problem Area/Constituents: Automated interchangeability of tools on a universal end effector that also provides services to the tool would find many applications and would improve the efficiency of dismantlement, decontamination, and other tasks.

Reference Requirements: Refer to the Regulatory Compliance chapter of Vol. 1 for potentially applicable proposed and promulgated environmental laws, signed and pending agreements for the Oak Ridge Reservation, radiation protection standards, DOE orders, and nonregulatory guidance. As site- and waste-specific characteristics are provided for each technology, specific regulatory requirements will be specified.

Subelement: Robotics/automation

Alternatives: Mechanical; end-of-arm tooling

Technology: End-effector changeout mechanisms. To allow a single robotic system to use a number of different sensors/tools, a remote automated end-effector changeout mechanism is required. Such a system should provide mechanical, electrical, data, and hydraulic interfaces between the manipulator and the sensors/tools. Quick-change devices are commercially available and have been used for a variety of tool-to-manipulator interfaces. Modification may be required for the special purpose sensors and tooling used for D\&D, RA, and WM.

\section{Status: Demonstration}

Efficacy-Quick-change devices are commercially available and have been used for a variety of tool-to-manipulator interfaces. Modification may be required for the special purpose sensors and tooling used for D\&D, RA, and WM.

Waste-Secondary waste may be generated during decontamination after use.

Science/Technology Needs: None. No science or technology needs exist for the end-effector remote changeout technology area.

Implementation Needs: Engineering modifications of existing commercial systems, as well as integration and testing with specific robotic systems, will be required for implementation. Additional costs are associated with tool holders compatible with automated tool exchange. Total development costs are estimated at $\$ 0.5 \mathrm{M}$. 
Author: D. C. Haley/615-576-4388

References: MMES staff members' personal experiences/opinions 
EM Problem: Decontamination and decommissioning; remedial action; waste management

Y-12 Plant Problem: Buildings, equipment, buried waste, and waste packaging. Building 9201-4 (Alpha-4)

Problem Area/Constituents: The application is secure gripping of tools and, perhaps, sensors using a standard interface.

Reference Requirements: Refer to the Regulatory Compliance chapter of Vol. 1 for potentially applicable proposed and promulgated environmental laws, signed and pending agreements for the Oak Ridge Reservation, radiation protection standards, DOE orders, and nonregulatory guidance. As site- and waste-specific characteristics are provided for each technology, specific regulatory requirements will be specified.

Subelement: Robotics/automation

Alternatives: Mechanical; end-of-arm tooling

Technology: General gripper/tool interface. As an alternative to using a quick-change interface as described in ROBA-24-OL, standard interfaces between tools/sensors and the manipulator gripper can be established and used. Such interfaces as " $T$ " handles and " $\mathrm{H}$ " handles are candidate interfaces. The advantage of such an interface is that the manipulator uses a single gripper continuously and uses that gripper to pick up and maneuver sensors/tools. Each sensor/tool must be fitted with the interface "handle," which, in general, is a simple, low-cost, passive hardware device. The disadvantage of this approach is that resources (power, data, etc.) for the sensor/tool must be supplied via an umbilical, which poses problems of cable management during manipulation.

\section{Status: Demonstration}

Efficacy-This option provides a reliable, simple, passive hardware interface that has been proven in many applications.

Waste-Secondary waste may be generated during decontamination after use.

Science/Technology Needs: None. No science or technology needs exist for the tool-arm interface technology area.

Implementation Needs: These interfaces should be designed so that forces and torque at the interface do not tend to drive the gripper open and cause the sensor/tool to be dropped. Engineering modifications of existing systems, as well as integration and testing with specific robotic systems, will be required for implementation. Costs associated with an appropriate gripper and the required hardware interface will be minimal and are estimated to be no more than $\$ 0.5 \mathrm{M}$.

Author: D. C. Haley/615-576-4388 
References: MMES staff members' personal experiences 
EM Problem: Decontamination and decommissioning; remedial action; waste management

Y-12 Plant Problem: Buildings and equipment. Building 9201-4 (Alpha-4)

Problem Area/Constituents: Methods of limiting the force applied to the workpiece will be required.

Reference Requirements: Refer to the Regulatory Compliance chapter of Vol. 1 for potentially applicable proposed and promulgated environmental laws, signed and pending agreements for the Oak Ridge Reservation, radiation protection standards, DOE orders, and nonregulatory guidance. As site- and waste-specific characteristics are provided for each technology, specific regulatory requirements will be specified.

Subelement: Robotics/automation

Alternatives: Mechanical; end-of-arm tooling

Technology: Force-limiting/compliant mechanisms. Because many of the manipulation tasks require contact between sensors/tools and the environment, some means is required to limit the forces that are applied. Mechanical compliance devices can be used to provide passive compliance during contact. Some form of active force sensing should also be present for control feedback for active compliance or for safety limit shutdown. A less desirable mechanical solution is the design of an interface structure to break at predetermined load limits to avoid damage to the overall robotic system.

Status: Demonstration

Efficacy-Both passive and active force limitings have been demonstrated and incorporated into production systems.

Waste-Secondary waste may be generated during decontamination after use.

Science/Technology Needs: None. No science or technology needs exist for the forcelimiting technology area. This data sheet covers only the mechanical (passive) approach to force limiting. Active force control is covered in the Control Algorithm area.

Implementation Needs: None. Engineering modifications of existing systems, as well as integration and testing with specific robotic systems, will be required for implementation. Minimal costs-estimated at no more than $\$ 0.5 \mathrm{M}$-are associated with active force/torque sensor systems and passive hardware compliance mechanisms.

Author: D. C. Haley/615-576-4388

References: MMES staff members' personal experiences/opinions 


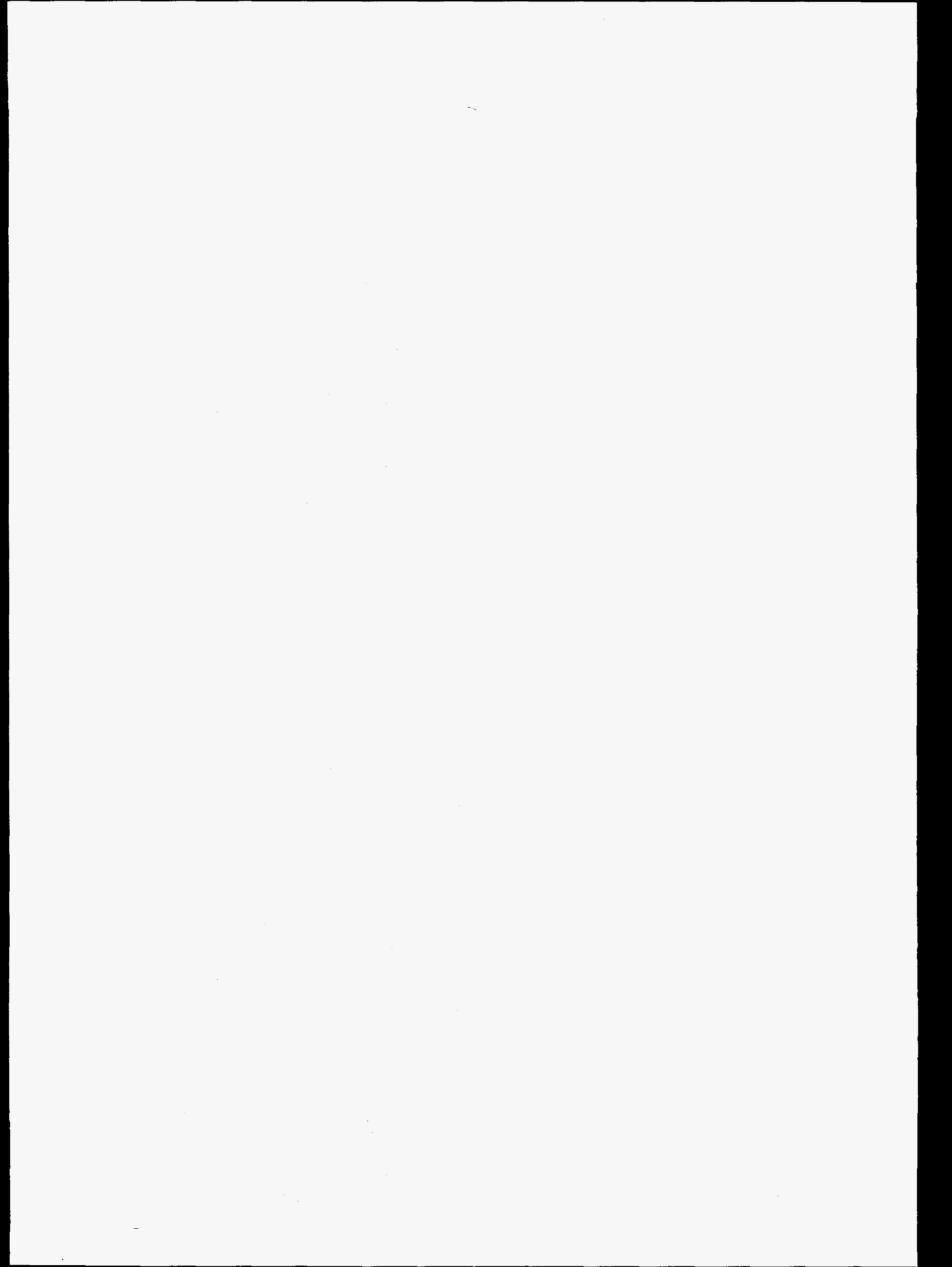


EM Problem: Decontamination and decommissioning (D\&D); remedial action (RA); waste management (WM)

Y-12 Plant Problem: D\&D, RA, and WM required for buildings, equipment, buried wastes, and waste packaging. Building 9201-4 (Alpha-4)

Problem Area/Constituents: Support of D\&D, RA, and WM activities will require a variety of control modes to most efficiently implement robotics and automation.

Reference Requirements: Refer to the Regulatory Compliance chapter of Vol. 1 for potentially applicable proposed and promulgated environmental laws, signed and pending agreements for the Oak Ridge Reservation, radiation protection standards, DOE orders, and nonregulatory guidance. As site- and waste-specific characteristics are provided for each technology, specific regulatory requirements will be specified.

Subelement: Robotics/automation

Alternatives: Controls; algorithms

Technology: Control modes. Robotics and automated systems can be operated in a variety of modes. These control modes include teleoperation, shared control, programmed (offline programming, teach/playback), supervised autonomy, and autonomous modes of operation. The control algorithms required to operate robotic hardware depend upon the level of direct operator (human) interaction and on the complexity and structure of the physical environment in which the systems must operate. Teleoperation includes all modes where an operator provides direct control of the system by manipulation of joysticks or hand controllers. Teleoperation provides for remote operation of hardware to do fairly complex tasks but requires a dedicated and well-trained operator. Pure replica master/slave teleoperation requires the simplest control algorithms. Introduction of force reflection, gravity compensation, or operator aids can greatly increase the complexity of the algorithms to support even straightforward teleoperation. For very well-structured environments, programmed control modes such as offline programming or teach/playback can be used. These modes are most effective in workcell setups similar to factory automation. In general for unstructured environments, the more autonomous the control modes, the more computationally complex the required algorithms become. In shared control modes, the operator directly controls one or more degrees of freedom while the control system controls other aspects of the system operation. Supervised autonomy refers to systems which can operate without direct operator control and have sufficient monitoring capability to detect problems, safely stop the current process and request operator assistance. This mode allows a single operator to control many systems and provide the problem-solving capability when required. Fully autonomous modes require extremely sophisticated control algorithms to detect exception events, evaluate alternative actions, replan tasks and continue operation toward a desired goal.

Status: Accepted-teleoperation. Demonstration-preprogrammed. 
Predemonstration—shared control, supervised autonomy, and autonomous control.

Efficacy/strengths/weaknesses-Teleoperation and preprogrammed modes are very effective, well-proven control modes. Teleoperation requires a dedicated operator. Preprogrammed control requires a well-structured and repetitive task. Shared control and supervised autonomy increase the effectiveness of robotic systems while relying on human intervention or control to handle problems. Highly capable autonomous systems can be extremely complex and are not in operational use.

Waste-Not applicable for control algorithm technologies. The systems which these algorithms control are covered in the mechanical technologies.

Overall cost-The recurring costs for software (control algorithms) are small after development and testing are complete. As the complexity of the control modes increases, the processing hardware and support hardware (sensors, etc.) required increase considerably and can become significant cost items. Preprogrammed control modes provide the minimum cost, maximum payback potential for high volume, repetitive applications. Such applications are not expected within most D\&D or RA activities. Some WM activities such as periodic inspections may utilize preprogrammed control. Teleoperation or shared control implementations, are operationally costly due to the single operator/single machine operational scenario. Teleoperated tasks times are, in general, slower than direct manual performance of the same task. Supervised autonomy holds the greatest potential for cost savings and payback by greatly increasing the efficiency of both the operator and the robotic systems.

Science/Technology Needs: Technology Development Needs-Technology development is required in the areas related to increasing the robotic system capabilities to operate more autonomously. These include operator assistance technologies such as graphics enhancements and tools. Development activities in perception and control sensor data acquisition and interpretation, and task and path planning are also required.

Technology Improvement Needs-Improvements in human-machine interfaces would increase the effectiveness of all control modes.

Implementation Needs: Development of various control mode technologies is an active area of DOE laboratory, university, and industry research. This activity is primarily software development in algorithm design, implementation, and verification. This area generally requires degree-level-personnel in servo-control systems (generally an electrical engineering discipline). Deployment requires implementation of control algorithms, processing (often distributed) systems, sensors systems and operator control station capabilities. Development costs are difficult to estimate or even bracket because the cost depends on the control mode selected and the complexity of the task.

Author: D. C. Haley/615-576-4388

References: MMES staff members' personal experiences/opinions

September 1994

Decontamination and Decommissioning 
EM Problem: Decontamination and decommissioning $(D \& D)$; remedial action; waste management

Y-12 Plant Problem: Buildings and waste fields. Building 9201-4 (Alpha-4)

Problem Area/Constituents: Support of characterization, decontamination, dismantlement, and retrieval

Reference Requirements: Refer to the Regulatory Compliance chapter of Vol. 1 for potentially applicable proposed and promulgated environmental laws, signed and pending agreements for the Oak Ridge Reservation, radiation protection standards, DOE orders, and nonregulatory guidance. As site- and waste-specific characteristics are provided for each technology, specific regulatory requirements will be specified.

Subelement: Robotics/automation

Alternatives: Controls; algorithms

Technology: Vehicle/mobility driving. Algorithms associated with driving, primarily deterministically based, have mandated line-of-sight functionality. Little use has been made of neural network-based or fuzzy control-based algorithms for telerobotic driving support. Driving performance is a primary implementation concern and requires a high degree of integration between driving algorithms and human-machine interface technologies. Without such integration, neither good driving algorithms nor good driving interfaces could function optimally. Higher speed and more efficient algorithmic processing would facilitate enhanced implementation. Autonomous driving capabilities reflect a greater degree of research needs; however, for facility $\mathrm{D} \& \mathrm{D}$, the needs for such autonomy is minimal.

Status: Demonstrated

Efficacy-High. This technology will enhance overall driving performance. Since much of the envisioned telerobotic activity involves driving of the telerobotic platform, a significant potential exists for performance improvement.

Waste-No secondary waste streams generated.

Science/Technology Needs: (1) Advanced integration of driving algorithms with humanmachine interface technologies, (2) fuzzy logic driving algorithms in support of man-in-theloop driving (most fuzzy logic work has addressed autonomous driving), and (3) development of non-line-of-sight driving support

Implementation Needs: Emphasis should be placed on higher speed and more efficient algorithmic processing. Development costs are estimated at $\$ 0.5 \mathrm{M}$.

Author: H. E. Knee/615-574-6163 
References: MMES staff members' personal experiences/opinions; personal communication 
EM Problem: Decontamination and decommissioning; remedial action; waste management

Y-12 Plant Problem: Building and waste fields. Building 9201-4 (Alpha-4)

Problem Area/Constituents: Support of characterization, decontamination, dismantlement, and retrieval

Reference Requirements: Refer to the Regulatory Compliance chapter of Vol. 1 for potentially applicable proposed and promulgated environmental laws, signed and pending agreements for the Oak Ridge Reservation, radiation protection standards, DOE orders, and nonregulatory guidance. As site- and waste-specific characteristics are provided for each technology, specific regulatory requirements will be specified.

Subelement: Robotics/automation

Alternatives: Controls; algorithms

Technology: Vehicle/mobility navigation. Path planning and self-location are the primary elements of the navigation problem. Much emphasis has been placed on sensor-based selflocation, and the use of preplanned trajectories for navigation. More sophisticated navigation systems involve dynamic sensing and path planning based on sensor feedback. Real-time and dynamic navigation support to operators, especially for situations involving nonholonomic constraints, is an on-going research area. Some emphasis is being placed on implementation of navigation systems utilizing satellite global positioning systems

Status: Evolving

Efficacy-Medium. This technology will allow systems to engage in more sophisticated navigational activities. In general, preprogrammed trajectories are too inflexible for complex navigational problems, and existing man-in-the-loop navigation is too inefficient. Advances in real-time, dynamic navigation planning support systems could broaden the spectrum of navigational activities.

Waste-No secondary waste streams are generated.

Science/Technology Needs: (1) Path planning with nonholonomic constraints and (2) realtime sensor feedback

Implementation Needs: Enhanced usage of global positioning systems. Development costs are estimated to be $\$ 0.5 \mathrm{M}$.

Author: H. E. Knee/615-574-6163

References: MMES staff members' personal experiences/opinions; personal communication 


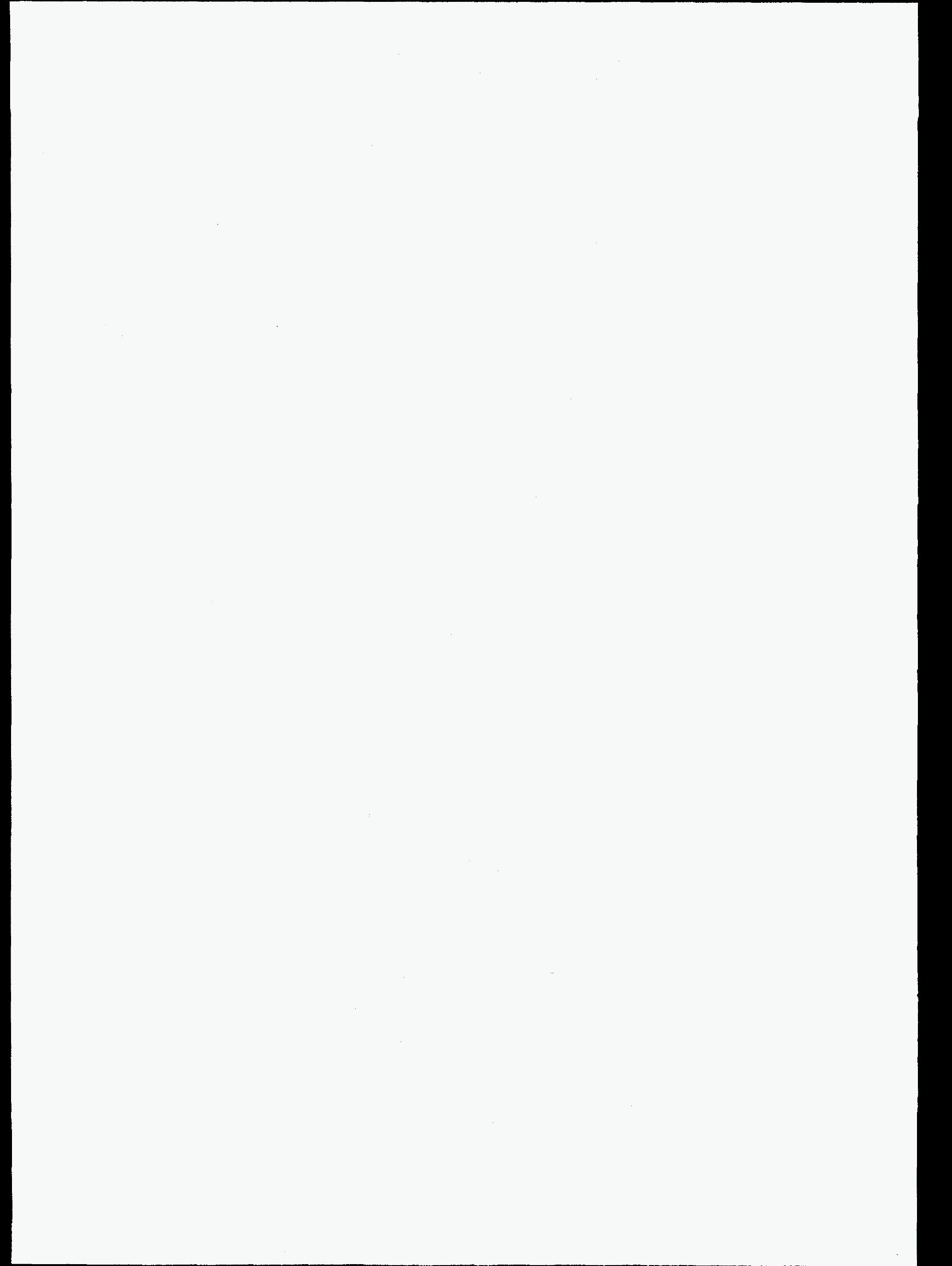


EM Problem: Decontamination and decommissioning (D\&D), remedial action (RA), waste management (WM)

Y-12 Plant Problem: D\&D, RA, and WM. Building 9201-4 (Alpha-4)

Problem Area/Constituents: Support of D\&D, RA, and WM activities will require a variety of control algorithms, including joint control, to most efficiently implement robotics and automation.

Reference Requirements: Refer to the Regulatory Compliance chapter of Vol. 1 for potentially applicable proposed and promulgated environmental laws, signed and pending agreements for the Oak Ridge Reservation, radiation protection standards, DOE orders, and nonregulatory guidance. As site- and waste-specific characteristics are provided for each technology, specific regulatory requirements will be specified.

Subelement: Robotics/automation

Alternative: Controls; algorithms

Technology: Manipulation (joint control). Joint-based control schemes assume that the desired trajectory is available in terms of time histories of joint position, velocity, and acceleration. Trajectory errors are generated by differencing desired and actual quantities expressed in joint space.

\section{Status: Demonstration}

Efficacy/strengths/weaknesses-Joint control is computationally efficient, can be implemented with minimal processing support, and can be run at very high rates. In general, however, tasks are not specified, or easily converted for description, in joint space.

Amount of waste produced-Not applicable for control-algorithm technologies. The systems these algorithms control are covered in the mechanical technologies.

Overall cost-The recurring costs for software (control algorithms) are small after development and testing are complete. As the complexity of the control algorithms increases, the processing hardware and support hardware (sensors, etc.) required increase considerably and can become significant cost items. Effective joint-space algorithms exist and can be implemented with minimal processing and sensing support. Total development costs are estimated to be $\$ 0.5 \mathrm{M}$.

Science/Technology Needs: Technology development needs-Most development activity is required in special purpose control algorithms [See Special Purpose Control (ROBA-32) and Redundancy Control (ROBA-33)].

Technology improvement needs-Improvement of control-algorithm technologies is an active area of DOE laboratory, university, and industry research. 
Implementation Needs: This activity is primarily software development in algorithm design, implementation, and verification. This area generally requires degree-level personnel in servocontrol systems (generally an electrical engineering discipline). Deployment requires implementation of control algorithms, processing (often distributed) systems, sensors systems, and operator control-station capabilities.

Author: D. C. Haley/615-576-4388

References: MMES staff members' personal experiences/opinions 
EM Problem: Decontamination and decommissioning (D\&D), remedial action (RA), waste management (WM)

Y-12 Plant Problem: D\&D, RA, and WM. Building 9201-4 (Alpha-4)

Problem Area/Constituents: Support of D\&D, RA, and WM activities will require a variety of control algorithms, including Cartesian control, to most efficiently implement robotics and automation.

Reference Requirements: Refer to the Regulatory Compliance chapter of Vol. I for potentially applicable proposed and promulgated environmental laws, signed and pending agreements for the Oak Ridge Reservation, radiation protection standards, DOE orders, and nonregulatory guidance. As site- and waste-specific characteristics are provided for each technology, specific regulatory requirements will be specified.

\section{Subelement: Robotics/automation}

Alternatives: Controls; algorithms

Technology: Manipulation (Cartesian control). Cartesian-based control schemes allow the desired trajectory to be specified in terms of time histories of Cartesian position, velocity, and acceleration, usually of the manipulator end point or a specified tool point. The specified or desired Cartesian motion must be converted to individual joint motions for actual manipulator control [see Joint Control (ROBA-30)]. Trajectory conversion, using the inverse kinematics of the manipulator, can be used to compute the time histories of the joint-space trajectory that correspond to the desired Cartesian path. The joint-space trajectories are then followed using joint-space control, basing errors on joint-space errors from the computed joint-space trajectories. An alternate approach is the use of coordinate conversion within the servo loop. The sensed position of the manipulator is transformed to Cartesian space by means of the forward kinematics, and this value is compared to the desired Cartesian value to form errors in Cartesian space.

\section{Status: Demonstration}

Efficacy/strengths/weaknesses-Cartesian control is computationally more demanding than joint-space control. Cartesian control systems run at a lower sampling frequency compared to joint-based systems, given the same processing resources. Tasks are generally much more easily specified in Cartesian space, and operator interaction with Cartesian systems are more intuitive.

Amount of waste produced-Not applicable for control algorithm technologies. The systems these algorithms control are covered in the mechanical technologies.

Overall cost-The recurring costs for software (control algorithms) are small after development and testing are complete. As the complexity of the control algorithms increases, the processing hardware and support hardware (sensors, etc.) required increase considerably and can become significant cost items. Effective Cartesian-space algorithms exist for both position 
and rate control. Implementation requires more processing and sensing support than required for joint control. Total development costs are estimated to be $\$ 1 \mathrm{M}$.

Science/Technology Needs: Technology development needs-Most development activity is required in special purpose control algorithms [see Special Purpose Control (ROBA-32) and Redundancy Control (ROBA-33)].

Technology improvement needs-Improvement of control-algorithm technologies is an active area of DOE laboratory, university, and industry research.

Implementation Needs: This activity is primarily software development in algorithm design, implementation and verification. This area generally requires degree-level personnel in servocontrol systems (generally an electrical engineering discipline). Deployment requires implementation of control algorithms, processing (often distributed) systems, sensors systems, and operator control station capabilities.

Author: D. C. Haley/615-576-4388

References: MMES staff members' personal experiences/opinions 
EM Problem: Decontamination and decommissioning (D\&D); remedial action (RA); waste management (WM)

Y-12 Plant Problem: Buildings, equipment, buried waste, and contaminated soils. Building 9201-4 (Alpha-4)

Problem Area/Constituents: Support of D\&D, RA, and WM activities will require a variety of control algorithms, including special purpose control algorithms, to most efficiently implement robotics and automation.

Reference Requirements: Refer to the Regulatory Compliance chapter of Vol. 1 for potentially applicable proposed and promulgated environmental laws, signed and pending agreements for the Oak Ridge Reservation, radiation protection standards, DOE orders, and nonregulatory guidance. As site- and waste-specific characteristics are provided for each technology, specific regulatory requirements will be specified.

\section{Subelement: Robotics/automation}

\section{Alternatives: Controls; algorithms}

Technology: Manipulation (special purpose control). Research and development in recent years has focused on many special purpose control algorithms to enhance system performance relative to more common joint or Cartesian position or rate control schemes. Hybrid position/force control algorithms allow position control of some task space degrees of freedom (DOF) while allowing the force generated in other DOF to be controlled. A class of controllers (force control, compliant control, impedance control) allows the system to actively respond to unwanted forces and torque and apply desired forces and torque in the performance of tasks. Force reflection allows sensed forces to be transmitted to an operator to provide a "feel" for forces encountered during task execution. Systems with more than 6 DOF are classified as kinematically redundant and require special control algorithms. This area is covered separately [see Manipulation (redundancy control), ROBA-33-0L]. All of these special purpose control algorithms provide capabilities that enhance the performance of robotic systems.

Algorithms related to high-level control functions are required as systems become more independent of direct operator control. Algorithms for task planning, sequencing and scheduling, path planning, and collision avoidance are required. High-level control capabilities also require more sophisticated operator interface functions.

\section{Status: Predemonstration}

Efficacy-Special purpose control algorithms have proven very effective in increasing system performance, versatility, and safety in laboratory implementations. In general, these algorithms require more complex processing capability, more support sensing, and more sophisticated operator interfaces. 
Waste-Not applicable for control algorithm technologies. The systems these algorithms control are covered in the mechanical technologies.

Overall Cost-The recurring costs for software (control algorithms) are small after development and testing are complete. As the complexity of the control algorithms increases, the processing hardware and support hardware (sensors, etc.) required increase considerably and can become significant cost items. Total development cost is estimated as $\$ 1 \mathrm{M}-\$ 2 \mathrm{M}$.

Science/Technology Needs: Technology Development Needs-Control algorithm development requires robotic system testbeds with extensive manipulation, mobility, and sensing and data acquisition capabilities for implementing, testing, and evaluating candidate algorithms.

Technology Improvement Needs-Improvement of control algorithm technologies is an active area of DOE laboratory, university, and industry research.

Implementation Needs: This activity is primarily software development in algorithm design, implementation, and verification. This area generally requires degree-level personnel in servocontrol systems (generally an electrical engineering discipline). Deployment requires implementation of control algorithms, processing (often distributed) systems, sensor systems, and operator control station capabilities.

Author: D. C. Haley/615-576-4388

References: MMES staff members personal experience/opinion

September 1994

Decontamination and Decommissioning 
EM Problem: Decontamination and decommissioning (D\&D); remedial action (RA); waste management (WM)

Y-12 Plant Problem: D\&D, RA, and WM. Building 9201-4 (Alpha-4)

Problem Area/Constituents: Support of D\&D, RA, and WM activities will require a variety of control algorithms, including special purpose redundancy control algorithms, to most efficiently implement robotics and automation.

Reference Requirements: Refer to the Regulatory Compliance chapter of Vol. 1 for potentially applicable proposed and promulgated environmental laws, signed and pending agreements for the Oak Ridge Reservation, radiation protection standards, DOE orders, and nonregulatory guidance. As site- and waste-specific characteristics are provided for each technology, specific regulatory requirements will be specified.

Subelement: Robotics/automation

Alternatives: Controls; algorithms

Technology: Manipulation (redundancy control). Robotic systems, primarily manipulator systems, which have more than 6 degrees of freedom (DOF) are considered to be kinematically redundant. Such systems can be used to reach a desired position and orientation from many possible paths. Such redundancy provides considerably increased versatility in performing tasks, especially in cluttered or confined spaces. An example of a redundant system is a 7-DOF manipulator that might be proposed for many dexterous tasks. A long-reach manipulator with a dexterous manipulator as an end effector is also an example of a kinematically redundant system. A special class of control algorithms are required to deal with the redundant systems and provide a basis for the selection of a solution from the many possible solutions that redundancy implies. Algorithms are required to support both teleoperation and robotic control modes for redundant systems.

\section{Status: Predemonstration}

Efficacy-Redundancy control algorithms are being tested in laboratory implementations. These algorithms have the potential to greatly increase the applicability and safety of kinematically redundant systems. In general these algorithms require complex processing capability, support sensing, and sophisticated operator interfaces.

Amount of waste produced-Not applicable for control algorithm technologies. The systems these algorithms control are covered in the mechanical technologies.

Overall cost-The recurring costs for software (control algorithms) are small after development and testing are complete. As the complexity of the control algorithms increases, the processing hardware and support hardware (sensors, etc.) required increase considerably and can become significant cost items. Total development costs are estimated to be $\$ 2 \mathrm{M}$. 
Science/Technology Needs: Technology Development Needs-Control algorithm development requires robotic system testbeds with extensive manipulation, mobility, sensing, and data acquisition capabilities for implementing, testing, and evaluating candidate algorithms.

Implementation Needs: This activity is primarily software development in algorithm design, implementation, and verification. This area generally requires advanced-degree-level personnel in servo-control systems (generally an electrical engineering discipline). Deployment requires implementation of control algorithms, processing (often distributed) systems, sensors systems, and operator control station capabilities.

Author: D. C. Haley/615-576-4388

References: MMES staff members' personal experiences/opinions 
EM Problem: Decontamination and decommissioning; remedial action; waste management

Y-12 Plant Problem: Buildings and waste sites. Building 9201-4 (Alpha-4)

Problem Area/Constituents: Support of characterization, decontamination, dismantlement, and retrieval

Reference Requirements: Refer to the Regulatory Compliance chapter of Vol. 1 for potentially applicable proposed and promulgated environmental laws, signed and pending agreements for the Oak Ridge Reservation, radiation protection standards, DOE orders, and nonregulatory guidance. As site- and waste-specific characteristics are provided for each technology, specific regulatory requirements will be specified.

Subelement: Robotics/automation

Alternatives: Controls; algorithms

Technology: Combined mobility/manipulation. This technology would permit parallel motion/mobility functions in the performance of a work task and would improve the speed of operations. One of the primary areas of research involves redundancy resolution. This research area is being addressed in a generic fashion to solve classes of redundancy resolution problems. Real-time task planning to support combined mobility and manipulation is required.

\section{Status: Evolving}

Efficacy-High. This technology will support the parallelization of activities currently done in series. Single-channel robotic functions tend to be ineffective.

Waste-No secondary waste streams generated.

Science/Technology Needs: (1) Research to support redundancy resolution and (2) realtime task planning

Implementation Needs: Software for simultaneous mobility and manipulation functions needs to be developed. Total development costs are estimated to be $\$ 1 \mathrm{M}$.

Author: H. E. Knee/615-574-6163

References: MMES staff members' personal experiences/opinions; personal communication 

EM Problem: Decontamination and decommissioning; remedial action; waste management

Y-12 Plant Problem: Buildings and waste sites. Building 9201-4 (Alpha-4)

ProblemArea/Constituents: Support of characterization, decontamination, dismantlement, and retrieval

Reference Requirements: Refer to the Regulatory Compliance chapter of Vol. 1 for potentially applicable proposed and promulgated environmental laws, signed and pending agreements for the Oak Ridge Reservation, radiation protection standards, DOE orders, and nonregulatory guidance. As site- and waste-specific characteristics are provided for each technology, specific regulatory requirements will be specified.

Subelement: Robotics/automation

Alternatives: Controls; algorithms

Technology: Safety (collision avoidance). This technology is primarily sensor based and is necessary to avoid unplanned interactions between the telerobotic system and objects in its environment (including itself). Two areas of concern are (1) selection of an appropriate sensor suite and (2) distributed intelligence (to the sensor level) to facilitate obstacle avoidance. Localized intelligence to ensure obstacle avoidance would minimize sensor processing times. Appropriate sensor suites will minimize the total telerobotic sensor processing requirements.

\section{Status: Demonstrated}

Efficacy-Medium. This technology will enhance the efficiency associated with uneventful driving/navigation/manipulation. Furthermore, it will minimize system downtime due to repairs associated with unavoided obstacle interaction.

Waste-No secondary waste streams generated.

Science/Technology Needs: There are no science/technology needs.

Implementation Needs: Emphasis should be placed on the implementation of architectures to support distributed and local sensor processing. In addition, implementation would benefit through the use of customized sensor suites. Total development costs are estimated to be $\$ 1 \mathrm{M}$.

Author: H. E. Knee/615-574-6163

References: MMES staff members' personal experiences/opinions; personal communication 


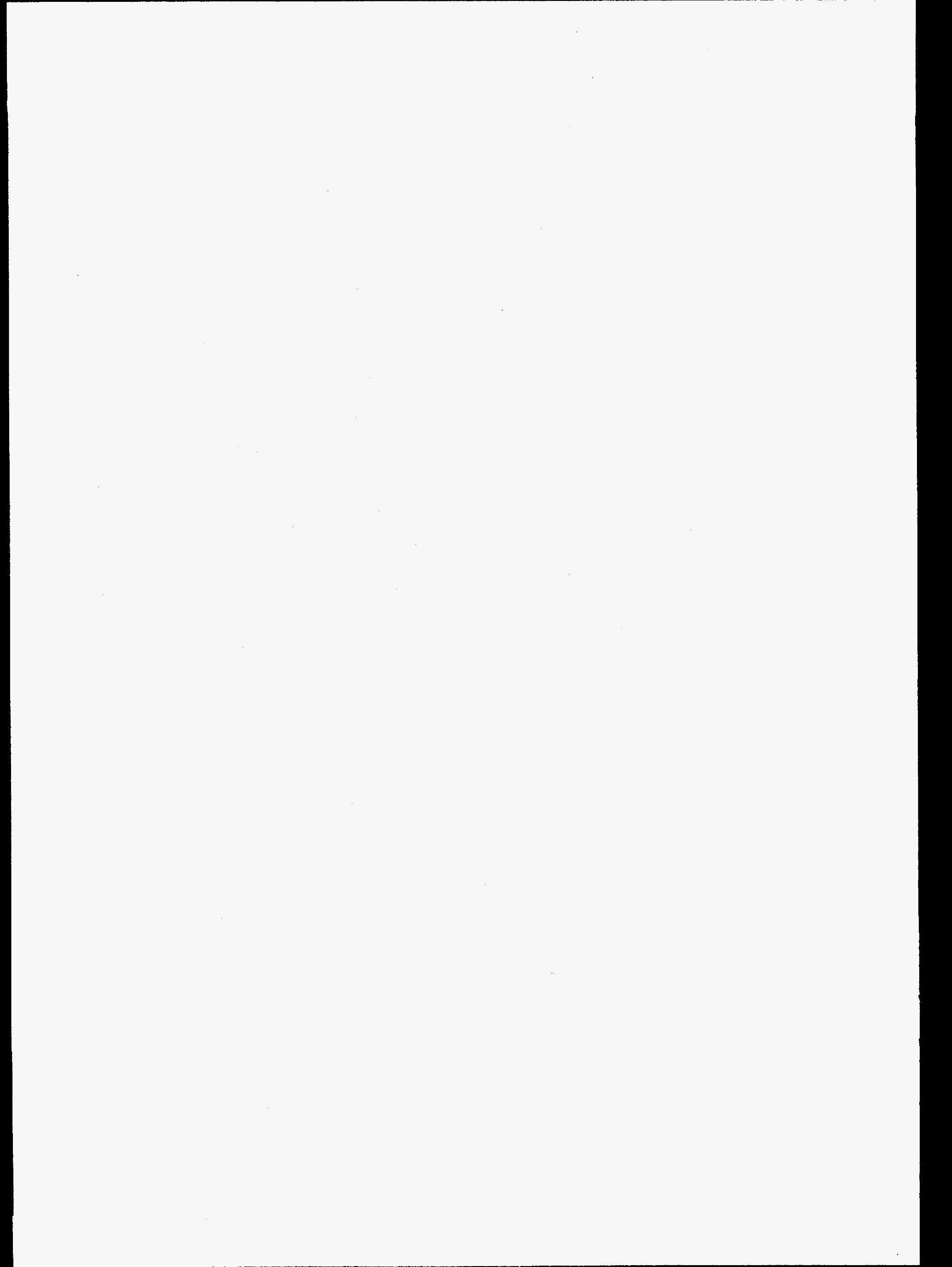


EM Problem: Decontamination and decommissioning; remedial action; waste management

T-12 Plant Problem: Buildings and equipment.Building 9201-4 (Alpha-4)

Problem Area/Constituents: Support of characterization, decontamination, dismantlement, remediation, packaging, handling, and inspection. Algorithms for load and rate limiting are required to provide safety features within the control systems of robotic systems.

Reference Requirements: Refer to the Regulatory Compliance chapter of Vol. 1 for potentially applicable proposed and promulgated environmental laws, signed and pending agreements for the Oak Ridge Reservation, radiation protection standards, DOE orders, and nonregulatory guidance. As site- and waste-specific characteristics are provided for each technology, specific regulatory requirements will be specified.

Subelement: Robotics/automation

Alternatives: Controls; algorithms

Technology: Safety (load/rate limiting). Algorithms within the manipulator control system are used to control the maximum load (force) produced by the manipulator on the workpiece. These algorithms use force sensor data (see ROBA-46, ROBA-50) to sense directly or compute the current load and restrict or stop motion as specified limits are approached or reached. Additional algorithms are used to limit the individual joint velocities (rate) or the manipulator end-effector velocity. Position or velocity sensor data are also used for either direct or computed measurement, and joint or manipulator motion is restricted or stopped as limits are approached. In cases where limits are exceeded, emergency shutdown (see ROBA-37) can be initiated to totally prohibit robotic system motion.

\section{Status: Predemonstration}

Efficacy-Algorithms for load and rate limiting are available and have been implemented in development systems. Simple algorithms based on direct measurements are most effective. Algorithms that require computed values need more computational processing resources and computational time and may limit reaction times.

Waste-None

Science/Technology Needs: Technology improvement is required in the development and testing of load- and rate-limiting algorithms requiring computed values.

Implementation Needs: This activity is primarily software development in algorithm design, implementation, and verification. This area generally requires degree-level personnel. Deployment requires implementation of control algorithms, processing (often distributed) systems, and sensor systems. Engineering modifications of development systems, as well as 
integration and testing for specific applications, will be required. Total development costs are estimated to be $\$ 0.5 \mathrm{M}$.

Author: D. C. Haley/615-576-4388

References: MMES staff members' personal experiences/opinions; personal communication; and vendor literature 
EM Problem: Decontamination and decommissioning; remedial action; waste management

Y-12 Plant Problem: Buildings and equipment. Building 9201-4 (Alpha-4)

Problem Area/Constituents: Support of characterization, decontamination, dismantlement, remediation, packaging, handling, and inspection. Algorithms for emergency shutdown are required to provide safety features within the control systems of robotic systems.

Reference Requirements: Refer to the Regulatory Compliance chapter of Vol. 1 for potentially applicable proposed and promulgated environmental laws, signed and pending agreements for the Oak Ridge Reservation, radiation protection standards, DOE orders, and nonregulatory guidance. As site- and waste-specific characteristics are provided for each technology, specific regulatory requirements will be specified.

\section{Subelement: Robotics/automation}

Alternatives: Controls; algorithms

Technology: Emergency shutdown. Algorithms within the manipulator control system are used to initiate emergency shutdown to prevent further motion when specified limits are reached or when the operator initiates an emergency shutdown from the operator console. Emergency shutdown algorithms are designed to remove power from actuator subsystems while continuing system sensing, data collection, and processing functions to allow system diagnostics and system recovery or restart.

\section{Status: Predemonstration}

Efficacy-Emergency shutdown will be required for all robotic systems. Algorithms for emergency shutdown are available and have been implemented in development systems. Simple algorithms based on direct measurements are most effective. Algorithms involving computed values require more computational processing resources and computational time and may limit reaction times.

Waste-None

Science/Technology Needs: Technology improvement is needed in the development and testing of emergency shutdown algorithms requiring computed values.

Implementation Needs: This activity is primarily software development in algorithm design, implementation, and verification. This area generally requires degree-level personnel. Deployment requires implementation of control algorithms, processing (often distributed) systems, and sensor systems. Engineering modifications of development systems, as well as integration and testing for specific applications, will be required. Total development costs are estimated to be $\$ 0.5 \mathrm{M}$.

Author: D. C. Haley/615-576-4388 
References: MMES staff members' personal experiences/opinions; personal communication; and vendor literature 
EM Problem: Decontamination and decommissioning; remedial action; waste management

Y-12 Plant Problem: Buildings and waste sites. Building 9201-4 (Alpha-4)

ProblemArea/Constituents: Support of characterization, decontamination, dismantlement, and retrieval

Reference Requirements: Refer to the Regulatory Compliance chapter of Vol. 1 for potentially applicable proposed and promulgated environmental laws, signed and pending agreements for the Oak Ridge Reservation, radiation protection standards, DOE orders, and nonregulatory guidance. As site- and waste-specific characteristics are provided for each technology, specific regulatory requirements will be specified.

Subelement: Robotics/automation

Alternatives: Controls; human-machine interface

Technology: Console (fixed/mobile/suitcase). Effective telerobotic control requires appropriately designed interface consoles that will function as "natural" translators of intention into action. Going from fixed to mobile and, finally, to suitcase follows console technology from the domain of demonstrated to evolving.

Status: Demonstration to evolving

Efficacy-Medium. This technology will support an optimum human-system interface, minimize human errors, and increase the effectiveness of the telerobotic activity.

Waste-No secondary waste streams generated.

Science/Technology Needs: (1) The reliability and (2) capacities of data links between the console and the robotic device need improvement; (3) attention to emerging interface technologies, including (4) embedded intelligence and (5) operator support/associate concepts, will facilitate more effectiveness through minimization of human error; (6) interface display design is of significance for suitcase consoles that have minimal interface real estate.

Implementation Needs: Emphasis should be placed on enhancing console durability. Total development costs are estimated to be $\$ 0.5 \mathrm{M}$.

Author: H. E. Knee/615-574-6163

References: MMES staff members' personal experiences/opinions; personal communication 


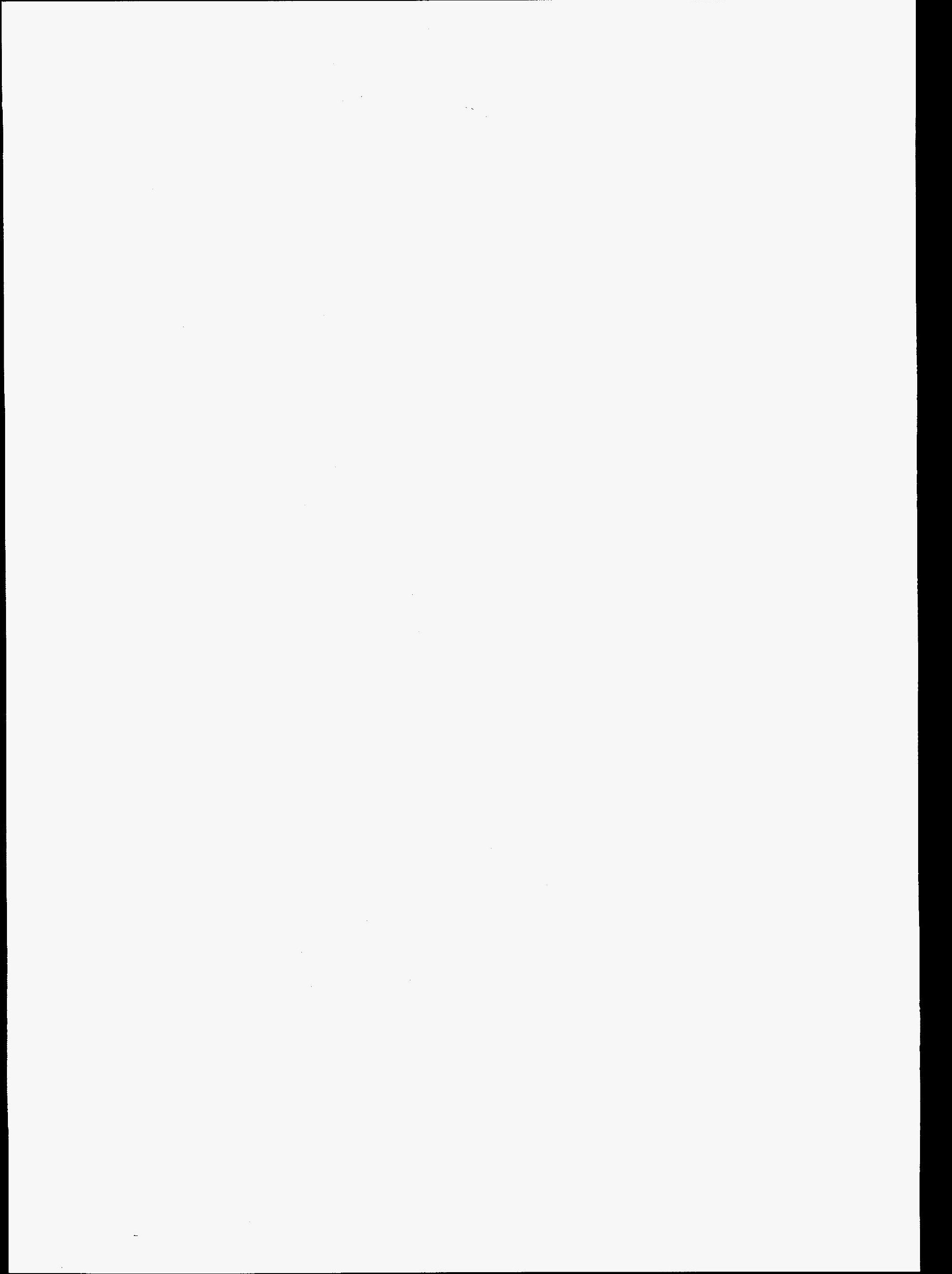


EM Problem: Decontamination and decommissioning (D\&D); remedial action (RA); waste management (WM)

Y-12 Plant Problem: Buildings and waste sites. Building 9201-4 (Alpha-4)

Problem Area/Constituents: Support of characterization, decontamination, dismantlement, and retrieval

Reference Requirements: Refer to the Regulatory Compliance chapter of Vol. 1 for potentially applicable proposed and promulgated environmental laws, signed and pending agreements for the Oak Ridge Reservation, radiation protection standards, DOE orders, and nonregulatory guidance. As site- and waste-specific characteristics are provided for each technology, specific regulatory requirements will be specified.

Subelement: Robotics/automation

Alternatives: Controls; human-machine interface

Technology: Console (data handling methods). Data handling and processing is at the heart of telerobotic activities associated with $\mathrm{D} \& \mathrm{D}$ of selected facilities. There are two areas of concern involving this technology. The first concern is the issue of appropriate data base management. A large amount of data in heterogeneous forms must be handled effectively. Second is the issue of data fusion. Making good sense (science) out of large data sets requires appropriate data integration (fusion).

\section{Status: Evolving}

Efficacy-High. This technology is a key element of the deployment of telerobotic systems. Without effective data handling, telerobotic and, subsequently, overall D\&D, RA, and WM activities would be less efficient

Waste-No secondary waste streams are generated.

Science/Technology Needs: (1) Design of effective heterogeneous data base management systems, (2) research associated with effective integration of heterogeneous data sources, (3) data fusion research, and (4) mathematical and intelligent navigation in large data sets

Implementation Needs: Total development costs are estimated to be $\$ 1 \mathrm{M}$.

Author: H. E. Knee/615-574-6163

References: MMES staff members' personal experiences/opinions; personal communication 
EM Problem: Decontamination and decommissioning (D\&D); remedial action (RA); waste management (WM)

Y-12 Plant Problem: All activities associated with the use of robotic devices for D\&D, RA, and WM tasks. Building 9201-4 (Alpha-4)

Problem Area/Constituents: Support of characterization, decontamination, dismantlement, retrieval, and waste-handling activities

Reference Requirements: Refer to the Regulatory Compliance chapter of Vol. 1 for potentially applicable proposed and promulgated environmental laws, signed and pending agreements for the Oak Ridge Reservation, radiation protection standards, DOE orders, and nonregulatory guidance. As site- and waste-specific characteristics are provided for each technology, specific regulatory requirements will be specified.

\section{Subelement: Robotics/automation}

Alternatives: Controls; human-machine interface

Technology: Operator interface (command input). This technology includes multimodal input-output, tactile control, voice control, eye-gaze control, hand controllers, and touch screens. Interface input devices are critical for effective telerobotic control. Such devices can be utilized as single-channel input to telerobotic platforms, such as a joystick, mouse, or other pointing device; or they can be utilized in a multimodal fashion that integrates the various human processing channels (tactile, aural, and visual) simultaneously. Positive examples of multimodal control (e.g., joy-stick/touch screen and voice) have been cited in the literature. Other human-to-system input channels (e.g., controlling an interface through the use of human eye-gaze measures) are still in the research stage. Because human tactile, aural, and visual systems are relatively independent from one another from a human processing perspective, relatively sophisticated control via multimodal input devices may be achievable and promises more efficient and error-free control.

Status: Evolving

Efficacy-High. Effective human-to-system interface capabilities will minimize operator loading, minimize human error, and maximize system efficiency.

Waste-No secondary waste streams are generated.

Science/Technology Needs: (1) Expansion of language for voice interface; (2) validation of eye-gaze measures/parameters; (3) head tracking in support of eye-gaze input; (4) research to support effective, simultaneous integration of tactile, aural, and eye-gaze input

Implementation Needs: Specialized operator training will be necessary for facilitating the simultaneous utilization of multiple human-to-system input channels. Development costs are estimated to be $\$ 0.5 \mathrm{M}$. 
Author: H. E. Knee/615-574-6163

References: MMES staff members' personal experiences/opinions; personal communication 
EM Problem: Decontamination and decommissioning; remedial action; waste management

Y-12 Plant Problem: Buildings and waste sites. Building 9201-4 (Alpha-4)

Problem Area/Constituents: Support of characterization, decontamination, dismantlement, and retrieval

Reference Requirements: Refer to the Regulatory Compliance chapter of Vol. 1 for potentially applicable proposed and promulgated environmental laws, signed and pending agreements for the Oak Ridge Reservation, radiation protection standards, DOE orders, and nonregulatory guidance. As site- and waste-specific characteristics are provided for each technology, specific regulatory requirements will be specified.

Subelement: Robotics/automation

Alternatives: Controls; human-machine interface

Technology: Operator interface (operator assistance). This technology includes, for example, computer-based operator support systems, scientific and data visualization, virtual reality, human-robot symbiosis, operator associate/partner concepts, and supervisory control. Assistance to human operators in complex environments spans technologies from manual (hard-copy) procedures to relatively sophisticated (interactive, supportive, and dynamic computer-based operator associate/partner) concepts. In between there are several technological support systems that include expert system advisors, neural network classifiers, and various other computer-aided applications. Operator assistance, in general, remains relatively passive (i.e., it provides, in a timely fashion, advice and guidance that human operators may choose to address or ignore). When differences exist between the control strategy selected by an intelligent control system, some forms of supervisory control may require some negotiation between an intelligent control system and a human operator (for these systems, however, the human operator eventually has the ultimate control authority). Other emerging systems involve a dymamic sharing of functions based on the current loading and available resources of each control agent within a control system (e.g., an intelligent control system may be one agent while a human operator is another). Such dynamic sharing of task functions is the basis for associate/partner concepts. Because teleoperation tends to be relatively complex, especially when addressing homogeneous facilities such as those at ORNL, great benefit could be achieved through the development and utilization of operator assistance for telerobotic control.

Status: From demonstration to evolving

Efficacy-High. This technology will allow the most efficient use of humans in the loop. It will therefore maximize efficiency and minimize human errors.

Waste-No secondary waste streams generated. 
Science/Technology Needs: (1) Means for establishing the validity and comprehensiveness of associated knowledge bases, (2) integration of multiple and diverse assistance, (3) research in conflict resolution, (4) hybrid artificial intelligence architectures, (5) dynamic allocation of function research, and (6) machine learning research

Implementation Needs: Specialized operator training will be necessary for effectively using computer-based operator assistance, especially simultaneous multiple assistance. Total development costs are estimated to be $\$ 1.5 \mathrm{M}$.

Author: H. E. Knee/615-574-6163

References: MMES staff members' personal experiences/opinions; personal communication 
EM Problem: Decontamination and decommissioning (D\&D); remedial action (RA): waste management (WM)

Y-12 Plant Problem: Those D\&D, RA, and WM activities that must be performed remotely and those activities for which control system oversight is required to alert operators. Building 9201-4 (Alpha-4)

Problem Area/Constituents: Support of characterization, decontamination, dismantlement, retrieval, surveillance, and waste handling activities

Reference Requirements: Refer to the Regulatory Compliance chapter of Vol. 1 for potentially applicable proposed and promulgated environmental laws, signed and pending agreements for the Oak Ridge Reservation, radiation protection standards, DOE orders, and nonregulatory guidance. As site- and waste-specific characteristics are provided for each technology, specific regulatory requirements will be specified.

Subelement: Robotics/automation

Alternatives: Controls; human-machine interface

Technology: Operator interface (status/alert system). The status and alert system are the primary input to human operators. Technology has provided the capability to provide operators with a quantity of data and information that far exceeds human processing capabilities. Because of this, special emphasis is needed in the design of interfaces to provide human operators with high-quality and (perhaps graphically) integrated status and alert data and information. Numerous and generally application-specific approaches for alarm handling have been researched. Other display technologies (e.g., multiple CRT-based displays and even large panel displays) are achieving good success in supporting complex process control environments. Visualization and virtual reality offer new opportunities involving the display of data and information to human visual systems. Improperly designed status/alert systems have a tendency to very quickly overload a human operator's cognitive system. Sophisticated telerobotic capabilities require that high-quality, integrated data and information be provided to the human operator on a timely basis.

Status: From demonstration to evolving

Efficacy-High. This technology will allow the human operator to function optimally. It will allow the human operator to have greater insight into system states and to be in a better position to plan and accomplish subsequent tasks. It will, therefore, maximize efficiency and minimize human errors.

Waste-No secondary waste streams generated.

Science/Technology Needs: (1) Research on display navigation, (2) research on new metaphors for information display, (3) sensor/data fusion, and (4) research on navigation in virtual worlds 
Implementation Needs: Specialized operator training will be necessary for effectively using advanced status/alert system data and information. Total development costs are estimated to be $\$ 0.5 \mathrm{M}$.

Author: H. E. Knee/615-574-6163

References: MMES staffmembers' personal experiences/opinions; personal communication 
EM Problem: Decontamination and decommissioning (D\&D); remedial action (RA); waste management (WM)

Y-12 Plant Problem: All activities associated with D\&D, RA, and WM that require robotic involvement. Building 9201-4 (Alpha-4)

Problem Area/Constituents: Support of characterization, surveying, decontamination, dismantlement, retrieval, and waste handling activities

Reference Requirements: Refer to the Regulatory Compliance chapter of Vol. 1 for potentially applicable proposed and promulgated environmental laws, signed and pending agreements for the Oak Ridge Reservation, radiation protection standards, DOE orders, and nonregulatory guidance. As site- and waste-specific characteristics are provided for each technology, specific regulatory requirements will be specified.

Subelement: Robotics/automation

Alternatives: Sensors; perception

Technology: Vision/aural systems. Appropriate visual and aural systems are necessary for robotic systems for a number of reasons. These include effective mobility, manipulation, planning, decision making, self-location, navigation, and obstacle avoidance. The vision area involves two facets of research. The first deals with robotic capabilities to sense threedimensional (3-D) objects in order to carry out telerobotic functions. The second deals with appropriately displaying 3-D data and information to a human operator in a fashion that is sensitive to all other data and information to be managed by the human operator. Highdefinition television (HDTV) is a technology that will facilitate enhanced visual recognition capabilities and would minimize uncertainties associated with the processing of visual data. For aural systems, the technology of directional audio promises to provide vector data rather than just magnitude data to support self-location, navigation, and obstacle avoidance.

Status: Demonstration (for 3-D vision and HDTV); predemonstration (for directional audio)

Efficacy-Medium. This technology will minimize the cognitive load on human operators and allow consideration of automating activities that now require human systems to function in the vision and aural domains. Efficiency would be increased and human errors reduced.

Waste-No secondary waste streams generated.

Science/Technology Needs: (1) Sensor fusion, (2) advanced visualization capabilities, and (3) increased processing speed

Implementation Needs: Total development costs are estimated to be $\$ 1 \mathrm{M}$.

Author: H. E. Knee/615-574-6163 
References: MMES staff members' personal experiences/opinions; personal communication 
EM Problem: Decontamination and decommissioning (D\&D); remedial action; waste management

Y-12 Plant Problem: D\&D of buildings and equipment, and waste handling. Building 9201-4 (Alpha-4)

Problem Area/Constituents: Dismantlement and decontamination

Reference Requirements: Refer to the Regulatory Compliance chapter of Vol. 1 for potentially applicable proposed and promulgated environmental laws, signed and pending agreements for the Oak Ridge Reservation, radiation protection standards, DOE orders, and nonregulatory guidance. As site- and waste-specific characteristics are provided for each technology, specific regulatory requirements will be specified.

Subelement: Robotics/automation

Alternatives: Sensors; perception

Technology: Range finders. A number of approaches are utilized to estimate range in telerobotic systems. Over the past several years, laser range finders, sonar, and LEDs have increased in both speed and accuracy, and the price has significantly decreased.

Status: Demonstrated

Efficacy-Medium. This technology will add to efficient telerobotic activities.

Waste-No secondary waste streams generated.

Science/Technology Needs: There are no science/technology needs.

Implementation Needs: Continued emphasis on speed and accuracy improvement; perhaps some emphasis on durability. Total development costs are estimated to be $\$ 1.5 \mathrm{M}$.

Author: H. E. Knee/615-574-6163

References: MMES staff members' personal experiences/opinions; personal communication 


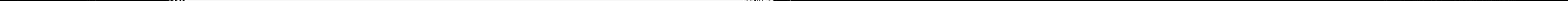


EM Problem: Decontamination and decommissioning (D\&D); remedial action (RA); and waste management (WM)

Y-12 Plant Problem: Buildings, equipment, buried waste, and contaminated soils. Building 9201-4 (Alpha-4)

Problem Area/Constituents: Radioactive and hazardous materials encountered in characterization, decontamination, dismantlement, and retrieval operations.

Reference Requirements: Refer to the Regulatory Compliance chapter of Vol. 1 for potentially applicable proposed and promulgated environmental laws, signed and pending agreements for the Oak Ridge Reservation, radiation protection standards, DOE orders, and nonregulatory guidance. As site- and waste-specific characteristics are provided for each technology, specific regulatory requirements will be specified.

Subelement: Robotics/automation

Alternatives: Sensors; perception

Technology: Proximity probes. These probes are required on robotic devices to prevent collisions with obstacles in the environment. These probes can provide information to the operator of a teleoperated device or to the control system for telerobotically operated or autonomous devices.

Status: Predemonstration. Additional Comments-These probes will be particularly useful for D\&D tasks in cluttered environments. They will have less application in RA or WM activities.

Efficacy-Medium. Alternatives for obstacle avoidance (e.g., vision systems) may be more costeffective.

Waste-None

Overall Cost-Probes for large volume coverage will require a development cost of $\$ 1 \mathrm{M}-\$ 2 \mathrm{M}$.

Science/Technology Needs: Present proximity probes are limited in both range and signal quality. This limitation results in artificially large boundaries being drawn around potential collision areas in the work space. Development of probes with increased range and better presentation to the operator are science/technology needs.

Implementation Needs: None

Author: J. N. Herndon/615-574-7121

References: Personal communications with other MMES personnel 


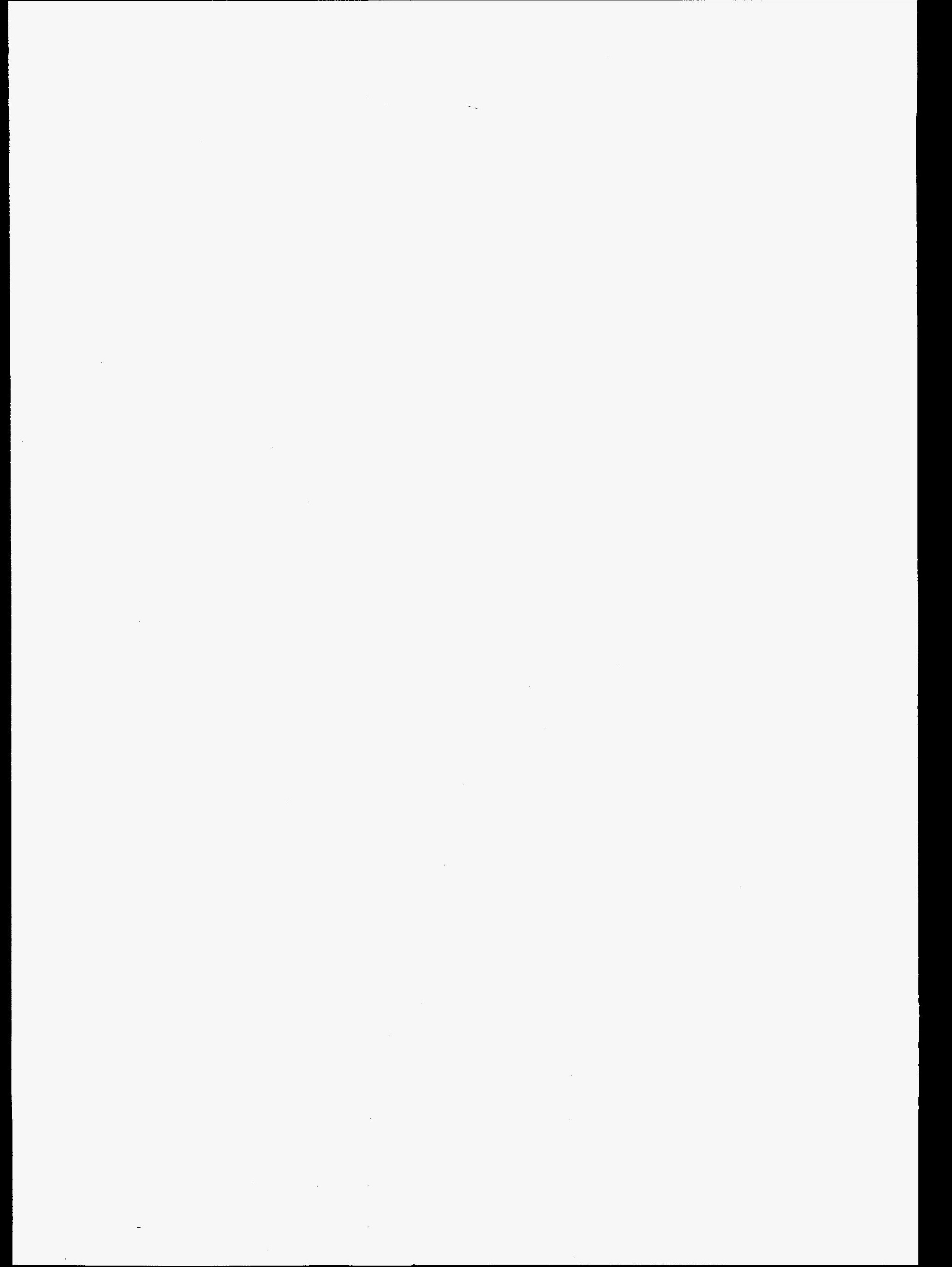


EM Problem: Decontamination and decommissioning; remedial action; waste management

Y-12 Plant Problem: Buildings and equipment. Building 9201-4 (Alpha-4)

Problem Area/Constituents: Support of characterization, decontamination, dismantlement, remediation, packaging, handling, and inspection. Sensors for sensing forces and torque are required to provide safety features within the control systems of robotic systems.

Reference Requirements: Refer to the Regulatory Compliance chapter of Vol. 1 for potentially applicable proposed and promulgated environmental laws, signed and pending agreements for the Oak Ridge Reservation, radiation protection standards, DOE orders, and nonregulatory guidance. As site- and waste-specific characteristics are provided for each technology, specific regulatory requirements will be specified.

Subelement: Robotics/automation

Alternatives: Sensors; perception

Technology: Force/torque sensors. Force and torque sensing can be implemented within robotic joints and via wrist or end-effector sensors. These sensors provide information regarding the forces being generated within the robotic system and the forces being applied to the workpiece (see ROBA-17). These forces can be used for control (see ROBA-32, ROBA50) or within safety systems (see ROBA-36, ROBA-37).

\section{Status: Demonstration}

Efficacy-Effective sensors for forces and torque at joints, the wrist, and tooling are available. Waste-None

Science/Technology Needs: Technology development is required in the development and testing of tactile sensing systems.

Technology improvement is required in the development and testing of force sensing systems.

Implementation Needs: Engineering modifications of development systems, as well as integration and testing for specific applications, will be required for implementation. Total development costs are estimated to be $\$ 0.5 \mathrm{M}$.

Author: D. C. Haley/615-576-4388

References: MMES staff members' personal experiences/opinions; personal communication; and vendor literature 


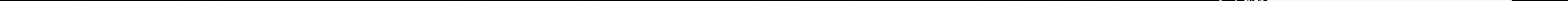


EM Problem: Decontamination and decommissioning; remedial action; waste management

Y-12 Plant Problem: Buildings, equipment, buried waste, and contaminated soils. Building 9201-4 (Alpha-4)

Problem Area/Constituents: Radioactive and hazardous materials encountered in characterization, decontamination, dismantlement, and retrieval operations

Reference Requirements: Refer to the Regulatory Compliance chapter of Vol. 1 for potentially applicable proposed and promulgated environmental laws, signed and pending agreements for the Oak Ridge Reservation, radiation protection standards, DOE orders, and nonregulatory guidance. As site- and waste-specific characteristics are provided for each technology, specific regulatory requirements will be specified.

Subelement: Robotics/automation

Alternatives: Sensors; control

Technology: Position/velocity sensors. Present industrial-grade position and velocity sensors are adequate for most expected applications in the remediation tasks. These available technologies are anticipated to be sufficient for any applications related to manipulator-arm and end-effector position and/or velocity sensor requirements.

Status: Accepted. The potential applications for these sensors are somewhat limited and may find their major usage in an automated analytical laboratory.

Efficacy-High for application in an analytical laboratory or possibly for sensor sweeps for characterization tasks

Waste-None

Overall Cost-Development costs associated with this technology are minimal and are estimated to be no more than $\$ 0.5 \mathrm{M}$.

Science/Technology Needs: None

Implementation Needs: None

Author: J. N. Herndon/615-574-7121

References: Discussions with MMES staff members 


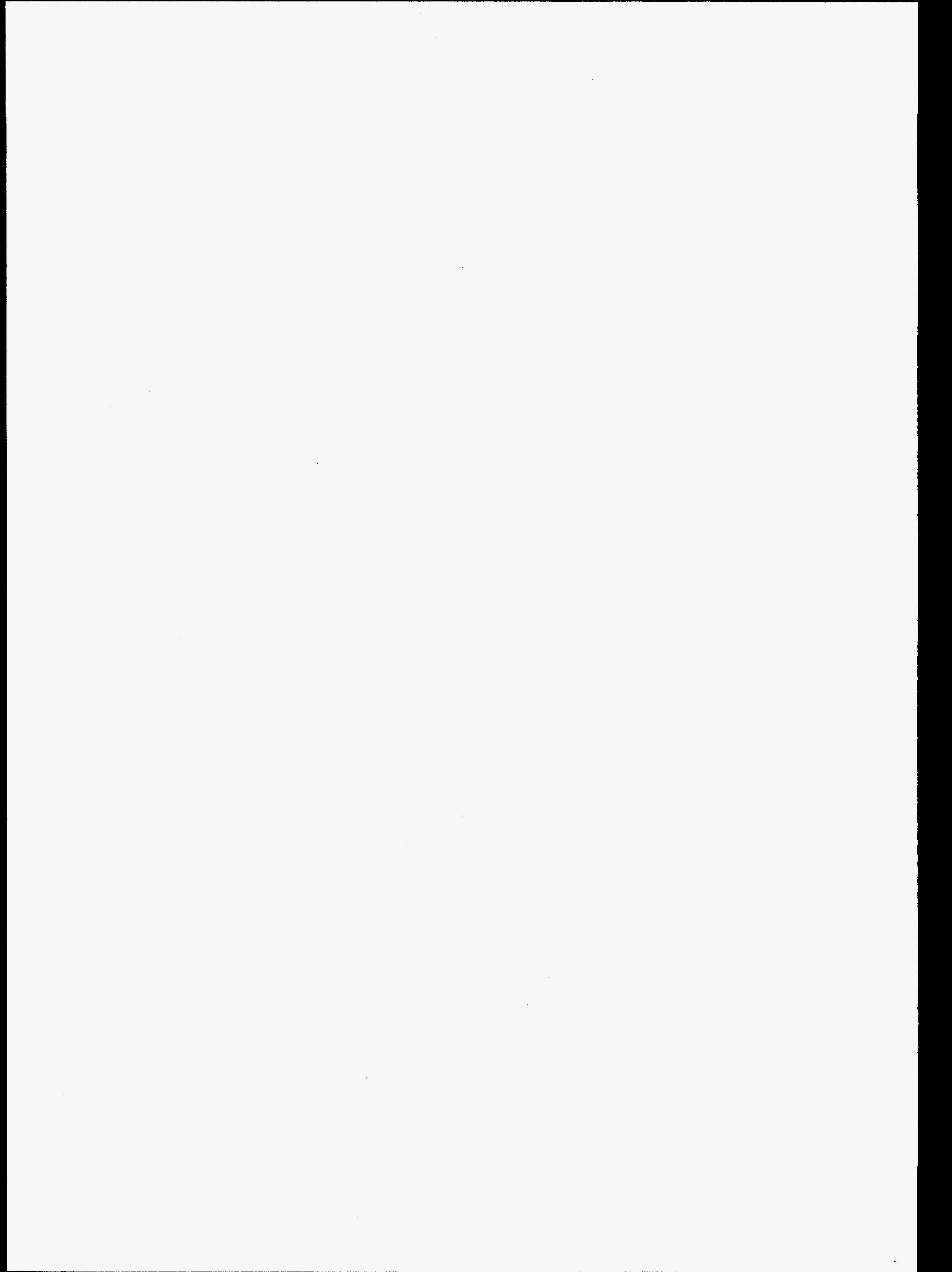


EM Problem: Decontamination and decommissioning (D\&D); remedial action; waste management

Y-12 Plant Problem: D\&D of buildings and equipment and waste handling and packaging. Building 9201-4 (Alpha-4)

Problem Area/Constituents: Support of characterization, dismantlement, decontamination, and waste retrieval operations

Reference Requirements: Refer to the Regulatory Compliance chapter of Vol. 1 for potentially applicable proposed and promulgated environmental laws, signed and pending agreements for the Oak Ridge Reservation, radiation protection standards, DOE orders, and nonregulatory guidance. As site- and waste-specific characteristics are provided for each technology, specific regulatory requirements will be specified.

Subelement: Robotics/automation

Alternatives: Controls; control

Technology: Force/torque. Any nontrivial telerobotic handling situation will require combined force/torque control. One of the main constituents of this technology is quality feedback. Force/torque control and feedback will facilitate more efficient interfacing with telerobotic environments. Stability of the system and resolution of impulse forces are two associated research issues.

Status: Demonstration

Efficacy-Medium. This technology will support more effective telerobotic interface capabilities.

Waste-No secondary waste streams generated.

Science/Technology Needs: (1) Stability and (2) impulse force resolution

Implementation Needs: No unusual implementation needs. Total development costs are estimated to be no more than $\$ 0.5 \mathrm{M}$.

Author: H. E. Knee/615-574-6163

References: MMES staff members' personal experiences/opinions; personal communication 


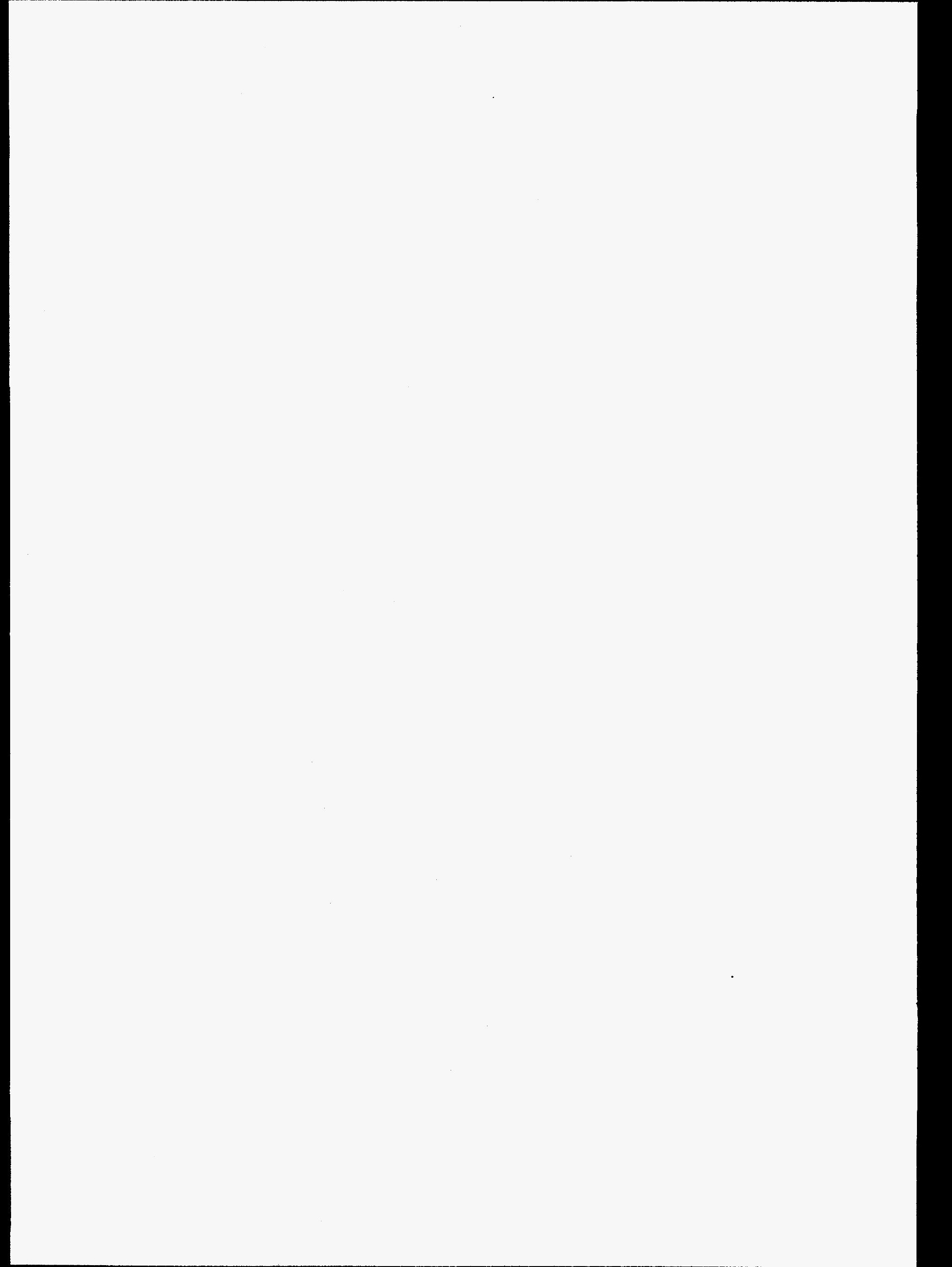


EM Problem: Decontamination and decommissioning; remedial action; waste management

Y-12 Plant Problem: Buildings and equipment. Building 9201-4 (Alpha-4)

Problem Area/Constituents: Support of characterization, decontamination, dismantlement, remediation, packaging, handling, and inspection. Sensors for end-effectors and tooling are required to enhance the available control capability.

Reference Requirements: None

Subelement: Robotics/automation

Alternatives: Sensors; control

Technology: Tooling sensors. End effectors and tooling can incorporate a variety of sensing systems to provide more information or control capability. Force sensors within the end effector of tactile sensing on the fingers can be used to measure and control the forces applied to the workpiece. Video cameras can be imbedded in or mounted externally on the end effector or tooling to provide visual feedback to allow more precise control of operations. Additional environmental sensors can be integrated into end effectors or tooling to measure various parameters (e.g., temperature). Proximity sensors can be deployed around the perimeter of end effectors and tooling to provide precise position control or to indicate impending collisions.

Status: Predemonstration

Efficacy-Development testing has shown a great increase in the effectiveness and ability for precise control of end effectors and robotically deployed tooling when sensing systems are added.

Waste-None

Science/Technology Needs: Technology development is required in the development and testing of tactile sensing systems.

Technology improvement is required in the development and testing of force and proximity sensing systems.

Implementation Needs: Engineering modifications of development systems, as well as integration and testing for specific applications, will be required for implementation. Total development costs are estimated to be $\$ 0.5 \mathrm{M}$.

Author: D. C. Haley/615-576-4388

References: MMES staff members' personal experiences/opinions; personal communication; and vendor literature 


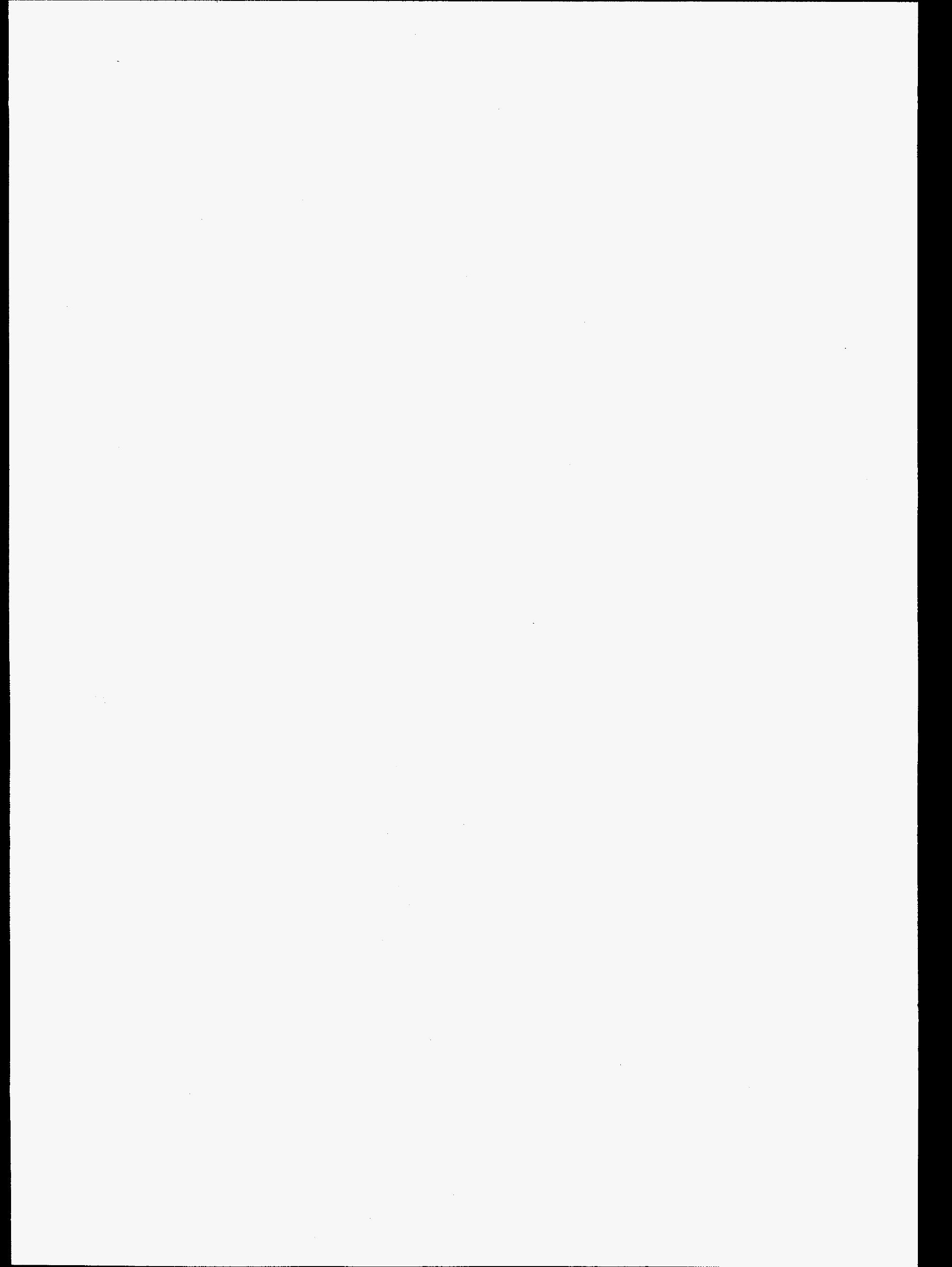


EM Problem: Decontamination and decommissioning (D\&D); remedial action (RA); waste management (WM)

Y-12 Plant Problem: Buildings and equipment. Building 9201-4 (Alpha-4)

Problem Area/Constituents: Support of characterization, decontamination, dismantlement, remediation, packaging, handling, and inspection. Field hardening of robotic systems will be required for many applications.

Reference Requirements: None

Subelement: Robotics/automation

Alternatives: Other technologies

Technology: Field hardening. Field hardening of robotic systems will be required for many applications. Robotic systems will be exposed to high heat, high humidity, radiological contamination, dust, and possibly caustic chemicals. Some applications may require underwater operation. The need for decontamination of robotic systems will also require modifications. Modifications such as smooth exterior surfaces, seals, and special lubricants will be required.

\section{Status: Demonstration}

Efficacy-Most of the modifications required to field harden robotic systems are in use in military systems. Commercially available systems are not generally designed for the range of environments that may be encountered in D\&D, RA, and WM activities. Specifically, these systems do not utilize design principles that facilitate decontamination (e.g., no crevices, blind holes, treads, etc.).

Waste-None

Science/Technology Needs: None

Implementation Needs: Engineering modifications of development systems, as well as integration and testing for specific applications, will be required for implementation. Total development costs are estimated to be $\$ 0.5 \mathrm{M}$.

Author: D. C. Haley/615-576-4388

References: MMES staff members' personal experiences/opinions; personal communication; and vendor literature 

EM Problem: Decontamination and decommissioning; remedial action; and waste management

Y-12 Plant Problem: Characterization and analysis of samples. Building 9201-4 (Alpha-4)

Problem Area/Constituents: Support of characterization and decommissioning

Reference Requirements: Refer to the Regulatory Compliance chapter of Vol. 1 for potentially applicable proposed and promulgated environmental laws, signed and pending agreements for the Oak Ridge Reservation, radiation protection standards, DOE orders, and nonregulatory guidance. As site- and waste-specific characteristics are provided for each technology, specific regulatory requirements will be specified.

Subelement: Robotics/automation

Alternatives: Other technologies

Technology: Integrated automated analytical laboratory

Status: The technology required for a totally integrated and automated analysis laboratory is being developed as part of a mult-DOE-site project entitled "Contaminant Analysis Automation" (CAA). Various hardware elements of this technology are in the prototype development stage, while other elements are in the conceptual design phase. Industrial partners are working with the CAA project through cooperative research and development agreements (CRADAs) to quickly transfer the technology to the commercial sector. The CAA project team is involved with the National Institute of Standards and Technology (NIST), through the Consortium on Automated Analytical Laboratory Systems, to develop standards required for implementation of a totally integrated and automated analysis laboratory that is independent of equipment and software vendors.

The CAA project is focusing on the development of systems that will standardize the hardware and software required to perform automated chemical analyses. This is being accomplished by designing and producing analysis systems call Standard Analysis Methods (SAMs). Each SAM will automate a specific chemical analysis method, including sample preparation, constituent(s) analysis, and the data interpretation by using a building block known as the standard laboratory module (SLM). An SLM is defined as a logical grouping of laboratory unit operations that perform a subtask of an analytical protocol. Each SLM interacts with a controller through a standard interface as part of a SAM or can function stand-alone. The stand-alone capability of the SLMs will allow each laboratory to select the level of automation and integration required to meet specific analysis needs. For sample preparation functions the SLMs tend to be hardware intensive, while data handling and interpretation SLMs are software intensive. Sample movement between SLMs will be handled by a robot, which is being implemented with a generic "move sample" command. Analytical instruments will also be configured as an SLM. The SAM concept will allow the chemist to assemble a totally automated analytical method using standardized SLMs in a "plug-and-play" fashion without 
the need to be concerned with hardware compatibility or the need to generate complicated control programs.

Prototypes of SLMs to implement U.S. Environmental Protection Agency (EPA) Methods 3540 and 3550 have been developed. Chemical validation testing of these systems will be completed during FY 1993. Validated SLMs will gain approval from the Laboratory Management Branch of DOE for their use in the DOE Methods Compendium. The Laboratory Management branch of DOE will also pursue EPA approval for the use of these devices to carry out EPA methods. Design of acid digestion modules is under way. An expert system-driven data interpretation module (DIM) is under development; EPA Method 8080, "Organochlorine Pesticides and PCBs," is the target protocol for the initial DIM development. A detailed cost/benefit analysis of the CAA technology will be completed at the end of FY 1993. An industrial partner to assist in the SAM integration effort and to commercialize the CAA technology was selected in February 1993, and the negotiation of a project umbrella CRADA is in progress. A demonstration of an integrated SAM that will analyze soil samples for polychlorinated biphenyls (PCBs) is scheduled for December 1993.

Science/Technology Needs: The need for integrated and automated analysis systems is driven by two major factors: (1) the number of samples that characterization, decommissioning, remediation, and waste management activities will generate exceeds the combined DOE and private contract laboratory capacity; and (2) the lack of sufficient manpower in the educational pipeline to allow expansion of laboratory capacity along the lines of exdsting manual laboratory technology. Specific areas needing significant development effort include the following:

\section{Sample Preparation SLMs}

The process of sample preparation is a labor- and time-intensive operation, and relatively little effort has been devoted to this aspect of laboratory automation. The sample preparation modules take a specimen submitted to the laboratory for analysis and perform those laboratory unit operations to prepare the sample for measurement by the appropriate instrumentation. A typical unit operation involves weighing the sample, adding reagents, adding spike and/or surrogate compounds, dissolving the sample extract constituents of interest, separating individual constituents, removing water, concentrating the extract, removing interferences, etc. The specific set of laboratory unit operations required for a given sample are determined by the type of information being sought by the customer of the analysis request.

\section{Analytical Instrument SLMs}

Analytical instrument SLMs have received considerable attention in the commercial market. However, it is virtually impossible to integrate instruments from different vendors into a unified laboratory operation, and it is often impossible to integrate newer models of an

September 1994

Decontamination and Decommissioning 
instrument from a single vendor into a unified laboratory operation. This incompatibility results in islands of automation within laboratories that operate totally independently of each other.

The private sector is beginning to address the issue of data exchange between chromatographic equipment from different vendors. The need is to develop standards for instrument control, data exchange, and related issues so analytical instrumentation from any vendor can be used to construct an SAM. The CAA involvement with NIST is addressing this issue. The CAA project will demonstrate an SLM version of a commercial gas chromatograph in December 1993.

\section{Automated Data Interpretation SLMs}

Automation of the sample preparation and analytical instrument elements of the laboratory will only move the productivity bottle neck to the data interpretation and review phase. The data interpretation and review element of the laboratory operation requires well-trained chemists. Discussions with staff in several environmental analysis laboratories indicate that $30-50 \%$ of staff time is devoted to this element of laboratory operation. The CAA project is pursuing the following approach to automate data interpretation.

DIM will be an expert system-driven SLM. The DIM will have two operational modes: (1) an on-line mode that performs data analysis and is controlled by the SAM task sequence controller via the standard SLM protocols and (2) an off-line mode that provides the analytical chemist with the tools required to build automated data analysis methods and review the data and analysis process. Other tasks required of DIM include the validation of the integrity of the raw data, the feedback of information to the SAM task sequence controller to ensure proper sample processing, automated quality control and quality assurance functions, and an interface to the SAM data base. DIM will include existing data analysis tools familiar to analytical chemists. A need for pattern recognition-based data analysis tools has been identified. This aspect of DIM is particularly important when dealing with multicomponent analyses (e.g., for PCBs, a major contaminant at the DOE sites), as well as classifying the sample source. A prototype of a pattern recognition-based DIM that will analyze soil samples for Aroclor content will be demonstrated in December 1993.

Other issued identified by the CAA project include electronic records systems that meet regulatory protocol and legal requirements, integrated control systems for the automated laboratory, data exchange between SAM and the laboratory information management system, and the deployment of automated laboratory systems at the work site. Effort within the private sector that may address the electronic records issue has been identified. Integrated control of the automated laboratory is being addressed by the CAA team in cooperation with the commercial system integrator. Deployment of the automated laboratory at the work site can be provided by the commercial sector once the integrated automated laboratory system has been demonstrated.

Implementation Needs: Training of laboratories' technicians will be required. EPA approval 
of SLMs is also required. Total development costs of $\$ 3 M$ are estimated.

Author: L. N. Klatt/615-574-6588

References: T. J. Beugelsdijk, J. W. Elling, T. H. Erkkila, and R. M. Hollen, "Contaminant Analysis Automation," in Environ. Test. Anal. 2(2), 68 (1993). 
EM Problem: Decontamination and decommissioning (D\&D); remedial action (RA); waste management (WM)

Y-12 Plant Problem: All remediation activities that require remote sampling for subsequent characterization. Building 9201-4 (Alpha-4)

Problem Area/Constituents: Support of characterization for D\&D, RA, and WM

Reference Requirements: Refer to the Regulatory Compliance chapter of Vol. 1 for potentially applicable proposed and promulgated environmental laws, signed and pending agreements for the Oak Ridge Reservation, radiation protection standards, DOE orders, and nonregulatory guidance. As site- and waste-specific characteristics are provided for each technology, specific regulatory requirements will be specified.

Subelement: Robotics/automation

Alternatives: Other technologies

Technology: Information management for sample handling. Information management systems for specimen acquisition and preparation prior to submission to characterization laboratory is required for proper interfacing with analytical laboratory.

Status: Demonstration

Science/Technology Needs: Fusion of this information with the characterization laboratory information management system, work site data base systems, and other project data base systems across different computing platforms needs to be accomplished. Automatic data entry systems at all levels of data input must be developed. Typical systems include bar code, machine readable symbologies, document scanners, image digitizers, and image analyzers. The reliability and auditability of the information management system must be established.

Implementation Needs: A pilot program will be required to demonstrate an information management system for sample submittal to analytical laboratories. Total development costs are estimated to be $\$ 1 \mathrm{M}$.

Author: L. N. Klatt/615-574-6588

References: MMESstaff members' personal experiences/opinions; personal communication 

EM Problem: Decontamination and decommissioning; remedial action; waste management

Y-12 Plant Problem: Sample collection for characterization for D\&D, RA, and WM. Building 9201-4 (Alpha-4)

Problem Area/Constituents: Support of characterization and decommissioning activities

Reference Requirements: Various regulations and guidelines define sample collection requirements (e.g., surface area covered by swipe samples). Refer to the Regulatory Compliance chapter of Vol. 1 for potentially applicable proposed and promulgated environmental laws, signed and pending agreements for the Oak Ridge Reservation, radiation protection standards, DOE orders, and nonregulatory guidance. As site- and waste-specific characteristics are provided for each technology, specific regulatory requirements will be specified.

Subelement: Robotics/automation

Alternatives: Other technologies

Technology: Sample collection. Robotic devices and tools modified for remote operation are required to collect the many samples that will be generated by D\&D, RA, and WM. These samples must be of a form and size suitable for characterization.

\section{Status: Evolving}

Science/Technology Needs: The types of specimens include substrates to determine smearable and nonsmearable contamination, liquid samples, core samples (high-density and normal building construction-grade concrete, soils, salt cake, slurries, and sludges), and airborne hazardous materials. Robotic systems to remotely deploy and manipulate the sample collection tools are required. The development of suitable robotic end effectors and the adapting of existing devices to robotic applications are required.

Implementation Needs: Extensive testing of the collection tools will be required prior to putting them into use. Total development costs are estimated to be $\$ 1.5 \mathrm{M}$.

Author: L. N. Klatt/615-574-6588

References: MMES staff members and personal communication 


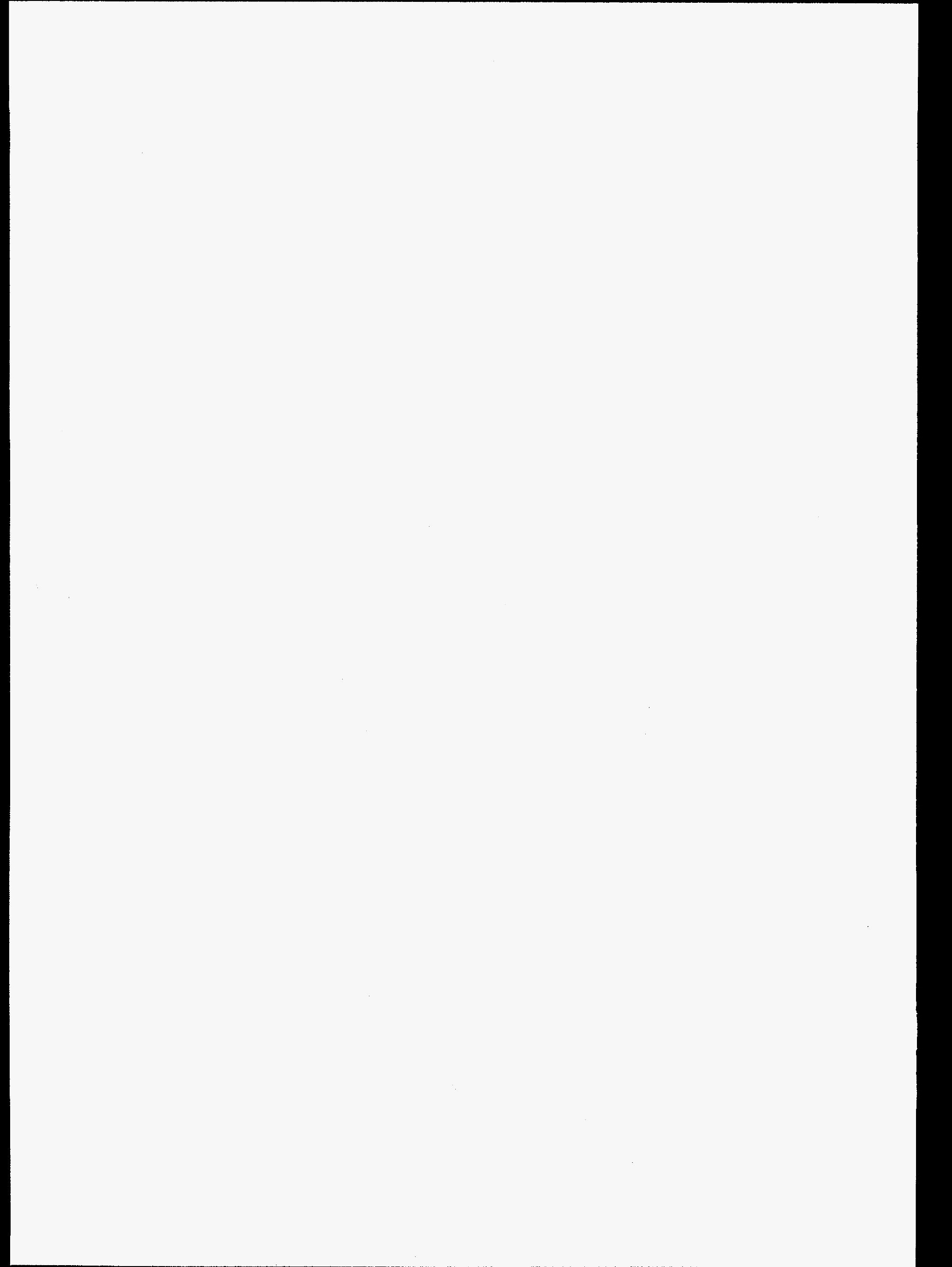


EM Problem: Decontamination and decommissioning; remedial action; waste management

Y-12 Plant Problem: All remediation tasks requiring sensor sweeps for characterization. Building 9201-4 (Alpha-4)

Problem Area/Constituents: Support of characterization and decommissioning activities

Reference Requirements: Refer to the Regulatory Compliance chapter of Vol. 1 for potentially applicable proposed and promulgated environmental laws, signed and pending agreements for the Oak Ridge Reservation, radiation protection standards, DOE orders, and nonregulatory guidance. As site- and waste-specific characteristics are provided for each technology, specific regulatory requirements will be specified.

Subelement: Robotics/automation

Alternatives: Other technologies

Technology: Characterization sensor integration

Status: Demonstration

Science/Technology Needs: Numerous sensors systems for remote measurements have been demonstrated at the laboratory stage. Examples of these systems range from the fiberoptic-based devices for direct measurements of trace constituents in monitoring wells to inline photometers for process control. Integration of this technology needs to be accomplished to allow use of these devices with robotic systems. Specific needs include signal conditioning at the end of the robotic end effector (e.g., high-impedance amplifiers for $\mathrm{pH}$ measurements), signal transmission to the operator console (tethered, low-voltage power cables and fiber-optic cables), and transfer of the relevant information to the appropriate work-site data base.

Implementation Needs: Testing of the system is necessary before putting it into use. Total development costs are estimated to be \$1M.

Author: L. N. Klatt/615-574-6588

References: MMES staff members, personal experience, and personal communication 


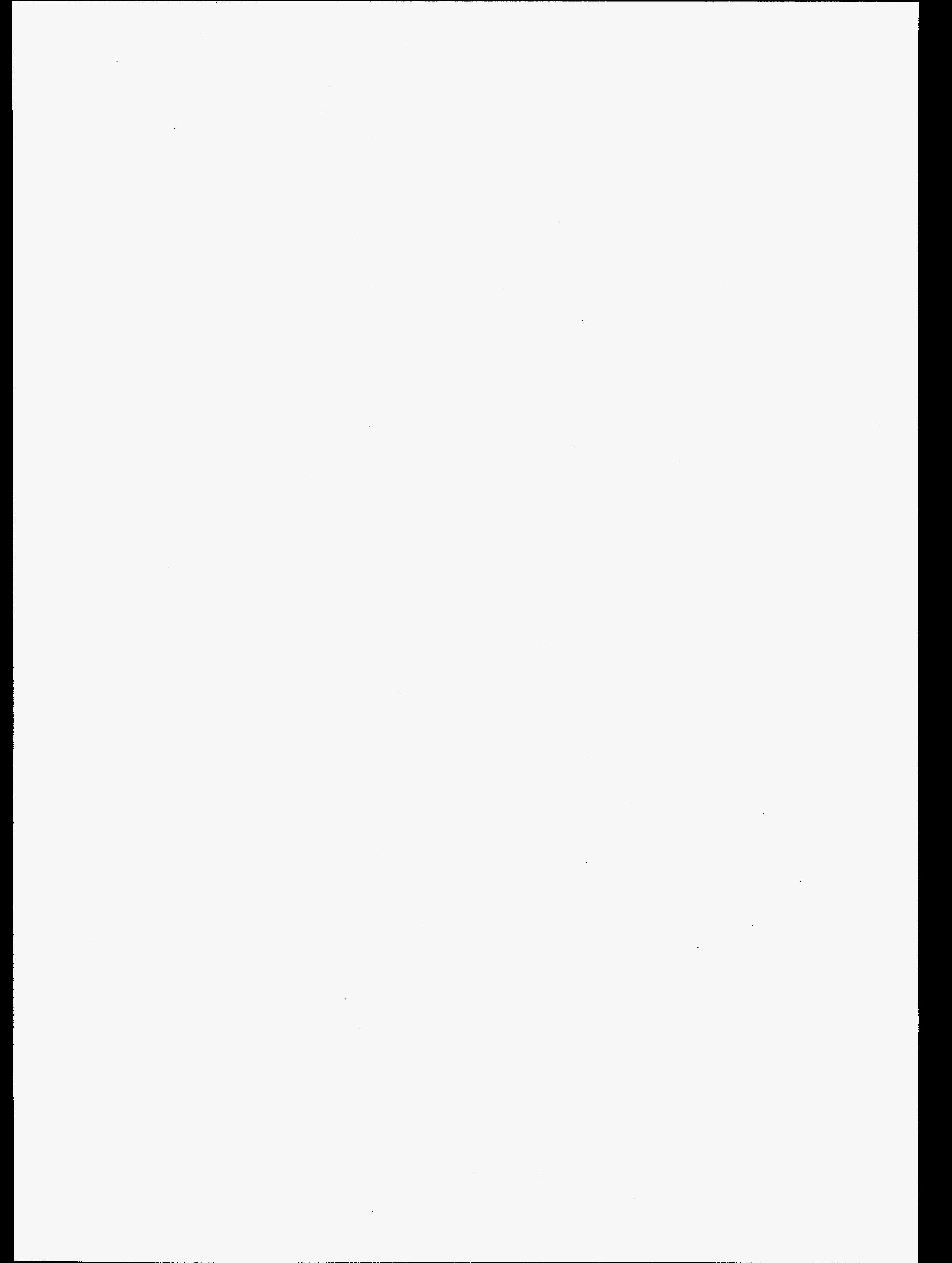


EM Problem: Decontamination and decommissioning; remedial action; waste management

Y-12 Plant Problem: Sample preparation for characterization for D\&D, RA, and WM. Building 9201-4 (Alpha-4)

Problem Area/Constituents: Support of characterization during remediation activities

Reference Requirements: Various U.S. Environmental Protection Agency (EPA) regulations define acceptable sample preparation techniques. Refer to the Regulatory Compliance chapter of Vol. 1 for potentially applicable proposed and promulgated environmental laws, signed and pending agreements for the Oak Ridge Reservation, radiation protection standards, DOE orders, and nonregulatory guidance. As site- and waste-specific characteristics are provided for each technology, specific regulatory requirements will be specified.

Subelement: Robotics/automation

Alternatives: Other technologies

Technology: Sample preparation. Characterization laboratories require samples to be prepared by standard techniques. Samples may have to be blended, reduced in size, etc.

\section{Status: Evolving}

Science/Technology Needs: Robotic and/or automatic systems to prepare a retrieved specimen for submission to the laboratory. Devices are required to cut small samples from large specimens (segments of pipe, duct work, building structural materials, etc.), blend sludges and slurries into a composite sample, section core specimens, and transfer small portions of liquids to sample vials. Modification and adaptation of current systems to remote operations are required.

Implementation Needs: Extensive testing of the automated systems will be required to ensure compliance with EPA regulations. Total development costs are estimated to be $\$ 1 \mathrm{M}$.

Author: L. N. Klatt/615-574-6588

References: MMES staff members, personal experience, and personal communication 

WASTE MANAGEMENT 


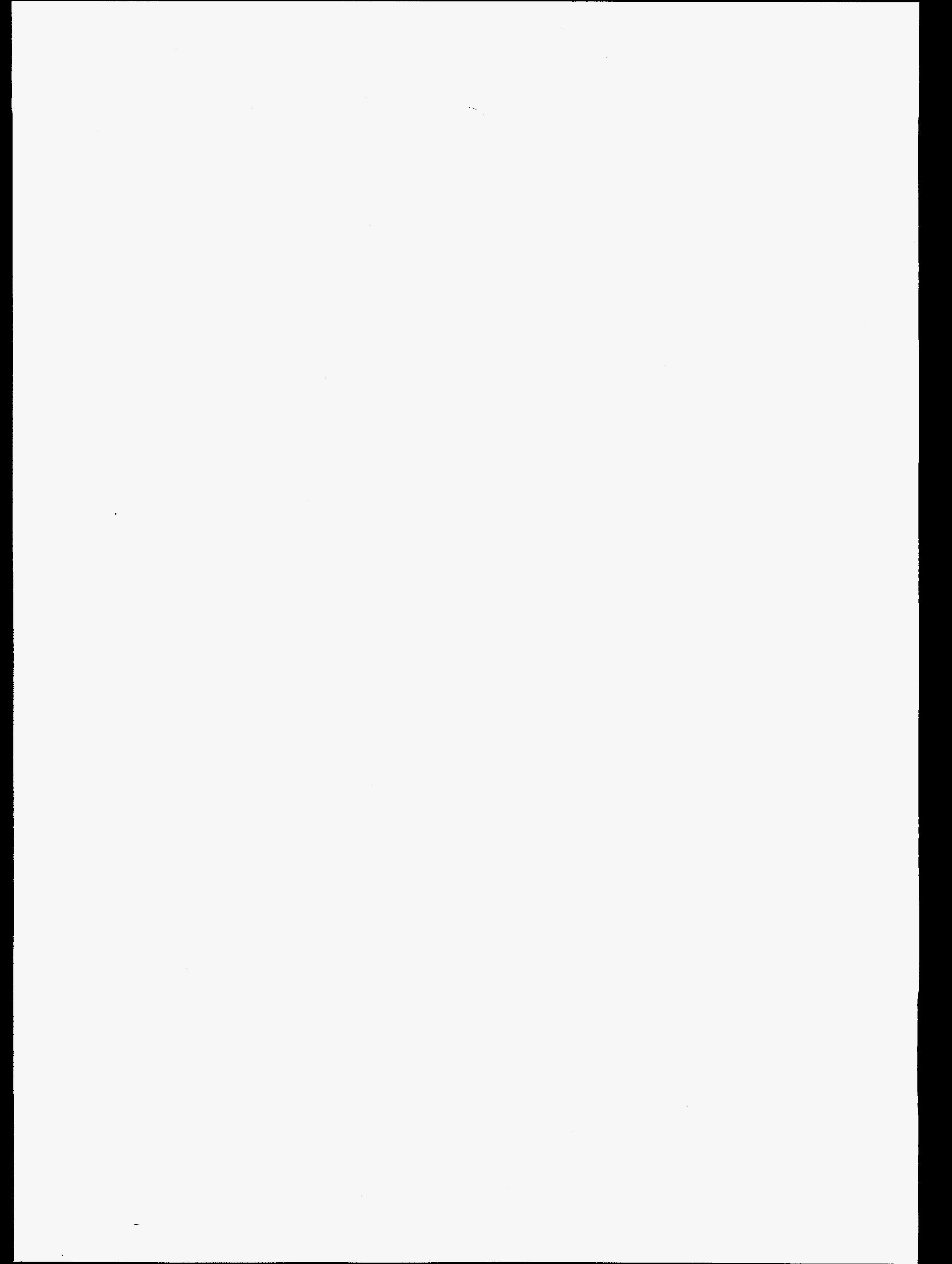


EM Problem: Waste management

Y-12 Plant Problem: Building 9201-4 (Alpha-4). Disposal of final residues from decontamination and decommissioning, environmental restoration, and waste management operations

Problem Area/Constituents: Permanent disposal of uranium, low-level, mixed, and special case wastes in a matrix of boreholes in clay-rich rocks.

\section{Reference Requirements:}

- Resource Conservation and Recovery Act of 1976, as amended (RCRA) Land Disposal Restriction (LDR) Federal Facilities Compliance Agreement (FFCA) dated 12 June 1992.

- Federal Facilities Agreement (FFA) for Oak Ridge Reservation.

- Clean Air Act regulations under 40 CFR 60-65.

- Clean Water Act regulations under 40 CFR 110-146 and 400-471.

- DOE orders.

Refer to the Regulatory Compliance Chapter of Vol. 1 applicable proposed and promulgated environmental laws, signed and pending agreements for the Oak Ridge Reservation, radiation protection standards, DOE orders, and nonregulatory guidance. As site- and waste-specific characteristics are provided for each technology, specific regulatory requirements will be specified.

Subelement: Waste disposal

Technology: Borehole disposal. The concept of disposal in a matrix of boreholes in clay-rich rocks is a relatively inexpensive method for the permanent disposition of uranium, low-level, mixed, and special case wastes. Its unique features are its complete flexibility and modular nature in operational conditions. A limited area is used at a time, and a complete borehole site can be rapidly returned to normal land use. Disposal depths may vary from a few hundred meters to $1000 \mathrm{~m}$. For the purpose of preliminary demonstration design, the borehole would be assumed to be $0.52 \mathrm{~m}$ in diameter and extending vertically to a depth of about $250 \mathrm{~m}$. The depth from the surface to the disposal zone would be about $80 \mathrm{~m}$. Because the lateral homogeneity of host material cannot be assumed for more than a few thousand meters, the general repository design practice would be to minimize lateral extent to not more than $1000 \mathrm{~m}$ in any direction. The bore holes would be laid out in hexagonal configuration.

Status: Predemonstration.

Science/Technology Needs: The concept is practicable by efficient use of existing experience from civil and mining engineering technology. However, the satisfactory application of the technology for mixed wastes disposal needs to be demonstrated. 
Although the complete evaluation of clay-rich rocks for disposal of radioactive and hazardous wastes includes consideration of the hydrologic, geologic, rock mechanical, geochemical, and thermal factors that would affect the construction, operation, and performance of these rocks, the findings related to their hydrologic elements are believed to be of overriding importance in this work. Because excessive groundwater inflow into the borehole workings is probably going to be the most important consideration that would lead to the need for nonroutine engineering measures, this groundwater inflow would likely be selected as the principal criterion for the hydrologic evaluation of prospective borehole disposal sites.

Implementation Needs: Development and demonstration of the use of the technology to dispose of radioactive mixed-wastes are estimated to require $\$ 2 M-\$ 3 M$ (1992 dollars). However, the payback could be significant when compared to the cost of alternate permanent disposal options.

Author: T. F. Lomenick/615-576-2583

\section{References:}

1. T. F. Lomenick, "The Development of Technologies for the Long-Term Containment of Low-Level Radioactive and Hazardous Wastes Into Geologic Formations," Chapter 15 in Effective and Safe Waste Management, R. L. Jolley and R. G. M. Wang, eds., Lewis Publishers, Boca Raton, Florida, 1993. 
EM Problem: Waste management

Y-12 Plant Problem: Building 9201-4 (Alpha-4). Disposal of final residues from decontamination and decommissioning, remedial action, and waste management operations

Problem Area/Target Constituents: Low level solid radioactive waste (uranium contamination).

Reference Requirements: Refer to Vol. 1, Chap. 8, for potentially applicable proposed and promulgated environmental laws, signed and pending agreements for the Oak Ridge Reservation, radiation protection standards, DOE orders, and nonregulatory guidance. As site- and waste-specific characteristics are provided for each technology, regulatory requirements will be specified.

Subelement: Waste disposal

Alternative: Low level waste disposal under DOE administration off Oak Ridge Reservation

Technology: Nevada Test Site. ${ }^{1}$ This site has been earmarked for the disposal of DOE lowlevel radioactive wastes.

Status: Accepted for some U.S. sites but disposal process needs to be demonstrated for Oak Ridge wastes. DOE Field Office, Nevada specifies that only waste containing radioactive constituents is accepted from off-site generators. Currently accepting low level waste from 6-8 off-site generators (not including Oak Ridge). Waste disposal conducted under an interim permit from the state of Nevada. Mixed waste is currently being accepted from only Rocky Flats. A draft Performance Assessment and Environmental Assessment were issued September, 1992. These made waste characterization criteria much more stringent than previously. Waste transportation to NTS is by highway only.

\section{Science/Technology Needs: Unknown}

Implementation Needs: Implementation needs for disposing the wastes include obtaining regulatory approval for the disposal of the specific low-level radioactive wastes at the site.

Disposal Costs: $\$ 10 / \mathrm{ft}^{3}$ but does not include programmatic waste management costs or packaging/handling/transportation costs. Constraints posed by waste certification and waste acceptance may make costs prohibitive for shipments from Oak Ridge Reservation.

Author: S. P. N. Singh/615-574-6639 and T. L. Sams/615-241-2409 


\section{References:}

1. T. L. Sams, R. C. Jones, R. J. Sams, Evaluation of Potential Disposal Sites for Environmental Restoration Waste Streams, Report ES/ER/TM-109, March 1994.

2. NVO-325 Awareness Training, DOE/NV Defense Waste Generator's Workshop, Las Vegas, Nevada, September, 1993. Revision 0 
EM Problem: Waste management

Y-12 Plant Problem: Building 9201-4 (Alpha-4). Disposal of final residues from decontamination and decommissioning, remedial action, and waste management operations

Problem Area/Target Constituents: Low level solid radioactive waste (uranium contamination).

Reference Requirements: Refer to Vol. 1, Chap. 8, for potentially applicable proposed and promulgated environmental laws, signed and pending agreements for the Oak Ridge Reservation, radiation protection standards, DOE orders, and nonregulatory guidance. As site- and waste-specific characteristics are provided for each technology, regulatory requirements will be specified.

Subelement: Waste disposal

Alternative: Low level waste, Mixed waste disposal at commercial, off-site facility.

Technology: Envirocare, Utah Landfill. ${ }^{1-2}$ The low-level mixed waste residues are permanently disposed at Envirocare's landfill near Clive, Utah. This landfill accepts low-level wastes, and mixed waste for disposal that meet its waste acceptance criteria (see Implementation Needs below).

Status: Accepted. The site is currently available for disposal of Oak Ridge wastes. 5822A Exemption Request for disposal of Oak Ridge stabilized sludge approved by DOE on October 13, 1993. Environment assessment final approval pending on Oak Ridge Reservation packaging, handling and transportation plan to Clive. Transportation contract in place. Waste shipments anticipated 1994. State of Utah has been an agreement state with the NRC since 1984. The Division of Radiation Control, and the Division of Solid and Hazardous Waste serve as the regulatory agencies that monitor the Envirocare facility. Envirocare has a rail spur and a railcar inversion unloading facility.

Science/Technology Needs: None

Implementation Needs: Implementation needs for disposing the wastes include obtaining regulatory approval for the disposal of the specific wastes at the site, meeting the site's WAC, treatment to RCRA LDR standards, and funding.

Expected disposal Costs: $\$ 30-\$ 50 / \mathrm{ft}^{3}$ 
Waste Acceptance Criteria: list of acceptable isotopes and concentrations.

\section{Radioactive Material}

Silver-110m

Americium-241

Americium-243

Beryllium-7

Calcium-45

Cadmium-109

Cobalt-56

Cobalt-57

Cobalt-58

Cobalt-60

Chromium-51

Cesium-134

Cesium-137

Europium-152

Europium-154

Iron-55

Mercury-203

Potassium-40

Iridium-192

Manganese-54

Niobium-94

Nickel-59

Nickel-63

Lead-210

Polonium-210

Radium-226

Radium-228

Radium-228 (1 year)

Radium-228 (5 years)

Radium-228 (10 years)

Ruthenium-106

Antimony-124

Antimony-125

Tin-113

\section{Concentration}

$5.6 \mathrm{E}+02 \mathrm{pCi} / \mathrm{g}$

2.3E+02 Pci/g

$1.7 \mathrm{E}+03 \mathrm{pCi} / \mathrm{g}$

$3.8 \mathrm{E}+04 \mathrm{pCi} / \mathrm{g}$

$4.0 \mathrm{E}+08 \mathrm{pCi} / \mathrm{g}$

4.6E+04 $\mathrm{pCi} / \mathrm{g}$

$3.6 \mathrm{E}+02 \mathrm{pCi} / \mathrm{g}$

$1.9 \mathrm{E}+04 \mathrm{pCi} / \mathrm{g}$

$1.6 \mathrm{E}+02 \mathrm{pCi} / \mathrm{g}$

$3.6 \mathrm{E}+02 \mathrm{pCi} / \mathrm{g}$

$6.8 \mathrm{E}+04 \mathrm{pCi} / \mathrm{g}$

1.2E $+03 \mathrm{pCi} / \mathrm{g}$

$5.6 \mathrm{E}+02 \mathrm{pCi} / \mathrm{g}$

$1.7 \mathrm{E}+03 \mathrm{pCi} / \mathrm{g}$

$1.4 \mathrm{E}+03 \mathrm{pCi} / \mathrm{g}$

$1.8 \mathrm{E}+06 \mathrm{pCi} / \mathrm{g}$

$1.0 \mathrm{E}+04 \mathrm{pCl} / \mathrm{g}$

1.0E $+04 \mathrm{pCi} / \mathrm{g}$

2.5E+03 pCi/g

$5.6 \mathrm{E}+03 \mathrm{pCi} / \mathrm{g}$

$1.6 \mathrm{E}+02 \mathrm{pCl} / \mathrm{g}$

$7.0 \mathrm{E}+02 \mathrm{pCl} / \mathrm{g}$

2.0E+06 pCi/g

2.3E+05 pCi/g*

2.0E+04 pCi/g

$20 \mathrm{E}+03 \mathrm{pCi} / \mathrm{g}^{*}$

$1.8 \mathrm{E}+03 \mathrm{pCi} / \mathrm{g}$

$1.2 \mathrm{E}+03 \mathrm{pCi} / \mathrm{g}^{*}$

6.7E+02 pCi/g*

$5.6 \mathrm{E}+02 \mathrm{pCi} / \mathrm{g}^{*}$

$1.9 \mathrm{E}+04 \mathrm{pCi} / \mathrm{g}^{*}$

$7.9 \mathrm{E}+02 \mathrm{pCi} / \mathrm{g}$

$5.3 \mathrm{E}+03 \mathrm{pCl} / \mathrm{g}$

7.3E+05 $\mathrm{pCi} / \mathrm{g}$
Radioactive Material

Strontium-90

Thorium-230

Thorium-232

Uranium-234

Uranium-235

Uranium-236

Uranium-238

Uranium-natural

Uranium-depleted

Zinc-65

+Curium-242

+Curium-242*

+Curium-243

+Curium-243*

+Curium-244

+Curium-244*

+Neptunium-237

+Plutonium-238

+Plutonium-238*

+Plutonium-239

+ Plutonium-240

+Plutonium-241

+Plutonium-241*

+Plutonium-242

+Carbon-14

+Hydrogen-3

+lodine-129

+ Sodium-22

+Technicium-99

\section{Concentration}

2.0E+04 $\mathrm{pCi} / \mathrm{g}$

$1.5 \mathrm{E}+04 \mathrm{pCl} / \mathrm{g}$

$6.8 \mathrm{E}+02 \mathrm{pCi} / \mathrm{g}^{*}$

3.7E $+04 \mathrm{pCi} / \mathrm{g}$

7.7E+02 $\mathrm{pCl} / \mathrm{g}$

$3.6+\mathrm{E} 04 \mathrm{pCi} / \mathrm{g}$

$2.8 \mathrm{E}+04 \mathrm{pCi} / \mathrm{g}$

$1.8 \mathrm{E}+04 \mathrm{pCi} / \mathrm{g}$

1.1E+05 $\mathrm{pCi} / \mathrm{g}$

$1.1 \mathrm{E}+04 \mathrm{pCi} / \mathrm{g}$

1.4E+06 $\mathrm{pCl} / \mathrm{g}$

$8.1 \mathrm{E}+03 \mathrm{pCi} / \mathrm{g}$

$1.5 \mathrm{E}+03 \mathrm{pCi} / \mathrm{g}$

1.3E+05 $\mathrm{pCl} / \mathrm{g}$

1.0E+04 $\mathrm{pCi} / \mathrm{g}$

7.4E+03 $\mathrm{pCi} / \mathrm{g}$

$2.0 \mathrm{E}+03 \mathrm{pCi} / \mathrm{g}$

$1.0 \mathrm{E}+04 \mathrm{pCi} / \mathrm{g}$

$8.2 \mathrm{E}+03 \mathrm{pCi} / \mathrm{g}$

$9.9 \mathrm{E}+03 \mathrm{pCi} / \mathrm{g}$

1.0E+04 $\mathrm{pCl} / \mathrm{g}$

$3.5 \mathrm{E}+05 \mathrm{pCl} / \mathrm{g}$

$1.1 \mathrm{E}+03 \mathrm{pCl} / \mathrm{g}$

1.0E+04 $\mathrm{pCi} / \mathrm{g}$

$5.0 \mathrm{E}+05 \mathrm{pCi} / \mathrm{g}$

$2.5 \mathrm{E}+07 \mathrm{pCl} / \mathrm{g}$

$3.1 \mathrm{E}+03 \mathrm{pCi} / \mathrm{g}$

$7.8 \mathrm{E}+02 \mathrm{pCi} / \mathrm{g}$

$1.9 \mathrm{E}+05 \mathrm{pCi} / \mathrm{g}$

+ Isotopes to be accepted in the future.

* Daughters are assumed to be present at same concentrations in equilibrium

September 1994

Decontamination and Decommissioning 
Author: S. P. N. Singh/615-574-6639, T. L. Sams/615-241-2409, R. L. Fellows/615-5765632

\section{References:}

1. T. L. Sams and R. C. Jones, Evaluation of Potential Disposal Sites for Environmental Restoration Waste Streams, to be published at the Oak Ridge K-25 Site.

2. T. L. Sams, R. C. Jones, and J. T. Grumski, Evaluation of Available Mixed-Waste Disposal Sites, ES/ER/TM-45 (Draft), Martin Marietta Energy Systems, Inc., Oak Ridge K-25 Site, August 1992. 


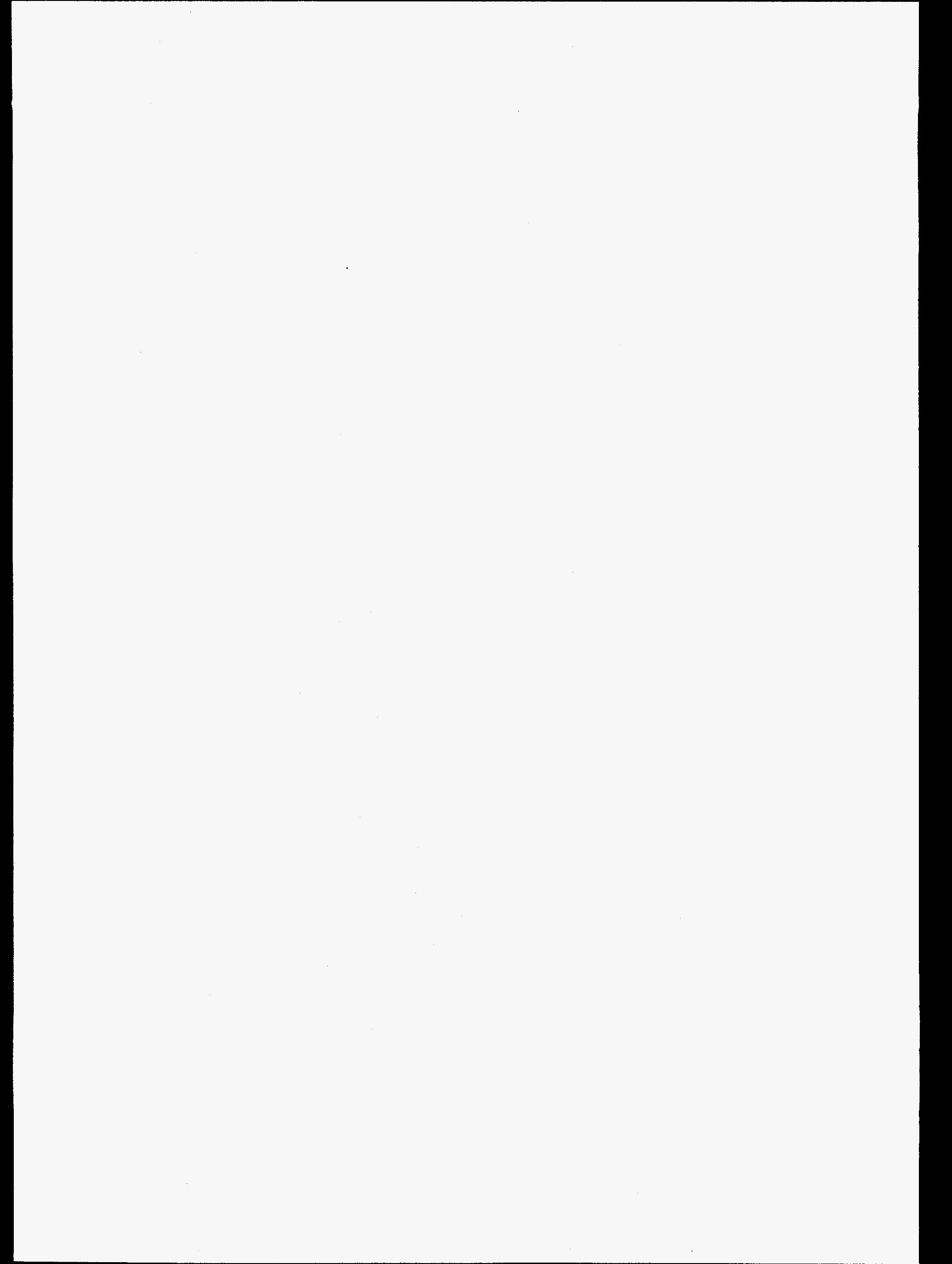


EM Problem: Waste management

Y-12 Plant Problem: Building 9201-4 (Alpha-4). Disposal of low-level radioactive waste

Problem Area/Target Constituents: Permanent disposal of low-level radioactive waste from decontamination and decommissioning, remedial action, and current waste management operations that meets the waste disposal site's waste acceptance criteria (WAC)

\section{Reference Requirements:}

- Resource Conservation and Recovery Act of 1976, as amended (RCRA) Land Disposal Restriction (LDR) Federal Facilities Compliance Agreement (FFCA) dated 12 June 1992

- Federal Facilities Agreement (FFA) for Oak Ridge Reservation

- Clean Air Act regulations under 40 CFR 60-65

- Clean Water Act regulations under 40 CFR 110-146 and 400-471

- DOE orders.

Refer to the Regulatory Compliance Chapter of Vol. 1 applicable proposed and promulgated environmental laws, signed and pending agreements for the Oak Ridge Reservation, radiation protection standards, DOE orders, and nonregulatory guidance. As site- and waste-specific characteristics are provided for each technology, specific regulatory requirements will be specified.

Subelement: Waste disposal

Alternative: Landfill

Technology: Oak Ridge Reservation (ORR) class L-I low-level-waste landfill (disposal facility).$^{1.2}$ This is a trench-type disposal facility being developed for the disposal of ORR Class L-I solid low-level wastes containing long half-life radionuclides such as uranium and technetium. The facility will have a proposed waste disposal capacity of 1.6 million cubic feet and an expected useful life of $\mathbf{3 0}$ to $\mathbf{4 0}$ years. The facility is currently undergoing engineering development. This facility is being developed in case the ORR low-level wastes cannot be disposed at an acceptable out-of-state facility.

Status: Predemonstration-This facility is currently being developed and designed. Additional details such as the site's WAC could be provided at a later date after they are finalized.

Science/Technology Needs: Additional research, development, and demonstration (RD\&D) still needs to be conducted to determine the environmental acceptability of this disposal option for the low-level radioactive wastes. 
Implementation Needs: The ORR Class L-I disposal facility is projected to be ready in early 1999. The capital cost for the facility is estimated to be \$90M (1992 dollars) and the annual operating costs are estimated to be around $\$ 8 \mathrm{M}$ (1992 dollars).

Implementation needs for disposing the wastes (after the facility is ready to accept wastes) include obtaining regulatory approval for the disposal of the specific low-level radioactive wastes at the site and funding.

Funding would be required for performing waste analyses (to ensure compliance with the site WAC) and waste packaging, handling, and transportation (WPH\&T) to the site. Estimated funding requirements cannot be forecast because they are dependent upon several factors such as waste volumes, tipping fees, analytical requirements, and WPH\&T costs.

Author: S.P.N. Singh/615-574-6639

\section{References:}

1. Systems Requirements Document for Low-Level Waste Disposal Facilities, X-OE-612, July 1992.

2. Conceptual Design Report for Low-Level Waste Disposal Facilities, K/D-5832-R1, Martin Marietta Energy Systems, Inc., Oak Ridge K-25 Site, July 1992. 


\section{EM Problem: Waste management}

Y-12 Plant Problem: Building 9201-4 (Alpha-4). Disposal of final residues from decontamination and decommissioning.

Problem Area/Target Constituents: Low level radioactive waste (uranium contamination).

Reference Requirements: Refer to Vol. 1, Chap. 8, for potentially applicable proposed and promulgated environmental laws, signed and pending agreements for the Oak Ridge Reservation, radiation protection standards, DOE orders, and nonregulatory guidance. As site- and waste-specific characteristics are provided for each technology, regulatory requirements will be specified.

Subelement: Waste disposal

Alternative: Low level waste, mixed waste at DOE-administered site

Technology: Hanford Disposal Site, Washington

Status: Accepted. In use. DOE Field Office, Richland specifies only wastes containing radioactive constituents or radioactive wastes mixed with hazardous constituents are accepted from off-site generators for disposal. The Hanford Site is currently accepting mixed and $L L W$ from 8-10 off-site generators (Oak Ridge is not included). Waste disposal operations are being conducted with an interim permit from the state of Washington. A draft Performance Assessment has been issued and partially implemented with the final version expected in 1994. Waste transportation to Hanford is by highway only.

Science/Technology Needs: Unknown

\section{Implementation Needs:}

Disposal Costs:

LLW $\$ 180-\$ 220 / \mathrm{ft}^{3}$

Mixed Waste- $\$ 180-\$ 220 / \mathrm{ft}^{3}$ and Hanford will only accept mixed waste provided the generator sends LLW to Hanford as well.

Waste Acceptance Criteria (WAC):

Mixed Waste - Radioactive and Hazardous Constituents

- Radioactive constituent must generally be Class L-2 or less

- Alpha-emitting transuranics with half-lives greater than 5 years and concentrations less than $100 \mathrm{nCi} / \mathrm{g}$ are acceptable

- Concentrations of hazardous constituents must not exceed those allowed in 40 CFR-268.

- Classified waste must have been treated to remove the classified characteristics.

- Mixed waste must not contain any rad constituents that are classified as greater than Class L-1 rad material per the NRC's classification system. 
Mixed Waste - Waste forms

- All waste must be in stable forms such as: cemented, compacted etc. The following special considerations apply:

- No free liquid wastes accepted

- Asbestos may be accepted

- Alkali metals subject to special restrictions

- Long-lived rad waste (including $\mathrm{C}^{14}, \mathrm{Se}^{79}, \mathrm{Tc}^{99}, \mathrm{I}^{129}$ ) accepted on a case-by-case basis

- No explosives or pyrophorics

- Mercury accepted with special restrictions

- PCB-contaminated waste accepted on case-by-case basis.

Mixed Waste - Waste Packaging

- Transported waste must be in containers which at least meet DOT standards

- Waste must be contained by two barriers to prevent release

Authors: T. L. Sams/615-241-2409

\section{References:}

1. T. L. Sams, R. C. Jones, R. J. Sams, Evaluation of Potential Disposal Sites for Enwironmental Restoration Waste Streams, Report ES/ER/TM-109, March 1994. 
EM Problem: Waste management

Y-12 Plant Problem: Building 9201-4 (Alpha-4). Disposal of final residues from decontamination and decommissioning.

Problem Area/Target Constituents: Low level radioactive waste (uranium contamination).

Reference Requirements: Refer to Vol. 1, Chap. 8, for potentially applicable proposed and promulgated environmental laws, signed and pending agreements for the Oak Ridge Reservation, radiation protection standards, DOE orders, and nonregulatory guidance. As site- and waste-specific characteristics are provided for each technology, regulatory requirements will be specified.

Subelement: Waste disposal

Alternative: Low level waste disposal on Oak Ridge Reservation

Technology: Interim Waste Management Facility Tumulus. This is a solid storage area in SWSA 6 (Solid Waste Storage Area 6) at X-10.

Status: Accepted. In use but closing is scheduled by December, 1993. SWSA 6 comprises 68 acres of which 15 are used for waste disposal. Since 1986 only LLW has been handled with waste being handled in silos and tumuli. Approximate capacity remaining is 1000 55gal drums. Presently certified for a small quantity of sludge stabilized at X-10. Minimum of two years expected for certification of a new waste stream.

Science/Technology Needs: None

Implementation Needs: Closing is scheduled for December, 1993, and the capacity is much too small, precluding the use of this interim storage area.

Authors: T. L. Sams/615-241-2409

References:

1. T. L. Sams, R. C. Jones, R. J. Sams, Evaluation of Potential Disposal Sites for Environmental Restoration Waste Streams, Report ES/ER/TM-109, March 1994. 


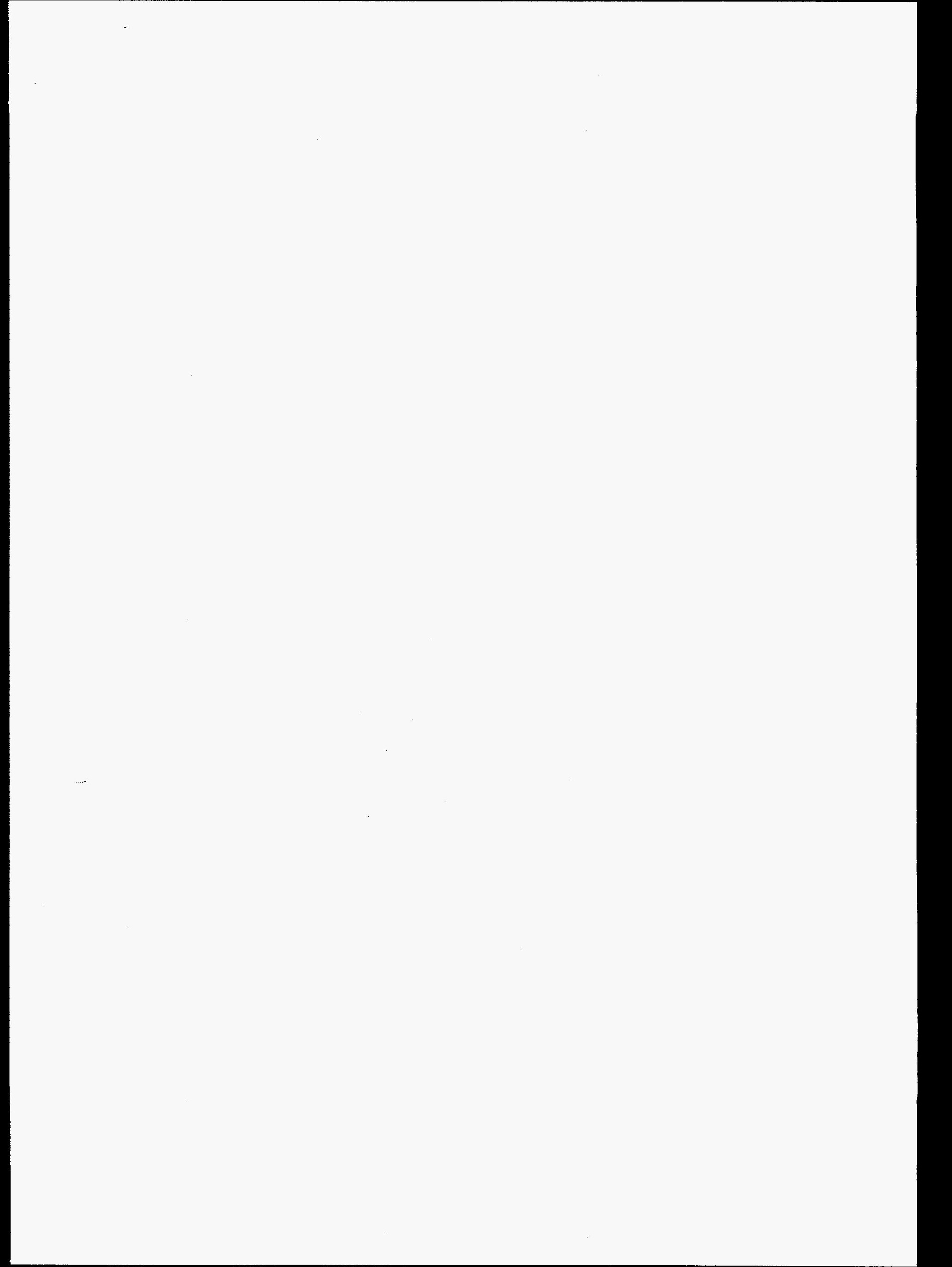


EM Problem: Waste management

Y-12 Plant Problem: Building 9201-4 (Alpha-4). Disposal of final residues from decontamination and decommissioning.

Problem Area/Target Constituents: Low level radioactive waste (uranium contamination).

Reference Requirements: Refer to Vol. 1, Chap. 8, for potentially applicable proposed and promulgated environmental laws, signed and pending agreements for the Oak Ridge Reservation, radiation protection standards, DOE orders, and nonregulatory guidance. As site- and waste-specific characteristics are provided for each technology, regulatory requirements will be specified.

Subelement: Waste disposal

Alternative: Low level waste, commercial, off Oak Ridge Reservation

Technology: Chem-Nuclear, Barnwell, SC. Low level waste disposal.

Status: Accepted. In operation. All waste materials reviewed based upon South Carolina Radioactive Materials License \#097 and the Barnwell Waste Management Facility Site Disposal Criteria. The South Carolina Department of Health and Environmental Control monitors the site. Waste transportation to Barnwell is by highway only.

Science/Technology Needs: Unknown

Implementation Needs:

Disposal Costs: Not Available

Waste Acceptance Criteria (WAC):

Radioactive Materials:

- Special Nuclear Material, $\mathrm{U}^{233}$, is accepted in amounts less than $200 \mathrm{~g} /$ package. $\mathrm{U}^{235}$ is accepted in amounts less than $350 \mathrm{~g} /$ package.

- $\mathrm{Pu}^{241}$ and $\mathrm{Cm}^{242}$ and alpha-emitting transuranics with half-lives less than 5 years accepted. See license for amounts.

- Naturally occurring Radioactive Materials (NORM) accepted subject to concentration limits listed in license.

Waste Form Requirements:

- No liquid waste

- Waste containing oil may be accepted providing it is less than $1 \%$ of total waste volume in container.

- No pyrophoric or flammable solids

- Wastes containing hazardous materials (mixed waste) accepted providing the radioactive hazard clearly exceeds the toxic hazard

- Waste containing greater than $8 \%$ (weight) chelating agents not accepted 
- PCB-containing waste not accepted

- Asbestos may be accepted

- Animal carcasses accepted

Waste Packaging

- Waste must be containerized

- Waste containers must have a High Integrity Container Certification from the South Carolina Department of Health and Environmental Control

- Waste must be packaged according to Barnwell-specific criteria

Authors: T. L. Sams/615-241-2409, R. L. Fellows/615-576-5632

References:

1. T. L. Sams, R. C. Jones, R. J. Sams, Evaluation of Potential Disposal Sites for Environmental Restoration Waste Streams, Report ES/ER/TM-109, March 1994. 
EM Problem: Waste management

Y-12 Plant Problem: Building 9201-4 (Alpha-4). Disposal of final residues from decontamination and decommissioning.

Problem Area/Target Constituents: Low level solid radioactive waste (uranium contamination).

Reference Requirements: Refer to Vol. 1, Chap. 8, for potentially applicable proposed and promulgated environmental laws, signed and pending agreements for the Oak Ridge Reservation, radiation protection standards, DOE orders, and nonregulatory guidance. As site- and waste-specific characteristics are provided for each technology, regulatory requirements will be specified.

Subelement: Waste disposal

Alternative: Low level waste, commercial, off Oak Ridge Reservation

Technology: U.S. Ecology, Hanford, WA. Low level waste disposal facility is located on the Hanford Nuclear Reservation on land leased to the State of Washington by DOE.

Status: Accepted. In operation. More than 8 million $\mathrm{ft}^{3}$ of commercial LLW have been disposed since 1965. The state of Washington collects fees to cover the costs of environmental monitoring and facility maintenance and closure after it ceases operations. The facility is monitored by the Washington Department of Health. Waste transportation is by highway only.

\section{Science/Technology Needs: Unknown}

\section{Implementation Needs:}

Disposal Costs: Not Available

Waste Acceptance Criteria

Radioactive Materials:

- Special Nuclear Material, ${ }^{233} \mathrm{U}$, is accepted in amounts less than $60 \mathrm{~g} / \mathrm{package} .{ }^{235} \mathrm{U}$ is accepted in amounts less than $100 \mathrm{~g} /$ package, $\mathrm{Pu}$ is accepted in amounts less than $600 \mathrm{~g} /$ package.

- ${ }^{241} \mathrm{Pu},{ }^{226} \mathrm{Ra}$ and ${ }^{242} \mathrm{Cm}$ and alpha-emitting transuranics with half-lives less than 5 years accepted. See license for amounts.

- Naturally occurring Radioactive Materials (NORM) accepted subject to concentration limits listed in license, pages 25 and 26.

Waste Form Requirements:

- No untreated liquid or sludge waste

- Solidified liquids may be accepted if an approved solidification medium or sorption medium is used 
- No scintillation liquids containing RCRA-regulated materials

- No pyrophoric or explosive solids

- Asbestos may be accepted

- Animal carcasses accepted

Waste Packaging

- Waste must be containerized according to DOT, NRC and state regulations

- Void space within the container must be reduced to the maximum extent possible

Authors: T. L. Sams/615-241-2409, R. L. Fellows/615-576-5632

References:

1. T. L. Sams, R. C. Jones, R. J. Sams, Evaluation of Potential Disposal Sites for Environmental Restoration Waste Streams, Report ES/ER/TM-109, March 1994. 
EM Problem: Waste management

Y-12 Plant Problem: Building 9201-4 (Alpha-4). Disposal of final residues from decontamination and decommissioning.

Problem Area/Target Constituents: Low level solid radioactive waste (uranium contamination).

Reference Requirements: Refer to Vol. 1, Chap. 8, for potentially applicable proposed and promulgated environmental laws, signed and pending agreements for the Oak Ridge Reservation, radiation protection standards, DOE orders, and nonregulatory guidance. As site- and waste-specific characteristics are provided for each technology, regulatory requirements will be specified.

Subelement: Waste disposal

Alternative: Mixed Waste, on Oak Ridge Reservation

Technology: “Oak Ridge Mixed Waste Disposal Unit”, a mixed waste disposal facility located on the Oak Ridge Reservation and administered by DOE.

Status: Conceptual. No design work or funding currently available.

Science/Technology Needs: Unknown

Implementation Needs: Unknown. No waste acceptance criteria developed

Authors: T. L. Sams/615-241-2409, R. L. Fellows/615-576-5632

\section{References:}

1. T. L. Sams, R. C. Jones, R. J. Sams, Evaluation of Potential Disposal Sites for Environmental Restoration Waste Streams, Report ES/ER/TM-109, March 1994. 


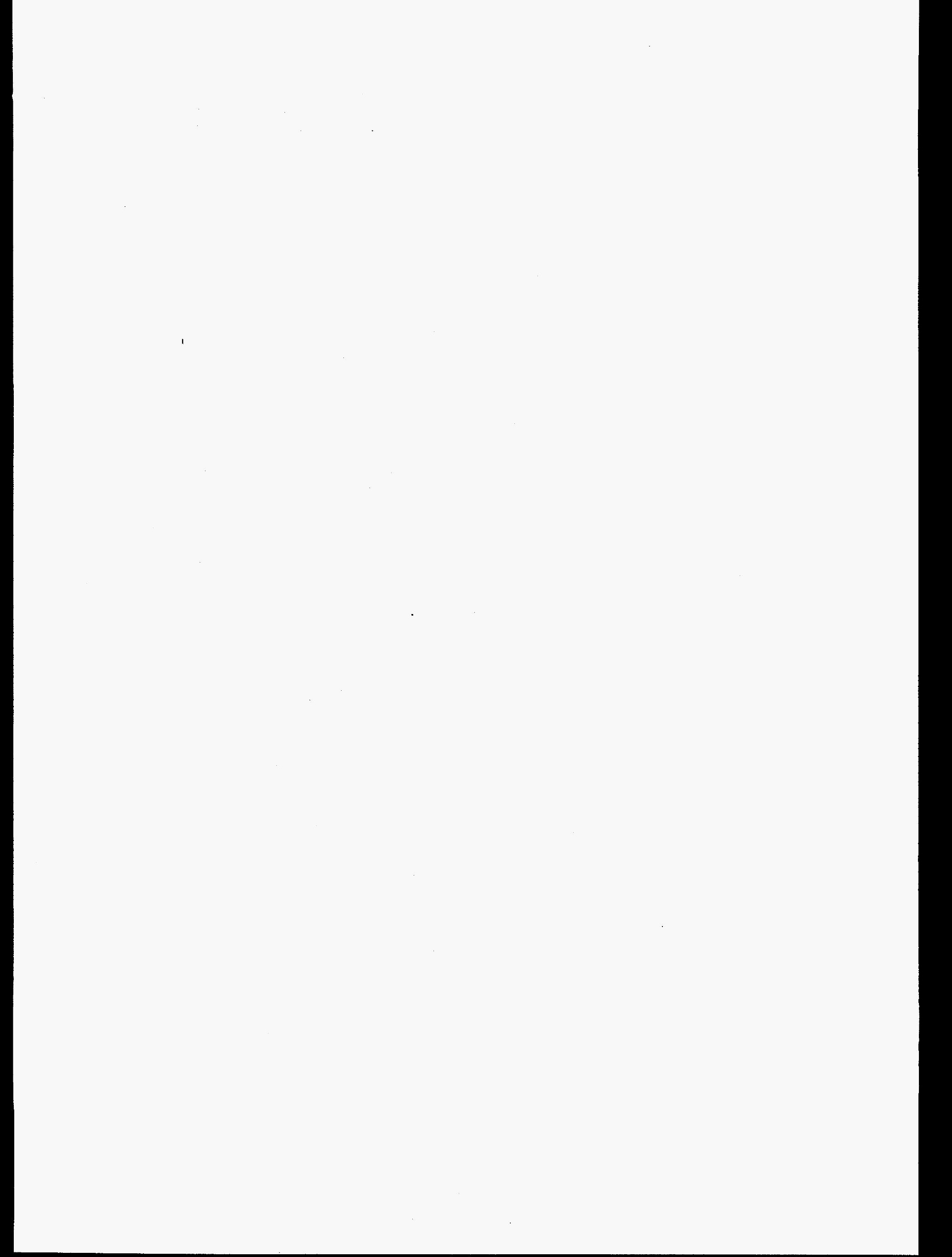


EM Problem: Waste management

Y-12 Plant Problem: Building 9201-4 (Alpha-4). Minimize the generation of hazardous and mixed wastes

ProblemArea/Constituents: Plantwide wastes from decontamination and decommissioning (D\&D), remedial action, and waste management operations at the site

\section{Reference Requirements:}

- Resource Conservation and Recovery Act of 1976 (RCRA), as amended, Land Disposal Restriction (LDR) Federal Facilities Compliance Agreement (FFCA) dated June 12, 1992

- Federal Facilities Agreement (FFA) for the Oak Ridge Reservation

- Federal Facilities Compliance Agreement-Toxic Substances Control Act (FFCA-TSCA) dated February 20, 1992

- Clean Air Act regulations under 40 CFR 60-65

- Clean Water Act regulations under 40 CFR 110-146 and 400-471

- DOE orders

Refer to the Regulatory Compliance Chapter of Vol. 1 applicable proposed and promulgated environmental laws, signed and pending agreements for the Oak Ridge Reservation, radiation protection standards, DOE orders, and nonregulatory guidance. As site- and waste-specific characteristics are provided for each technology, specific regulatory requirements will be specified.

Subelement: Waste minimization

\section{Alternatives:}

Technology: Waste Minimization Evaluation System (WMES). An ORNL-developed computerized process simulation model, WMES can be used as an effective waste minimization evaluation tool. Effective waste minimization and pollution prevention techniques should be used to minimize the toxicity and quantity of wastes and pollutants resulting from normal operations as well as D\&D activities at Bldg. 9201-4. ${ }^{\text {' }}$ Previous applications of the WMES model have yielded significant reduction of wastes and pollutants because of application of waste minimization techniques. This has, in turn, resulted in substantial savings in both operating and clean-up costs., ${ }^{2,3}$ The WMES can be customized to model any specific waste management operation. Briefly, the WMES integrates the following essential elements to develop waste minimization strategy for a given application:

- systems analysis,

- process modeling,

- waste minimization experiences,

- knowledge of avallable treatment options,

- regulatory requirements, and 
- alternative evaluation and selection processes.

Status: Demonstration

Science/Technology Needs: The generic methodology for the evaluation system has been developed along with a computerized prototype. The evaluation system is implemented using a modular approach. Several modules must be further developed to provide a full-scale application, including the waste minimization experience data base, the regulations data base, basic process models, the treatment selection process, and the data exchange mechanism. Customization for the particular application at the Alpha-4 site will require additional treatment and other process models, waste characterization data bases, and specific reporting features. When customized, the evaluation system will be used to perform waste minimization studies for specific problems associated with normal operations and/or D\&D activities at Bldg. 9201-4 to demonstrate the efficacy of the system.

Implementation Needs: Development of the evaluation system is expected to require a team effort over 2 years and funding of about $\$ 3 M-\$ 5 M$ (1992 dollars). Previous waste minimization experiences have shown that an appreciable reduction in waste production ${ }^{4.5}-$ ranging from $15 \%$ to $50 \%$ of volume generation-can be achieved. For example, the cost of D\&D for the K-25 Site has been estimated at about $\$ 7$ billion. ${ }^{6}$ Hence, the impact of an effective waste minimization effort could be expected to produce savings of about $\$ 1$ billion or more.

Author: J. J. Aerrada/615-574-4998

References:

1. Martin Marietta Energy Systems, Inc., Pollution Prevention, Draft Standard No. ESS-EP137, Rev. 0, October 1992.

2. U. S. EPA, Experience with the EPA Manual for Waste Minimization Opportunity Assessments, PB91-137133.

3. J. M. Hoegler, "Magnetic Separation of Uranium from Waste Materials," Waste Minimization Practice.

4. T. C. Keener, "The Application of a Mobile Solvent Recovery Process to Minimize Hazardous Waste," Waste Minimization Practice.

5. P. F. Mahoney and J. F. Muller, "Utilization of Ash Products from Combustion of Shredded Solid Waste," Waste Minimization Practice.

6. Ebasco Services, Inc., Environmental Restoration of the Gaseous Diffusion Plants, report prepared for the U. S. DOE under Contract No.: DE-AC05-910R21928, October 1991. 
EM Problem: Waste management

Y-12 Plant Problem: Building 9201-4 (Alpha-4). Waste packaging, handling, and transportation

Problem Area/Constituents: The specific needs for packaging, handling, and transportation of wastes (radioactive, other hazardous, mixed waste, etc.) have not been clearly defined for ORNL. The waste/material forms, quantities, locations of origination and destination, and time-period when packaging, handling, and transportation must occur need to be clearly specified. Then the availability of acceptable packagings; the need for package design, development, testing, and certification; and the physical interfacing of the package/ transportation system with the physical plant and materials can be defined.

\section{Reference Requirements:}

- Hazardous Materials Transportation Act (HMTA) regulations under 49 CFR 171-179

- Atomic Energy Act

- Packaging and Transportation of Radioactive Materials regulations under 10 CFR 71

- DOE Orders $1540,4700.1$, and 5480

- IAEA Safety Series No. 6 (1990)

- ICAO, LATA, and IMO codes and regulations

- Transportation and Traffic Management regulations under 41 CFR 109-40

- ANSI, ASME, ASTM standards

Refer to the Regulatory Compliance Chapter of Vol. 1 of Vol. 1 for potentially applicable proposed and promulgated environmental laws, signed and pending agreements for the Oak Ridge Reservation, radiation protection standards, DOE orders, and nonregulatory guidance. As site- and waste-specific characteristics are provided for each technology, specific regulatory requirements will be specified.

Subelement: Waste packaging, handling, and transportation.

Alternative: Interface definition. Define physical interfaces between the waste generators and the transportation system.

Technology: Interface definition. DOE 4700.1 defines the systems engineering requirements that must be satisfied in defining the administrative and physical interfaces. These interfaces need to be defined early in the ORNL remediation and waste management programs and then continually tracked to ensure adequate development of the packaging and transportation system and any ancillary equipment needed to facilitate the on-site and off-site movements of the waste materials.

Status: Technology is generally available. Efforts are needed to adequately, safely, and economically apply the technologies. Also needed are interface working groups involving waste generators and developers/operators of packaging/transportation systems. 


\section{Science/Technology Needs: None}

Implementation Needs: Because interface working groups involving waste generators and developers/operators of packaging/transportation systems are needed, implementation costs associated with this problem are estimated at $\$ 400 \mathrm{~K}$ over a 2- to 3-year period, with follow-on funding of about $\$ 100 \mathrm{~K} /$ year to ensure interfaces remain properly defined as the remediation effort proceeds. Thus a total implementation funding of about $\$ 1 \mathrm{M}$ is anticipated over the lifetime of the program.

Author: R. B. Pope/615-574-6461

\section{References:}

1. "Shippers-General Requirements for Shipments and Packagings," 49 CFR Pt. 173 (periodically updated).

2. "Packaging and Transportation of Radioactive Material," $10 \mathrm{CFR}$ Pt. 71 (periodically updated).

3. Regulations for the Safe Transport of Radioactive Material, 1985 Edition (as amended), Safety Series, No. 6, International Atomic Energy Agency, Vienna, Austria, 1990.

4. "Transportation Regulations; Compatibility With the International Atomic Energy Agency (IAEA)," Federal Register, Vol. 53, No. 110, Wednesday, June 8, 1988, Proposed Rule (Nuclear Regulatory Commission).

5. "Transportation Regulations; Compatibility with Regulations of the International Atomic Energy Agency; Notice of Proposed Rule," HM-169A, Federal Register, Vol. 54, No. 218, Tuesday, November 14, 1989 (Department of Transportation). 
EM Problem: Waste management

Y-12 Plant Problem: Building 9201-4 (Alpha-4). On-site and off-site shipments of radioactive materials

Problem Area/Constituents: Excepted quantities of radioactive materials. Packaging, handling, and transportation, using excepted packages, for radioactive materials whose activity per package does not exceed the limits as specified in the regulations.

\section{Reference Requirements:}

- Hazardous Materials Transportation Act regulations under 49 CFR 171-179

- Atomic Energy Act

- DOE Orders $1540.1,1540.2,1540.3,5700.6 \mathrm{C}$

- IAEA Safety Series, Nos. 6, 7, and 37 (1990)

- International Maritime Dangerous Goods Code

- Transportation and Traffic Management regulations under 41 CFR 109.40

Refer to the Regulatory Compliance Chapter of Vol. 1 of Vol. 1 for potentially applicable proposed and promulgated environmental laws, signed and pending agreements for the Oak Ridge Reservation, radiation protection standards, DOE orders, and nonregulatory guidance. As site- and waste-specific characteristics are provided for each technology, specific regulatory requirements will be specified.

Subelement: Waste packaging, handling, and transportation.

Alternatives: Packaging, handling, and transportation in packages satisfying at least the design requirements for excepted packages

Technology: Excepted packages. Strong, tight containers shall be used for excepted materials. These strong, tight packages will not leak any of the radioactive materials during conditions normally incident to routine conditions of transport. Adequate technology currently exists for such strong, tight packages.

Status: Accepted. Limited quantities of radioactive materials, as defined in the regulations, are authorized to be packaged and shipped as excepted materials in strong, tight containers if the provisions made in the regulations are met. Strong, tight packages are currently used throughout DOE to ship limited quantities of radioactive materials.

Science/Technology Needs: New strong, tight package designs may be required if current designs are not suitable. This can only be defined when the waste generator/packaging/ transportation interface effort is undertaken. However, should new designs be required, current technology will be sufficient for the new designs. 
Implementation Needs: Because the technology for strong, tight packages is adequate, there are currently no perceived implementation needs defined other than the specific costs of acquiring, preparing, and shipping packages. These costs can only be defined after the waste generator/packaging/transportation interface effort has reached initial conclusions.

Author: K. S. Boes/615-574-8067 and R. B. Pope/615-574-6461

\section{References:}

1. "Shippers-General Requirements for Shipments and Packagings," 49 CFR Pt. 173 (periodically updated).

2. "Transportation Regulations; Compatibility with Regulations of the International Atomic Energy Agency; Notice of Proposed Rule," HM-169A, Federal Register, Vol. 54, No. 218, Tuesday, November 14, 1989 (Department of Transportation). 
EM Problem: Waste management

X-12 Plant Problem: Building 9201-4 (Alpha-4). On-site and off-site shipments of radioactive materials

Problem Area/Constituents: Low-specific-activity (LSA) materials. Emerging federal regulations (10 CFR 71 and 49 CFR 173) will require development of methods for preparing and/or verifying wastes to be in the adjusted categories of LSA forms, to develop methods for satisfying requirements and limitations of the pending changed Department of Transportation (DOT) and Nuclear Regulatory Commission (NRC) regulations, and to provide packaging to satisfy the new Industrial Packages (IP) types IP-I, IP-2 and IP-3.

\section{Reference Requirements:}

- Hazardous Materials Transportation Act (HMTA) regulations under 49 CFR 171-179

- Atomic Energy Act

- Packaging and Transportation of Radioactive Materials regulations under 10 CFR 71

- DOE Orders 1540 and 5480

- IAEA Safety Series No. 6 (1990)

- ICAO, IATA, and IMO codes and regulations

- Transportation and Traffic Management regulations under 41 CFR 109-40

- ANSI, ASME, and ASTM standards

Refer to the Regulatory Compliance Chapter of Vol. 1 of Vol. 1 for potentially applicable proposed and promulgated environmental laws, signed and pending agreements for the Oak Ridge Reservation, radiation protection standards, DOE orders, and nonregulatory guidance. As site- and waste-specific characteristics are provided for each technology, specific regulatory requirements will be specified.

Subelement: Waste packaging, handling, and transportation

Alternative: Ship in bulk, unpackaged (as allowed by regulation) or ship in industrial packagings (IP) types IP-1, IP-2, and IP-3 (as allowed by regulation). Many alternatives are available for handling and transportatation (e.g, manual, mechanical, or automated handling; road, rail, or waterborne transport).

Technology: Low-specific-activity materials. Includes processing; preparation; and packaging, handling, and transportation.

Status: The preparation and processing of LSA materials has been well developed over the years; however, the categorization of these materials and the packaging requirements are being changed by federal regulators in response to changes made in 1985 by the International Atomic Energy Agency. The preparation, processing, and/or verification of LSA wastes to satisfy the new DOT/NRC requirements will necessitate the development of procedures and possibly some instrumentation and/or equipment. The new regulations are 
expected to implemented by DOT and NRC during 1993.

Science/Technology Needs: New package designs will be required, especially for the newly redefined IP-2 category. Packages in this category are subject to criteria that are midway between those for the existing packages, which have been regrouped under categories IP-1 and IP-3. In addition, package designs to specifically accommodate LSA materials arising from ORNL remediation activities, be they IP-1, IP-2 or IP-3, could be required. The preparation of new package designs to satisfy the IP-2 requirements will also require a multistep engineering process involving identification of the characteristics of the LSA materials to be transported in IP-2 packagings; the design, testing and qualification of the package designs; and the preparation of documentation to facilitate transfer of the designed package technology to private industry for fabrication and possible use elsewhere.

Implementation Needs: Development costs are estimated to be \$ IM (1992 dollars). These are the costs for the definition of waste forms to be packaged and transported; for specific design requirements; and for the development and technology transfer of tested designs to private industry of the IP package designs acceptable to these waste forms. Implementation costs cannot be estimated at this time because these require the definition of specific costs of acquiring, preparing, and shipping packages. These costs can only be defined after the waste generator/packaging/transportation interface effort has reached initial conclusions.

Author: R. B. Pope/615-574-6461

\section{References:}

1. "Shippers-General Requirements for Shipments and Packagings," 49 CFR Pt. 173 (periodically updated).

2. "Packaging and Transportation of Radioactive Material," 10 CFR Pt. 71 (periodically updated).

3. "Regulations for the Safe Transport of Radioactive Material," 1985 Edition (as amended), Safety Series, No. 6, International Atomic Energy Agency, Vienna, Austria, 1990.

4. "Transportation Regulations; Compatibility With the International Atomic Energy Agency (IAEA)," Federal Register, Vol. 53, No. 110, Wednesday, June 8, 1988, Proposed Rule (Nuclear Regulatory Commission).

5. "Transportation Regulations; Compatibility with Regulations of the International Atomic Energy Agency; Notice of Proposed Rule," HM-169A, Federal Register, Vol. 54, No. 218, Tuesday, November 14, 1989 (Department of Transportation).

September 1994

Decontamination and Decommissioning 
EM Problem: Waste management

Y-12 Plant Problem: Building 9201-4 (Alpha-4). On-site and off-site shipments of radioactive materials

Problem Area/Constituents: Surface-contaminated objects (SCOs). Emerging federal regulations (10 CFR 71 and 49 CFR 173) will require development of methods for preparing and/or verifying wastes to be in the adjusted categories of Low Specific Activity (LSA) forms, to develop methods for satisfying requirements and limitations of the pending changed Department of Transportation (DOT) and Nuclear Regulatory Commission (NRC) regulations, and to provide packagings to satisfy the new industrial packages (IP) types IP-I, IP-2, and IP-3.

\section{Reference Requirements:}

- Hazardous Materials Transportation Act (HMTA) regulations under 49 CFR 171-179

- Atomic Energy Act

- Packaging and Transportation of Radioactive Materials regulations under 10 CFR 71

- DOE Orders 1540 and 5480

- IAEA Safety Series, No. 6 (1990)

- ICAO, IATA, and IMO codes and regulations

- Transportation and Traffic Management regulations under 41 CFR 109.40

- ANSI, ASME, and ASTM standards

Refer to the Regulatory Compliance Chapter of Vol. 1 of Vol. 1 for potentially applicable proposed and promulgated environmental laws, signed and pending agreements for the Oak Ridge Reservation, radiation protection standards, DOE orders, and nonregulatory guidance. As site- and waste-specific characteristics are provided for each technology, specific regulatory requirements will be specified.

Subelement: Waste packaging, handling, and transportation

Alternative: Ship in industrial packaging types IP-1 and IP-2 (as allowed by regulation) or in IP-3 if readily available. Many alternatives available for handling and transport (e.g, manual, mechanical, or automated handling; road, rail, or waterborne transport).

Technology: Surface-contaminated-object processing, preparation and packaging, and handling and transportation

Status: The preparation and processing of surface-contaminated objects has been developed to some extent because this new category of materials has been adapted from one of the former groupings of LSA materials. However, the actual qualification of such objects as SCO-I or SCO-II has not been undertaken because this is specifically a new category with some new constraints and restrictions. In addition, the packaging requirements are being changed by federal regulators in response to changes made in 1985 by the International Atomic Energy 
Agency. Specifically, the former package designs for carriage of LSA materials have been modified and increased to become industrial packages of three categories. Efforts will be needed to adapt current knowledge, designs, and/or technology to the new requirements. The preparation, processing, and/or verification of SCO wastes (nonradioactive objects with surface contamination to specified levels) to satisfy the new DOT/NRC requirements will necessitate the development of processing procedures and possibly some instrumentation and/or equipment. The new regulations are expected to implemented by DOT and NRC during 1993.

Science/Technology Needs: New package designs may be required for carriage of SCO-I and SCO-II in IP-1; and new package designs will be required for the IP-2 category, which is a new category for the regulations. Packages in this category are subject to criteria that are midway between those for the existing packages, which have been regrouped under categories IP-1 and IP-3. Package designs to specifically accommodate SCO-I and SCO-II arising from ORNL remediation activities, be they IP-1 or IP-2 (or even, more conservatively, IP-3), could be required.

The preparation of new package designs to satisfy the IP-2 requirements specifically, and possibly new IP-1 package designs for the new SCO forms, will require a multistep engineering process involving identification of the characteristics of the LSA materials to be transported in IP-1 and IP-2 packagings; the design, testing, and qualification of the package designs; and the preparation of documentation to facilitate transfer of the designed package technology to private industry for fabrication and possible use elsewhere.

Implementation Needs: Development costs are estimated to be $\$ 1 M-\$ 2 M$ (1992 dollars). These are the costs for the definition of SCO waste forms to be packaged and transported; specific package design requirements; and the development and technology transfer of tested designs to the private industry of the IP package designs acceptable for these waste forms. Implementation costs cannot be estimated at this time because these require the definition of specific costs of acquiring, preparing and shipping packages. These costs can only be defined after the waste generator/packaging/transportation interface effort has reached initial conclusions.

Author: R. B. Pope/615-574-6461

\section{References:}

1. "Shippers - General Requirements for Shipments and Packagings," 49 CFR Pt. 173 (periodically updated).

2. "Packaging and Transportation of Radioactive Material," $10 \mathrm{CFR}$ Pt. 71 (periodically updated).

3. "Regulations for the Safe Transport of Radioactive Material," 1985 Edition (as amended), Safety Series, No. 6, International Atomic Energy Agency, Vienna, Austria, 1990.

4. "Transportation Regulations; Compatibility With the International Atomic Energy Agency (LAEA)," Federal Register, Vol. 53, No. 110, Wednesday, June 8, 1988, Proposed Rule

September 1994

Decontamination and Decommissioning 
(Nuclear Regulatory Commission).

5. "Transportation Regulations; Compatibility with Regulations of the International Atomic Energy Agency; Notice of Proposed Rule," HM-169A, Federal Register, Vol. 54, No. 218, Tuesday, November 14, 1989 (Department of Transportation). 
EM Problem: Waste management

Y-12 Plant Problem: Building 9201-4 (Alpha-4). On-site and off-site shipments of radioactive materials

Problem Area/Constituents: Type-A radioactive material transportation packages. Multiuse Type-A packaging design, development, and qualification for packaging, handling, and transportation of nonfissile concentrated wastes having total activities less than Type-A package limits, as specified in the regulations.

\section{Reference Requirements:}

- Hazardous Materials Transportation Act (HMTA) regulations under 49 CFR 171-179

- Atomic Energy Act

- Packaging and Transportation of Radioactive Materials regulations under 10 CFR 71

- DOE Orders 1540 and 5480

- IAEA Safety Series, No. 6 (1990)

- ICAO, IATA, and IMO codes and regulations

- Transportation and Traffic Management regulations under 41 CFR 109.40

- ANSI, ASME, and ASTM standards

Refer to the Regulatory Compliance Chapter of Vol. 1 of Vol. 1 for potentially applicable proposed and promulgated environmental laws, signed and pending agreements for the Oak Ridge Reservation, radiation protection standards, DOE orders, and nonregulatory guidance. As site- and waste-specific characteristics are provided for each technology, specific regulatory requirements will be specified.

Subelement: Waste packaging, handling, and transportation

Alternative: Ship in packages whose design at least satisfies the Type-A package design requirements specified in the federal regulations. Many alternatives are available for handling and transport (e.g, manual, mechanical, or automated handling; road, rail, or waterborne transport).

Technology: Type-A packaging, handling and transportation.

Status: Evolving technology. Standardized, multiuse packagings are needed to improve compliance in Type-A shipments in general. Improved, standardized Type-A packaging may be needed for shipping radioactive materials during ORNL cleanup efforts. Design emphasis will be placed on incorporating human factors design features to minimize the potential for human error. A standardized multiuse Type-A package will help ensure safe, effective, and efficient transportation of Type-A quantities of radioactive materials during decommissioning and decontamination operations at ORNL. Safe transport, in turn, will support environmental restoration efforts and aid in the prevention of environmental damage occurring from 
transportation-related releases of radioactive materials. An acceptable multiuse Type-A packaging should be low cost, light weight, widely applicable, and user friendly; it should also be reusable and/or recyclable. It must meet Department of Transportation and international regulations for transport by all modes.

Science/Technology Needs: Multiuse, standardized Type-A packaging designs need to be developed. An operationally efficient multiuse Type-A package, or packages, for radioactive materials needs to be developed to support decommissioning and decontamination efforts. This technology will provide better, standardized packaging for small quantities of radioactive liquids, solids, and gasses. This design should be as widely applicable as possible and made available, where appropriate, for cleanup efforts at ORNL.

Implementation Needs: Development costs: Funding required for development, testing, and qualification of multiuse Type-A packaging designs is estimated at \$1M. Implementation costs cannot be estimated at this time because these require the definition of specific costs of acquiring, preparing, and shipping packages. These costs can only be defined after the waste generator/packaging/transportation interface effort has reached initial conclusions.

Author: K. S. Boes/615-574-8067 and R. B. Pope/615-574-6461

\section{References:}

1. "Shippers-General Requirements for Shipments and Packagings," 49 CFR Pt. 173 (periodically updated).

2. "Packaging and Transportation of Radioactive Material," 10 CFR Pt. 71 (periodically updated).

3. "Regulations for the Safe Transport of Radioactive Material," 1985 Edition (as amended), Safety Series, No. 6, International Atomic Energy Agency, Vienna, Austria, 1990.

4. 'Transportation Regulations; Compatibility With the International Atomic Energy Agency (IAEA)," Federal Register, Vol. 53, No. 110, Wednesday, June 8, 1988, Proposed Rule (Nuclear Regulatory Commission).

5. "Transportation Regulations; Compatibility with Regulations of the International Atomic Energy Agency; Notice of Proposed Rule," HM-169A, Federal Register, Vol. 54, No. 218. Tuesday, November 14, 1989 (Department of Transportation). 
EM Problem: Waste management

Y-12 Plant Problem: Building 9201-4 (Alpha-4). On-site and off-site shipments of radioactive materials

Problem Area/Constituents: Type-B radioactive material packages. Packaging, handling, and transportation using Type-B packages for nonfissile radioactive materials having total activities greater than Type-A package limits, as specified in the regulations.

\section{Reference Requirements:}

- Hazardous Materials Transportation Act (HMTA) regulations under 49 CFR 171-179

- Atomic Energy Act

- Packaging and Transportation of Radioactive Materials regulations under 10 CFR 71

- DOE Orders 1540 and 5480

- IAEA Safety Series, No. 6 (1990)

- ICAO, IATA, and IMO codes and regulations

- Transportation and Traffic Management regulations under 41 CFR 109.40

- ANSI, ASME, and A STM standards

Refer to the Regulatory Compliance Chapter of Vol. 1 of Vol. 1 for potentially applicable proposed and promulgated environmental laws, signed and pending agreements for the Oak Ridge Reservation, radiation protection standards, DOE orders, and nonregulatory guidance. As site- and waste-specific characteristics are provided for each technology, specific regulatory requirements will be specified.

Subelement: Waste packaging, handling, and transportation

Alternative: Ship in packages whose design satisfies the Type-B package design requirements specified in the federal regulations. Many alternatives are available for handling and transport (e.g, manual, mechanical, or automated handling; road, rail, or waterborne transport).

Technology: Type-B packaging, handling, and transportation

Status: Accepted. Type-B packages are currently used throughout DOE to ship radioactive materials.

Science/Technology Needs: New package designs may be required if current designs are not suitable; however, current technology will be used for the new designs. Type-B packaging is used to package nonfissile concentrated wastes having total activities greater than TypeA package limits. Adequate technology currently exists for Type-B packaging. Certified package designs can be identified from the RAMPAC data base and Packaging Management Transportation System (PMTS) data base, which will identify numbers and status of packagings available or under development by EM-561. 
Implementation Needs: Because the technology for Type-B packages is adequate, no implementation needs are currently defined in terms of technology development or package design. Other implementation costs cannot be estimated at this time because these require the definition of specific costs of acquiring, preparing, and shipping packages. These costs can only be defined after the waste generator/packaging/transportation interface effort has reached initial conclusions.

Author: K. S. Boes/615-574-8067 and R. B. Pope/615-574-6461

\section{References:}

1. "Shippers-General Requirements for Shipments and Packagings," 49 CFR Pt. 173 (periodically updated).

2. "Packaging and Transportation of Radioactive Material," 10 CFR Pt. 71 (periodically updated). 
EM Problem: Waste management

Y-12 Plant Problem: Building 9201-4 (Alpha-4). On-site and off-site shipments of radioactive materials

Problem Area/Constituents: Fissile material packaged and transported in Type-A or Type-B fissile radioactive material packages. Packaging, handling, and transportation using Type-A fissile certified and Type-B fissile certified packages for fissile radioactive materials having total activities less than or greater than Type-A package limits, respectively, as specified in the regulations.

\section{Reference Requirements:}

- Hazardous Materials Transportation Act (HMTA) regulations under 49 CFR 171-179

- Atomic Energy Act

- Packaging and Transportation of Radioactive Materials regulations under 10 CFR 71

- DOE Orders 1540 and 5480

- IAEA Safety Series, No. 6 (1990)

- ICAO, IATA, and IMO codes and regulations

- Transportation and Traffic Management regulations under 41 CFR 109.40

- ANSI, ASME, and ASTM standards

Refer to the Regulatory Compliance Chapter of Vol. 1 of Vol. 1 for potentially applicable proposed and promulgated environmental laws, signed and pending agreements for the Oak Ridge Reservation, radiation protection standards, DOE orders, and nonregulatory guidance. As site- and waste-specific characteristics are provided for each technology, specific regulatory requirements will be specified.

Subelement: Waste packaging, handling, and transportation

Alternative: Ship in packages whose design satisfies the Type-A fissile package design or the Type-B fissile package design requirements specified in the federal regulations. Many alternatives are available for handling and transport (e.g, manual, mechanical, or automated handling; road, rail, or waterborne transport).

Technology: Type-A fissile certified and Type-B fissile certified packaging, handling, and transportation.

Status: Accepted. Both Type-A fissile certified packages and Type-B fissile certified packages are currently used throughout DOE to ship fissile radioactive materials. Type-A fissile certified packaging is used to package concentrated wastes containing fissile material with total activities less than Type-A package limits. Type-B fissile certified packaging is used to package concentrated wastes containing fissile material with total activities greater than Type-A package limits. Adequate technology currently exists for both Type-A fissile certified 
packaging and Type-B fissile certified packaging. Certified package designs can be identified from the RAMPAC data base and Packaging Management Transportation System (PMTS) data base, which will identify numbers and status of packagings available or under development by EM-561.

Science/Technology Needs: New package designs may be required if current designs are not suitable; however, current technology will be used for the new designs.

Implementation Needs: Because the technology for Type-A fissile certified and Type-B fissile certified packages is adequate, no implementation needs exist currently. Other implementation costs cannot be estimated at this time because these require the definition of specific costs of acquiring, preparing, and shipping packages. These costs can only be defined after the waste generator/packaging/transportation interface effort has reached initial conclusions.

Author: K. S. Boes/615-574-8067 and R. B. Pope/615-574-6461

\section{References:}

1. "Shippers-General Requirements for Shipments and Packagings," 49 CFR Pt. 173 (periodically updated).

2. "Packaging and Transportation of Radioactive Material," 10 CFR Pt. 71 (periodically updated).

3. "Regulations for the Safe Transport of Radioactive Material," 1985 Edition (as amended), Safety Series, No. 6, International Atomic Energy Agency, Vienna, Austria, 1990.

4. "Transportation Regulations; Compatibility With the International Atomic Energy Agency (IAEA)," Federal Register, Vol. 53, No. 110, Wednesday, June 8, 1988, Proposed Rule (Nuclear Regulatory Commission).

5. "Transportation Regulations; Compatibility with Regulations of the International Atomic Energy Agency; Notice of Proposed Rule," HM-169A. Federal Register, Vol. 54, No. 218, Tuesday, November 14, 1989 (Department of Transportation). 
EM Problem: Waste management

Y-12 Plant Problem: Building 9201-4 (Alpha-4). On-site and off-site shipments of hazardous materials other than radioactive materials

Problem Area/Constituents: Polychlorinated biphenyl (PCB) materials. Packaging, handling, and transportation using performance-oriented packaging (POP) for PCBcontaminated wastes.

\section{Reference Requirements:}

- Hazardous Materials Transportation Act (HMTA) regulations under 49 CFR 171-179

- DOE Orders $1540.1,1540.2$, and 5700.6C

- International Maritime Dangerous Goods Code

- Transportation and Traffic Management regulations under 41 CFR 109.40

- 49 U.S.C. 1803 et. seq.

- ANSI, ASME, and ASTM standards

Refer to the Regulatory Compliance Chapter of Vol. 1 of Vol. 1 for potentially applicable proposed and promulgated environmental laws, signed and pending agreements for the Oak Ridge Reservation, radiation protection standards, DOE orders, and nonregulatory guidance. As site- and waste-specific characteristics are provided for each technology, specific regulatory requirements will be specified.

Subelement: Waste packaging, handling, and transportation

Alternative: Waste packaging, handling, and transportation. Ship in POP containers satisfying applicable DOT requirements, as required by material quantity and type. Many alternattves are available for handling and transport (e.g, manual, mechanical, or automated handling; road, rail, or waterborne transport).

Technology: Performance-oriented packaging, packing groups II and III, for PCBs. This technology includes POP packing group II and III containers and bulk strong, tight containers. PCBs are normally regulated by air and water only and are authorized to be shipped in POP packing group II containers unless there is a reportable quantity. If there is a reportable quantity of PCBs (the reportable quantity is $1 \mathrm{lb}$ ), then all modes are regulated. When rail and highway shipments are regulated, POP packing group III containers are authorized. Bulk shipments are authorized to be made in strong, tight containers, per the regulations. Adequate technology currently exists for packaging and shipping PCBs.

Status: Accepted. Performance-oriented packaging and strong, tight packages are currently used throughout DOE to ship PCBs.

Science/Technology Needs: New package designs may be required if current designs are

September 1994

Decontamination and Decommissioning 
not suitable; however, current technology will be used for the new designs.

Implementation Needs: Because the technology for POP and strong, tight packages is adequate, no implementation needs exist currently. Other implementation costs cannot be estimated at this time because these require the definition of specific costs of acquiring, preparing, and shipping packages. These costs can only be defined after the waste generator/packaging/transportation interface effort has reached initial conclusions.

Author: K. S. Boes/615-574-8067 and R. B. Pope/615-574-6461

\section{References:}

1. "Shippers-General Requirements for Shipments and Packagings," 49 CFR Pt. 173 (periodically updated).

2. "Performance-Oriented Packaging Standards; Changes to Classification, Hazard Communication, Packaging and Handling Requirements Based on UN Standards and Agency Initiative," HM-181 Final Rule, Federal Register, Vol. 55, No. 246, Friday, December 21, 1990 (Department of Transportation). 


\section{EM Problem: Waste management}

Y-12 Plant Problem: Building 9201-4 (Alpha-4). On-site and off-site shipments of hazardous materials other than radioactive materials

Problem Area/Constituents: Asbestos materials. Packaging, handling, and transportation using performance-oriented packaging (POP) for asbestos material.

\section{Reference Requirements:}

- Hazardous Materials Transportation Act (HMTA) regulations under 49 CFR 171-179

- DOE Orders $1540.1,1540.2$, and 5700.6C

- International Maritime Dangerous Goods Code

- Transportation and Traffic Management regulations under 41 CFR 109.40

- 49 U.S.C. 1803 et. seq.

- National Emission Standards for Hazardous Air Pollutants (NESHAP) regulations

- ANSI, ASME, and ASTM standards

Refer to the Regulatory Compliance Chapter of Vol. 1 of Vol. 1 for potentially applicable proposed and promulgated environmental laws, signed and pending agreements for the Oak Ridge Reservation, radiation protection standards, DOE orders, and nonregulatory guidance. As site- and waste-specific characteristics are provided for each technology, specific regulatory requirements will be specified.

Subelement: Waste packaging, handling, and transportation

Alternative: Ship in POP packing group II and III containers satisfying applicable Department of Transportation requirements, as required by material quantity and type. Many alternatives are available for handling and transport (e.g, manual, mechanical, or automated handling; road, rail, or waterborne transport).

Technology: Performance-oriented packaging, packing groups II and III, for asbestos.

Status: Accepted. POP and strong, tight packages are currently used throughout DOE to ship asbestos. Friable, white asbestos is authorized to be shipped in POP packing group III containers. Friable, blue, or brown asbestos is authorized to be shipped in POP packing group II containers. Asbestos that is immersed or fixed in a natural or artificial binder material (such as cement, plastic, asphalt, resins or mineral ore) and manufactured products containing asbestos are not subject to the requirements of the packaging regulations.

Science/Technology Needs: Adequate technology currently exists for packaging and shipping all types of asbestos.

Implementation Needs: Because the technology for POP and strong, tight packages is 
adequate, no implementation needs exist currently. Other implementation costs cannot be estimated at this time because these require the definition of specific costs of acquiring, preparing, and shipping packages. These costs can only be defined after the waste/ generator/packaging/transportation interface effort has reached initial conclusions.

Author: K. S. Boes/615-574-8067 and R. B. Pope/615-574-6461

\section{References:}

1. "Shippers-General Requirements for Shipments and Packagings," 49 CFR Pt. 173 (periodically updated).

2. "Performance-Oriented Packaging Standards; Changes to Classification, Hazard Communication, Packaging and Handling Requirements Based on UN Standards and Agency Initiative," HM-181 Final Rule, Federal Register, Vol. 55, No. 246, Friday, December 21, 1990 (Department of Transportation). 
EM Problem: Waste management

Y-12 Plant Problem: Building 9201-4 (Alpha-4). On-site and off-site shipments of hazardous materials other than radioactive materials

Problem Area/Constituents: Packaging, handling, and transportation for nonradioactive hazardous waste

\section{Reference Requirements:}

- Hazardous Materials Transportation Act regulations under 49 CFR 171-179

- DOE Orders $1540.1,1540.2,5700.6 \mathrm{C}$

- International Maritime Dangerous Goods Code

- Transportation and Traffic Management regulations under 41 CFR 109

- 49 U.S.C. 1803 et. seq.

Refer to the Regulatory Compliance Chapter of Vol. 1 of Vol. 1 for potentially applicable proposed and promulgated environmental laws, signed and pending agreements for the Oak Ridge Reservation, radiation protection standards, DOE orders, and nonregulatory guidance. As site- and waste-specific characteristics are provided for each technology, specific regulatory requirements will be specified.

Subelement: Waste packaging, handling, and transportation

Alternatives: Ship in Performance-Oriented Packaging (POP) satisfying applicable Department of Transportation requirements, as required by material quantity and type. Many alternatives are available for handling and transport (e.g, manual, mechanical, or automated handling; road, rail, or waterborne transport).

Technology: Performance-oriented packaging (POP); specification packaging; strong, tight containers

Status: Accepted. POP; specification packaging; and strong, tight containers are currently used throughout DOE to ship nonradioactive hazardous waste.

Science/Technology Needs: POP packing groups I, II, and III containers; specification packaging; and strong, tight containers. Nonradioactive RCRA hazardous waste is authorized to be shipped in POP group I, II, and III packages for nonbulk shipments, per the regulations. For bulk shipments, specification packaging and strong, tight containers are authorized, per the regulations. Adequate technology currently exists for packaging and shipping RCRA nonradioactive hazardous waste.

Implementation Needs: Because the technology for POP and strong, tight containers is adequate, no implementation needs exist currently. Other implementation costs cannot be 
estimated at this time because these require the definition of specific costs of acquiring, preparing, and shipping packages. These costs can only be defined after the waste/ generator/packaging/transportation interface effort has reached initial conclusions.

Author: K. S. Boes/615-574-8067

\section{References:}

1. "Shippers-General Requirements for Shipments and Packagings," 49 CFR Pt. 173 (periodically updated).

2. "Performance-Oriented Packaging Standards; Changes to Classification, Hazard Communication, Packaging and Handling Requirements Based on UN Standards and Agency Initiative," HM-181 Final Rule, Federal Register, Vol. 55, No. 246, Friday, December 21, 1990 (Department of Transportation). 
EM Problem: Waste management

Y-12 Plant Problem: Building 9201-4 (Alpha-4). On-site shipments of hazardous materials other than radioactive

Problem Area/Constituents: On-site packaging, handling, and transportation of hazardous and/or radioactive materials and wastes

\section{Reference Requirements:}

- Resource Conservation and Recovery Act of 1976 (RCRA), as amended, Land Disposal Restriction (LDR) Federal Facilities Compliance Agreement (FFCA) dated June 12, 1992

- Hazardous Materials Transportation Act regulations under 49 CFR 171-179

- Atomic Energy Act

- Nuclear Regulatory Commission regulations under 10 CFR 71

- DOE Orders $1540.1,1540.2,1540.3,5610.1,5700.6 C, 5480 . X$ (draft)

- NRC Regulatory Guides-Series 7

- International Maritime Dangerous Goods Code

- Transportation and Traffic Management regulations under 41 CFR 109

- 49 U.S.C. 1803 et. seq.

- LAEA Safety Series, No. 6 (1990)

- ICAO, IATA, and IMO codes and regulations

- ANSI, ASME, and ASTM standards

Refer to the Regulatory Compliance Chapter of Vol. 1 of Vol. 1 for potentially applicable proposed and promulgated environmental laws, signed and pending agreements for the Oak Ridge Reservation, radiation protection standards, DOE orders, and nonregulatory guidance. As site- and waste-specific characteristics are provided for each technology, specific regulatory requirements will be specified.

Subelement: Waste packaging, handling, and transportation

Alternatives: Packaging, handling, and transportation on-site would be done to comply with regulations for off-site transport, or performed in alternate ways that provide equivalent levels of safety.

Technology: On-site packaging, handling, and transportation. This technology includes Type-A; Type-B; fissile; strong, tight; industrial packaging (IP); performance-oriented packaging (POP); and bulk packaging or packaging equivalent in safety. Full adherence to federal regulations applicable to off-site packaging and transportation of hazardous materials is an acceptable approach to meeting the requirements. Packaging that has the equivalent safety of off-site packaging is also acceptable. Assuring equivalent safety can be achieved by relying on package performance or incorporating specific hazardous communications and enroute control measures along with package performance. Adequate technology currently exists for on-site packaging and transportation. 
Status: Accepted. On-site shipments of hazardous and radioactive materials and wastes are currently made throughout DOE. As DOE Order 5480.X is made final, the compliance with this order will need to be assessed and technologies developed as needed.

Science/Technology Needs: New package designs may be required if current designs are not suitable; however, current technology will be used for the new designs.

Implementation Needs: Because the technology for on-site packaging and transportation is adequate, no implementation needs are identified currently; however, as the pending DOE order $5480 . \mathrm{X}$ is finalized and implemented, various needs may be identified.

Author: K. S. Boes/615-574-8067 and R. B. Pope/615-574-6461

\section{References:}

1. "Shippers-General Requirements for Shipments and Packagings," 49 CFR Pt. 173 (periodically updated).

2. "Packaging and Transportation of Radioactive Material," 10 CFR Pt. 71 (periodically updated).

3. "Regulations for the Safe Transport of Radioactive Material," 1985 Edition (as amended), Safety Series, No. 6, International Atomic Energy Agency, Vienna, Austria, 1990.

4. "Transportation Regulations; Compatibility With the International Atomic Energy Agency (IAEA)," Federal Register, Vol. 53, No. 110, Wednesday, June 8, 1988, Proposed Rule (Nuclear Regulatory Commission).

5. "Transportation Regulations; Compatibility with Regulations of the International Atomic Energy Agency; Notice of Proposed Rule," HM-169A, Federal Register, Vol. 54, No. 218, Tuesday, November 14, 1989 (Department of Transportation).

6. "Performance-Oriented Packaging Standards; Changes to Classification, Hazard Communication, Packaging and Handling Requirements Based on UN Standards and Agency Initiative," HM-181 Final Rule, Federal Register, Vol. 55, No. 246, Friday, December 21, 1990 (Department of Transportation).

7. Onsite Packaging and Transportation of Hazardous Materials, Substances, and Wastes, DOE Order 5480.X (draft), October 16, 1992, Department of Energy. 
EM Problem: Waste management

Y-12 Plant Problem: Building 9201-4 (Alpha-4). On-site and off-site shipments of radioactive materials

Problem Area/Constituents: Mixed Wastes. Packaging, handling, and transportation of mixed waste (waste containing both radioactive and hazardous components as defined by the Atomic Energy Act and the Resource Conservation and Recovery Act, respectively) or material having more than one hazard as defined in the regulations.

\section{Reference Requirements:}

- Resource Conservation and Recovery Act of 1976 (RCRA), as amended, Land Disposal Restriction (LDR) Federal Facilities Compliance Agreement (FFCA) dated June 12, 1992

- Hazardous Materials Transportation Act (HMTA) regulations under 49 CFR 171-179

- Atomic Energy Act

- Packaging and Transportation of Radioactive Materials regulations under 10 CFR 71

- DOE Orders $1540.1,1540.2,1540.3,5610.1,5820.2 \mathrm{~A}$, and 5700.6C

- NRC Regulatory Guides-Series 7

- International Maritime Dangerous Goods Code

- Transportation and Traffic Management regulations under 41 CFR 109.40

- IAEA Safety Series, No. 6 (1990)

- ICAO, IATA, and IMO codes and regulations

- ANSI, ASME, and ASTM standards

Refer to the Regulatory Compliance Chapter of Vol. 1 of Vol. 1 of Vol. 1 for potentially applicable proposed and promulgated environmental laws, signed and pending agreements for the Oak Ridge Reservation, radiation protection standards, DOE orders, and nonregulatory guidance. As site- and waste-specific characteristics are provided for each technology, specific regulatory requirements will be specified.

Subelement: Waste packaging, handling, and transportation

Alternative: Ship in packages whose design satisfies the radioactive material package design requirements according to the radioactive nature of the contents as specified in the federal regulations. Many alternatives are available for handling and transport (e.g, manual, mechanical, or automated handling; road, rail, or waterborne transport).

Technology: Waste packaging, handling, and transportation for mixed wastes. Includes wastes having more than one hazard.

Status: Accepted. Both mixed waste and waste having more than one hazard are currently being packaged for transport. Material having more than one hazard as defined in the regulations must be classed for packaging and transportation according to the order of 
hazards table in the regulations. The packaging used shall be in accordance with the hazard that takes precedence. The regulations provide some exceptions for certain materials. Mixed waste shall be classed according to the radioactive material hazard class except for limited quantities. The regulations provide some exceptions for limited quantities of radioactive material. Radioactive materials shall be packaged in Industrial, Type-A, Type-B, Type-A fissile, or Type-B fissile packagings as appropriate.

Science/Technology Needs: Because the technology for handling, packaging, and transporting mixed waste and waste having more than one hazard is adequate, current technology will be used. Where current designs may prove to be unsuitable, current technology will be used to develop new designs.

Implementation Needs: No implementation needs exist because current technology is adequate or covered by other waste packaging, handling and transportation problem elements.

Author: K. S. Boes/615-574-8067 and R. B. Pope/615-574-6461

\section{References:}

1. "Shippers-General Requirements for Shipments and Packagings," 49 CFR Pt. 173 (periodically updated).

2. "Packaging and Transportation of Radioactive Material," 10 CFR Pt. 71 (periodically updated).

3. "Regulations for the Safe Transport of Radioactive Material," 1985 Edition (as amended), Safety Series, No. 6, International Atomic Energy Agency, Vienna, Austria, 1990.

4. "Transportation Regulations; Compatibility With the International Atomic Energy Agency (IAEA)," Federal Register, Vol. 53, No. 110, Wednesday, June 8, 1988, Proposed Rule (Nuclear Regulatory Commission).

5. "Transportation Regulations; Compatibility with Regulations of the International Atomic Energy Agency; Notice of Proposed Rule," HM-169A, Federal Register, Vol. 54, No. 218, Tuesday, November 14, 1989 (Department of Transportation).

6. "Performance-Oriented Packaging Standards; Changes to Classification, Hazard Communication, Packaging and Handling Requirements Based on UN Standards and Agency Initiative," HM-181 Final Rule, Federal Register, Vol. 55, No. 246, Friday, December 21, 1990 (Department of Transportation).

7. Onsite Packaging and Transportation of Hazardous Materials, Substances, and Wastes, DOE Order 5480.X (draft), October 16, 1992, Department of Energy. 
EM Problem: Waste management

Y-12 Plant Problem: Building 9201-4 (Alpha-4). Off-site shipments of radioactive materials

Problem Area/Constituents: Waste shipment tracking. Satellite tracking of and communications for off-site shipments of spent nuclear fuel, high-level waste, transuranic (TRU) waste shipments to the Waste Isolation Pilot Plant (WIPP), and other high-visibility radioactive material shipments from ORNL.

Reference Requirements: DOE Order 1540. Refer to the Regulatory Compliance Chapter of Vol. 1 of Vol. 1 for potentially applicable proposed and promulgated environmental laws, signed and pending agreements for the Oak Ridge Reservation, radiation protection standards, DOE orders, and nonregulatory guidance. As site- and waste-specific characteristics are provided for each technology, specific regulatory requirements will be specified.

Subelement: Waste packaging, handling, and transportation

Alternative: Waste transportation

Technology: Waste shipment tracking. DOE has developed and operates a satellite-based shipment tracking and communication system (TRANSCOM) that provides for tracking and communications for off-site shipments of spent nuclear fuel, high-level and TRU waste shipments to WIPP, and other high-visibility radioactive materials shipments. A computerized information processing system provides information to the TRANSCOM Communication Center (TCC) on the status of the shipment. Operators of transport vehicles can be queried by and communicate with the TCC, and information on shipment location is made available to state Emergency Operations Centers, the Nuclear Regulatory Commission, and the Department of Transportation.

Status: Accepted. The TRANSCOM system has been developed and is in operation. The sponsor for this is the Transportation Management Division (TMD/EM-561), and the system is operated in Oak Ridge, Tennessee, under the direction of ORNL.

Science/Technology Needs: None. The system is fully operational and has undergone and been qualified for operation following a WIPP Operational Readiness Review.

Implementation Needs: Because the TRANSCOM is fully operational, no implementation needs exist, except that funding could be required to supply additional TCC operational staff and transponders for transport vehicles.

Author: R. B. Pope/615 574-6461

References: DOE Order 1540 


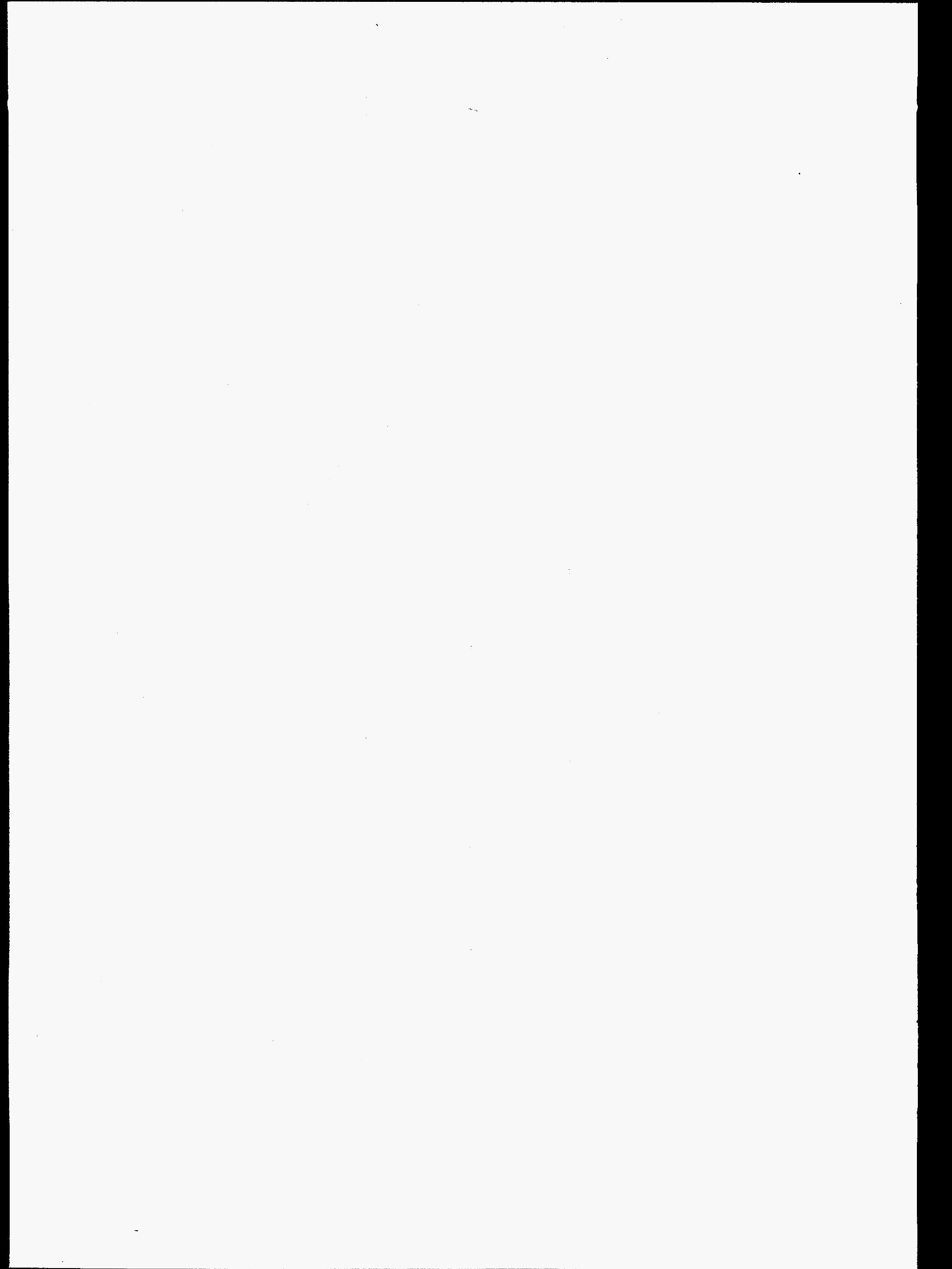




\section{EM Problem: Waste Management}

Y-12 Plant Problem: Building 9201-4 (Alpha-4). Contaminated wastewater, contaminated oil, contaminated solvents, contaminated concrete rubble, scrap transite, scrap asbestos, pipe and insulation, other scrap building materials, scrap metal, other scrap materials, contaminated clothing and rags, contaminated soil

Problem Area/Constituents: Polychlorinated biphenyls (PCBs) and other halogenated organics in water, PCBs in solvents, PCBs in oil, contaminated concrete rubble, scrap transite, metals, soll, etc., contaminated with PCBs or other halogenated or aromatic solvents.

\section{Reference Requirements:}

- Resource Conservation and Recovery Act of 1976 (RCRA), as amended, Land Disposal Restriction (LDR) Federal Facilities Compliance Agreement (FFCA) dated June 12, 1992.

- Federal Facilities Agreement (FFA) for the Oak Ridge Reservation.

- Federal Facilities Compliance Agreement-Toxic Substances Control Act (FFCA-TSCA) dated February 20, 1992.

- Clean Air Act regulations under 40 CFR 60-65.

- Clean Water Act regulations under 40 CFR 110-146 and 400-471.

- DOE orders.

Refer to the Regulatory Compliance Chapter of Vol. 1 for potentially applicable proposed and promulgated environmental laws, signed and pending agreements for the Oak Ridge Reservation, radiation protection standards, DOE orders, and nonregulatory guidance. As site- and waste-specific characteristics are provided for each technology, specific regulatory requirements will be specified.

\section{Subelement: Waste processing}

Alternative: Chemical treatment

Technology: Gamma radiolysis. In-situ/in-drum gamma radiolysis of PCBs, TCE, and other halogenated contaminants can be useful for destroying the halogenated species by direct removal of halogens by solvated electrons, as reported, ${ }^{1-3}$ or by attack of hydroxyl free radicals (and perhaps other free radicals) on the unhalogenated portion of the PCB ring structure.4-6 The generation of free radicals is enhanced by irradiation of water-containing materials (wet) or by addition of such chemicals as hydrogen peroxide, alcohol, etc. ${ }^{7,8}$ The irradiation could proceed until the complete destruction of the contaminants into innocuous species or could be complemented by subsequent bioremediation of the biodegradable fragments.

Status: Evolving technology

September 1994

Decontamination and Decommissioning 
Science/Technology Needs: The radiolytic destruction of halogenated and aromatic compounds is well documented. Issues to be addressed are the complete characterization of the fragments under different conditions and different materials and their relative toxicities. ${ }^{9}$ Another important issue is the possibility of combining radiolysis with enhanced biodegradation of the radiolytic byproducts. ${ }^{10}$

Implementation Needs: Development costs are estimated to be $\$ 1.25 \mathrm{M}$ (1992 dollars). These are the costs for development and technology transfer to the private industry of "Insitu/in-drum radiolytic destruction of PCBs and other halogenated substances from mixed wastes, TSCA wastes, contaminated soil, concrete, gaskets, pipes, etc." These costs are based on TTP No. OR-4TAK-93 by G. D. Del Cul, et al., submitted to DOE-OTD. A similar proposal (OR-PCBS-UT, G. D. Del Cul and D. C. White) would utilize gamma radiolysis followed by bioremediation with an estimated funding requirement of $\$ 2.5 \mathrm{M}$.

Author: Del Cul/615-576-5743

\section{References:}

1. R. E. Arbon et al., "Gamma radiolysis of chlorinated hydrocarbons," Spectrum '92, International Topical Meeting, Nuclear and Hazardous Waste Management, 1266-1268, Boise, Idaho, August 23-27, 1992.

2. F. Lepine and R. Masse, "Effect of gamma irradiation on a PCB mixture in organic solvents," Bull. Enwiron. Contam. Toxicol, 44, 549-554, 1990.

3. F. Lepine and R. Masse, "Degradation Pathways of PCB upon gamma irradiation," Environmental Health Perspectives, 89, 183-187, 1990.

4. D. L. Sedlak and A. W. Andren, "Aqueous-phase oxidation of polychlorinated biphenyls by hydroxyl radical," Environ. Sc. Technol, 25, 1419-1427, 1991.

5. R. F. Cichy et al., "Polychlorinated biphenyl reduction in lake trout by irradiation and broiling," Bull Enwiron. Contam. Toxicol, 22, 807-812, 1979.

6. A. Singh et al., "Radiolytic dechlorination of polychlorinated biphenyl," Radiat. Physics. Chem., 25, 11, 1985.

7. Evans et al., "Mechanism of the radiation induced dechlorination of 1,1,1-trichloro-2,2bis(p-chlorophenyl) ethane in alcoholic solutions," J. Phys. Chem, 75, 2762, 1971.

8. T. Sawai et al., "The radiolytic-chain dechlorination of polychlorinated biphenyls in alkaline 2-propanol solutions," Bull Chem. Soc. Japar, 47, 1989, 1974.

9. G. D. Del Cul et al., In-situ/in-drum radiolytic destruction of PCBs and other halogenated substances from mixed wastes, TSCA wastes, contaminated soil, concrete, gaskets, pipes, etc., OTD-TTP No. OR-4TAK-93.

10. G. D. Del Cul, D. C. White et al., In-situ/in drum PCB destruction: utilization of gamma irradiation for acceleration of bioremediation technologies for PCB destruction, OTD-TTP OR-PCBS-UT.

September 1994

Decontamination and Decommissioning 


\section{EM Problem: Waste management}

Y-12 Plant Problem: Building 9201-4 (Alpha-4). Radioactive and hazardous metals in waste; uranium tails

Problem Area/Constituents: Chemistry of fullerenes (bucky balls)/encapsulation of radioactive and hazardous metals in fullerenes

\section{Reference Requirements:}

- Resource Conservation and Recovery Act of 1976 (RCRA), as amended, Land Disposal Restriction (LDR) Federal Facilities Compliance Agreement (FFCA) dated June 12, 1992

- Federal Facilities Agreement (FFA) for the Oak Ridge Reservation

- Clean Air Act regulations under 40 CFR 60-65

- Clean Water Act regulations under 40 CFR 110-146 and 400-471

- DOE orders

Refer to the Regulatory Compliance Chapter of Vol. 1 for potentially applicable proposed and promulgated environmental laws, signed and pending agreements for the Oak Ridge Reservation, radiation protection standards, DOE orders, and nonregulatory guidance. As site- and waste-specific characteristics are provided for each technology, specific regulatory requirements will be specified.

\section{Subelement: Waste processing}

Altemative: Chemical treatment

Technology: Bucky ball chemistry. Involves the innovative use of fullerenes (also known as bucky balls) to treat and encapsulate radioactive and hazardous species in high-molecular-weight carbon cages. The process is envisaged to consist of laser and/or electrical arc or plasma treatment for trapping transuranium materials (and hazardous metals) within closed graphitic spheres (i.e., fullerene with transuranium metals inside). This is expected to result in the trapping of radionuclides (and toxic metals) inside a graphite or carbon moderator for indefinite storage.

Status: During the past 2 years, major advances in the new materials area have occurred following the discovery of facile synthetic routes to the production of macroscopic quantities of fullerene materials. Fullerenes are edgeless, hollow, all-carbon molecules that contain hexagonal and pentagonal arrangements of $\mathrm{sp}^{2}$ carbon atoms around the surface. The word fullerene is derived from the word buckminsterfullerene, which is the name given to the most symmetrical fullerene $\left(C_{60}\right)$, in honor of the late $R$. Buckminster Fuller, to recognize the similarity of the $\mathrm{C}_{60}$ 's geometry to his geodesic architectural style. Fullerenes can range in size from 20 carbon atoms to many thousands of carbon atoms. Those fullerenes that have been well characterized have approximately spherical shapes; however, tubular shapes of varying lengths and diameters are proposed to exist as well as fullerenes with negative curvature. 
Oak Ridge National Laboratory (ORNL) has instituted a major research activity in this area through the laboratory Directors Research Program. Scientists in many divisions throughout the laboratory are using their well-developed and powerful techniques to fully characterize this new class of molecules. Potential applications resulting from this new technological advance are many. Applied research also is devoted to tribology (frictional properties), nuclear medicine, batteries, catalysis, and other areas.

When fullerenes are produced using the electrical arc vaporization method, a build-up of material occurs on the negatively charged electrode. When this material is observed using electron microscopy, the structure revealed is that of concentric tubules of graphite. These materials, which are amicably called "bucky tubes," are shown to possess lengths which are centered at about one micron. Bucky tubes and the observation of the encapsulation of many atoms into coalesced fullerenes ${ }^{4}$ leads to a remarkable synthesis of observations: bucky tubes with numerous metal atoms inside. Current thinking within the fullerene research community is that metal atoms so trapped will never be released, except when exposed to extraordinarily harsh treatment. Because only metal atoms with low ionization potentials are encapsulated into the bucky tubes and spheres, this method may also represent a new way to reduce waste volume.

Recent observations by Ugarte using electron microscopy show that, when bucky tubes are exposed to a high flux of MeV electrons, they will anneal into structures that can be thought of as onions or "Russian dolls" of graphite: spheres within spheres. Ugarte, has recently shown that these species can trap small crystallites of metal species, specifically lanthanum. These crystallites are forever protected from the rest of the world by many layers of closed, edgeless graphitic spheres. These exciting new results suggest that it may be possible to mix radioactive waste together with "soot" from the carbon arc reactors. Irradiation of this mixture with high-energy electrons would allow encapsulation of these wastes into the spheres-within-spheres fullerene structures (bucky onions).

Fullerene technology is an evolving technology that could be applied to encapsulating radioactive and hazardous metals before their safe disposal in the environment. The technology is in an early phase of development and shows considerable promise. At present, development is at the bench-scale level. Considerable research, development, and demonstration will need to be undertaken before the technology reaches the implementation phase.

Science/Technology Needs: To fully evaluate the proposed encapsulation of radioactive and toxic metals in the all-carbon fullerene spheres, the following technical items must be acquired:

- A high-power, pulsed, multi-wavelength laser, such as a high-power, Nd:YAG laser with doubling crystals for development and use of the laser vaporization technique and qualification of recipes. A neodymium:yttrium-aluminum-garnet (Nd:YAG) laser is widely avallable commercially. This solid-state laser easily produces radiation at wavelengths of $1065 \mathrm{~nm}, 532 \mathrm{~nm}, 355 \mathrm{~nm}$, and $266 \mathrm{~nm}$ and has been proven to be effective in producing

September 1994

Decontamination and Decommissioning 
endohedral fullerene species. This laser would be used along with an existing $15 \mathrm{~kW} \mathrm{CO}$ laser, which is currently available at ORNL.

- An advanced, elevated-temperature target zone for tuning the annealing zone for the growing metallofullerenes. Such an apparatus would require oven and high-temperature vacuum and pressure materials for controlling the environment and position of the composite graphite target and for recovery of the products. This apparatus would constitute an improvement over our existing experimental designs. The desired temperatures would range from $1000^{\circ} \mathrm{C}$ to $2000^{\circ} \mathrm{C}$. Desired pressures: 1 microns to 2 atmospheres.

- Laser desorption mass spectrometry, notably fourier-transform mass spectrometry or time-of-flight mass spectrometry using a supersonic molecular beam source, has proven to be the most sensitive approach for initial analysis of the materials produced. One such device would be required to effectively study these materials.

- A new fullerene/metallofullerene generator based on the idea of the three-zone fullerene generator, where the atomic carbon and metal are generated using a novel approach currently being studied elsewhere. Power generators for such a device would be in the $20-30 \mathrm{~kW}$ range. Insulation, heaters, and/or cooling devices for tuning the annealing zone to the optimal temperature would be required. Also, provisions would be allowed for rapid removal of the products from the damaging temperatures required for annealing. Miscellaneous supplies for plumbing such a generator to various gas supplies, water cooling, and exhaust provisions would be required. Such a generator would be a (large) laboratory-scale test plant for the scaling-up of this process.

- Sufficient wet-chemistry supplies and equipment, such as a UV/VIS spectrophotometer, an IR spectrometer, a RAMAN spectrometer, fume hoods, balances, glassware, a glove box for air sensitive samples, and miscellaneous wet chemistry supplies.

The Health and Safety Research Division (HASRD) at ORNL possesses many of the necessary techniques required to examine the encapsulation of toxic or radioactive metals and actinides within the fullerenes. The High Flux Isotope Reactor (HFIR) neutron facility along with the High Temperature Materials Laboratory (HTML) possesses unique techniques which can be used to characterize these types of encapsulated species.

Implementation Needs: To scale up to include the encapsulation of large-scale wastes, new methodologies for encapsulation should be developed. To this end, a surplus $15 \mathrm{~kW} \mathrm{CO}$ laser from the Space Defense Initiative (SDI) program at ORNL is available to test the possible large-scale encapsulation of metal atoms in carbon clusters using the laser-based approach. We propose that this possibility be examined. The $\mathrm{CO}_{2}$ laser represents a $\$ 1 \mathrm{M}$ facility which could be devoted to this project. To implement this facility, the laser must first be put into operation. Trained operators continue to attend to this laser facility; therefore, putting the laser into operation will not be a problem. Also, it will be necessary to design and construct the actual radiation reactor and the separations technology for recovering the encapsulated waste material.

An estimated investment of $\$ 10 \mathrm{M}$ (1992 dollars) will be required to scale this process to a level where significant quantities of radioactive or hazardous materials can be safely fixed in 
graphitic spheres. The potential payback on this investment is expected to be in the range of $\$ 150 \mathrm{M}$, considering the waste treatment and disposal aspects of this technology and the potential spinoffs in other fields such as nuclear medicine and new materials development.

Authors: D. P. Armstrong/615-574-9327, R. E. Haufler/615-576-8644 and R. N. Compton/615-574-6233

\section{References:}

1. W. Kratschmer, L. D. Lamb, K. Fostiropoulos, and D. R. Huffman, "Solid C60: a New Form of Carbon," Nature, 347, 354, 1990.

2. H. W. Kroto, J. R. Heath, S. C. O'Brien, R. F. Curl, and R. E. Smalley, " $\mathrm{C}_{60}$ : Buckminsterfullerene," Nature, 318, 162, 1985.

3. Sumio lijima, "Helical Microtubules of Graphitic Carbon," Nature, 354, 56, 1991.

4. Sumio Ijima, Toshinari Ichihashi, and Yoshinori Ando, "Pentagons, Heptagons, and Negative Curvature in Graphite Microtubule Growth," Nature, 356, 767, 1992.

5. D. Ugarte, "Curling and Closure of Graphitic Networks Under Electron-Beam Irradiation," Nature, 359, 707, 1992. 
EM Problem: Waste management

Y-12 Plant Problem: Building 9201-4 (Alpha-4). Spent ion exchange media

Problem Area/Constituents: Ion exchange media are used extensively in liquid effluent treatment. While the media can be regenerated and recycled, they eventually loose their effectiveness and are called "spent ion exchange media." This spent media has to be disposed. To minimize hazardous waste volume, this media is treated to remove the undesirable components to permit disposal of the media as a nonhazardous waste. Before disposal, the spent media will be treated to remove, for example, inorganic, organic, or radioactive species.

\section{Reference Requirements:}

- Resource Conservation and Recovery Act of 1976 (RCRA), as amended, Land Disposal Restriction (LDR) Federal Facilities Compliance Agreement (FFCA) dated June 12, 1992

- Federal Facilities Agreement (FFA) for the Oak Ridge Reservation

- Federal Facilities Compliance Agreement-Toxic Substances Control Act (FFCA-TSCA) dated February 20, 1992

- Clean Air Act regulations under 40 CFR 60-65

- Clean Water Act regulations under 40 CFR 110-146 and 400-471

- DOE orders

Refer to the Regulatory Compliance Chapter of Vol. 1 for potentially applicable proposed and promulgated environmental laws, signed and pending agreements for the Oak Ridge Reservation, radiation protection standards, DOE orders, and nonregulatory guidance. As site- and waste-specific characteristics are provided for each technology, specific regulatory requirements will be specified.

Subelement: Waste Processing

Alternative: Chemical treatment

Technology: Elution. The removal of the adsorbed hazardous and/or radioactive compounds from spent ion exchange media using chemical agents such as acids, bases, and solvents. Elution is like the stripping operations that occur during the regeneration step.

Status: The status is labelled "demonstration," even though most elution operations are commercial technologies, because application of a particular elution operation to treat a given spent ion exchange medium may need to be demonstrated before it is used.

Science/Technology Needs: Because elution is closely linked to ion exchange operations, science/technology needs also are closely related. However, the efficacy of a given elution operation may need to be demonstrated for a given application before the operation is used full-scale. 
Implementation Needs: None, for the elution operations. However, treatment of the spent ion exchange medium before its disposal could significantly reduce waste disposal costs for spent media.

Author: S.P.N. Singh/615-574-6639

References: None 
EM Problem: Waste management

Y-12 Plant Problem: Building 9201-4 (Alpha-4). Scrap aluminum and liquid nitrate wastes

Problem Area/Constituents: Scrap aluminum generated during D\&D operations at Bldg. 9201-4.

\section{Reference Requirements:}

- Resource Conservation and Recovery Act of 1976 (RCRA), as amended, Land Disposal Restriction (LDR) Federal Facilities Compliance Agreement (FFCA) dated June 12, 1992

- Federal Facilities Agreement (FFA) for the Oak Ridge Reservation

- Clean Air Act regulations under 40 CFR 60-65

- Clean Water Act regulations under 40 CFR 110-146 and 400-471

- DOE orders

Refer to the Regulatory Compliance Chapter of Vol. 1 for potentially applicable proposed and promulgated environmental laws, signed and pending agreements for the Oak Ridge Reservation, radiation protection standards, DOE orders, and nonregulatory guidance. As site- and waste-specific characteristics are provided for each technology, specific regulatory requirements will be specified.

Subelement: Waste processing

Alternative: Chemical treatment

Technology: NAC process. According to Mattus et al., the NAC process can use contaminated scrap aluminum for denitration and disposal of nitrate wastes at several DOE facilities, such as those at Hanford, Idaho Falls, Oak Ridge, and Savannah River. The process causes aluminum to react with nitrate wastes to produce ammonia (which can be burned) and a ceramic-like alumina waste form (which can be disposed in land). Presently, this is a developmental technology. Mattus, et al., estimated that the process would require approximately $2.3 \mathrm{~kg}$ of aluminum per $\mathrm{kg}$ of nitrate waste. If aluminum is obtained at $\$ 0.88 / \mathrm{kg}$, the process is estimated to cost $\$ 2.01-\$ 2.66 / \mathrm{kg}$ of nitrate destroyed.

Status: Predemonstration.

Science/Technology Needs: The feasibility of the NAC process has been proven on a benchscale. However, further research and development needs to be conducted to establish the capabilities of the technology. For example, research needs to be conducted to determine if the final process waste form will encapsulate residual radioactive and hazardous components present in the contaminated aluminum and nitrate wastes. From a technology viewpoint, process capabilities need to be demonstrated on, for example, the 1-2 ton/hour pilot-plant scale. In addition, technology assessments need to be conducted to establish the viability and economics of the process. 
Implementation Needs: Further development of the process is expected to require $\$ 2 M-\$ 5 M$. However, because the process will dispose of two major, large volume, DOE waste streams (contaminated scrap aluminum and nitrates), its payback potential is expected to be at least one order of magnitude higher than the above-mentioned development costs. This is based on current disposal costs for these two streams.

Author: S.P.N. Singh/615-574-6639

\section{References:}

1. A. J. Mattus et al., A Low-Temperature Process for the Denitration of Hanford Single-Shell Tank Nitrate-Based Waste Utilizing the Nitrate to Ammonia and Ceramic (NAC) Process, ORNL/TM-12245 (draft report), October 1992. 
EM Problem: Waste management

Y-12 Plant Problem: Building 9201-4 (Alpha-4). Mercury contaminated concrete rubble and other solid wastes

Problem Area/Constituents: Volatile organic compounds (VOCs) in soil, contaminated concrete rubble, and PCBs in soil

\section{Reference Requirements:}

- Resource Conservation and Recovery Act of 1976 (RCRA), as amended, Land Disposal Restriction (LDR) Federal Facilities Compliance Agreement (FFCA) dated June 12, 1992

- Federal Facilities Agreement (FFA) for the Oak Ridge Reservation

- Federal Facilities Compliance Agreement-Toxic Substances Control Act (FFCA-TSCA) dated February 20, 1992

- Clean Air Act regulations under 40 CFR 60-65

- Clean Water Act regulations under 40 CFR 110-146 and 400-471

- DOE orders

Refer to the Regulatory Compliance Chapter of Vol. 1 for potentially applicable proposed and promulgated environmental laws, signed and pending agreements for the Oak Ridge Reservation, radiation protection standards, DOE orders, and nonregulatory guidance. As site- and waste-specific characteristics are provided for each technology, specific regulatory requirements will be specified.

Subelement: Waste processing

Alternative: Thermal treatment

Technology: Thermal desorption. Thermal treatment of wastes contaminated with organic compounds can be performed with many different treatment systems. However, the four main types of treatment systems are rotary dryer systems, indirect-fired systems, heated screw-type systems, and asphalt plant aggregate dryers. Each treatment system uses the same basic principal of operation. Each system consists of a furnace in which the organic compounds are desorbed from the waste feed. Desorbed organics are removed from the furnace by a purge gas and are destroyed in an afterburner or are collected by a physical/chemical treatment system. The systems are operated between 300 and $800^{\circ} \mathrm{F}$ and are fed with 5-100 tons of contaminated waste per hour. The desorbed organic compounds are removed from the furnace by a purge gas stream, which may flow co-current or countercurrent to the waste feed. A typical system consists of a feed pretreatment system, feed hoppers and conveyors, a co-current or counter-current furnace (directly or indirectly fired), a treated soil cooling system, cyclones, an afterburner, a bag house, an induced draft (ID) fan, and a stack. 
Status: Accepted by industry. Greater than $99 \%$ efficient in removing VOCs from soil. Costs range from $\$ 35 /$ ton (for low contamination-low moisture content soils) to $\$ 120 /$ ton. Thermal desorption of contaminated solids is in use at various remediation sites around the U.S. (some clean-ups have been completed).

Science/Technology Needs: The following concerns may cause problems during operation:

- Limitations exist on the types of waste and contaminants that can be treated.

- The presence of some contaminants may cause permitting problems.

\section{Implementation Needs:}

- Process equipment (e.g., indirect- or direct-fired rotary kiln, heated screw kiln, etc.)

- Minimum $2000 \mathrm{ft}^{2}$ equipment battery limit

- All utilities, including reliable electricity, cooling water, natural gas, steam, air, nitrogen, etc.

- Drum and material handling equipment

- Trained chemical operators

- U.S. Environmental Protection Agency (EPA)-certified laboratory support

Author: C. H. Dukes/615-574-9561

\section{References:}

1. J.J. Cudahy et al., "Petroleum Contaminated Soils and Sludges-Markets, Permitting and Thermal Desorption Technologies," Proceedings of the 1992 Incineration Conference, Albuquerque, NM, May 1992.

2. R. K. Nielson and M. G. Cosmos, Low Temperature Thermal Treatment $\left(\mathrm{LT}^{3}\right)$ of Volatile Organic Compounds from Soil: A Technology Demonstration, paper presented at the 1988 Summer National Meeting of the AIChE, Denver, CO, August 1988. 
EM Problem: Waste management

Y-12 Plant Problems: Building 9201-4 (Alpha-4). Contaminated concrete rubble and other contaminated mixed waste solids and liquids

Problem Area/Constituents: Treatment of mixed-waste contaminated solids such as TSCA Incinerator wet ash and secondary solid effluents containing PCBs, organics, inorganics, radionuclides, and metals

\section{Reference Requirements:}

- Resource Conservation and Recovery Act of 1976 (RCRA), as amended, Land Disposal Restriction (LDR) Federal Facilities Compliance Agreement (FFCA) dated June 12, 1992

- Federal Facilities Agreement (FFA) for the Oak Ridge Reservation

- Federal Facilities Compliance Agreement-Toxic Substances Control Act (FFCA-TSCA) dated February 20, 1992

- Clean Air Act regulations under 40 CFR 60-65

- Clean Water Act regulations under 40 CFR 110-146 and 400-471

- DOE orders

Refer to the Regulatory Compliance Chapter of Vol. 1 for potentially applicable proposed and promulgated environmental laws, signed and pending agreements for the Oak Ridge Reservation, radiation protection standards, DOE orders, and nonregulatory guidance. As site- and waste-specific characteristics are provided for each technology, specific regulatory requirements will be specified.

Subelement: Waste processing

Alternative: Thermal treatment

Technology: Plasma Arc Furnace (PAF). This technology builds on plasma hearth melting developments. ${ }^{1}$ The plasma arc furnace is a new and promising technology that uses an electrically heated plasma gas to destroy organic contaminants and bind radionuclides and heavy metals in a glassy slag. The PAF represents an alternative to cementation and incineration that may be more acceptable to the public.

The plasma technology typically includes a continuous plasma torch operated in transfer mode (one end of the torch arc impinges on the material being heated) or non-transferred mode. The system includes an enclosed feeder, an afterburner, a slag removal system, a waste-gas chiller, a waste-gas scrubber, a continuous emissions monitoring system, stack samplers, and various controls and diagnostic equipment. The PAF can be operated under reducing or oxddizing conditions.

The term "plasma" refers to a highiy ionized gas. Plasmas can be generated by a variety of techniques and occur over a wide range of pressures and energy levels. Typically, a torch 
uses a flowing gas to stabilize an electrical discharge (arc) between two electrodes. One or both of these electrodes is contained within the torch. For treatment of solid materials, the second electrode is usually the material being processed (using arc welding terminology, this is called the "work piece"). Energy is dissipated as heat and light as the electrical current flows through the gas. Through resistance heating (Joule heating), this process creates a high-temperature gas as well as directly heats the work piece. ${ }^{2}$ Plasma torches have high energy densities with local temperatures up to $15,000^{\circ} \mathrm{K} .{ }^{1}$ Qualitative benefits of this technology are high-integrity final waste form, portability, and low off-gas and contamination control. Potential disadvantages are that plasma processes are very energy intensive, and power costs could limit applications to small-scale. ${ }^{6}$

Status: Demonstration; the following pilot-scale systems are installed:

- Centrifugal Retech Furnace-Switzerland

- Centrifugal Retech Furnace, DOE/EPA Site Program-Butte, Montana

- Fixed Hearth Retech Furnace-Ukiah, CA

- Plasma Facility-Charleston, S.C. ${ }^{3}$

- Westinghouse Pilot Facility-Pittsburgh, $\mathrm{PA}^{3}$

Some operating and emissions data exist for these facilities, but additional data are needed for process design and scale-up to meet DOE mixed waste management objectives for treatment of K-25 Site waste streams.

Science/Technology Needs: Research and development (R\&D) is required to assess metals carry-over from the primary chamber (hot zone) and optimize slag chemistry regarding metals stabilization, variations in waste input streams, reintroduction of condensed volatile metals into the slag phase, and radionuclide partitioning ${ }^{4}$ in the effluent streams, including metal specification studies in the entrained particulates in the off-gas as a function of particle size. Additional development is required to determine and improve electrode life, materials of construction in general, destruction and removal efficiency of hazardous/toxic organics, power efficiency, mass/energy balances to effect minimum secondary waste generation, and optimal safe operating methods as a function of heterogeneous waste processing.

Implementation Needs: The cost of demonstrating this technology to treat a wide range of low-to-high ash inorganic/organic waste feed streams to produce a low leachable slag is estimated to be $\$ 10 \mathrm{M}$. Estimated time to complete full-scale demonstration to treat specific K-25 Site waste streams at 1-2 tons/hour capacity is 3 years (Funding requests: FY 1993: \$0.5M; FY 1994: \$1.5M; and FY 1995: \$1.0M).

Author: W. H. Hermes/615-574-9568

September 1994

Decontamination and Decommissioning 


\section{References:}

1. W. Hoffelner et al., "Plasma Technology for Rapid Oxidation, Melting and Vitrification of Low/Medium Radioactive Waste," Nuclear Engineering International, October 1992.

2. M. I. Morris, The Plasma Hearth Process: The Fixed Hearth Arc Plasma Treatment Process for Mixed Waste Treatment Applications, draft TTP, December 10, 1992.

3. W. H. McCulla, personal communication with M. I. Morris, December 1, 1992.

4. J. B. Berry et al., Mixed Waste Treatment Research, Development, Demonstration, Testing and Evaluation, draft report, DOE Office of Technology Development, December 1992.

5. Retech, Inc., Plasma Centrifugal Furnace Applications Analysis Report, EPA/540/A591/007, June 1992.

6. L. C. Borduin et al., Thermal and Photochemical Processes Applicable to Environmental Restoration and Waste Management Operations, 1989.

7. C. G. Whiteworth et al., Slag Chemistry and Metals Volatilization in the Plasma Arc Furnace Experiment, background file.

8. W. H. Hermes, background file containing personal notes of discussions with SAIC regarding emissions from centrifugal furnace in Switzerland, Retech Lab-Scale System, and Fixed Hearth Plasma System at Ukiah, CA. 


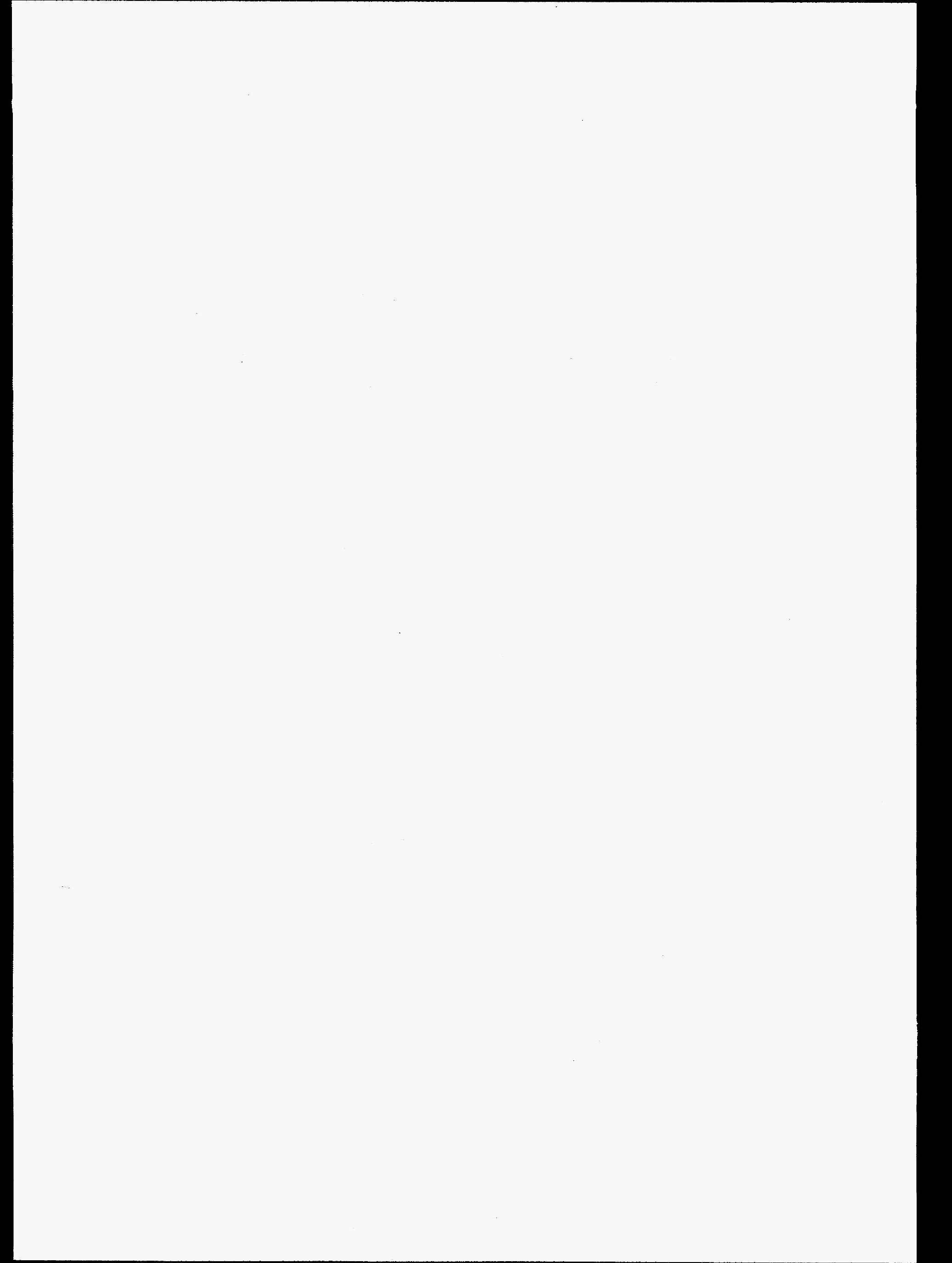




\section{EM Problem: Waste management}

Y-12 Plant Problem: Treatment and disposal of uncontaminated, hazardous, toxic and/or mixed-waste-contaminated solids, liquids, and gases generated from decontamination and decommissioning (D\&D), remedial action (RA), and waste management (WM) operations

Problem Area/Constituents: Uncontaminated and hazardous, toxic, and/or mixed-wastecontaminated solids, sludges, liquids, and gases generated as a result of D\&D, RA, and WM operations at the site

\section{Reference Requirements:}

- Resource Conservation and Recovery Act of 1976, as amended (RCRA) Land Disposal Restriction (LDR) Federal Facilities Compliance Agreement (FFCA) dated 12 June 1992

- Federal Facilities Agreement (FFA) for Oak Ridge Reservation (ORR)

- Federal Facilities Compliance Agreement-Toxic Substances Control Act (FFCA-TSCA) dated 20 February 1992

- Resource Conservation and Recovery Act and the Hazardous and Solid Waste Amendments of 1984, 40 CFR 260-270

- NEPA, 40 CFR 1500-1508

- National Emissions Standards for Hazardous Air Pollutants, 40 CFR 61, Subpart H

- DOE Order 5480.11, "Radiation Protection for Occupational Workers" (draft)

- DOE Order 5480.12, "Prevention, Control, and Abatement of Environmental Pollution"

- $\quad 40$ CFR 270.32 ("omnibus" authority)

- $\quad 40$ CFR 264.342(b) (POHC selection)

- Clean Air Act regulations under 40 CFR 60-65

- Clean Water Act regulations under 40 CFR 110-146 and 400-471

- Toxic Substances Control Act

- RCRA Part 64 Subpart 0

Refer to the Regulatory Compliance chapter of Vol. 1, Pt. C, for potentially applicable proposed and promulgated environmental laws, signed and pending agreements for the $O R R$, radiation protection standards, DOE orders, and nonregulatory guidance. As site- and wastespecific characteristics are provided for each technology, specific regulatory requirements will be specified.

Subelement: Waste processing

Alternatives: Thermal treatment

Technology: Incineration. Incineration is an engineered process that employs decomposition via thermal axidation at high temperature (usually $900^{\circ} \mathrm{C}$ or greater) to destroy the organic fraction of the waste and reduce volume. ${ }^{1}$ Generally, combustible wastes or wastes with 
significant organic content are considered most appropriate for incineration. Technically, however, any waste with an organic fraction is a candidate for incineration. For instance, significant amounts of contaminated water were being incinerated in the United States during the early $1980 \mathrm{~s}^{2}$ Contaminated soils are also being incinerated with increasing frequency. The U. S. Environmental Protection Agency (EPA), for example, has employed a mobile incinerator to decontaminate 40 tons of Missouri soil that had been contaminated with $4 \mathrm{lb}$ of chlorinated dioxin compounds. ${ }^{3-6}$

The key element of any incineration system is the combustion reactor, or combustor. The combustor is supported by several subsystems that together with the combustion reactor make up a total incineration system. These subsystems include feed preparation and delivery systems, ash removal and packaging systems, off-gas cleaning systems, system controls, and auxiliary utility supply systems. The state of technology of incineration systems could be made sufficient to provide safe and effective treatment of low-level radioactive, hazardous, and toxic wastes. Not all proven incineration systems are designed to handle all these waste feed types. ${ }^{7}$ The incinerator design is based on specific waste profiles to establish basis flows, temperatures, residence times, and mixing requirements to meet waste treatment processing objectives and pollutant destruction and removal efficiencies (DREs).

Several different types of incinerators are available for waste destruction [e.g., rotary kiln, fluidized bed, multiple hearth (or Herreshoff furnace), liquid injection, and controlled air incinerators, to name a few]. Brief descriptions of these incinerators follow:

Rotary kiln. A rotary kiln incinerator is a slowly-rotating, refractory-lined horizontal cylindrical shell mounted at a slight slope. Waste can move concurrent or countercurrent to gas flow. The primary function is to destroy the wastes. This occurs through a series of steps including volatilization, destructive distillation, and combustion reactions. A secondary chamber is generally required for complete destruction of the wastes. The advantages of a rotary kiln are that it is robust and highly versatile and that it can handle solids, slurries, liquids, and gases. The disadvantages of a rotary kiln incinerator include difficulty in controlling the incineration process, seals in-leakage, and refractory replacement.

Fluidized bed. A fluidized-bed incinerator is basically a vertical single refractory-lined vessel partially filled with sand, alumina, or crushed lime or limestone. Fluidized bed incinerators can be either circulating or bubbling bed in design. Combustion air is supplied through a distribution plate to fluidize the bed of particles. Wastes to be incinerated are usually fed into the bed of particles. Circulating bed incinerators usually require a cyclone to return the entrained particles to the bed. The advantages of the fluidized bed incinerator include the uniformity of the bed temperature (thus ensuring better control of the waste DRE), its ease of operation in the range of 1400 to $1600^{\circ} \mathrm{F}$ with wide range in excess air to allow better metal volatility control, and its compact size. Some of the disadvantages of the fluidized bed incinerator include the requirement that the solid wastes generally be $<2$ in. in size (thus it cannot be used for incinerating large bulk solids), significant carryover of the bed material, and the presence of tarry wastes can cause operational problems due to "slumping" or defluidization of the bed.

September 1994

Decontamination and Decommissioning 
Multiple hearth. The multiple hearth incinerator ${ }^{8}$ (also called the Herreshoff furnace) basically consists of a vertical cylindrical refractory-lined furnace or combustion chamber containing several hearths. These hearths are stacked one on top of the other inside the furnace. A slowly rotating hollow vertical shaft passes through the center of the hearths. Hollow rabble arms are attached to this central shaft on each hearth to rake the wastes on the hearth. The rabble arms and shaft are cooled by passing combustion air through the assembly. This combustion air is then fed to the bottom of the furnace below the lowest hearth. Temperatures in the furnace can reach upwards of $1800^{\circ} \mathrm{F}$. The wastes to be incinerated are usually fed on the top hearth. The wastes then pass through the many hearths in the furnace and the uncombusted residue is discharged at the bottom. The advantages of a multiple hearth incinerator include its versatility, large throughput, and ability to control the residence time of the solid wastes in the incinerator. The disadvantages include higher capital, operating, and maintenance costs.

Liquid injection. A liquid injection incinerator consists of a hollow refractory-lined horizontal or vertical cylinder equipped with one or more burners where liquids are atomized into droplets and burned in suspension. The incinerator is especially designed for the destruction of fluid wastes. The advantages of the incinerator include excellent control of the destruction of the liquid wastes. The disadvantages include its limitation to the destruction of pumpable liquids only and the need to ensure excellent atomization of the liquid wastes to obtain the required DREs.

Controlled air. A controlled-air incinerator ${ }^{8}$ is also known as a fixed-hearth incinerator or pyrolytic furnace. Briefly, it consists of a simple incinerator employing two combustion chambers. In the first chamber the waste is pyrolyzed by operating at 50 to $80 \%$ stoichiometric air. This causes the volatile fraction of the waste to be vaporized by the exothermic heat provided by the oxidation of the fixed carbon fraction. Complete combustion of the pyrolyzed waste is then obtained in the second chamber by operating at significant excess air. The advantages of the controlled air incinerator include minimization of the particulate entrainment from the primary to the secondary combustion chamber and the generally compact size of the incinerator. Disadvantages include its smaller capacity due to physical limitations in feeding and transporting the waste through the incinerator.

Status: Accepted. The EPA considers incineration to be the best demonstrated accepted technology (BDAT) for the destruction and disposal of combustible hazardous and/or toxic wastes.

Science/Technology Needs: Incineration is proven technology for the disposal of combustible hazardous, toxic, and low-level radioactive mixed wastes. However, off-gas particulate emission regulations, overall stack emissions of metals, and final ash waste form leachability characteristics are general areas where applying research, development, and demonstration (RD\&D) could prove to be cost-effective. The RD\&D effort is both regulation and public perception driven. Understanding steady-state and upset trace emissions from incinerators (and thereby instituting mitigative feed and operational controls to reduce emissions) are other science and technology needs. 
Implementation Needs: It is impractical to predict the cost of a new incinerator without having information on the incineration system design. However, it is instructional to note that the capital cost for the 30MBtu/h K-25 Site Toxic Substances Control Act (TSCA) Incinerator was \$26M (1987 dollars) and that it took 8-10 years to obtain the necessary regulatory permits for operation. The current (circa 1993) waste destruction costs at the K-25 Site TSCA incinerator are estimated to be $\$ 6 / \mathrm{lb}$ of waste.

The estimated costs for an RD\&D program to address some of the science and technology needs is \$5M (1992 dollars) for a three-year program.

Authors: W. H. Hermes/615-574-9568 and S. P. N. Singh/615-574-6639

\section{References:}

1. C. R. Dempsey et al., "Incineration of Hazardous Waste: A Critical Review Update," Air Waste Manage. 43 (January 1993).

2. G. Vogel et al., Composition of Hazardous Waste Streams Currently Incinerated, report by Mitre Corp. for the U.S. Environmental Protection Agency, April 1983.

3. S. Tejada, "The Blue Goose Flies!" EPA Journal, October 1985.

4. J.J. Yezzi et al., "Results of the Initial Trial Burn of the EPA-ORD Mobile Incineration System," in Proceedings of the ASME 1984 National Waste Processing Conference, Orlando, Fla., June 1984.

5. H. Mortensen et al., Destruction of Diaxin Contaminated Solids and Liquids by Mobile Incineration, EPA Final Report EPA/600/R2-87/033, U.S. Environmental Protection Agency, June 1987.

6. F. Freestone et al., "Evaluation of On-Site Incineration for Clean-Up of DioxinContaminated Materials," Nucl Chem. Waste Manage. 7, 3-20 (1987).

7. R. A. Koernig, Summary of Radioactive and Mixed Waste Incineration Technologies Within DOE Nuclear Weapons Complex, prepared by Congress of the United States Office of Technology Assessment, Merlin CO/Boulder, Inc., February 1991.

8. C. R. Bruner, Incineration Systems Selection and Design, Incinerator Consultants, Inc., Reston, Va., 1988. 
EM Problem: Waste management

ORNL Problem: Organically contaminated gaseous and liquid effluents and spent ion exchange media

Problem Area/Constituents: Destruction of organics (including halogenated organics) in gas or liquid effluents and radioactively contaminated spent ion exchange resins

\section{Reference Requirements:}

- Resource Conservation and Recovery Act of 1976 (RCRA), as amended, Land Disposal Restriction (LDR) Federal Facilities Compliance Agreement (FFCA) dated June 12, 1992

- Federal Facilities Agreement (FFA) for the Oak Ridge Reservation (ORR)

- Federal Facilities Compliance Agreement-Toxic Substances Control Act (FFCA-TSCA) dated February 20, 1992

- Clean Air Act regulations under 40 CFR 60-65

- Clean Water Act regulations under 40 CFR 110-146 and 400-471

- DOE orders

Refer to the Regulatory Compliance chapter of Vol. 1, Pt. C, for potentially applicable proposed and promulgated environmental laws, signed and pending agreements for the ORR, radiation protection standards, DOE orders, and nonregulatory guidance. As site- and wastespecific characteristics are provided for each technology, specific regulatory requirements will be specified.

\section{Subelement: Waste processing}

Alternative: Thermal treatment

Technology: Catalytic destruction. Catalytic destruction of organics offers a number of advantages over other traditional methods of destruction or removal of these compounds, such as thermal incineration or carbon adsorption. The lower temperatures and shorter residence times required for catalytic oxidation as compared to thermal oxidation translate into lower capital and operating costs. Complete catalytic destruction minimizes the risk of formation of products of incomplete combustion (PICs), such as carbon monoxide (CO), and the production of nitrogen oxides $\left(\mathrm{NO}_{x}\right)$ is much lower at the temperatures required for catalytic oxidation. Adsorption on granular actived carbon is often a process that is used because of low capital requirement and operating simplicity. However, when the quantity of the contaminant is large or has a low adsorption factor on the carbon, the operating cost for the adsorption process becomes noncompetitive, particularly when the need to regenerate or dispose of contaminated spent carbon is considered.

Catalytic destruction techniques in various media (gaseous, liquid, solid) are presented as follows: 


\section{Removal of organics in gaseous effluents}

Pulse combustion-based technique..$^{1,2}$ A significant amount of research and development has been performed internationally on pulse combustors in recent years. Maximum volatile organic compound (VOC) destruction efficiency greater that $99 \%$ was achieved in some experiments. The concept relies on unique characteristics of pulse combustors to effect a twostage (thermal and catalytic) destruction process by exploiting both the high combustion intensity in the pulse combustor chamber and the acoustic resonances in the combustor tailpipe. High combustion intensity results from rapid fluid dynamic mixing and will lead to compact, transportable designs; acoustic resonances result in significantly increased mass transport to the catalyst, leading to high destruction efficiencies.

Conventional catalytic axidation technique..$^{3-6}$ Halogenated organic compounds, such as C-1 chlorocarbons, trichloroethylene, O-dichlorobenzene, dioxins, furans, etc., can be catalytically destructed and removed from the gaseous effluents at varied temperatures, about $200-500^{\circ} \mathrm{C}$, substantially lower than regular incineration without the application of a catalyst. Optimal cracking temperatures vary, depending on factors such as the type of catalyst used and the chemical composition of the gaseous stream. A potential disadvantage of catalytic oxidizers, however, is that the conventional precious metal catalysts may be deactivated, or poisoned, by various volatile materials. During recent years, different halohydrocarbon destruction catalysts (HDCs) have been developed, some successfully implemented in the industrial applications. An HDC is designed to tolerate chlorine and other halogens and is expected to be very effective in promoting the oxidation of halohydrocarbons at temperatures below $450^{\circ} \mathrm{C}$. Some catalysts tested in the laboratory were prepared by washcoating ceramic honeycomb supports with either the HDC formulation or with a state-of-the-art chromiaalumina catalyst powder.

2. Catalytic destruction of organics in liquid effluents ${ }^{4}$

A catalytic process has been developed at Pacific Northwest Laboratory for destroying hazardous organics and chlorinated organics, including spent solvents, in aqueous waste streams. Experiments have been conducted in a batch reactor, a bench-scale continuous stirred tank, and a continuous flow tubular reactor. The process can destroy a wide variety of organic compounds at $300^{\circ} \mathrm{C}$ and $2000-3000$ psig in the presence of a nickel catalyst. It was originally conceived to treat industrial wastewater with high chemical- and biochemicaloxygen-demand loadings caused by the presence of organics. It is most applicable to waste streams that are too dilute for incinceration but too concentrated for conventional biological treatment (typically 1-10\% organic). The process can also be used to treat aqueous wastes resulting from cleanup of hazardous waste sites and condensates, as well as wastewater contaminated with organics from washing, flushing, and stripping.

3. Incineration of spent ion exchange resins in combination with a catalytic fluid bed ${ }^{7}$

This is a waste minimization process for handling large quantities of radioisotopecontaminated spent ion exchange resins generated from nuclear power plant operations. The

September 1994

Decontamination and Decommissioning 
Oarai Research Establishment of the Japan Atomic Energy Research Institute has operated a pilot plant that consists of an incineration system, resin feed system, and off-gas cleaning system. In the incinerator, activated alumina is used as fluid bed material, and the catalyst is copper oxide coated on the surface of a porous ceramic frame made from $\mathrm{Al}_{2} \mathrm{O}_{3}, \mathrm{ZrO}_{2}$, and $\mathrm{SiO}_{2}$. Experimental data indicate that the pilot unit could confine almost all radioactivities in itself during the incineration so that radioactivities released into the operation area were few, and those to the environment were below regulatory limits.

\section{Status: Predemonstration}

Science/Technology Needs: A complete investigative study of all experimental work done on the subject process must be conducted. Development work at the bench scale then will be performed for VOCs of interest, and their combustion chemistry will be established. If technical viability and economics justify further work, scale-up to pilot- or full-scale processes will follow.

Implementation Needs: The above-mentioned development study is expected to cost $\$ 0.75 \mathrm{M}-\$ 1.5 \mathrm{M}$ (1992 dollars). As development work progresses and technical promise is clearly demonstrated, collaborative research efforts with the private sector can be established. As a result, lower implementation costs may be possible, particularly for the latter part of the development activities.

Author: J. M. Chiang/615-574-9659

\section{References:}

1. T. T. Bramlette, J. O. Keller, M. A. Allendorf, and P. K. Barr, A Pulse Combustion-Based VOC Destruction Technique, Sand-92-8429C.

2. Anon., Air Pollution Control Feasibility Study, Report No. 4998.006 (prepared by O'Brien \& Gere Engineers, Inc.), Westinghouse Savannah River Co., Aiken, S.C., August 1991.

3. E. Baker, D. C. Elliot, L. Sealock, and G. Neuenschwander, "Catalytic Destruction of Organics and Chlorinated Organics with TEES II," International Incineration Conference Proceedings, Albuquerque, N. M., 1992.

4. F. Buck and C. Hauck, "Vapor Extraction and Catalytic Oxidation of Chlorinated VOC," King, Buck \& Associates, Inc., San Diego, 1992.

5. I. M. Freidel, "Catalytic Destruction of Chlorinated Organics Including PCDDs/PCDFs in Flue Gas from Waste Incinerators," Allied Signal Industrial Catalysts, Tulsa, Okla., 1992.

6. M. Hiraoka and N. Takeda, "Catalytic Destruction of PCDDs/PCFFs in Municipal Solid Waste Flue Gas," Department of Environmental and Sanitary Engineering, Kyoto University, Japan, 1991.

7. T. Yahata, S. Kihara, M. Hirata, and M. Abe, Incineration of Spent Ion Exchange Resins in Combination with Fluidized Bed and Catalyst, Japan Atomic Energy Research Institute, Japan, 1992. 


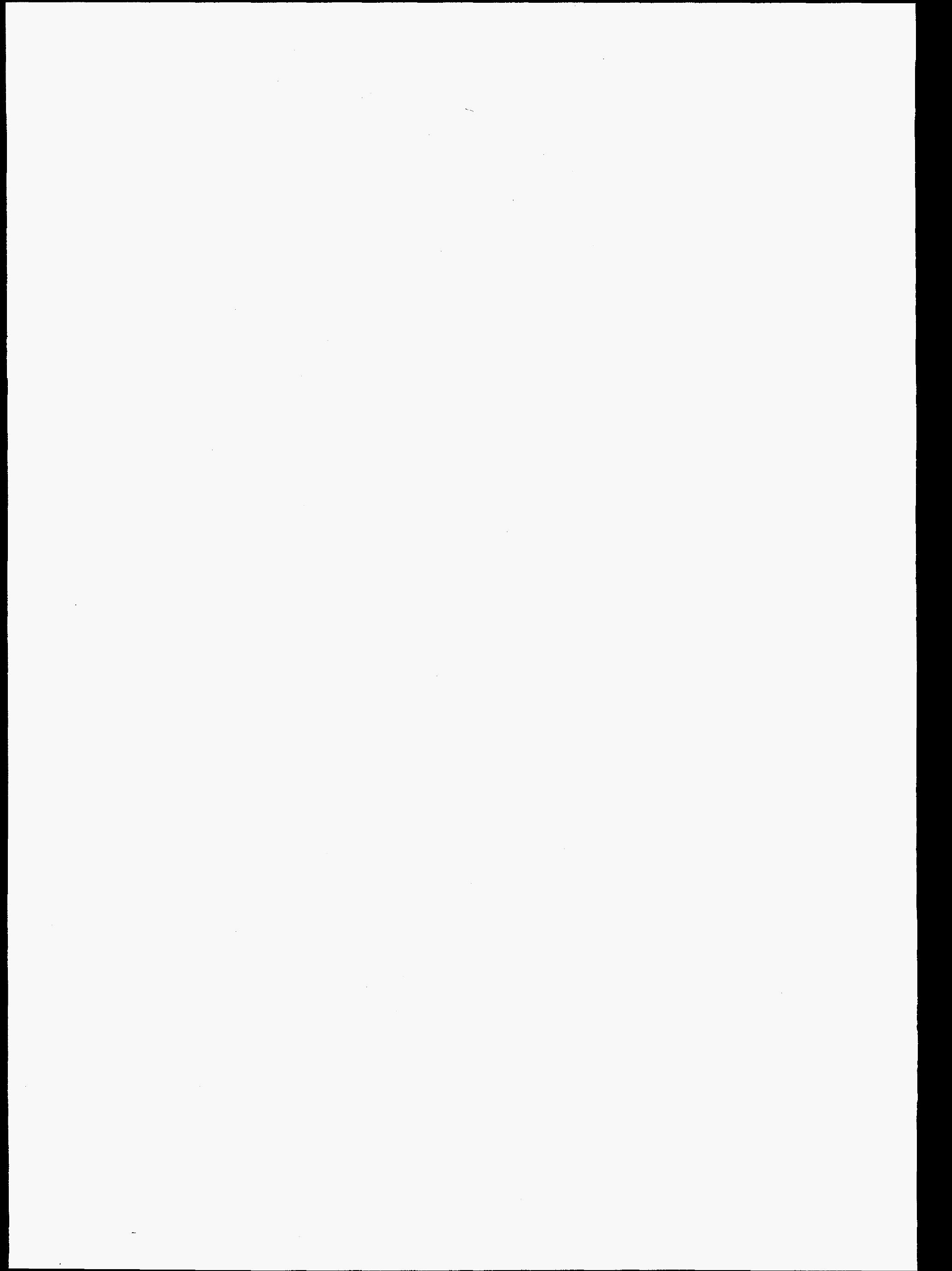


EM Problem: Waste management

Y-12 Plant Problem: Building 9201-4 (Alpha-4). Other scrap building materials (e.g., wood, tile, roofing, fixtures, gaskets)

Problem Area/Constituents: Treatment of other scrap building materials (e.g., wood, tile, roofing, fixtures, gaskets, etc.). Pyrolysis is most applicable for the following types of waste:

- Waste stored in containers or drums that cannot be easily drained

- Sludges or liquids that contain (1) high ash content; (2) volatile inorganics (e.g., $\mathrm{NaCl}$, $\mathrm{FeCl}_{2}, \mathrm{Zn}$, and $\mathrm{Pb}$ ); and (3) high levels of chlorine, sulfur, and/or nitrogen

\section{Reference Requirements:}

- Resource Conservation and Recovery Act of 1976 (RCRA), as amended, Land Disposal Restriction (LDR) Federal Facilities Compliance Agreement (FFCA) dated June 12, 1992

- Federal Facilities Agreement (FFA) for the Oak Ridge Reservation

- Federal Facilities Compliance Agreement-Toxic Substances Control Act (FFCA-TSCA) dated February 20, 1992

- Clean Air Act regulations under 40 CFR 60-65

- Clean Water Act regulations under 40 CFR 110-146 and 400-471

- DOE orders

Refer to the Regulatory Compliance Chapter of Vol. 1 for potentially applicable proposed and promulgated environmental laws, signed and pending agreements for the Oak Ridge Reservation, radiation protection standards, DOE orders, and nonregulatory guidance. As site- and waste-specific characteristics are provided for each technology, specific regulatory requirements will be specifled.

Subelement: Waste processing

Aternative: Thermal treatment

Technology: Pyrolysis combustion. Pyrolysis is defined as the chemical decomposition or change brought about by heating in the absence of oxygen. Application of pyrolysis to hazardous-waste treatment leads to a two-step process for disposal. In the first step, wastes are heated, separating the volatile components (combustible gases, water vapor, etc.) from nonvolatile char and ash. In the second step, volatile components are destroyed under proper conditions to assure incineration of all hazardous components.

The first step in pyrolysis treatment of hazardous waste is endothermic and generally is done at $425-760^{\circ} \mathrm{C}\left(800-1400^{\circ} \mathrm{F}\right)$. Normal feed rate is $0.25-5$ tons of contaminated waste per hour. The heating chamber is called the pyrolyzer. The endothermic property makes the process much easier to control. Hazardous organic compounds can be volatilized at this low temperature, leaving a clean residue. The volatiles are burned (second step) in a fume 
incinerator at conditions that make it possible to achieve destruction efficiency of more than

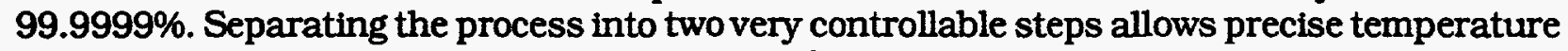
control and makes possible the construction of smaller equipment.

Status: Accepted by industry, and units are in use. Removal efficiencies of much greater than $99 \%$ are expected. Cost is unknown, but it is expected to be like thermal desorption (\$35-\$120/ton).

Science/Technology Needs: The following may cause problems during operation:

- Energy content in a waste feed may be retained by the char.

- Products of incomplete combustion (PICs), principal organic hazardous constituents (POHCs), and carcinogens present in the waste require fume incineration.

- Solids may prematurely enter the second stage resulting in shutdown.

- High water content in the waste may quench, or high plastic content may overwhelm the combustion process.

\section{Implementation Needs:}

- Process equipment (e.g., indirect- or direct-fired rotary kiln, heated screw kiln, etc.)

- Minimum 2,000 $\mathrm{ft}^{2}$ equipment battery limit

- All utilities, including reliable electricity, cooling water, natural gas, steam, air, nitrogen, etc.

- Drum and material handling equipment

- Trained chemical operators

- EPA-certified laboratory support

Author: C. H. Dukes/615-574-9561

\section{References:}

1. H. M. Freeman, ed., Standard Handbook of Hazardous Waste Treatment and Disposal, McGraw-Hill, New York, p. 8.91-8.104, 1989. 
EM Problem: Waste management

T-12 Plant Problem: Treatment of contaminated ferrous and un-ferrous metals generated during D\&D of Alpha-4.

Problem Area/Constituents: Removal of inorganic, organic, and radioactive compounds from scrap aluminum, copper, and other ferrous and nonferrous metals

\section{Reference Requirements:}

- Resource Conservation and Recovery Act of 1976 (RCRA), as amended, Land Disposal Restriction (LDR) Federal Facilities Compliance Agreement (FFCA) dated June 12, 1992

- Federal Facilities Agreement (FFA) for the Oak Ridge Reservation

- Federal Facilities Compliance Agreement-Toxic Substances Control Act (FFCA-TSCA) dated February 20, 1992

- Clean Air Act regulations under 40 CFR 60-65

- Clean Water Act regulations under 40 CFR 110-146 and 400-471

- DOE orders

Refer to the Regulatory Compliance Chapter of Vol. 1 for potentially applicable proposed and promulgated environmental laws, signed and pending agreements for the Oak Ridge Reservation, radiation protection standards, DOE orders, and nonregulatory guidance. As site- and waste-specific characteristics are provided for each technology, specific regulatory requirements will be specified.

Subelement: Waste processing

Alternative: Thermal treatment

Technology: Smelting. ${ }^{1.2}$ A pyrometallurgical process for decontaminating and treating scrap metals such as aluminum, copper, and other ferrous and non-ferrous metals generated during decontamination of the site. Basically, smelting consists of heating contaminated scrap metal in a furnace with various fluxes (such as limestone and coke) to high temperatures (generally, $700-2000^{\circ} \mathrm{C}$ ) in the presence of reducing gases to yield molten metal and a slag or dross. Organic contaminants are destroyed during the smelting operation, and nonvolatile inorganic and radioactive species are (hopefully) removed with the slag. The slag can be further processed (if necessary) and disposed while the metal is cooled, cast in molds, and recycled (provided that radioactive contamination is within regulatory limits).

Status: Accepted by industry. However, satisfactory application of the technology to treat radioactively-contaminated metals at the site needs to be demonstrated.

Science/Technology Needs: The major science/technology needs for using smelting to treat mixed-waste-contaminated metals consists of demonstrating that the radioactive species are removed preferentially with the slag or dross resulting from the smelting operations and that 
the slag can be disposed in compliance with environmental regulations. This may require bench-scale studies and demonstrations to prove its applicability.

Implementation Needs: Development and demonstration of the technology to treat mixedwaste contaminated metals are estimated to cost $\$ 3 \mathrm{M}-\$ 5 \mathrm{M}$ (1992 dollars). However, the payback could be significant when compared to the cost of alternative treatment options. Also, the process could result in significant waste minimization because the metals could be recovered and recycled.

Author: S.P.N. Singh/615-574-6639

\section{References:}

1. D. M. Considine, ed., Chemical and Process Technology Encyclopedia, McGraw-Hill, New York, 1974.

2. R. N. Shreve, Chemical Process Industries, McGraw-Hill, New York, 1967. 
EM Problem: Waste management

Y-12 Plant Problem: Building 9201-4 (Alpha-4). Scrap contaminated nickel

Problem Area/Constituents: Contaminated nickel generated during D\&D operations at Building 9201-4

\section{Reference Requirements:}

- Resource Conservation and Recovery Act of 1976 (RCRA), as amended, Land Disposal Restriction (LDR) Federal Facilities Compliance Agreement (FFCA) dated June 12, 1992

- Federal Facilities Agreement (FFA) for the Oak Ridge Reservation

- Clean Air Act regulations under 40 CFR 60-65

- Clean Water Act regulations under 40 CFR 110-146 and 400-471

- DOE orders

Refer to the Regulatory Compliance Chapter of Vol. 1 for potentially applicable proposed and promulgated environmental laws, signed and pending agreements for the Oak Ridge Reservation, radiation protection standards, DOE orders, and nonregulatory guidance. As site- and waste-specific characteristics are provided for each technology, specific regulatory requirements will be specified.

Subelement: Waste processing

Alternative: Thermal treatment

Technology: Mond process. Also known as the carbonyl process. In this process, contaminated nickel reacts with carbon monoxide $(\mathrm{CO})$ at around $100^{\circ} \mathrm{C}$ to form nickel carbonyl $\left[\mathrm{Ni}(\mathrm{CO})_{4}\right]$. The nickel carbonyl is taken to another reactor containing uncontaminated nickel pellets and heated to around $200-300^{\circ} \mathrm{C}$. The nickel carbonyl decomposes at these temperatures to yield nickel metal and $\mathrm{CO}$. The nickel is deposited on the pellets, and the $\mathrm{CO}$ is recycled to the first reactor. This process is highly selective for the recovery of nickel, because carbonyls of the other elements present in the contaminated nickel are not likely to be formed under the above process's operating conditions. ${ }^{1}$

\section{Status: Demonstration}

Science/Technology Needs: Although the Mond process is commercial technology, its applicability to satisfactory treatment of the porous nickel recovered from D\&D operations at the K-25 Site needs to be demonstrated.

Implementation Needs: Technology demonstration is estimated to cost \$1M-\$2M (1992 dollars). However, if the process can satisfactorily decontaminate the nickel to the desired level, the payback for the process is expected to be greater than $\$ 20 \mathrm{M}-\$ 30 \mathrm{M}$, based on estimates of current disposal costs.

Author: S.P.N. Singh/615-574-6639 


\section{References:}

1. "Nickel and Nickel Alloys," Kirk-Othmer Encyclopedia of Chemical Technology, 3rd Edition, Vol. 15, John Wiley and Sons, New York, New York, 1982. 
EM Problem: Waste management

Y-12 Plant Problem: Building 9201-4 (Alpha-4). Scrap plastics, paper, and other organic contaminated wastes

Problem Area/Constituents: Organic ion-exchange media, radionuclides and technetium in oil, heavy metals in oil, PCB in oil, nonhalogenated organics in oil, mercury in oil, radionuclides in solvents, heavy metals in solvents, PCB in solvents, plastics, paper, cloth, organics, wood, combustible residues, organics.

\section{Reference Requirements:}

- Resource Conservation and Recovery Act of 1976 (RCRA), as amended, Land Disposal Restriction (LDR) Federal Facilities Compliance Agreement (FFCA) dated June 12, 1992

- Federal Facilities Agreement (FFA) for the Oak Ridge Reservation

- Federal Facilities Compliance Agreement-Toxic Substances Control Act (FFCA-TSCA) dated February 20, 1992

- Clean Air Act regulations under 40 CFR 60-65

- Clean Water Act regulations under 40 CFR 110-146 and 400-471

- DOE orders

Refer to the Regulatory Compliance Chapter of Vol. 1 for potentially applicable proposed and promulgated environmental laws, signed and pending agreements for the Oak Ridge Reservation, radiation protection standards, DOE orders, and nonregulatory guidance. As site- and waste-specific characteristics are provided for each technology, specific regulatory requirements will be specified.

Subelement: Waste processing

Alternative: Thermal treatment

Technology: Molten salt oxidation. The basic concept is to (1) introduce waste and air into a ceramic-lined vessel containing a bed of molten salt, (2) oxidize organic wastes in the molten salt, (3) use the heat of oxidation to keep the salt molten, and (4) remove spent salt for processing and recycling or for disposal. ${ }^{1}$

Molten salt oxidation is a combination of chemical and thermal treatment and is perceived as having several advantages: the molten salt (1) acts as a dispersing medium for both waste and air, (2) enhances oxidation reactions, (3) enhances completeness by providing a better contact over a relatively long period, (4) neutralizes and retains acid gases and is selfscrubbing, (5) retains soot, chars, and ashes, and (6) can be designed as a mobile unit. A potential problem is generation of a spent salt that has to be processed (and recycled) or disposed. For particular waste streams, the waste volume reduction can be significant or, for others, minimal or negative. 
Status: Demonstration

Science/Technology Needs: Molten salt oxidation is not a new process. Small prototype units were operated and tested during the $1970 \mathrm{~s},{ }^{2}$ and $R \& D$ efforts continue today. ${ }^{3-6} \mathrm{~A}$ technology evaluation of molten salt process technology recently was conducted, ${ }^{7}$ and a 5-year implementation plan is under way. ${ }^{1}$ Sodium carbonate-based molten salt beds are the most developed, but nitrate salts ${ }^{4}$ or other species are being tested. ${ }^{5}$ The molten salt oxidation process also may be suitable as an off-gas treatment operation for other thermal processes. ${ }^{1}$

Implementation Needs: The 5-year implementation plan, ${ }^{1}$ which includes bench-scale testing, prototype construction and demonstrations, and basic and applied research, is expected to cost $\$ 35.6 \mathrm{M}$. The estimated cost is $\$ 1.4 \mathrm{M}$ for a 3-year development plan and technology transfer for the destruction of ion-exchange resins using molten salts, recovery of special materials, and salt recycling based on DOE-OTD-TTP No. OR-4TAH-93 by G. D. Del Cul and C. E. Bamberger. ${ }^{8}$

Author: G. D. Del Cul/615-576-5743

\section{References:}

1. Task Force Report, Molten Salt Oxidation Technology Five-Year Implementation Plan, PGJPO-650, May 1992.

2. D. E. McKenzie et al., Disposal of Transuranic Solid waste Using Molten Salt Combustion Process, AI-ERDA-13151, 1975.

3. O. H. Krikorian, A Preliminary Assessment of Salt and Radionuclide Volatilities in the Molten Salt Reactor, UCRL-ID-105173, 1990.

4. R. W. Bradshaw, "Hazardous Waste Treatment Using Oxidizing Molten Salts," Emerging Technologies Meeting, Atlanta, Georgia, October 1991.

5. G. D. Del Cul and C. E. Bamberger, "Catalyzed Molten Salt Oxidation of Spent IonExchange Resins," Gaseous Diffusion Process and Long Range Technical Support, Milestone report, 1993.

6. R. S. Upadhye and J. F. Cooper, "Molten-Salt Destruction Process for Mixed Wastes," Chemistry \& Material Science 1990-1991, UCRL-53943-91, 1991.

7. Evaluation of the Molten Salt Oxidation Process Technology, DOE/ID/12584-97, GJPO105, January 1992.

8. G. D. Del Cul and C. E. Bamberger, Catalyzed Resin Destruction: Catalyzed LowTemperature Combustion of Spent Organic Ion-Exchange Resins, DOE-OTD TTP-OR4TAH-93, 1993. 
EM Problem: Waste management

Y-12 Plant Problem: Building 9201-4 (Alpha-4) contaminated wastes

Problem Area/Constituents: Treatment of refractory organohalides; wastes containing heavy metals along with refractory organic materials; mixed wastes containing organic sludges with radionuclides; spent, radioactive, fon-exchange resins and contaminated soils

\section{Reference Requirements:}

- Resource Conservation and Recovery Act of 1976 (RCRA), as amended, Land Disposal Restriction (LDR) Federal Facilities Compliance Agreement (FFCA) dated June 12, 1992

- Federal Facilities Agreement (FFA) for the Oak Ridge Reservation

- Federal Facilities Compliance Agreement-Toxic Substances Control Act (FFCA-TSCA) dated February 20, 1992

- Clean Air Act regulations under 40 CFR 60-65

- Clean Water Act regulations under 40 CFR 110-146 and 400-471

- DOE Orders

Refer to the Regulatory Compliance Chapter of Vol. 1 for potentially applicable proposed and promulgated environmental laws, signed and pending agreements for the Oak Ridge Reservation, radiation protection standards, DOE orders, and nonregulatory guidance. As site- and waste-specific characteristics are provided for each technology, specific regulatory requirements will be specified.

Subelement: Waste processing

Alternative: Thermal treatment

Technology: Chem char process. ${ }^{1-4}$ Destroys hazardous wastes by reverse-burn (where flame and oxidant move in opposite direction) gasification. The wastes to be treated are mixed with char, and the mixture is mechanically pushed upward through a gasifier. The char, prepared by reverse-burn gasification of subbituminous coal, has a macroporous structure and a surface area of approximately $200 \mathrm{~m}^{2} / \mathrm{g}$, which can effectively sorb and immobilize waste liquids and sludges and provide an ideal physical matrix for treatment. Limited oxygen is supplied from the bottom of the gasifier so that a thin layer of flame travelling upward is created in the middle of the gasifier. Flame zone temperature is about $1500^{\circ} \mathrm{C}$. The flame supplies the energy needed to gasify the wastes. The gaseous products then flow upward to pass through a heated char bed, which is claimed to provide a strong reducing atmosphere that will effectively destroy the refractory organic compounds without producing undesirable by-products.

This gasification process is claimed to be a significant breakthrough for thermal treatment of environmental wastes. No inorganic particulates are present in the gas effluent, and metal emissions are minimal, probably because of capture of the volatilized metal vapors by the 
char bed, either through condensation or chemisorption. Char residue is a dry, inert, carbonaceous solid which is either nonhazardous or can be mixed readily with cement to prevent leaching of radioactive, toxic, or heavy metal constituents which are retained in the char residue or ash. Char residue can be gasified further in a forward-burn mode to produce a highly stable, low volume, glassy slag.

\section{Status: Predemonstration}

Science/Technology Needs: Development work will be initiated with a thorough review of the chem char process in collaboration with researchers of Chem Char Research, Inc. (CRI), holder of a patent on this process. Experimental studies on treating various liquid and solid wastes then will be conducted. Work will occur in conjunction with CRI (or independently at the K-25 Site) and will involve design and installation of a pilot gasifier system. Experiments will be planned to collect data, such as gasifier temperature profile, product gas composition, particle size, mixture flow rate and residence time, and treatment quality. Potential operational problems also will be investigated (e.g., channeling of oxygen and product gases, controllability of a stable and uniform flame front in the gasifier, incomplete combustion, and non-uniformity of treatment quality). Subsequently, an assessment of this process will be made regarding its technical and economic viability, and the result of this assessment will determine whether a larger facility (and its capacity) can be justified.

Implementation Needs: Development work is estimated to cost $\$ 0.75 \mathrm{M}-\$ 1.5 \mathrm{M}$.

Author: J. M. Chiang/615-574-9659

\section{References:}

1. The Chem Char Process for Hazardous Waste Treatment, technical bulletin, Chem Char Research, Inc.

2. C. D. Reach, personal communication with W. D. Altman, Martin Marietta Energy Systems, Inc., March 2, 1992.

3. T. C. Ho, "Comments on the Chem Char Process," personal notes, July 8, 1992.

4. L. L. Kinner and D. W. Lawsen, "Reverse-Burn Gasification for Treatment of Hazardous Wastes: Contaminated Soll, Mixed Wastes, and Spent Activated Carbon Regeneration," Environ. Sci. Technol, 27, 1993.

September 1994

Decontamination and Decommissioning 
EM Problem: Waste management

Y-12 Plant Problem: Building 9201-4 (Alpha-4). Waste processing sludges and other contaminated solids

Problem Area/Constituents: Mixed-waste-contaminated ashes, sludges, and other waste processing solid residues

\section{Reference Requirements:}

- Resource Conservation and Recovery Act of 1976 (RCRA), as amended, Land Disposal Restriction (LDR) Federal Facilities Compliance Agreement (FFCA) dated June 12, 1992

- Federal Facilities Agreement (FFA) for the Oak Ridge Reservation

- Federal Facilities Compliance Agreement-Toxic Substances Control Act (FFCA-TSCA) dated February 20, 1992

- Clean Air Act regulations under 40 CFR 60-65

- Clean Water Act regulations under 40 CFR 110-146 and 400-471

- DOE orders

Refer to the Regulatory Compliance Chapter of Vol. 1 for potentially applicable proposed and promulgated environmental laws, signed and pending agreements for the Oak Ridge Reservation, radiation protection standards, DOE orders, and nonregulatory guidance. As site- and waste-specific characteristics are provided for each technology, specific regulatory requirements will be specified.

Subelement: Waste processing/waste stabilization

Alternative: Thermal treatment

Technology: Microwave heating. ${ }^{1-4}$ Microwaves are electromagnetic energy waves having frequencies between 300 megahertz ( $\mathrm{MHz}$ ) and 300 gigahertz (GHz). When microwaves interact with matter, energy is transferred into vibrational modes. This vibrational energy is dissipated in the form of heat. This heating can take place inside the bulk of the material or near the surface, depending on how much the microwaves are absorbed. Several groups in Japan, Europe, and the U.S. currently are developing processes that use microwave energy to thermally treat radioactive wastes. ${ }^{1,5} \mathrm{~A}$ pilot plant-scale operation in Japan is using microwaves to melt plutonium-contaminated ash from conventional radioactive waste incinerators. In this process, microwaves are fed directly to the ash, causing the ash particles to fuse together to form a monolithic solid that, upon cooling, forms a leach-resistant waste form suitable for long-term storage in a geologic repository. ${ }^{3}$

Status: Demonstration

Science/Technology Needs: Development is necessary to establish the feasibility of scaling up the microwave heating process from laboratory scale to pilot-plant scale. At the same time, 
development is required to treat off-gas effluents in particular, when the wastes to be treated contain, for example, technetium, beryllium, PCBs, and organics.

Implementation Needs: Development costs are expected to be $\$ 2.5 \mathrm{M}$ (1992 dollars). These are the costs for developing bench-scale units, designing an industrial-scale prototype, and transferring microwave solidification/sintering technology to private industry. These costs are based on TTP OR-4WAA-93, by T. L. White and G. D. Del Cul, entitled Microwave Sludge Stabilization: Dewatering of Contaminated Mixed-Waste Sludges and Ashes using Microwave Heating, submitted to DOE-OTD.

Author: G. D. Del Cul/615-576-5743

\section{References:}

1. T. L. White et al., "Microwave Processing of Radioactive Materials," Emerging Technologies for Hazardous Waste Treatment, American Chemical Society, Atlanta, Georgia, May 1989.

2. M. S. Morrell et al., "A Pilot Plant Demonstration of the Vitrification of Radioactive Solutions Using Microwave Power," Nuclear and Chemical Waste Management, 6, p. 193-195, 1986.

3. Y. Ogata et al., Processing at the Plutonium-Contaminated Waste Treatment Facility, LAEASM-303/68, 1989.

4. R. D. Petersen, Microwave Vitrification of Rocky Flats TRU Sludge, RFP-4414, 1990.

5. E. P. Bescher et al., Microwave Sintering of Ashes, Air \& Waste Management Association, 84th Annual Meeting \& Exhibition, Vancouver, Canada, June 1991. 
EM Problem: Waste management

Y-12 Plant Problem: Building 9201-4 (Alpha-4). Waste processing sludges

Problem Area/Constituents: Solid and liquid organics, nitrate-bearing chemicals, PCB-contaminated liquids and solids

\section{Reference Requirements:}

- Resource Conservation and Recovery Act of 1976 (RCRA), as amended, Land Disposal Restriction (LDR) Federal Facilities Compliance Agreement (FFCA) dated June 12, 1992

- Federal Facilities Agreement (FFA) for the Oak Ridge Reservation

- Federal Facilities Compliance Agreement-Toxic Substances Control Act (FFCA-TSCA) dated February 20, 1992

- Clean Air Act regulations under 40 CFR 60-65

- Clean Water Act regulations under 40 CFR 110-146 and 400-471

- DOE Orders

Refer to the Regulatory Compliance Chapter of Vol. 1 for potentially applicable proposed and promulgated environmental laws, signed and pending agreements for the Oak Ridge Reservation, radiation protection standards, DOE orders, and nonregulatory guidance. As site- and waste-specific characteristics are provided for each technology, specific regulatory requirements will be specified.

\section{Subelement: Waste processing}

\section{Alternative: Thermal treatment}

Technology: Molten glass combustor. ${ }^{1.2}$ This device adds glass-forming constituents to waste feed to produce a pool of molten glass at the bottom of a reactor in which solid wastes react. Gases produced during combustion are released from the surface of the glass pool. Ash remains in the pool and is periodically removed with the carrier glass matrix. The removed glass is collected in storage containers and solidifies upon cooling.

The glass pool is melted and brought to operating temperature by passing a current between electrodes immersed in the glass at the sides of the combustor. The resistance of the glass to the flow of electrical current creates the needed rise in temperature in the molten-glass pool. Typical materials used to form a base glass are oxides of silicon, boron, and phosphorus. Sodium and potassium oxides are added to adjust glass melting temperature and viscosity. Aluminum axide is added as required to decrease leachability of the glass.

Volatile constituents of solid and liquid waste feed streams are combusted in the volume of the combustor above the molten-glass pool. Combustion air typically is supplied through ports in the sides of this section of the combustor. Auxiliary fuel burners are used to ensure 
that the volume above the molten-glass pool is maintained at proper temperature for combustion of the volatiles.

The thermal vitrification process involves heating the materials by electricity or fossil fuels to temperatures beyond $1000^{\circ} \mathrm{C}$. At these temperatures, any free liquid or organic material is evaporated, pyrolyzed, or oxidized, and residual inorganics are vitrified to form a molten glass. Thermally formed glasses are produced by fusing or melting crystalline materials and/or amorphous materials at elevated temperatures to produce liquids. Subsequently, these liquids are cooled to a rigid condition without crystallization.

Normally, glass waste forms are obtained by mixing one or more waste streams containing radioactive and hazardous inorganic chemical compounds with other glass-forming materials and melting these materials during a vitrification process. The glass-forming materials may be commercially melted glass frit, select chemicals, or in some cases, materials that are present in the waste streams. Generally, silicon oxide $\left(\mathrm{SiO}_{2}\right)$ is the major component and provides the three-dimensional network which strongly influences the glass-waste-form character and properties.

The glass waste form discussed in the vast majority of references is borosilicate glass $\left(\mathrm{B}_{2} \mathrm{O}_{3} \cdot \mathrm{SiO}\right)$, as employed in the high-level radioactive waste program. The waste form usually contains less than $30 \mathrm{wt} \%$ of waste solids; however, the vitrification process generally reduces the volume significantly. Specific waste streams can be added at higher levels and, under conditions where the waste contains sufficient glass formers, a glass containing only waste can be produced. A wide variety of glass compositions may be suitable as glass waste forms and may provide advantages for the vitrification of a particular waste stream.

Because glass can accept a wide variety and amount of radioactive or hazardous materials and because its physical properties normally are consistent with time, glass has been selected to stabilize a variety of waste streams. It should be noted that the EPA has determined that vitrification is the "best-demonstrated available technology" for chemically hazardous constituents in high-level nuclear waste.

Status: Molten glass combustors are accepted by industry, and units are in use. Removal efficiencies of greater than $99 \%$ can be obtained for most waste types. Cost is not well established, but is expected to exceed $\$ 200$ per ton. The vitrification process has proven effective, but the costs of operation are not well known.

Science/Technology Needs: The following may cause problems during operation:

- Monitoring the need for changes in additives can be difficult.

- The presence of certain organics and/or metals can result in problems caused by changes in the valance of the glass. This may result in precipitates forming in the glass matrix.

- Certain elements (eg., chromium, ruthenium, and noble metals) have limited solubilities in specific glass structures.

September 1994

Decontamination and Decommissioning 


\section{Implementation Needs:}

- Process equipment (e.g., indirect or direct-fired rotary kiln, heated screw kiln, etc.)

- Minimum 2,000 $\mathrm{ft}^{2}$ equipment battery limit

- All utilities, including electricity, cooling water, natural gas, steam, air, nitrogen, etc.

- Drum and material handling equipment

- Trained chemical operators

- EPA-certified laboratory support

Author: C. H. Dukes/615-574-9561

\section{References:}

1. H. M. Freeman, ed., Standard Handbook of Hazardous Waste Treatment and Disposal, McGraw-Hill, New York, p. 8.91-8.104, 1989.

2. J. B. Berry, P. W. Lurk, and G. J. Coyle, Jr., Mixed-Waste Treatment Research, Development, Demonstration, Testing, and Evaluation, pp. 13-16, U. S. Department of Energy, Office of Technology Development, 1992, pp. 13-16. 


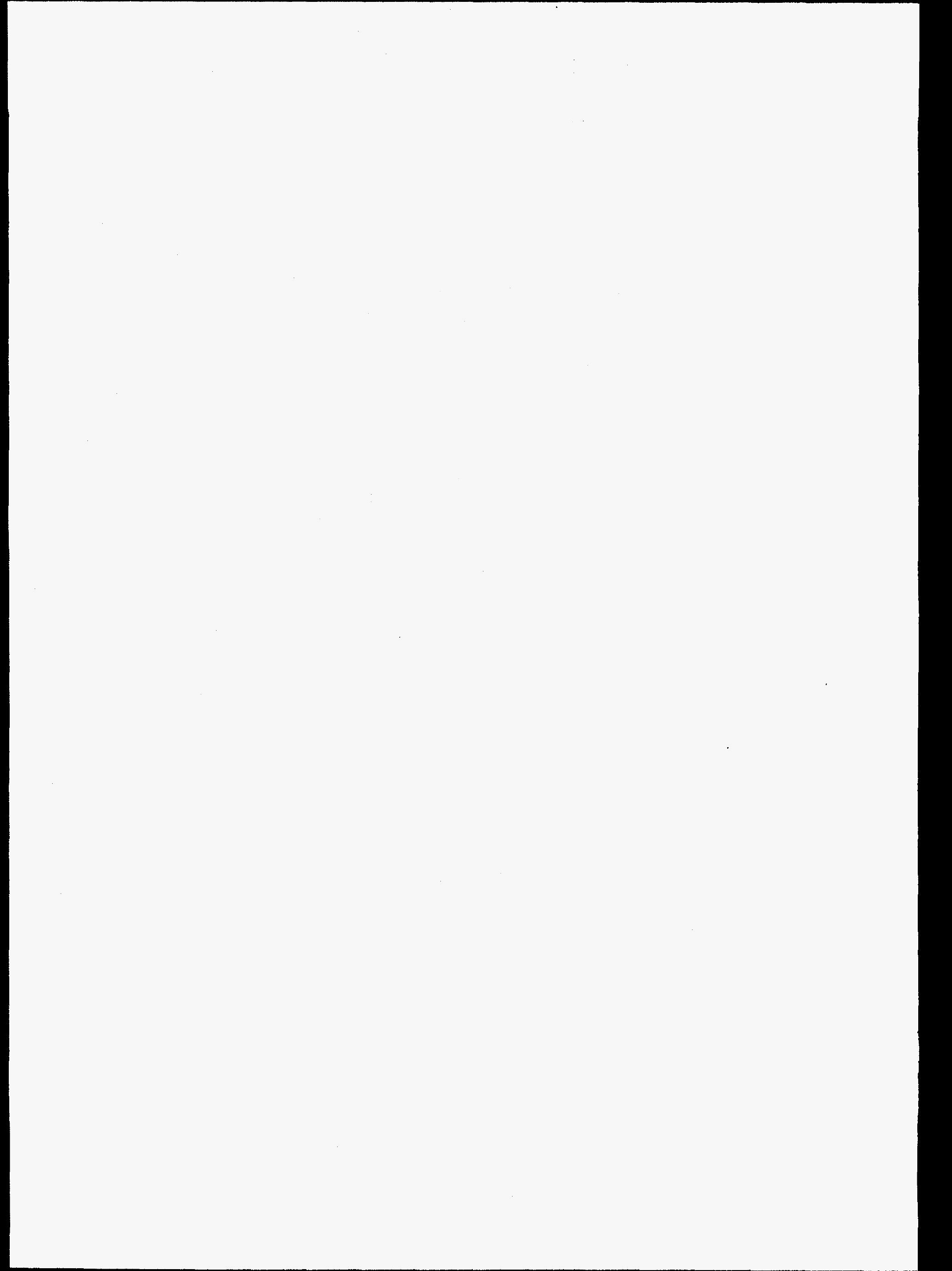


EM Problem: Waste management

Y-12 Plant Problem: Building 9201-4 (Alpha-4). Waste processing sludges

Problem Area/Constituents: Sludges generated as a result of other waste treatment operations. These sludges could contain organics, heavy metals, inorganic salts, and radionuclides such as uranium and technetium.

\section{Reference Requirements:}

- Resource Conservation and Recovery Act of 1976 (RCRA), as amended, Land Disposal Restriction (LDR) Federal Facilities Compliance Agreement (FFCA) dated June 12, 1992

- Federal Facilities Agreement (FFA) for the Oak Ridge Reservation

- Federal Facilities Compliance Agreement-Toxic Substances Control Act (FFCA-TSCA) dated February 20, 1992

- Clean Air Act regulations under 40 CFR 60-65

- Clean Water Act regulations under 40 CFR 110-146 and 400-471

- DOE orders

Refer to the Regulatory Compliance Chapter of Vol. 1 for potentially applicable proposed and promulgated environmental laws, signed and pending agreements for the Oak Ridge Reservation, radiation protection standards, DOE orders, and nonregulatory guidance. As site- and waste-specific characteristics are provided for each technology, specific regulatory requirements will be specified.

\section{Subelement: Waste processing}

\section{Alternatives: Biotreatment}

Technology: Biodegradation..$^{1,2}$ Consists of the molecular decomposition of organics and/or the assimilation of inorganic elements by the complex reactions of living organisms, such as naturally occurring or genetically engineered microbes. Given the proper environment, these microbes often can destroy complex hazardous molecules. Often, the process is slow, but destruction efficiency is impressive. Aerobic and anaerobic digestion historically have been used for treatment of wastewaters and resulting sludges. Much research is being conducted to genetically engineer microbes (e.g., white rot fungus) to treat specific, recalcitrant, hazardous pollutants such as chlorinated organics.

Status: Demonstration/evolving technology. The status is partially labeled "demonstration," even though aerobic/anaerobic digestion is commercially available, because the microbes' suitability for treatment of a particular K-25 Site waste stream may need to be demonstrated. The evolving technology status is for the biodegradation processes that would use geneticallyengineered microbes. 
Science/Technology Needs: Certain variations of biodegradation, such as aerobic/ anaerobic treatment, are commercial technologies; therefore, their additional science/ technology needs will be minimal. However, development of engineered microorganisms to treat specific pollutants is still in its infancy and has significant science/technology needs. These needs range from fundamental research to pilot-plant-scale demonstrations of the treatment process(es).

Implementation Needs: Costs for developing the technology for treating the waste sludges likely to be generated at the site are estimated to be around $\$ 1 \mathrm{M}-\$ 10 \mathrm{M}$ (1992 dollars). If a successful technology can be demonstrated, the payback could be at least one order of magnitude higher than development costs, when compared to the costs of treatment and waste disposal alternative.

Author: S.P.N. Singh/615-574-6639

\section{References:}

1. H. M. Freeman, ed., Standard Handbook of Hazardous Waste Treatment and Disposal, McGraw-Hill, New York, 1989.

2. J. B. Berkowitz et al., Unit Operations for Treatment of Hazardous Industrial Wastes, Noyes Data Corporation, Park Ridge, New Jersey, 1978. 
EM Problem: Waste management

Y-12 Plant Problem: Disposal of permitted uncontaminated (i.e., not containing radioactive or RCRA-hazardous components) wastes that cannot be recycled, reused, sold as scrap, or incinerated. This option could apply to wastes such as uncontaminated concrete rubble, yard wastes, and refuse

Problem Area/Constituents: Uncontaminated wastes and residuals generated as a result of decontamination and decommissioning, remedial action, or waste management operations that cannot be recycled, reused, sold as scrap, or incinerated

\section{Reference Requirements:}

- Resource Conservation and Recovery Act of 1976 (RCRA), as amended, Land Disposal Restriction (LDR) Federal Facilities Compliance Agreement (FFCA) dated June 12, 1992

- Federal Facilities Agreement (FFA) for the Oak Ridge Reservation (ORR)

- Clean Air Act regulations under 40 CFR 60-65

- Clean Water Act regulations under 40 CFR 110-146 and 400-471

- DOE Orders

Refer to the Regulatory Compliance chapter of Vol. 1, Pt. C, for more detailed discussions of potentially applicable proposed and promulgated environmental laws, signed and pending agreements for the ORR, radiation protection standards, DOE orders, and nonregulatory guidance. As site- and waste-specific characteristics are provided for each technology, specific regulatory requirements will be specified.

Subelement: Waste processing

Alternatives: Physical treatment

Technology: Landfill disposal. This waste management option consists of the following steps:

- identifying, collecting, performing waste analyses, and packaging these uncontaminated wastes;

- evaluating the potential waste management options for these wastes and determining that landfll disposal is the optimum option for these wastes; and

- then, if they meet the waste acceptance criteria of the disposal site, disposing of these wastes in the permitted landfills on the ORR.

Status: Accepted

Science/Technology Needs: These are expected to be minimal. They could include, for example, assessing alternate disposal options for these wastes other than landfill disposal such as recycle/reuse. 
Implementation Needs: These are estimated to be about $\$ 0.5 \mathrm{M}-\$ 1 \mathrm{M}$ (1992 dollars) for conducting studies to examine optimum disposal options for these waste residuals. These costs do not include costs for waste collection, analyses, or tipping fees at the landfill site(s).

Author: S. P. N. Singh/615-574-6639

References: None 
EM Problem: Waste management

Y-12 Plant Problem: Building 9201-4 (Alpha-4). Treatment of contaminated wastewater, liquids, and gas streams

Problem Area/Constituents: Removal of inorganic, organic, and radioactive compounds and particulates from various liquids and gaseous streams at Bldg. 9201-4

\section{Reference Requirements:}

- Resource Conservation and Recovery Act of 1976 (RCRA), as amended, Land Disposal Restriction (LDR) Federal Facilities Compliance Agreement (FFCA) dated June 12, 1992

- Federal Facilities Agreement (FFA) for the Oak Ridge Reservation

- Federal Facilities Compliance Agreement-Toxic Substances Control Act (FFCA-TSCA) dated February 20, 1992

- Clean Air Act regulations under 40 CFR 60-65

- Clean Water Act regulations under 40 CFR 110-146 and 400-471

- DOE orders

Refer to the Regulatory Compliance Chapter of Vol. 1 for potentially applicable proposed and promulgated environmental laws, signed and pending agreements for the Oak Ridge Reservation, radiation protection standards, DOE orders, and nonregulatory guidance. As site- and waste-specific characteristics are provided for each technology, specific regulatory requirements will be specified.

Subelement: Waste processing

Alternative: Physical treatment

Technology: Filtration. Generic treatment technology for the physical removal of solid particulates from liquid or gaseous streams, often using a porous medium. ${ }^{1}$ This waste management operation includes various forms of solids separation such as decantation, centrifugation, gravity separation, foaming sedimentation, cake filtration, bag house and HEPA filtration, and electrostatic precipitation. At Bldg. 9201-4, filtration may be used for removal of inorganics, organics, and particulates from the various liquid and gaseous effluent streams generated, for example, in $D \& D$, remedial action, and other site waste processing operations.

Status: Demonstration, even though most filtration processes are commercial technologies, because application of a particular filtration process to treat an Alpha-4 Site stream may need to be demonstrated before it is used.

Science/Technology Needs: Because filtration is a commercial technology, science/ technology needs generally are minimal. However, the efficacy of a given filtration process may need to be demonstrated for a given application before its full-scale use. 
Implementation Needs: Because of the wide range of processes covered under filtration and because its cost depends on numerous factors, it is impractical to provide a meaningful cost for this technology. In general, implementation costs for technology demonstration of a filtration process may range up to $\$ 1 \mathrm{M}$. However, because filtration often enables the discharge of large volume waste streams (by removing the contaminants), the payback for use of the technology often is quite significant compared with the cost of alternatives.

Author: S.P.N. Singh/615-574-6639

\section{References:}

1. H. M. Freeman, ed., Standard Handbook of Hazardous Waste Treatment and Disposal, McGraw-Hill, New York, New York, 1989. 
EM Problem: Waste management

Y-12 Plant Problem: Treatment of hazardous-, radioactive-, and/or mixed-wastecontaminated wastewaters liquids, and gases from D\&D of Alpha-4.

Problem Area/Constituents: These include process wastewater, liquid wastes, and gases contaminated with hazardous and radioactive components

\section{Reference Requirements:}

- Resource Conservation and Recovery Act of 1976 (RCRA), as amended, Land Disposal Restriction (LDR) Federal Facilities Compliance Agreement (FFCA) dated June 12, 1992

- Federal Facilities Agreement (FFA) for the Oak Ridge Reservation

- Clean Air Act regulations under 40 CFR 60-65

- Clean Water Act regulations under 40 CFR 110-146 and 400-471

- DOE orders

Refer to the Regulatory Compliance chapter of Vol. 1, Pt. C, for more detailed discussions of potentially applicable proposed and promulgated environmental laws, signed and pending agreements for the Oak Ridge Reservation, radiation protection standards, DOE orders, and nonregulatory guidance. As site- and waste-specific characteristics are provided for each technology, specific regulatory requirements will be speciffed.

\section{Subelement: Waste processing}

\section{Alternatives: Chemical treatment}

Technology: Adsorption (including Chemisorption). ${ }^{1,2}$ This involves the process of transfer of contaminant species from one medium (e.g., supernates or wastewater) to another where they are chemically adsorbed. The potential applications of chemisorption for treating the Alpha-4 wastes are listed below:

- removal of mercury, using crown ethers, from MVST supernates and other highly alkaline wastewaters; ${ }^{1}$

- removal of mercury, using sulfur-impregnated activated carbon, from process wastewater; ${ }^{1}$

- removal of radionuclides from liquid low-level wastes and contaminated gases. ${ }^{3-8}$

Status: Predemonstration

Science/Technology Needs: Significant additional research, development, and demonstration (RD\&D) is required to develop acceptable processes using chemisorption to achieve the above contaminant removals from the wastes at the ORNL Site. 
Implementation Needs: The additional RD\&D to develop the chemisorption separation processes is estimated to cost about \$2M (1992 dollars) and take 2-3 years to accomplish.

Authors: D. A. Bostick/615-576-7695, W. D. Arnold/615-576-7965, and J. J. Perona/ 615-574-5006

\section{References:}

1. J. J. Perona, ORNL, personal communication with S. P. N. Singh, March 26, 1993.

2. D. A. Bostick and W. D. Arnold, ORNL, personal communication with S. P. N. Singh, March 26, 1993.

3. D. O. Campbell, D. D. Lee, and T. A. Dillow, Development Studies for Treatment of ORNL Low-Level Liquid Waste, ORNL/TM-11798, Martin Marietta Energy Systems, Inc., Oak Ridge Natl. Lab., November 1991.

4. D. D. Lee and D. O. Campbell, Treatment Requirements for Decontamination of ORNL Low-Level Liquid Waste, ORNL/TM-11799, Martin Marietta Energy Systems, Inc., Oak Ridge Natl. Lab., October 1991.

5. D. O. Campbell and D. D. Lee, Treatment Options and Flowsheets for ORNL Low-Level Liquid Waste Supernate, ORNL/TM-11800, Martin Marietta Energy Systems, Inc., Oak Ridge Natl. Lab., December 1991.

6. T. E. Kent, W. D. Arnold, Jr., W. D. Bond, J. L. Collins, D. J. Davidson, P. A. Taylor, and D. F. Williams, "Overview of Laboratory Treatment Studies for Decontamination of Liquid Low-Level Waste at Oak Ridge National Laboratory," Proceedings of Waste Management '93 Conference, Tucson, Ariz., 1993.

7. K. R. Balasubramanian, K. L. N. Rao, C. Mathai, R. N. Varma, and V. I. Dhiwar, Separation and Purification of Short Lived Fission Products from Irradiated Uranium, Report No: BARC-1991/E/009, 1991.

8. D. M. Camaioni, N. G. Colton, and R. L. Bruening, Investigation of the Potential of SilicaBonded Macrocyclic Ligands for Separation of Metal Ions from Nuclear Waste, PNL-7894, January 1992.

September 1994

Decontamination and Decommissioning 
EM Problem: Waste management

Y-12 Plant Problem: Building 9201-4 (Alpha-4). Contaminated wastewater

Problem Area/Constituents: Volatile and nonvolatile organics, PCBs, benzene, toluene, ethylbenzene, and xylene (BTEX), other halogenated and nonhalogenated solvents in water

\section{Reference Requirements:}

- Resource Conservation and Recovery Act of 1976 (RCRA), as amended, Land Disposal Restriction (LDR) Federal Facilities Compliance Agreement (FFCA) dated June 12, 1992

- Federal Facilities Agreement (FFA) for the Oak Ridge Reservation

- Federal Facilities Compliance Agreement-Toxic Substances Control Act (FFCA-TSCA) dated February 20, 1992

- Clean Air Act regulations under 40 CFR 60-65

- Clean Water Act regulations under 40 CFR 110-146 and 400-471

- DOE orders

Refer to the Regulatory Compliance Chapter of Vol. 1 for potentially applicable proposed and promulgated environmental laws, signed and pending agreements for the Oak Ridge Reservation, radiation protection standards, DOE orders, and nonregulatory guidance. As site- and waste-specific characteristics are provided for each technology, specific regulatory requirements will be specified.

Subelement: Waste processing

Alternatives: Physical treatment

Technology: Activated carbon. Removes organic contaminants from water by adsorption or the attraction and accumulation of one substance on the surface of another. In general, high surface area and pore structure are the prime considerations in adsorption of organics from water; whereas, the chemical nature of the carbon surface is of relatively minor significance. Granular activated carbons typically have surface areas of $500-1,400 \mathrm{~m}^{2} / \mathrm{g}$. Activated carbon has a preference for organic compounds and, because of this selectivity, is particularly effective in removing organic compounds from aqueous solutions.

Status: Accepted

Science/Technology Needs: Adsorption properties of activated carbon are influenced by the material from which the carbon is manufactured. Additional investigation is necessary to isolate the carbon type applicable to the specific contaminant. 
Implementation Needs: Some bench-scale studies are needed to identify the specific activated carbon required to remove the contaminant in question. Activated carbon is commonly used as a polishing unit in removal of organics from waste streams.

Author: D. P. Schaefferkoetter/615-574-7561

\section{References:}

1. United States EPA, Process Design Manual for Carbon Adsorption, Technology Transfer, October 1983.

2. K. J. Deeny, J. A. Heidman, and A. J. Condren, Project Summary, Performance of Actwated Sludge - Powdered Activated Carbon - Wet Air Regeneration Systems, EPA/600/52-90/012, August 1990. 
EM Problem: Waste management

Y-12 Plant Problem: Building 9201-4 (Alpha-4). Contaminated wastewater

Problem Area/Constituents: Wastewater, waste oils, spent solvents, and waste gases generated at Bldg. 9201-4 which are contaminated with radioactive species and magnetic particulates (e.g., iron, nickel, chromium, and manganese)

\section{Reference Requirements:}

- Resource Conservation and Recovery Act of 1976 (RCRA), as amended, Land Disposal Restriction (LDR) Federal Facilities Compliance Agreement (FFCA) dated June 12, 1992

- Federal Facilities Agreement (FFA) for the Oak Ridge Reservation

- Clean Air Act regulations under 40 CFR 60-65

- Clean Water Act regulations under 40 CFR 110-146 and 400-471

- DOE orders

Refer to the Regulatory Compliance Chapter of Vol. 1 for potentially applicable proposed and promulgated environmental laws, signed and pending agreements for the Oak Ridge Reservation, radiation protection standards, DOE orders, and nonregulatory guidance. As site- and waste-specific characteristics are provided for each technology, specific regulatory requirements will be specified.

\section{Subelement: Waste processing}

Alternative: Physical treatment

Technology: High gradient magnetic separation (HGMS). A process that uses a highintensity magnetic field (up to 20,000 gauss) to remove and recover radioactive and weakly paramagnetic materials from contaminated wastewater, waste oils, spent solvents, and gas streams before their disposal. Use of the process can help reduce (minimize) the volume of hazardous or mixed wastes for disposal, thereby lowering disposal costs. HGMS can be used to recover nonmagnetic waste species (by seeding the waste stream with a magnetic floc, such as $\mathrm{Fe}_{3} \mathrm{O}_{4}$; however, using the other conventional filtration techniques for this purpose may be more cost effective). HGMS is best suited to treating large volume or high flow-rate waste streams or streams contaminated with paramagnetic materials. Where applicable, the process can recover particulates as small as one micron in diameter; however, it is most effective for recovering 30-100-micron particulates. ${ }^{1}$ HGMS cost estimates (in 1978 dollars) for treating various industrial wastewaters range from $\$ 0.10-\$ 0.50 / 1000 \mathrm{gal}$ (for removing highly magnetic materials) to $\$ 1-\$ 4 / 1000$ gal (for removing weakly magnetic contaminants).

Status: Demonstration

Science/Technology Needs: Application of the technology to treat the appropriate liquid and gaseous waste streams at the K-25 Site needs to be demonstrated. 
Implementation Needs: The above-mentioned technology demonstration of the HGMS process could cost from $\$ 500 \mathrm{~K}$ to $\$ 1 \mathrm{M}$ (in 1992 dollars). If the process could be demonstrated to apply to treatment of certain K-25 Site waste streams, the technology could prove to be cost-effective, because its major advantage is that it can readily process large volumes of lightly contaminated waste streams.

Author: S.P.N. Singh/615-574-6639

\section{References:}

1. J. B. Berkowitz et al., Unit Operations for Treatment of Hazardous Industrial Wastes, Noyes Data Corporation, Park Ridge, New Jersey, pp. 590-609, 1978. 
EM Problem: Waste management

Y-12 Plant Problem: Building 9201-4 (Alpha-4). Contaminated wastewater

Problem Area/Constituents: Radionuclides, heavy metals, and mercury in wastewater.

\section{Reference Requirements:}

- Resource Conservation and Recovery Act of 1976, as amended (RCRA) Land Disposal Restriction (LDR) Federal Facilities Compliance Agreement (FFCA) dated 12 June 1992.

- Federal Facilities Agreement (FFA) for Oak Ridge Reservation.

- Clean Air Act regulations under 40 CFR 60-65.

- Clean Water Act regulations under 40 CFR 110-146 and 400-471.

- DOE orders

Refer to the Regulatory Compliance Chapter of Vol. 1 for potentially applicable proposed and promulgated environmental laws, signed and pending agreements for the Oak Ridge Reservation, radiation protection standards, DOE orders, and nonregulatory guidance. As site- and waste-specific characteristics are provided for each technology, specific regulatory requirements will be specified.

Subelement: Waste processing

Technology: Electrolysis. The term electrolysis or electrolyte reaction are applied to those reactions of axidation or reduction that take place at the surface of conductive electrodes immersed in a chemical medium under the influence of an applied potential. Reductive reactions take place at the cathode while oxidation reactions take place at the anode. The voltage range for the treatment of aqueous wastes is limited to conditions below the voltage that decomposes water into oxygen and hydrogen (i.e., slightly above 1.23 volts). Electroflotation is a process that utilizes electro-generated bubbles for the separation of oilwater mixtures.

For conventional electrolysis, the transport toward and from the electrodes is limited by standard concentration-gradient diffusion and stirring. On the other hand, for electrophoresis and electromigration processes, the transport takes place as a result of a high voltage electric field applied on charged species.

Successful uses of electrolysis in waste treatment may be divided in three broad categories namely, reduction of a dissolved species at the cathode, oxidation of a dissolved species at the anode, or the useful electrolysis of a solvent, as in electroflotation. ${ }^{1}$ Electrolytic processes may be envisioned for the removal of heavy metals and radionuclides (including uranium and technetium) from contaminated wastewater and for the decontamination of metallic surfaces.

Status: Demonstration 
Science/Technology Needs: Many electrochemical waste treatment processes can be envisioned for the removal of heavy metals and radionuclides. The efficiency, applicability, and the process economics for a specific application need to be evaluated.

Implementation Needs: Development Costs: The development costs are estimated to be $\$ 1.25 \mathrm{M}$ (1992 dollars) for the use of electrolysis to treat wastewaters at Bldg. 9201-4. These costs are for a three-year development plan and technology transfer to private industry of advanced electrolysis treatment processes. Electrical energy costs range from $10 \%$ to $35 \%$ of the total direct operating costs.

Author: G. D. Del Cul/615-574-5743

\section{References:}

1. J. B. Berkowitz et al., Unit Operations for Treatment of Hazardous Industrial Wastes, Noyes Data Corp., New Jersey, 1978. 
fEM Problem: Waste management

Y-12 Plant Problem: Building 9201-4 (Alpha-4). Contaminated wastewater

Problem Area/Constituents: Radionuclides in water, heavy metals in water, PCBs in water, other nonvolatile organics in water

\section{Reference Requirements:}

- Resource Conservation and Recovery Act of 1976 (RCRA), as amended, Land Disposal Restriction (LDR) Federal Facilities Compliance Agreement (FFCA) dated June 12, 1992

- Federal Facilities Agreement (FFA) for the Oak Ridge Reservation

- Federal Facilities Compliance Agreement-Toxic Substances Control Act (FFCA-TSCA) dated February 20, 1992

- Clean Air Act regulations under 40 CFR 60-65

- Clean Water Act regulations under 40 CFR 110-146 and 400-471

- DOE orders

Refer to the Regulatory Compliance Chapter of Vol. 1 for potentially applicable proposed and promulgated environmental laws, signed and pending agreements for the Oak Ridge Reservation, radiation protection standards, DOE orders, and nonregulatory guidance. As site- and waste-specific characteristics are provided for each technology, specific regulatory requirements will be specified.

Subelement: Waste processing

Alternative: Physical treatment

Technology: Membranes. This data sheet includes a discussion of reverse osmosis (RO), ultrafiltration (UF), advanced membrane filtration (AMF), supported liquid membranes, and electrodialysis systems.

Membrane systems are replacing several older, costly separation technologies because of the lower cost and greater flexibility of these systems. ${ }^{1}$ Breakthroughs in developing membrane materials have led to commercialization of membrane separation systems for waste treatment, desalinization, gas-mixture separations, and other high-volume applications. ${ }^{2}$

Reverse osmosis (RO) is a separation technology based on the preferential transport of water through a membrane, from a concentrated solution to a more diluted solution, using a pressure exceeding the osmotic pressure. The process can produce highly purified water (permeate); however, rejection of salts is a function of salt concentration in the feed. The combined advantage and disadvantage of $\mathrm{RO}$ is that it removes all of the ions present. This is a disadvantage, because the volume of the secondary waste (consisting of the concentrate or reject stream) is increased by the presence of ions, such as sodium and calcium, that need 
not be removed. The concentrate may have to be treated using other technologies, such as evaporation, to remove or recover the water and produce a saltcake for disposal.

Ultrafiltration (UF) is very similar to $R O$, but UF will not remove solutes of low-to-intermediate molecular weight.

Advanced membrane filtration $(\mathrm{AMF})^{3}$ is a technology that uses membranes with pore sizes larger than those typical of UF and RO. AMF has been used as a final step in the treatment of industrial laundry wastewater for removing suspended solids of low-level radioactive wastes containing uranium and heavy metals.

A supported liquid membrane consists of an organic phase which is held by capillary forces within the pores of a microporous polymeric membrane.

Electrodialysis is a membrane separation process by which aqueous solutions are deionized by the electromigration of cations and anions through semipermeable membranes. ${ }^{5}$

$$
\text { Physical parameters of membranes for RO, UF and AMF }{ }^{3}
$$

\begin{tabular}{|c|c|c|c|}
\cline { 2 - 4 } \multicolumn{1}{c|}{} & $\begin{array}{c}\text { Reverse Osmosis } \\
\text { (RO) }\end{array}$ & Ultrafiltration (UF) & $\begin{array}{c}\text { Advanced } \\
\text { Membrane } \\
\text { Filtration }\end{array}$ \\
\hline Pore size $(\mu \mathrm{m})$ & 0.001 & $0.05-0.1$ & $>0.1$ \\
\hline Pressure (psi) & $300-400$ & $50-100$ & $40-50$ \\
\hline $\begin{array}{c}\text { Flow rate (gal } / \mathrm{ft}^{2} \text { of } \\
\text { membrane/day) }\end{array}$ & $10-25$ & $30-75$ & $200-400$ \\
\hline
\end{tabular}

Several reverse osmosis and ultrafiltration systems have been evaluated in the literature, including the following.

- Full-scale treatment system have been developed for removing Ra-226 and several heavy metals, ${ }^{6}$ uranyl nitrate solution, ${ }^{7}$ and actinides. ${ }^{8-10}$

- Advanced membrane filtration units have been used for treating industrial laundry wastewater and for removing uranium and heavy metals from low-level radioactive wastewater. ${ }^{3}$

- Supported liquid membranes were used for removing uranium from contaminated groundwater. ${ }^{11}$

- Electrodialysis has been used for removing several radionuclides including cesium-137, strontium-90, cobalt-60, ruthenium-106, and heavy metals such as mercury and chromium. ${ }^{12,13}$

Status: Demonstration

September 1994

Decontamination and Decommissioning 
Science/Technology Needs: Development is necessary to establish the feasibility of scaling up from a pilot-plant scale to an industrial scale. Development also is needed to prevent or minimize membrane fouling caused by bacteria and colloidal materials. ${ }^{14}$ Laboratory-scale development is proposed for novel inorganic membranes ${ }^{15}$ that may be readily cleanable, and this development could alleviate the fouling problem.

Implementation Needs: RO was identified as the baseline treatment technology for total dissolved solids removal at the $\mathrm{K}-25$ Central Neutralization Facility. ${ }^{16}$ The estimated installed cost for this 100,000-gal/day unit is about \$10M (1992 dollars). Estimated cost to develop the novel inorganic membranes is about $\$ 750 \mathrm{~K}$ (1992 dollars). This cost is based on the Technical Task Plan submitted by D. E. Fain (DOE-OTD TTP OR-1211-08, Development of Inorganic Membranes for Mixed Waste Landfill Cleanup).

Author: G. D. Del Cul/615-576-5743

\section{References:}

1. W. F. Weber and W. Bowman, "Membranes Replacing Other Separation Technologies," Chemical Engineering Progress, November 23, 1986.

2. J. Hagging, "New Generation of Membranes Developed for Industrial Separations," Chemical Engineering News, 7, June 6, 1988.

3. T. V. Tran, "Advanced Membrane Filtration Process Treats Industrial Wastewater Efficiently," Chemical Engineering Progress, March 29, 1985.

4. P. Danesi et al., "Mass Transfer Rate Through Liquid Membranes: Interfacial Chemical Reactions and Diffusion as Simultaneous Permeability Controlling Factors," Separation Science and Technology, 16(2), 201-211, 1981.

5. K. M. Hodgson, Demonstration of Technologies to Remove Contamination From Groundwater, WHC-SA-367, 1989.

6. T. J. Sorg and G. S. Logsdon, Treatment Technology to Meet the Interim Primary Drinking Water Regulations for Inorganics: Part 5," Research and Technology, 412, July 1980.

7. S. Prabhakar et al., "Studies on the Reverse Osmosis Treatment of Uranyl Nitrate Solutions," Separation Science and Technology, 27(3), 349, 1992.

8. R. C. Roberts and W. R. Herald, Development of Ultrafiltration and Adsorbents, MLM$2684,1979$.

9. (Missing)

10. C. M. Colvin et al., Summary of the Ultrafiltration, Reverse Osmosis, and Adsorbents Project, MLM-3033, 1983.

11. R. Chiarizia and E. P. Horwitz, "Study of Uranium Removal from Groundwater by Supported Liquid Membranes," Solvent Extraction and Ion Exchange, 8(1), 65-98, 1990.

12. Staff UNC Nuclear Industries, Technical Feasibility Study of Electrolytic Ion Transfer Membranes for Radioactive Liquid Membrane Processing, UNI-1692, 1982.

13. J. A. Del Debbio, Removal of Trace Radionuclides and Chemical Contaminants From Waste Evaporator Condensates by Electrodialysis, WINCO-1045, 1987.

14. W. H. Bond, Ultrafiltration/Reverse Osmosis, MLM-2987, 1982. 
15. J. L. Siler et al., Fouling of Ceramic Filters and Thin-Film Composite Reverse Osmosis Membranes by Inorganic and Bacteriological Constituents, WSRC-MS-91-350, 1991.

16. MMES Central Engineering, Feasibility Study Phase I: Scoping Study for the Central Neutralization Facility Upgrade/Replacement Line Item, K/D-6051, 1992. 
EM Problem: Waste management

T-12 Plant Problem: Building 9201-4 (Alpha-4). Contaminated wastewater and gases containing particulates

Problem Area/Constituents: Surface cleaning of contaminated surfaces by high pressure liquids or dry blasting processes during D\&D operations generates enormous quantities of contaminated liquids or gases which must be reduced in volume for cost-effective waste management.

\section{Reference Requirements:}

- Resource Conservation and Recovery Act of 1976 (RCRA), as amended, Land Disposal Restriction (LDR) Federal Facilities Compliance Agreement (FFCA) dated June 12, 1992

- Federal Facilities Agreement (FFA) for the Oak Ridge Reservation

- Federal Facilities Compliance Agreement-Toxic Substances Control Act (FFCA-TSCA) dated February 20, 1992

- Clean Air Act regulations under 40 CFR 60-65

- Clean Water Act regulations under 40 CFR 110-146 and 400-471

- DOE orders

Refer to the Regulatory Compliance Chapter of Vol. 1 for potentially applicable proposed and promulgated environmental laws, signed and pending agreements for the Oak Ridge Reservation, radiation protection standards, DOE orders, and nonregulatory guidance. As site- and waste-specific characteristics are provided for each technology, specific regulatory requirements will be specified.

Subelement: Waste processing

Alternative: Physical treatment

Technology: Inorganic microporous filters. These can be optimized to filter contaminated particulates from surface cleaning liquids and gases. Development is progressing well on an inorganic HEPA filter that can be used in strong detergents and solvents and can be cleaned and reused indefinitely. The filter will prove valuable in solving these problems.

Status: Predemonstration. Laboratory testing of a HEPA filter that approaches target criteria is under way. Tests indicate that target permeabilities with design pore sizes are close, and this has instilled confidence in the efficacy of the technology.

Science/Technology Needs: Ongoing development efforts for the HEPA filter will prove enormously helpful in optimizing inorganic filters to remove and concentrate contaminated particulates from waste fluids. The main goal of these development efforts will be to develop the criteria necessary for achieving separations, designing the filters, and then fabricating the filters with high permeabilities to achieve effective separation with minimum pressure drops. 
The technology needs to be demonstrated on a pilot-plant scale with a filter for liquids and for gases. Also, technology assessments need to be conducted to establish the viability and the economics of the process.

Implementation Needs: Development of the process through the demonstration phase is expected to cost $\$ 0.75 \mathrm{M}-\$ 1 \mathrm{M}$ (1992 dollars). Cost reductions provided by the process are expected to be $\$ 20 \mathrm{M}-\$ 25 \mathrm{M}$, based on simplified waste management operations and significantly reduced waste disposal costs. ${ }^{1}$

Author: J. M. Vance/615-576-0159

\section{References:}

1. S. P. N. Singh, personal communication with J. M. Vance, The Oak Ridge K-25 Site, November 30, 1992. 
EM Problem: Waste management

T-12 Plant Problem: Building 9201-4 (Alpha-4). Contaminated wastewater

Problem Area/Constituents: Oils, solvents, soils, and (perhaps) debris contaminated with $\mathrm{PCBs}$ and/or other halogenated organics

\section{Reference Requirements:}

- Resource Conservation and Recovery Act of 1976 (RCRA), as amended, Land Disposal Restriction (LDR) Federal Facilities Compliance Agreement (FFCA) dated June 12, 1992

- Federal Facilities Agreement (FFA) for the Oak Ridge Reservation

- Federal Facilities Compliance Agreement-Toxic Substances Control Act (FFCA-TSCA) dated February 20, 1992

- Clean Air Act regulations under 40 CFR 60-65

- Clean Water Act regulations under 40 CFR 110-146 and 400-471

- DOE orders

Refer to the Regulatory Compliance Chapter of Vol. 1 for potentially applicable proposed and promulgated environmental laws, signed and pending agreements for the Oak Ridge Reservation, radiation protection standards, DOE orders, and nonregulatory guidance. As site- and waste-specific characteristics are provided for each technology, specific regulatory requirements will be specified.

Subelement: Waste processing

\section{Alternative: Chemical treatment}

Technology: Chemical dechlorination. ${ }^{1}$ PCBs and other chlorinated organics are easily and rapidly dechlorinated by "solvated electrons" generated by dissolution of elemental metallic calcium in liquid ammonia. The products of the reaction are calcium chloride, calcium hydroxide, and the organic backbone of the chlorinated waste (e.g., biphenyl). This chemistry has been well known for some time; however, its application to the treatment of hazardous wastes is novel. Furthermore, liquid ammonia is a good solvent for many metals and radionuclides; thus, this process may be able to separate these constituents as well as degrade the chlorinated organics. Liquid ammonia also is an effective dispersant for clayey soils. The concept for a hazardous waste treatment process is a batch reactor. Reaction times are on the order of several minutes or less. It may be possible to treat contaminated solid debris, depending on the presence of other chemicals that may quench the solvated electrons.

Status: Predemonstration. A. L. Sandpiper Corporation, a small R\&D company in Columbus, Ohio, has a patent on the process and has done many treatability studies for various clients using a bench-scale apparatus. The company is planning to scale up soon to a capacity of $50-100 \mathrm{~kg}$ of soil per batch. 
Science/Technology Needs: The ability of the process to destroy PCBs is proven. The primary need for DOE applications is determination of the fate of heavy metals and radionuclides and characterization of the waste streams for ultimate disposal. These data can be obtained easily from laboratory-scale treatability tests.

Implementation Needs: Implementation needs for further development of the process are minimal. The cost for lab verification studies and process scale-up and design are expected to be $\$ 200 \mathrm{~K}-\$ 500 \mathrm{~K}$. Cost for a pilot-scale demonstration of the process on DOE wastes at a DOE site is expected to be $\$ 1 M-\$ 2 M$ (1992 dollars). However, should the technology prove successful, the payback on the process could be about $\$ 1$ billion, based on disposal costs for the applicable waste streams.

Author: T. L. Donaldson/615-574-2210

\section{References:}

1. Technical Report on Agent $313^{\mathrm{TM}}$ Remediation Technology, A. L. Sandpiper Corporation, P. O. Box 16423, Columbus, Ohio, 1992. 
EM Problem: Waste management

Y-12 Plant Problem: Building 9201-4 (Alpha-4). Contaminated wastewater

Problem Area/Constituents: Radionuclides (RAD) and metals in water

\section{Reference Requirements:}

- Resource Conservation and Recovery Act of 1976 (RCRA), as amended, Land Disposal Restriction (LDR) Federal Facilities Compliance Agreement (FFCA) dated June 12, 1992

- Federal Facilities Agreement (FFA) for the Oak Ridge Reservation

- Clean Air Act regulations under 40 CFR 60-65

- Clean Water Act regulations under 40 CFR 110-146 and 400-471

- DOE orders

Refer to the Regulatory Compliance Chapter of Vol. 1 for potentially applicable proposed and promulgated environmental laws, signed and pending agreements for the Oak Ridge Reservation, radiation protection standards, DOE orders, and nonregulatory guidance. As site- and waste-specific characteristics are provided for each technology, specific regulatory requirements will be specified.

\section{Subelement: Waste processing}

Alternative: Chemical treatment

Technology: Chemical precipitation. Precipitation refers to the process of removing some chemical species in solution by formation and segregation of a solid (either crystalline or amorphous) which can be considered a primarily pure phase. Other species may be incorporated into the crystal or polymeric structure during the growing process of the solid phase. Some constituents also may be adsorbed or physically entrapped in the evolving matrix. Coprecipitation is the name used to identify these kinds of phenomena. To thoroughly remove the generated particles (by filtering, by settling, or by other means), induction of optimal conditions is essential for small particles to coalesce into larger ones.

For crystalline particles, the usual procedure is to precipitate under conditions of low supersaturation. Under these circumstances, few nucleation sites are generated, leading to the growth of fewer and larger crystals. Good agitation, use of complexing agents, and appropriate choice of parameters, such as $\mathrm{pH}$ and temperature, are some of the techniques commonly used to improve removal. Aging of the precipitate also will induce larger particles by dissolution of small crystals and growth of bigger, more stable ones.

For gelatinous products, coalescence of the precipitate is accomplished by using two mechanisms: flocculation and coagulation. The process of agglomeration that occurs when particles collide and stick together is known as flocculation. This mechanism is enhanced by slow mixing and/or heating. If the particles are very small, their agglomeration usually is 
hindered by mutual electrical repulsion. Coagulation is the process that occurs upon suppression of the repelling forces preventing small particle coalescence. Several coagulation mechanisms that reduce the interparticle repulsion have been described in the literature. Among these are adsorption, enmeshment, and bridging.

A charged surface tends to surround itself with an atmosphere of oppositely charged ions. Small charged particles cannot coalesce into larger ones because of the mutual electrical repulsion between them and their charged surroundings. Neutralization of the surface charge by adsorption of oppositely charged species will eliminate the repulsion. These neutral particles, which no longer repel each other, will tend to flocculate by collision. Adsorptioncoagulation is the predominant mechanism of removal when, for example, ferric-iron is used at low concentrations to form soluble iron-hydroxy complexes. Bulky particles can trap or enmesh small particles. The enmeshment process is the primary mechanism of removal when insoluble, bulky coagulant is added, as when ferric iron is added at doses exceeding the solubility of iron oxyhydroxide. (Such enmeshment is the predominant process used in iron treatments.) Bridging is typical of polymeric coagulants. In this process, a charged, longchain polymer (anionic or cationic) bridges several smaller particles to form a larger conglomerate.

- Several iron compounds were evaluated in the literature for the adsorptioncoprecipitation of radionuclides from wastewaters.

- Addition of ferrous salts (usually sulfate) will remove metal ions by chemical reduction and adsorption-coprecipitation. Fe (II) can, under certain conditions, reduce uranyl ions to highly insoluble U (IV) species, chromium (VI) will be reduced to chromium (III), and Tc (VI), to Tc (IV). The presence of carbonate complexes will interfere and keep the uranium in solution. ${ }^{1}$ To minimize this problem, the solution to be treated usually is acidified ( $\mathrm{pH} 2-4)$ and sparged to remove the carbon dioxide. ${ }^{2}$ Because the precipitated solids from ferric ions are more effective for radionuclides removal, ferrous iron addition usually is followed by air oxidation to ferric iron under alkaline conditions ( $\mathrm{pH}$ 8.5-12) using sodium hydroxide or lime. Insoluble chromium (III) will change into soluble chromate (VI) at high $\mathrm{pH}^{3}$ It was found that $12 \mathrm{mg}$ of $\mathrm{FeSO}_{4}$ were needed to remove each milligram of technetium. ${ }^{4}$

- The addition of $\mathrm{Fe}$ (III) at $\mathrm{pHs}$ of 4-12 is a very common practice for coprecipitating/adsorbing several heavy metals and radionuclides. Removal efficiency is about the same whether the iron was precipitated before being added to the waste (adsorption) or afterwards (coprecipitation). Removal of anions is enhanced at low $\mathrm{pH}$ and is higher at high $\mathrm{pH}$ for cations. ${ }^{5}$

- Potassium ferrate (a strong oxidant) can be used as a coagulant for removing uranium, transuranic elements, and several heavy metals from aqueous waste streams. Advantages claimed for this technique are homogeneous dispersion of reagent and reduced sludge volume. ${ }^{6,7}$ Limited testing at the K-25 Site did not show satisfactory uranium and technetium removal from incinerator liquid effluent (further development work to show applicability may be required).

September 1994

Decontamination and Decommissioning 
- Uranium can be precipitated from solutions of uranyl salts by hydrogen peroxide as $\mathrm{UO}_{4} .2 \mathrm{H}_{2} \mathrm{O}$ at $\mathrm{pH}$ values of $0.5-3.5 .^{8-10}$ Any uranium present in the lower oxidation states will be oxidized by hydrogen peroxide. The global reaction can be written as

$$
\mathrm{UO}_{2}^{++}+\mathrm{H}_{2} \mathrm{O}_{2}+2 \mathrm{H}_{2} \mathrm{O} \rightarrow \mathrm{UO}_{4} 2 \mathrm{H}_{2} \mathrm{O}+2 \mathrm{H}^{+} .
$$

An excess of $\mathrm{H}_{2} \mathrm{O}_{2}$ is necessary, but adding more than twice the stoichiometric quantity is counterproductive. The peroxide precipitation is rather selective, and the final product is predominantly pure. Complexing ions, such as fluoride, will interfere mildly with the precipitation. The complexing effect can be greatly reduced by keeping the $\mathrm{pH}$ low $(0.5-1.0)$. However, at a $\mathrm{pH}<0.5$, precipitation of uranyl lons will be incomplete. After treatment at optimal conditions, residual uranium concentration will be in the ppm range. The $\mathrm{H}_{2} \mathrm{O}_{2}$ excess will tend to self-decompose; however, complete removal can be achieved by contacting the $\mathrm{H}_{2} \mathrm{O}_{2}$ with activated charcoal in a vented system.

- Lime softening: This process consists of adding lime to create a final pH between 9 and 12. This technique is particularly useful when fluoride ions are present, because it will remove them as calcium fluoride. Uranium (IV) or (V) will precipitate as axyhydroxides that include calcium and will coprecipitate with the calcium fluoride. The presence of carbonate complexes will interfere and keep uranium in the solution. To minimize this interference, the solution to be treated must be previously acidified and agitated or sparged to remove the dissolved carbon dioxide. Lime softening can be complemented with iron coprecipitation.

- Sulfide precipitation: Several metallic ions [particularly technetium (VII)] form rather insoluble sulfides (pertechnetate).

Status: Accepted

Science/Technology Needs: Precipitation technologies are fairly mature and iron coprecipitation presently is being used at the K-25-CNF (Central Neutralization Facility at the K-25 Site). However, significant development still is necessary to resolve the new challenges originated by increasingly lower regulatory limits. Potassium ferrate is not a demonstrated alternative, and further development work is needed to show its applicability and relative performance.

Implementation Needs: Iron coprecipitation is the baseline treatment at the K-25-CNF for heavy metals and radionuclides removal. According to a proposed treatabllity study to upgrade the K-25-CNF treatment facility, the estimated cost to develop, test, and document the different upgraded treatment options is about $\$ 400 \mathrm{~K}^{11}$ 
Author: G. D. Del Cul/615-576-5743

References:

1. L. Maya, "Dioxo Uranium(VI) Carbonate Complexation in Uranium Recovery by Reactive Ion-Exchange," Joumal of Inorganic Nuclear Chemistry, 43, $2133,1981$.

2. G. D. Del Cul et al., Evaluation of Alternatives for Best Available Technology Treatment and Retreatment of Uranium-Contaminated Solutions at the Paducah Gaseous Diffusion Plant C-400 Facilty, Final Report, K/QT-394 Part 3, 1991.

3. M. Pourbaix, Electrochemical Equilibria in Aqueous Solutions, Pergamon Press, Oxford, New York, 1966.

4. S. F. Seltzer, Removal of Trace Quantities of Technetium from Technetium-Bearing Liquid Wastes, KY/L-962, 1979.

5. M. A. Manzione and D. T. Merrill, Trace Metal Removal by Iron Coprecipitation: Field Evaluation, EPRI GS-6438, 1989.

6. M. E. Potts et al., "Removal of uranium, transuranic elements and priority pollutant metals from wastewaters utilizing potassium ferrate (VI)," Emerging Technologies for Hazardous Waste Management, 294, Atlanta, Georgla, October 1-3, 1991.

7. M. E. Potts et al., Treatment of Uranium Containing Wastewaters," 92-38.07, 85th Annual Meeting \& Exhibition, AWMA, Kansas City, Missouri, June 21-26, 1992.

8. P. N. Palei, Analytical Chemistry of Uranium, London-Ann Arbor, Michigan, 1970.

9. H. A. C. McKay, Uranium Chemistry in the Nuclear Industry, AERE-R-10255, 1981.

10. Gmelin Handbuch der Anorganischen Chemie, Uranium, Vol. 55 (Suppl. C3), SpringerVerlag, Berlin-New York, 1975.

11. W. D. Bostick, Proposed Treatability Study in Support of the Central Neutralization Facility Upgrade/Replacement Line Item, Interoffice Memorandum, October 1992. 
EM Problem: Waste management

Y-12 Plant Problem: Building 9201-4 (Alpha-4). Contaminated wastewater

Problem Area/Constituents: Halogenated and nonhalogenated volatiles and semivolatiles, organics, pesticides, dioxins, PCBs, solvents, BTEX, cyanides/corrosives in solids, soils, sludges, sewage, and oil; radionuclides in solids, soils, sludges, sewage, and oil

\section{Reference Requirements:}

- Resource Conservation and Recovery Act of 1976 (RCRA), as amended, Land Disposal Restriction (LDR) Federal Facilities Compliance Agreement (FFCA) dated June 12, 1992

- Federal Facilities Agreement (FFA) for the Oak Ridge Reservation

- Federal Facilities Compliance Agreement-Toxic Substances Control Act (FFCA-TSCA) dated February 20, 1992

- Clean Air Act regulations under 40 CFR 60-65

- Clean Water Act regulations under 40 CFR 110-146 and 400-471

- DOE orders

Refer to the Regulatory Compliance Chapter of Vol. 1 for potentially applicable proposed and promulgated environmental laws, signed and pending agreements for the Oak Ridge Reservation, radiation protection standards, DOE orders, and nonregulatory guidance. As site- and waste-specific characteristics are provided for each technology, specific regulatory requirements will be specified.

\section{Subelement: Waste processing}

Alternative: Chemical treatment

Technology: Solvent extraction, general. ${ }^{1-7}$ Various forms of solvent extraction technology are available or under development. These include:

- Continuous solvent extraction technology using common organic solvents to extract pollutants from soils, sediments and sludges and a second solvent to concentrate them. The second solvent, containing all of the contaminants, is removed from the process for final destruction. Decontaminated solids and water are returned to the environment. Contaminants are separated from the solids in a multistage countercurrent, solid-liquid contactor. The contaminant-rich leaching solvent subsequently is contacted with a second solvent in a liquid-liquid counter-current extractor. Residual leaching solvent in the decontaminated soil is recovered in a screw dryer and recycled back to the leaching step. The contaminant-free leaching solvent mixture from the liquid-liquid extraction step is sent to a solvent recovery unit and then returned to the leaching step.

- Solvent extraction technology using triethylamine as the solvent. Triethylamine is a biodegradable solvent formed by reacting ammonia and ethyl alcohol. The key to success of the process is triethylamine's property of inverse miscibility; below $65^{\circ} \mathrm{F}$, triethylamine 
is soluble in water (hydrophilic) and, above $65^{\circ} \mathrm{F}$, triethylamine is insoluble in water (hydrophobic). Therefore, cold triethylamine can extract water and water-soluble compounds, and warm triethylamine can extract organic contaminants such as PCBs, pesticides, semi-volatile organics, and VOCs. When treating nonpumpable wastes (soils), the process is configured as a batch extraction system. Extraction is continuous when treating pumpable wastes (sludges and sediments). Solvent recovery and recycling is continuous.

- Solvent extraction is an effective process to treat wet sludges containing volatile/semivolatile organics, oils, grease, and coal-tar compounds. The key components of this process are the solvent system and a multistage, counter-current extractor. The special solvent system is a mixture of polar and nonpolar components which help in breaking the emulsion of oil, water, and inorganic constituents. The process consists of three unit operations. The process extracts organics from a concentration as high as $40 \%$ by weight and discharges a relatively nonhazardous stream of inorganics with less than $0.1 \%$ organics. The water stream (in the case of wet sludges) containing organics dissolved in the lower ppm range can be biotreated. The organic phase containing solvent and contaminants from the extractor is distilled. The solvent is recovered and recycled back to the process. Contaminants are collected for further disposal.

- Heteroatom extraction technology can treat multiple wastes including crude oils, oil fractions, heavy oils, shale oils, and coal liquids which are present in soil, waste streams, etc. The process requires only $\mathrm{CO}_{2}$ and water at low temperatures and pressures. Extractive components can be recycled, which reduces costs and waste discharges. Treated and extracted oil remain unchanged. This nondestructive extraction procedure selectively separates the most toxic fractions of wastes, such as the nitrogen and sulfur containing compounds.

Status: Predemonstration or demonstration, depending on the particular application

Science/Technology Needs: Technical application depends on the contaminant(s) and the base matrix. Much of the development is on a pilot scale. R\&D (ranging from bench-scale to demonstration) may be required to develop (identify) the appropriate solvent and removal technology.

Implementation Needs: Solvent extraction applies to removal of multiple contaminants from D\&D and RA-derived solid wastes. Estimated cost could range from $\$ 75$ to $\$ 400 /$ ton for plants treating 5-30 tons/day of solid wastes. Payback for the technology is difficult to evaluate without information on the specific application. 
Author: D. P. Schaefferkoetter/615-574-7561. Reviewed by K. E. Plummers/615-574-7069

\section{References:}

1. W. Steiner, "New Technologies Offer Site Cleanup Alternatives," Civil Engineering News, p. 24, September 1991.

2. D. Hall, "An Overview of Solvent Extraction Treatment Technologies," Environmental Progress, 9, No. 2, May 1990.

3. Extraction and Drying of Superfund Wastes with the Carver-Greenfield Process, U.S. EPA Forum, June 11-13, 1991.

4. T. C. Halcombe, Process for Recovering Dry Solids from Aqueous Solids Mixtures, U.S. Patent No. 4,702,798, October 27, 1987.

5. U.S. EPA, Engineering Bulletin-Solvent Extraction Treatment, EPA/540/2-90/013.

6. R. L. Fellows, W. D. Bostick, and J. L. Shoemaker, Extraction of Organics From Sludge: CPCF Sludge Extraction Evaluation Bench-Scale Chemistry Tests, K-25 Site, Oak Ridge, TN, April 1991.

7. S. I. Saferman and S. K. Bhattacharya, Treatability of RCRA Compounds in BOD/Nitrification Wastewater Treatment System with Dual Media Filtration, EPA/600/S2/90/013, August 1990 

EM Problem: Waste management

Y-12 Plant Problem: Building 9201-4 (Alpha-4). Contaminated wastewater, liquids, and sludges

Problem Area/Constituents: Radionuclides, technetium, heavy metals, PCBs, solvents, and oils in various media such as solid wastes, wastewater, and spent oils and solvents

\section{Reference Requirements:}

- Resource Conservation and Recovery Act of 1976 (RCRA), as amended, Land Disposal Restriction (LDR) Federal Facilities Compliance Agreement (FFCA) dated June 12, 1992

- Federal Facilities Agreement (FFA) for the Oak Ridge Reservation

- Clean Air Act regulations under 40 CFR 60-65

- Clean Water Act regulations under 40 CFR 110-146 and 400-471

- DOE orders

Refer to the Regulatory Compliance Chapter of Vol. 1 for potentially applicable proposed and promulgated environmental laws, signed and pending agreements for the Oak Ridge Reservation, radiation protection standards, DOE orders, and nonregulatory guidance. As site- and waste-specific characteristics are provided for each technology, specific regulatory requirements will be specified.

Subelement: Waste processing

Alternative: Chemical treatment

Technology: Solvent extraction/centrifugal contactor technology. ${ }^{1-3}$ This process uses solvent extraction and centrifugal contactor technology for separation of radioactive and hazardous waste components from the various waste media generated in the course of performing D\&D and remedial action operations.

Status: Demonstration/predemonstration. The separation of heavy metals, uranium, actinides, and other radionuclides using centrifugal contactor-based solvent extraction technology has been an accepted process at DOE nuclear sites for over 25 years. However, other possible applications of this technology may require considerable R\&D.

\section{Science/Technology Needs:}

- Configuration of a chemical process flow sheet for dilute concentrations of contaminants (e.g., heavy metals, uranium, other radionuclides, etc.) relative to the potentially higher concentrations of other contaminants in the waste stream

- Determination of extraction stages required to reduce waste content of the liquid phase to below regulatory limits for release 
- Demonstration of closed-loop operation, in which extractant is recovered for reuse with surrogate waste stream

- Determination of materials of construction (if required) due to composition of the waste stream

Implementation Needs: R\&D costs for developing the technology for a specific application are estimated to range between $\$ 1 \mathrm{M}$ and $\$ 2.5 \mathrm{M}$ (1992 dollars). Payback for the technology is application-specific but could be significant considering alternate treatment technologies and waste disposal costs.

Authors: K. E. Plummer/615-574-7069 and R. T. Jubin/615-574-6566

\section{References:}

1. U.S. EPA, Test Methods for Evaluating Solid Waste, Volume 1B: Laboratory Manual for Physical/Chemical Methods, PB88-239223, Part 2 of 4, November 1986.

2. M. Germain and P. Pluot, "Diluent Washing of Aqueous Phase Using Centrifugal Contactors," 80-219, Proceedings of International Solvent Extraction Conference, 1980.

3. M. E. Whatley, The Removal of Residual Tributyl Phosphate from Aqueous Solvent Extraction Effluent Streams by Organic Diluent Washing, ORNL/CFRP-88/24, September 1988. 
EM Problem: Waste management

T-12 Plant Problem: Building 9201-4 (Alpha-4). Waste processing solid residuals

Problem Area/Constituents: Solids and liquids contaminated with RAD, technetium, heavy metals, and hazardous organics and inorganics

\section{Reference Requirements:}

- Resource Conservation and Recovery Act of 1976, as amended (RCRA) Land Disposal Restriction (LDR) Federal Facilities Compliance Agreement (FFCA) dated 12 June 1992.

- Federal Facilities Agreement (FFA) for Oak Ridge Reservation.

- Federal Facilities Compliance Agreement-Toxic Substances Control Act labbreviated as FFCA-TSCA) dated 20 February 1992.

- Clean Air Act regulations under 40 CFR 60-65.

- Clean Water Act regulations under 40 CFR 110-146 and 400-471.

- DOE orders.

Refer to the Regulatory Compliance Chapter of Vol. 1 for potentially applicable proposed and promulgated environmental laws, signed and pending agreements for the Oak Ridge Reservation, radiation protection standards, DOE orders, and nonregulatory guidance. As site- and waste-specific characteristics are provided for each technology, specific regulatory requirements will be specified.

Subelement: Waste processing

\section{Alternative: Chemical treatment}

Technology: Leaching/stripping. Leaching and stripping are processes that consist of the contacting of a fluid (usually an aqueous solution) containing one or more active species (i.e., the leaching solvent or leachant) with the contaminated material. This operation results in a partial or total transfer of the contaminants to the leachant.

There are various ways of performing leaching/stripping operations ${ }^{1}$ namely: (1) batch leaching. This involves contacting the contaminated material and the leaching solvent in a batch until leaching is complete and then separating the phases; (2) cocurrent continuous leaching. This process involves the continuous addition of leachant and contaminated material to a stirred mixer and co-current discharge of the reaction products; (3) countercurrent continuous leaching. This involves counter-current flow of the leachant and the contaminated material; and, (4) percolating leaching. This is a process in which the leachant passes down through a column or pile of the contaminated material and the leachate is collected at the bottom of the column or pile.

A wide variety of reagents can be used. For example, acids (e.g., sulfuric, hydrochloric, nitric, phosphoric and carbonic), bases (e.g., sodium hydroxide, lime-sodium sulfate, and ammonia), 
complexants (e.g., EDTA, ammonium salts, and sodium cyanide), and oxidants (e.g., air, oxygen, chlorine, hydrogen peroxide, and steam).

Substantial experience exists on the recovery of relatively high-value metals from relatively low-value ores and scrap materials. The experience of selective leaching or removal of small or trace amounts of contaminants from diverse waste materials however, is scarce. Development of specific technologies to achieve this objective is required. For stripping and leaching to be successful, a thorough knowledge of the waste to be treated is essential. The choice of a particular leachant and the operating conditions will depend on the specific waste or range of wastes to be treated.

Status: Demonstration. Use of leaching/stripping technologies for the removal of contaminants can be used as a generic technique. However, each application or range of applications needs development to determine the optimum leachant composition and treatment conditions.

Science/Technology Needs: Specific waste streams to be treated will need development work to verify the applicability of the technologies and determine the treatment characteristics and conditions.

Implementation Needs: Different types of processing equipment are already commercially available. The experience of selective leaching or removal of small amounts of contaminants from diverse waste materials is scant and development of specific technologies is required. For these technologies to be successful, a thorough knowledge of the waste to be treated is essential.

The choice of a particular leachant and the operating conditions will depend on the specific waste or range of wastes to be treated. The cost of researching and developing specific applications is estimated to be about $\$ 1 \mathrm{M}-\$ 2 \mathrm{M}$ (1992 dollars). The potential payback for the use of the technology could be significant for certain applications.

Author: G. D. Del Cul/615-576-5743

\section{References:}

1. J. B. Berkowitz et al., Unit Operations for Treatment of Hazardous Industrial Wastes, Noyes Data Corporation, New Jersey, 1978

September 1994

Decontamination and Decommissioning 
EM Problem: Waste management

Y-12 Plant Problem: Building 9201-4 (Alpha-4). Organic contaminated wastewater

Problem Area/Constituents: Organics in water, PCBs in water

\section{Reference Requirements:}

- Resource Conservation and Recovery Act of 1976 (RCRA), as amended, Land Disposal Restriction (LDR) Federal Facilities Compliance Agreement (FFCA) dated June 12, 1992

- Federal Facilities Agreement (FFA) for the Oak Ridge Reservation

- Federal Facilities Compliance Agreement-Toxic Substances Control Act (FFCA-TSCA) dated February 20, 1992

- Clean Air Act regulations under 40 CFR 60-65

- Clean Water Act regulations under 40 CFR 110-146 and 400-471

- DOE orders

Refer to the Regulatory Compliance Chapter of Vol. 1 for potentially applicable proposed and promulgated environmental laws, signed and pending agreements for the Oak Ridge Reservation, radiation protection standards, DOE orders, and nonregulatory guidance. As site- and waste-specific characteristics are provided for each technology, specific regulatory requirements will be specified.

Subelement: Waste processing

Alternative: Chemical treatment

Technology: Ozonation-photolysis. Chemical treatment (also known as advanced oxidation)-Ozonation, photolysis, ultraviolet (UV) $/ \mathrm{H}_{2} \mathrm{O}_{2}, \mathrm{Fe}^{+2 /+3} / \mathrm{H}_{2} \mathrm{O}_{2}$ (Fenton Reagent), etc. Advanced oxidation technologies can be very useful for destroying organic contaminants in soil and water. Several processes can be found in the literature, ${ }^{1}$ such as ozonation at acidic $\mathrm{pH}$, ozonation at near neutral $\mathrm{pH}, \mathrm{UV} / \mathrm{H}_{2} \mathrm{O}_{2}, \mathrm{Fe}^{+2 /+3} / \mathrm{H}_{2} \mathrm{O}_{2}$ (Fenton Reagent), photocatalysis, etc. Except for ozone under acidic conditions $(\mathrm{pH}<3)$, where the primary oxidant is molecular ozone itself, ${ }^{2}$ axidation of organic compounds occurs mainly through hydroxyl-free radicals $\mathrm{OH}^{3}$ Hydroxyl-free radicals are produced during decomposition of ozone under neutral or basic $\mathrm{pH}$, ultraviolet (UV)-light photocatalysis using titanium dioxide, ${ }^{4} \mathrm{UV}$-light photocatalysis of $\mathrm{H}_{2} \mathrm{O}_{2}{ }^{1}$, oxidation of $\mathrm{Fe}^{+2}$ with $\mathrm{H}_{2} \mathrm{O}_{2}$ (Fenton Reagent), ${ }^{5}$ etc. Hydroxyl-free radicals have been shown to be powerful oxidants that react nonspecifically with organic compounds. ${ }^{5}$ Although some organic species are easily destroyed by oxidation agents, they are rarely converted completely to carbon dioxide and water, and some rather hazardous compounds can be produced as "end products of oxidation." For these technologies to be successful, a thorough knowledge of the waste to be treated is essential.

The choice of a particular oxidation system over others will depend on the specific waste or range of wastes to be treated. 
Status: Demonstration. Use of oxidation technologies for destroying organics cannot be used as a generic technique, and each application or range of applications needs $R \& D$.

Science/Technology Needs: As previously mentioned, although some organic species are easily destroyed by oxidation agents, they are rarely completely converted to carbon dioxide and water, and some rather hazardous compounds can be produced as "end products of oxidation." The equipment needed to implement these technologies is relatively simple and can be coupled easily to other chemical treatments, such as coprecipitation (e.g., Fenton reagent, $\mathrm{Fe}^{+2 /+3} / \mathrm{H}_{2} \mathrm{O}_{2}$, could be used to destroy organic matter and could be followed by $\mathrm{pH}$ adjustment for iron-coprecipitation of radionuclides and heavy metals). Specific waste streams to be treated will need some development work to verify the applicability of oxidation technologies.

Implementation Needs: The cost of demonstrating applicability of the technology to treat a given waste stream is estimated to be $\$ 1 M-\$ 2 M$ (1992 dollars). If use of the process proves successful, payback for the technology is likely to be significant (on the order of tens to hundreds of millions of dollars), especially when considering the cost of waste disposal alternatives for some waste streams containing relatively refractory compounds.

Author: G. D. Del Cul/615-576-5743

References:

1. C. C. D. Yao et al., Kinetic Features of Advanced Oxidation Processes for Treating Aqueous Chemical Mixtures, Second International Symposium: Chemical Oxidation, Technology for the Nineties, Nashville, Tennessee, 1992.

2. R. G. Rice, Ozone Chemistry Applied to Cooling Tower Water Treatment, Second International Symposium: Chemical Oxidation, Technology for the Nineties, Nashville, Tennessee, 1992.

3. C. Munz et al., Oxidative treatment of Process Water in a Soil Decontamination Plant: I. Laboratory Studies, Second International Symposium: Chemical Oxidation, Technology for the Nineties, Nashville, Tennessee, 1992.

4. C. S. Turchi and M. S. Mehos, Solar Photocatalytic Detoxification of Groundwater: Developments in Reactor Design, Second International Symposium: Chemical Oxidation, Technology for the Nineties, Nashville, Tennessee, 1992.

5. K. A. Flaherty and C. P. Huang, Continuous Flow Applications of Fenton's Reagent for the Treatment of Refractory Wastewaters, Second International Symposium: Chemical Oxidation, Technology for the Nineties, Nashville, Tennessee, 1992. 
EM Problem: Waste management

Y-12 Plant Problem: Building 9201-4 (Alpha-4). Contaminated wastewater

Problem Area/Constituents: Organics in water, PCBs in water, organic residues, and organics in sludges

\section{Reference Requirements:}

- Resource Conservation and Recovery Act of 1976 (RCRA), as amended, Land Disposal Restriction (LDR) Federal Facilities Compliance Agreement (FFCA) June 12, 1992

- Federal Facilities Agreement (FFA) for the Oak Ridge Reservation

- Federal Facilities Compliance Agreement-Toxic Substances Control Act (FFCA-TSCA) dated February 20, 1992

- Clean Air Act regulations under 40 CFR 60-65

- Clean Water Act regulations under 40 CFR 110-146 and 400-471

- DOE orders

Refer to the Regulatory Compliance Chapter of Vol. 1 for potentially applicable proposed and promulgated environmental laws, signed and pending agreements for the Oak Ridge Reservation, radiation protection standards, DOE orders, and nonregulatory guidance. As site- and waste-specific characteristics are provided for each technology, specific regulatory requirements will be specified.

\section{Subelement: Waste processing}

\section{Alternative: Chemical treatment}

Technology: Wet air oxidation (WAO). Wet air oxidation is the process of oxidizing dissolved or suspended contaminants in aqueous wastes streams at elevated temperatures and pressures. ${ }^{1}$ It is generally considered applicable for treating certain organic-containing media that are too toxic to treat biologically and yet too dilute to incinerate economically. ${ }^{2,3} \mathrm{~A}$ leading manufacturer of commercially avallable WAO equipment reports that WAO occurs at temperatures of $175-320^{\circ} \mathrm{C}$ and at pressures of 300-3000 psig. The reaction proceeds, without need for auxiliary fuel, at feed chemical oxygen demand (COD) concentrations of $20-30 \mathrm{~g} / \mathrm{L}$. ${ }^{4}$ The extent of contaminant destruction depends on the wastes to be oxidized and reaction conditions. Typically, $80 \%$ of the organics will be oxidized to $\mathrm{CO}_{2}$ and water. Although some organic species are easily destroyed by WAO, they are rarely completely converted to carbon dioxide and water. Residual organics generally will be low-molecular-weight, biodegradable compounds such as acetic and formic acids. Nevertheless, with some waste streams, hazardous compounds can be produced as "end products of oxidation." For this technology to be successful, thorough knowledge of the waste to be treated is essential. For the destruction of halogenated aromatics such as PCBs, more energetic conditions and catalysts are necessary, and the destruction is likely to be incomplete. 
Status: Demonstration. The technology is commercially available; however, satisfactory destruction of highly refractory organics requires treatability studies and development. Also, use of the technology to treat a specific site waste stream will need to be demonstrated.

Science/Technology Needs: As previously mentioned, although some organic species are easily and completely destroyed by WAO, they are rarely completely converted to carbon dioxide and water, and some rather hazardous compounds can be produced as "end products of oxidation." Specific waste streams to be treated will need development work to verify applicability of the process to treat the problem waste stream.

Implementation Needs: A comparison between a $20-\mathrm{gal} / \mathrm{min}$ WAO system and a comparable incinerator for wastewater containing 7\% COD showed an installation cost 50\% higher than that of an incinerator. However, the operating costs for WAO were only one fourth that of an incinerator [ $\$ 163,000$ for WAO vs $\$ 463,500$ for incineration (1979 dollars)]. ${ }^{5}$ Cost estimates of $\$ 0.1-\$ 0.5 / \mathrm{gal}$ of waste have been proposed. Development costs to determine the treatability of Alpha-4 (Bldg. 9201-4) wastes by WAO are estimated to be \$2M (1992 dollars).

Author: G. D. Del Cul/615-576-5743

\section{References:}

1. M. Breton et al., Treatment Technologies for Solvent Containing Wastes, Pollution Technology Review No. 149, Noyes Data Corporation, New Jersey, 1988.

2. M. J. Dioptric et al., "Wet Air Oxidation of Hazardous Organics in Wastewater," Environmental Progress, 4(3), 1985.

3. H. Freeman, Innovative Thermal Hazardous Treatment Processes, U.S. EPA, Hazardous Waste Engineering Research Laboratory, Cincinnati, Ohio, 1985.

4. California Air Resources Board, Air Pollution Impacts of Hazardous Waste Incineration: A California Prospective, December 1983.

5. A. R. Wilhelm and D. G. Datums, "Wet Air Oxidation, An Alternative to Incineration," Chemical Engineering Progress, August 1989.

September 1994

Decontamination and Decommissioning 
EM Problem: Waste management

Y-12 Plant Problem: Building 9201-4 (Alpha-4). Contaminated oil

Problem Area/Constituents: Organic ion-exchange media, radionuclides and technetium in oil, heavy metals in oil, PCB in oil, nonhalogenated organics in oil, radionuclides in solvents, heavy metals in solvents, PCB in solvents, organics

\section{Reference Requirements:}

- Resource Conservation and Recovery Act of 1976 (RCRA), as amended, Land Disposal Restriction (LDR) Federal Facilities Compliance Agreement (FFCA) dated June 12, 1992

- Federal Facilities Agreement (FFA) for the Oak Ridge Reservation

- Federal Facilities Compliance Agreement-Taxic Substances Control Act (FFCA-TSCA) dated February 20, 1992

- Clean Air Act regulations under 40 CFR 60-65

- Clean Water Act regulations under 40 CFR 110-146 and 400-471

- DOE orders

Refer to the Regulatory Compliance Chapter of Vol. 1 for potentially applicable proposed and promulgated environmental laws, signed and pending agreements for the Oak Ridge Reservation, radiation protection standards, DOE orders, and nonregulatory guidance. As site- and waste-specific characteristics are provided for each technology, specific regulatory requirements will be specified.

Subelement: Waste processing

Alternative: Chemical treatment

Technology: Supercritical water oxidation. The basic concept of supercritical oxidation technology is based on the complete miscibility of oxygen, carbon dioxide, and many organics with dense supercritical water (the critical point for pure water is $374^{\circ} \mathrm{C}$ and $221 \mathrm{Bar}$ ). Under supercritical conditions, both flameless oxidation and flaming combustion can occur. ${ }^{1-4}$ Work conducted in the mid-1980s by Modell ${ }^{4}$ and his colleagues showed that many organic compounds can be almost completely destroyed in a supercritical homogeneous phase and that the heat of reaction can substantially reduce, and sometimes completely eliminate, the need for additional fuel to sustain the process. ${ }^{5}$ The perceived advantages of a supercritical water reactor follow. (1) The reactor is a closed system, and there are no emissions to the atmosphere. (2) If it malfunctions, it can be shut down relatively easily. (3) The prototypes to treat wastes run at $500-600^{\circ} \mathrm{C}$, considerably lower than conventional thermal methods. (4) If carbon content of the waste is at least $10 \%$, the supercritical process can be energysufficient. The potential problems are: (1) corrosion of the reactor parts, (2) plugging and caking, and (3) separation of solids under high-pressure, high-temperature conditions.

Status: Demonstration 
Science/Technology Needs: Small laboratory demonstration units (a few gallons of waste per day) were operated and tested, and R\&D efforts continue today. ${ }^{6}$ Significant development is needed to solve the corrosion, plugging, and caking of the reactors. Overall experience is needed in the specific problems related to particular types of wastes to generate a data base to support the applicability and relative merits of this promising technology.

Implementation Needs: The estimated cost for a 3-year development plan and technology transfer for destruction of spent organic ion-exchange resins using supercritical water oxidation, recovery of special materials, and recycling, based on DOE-OTD-TTP No. OR-4TAJ93 by G. D. Del Cul and C. E. Bamberger, is $\$ 1.6 \mathrm{M} .{ }^{8} \mathrm{~A}$ full-scale implementation program for designing, testing, and developing bench-scale and industrial-scale prototypes would cost approximately $\$ 3 \mathrm{M}$.

Author: G. D. Del Cul/615-576-5743

References:

1. R. K. Helling and J. W. Tester, "Oxidation of Simple Compounds and Mixtures in Supercritical Water: Carbon Monoxide, Ammonia and Ethanol," Environ. Sct. Technol, $22,1319,1988$.

2. T. J. Houser et al., "Reactivity of Some Organic Compounds with Supercritical Water," Fuel, 65, 827, 1986.

3. C. F. Melius et al., Effects of Water on Combustion Kinetics at High Pressure, 23rd Symposium (International) on Combustion, The Combustion Institute, 1991.

4. T. B. Thomason and M. Modell, "Supercritical Water Destruction of Aqueous Wastes," Hazardous Waste, 1, 453, 1984.

5. R. W. Shaw et al., "Supercritical Water a Medium for Chemistry," C\&EN, December 1991.

6. R. D. McFarland et al., Design and Operational Parameters of Transportable Supercritical Water Oxidation Waste Destruction Unit, LA-12216-MS, 1991.

7. S. J. Buelow, "Destruction of Hazardous Wastes by Supercritical Water Oxidation," Second International Symposium: Chemical Oxidation, Technology for the Nineties, Nashville, Tennessee, 1992.

8. G. D. Del Cul and C. E. Bamberger, Supercritical Water Oxidation: Low Temperature Destruction of Organic Polymeric Resins Using a Supercritical Water Reactor, OTD-DOE OR-4TAJ-93, 1992. 
EM Problem: Waste management

Y-12 Plant Problem: Building 9201-4 (Alpha-4). Contaminated wastewater

Problem Area/Constituents: Radionuclides in water

\section{Reference Requirements:}

- Resource Conservation and Recovery Act of 1976 (RCRA), as amended, Land Disposal Restriction (LDR) Federal Facilities Compliance Agreement (FFCA) dated June 12, 1992

- Federal Facilities Agreement (FFA) for the Oak Ridge Reservation

- Clean Air Act regulations under 40 CFR 60-65

- Clean Water Act regulations under 40 CFR 110-146 and 400-471

- DOE orders

Refer to the Regulatory Compliance Chapter of Vol. 1 for potentially applicable proposed and promulgated environmental laws, signed and pending agreements for the Oak Ridge Reservation, radiation protection standards, DOE orders, and nonregulatory guidance. As site- and waste-specific characteristics are provided for each technology, specific regulatory requirements will be specified.

Subelement: Waste Processing

\section{Alternative:}

Technology: Ion-exchange. A process involving the mass transfer of ions between a polar solvent and an inert liquid or solid matrix containing highly polar groups which can be ionized.

Several different ion-exchange media have been evaluated in the literature including:

- Strong-base anion exchange resins. Dowex 2 X-8 resin has been reported as having a very high affinity for the uranyl ion complexed with carbonate as a tricarbonate complex in the presence of competing anions. This carbonate complex is the predominant uranium species in groundwater. ${ }^{1}$

- Inorganic ion-exchange media. Inorganic hydrous oxides and phosphates can be used to remove uranium and cesium from contaminated groundwater and brines. ${ }^{2}$

Titanium monohydrogen phosphate microspheres can be used to remove uranium from nitrate solutions. ${ }^{3}$ Glass-based materials (e.g. Durasil media) were used to remove radionuclides from contaminated wastewaters. ${ }^{4}$

- Polyvinylpyridine polymer-based resins. Reillex 1-methyl-4-vinylpyridine polymer-based resins are good sorbers of plutonium (iv) from nitric acid with high capacity and rapid sorption kinetics. This resin has a greater stability to chemical and radiolytic degradation than conventional anion exchange resins. ${ }^{5}$ 
- Cation exchange resins. Cesium-137 and strontium-90 can be removed from aqueous solutions using cation-exchange resins such as Duolite CS-100 (a bifunctional phenoliccarboxylate resin in the sodium form) or Dowex HGR-W2 sodium-form. ${ }^{6.7}$

\section{Status: Demonstration}

Science/Technology Needs: Use of organic ion-exchange resins is a standard unit operation for radionuclides removal from contaminated wastewater. Inorganic ion-exchangers-sorbers are in the development/predemonstration stage, and their applicability and process economics will need to be evaluated.

Implementation Needs: Development costs are estimated to be $\$ 1.25 \mathrm{M}$ (1992 dollars). These are costs for development and technology transfer to private industry of advanced inorganic sorbents for selective removal of technetium and other contaminants. These costs are based on TTP No. OR-4DAH-93, by G. D. Del Cul, C. E. Bamberger, and J. L. Collins, submitted to DOE-OTD for Technetium Removal.

Author: W. D. Bostick/615-574-6827

References:

1. L. Maya, "Dioxo uranium(vi) carbonate complexation in uranium recovery by reactive ionexchange," Journal of Inorganic Nuclear Chemistry, 43, 2133-2137, 1981.

2. J. L. Collins et al., "Sorption of metal ions from groundwaters using hydrous titanium oxide microspheres," Emerging Technologies for Hazardous Waste Management, Atlanta, Georgia, October 1-3, 1991.

3. T. J. Takacs, "Removal of Uranium from Nitrate Wastes," Y-12 Development Division, Technical Progress Report, Part. 6, Period Ending June 15, 1992, Y-2455-6, 1992.

4. S. P. N. Singh and T. F. Lomenick, Summary Report on the Demonstration of the Duratek Process for Treatment of Mixed-Waste Contaminated Groundwater, ORNL/TM-11927, April 1992.

5. S. F. Marsh, Evaluation of a New, Macroporous Polyvinylpyridine Resin for Processing Plutonium Using Nitrate Anion Exchange, LA-11490, 1989.

6. E. D. Collins et al., An Improved Ion-Exchange Method for Treatment of Slightly Contaminated Wastewaters, CONF-8809116-1, 1988.

7. R. Hall, Treatment of Low-Activity-Level Process Wastewaters by Continuous Countercurrent Ion Exchange, CONF-900210, 2, 429 (1990). 
EM Problem: Waste management

Y-12 Plant Problem: Treatment of process wastewater, Melton Valley supernates, and other highly alkaline wastewaters

Problem Area/Constituents: Removal of mercury contamination from the wastewater

\section{Reference Requirements:}

- Resource Conservation and Recovery Act of 1976 (RCRA), as amended, Land Disposal Restriction (LDR) Federal Facilities Compliance Agreement (FFCA) dated June 12, 1992

- Federal Facilities Agreement (FFA) for the Oak Ridge Reservation (ORR)

- Clean Air Act regulations under 40 CFR 60-65

- Clean Water Act regulations under 40 CFR 110-146 and 400-471

- DOE orders

Refer to the Regulatory Compliance chapter of Vol. 1, Pt. C, for more detailed discussions of potentially applicable proposed and promulgated environmental laws, signed and pending agreements for the ORR, radiation protection standards, DOE orders, and nonregulatory guidance. As site- and waste-specific characteristics are provided for each technology, specific regulatory requirements will be specified.

\section{Subelement: Waste processing}

\section{Alternatives: Chemical treatment}

Technology: Ion exchange (mercury removal)..$^{1-5}$ Briefly, ion exchange is a process for the removal of ionic contaminants from aqueous solutions and liquids containing ionic radioactive and/or hazardous species. The contaminant(s) are removed from the liquid by being trapped on a solid matrix known as an ion-exchange medium or resin. When the ionexchange medium becomes saturated with the contaminant, it can be regenerated separately to recover the contaminant for further treatment or disposal. The regenerated medium can often be recycled to treat additional contaminated waste liquids. Given below are descriptions of two ion-exchange media or resins for mercury removal from contaminated wastewaters:

- Mercury can be removed by ion exchange. Resins containing the iminodiacetic acid group will pick up cationic mercury selectively from calcium and magnesium, but copper and cobalt are also picked up. Mercury in the form of anionic complexes (e.g. $\mathrm{HgCl}_{3}{ }^{-}$or $\mathrm{HgCl}_{4}{ }^{-}$) can be picked up by anion-exchange resins but not selectively. Savannah River Plant has used a thiol resin that is very selective for mercury in the cationic form. A thiol resin available commercially is Ionac SR-4.

- Mercury can also be removed by using chelating resins. Processes using these resins would be similar to conventional ion-exchange operations, except chelating resins cannot be regenerated or are regenerated with some difficulty. A resin containing an 
isothiouronium was studied in 1971 for removal of both $\mathrm{Hg}^{2+}$ and $\mathrm{CH}_{3} \mathrm{Hg}$. The selectivity in the presence of dissolved iron, nickel, copper and zinc at concentrations up to $10 \mathrm{~g} / \mathrm{L}$ was complete. A second account of a chelate resin application was found in a U.S. patent assigned to a Japanese company. ${ }^{4}$

Status: Accepted. A vast variety of ion-exchange separations are carried out commercially.

Science/Technology Needs: Better characterization of ORNL and DOE wastes to identify waste stream compositions that are amenable to ion-exchange separations. A vendor survey is required to determine if chelating resins are available for mercury recovery operations. Uptake tests may be required. Better characterization of the wastes is needed to determine mercury speciation and competing ions.

Implementation Needs: The implementation needs for conducting the required research, development, and demonstration are estimated to cost aabout \$1M-\$2M (1992 dollars). This does not include the costs for the equipment or setting up the process to treat the above wastewaters.

Author: J. J. Perona/615-576-9280

\section{References:}

1. F. X. McGarvey, "Mercury Removal with Ionac Ion-Exchange Resins," in Arsenic and Mercury; Workshop on Removal, Recovery, Treatment and Disposal," R. J. Turner and J. Labiosa, eds., EPA/600/R-92/105, U.S. Environmental Protection Agency, August 1992.

2. J. Bibler, R. Wallace, and M. Ebra, "Mercury Removal from SRP Radioactive Waste Streams Using Ion Exchange," in Proceedings of the 1986 Conference on Waste Management, High-Level Waste, Vol. 2, p. 471.

3. S. L. Law, "Methyl Mercury and Inorganic Mercury Collection by a Selective Chelating Resin," Science 174, 285 (1971).

4. K. Motani et al., U.S. Patent Number 3,790,535 issued February 5, 1974; assigned to Tokuyama Soda KK, Japan.

5. F. McGarvey and E. Hauser, "Removal of Mercury from Industrial Wastes as Well as from Potable Water," Sixth International Conference on Chemistry for the Protection of the Enwironment, September 15-18, 1987, Torino, Italy. 
EM Problem: Waste management

Y-12 Plant Problem: Building 9201-4 (Alpha-4). Waste processing, solid residuals

Problem Area/Constituents: Wastes containing long-life radioisotopes

\section{Reference Requirements:}

- Resource Conservation and Recovery Act of 1976 (RCRA), as amended, Land Disposal Restriction (LDR) Federal Facilities Compliance Agreement (FFCA) dated June 12, 1992

- Federal Facilities Agreement (FFA) for the Oak Ridge Reservation

- Clean Air Act regulations under 40 CFR 60-65

- Clean Water Act regulations under 40 CFR 110-146 and 400-471

- DOE orders

Refer to the Regulatory Compliance Chapter of Vol. 1 for potentially applicable proposed and promulgated environmental laws, signed and pending agreements for the Oak Ridge Reservation, radiation protection standards, DOE orders, and nonregulatory guidance. As site- and waste-specific characteristics are provided for each technology, specific regulatory requirements will be specified.

Subelement: Waste processing

Alternative: Chemical treatment

Technology: Transmutation. This technology, as envisioned, would irradiate concentrated nuclear waste with a high-intensity thermal flux of accelerator-produced neutrons (in the range of $1016 \mathrm{n} / \mathrm{cm}^{2} . \mathrm{s}$ ). As a result of this irradiation, the initial radionuclides will transmute. Some of the radionuclides will transmute into stable or more stable nuclides, while some will transmute into shorter-lived radioisotopes that will then decay into stable nuclides. To produce the high intensity neutron flux needed for this technology to be practical (100 times larger than in large-scale thermal reactors), a proton linear accelerator with 1.5$\mathrm{GeV}$ energy and a $250 \mathrm{~mA} \mathrm{CW}$ current is proposed. ${ }^{1}$ The accelerated proton beam would collide with a lead or lead-bismuth spallation target and generate a high flux of neutrons.

Technetium-99 ( $\mathrm{T}=2.1 \times 10^{5}$ years) is a very good candidate for the transmutation concept. It has a relatively high cross section for the capture of thermal neutrons (19 Barns, 1 Barn $=10^{-24} \mathrm{~cm}^{2}$ and all of the higher technetium isotopes that could be generated by single or multiple neutron capture will rapidly decay into stable ruthenium, rhodium, or palladium. ${ }^{2}$

\section{Status: Conceptual}

Science/Technology Needs: Several national laboratories, including Los Alamos, Brookhaven, Hanford-PNL, etc., ${ }^{1,36}$ have conceptual plans for constructing "transmutation" 
reactors based on the hybrid proton accelerator subcritical lattice reactor. A detailed neutronic analysis of the proposed schemes also has been conducted. ${ }^{3}$ The next step should be design and construction of a prototype unit.

Implementation Needs: The future of an industrial-scale transmutation facility depends on availability of a permanent, high-level waste repository. Development and construction costs for a "national transmutation facility" would be in the billion dollar range and would take many years to complete and become operational. This waste treatment option has been suggested for treatment and disposal of the long half-life radionuclides (such as technetium-99) in a "national" facility.

Author: G. D. Del Cul/615-576-5743

\section{References:}

1. G. P. Lawrence, "High Power Linear Accelerators for Tritium Production and Transmutation," Nucl. Instrum. Methods Phys. Res., Sect. B, B56-B57 (Pt. 2), 1000-47 1$1,1992$.

2. W. Seelmann-Eggbert et al., Chart of the Nuclides, Kernforschungszentrum Karlsruhe, 1981.

3. M. A. Lone et al., An Overview of the ATW Reactor for Transmutation of Neptunium-237, AECL-10953, 1992.

4. C. D. Bowman et al., "Nuclear Energy Generation and Waste Transmutation Using an Accelerator-Driven Intense Thermal Neutron Source," Nucl. Instrum. Methods Phys. Res., Sect. A, A320(1-2), 336-67, 1992.

5. G. J. Van Tuyle et al., The Phoenix Concept: Proposed Transmutation of Long-Lived Radioactive Wastes to Produce Electric Power, BNL-52279, 1991.

6. CURE: Clean Use of Reactor Energy, WHC-EP-0268, 1990.

September 1994

Decontamination and Decommissioning 
EM Problem: Waste management

Y-12 Plant Problem: Building 9201-4 (Alpha-4). Waste off-gases from D\&D, RA, and WM operations

Problem Area/Constituents: Removal of inorganic, organic, and radioactive compounds and particulates from various gaseous streams at the $\mathrm{K}-25$ Site

\section{Reference Requirements:}

- Resource Conservation and Recovery Act of 1976 (RCRA), as amended, Land Disposal Restriction (LDR) Federal Facilities Compliance Agreement (FFCA) dated June 12, 1992

- Federal Facilities Agreement (FFA) for the Oak Ridge Reservation

- Federal Facilities Compliance Agreement-Toxic Substances Control Act (FFCA-TSCA) dated February 20, 1992

- Clean Air Act regulations under 40 CFR 60-65

- Clean Water Act regulations under 40 CFR 110-146 and 400-471

- DOE orders

Refer to the Regulatory Compliance Chapter of Vol. 1 for potentially applicable proposed and promulgated environmental laws, signed and pending agreements for the Oak Ridge Reservation, radiation protection standards, DOE orders, and nonregulatory guidance. As site- and waste-specific characteristics are provided for each technology, specific regulatory requirements will be specified.

Subelement: Waste processing

Alternative: Thermal treatment

Technology: Low-temperature separation. ${ }^{1}$ This process can be used for removing hazardous volatile contaminants [e.g., volatile organic compounds (VOCs)] from waste-gas streams. Briefly, low-temperature separation consists of cooling the waste gas (by refrigeration, if necessary) to temperatures where the volatile contaminants can condense out of the gas stream. The condensed liquids then can either be distilled to recover and recycle the individual VOCs or be disposed, for example, by incineration. The process can be used for separating, for example, freons, acetone, benzene, etc., from the waste-gas stream.

Status: Predemonstration

Science/Technology Needs: These are likely to be minimal, because the fundamentals of the process can be obtained from, for example, the petroleum industry. However, use of the process to treat waste-gas streams at the site may require bench-scale studies and demonstrations to prove its applicability. 
Implementation Needs: Development and demonstration of use of the technology to treat waste-gas streams are estimated to cost \$1M-\$2M (1992 dollars). However, payback could be significant when compared to the cost of treatment alternatives. Also, the process could result in significant waste minimization because of recovery and recycling of the hazardous compounds.

Author: S.P.N. Singh/615-574-6639

\section{References:}

1. R. H. Perry and D. W. Green, eds., Perry's Chemical Engineers' Handbook, 6th edition, McGraw-Hill, New York, 1984. 
EM Problem: Waste management

Y-12 Plant Problem: Building 9201-4 (Alpha-4). Waste off-gases from decontamination and decommissioning, remedial action, and waste management operations

Problem Area/Constituents: Waste streams containing volatile organic compounds (VOCs) such as methylene chloride, chloroform, carbon tetrachloride, etc., and gaseous wastes containing water-soluble gas contaminants

\section{Reference Requirements:}

- Resource Conservation and Recovery Act of 1976 (RCRA), as amended, Land Disposal Restriction (LDR) Federal Facilities Compliance Agreement (FFCA) dated June 12, 1992

- Federal Facilities Agreement (FFA) for the Oak Ridge Reservation

- Federal Facilities Compliance Agreement-Toxic Substances Control Act (FFCA-TSCA) dated February 20, 1992

- Clean Air Act regulations under 40 CFR 60-65

- Clean Water Act regulations under 40 CFR 110-146 and 400-471

- DOE orders

Refer to the Regulatory Compliance Chapter of Vol. 1 for potentially applicable proposed and promulgated environmental laws, signed and pending agreements for the Oak Ridge Reservation, radiation protection standards, DOE orders, and nonregulatory guidance. As site- and waste-specific characteristics are provided for each technology, specific regulatory requirements will be specified.

Subelement: Waste processing

Alternative: Thermal treatment

Technology: Steam stripping. An industrially accepted process for removing low-boiling, organic compounds from aqueous wastes. The process also can be used in gas treatment to remove water-soluble contaminants. It is a continuous fractional distillation process in which live steam is injected directly into the bottom of a packed or tray tower to separate VOCs from wastewater in multiple equilibrium stages. It is most effective in treating liquid wastes containing organic solvents with low boiling points and which are relatively immiscible in water.

\section{Status: Demonstration}

Science/Technology Needs: Specific waste treatment applications for this technology will be identified. A pilot facility then could be designed and installed or purchased as a skidmounted package. This package likely will consist of a fractionation column, heat exchanger(s), reboilers, tanks, instrumentation controls, and other accessory equipment. Optimum operating conditions will be established during pilot runs, and scale-up to a larger 
facility will follow, if required. The pilot unit can be a mobile unit for ease of transport to the waste sites.

Implementation Needs: The above-stated work is estimated to cost \$1M-\$2M (1992 dollars) to implement.

Author: J. M. Chiang/615-574-9659

\section{References:}

1. R. J. Turner, "Waste Treatability Tests of Spent Solvent and Other Organic Wastewaters," Environmental Progress, May 1989.

2. Field Measurements of Full-Scale Hazardous Waste Treatment Facilities-Organic Solvent Wastes, EPA Report No. PB89-138853, October 1988.

3. F. N. Kemmer, The Nalco Water Handbook, Nalco Chemical Co., 1979.

4. Handbook of Industrial Water Conditioning, Betz Laboratories, Inc., 1980.

5. R.H. Perry and D. W. Green, eds., Perry's Chemical Engineers' Handbook, 6th edition, McGraw-Hill, New York, 1984. 
EM Problem: Waste management

Y-12 Plant Problem: Building 9201-4 (Alpha-4). Contaminated liquid wastes

Problem Area/Constituents: Radionuclides in water, heavy metals in water, PCBs in water, other volatile organics in water, PCBs in oil, nonhalogenated organics in oil, radionuclides in solvents, radionuclides (including technetium) in mercury, and contaminated specialty freons

\section{Reference Requirements:}

- Resource Conservation and Recovery Act of 1976 (RCRA), as amended, Land Disposal Restriction (LDR) Federal Facilities Compliance Agreement (FFCA) dated June 12, 1992

- Federal Facilities Agreement (FFA) for the Oak Ridge Reservation

- Federal Facilities Compliance Agreement-Toxic Substances Control Act (FFCA-TSCA) dated February 20, 1992

- Clean Air Act regulations under 40 CFR 60-65

- Clean Water Act regulations under 40 CFR 110-146 and 400-471

- DOE orders

Refer to the Regulatory Compliance Chapter of Vol. 1 for potentially applicable proposed and promulgated environmental laws, signed and pending agreements for the Oak Ridge Reservation, radiation protection standards, DOE orders, and nonregulatory guidance. As site- and waste-specific characteristics are provided for each technology, specific regulatory requirements will be specified.

Subelement: Waste processing

Alternative: Thermal treatment

Technology: Distillation. ${ }^{1.2}$ Basic distillation involves application of heat to a liquid mixture, vaporization of part of the mixture, and removal of heat from the vaporized portion. The resulting condensed vapor, called distillate, is richer in the more volatile components, and the residual, unvaporized bottoms are richer in less volatile components.

In simple distillation, a single equilibrium stage is used to obtain a desired separation, and the operation may be either batch or continuous. Simple continuous distillation (also called flash distillation) has a continuous feed to an equilibrium stage; the liquid and vapor leaving the stage are in equilibrium. Flash distillation is used in applications where crude separation is adequate. The component separation in simple distillation is limited by thermodynamic partitioning constraints, and multiple staging must therefore be used to increase separation efficiency.

Multiple staging in column design is achieved by returning part of the condensed overhead vapors to the top of the column, thereby bringing this reflux liquid into intimate contact with 
the rising vapors. Either a tray or a packed column normally is used to provide the necessary gas-liquid interfacial area for mass transfer. The degree of component separation for a given system configuration depends on operating conditions, the number of stages, and the amount of reflux.

Status: Accepted by industry; many units are in use (solvent recovery and chemical purification for example). Capital costs are high.

Science/Technology Needs: The following may cause problems during operation:

- Feed to a column must be free-flowing with negligible solids content. Solids generally plug and foul the internals of a distillation column.

- Other compounds that can cause problems during distillation include organic peroxides, pyrophoric organics, most inorganic wastes, and feeds that tend to polymerize.

- Feeds should not exhibit large and/or frequent variations in composition.

Implementation Needs:

- Process equipment

- All utilities, including reliable electricity, air, water, etc.

- Drum and material handling equipment

- Trained chemical operators

- EPA-certified laboratory support

Author: C. H. Dukes/615-574-9561

References:

1. H. M. Freeman, ed., Standard Handbook of Hazardous Waste Treatment and Disposal, McGraw-Hill, New York, p. 6.23-6.38, 1989.

2. R. H. Perry and D. W. Green, eds., Chemical Engineers' Handbook, 6th edition, McGrawHill, New York, 1984.

September 1994

Decontamination and Decommissioning 
EM Problem: Waste management

Y-12 Plant Problem: Building 9201-4 (Alpha-4). Contaminated wastewater

Problem Area/Constituents: Radioactive species (including technetium), organics, and inorganic salts in water or other organic solvents

\section{Reference Requirements:}

- Resource Conservation and Recovery Act of 1976 (RCRA), as amended, Land Disposal Restriction (LDR) Federal Facilities Compliance Agreement (FFCA) dated June 12, 1992

- Federal Facilities Agreement (FFA) for the Oak Ridge Reservation

- Federal Facilities Compliance Agreement-Toxic Substances Control Act (FFCA-TSCA) dated February 20, 1992

- Clean Air Act regulations under 40 CFR 60-65

- Clean Water Act regulations under 40 CFR 110-146 and 400-471

- DOE orders

Refer to the Regulatory Compliance Chapter of Vol. 1 for potentially applicable proposed and promulgated environmental laws, signed and pending agreements for the Oak Ridge Reservation, radiation protection standards, DOE orders, and nonregulatory guidance. As site- and waste-specific characteristics are provided for each technology, specific regulatory requirements will be specified.

Subelement: Waste processing

Alternative: Thermal treatment

Technology: Evaporation. ${ }^{1,2}$ A process of bringing the contaminated liquids or slurries to their boiling point and vaporizing either pure water (for aqueous wastes) or the organic solvent (for wastes in an organic medium). The vapor either can be used for power production or condensed and either reused or disposed separately. The solids concentrate in the residue to the point where the residue either can be reused in the process (e.g. the use of sulfate liquors in paper processing) or can be packaged for disposal. Evaporation often is used as a waste reduction method in the disposal of radioactive wastes.

Status: Accepted by industry; many units are in use. Application of the technology may need to be demonstrated for the specific site problem.

Science/Technology Needs: The following may cause problems during operation:

- Scale formation on heating tubes can result in a decrease in the overall heat transfer coefficient.

- When evaporating organic-laden wastes, thermal degradation or coking of organics also may pose a problem. 
- Initial solute concentrations in the waste stream must be high enough to warrant use of evaporation (generally $>10,000 \mathrm{ppm}$ ).

\section{Implementation Needs:}

- Process equipment

- All utilities, including reliable electricity, natural gas, steam, air, water, etc.

- Drum and material handling equipment

- Trained chemical operators

- EPA-certified laboratory support

Author: C. H. Dukes/615-574-9561

\section{References:}

1. N. L. Nemerow and A. Dasgupta, Industrial and Hazardous Waste Treatment, Van Nostrand Reinhold, New York, p. 151-152, 1991.

2. H. M. Freeman, ed., Standard Handbook of Hazardous Waste Treatment and Disposal, McGraw-Hill, New York, Sec. 7.7, 1989. 
EM Problem: Waste management

Y-12 Plant Problem: Building 9201-4 (Alpha-4). Contaminated wastewater and liquid organic wastes

Problem Area/Constituents: Volatile organics and nonvolatile organics in water and soils; nonhalogenated organics in oil (if oil can be codegraded with organics)

\section{Reference Requirements:}

- Resource Conservation and Recovery Act of 1976 (RCRA), as amended, Land Disposal Restriction (LDR) Federal Facilities Compliance Agreement (FFCA) dated June 12, 1992

- Clean Air Act regulations under 40 CFR 60-65

- Clean Water Act regulations under 40 CFR 110-146 and 400-471

- DOE orders

Refer to the Regulatory Compliance Chapter of Vol. 1 for potentially applicable proposed and promulgated environmental laws, signed and pending agreements for the Oak Ridge Reservation, radiation protection standards, DOE orders, and nonregulatory guidance. As site- and waste-specific characteristics are provided for each technology, specific regulatory requirements will be specified.

Subelement: Waste processing

Alternative: Biological treatment

Technology: Aerobic Digestion. ${ }^{1}$ Many naturally occurring microorganisms can oxidize a wide variety of organic chemicals to carbon dioxide and water at ambient temperature, pressure, and neutral $\mathrm{pH}$. This is the basis for traditional biological treatment of municipal and industrial wastewaters. Approximately half of the carbon typically is incorporated into new biological cell mass; thus, significant biosludge may be generated for subsequent disposal. Various types of suspended-growth and fixed-film bioreactors are used. The rate of biooxidation, and thus the residence time required, depends highly on the individual organic species to be degraded.

Status: Demonstration. The status is labeled "demonstration," although aerobic digestion is commercially available (and used widely for treating various municipal and industrial wastewaters), because its suitability for treating a particular K-25 Site waste stream may need to be demonstrated before its use.

Science/Technology Needs: Because aerobic digestion is a commercial technology, science/technology needs generally are minimal. However, the efficacy of the process to treat a particular K-25 Site waste stream may need to be evaluated/demonstrated before its fullscale use at the site. 
Implementation Needs: Because of the wide spectrum of applying aerobic digestion, it is impractical to list a meaningful cost for the technology, because this depends on numerous factors. In general, implementation costs for bench-scale treatability studies and a technology demonstration may cost $\$ 1 \mathrm{M}-\$ 2 \mathrm{M}$. However, use of the process could enable the discharge of large volume waste streams (by removing the contaminants). Payback for the technology is quite significant compared to the cost of alternatives.

Author: T. L. Donaldson/615-574-2210

\section{References:}

1. C. P. L. Grady and H. C. Lim, Biological Wastewater Treatment, Theory, and Application, Marcel Dekker, New York, 1980. 
EM Problem: Waste management

Y-12 Plant Problem: Building 9201-4 (Alpha-4). Contaminated wastewater and liquid organic wastes

Problem Area/Constituents/media: PCBs in water; volatile and nonvolatile organics in water and soils; PCBs and nonhalogenated organics in oils (if the oil can be codegraded)

\section{Reference Requirements:}

- Resource Conservation and Recovery Act of 1976 (RCRA), as amended, Land Disposal Restriction (LDR) Federal Facilities Compliance Agreement (FFCA) dated June 12, 1992

- Federal Facilities Agreement (FFA) for the Oak Ridge Reservation

- Federal Facilities Compliance Agreement-Toxic Substances Control Act (FFCA-TSCA) dated February 20, 1992

- Clean Air Act regulations under 40 CFR 60-65

- Clean Water Act regulations under 40 CFR 110-146 and 400-471

- DOE orders

Refer to the Regulatory Compliance Chapter of Vol. 1 for potentially applicable proposed and promulgated environmental laws, signed and pending agreements for the Oak Ridge Reservation, radiation protection standards, DOE orders, and nonregulatory guidance. As site- and waste-specific characteristics are provided for each technology, specific regulatory requirements will be specified.

Subelement: Waste processing

Alternative: Biological treatment

Technology: Anaerobic digestion. ${ }^{1}$ Many organic chemicals can be degraded anaerobically (reduced) by naturally occurring microorganisms. The rates are usually slower than aerobic (biooxidation) degradation, but only about $10 \%$ of the carbon typically is converted to biological cell mass, compared with $\mathbf{5 0 \%}$ for biooxidation. Thus, much less biosludge is produced. Major products of anaerobic digestion are carbon dioxide and methane; the latter can be recovered economically from treatment of high-strength wastes. Suspended-growth and biofilm reactors are used. Anaerobic digestion also can affect reductive dehalogenation of organics; thus, it may be suitable for treatment of PCBs.

Status: Demonstration. The status is labeled "demonstration," although anaerobic digestion is commercially avallable for stabilizing (degrading) biosludge from aerobic wastewater treatment and for treating high-strength organic wastes from food processing and agricultural operations. This is so because the suitability of the technology for treating particular K-25 Site waste streams may need to be developed/demonstrated. For example, treatment of halogenated organics (e.g., PCBs) is still in the developmental phase, though treatment of chlorinated solvents has been demonstrated in bench- and pilot-scale studies. 
Science/Technology Needs: Opportunities exist for improving methane recovery/use and for improving degradation rates via clever bioreactor and process designs. However, although the technology is practiced commercially, its application to K-25 Site waste streams may need to be evaluated/demonstrated. More development work is needed on halogenated compounds (e.g., PCBs and solvents).

Implementation Needs: Treatability studies are always a good idea to evaluate the suitability of the technology to treat a particular waste stream. For chlorinated compounds, a pilot-scale demonstration should be conducted before commitment to a full-scale facility. Cost of the pilot-scale demonstration is likely to be $\$ 1.5 \mathrm{M}-\$ 2 \mathrm{M}$ at a DOE site. Biotreatment of PCBs is still at the predemonstration stage, and technical breakthroughs are needed to move confidently to the demonstration stage. As before, payback for the technology could be quite significant compared to the cost of alternatives.

Author: T. L. Donaldson/615-574-2210

\section{References:}

1. C. P. L. Grady and H. C. Lim, Biological Wastewater Treatment, Theory, and Application, Marcel Dekker, New York, 1980. 
EM Problem: Waste management

Y-12 Plant Problem: Treatment of mixed- and/or hazardous-waste-contaminated liquids, combustible debris, and toxic metal wastes

Problem Area/Constituents: Hazardous and/or mixed liquid wastes containing toxic or hazardous metals such as mercury, chromium, and radionuclides

\section{Reference Requirements:}

- Resource Conservation and Recovery Act of 1976 (RCRA), as amended, Land Disposal Restriction (LDR) Federal Facilities Compliance Agreement (FFCA) dated June 12, 1992

- Federal Facilities Agreement (FFA) for the Oak Ridge Reservation (ORR)

- Clean Air Act regulations under 40 CFR 60-65

- Clean Water Act regulations under 40 CFR 110-146 and 400-471

- DOE orders

Refer to the Regulatory Compliance chapter of Vol. 1, Pt. C, for potentially applicable proposed and promulgated environmental laws, signed and pending agreements for the ORR, radiation protection standards, DOE orders, and nonregulatory guidance. As site- and wastespecific characteristics are provided for each technology, specific regulatory requirements will be specified.

\section{Subelement: Waste processing}

\section{Alternative: Biotreatment}

Technology: Biosorption. 'Biosorption or bioprecipitation is an innovative or emerging technology for the biological removal of metals and radionuclides from wastes. Biological materials, consisting of naturally occurring microorganisms, will be incorporated into treatment processes for removing dissolved metals from recovered groundwater ex situ or from stored wastes. Synergistic combinations of microorganisms, immobilized within polymeric matrices to form macroscopic particulate resins, will be deployed in standard flowthrough reactors for continuous treatment of wastewaters. Dissolved contaminants will be retained within the reactor, either sorbed to or precipitated within biomass and thus rendered insoluble. The technology is particularly well suited for the treatment of dilute (low-ppmlevel) wastes. The selectivity of the biomass (which is developed for specific wastes) permits treatment of complex waste streams.

The ORNL team is currently developing a process for removal of dissolved uranium and nickel from waste streams generated at the Oak Ridge Y-12 Plant. The immobilized biomass has been shown to be more effective than activated carbon or typical ion exchange resins (e.g., Dionex and Amberlite) in this application, with respect to capacity and selectivity for uranium. Testing of authentic Y-12 wastes is under way. A pilot-scale demonstration at the 
Y-12 Plant is currently scheduled for July-August 1993. A final report will be prepared during September 1994.

A parallel project supported by DOE/EM-54 is aimed at the development of bioremoval technology for restoration of surface and groundwater present at a former uranium mining site in eastern Germany. The project focuses on removal of dissolved uranium, radium, and iron from aquatic systems. Process development efforts and bench-scale tests are currently under way. The technology will be demonstrated in the U.S. on the pilot scale during FY 1994. A field-scale demonstration will be carried out during FY 1995.

A third project supported by EM-43 focuses on bioremoval technology for treatment of secondary wastes consisting of spent hydrated molten salt (sodium carbonate) streams previously used for the oxidative destruction of the organic component of mixed wastes. The project goal is removal of dissolved uranium from the matrix, permitting recycling of the sodium carbonate. Technology development is currently underway. The technology will be demonstrated on the bench scale during FY 1994 and on the pilot scale during FY 1995.

Specific uranium bioremoval agents-active under realistic conditions of uranium concentration, $\mathrm{pH}$, and presence of other solutes-are being developed for each of the above tasks. Methods for improving the uranium sorptive capacity of these agents have been developed. Immobilization matrices compatible with the biomass and stable within the wastes have been identified. Standard packed-bed reactor configurations have been shown to be useful, and preliminary work has suggested the potential utility of a novel mode of reactor operation based on fluidized-bed reactor technology.

The cost of the immobilized biomass has been estimated at $\$ 100-\$ 150 / \mathrm{kg}$ (1992 dollars) when produced on a laboratory scale. This figure is expected to decrease significantly when the production method is scaled up. The sorbents can be regenerated by treatment with dilute $(0.1 \mathrm{~N})$ salt or acid solutions, suggesting that multiple cycles are possible, thereby minimizing overall cost. This treatment would result in the elution of the contaminants as a small volume of concentrated waste. Alternatively, the spent biomass may be disposed with the bound contaminants in place. The immobilized biomass is up to $96 \%$ water by weight; the spent material may be dewatered and/or incinerated for ready disposal. The cost of regenerating or destroying the spent material may be the least costly part of the technology. These costs may be less than $\$ 1 / \mathrm{kg}$. The final waste volume will depend on the amount of wastewater treated, the concentration of dissolved contaminants in the wastewater, the capacity of the biomass, and the number of cycles involved, among other parameters. Nonetheless, the final waste volume is expected to be moderate to low.

Status: Predemonstration. The technology has been proven at ORNL on the bench scale for the removal of uranium, strontium, copper, cadmium, and other contaminants.

Science/Technology Needs: This is an emerging technology with concomitant basic research needs. Many biomaterials will bind and/or precipitate metallic species. Optimal biomass must be identified for specific species. Other major questions concern the rate of

September 1994

Decontamination and Decommissioning 
removal, the maximum removal that can be achieved (i.e., binding or catalytic capacity), the range of contaminant concentrations over which the removal activity occurs, the stability of the bound or precipitated contaminants, selectivity for the targeted contaminant (incorporating studies of the effect of $\mathrm{pH}$ and potentially interfering ions), and the effective lifespan of the removal activity. Methods for maximizing metal removal efficiency and for minimizing biomass degradation or loss must be developed. Stable, inexpensive, and readily available immobilization matrices must be identified. Methods for the fabrication of the biomass into readily handled beads or other particulate forms via immobilization must be devised, with the goals of stabilizing the biomass, maximizing mass transfer rates, and minimizing dispersion within the reactor. Methods for regeneration and disposal of the biomass must be developed. The developmental efforts should focus on these issues.

Implementation Needs: The development costs are estimated to be \$2M (1992 dollars). Suitable immobilized biomass must be developed for the particular ORNL wastes. Subsequent implementation needs are expected to be normal. Standard reactor configurations are proposed for deployment of the biomass. No other unusual equipment needs are foreseen. Use of standard pumping schemes will allow ready integration of this technology into overall wastewater recovery, treatment, and disposal schemes. However, this technology is not developed sufficiently enough to enable meaningful cost estimates.

Author: B. D. Faison/615-576-0484

\section{References:}

1. H. Eccles and S. Hunt, Immobilization of Ions by Bio-Sorption, Ellis Horwood, Ltd, Chichester, England, 1986. 

EM Problem: Waste management

Y-12 Plant Problem: Building 9201-4 (Alpha-4). Contaminated solvents (halogenated and nonhalogenated)

Problem Area/Constituents: PCBs in water, solvents, oils (if the oils can be codegraded), and solls; other halogenated organics in the above-mentioned media.

\section{Reference Requirements:}

- Resource Conservation and Recovery Act of 1976 (RCRA), as amended, Land Disposal Restriction (LDR) Federal Facilities Compliance Agreement (FFCA) dated June 12, 1992

- Federal Facilities Agreement (FFA) for the Oak Ridge Reservation

- Federal Facilities Compliance Agreement-Toxic Substances Control Act (FFCA-TSCA) dated February 20, 1992

- Clean Air Act regulations under 40 CFR 60-65

- Clean Water Act regulations under 40 CFR 110-146 and 400-471

- DOE orders

Refer to the Regulatory Compliance Chapter of Vol. 1 for potentially applicable proposed and promulgated environmental laws, signed and pending agreements for the Oak Ridge Reservation, radiation protection standards, DOE orders, and nonregulatory guidance. As site- and waste-specific characteristics are provided for each technology, specific regulatory requirements will be specified.

Subelement: Waste processing

Alternative: Biological treatment

Technology: Microbial Dechlorination. ${ }^{1.2}$ Reductive (anaerobic) dechlorination of a variety of chlorinated organics has been demonstrated in laboratory experiments. Also, strong evidence exists for microbial dechlorination in natural environments. Compared to manufactured PCB Aroclors, weathered PCB-contaminated sediments show lower relative amounts of the higher-chlorinated congeners and higher amounts of the lower-chlorinated congeners, which is consistent with dechlorination. Weathered spills of trichloroethylene and perchloroethylene often show dichloroethylenes and vinyl chloride, which are believed to be products of dechlorination. Presently, the mechanisms and pathways for microbial dechlorination are poorly characterized and understood. However, much work is under way at numerous institutions. The leading conceptual process configuration is now probably a slurry bioreactor or a composting/landfarming system for contaminated soils and water. A system capable of treating oils is not obvious, although oils could be applied to a slurry bioreactor or a landfarming system. 
Status: Conceptual to development, depending on application. Phenomena are demonstrable at bench-scale, but process development work is only beginning. A few field tests have been attempted.

Science/Technology Needs: More basic understanding is needed to improve ability to manipulate and control the process and to ensure adequate performance. Then, pilot-scale demonstrations will be needed.

Implementation Needs: Development costs for the technology are estimated to be $\$ 5 \mathrm{M}$ to \$10M (1992 dollars). However, should the technology prove successful, payback for the process could be as much as $\$ 100-200 \mathrm{M}$, based on disposal costs for the waste streams in question.

Author: T. L. Donaldson/615-574-2210

\section{References:}

1. M. L. Rochkind-Dubinsky, G. S. Sayler, and J. W. Blackburn, Microbial Decomposition of Chlorinated Aromatic Compounds, Marcel Dekker, Inc., New York, 1986.

2. D. A. Abramowicz, "Aerobic and Anaerobic Biodegradation of PCBs: A Review," Critical Reviews in Biotechnology, 10, 241-251 (1990). 
EM Problem: Waste management

Y-12 Plant Problem: Building 9201-4 (Alpha-4). Waste off-gases from decontamination and decommissioning $(D \& D)$, remedial action, and waste management operations

Problem Area/Constituents: Mixed gases generated during D\&D operations

\section{Reference Requirements:}

- Resource Conservation and Recovery Act of 1976 (RCRA), as amended, Land Disposal Restriction (LDR) Federal Facilities Compliance Agreement (FFCA) dated June 12, 1992

- Federal Facilities Agreement (FFA) for the Oak Ridge Reservation

- Federal Facilities Compliance Agreement-Toxic Substances Control Act (FFCA-TSCA) dated February 20, 1992

- Clean Air Act regulations under 40 CFR 60-65

- Clean Water Act regulations under 40 CFR 110-146 and 400-471

- DOE orders

Refer to the Regulatory Compliance Chapter of Vol. 1 for potentially applicable proposed and promulgated environmental laws, signed and pending agreements for the Oak Ridge Reservation, radiation protection standards, $D O E$ orders, and nonregulatory guidance. As site- and waste-specific characteristics are provided for each technology, specific regulatory requirements will be specified.

Subelement: Waste treatment

Alternative: Physical treatment

Technology: Inorganic membrane separations. Inorganic membranes can be optimized to separate gases. Specifically, $\mathrm{ClF}_{3}$ can be separated from the contaminated gas stream for recycling to the decontamination process. $\mathrm{UF}_{6}$ gas can be removed in a subsequent separation stage for uncomplicated cold trapping and can be reclaimed in a pure state for useful purposes. Presently, this is a development technology. Development of an inorganic membrane to separate hydrogen from gas streams is progressing well and will prove very valuable to this development.

Status: Predemonstration. Technology development is at the bench-scale level, and testing of gas membranes is approaching target criteria. Tests show that theoretical analysis is on-target and that this has instilled confidence in the efficacy of the technology.

Science/Technology Needs: Ongoing development efforts for the hydrogen membrane will prove a giant step forward in developing a membrane for these gas separations. The main goal of these efforts will be to develop the criteria necessary for the separations, to design the membranes, and then to fabricate gas membranes with pore sizes sufficiently small to achieve effective separation by molecular sieving. A pore radius of 5-10 $\dot{A}$ is expected to be 
required. Pore radii of $7 \dot{A}$ have been achieved in ceramics membranes, but these membranes will have to be fabricated from materials that can survive in corrosive gases. The technology should be demonstrated with two membrane modules in series and should be at the pilot-plant scale. In addition, technology assessments need to be conducted to establish the viability and economics of the process.

Implementation Needs: Development of the process through the demonstration phase is estimated to cost $\$ 1.5 \mathrm{M}-\$ 2 \mathrm{M}$ (1992 dollars). However, the process will not generate any additional wastes $\left(\mathrm{NaF}\right.$, alumina, etc.), will reduce $\mathrm{ClF}_{3}$ usage, and will facilitate the uncomplicated recovery of $\mathrm{UF}_{6}$ for useful purposes. This membrane stage can be followed by a slurry system to scrub out the contaminated gases, with the slurry directed to another membrane stage to separate and concentrate the mixed waste. The cost benefits of this process are expected to exceed $\$ 20 \mathrm{M}^{1}$

Author: J. M. Vance/615-576-0159

\section{References:}

1. S.P. N. Singh, personal communication with J. M. Vance, K-25 Site, November 30, 1992. 
EM Problem: Waste management

Y-12 Plant Problem: Building 9201-4 (Alpha-4). Waste off-gases from D\&D, RA, and WM operations

Problem Area/Constituents: Removal of inorganic, organic, and radioactive compounds and particulates from various gaseous streams at Bldg. 9201-4

\section{Reference Requirements:}

- Resource Conservation and Recovery Act of 1976 (RCRA), as amended, Land Disposal Restriction (LDR) Federal Facilities Compliance Agreement (FFCA) dated June 12, 1992

- Federal Facilities Agreement (FFA) for the Oak Ridge Reservation

- Federal Facilities Compliance Agreement-Toxic Substances Control Act (FFCA-TSCA) dated February 20, 1992

- Clean Air Act regulations under 40 CFR 60-65

- Clean Water Act regulations under 40 CFR 110-146 and 400-471

- DOE orders

Refer to the Regulatory Compliance Chapter of Vol. 1 for potentially applicable proposed and promulgated environmental laws, signed and pending agreements for the Oak Ridge Reservation, radiation protection standards, DOE orders, and nonregulatory guidance. As site- and waste-specific characteristics are provided for each technology, specific regulatory requirements will be specified.

Subelement: Waste processing

Alternative: Physical treatment

Technology: Scrubbers. ${ }^{1,2}$ Mechanical devices used to remove solid and/or gaseous contaminants from gas streams using a solvent (usually water). Briefly, the solvent is intimately mixed with the contaminated gas stream in a countercurrent, cocurrent, or cross-flow configuration. The contaminants dissolve or are collected in the solvent stream. Rich or loaded solvent solution then is treated separately to recover contaminants from the solvent for recycling or disposal. Conventional scrubbers include packed-bed, tray-type, venturi, liquid-film, or cyclone scrubbers. When properly designed and operated, scrubbers are very efficient in removing contaminants from the waste-gas stream.

Status: Demonstration. The status is labeled "demonstration," although most scrubbers are commercially avallable, because application of a particular scrubber to treat a 9201-4 stream may need to be demonstrated before its full-scale use.

Science/Technology Needs: Because scrubbers are commercial technology, generally, science/technology needs are minimal. However, the efficacy of a particular scrubber may need to be demonstrated for a given application before its full-scale use. 
Implementation Needs: Because of the wide range of applications where scrubbers can be used, it is impractical to list a meaningful cost for the technology, because it depends on numerous factors. In general, implementation of a technology demonstration of a scrubber application may cost up to $\$ 1 \mathrm{M}$. However, because scrubbers enable the discharge of (often) large volume waste streams (by removing the contaminants), payback for use of the technology can be quite significant compared to the cost of alternatives.

Author: S.P.N. Singh/615-574-6639

\section{References:}

1. H. M. Freeman, ed., Standard Handbook of Hazardous Waste Treatment and Disposal, McGraw-Hill, New York, 1989.

2. R. H. Perry and D. W. Green, eds., Perry's Chemical Engineers' Handbook, 6th edition, McGraw-Hill, New York, 1984. 
EM Problem: Waste management

Y-12 Plant Problem: Building 9201-4 (Alpha-4). Waste off-gases from decontamination and decommissioning, remedial action, and waste management operations

Problem Area/Constituents: Removal of inorganic, organic, and radioactive compounds and particulates from various gaseous streams at Alpha-4 (Bldg. 9201-4)

\section{Reference Requirements:}

- Resource Conservation and Recovery Act of 1976 (RCRA), as amended, Land Disposal Restriction (LDR) Federal Facilities Compliance Agreement (FFCA) dated June 12, 1992

- Federal Facilities Agreement (FFA) for the Oak Ridge Reservation

- Federal Facilities Compliance Agreement-Toxic Substances Control Act (FFCA-TSCA) dated February 1992

- Clean Air Act regulations under 40 CFR 60-65

- Clean Water Act regulations under 40 CFR 110-146 and 400-471

- DOE orders

Refer to the Regulatory Compliance Chapter of Vol. 1 for potentially applicable proposed and promulgated environmental laws, signed and pending agreements for the Oak Ridge Reservation, radiation protection standards, DOE orders, and nonregulatory guidance. As site- and waste-specific characteristics are provided for each technology, specific regulatory requirements will be specified.

Subelement: Waste processing

Alternative: Physical treatment

Technology: Cyclone separators. ${ }^{1}$ Like scrubbers, cyclone separators are used to separate solids and entrained liquids from gas streams. Cyclone separators use centrifugal action to separate particulates and entrained liquids from the waste-gas stream. When properly designed and operated, cyclone separators are very efficient at removing contaminants from the waste-gas stream.

Status: Demonstration. The status is labeled "demonstration," although most cyclone separators are commercially available, because application of a particular separator to treat a 9201-4 stream may need to be demonstrated before its full-scale use.

Science/Technology Needs: Because cyclone separators are commercial technology, generally, science/technology needs are minimal. However, the efficacy of a particular separator may need to be demonstrated for a given application before its full-scale use.

Implementation Needs: Because of the wide range of applications where cyclone separators can be used, it is impractical to determine a meaningful cost for the technology, because it 
depends on numerous factors. In general, implementation of a technology demonstration may cost up to $\$ 1 \mathrm{M}$. However, because cyclone separators enable the discharge of (often) large volume waste streams (by removing the contaminants), payback for use of the technology is quite significant compared to the cost of alternatives.

Author: S.P.N. Singh/615-574-6639

\section{References:}

1. R. H. Perry and D. W. Green, eds., Perry's Chemical Engineers' Handbook, 6th edition, McGraw-Hill, New York, 1984. 
EM Problem: Waste management

Y-12 Plant Problem: Building 9201-4 (Alpha-4). Waste gases of unkmown characteristics from dcontamination and decommissioning (D\&D) and remedial action operations (some possibly in cylinders of uncertain integrity)

Problem Area/Constituents: Orphan gas cylinders lacking information of their contents

\section{Reference Requirements:}

- Resource Conservation and Recovery Act of 1976 (RCRA), as amended, Land Disposal Restriction (LDR) Federal Facilities Compliance Agreement (FFCA) dated June 12, 1992

- Federal Facilities Agreement (FFA) for the Oak Ridge Reservation

- Clean Air Act regulations under 40 CFR 60-65

- Clean Water Act regulations under 40 CFR 110-146 and 400-471

- DOE orders

Refer to the Regulatory Compliance Chapter of Vol. 1 for potentially applicable proposed and promulgated environmental laws, signed and pending agreements for the Oak Ridge Reservation, radiation protection standards, DOE orders, and nonregulatory guidance. As site- and waste-specific characteristics are provided for each technology, specific regulatory requirements will be specified.

Subelement: Waste processing

Alternative: Physical treatment

Technology: Recharacterize and repackage. Environmental restoration and D\&D operations at the site likely will uncover orphan gas cylinders of unknown content. Safe disposal of these gas cylinders in compliance with regulations is likely to require recharacterization of the contents of the cylinders and (depending on the physical condition of the cylinders) repackaging in approved cylinders for treatment, use, or disposal. This is likely to be an operation that can be managed with current technology.

Status: Accepted

Science/Technology Needs: Minimal to none

Implementation Needs: These are likely to be minimal. The estimated costs for conducting the operations will depend on the number of such cylinders found, their physical state, and the nature of their contents. Payback for this operation is expected to be high, considering the alternatives. 
Author: S.P.N. Singh/615-574-6639

References: None 
EM Problem: Waste management

T-12 Plant Problem: Building 9201-4 (Alpha-4). Waste off-gasses from decontamination and decommissioning, remedial action, and waste management operations

Problem Area/Constituents: Treatment of pollutants such as vinyl chloride, benzene, $\mathrm{H}_{2} \mathrm{~S}$, and methyl mercaptan and aqueous effluents containing hazardous organics such as benzene, carbon tetrachloride and trichloroethylene.

\section{Reference Requirements:}

- Resource Conservation and Recovery Act of 1976 (RCRA), as amended, Land Disposal Restriction (LDR) Federal Facilities Compliance Agreement (FFCA) dated June 12, 1992

- Federal Facilities Agreement (FFA) for the Oak Ridge Reservation

- Federal Facilities Compliance Agreement-Toxic Substances Control Act (FFCA-TSCA) dated February 20, 1992

- Clean Air Act regulations under 40 CFR 60-65

- Clean Water Act regulations under 40 CFR 110-146 and 400-471

- DOE orders

Refer to the Regulatory Compliance Chapter of Vol. I for potentially applicable proposed and promulgated environmental laws, signed and pending agreements for the Oak Ridge Reservation, radiation protection standards, DOE orders, and nonregulatory guidance. As site- and waste-specific characteristics are provided for each technology, specific regulatory requirements will be specified.

Subelement: Waste processing

Alternatives: Thermal treatment

Technology: Electron-beam (E-beam) destruction. ${ }^{1.2}$ The process is claimed to be a simple, energy-efficient method for reducing small concentrations of pollutants in air (or other carrier gases) to less than the $1 \mathrm{ppm}$ level using a high-energy electron beam.

E-beam irradiation ionizes the carrier gas; these ions then can react with the pollutant to destroy it while leaving the carrier gas relatively unreacted. This reaction is accomplished primarlly by rapid-charge transfer from the carrier gas to the pollutants, which normally have lower ionization potential. Pollutant ions subsequently are destroyed.

For wastewater treatment, E-beam destruction of organic molecules is a very effective mechanism, because irradiation of water with high-energy electrons results in production of oxidizing and reducing species, such as hydroxyl $(\mathrm{OH})$ and aqueous electron (e-aq) radicals. These reactive species then react with and destroy the organic molecules. Essentially, the E-beam process is insensitive to effluent quality, with effective removal of organic chemicals observed in both lightly contaminated-water effluent streams and raw sewage. 
Status: Predemonstration

Science/Technology Needs: A study must be conducted of all experimental work done involving E-beam destruction technology. Development work at the bench scale with the pollutants of interest then will begin. If technical viability and economics justify it, scale-up and testing on a larger scale unit will follow.

Implementation Needs: Implementing the development needs outlined above is estimated to cost $\$ 0.75 \mathrm{M}-\$ 1.5 \mathrm{M}$ (1992 dollars). As development work progresses and the application appears promising, collaborative research efforts with the private sector or with academia can be established. As a result, the potential may exist for lower implementation cost, particularly during the latter part of the development cycle.

Author: J. M. Chiang/615-574-9659

\section{References:}

1. R. C. Slater and D. H. Douglas-Hamilton, Electron-Beam-Initiated Destruction of Low Concentrations of Vinyl Chloride in Carrier Gases, AVCO Everett Research Laboratory, Everett, Mass., 1981.

2. T. D. Waite, C. N. Kurucz, and W. J. Cooper, Utilizing High Energy Electrons for Treatment of Industrial Wastes Containing Non-Biodegradable Organic Compounds, proceedings of the 22nd mid-Atlantic Industrial Waste Conference, 1990. 
EM Problem: Waste management

Y-12 Plant Problem: Building 9201-4 (Alpha-4). Waste off-gases from decontamination and decommissioning $(D \& D)$, remedial action, and waste management operations

Problem Area/Constituents: All environmental restoration and D\&D processes that emit volatile organic compounds (VOCs) exceeding the regulatory limits

\section{Reference Requirements:}

- Resource Conservation and Recovery Act of 1976 (RCRA), as amended, Land Disposal Restriction (LDR) Federal Facilities Compliance Agreement (FFCA) dated June 12, 1992

- Federal Facilities Agreement (FFA) for the Oak Ridge Reservation

- Federal Facilities Compliance Agreement-Toxic Substances Control Act (FFCA-TSCA) dated February 20, 1992

- Clean Air Act regulations under 40 CFR 60-65

- Clean Water Act regulations under 40 CFR 110-146 and 400-471

- DOE orders

Refer to the Regulatory Compliance Chapter of Vol. 1 for potentially applicable proposed and promulgated environmental laws, signed and pending agreements for the Oak Ridge Reservation, radiation protection standards, DOE orders, and nonregulatory guidance. As site- and waste-specific characteristics are provided for each technology, specific regulatory requirements will be specified.

\section{Subelement: Waste processing}

\section{Alternative: Thermal treatment}

Technology: Flameless thermal oxidizer. ${ }^{1}$ This oxidizer can achieve greater than $99.99 \%$ removal of VOCs from the waste streams with negligible innocuous by-product formation. The principal feature of the axidizer is the inert ceramic bed or "matrix," which provides thorough mixing of oxygen with the waste gases. Designed for optimal mixing and residence time, the matrix is preheated and controlled at a desirable temperature to achieve complete oxidation of the hydrocarbons, producing carbon dioxide and water. The large thermal mass of the matrix absorbs heat released during oxidation, preventing flames or flashback. If the inlet fume is laden with chlorinated or sulfur-containing organics, a system to remove the $\mathrm{HCl}$ or $\mathrm{SO}_{2}$ generated is incorporated, when necessary for regulatory compliance. Versatile in handling widely fluctuating flow rates and organic concentrations, the process provides advantages over other VOC control technologies, such as incineration, carbon adsorption, etc. It eliminates the drawbacks inherent in other alternate processes (i.e., process instability, byproduct of waste formation, flame safety issues, and poor treatment of chlorinated hydrocarbons).

Status: Demonstration 
Science/Technology Needs: The flameless thermal oxidation process will be thoroughly reviewed. A pilot-scale oxidizer will be designed, and experimental runs will be performed to optimize operating conditions. Optimal design of the ceramic matrix is crucial to achieving the desirable mixing of air and hydrocarbons and to provide proper residence time for complete oxidation. Instrumentation and control are to be incorporated into the system adequately to maintain desired bed temperature and to control air flow in response to varying hydrocarbon flow rates and concentrations.

Implementation Needs: $\mathrm{R} \& \mathrm{D}$ work is estimated to cost $\$ 1 \mathrm{M}-\$ 1.5 \mathrm{M}$. As this is a process covered by a patent, an additional user fee may be incurred for its full-scale application. The development cost estimated may be offset somewhat by contributions from the private sector or academia if collaborative research efforts are established. This process is envisioned to have the potential for being a viable option to eliminate toxic emissions from the oil and gas, chemical, and pharmaceutical industries.

Author: J. M. Chiang/615-574-9659

\section{References:}

1. Flameless Thermal Oxidizers for VOC Control, bulletin, Thermatrix, Inc., 1992.

2. Personal communication to Jim Pelmer, MMES Office of Technology Transfer, 1992.

September 1994

Decontamination and Decommissioning 
EM Problem: Waste Management

Y12 Plant Problem: Building 9204-1 (Alpha-4)

Problem Area/Constituents: Asbestos-containing materials contaminated with U and PCBs

\section{Reference Requirements:}

- Resource Conservation and Recovery Act of 1976 (RCRA), as amended, Land Disposal Restriction (LDR) Federal Facilities Compliance Agreement (FFCA) dated June 12, 1992

- Federal Facilities Agreement (FFA) for the Oak Ridge Reservation

- Clean Air Act regulations under 40 CFR 60-65

- Clean Water Act regulations under 40 CFR 110-146 and 400-471

Refer to the Regulatory Compliance chapter of Vol. 1 for potentially applicable proposed and promulgated environmental laws, signed and pending agreements for the Oak Ridge Reservation, radiation protection standards, DOE orders, and nonregulatory guidance. As site- and waste-specific characteristics are provided for each technology, specific regulatory requirements will be specified.

Subelement: Waste processing

Alternative: Chemical treatment

Technology: HF destruction of asbestos. Asbestos fibers can be converted to nonhazardous fluorosilicates by reaction with hydrofluoric acid in solution or hydrogen fluoride gas.

Status: Predemonstration. The HF solution process has been demonstrated at the Oak Ridge K-25 Site by DSI Inc. to be an effective method of asbestos destruction.

Efficacy-After asbestos was subjected to the treatment, no asbestos fibers were found.

Waste-DSI process leaves an acidic solution possibly contaminated with U or PCBs from the asbestos. Without other contamination, neutralization renders the solution nonhazardous. Solid sludge of nonhazardous fluorosilicate is removed from solution by filtering. This process is being conducted on a production scale at Griffiss Air Force Base.

A gas phase process has been patented at Martin Marietta Energy Systems, Oak Ridge based upon work by T. W. Selby of the K-25 Site. No further scale up studies have been conducted.

Science/Technology Needs: Technology assessments need to be conducted to establish the economics of the process.

Implementation Needs: Operating cost: est $<\$ 0.50 / \mathrm{lb}$, capital cost: $\mathrm{NA}$, development cost: $<\$ 0.2 \mathrm{M}$ 
Author: R. L. Fellows/615-576-5632

\section{References:}

1. DSI Demonstration of Asbestos Destruction, Center for Environmental Technology, K-25 Site, Oak Ridge. To be published (1994) 
EM Problem: Waste Management

Y12 Plant Problem: Building 9204-1 (Alpha-4)

Problem Area/Constituents: Corrosive Wastes

\section{Reference Requirements:}

- Resource Conservation and Recovery Act of 1976 (RCRA), as amended, Land Disposal Restriction (LDR) Federal Facilities Compliance Agreement (FFCA) dated June 12, 1992

- Federal Facilities Agreement (FFA) for the Oak Ridge Reservation

- Clean Air Act regulations under 40 CFR 60-65

- Clean Water Act regulations under 40 CFR 110-146 and 400-471

Refer to the Regulatory Compliance chapter of Vol. 1 for potentially applicable proposed and promulgated environmental laws, signed and pending agreements for the Oak Ridge Reservation, radiation protection standards, DOE orders, and nonregulatory guidance. As site- and waste-specific characteristics are provided for each technology, specific regulatory requirements will be specified.

Subelement: Waste processing

Alternative: Chemical treatment

Technology: Neutralization. A process to remove the corrosivity characteristic from liquid wastes. Corrosivity is defined on the acid/base $\mathrm{Ph}$ scale and a waste is defined as hazardous by the Resource Conservation and Recovery Act (RCRA) if its $\mathrm{pH}$ is greater than 12.5 (alkaline or caustic) or less than 2.0 (acidic). Neutralization involves adding sufficient acid or base to bring the $\mathrm{pH}$ within the range of 2.0 to 12.5 .

Status: Accepted. Neutralization is common practice.

Efficacy-Completely effective.

Waste-

Science/Technology Needs: None

Implementation Needs: Operating cost: est $<\$ 0.02 / \mathrm{lb}$, capital cost: mixing vessel. development cost: None

Author: R. L. Fellows/615-576-5632

\section{References:}

1. Standard elementary chemistry texts. 


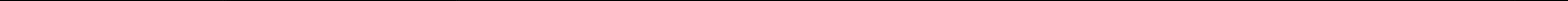




\section{EM Problem: Waste management}

\section{Y-12 Plant Problem: Building 9201-4 (Alpha-4). Contaminated solid wastes}

Problem Area/Constituents: Stabilization of final contaminated residues that result from decontamination and decommissioning, environmental restoration, and waste management operations performed at the site. These radioactive and/or hazardous wastes have to be prepared for final disposal in compliance with regulations.

\section{Reference Requirements:}

- Resource Conservation and Recovery Act of 1976 (RCRA), as amended, Land Disposal Restriction (LDR) Federal Facilities Compliance Agreement (FFCA) dated June 12, 1992

- Federal Facilities Agreement (FFA) for the Oak Ridge Reservation

- Federal Facilities Compliance Agreement-Toxic Substances Control Act (FFCA-TSCA) dated February 20, 1992

- Clean Air Act regulations under 40 CFR 60-65

- Clean Water Act regulations under 40 CFR 110-146 and 400-471

- DOE orders

Refer to the Regulatory Compliance Chapter of Vol. 1 applicable proposed and promulgated environmental laws, signed and pending agreements for the Oak Ridge Reservation, radiation protection standards, DOE orders, and nonregulatory guidance. As site- and waste-specific characteristics are provided for each technology, specific regulatory requirements will be specified.

Subelement: Waste stabilization

Alternatives: Solidification, macroencapsulation, microencapsulation, chemical fixation

Technology: Cementitious solidification/stabilization (CS/S). One of the most widely used techniques for treating and ultimately disposing of hazardous wastes and low-level radioactive wastes. Cementitious materials are the predominant materials of choice because of their low associated processing costs, compatibility with a wide variety of disposal scenarios, and ability to meet stringent processing and performance requirements. Cementitious materials include cement; granulated blast furnace slag; fly ash; lime; and fumed silica. Various clays and additives are used to help immobilize contaminants or otherwise enhance waste form properties. Soluble constituents in the waste chemically interact with the cementitious materials to form low-solubility products at the low $\mathrm{pH}$ and Eh prevailing in the waste form. These interactions usually affect cementitious hardening and properties to some degree. Testing with a specific waste or waste stream is required to tailor formulation to the desired properties. Sufficient attention must be given to characterizing the waste, developing the formulation to treat the waste, and implementing this formulation in the field to assure correct mixing of the formulation. Adding these dry ingredients inevitably increases the volume of the waste treated, which can add significantly to lifetime disposal 
costs. The volume decrease claimed by techniques such as thermoplastic encapsulation come from evaporation of water and encapsulation of the solids. The same evaporation pretreatment could be used with $\mathrm{CS} / \mathrm{S}$ to obtain a net volume decrease, but some of the simplicity of CS/S would be lost. Cementitious waste forms are porous, making them more leachable than polymeric or glass waste forms. The key to success has been controlling this leachability (by $\mathrm{pH}, \mathrm{Eh}$, and/or absorbents) within satisfactory limits for a simpler and cheaper treatment.

For relatively inert materials, such as rubble or scrap metal, cementitious materials are ideal for macroencapsulation. Encapsulated ion-exchange resins can lead to breakdown of the solid matrix because of the volume changes that result from wetting and drying of the resin. Proper formulation and pretreatment has succeeded in overcoming this problem for encapsulated resins.

\section{Status: Accepted}

Science/Technology Needs: Better understanding of immobilization mechanisms and chemistry of these waste forms can lead to improved performance and better predictions about their durability. This technique is currently in wide-scale use.

Implementation Needs: These are relatively minimal, because it is accepted technology. However, formulations need to be developed, or at least tested, for the specific wastes intended for treatment. Proper implementation is necessary to ensure that waste forms tested in the laboratory represent what will be produced in the field. This means having the necessary equipment and expertise to properly blend and mix these solid constituents and manage the operation properly, so that the right formulation is mixed.

Author: R. D. Spence/615-574-6782

\section{References:}

1. J. R. Conner, Chemical Fixation and Solidification of Hazardous Wastes, Van Nostrand Reinhold, New York, 1990.

2. I. L. Morgan and W. D. Bostick, Performance Testing of Grout-Based Waste Forms for the Solidification of Anion Exchange Resins, K/QT-382, October 1990.

3. A. van Dalen and J. E. Rijpkema, Modified Sulphwr Cement: A Low Porosity Encapsulation Material for Low, Medium and Alpha Waste, paper published by the Commission of the European Communities, EUR 12303 EN, 1989

September 1994

Decontamination and Decommissioning 
EM Problem: Waste management

T-12 Plant Problem: Building 9201-4 (Alpha-4). Contaminated solid wastes

Problem Area/Constituents: Stabilization of final contaminated residues that result from decontamination and decommissioning, environmental restoration, and waste management operations performed at the site. These radioactive and/or hazardous wastes must be prepared for final disposal in compliance with regulations.

\section{Reference Requirements:}

- Resource Conservation and Recovery Act of 1976 (RCRA), as amended, Land Disposal Restriction (LDR) Federal Facilities Compliance Agreement (FFCA) dated June 12, 1992

- Federal Facilities Agreement (FFA) for the Oak Ridge Reservation

- Federal Facilities Compliance Agreement-Toxic Substances Control Act (FFCA-TSCA) dated February 20, 1992

- Clean Air Act regulations under 40 CFR 60-65

- Clean Water Act regulations under 40 CFR 110-146 and 400-471

- DOE orders

Refer to the Regulatory Compliance Chapter of Vol. 1 applicable proposed and promulgated environmental laws, signed and pending agreements for the Oak Ridge Reservation, radiation protection standards, DOE orders, and nonregulatory guidance. As site- and waste-specific characteristics are provided for each technology, specific regulatory requirements will be specified.

Subelement: Waste stabilization

Alternatives: Solidification, macroencapsulation, microencapsulation, chemical fixation

Technology: Polymer impregnated concrete (PIC). Basically, this technique is an extra step to achieve waterproofing after cementitious solidification/stabilization. Polymer impregnation has been studied for many years, and usually it is restricted to treatment within a few millimeters of the surface. It has been used as a means of waterproofing and environmental protection for structural concrete. A technique was invented at ORNL to achieve essentially complete monomer permeation throughout a waste form by adding polystyrene foam during mixing of the cementitious waste form. This introduces porosity into the waste form, potentially making a weaker product (not a great concern for waste forms). The monomer is polymerized, giving a waterproofing component throughout the waste form. This treatment has protected cementitious waste form samples from attack by concentrated hydrochloric acid. Laboratory development is still needed to optimize treatment and test the properties of the resulting waste form. The product has the advantages of a cementitious waste form with the added protection of a waterproofing layer throughout the waste form. Leaching will still occur across the polymeric barrier from the porous cementitious waste form. Brookhaven 
National Laboratory (BNL) ${ }^{2}$ acquired a patent using polymer impregnation of cement as a means of disposing of tritiated water.

Status: Evolving technology/conceptual. Some laboratory work has been conducted on the technology.

Science/Technology Needs: These include further development of the technology to where it can be used to successfully stabilize some of the residues likely to be generated at the site.

Implementation Needs: Development and demonstration of the efficacy of this stabilization method are expected to cost $\$ 5 \mathrm{M}-\$ 10 \mathrm{M}$ (1992 dollars). However, payback for the process could be significant, if it is demonstrated that the technique can successfully stabilize radioactive and/or hazardous residues generated at the site.

Author: R. D. Spence/615-574-6782

\section{References:}

1. A. J. Mattus and R. D. Spence, ORNL, Process for Impregnating a Concrete or Cement Body with a Polymeric Material, U.S. patent 4,828,761, May 9, 1989.

2. Peter Colombo, Robert M. Neilson, Jr., and Walter W. Becker, BNL, Process for Disposal of Aqueous Solutions Containing Radioactive Isotopes, U.S. patent 4,174,293, November 13, 1979. 
EM Problem: Waste management

Y-12 Plant Problem: Building 9201-4 (Alpha-4). Contaminated solid wastes

Problem Area/Constituents: Stabilization of final contaminated residues that result from decontamination and decommissioning, environmental restoration, and waste management operations performed at the site. These radioactive and/or hazardous wastes must be prepared for final disposal in compliance with regulations.

\section{Reference Requirements:}

- Resource Conservation and Recovery Act of 1976 (RCRA), as amended, Land Disposal Restriction (LDR) Federal Facilities Compliance Agreement (FFCA) dated June 12, 1992

- Federal Facilities Agreement (FFA) for the Oak Ridge Reservation

- Federal Facilities Compliance Agreement-Toxic Substances Control Act (FFCA-TSCA) dated February 20, 1992

- Clean Air Act regulations under 40 CFR 60-65

- Clean Water Act regulations under 40 CFR 110-146 and 400-471

- DOE orders

Refer to the Regulatory Compliance Chapter of Vol. 1 applicable proposed and promulgated environmental laws, signed and pending agreements for the Oak Ridge Reservation, radiation protection standards, DOE orders, and nonregulatory guidance. As site- and waste-specific characteristics are provided for each technology, specific regulatory requirements will be specified.

\section{Subelement: Waste stabilization}

Altematives: Solidifcation, macroencapsulation, microencapsulation, chemical fixation

Technology: Thermoplastic encapsulation. Two thermoplastics, bitumen and polyethylene, have been developed as encapsulation waste forms. Ostensibly, thermoplastics do not interact with the waste, so extensive testing to tailor the waste form is not needed, and net volume reductions can result for liquid wastes. The waste must be dried and the dried solids encapsulated in the thermoplastic. The waste is exposed to higher temperatures during drying and mixing with the molten thermoplastic, so volatile species such as mercury may not be amenable to such treatment. The processing is more complex than cementitious solidification/stabilization (CS/S). The waste is not chemically immobilized or stabilized, but the thermoplastic is nonporous and, hence, less leachable. It is questionable whether such physical encapsulation waste forms will pass the required TCLP leachability test. Also, current EPA guidance states that chemical fixation, rather than just physical encapsulation, is required. For this reason, CS/S is the best demonstrated, available technology (BDAT) for hazardous RCRA metals. 
Bitumen has been used extensively in Europe, and a couple of commercial vendors have offered bitumen encapsulation in the U.S. However, bitumen creeps and requires a container (e.g., 55-gal drum or concrete vault) for structural integrity. Bitumen absorbs water, swelling as it does so. Encapsulated soluble salts will set up large osmotic pressures within thermoplastic waste forms upon contact with water, causing further expansion for bitumen waste forms. Concern also exists about encapsulating nitrate salts (known oxidizers) in thermoplastics and the biodegradability of these waste forms. Once ignited, such a mixture may burn without air. The combination of these problems has made bitumen less popular than cementitious waste forms, despite its superior leach resistance. Polyethylene may overcome most, if not all, of these problems, but presently it is only a laboratory development. Polyethylene offers the structural integrity that bitumen lacks. Also, BNL has studied polyethylene as a waste form for DOE and claims that nitrate salts encapsulated in polyethylene will pass fire and self-ignition tests. BNL has not developed the technology for drying the waste before encapsulation in polyethylene, nor has BNL encapsulated actual wastes. BNL has mainly studied the properties of dry salts encapsulated in polyethylene. So, the technology is mainly a laboratory development. It has yet to be demonstrated that the process can pass the required TCLP leaching test and that the process has production-scale capabilities with actual wastes.

Status: The bitumen stabilization process is at the demonstration-scale level, while the process using polyethylene is adjudged to be at the predemonstration scale of development.

Science/Technology Needs: These include developing the particular technology to where it can be used to stabilize residues generated at the site in compliance with regulations. These could include, for example, developing the waste form that will pass the TCLP test; techniques to dry the waste before encapsulation for the polyethylene process; developing the waste forms' resistance to biological degradation; and developing methods for handling volatile hazardous compounds that may be present in residues. Materials susceptibility to corrosion at the elevated temperatures for the processing equipment also needs to be explored, especially if chloride or fluoride species are present in the waste.

Implementation Needs: Developing and demonstrating the efficacy of this stabilization method are estimated to cost $\$ 2 M-\$ 5 M$ (1992 dollars). However, payback for the process could be significant, if it is demonstrated that the technique can successfully encapsulate or isolate radioactive and/or hazardous compounds from the environment for the required period.

Author: R. D. Spence/615-574-6782

\section{References:}

1. J. R. Conner, Chemical Fixation and Solidification of Hazardous Wastes, Van Nostrand Reinhold, New York, 1990.

September 1994

Decontamination and Decommissioning 
EM Problem: Waste management

Y-12 Plant Problem: Building 9201-4 (Alpha-4). Contaminated solid wastes

Problem Area/Constituents: Stabilization of the final contaminated residues that result from the decontamination and decommissioning, environmental restoration, and waste management operations performed at the site. These radioactive and/or hazardous wastes must be prepared for final disposal in compliance with regulations.

\section{Reference Requirements:}

- Resource Conservation and Recovery Act of 1976 (RCRA), as amended, Land Disposal Restriction (LDR) Federal Facilities Compliance Agreement (FFCA) dated June 12, 1992

- Federal Facilities Agreement (FFA) for the Oak Ridge Reservation

- Federal Facilities Compliance Agreement-Toxic Substances Control Act (FFCA-TSCA) dated February 20, 1992

- Clean Air Act regulations under 40 CFR 60-65

- Clean Water Act regulations under 40 CFR 110-146 and 400-471

- DOE orders

Refer to the Regulatory Compliance Chapter of Vol. 1 applicable proposed and promulgated environmental laws, signed and pending agreements for the Oak Ridge Reservation, radiation protection standards, DOE orders, and nonregulatory guidance. As site- and waste-specific characteristics are provided for each technology, specific regulatory requirements will be specified.

Subelement: Waste stabilization

Alternatives: Solidification, macroencapsulation, microencapsulation, chemical fixation

Technology: Thermoplastic encapsulation. Sulfur polymer cement (SPC) encapsulation is like thermoplastic encapsulation in that the dried waste solids are encapsulated in the molten sulfur. The advantages are similar in that little interaction is anticipated, a nonporous waste form results in less leaching, and drying the liquids results in a net volume decrease. Sulfur is resistant to acid attack, so that SPC has been used as a construction material in aggressive acid environments. On the other hand, sulfur cannot be used in other environments, such as high alkalinity. These deleterious environments have been identified and must be avoided. SPC has been studied as a waste form by BNL and in Europe. These studies have been on a laboratory scale, so pilot-scale testing and demonstrations still are needed. As with thermoplastics, it is questionable whether SPC will meet EPA's guidance regarding the use of chemical fixation, because it is a purely physical encapsulation technique. SPC is being touted as an acceptable waste form for disposing of radioactive wastes. The Europeans succeeded in pretreating ion-exchange resins, so that resins 
encapsulated in SPC could be immersed in water without resin swelling, causing a breakdown of the SPC matrix.

Status: Predemonstration

Science/Technology Needs: These include performance testing of the SPC waste form in the TCLP test; developing the technology for drying the waste before encapsulation; and demonstrating the technique on an industrial scale.

Implementation Needs: Those needed to develop this process to where it can be used to treat residues at Alpha-4 (Bldg. 9201-4) are given above. Development is estimated to cost \$2M-\$5M (1992 dollars).

Author: R. D. Spence/615-574-6782

\section{References:}

1. P. D. Kalb, J. H. Heiser III, and P. Colombo, Encapsulation of Mixed Radioactive and Hazardous Waste Contaminated Incinerator Ash in Modified Sulfur Cement, BNL-43691, undated (approximately 1991).

2. A. van Dalen and J. E. Rijpkema, Modified Sulphur Cement: A Low Porosity Encapsulation Material for Low, Medium and Alpha Waste, paper published by the Commission of the European Communities, EUR 12303 EN, 1989.

3. G. R. Damell, Sulfur Polymer Cement, A New Final Waste Form for Radioactive and Hazardous Wastes, paper published by Idaho National Engineering Laboratory, November 19, 1992. 
EM Problem: Waste management

Y-12 Plant Problem: Building 9201-4 (Alpha-4). Contaminated solid wastes

Problem Area/Constituents: Stabilization of final contaminated residues resulting from decontamination and decommissioning, environmental restoration, and waste management operations performed at the site. These radioactive and/or hazardous wastes must be prepared for final disposal in compliance with regulations.

\section{Reference Requirements:}

- Resource Conservation and Recovery Act of 1976 (RCRA), as amended, Land Disposal Restriction (LDR) Federal Facilities Compliance Agreement (FFCA) dated June 12, 1992

- Federal Facilities Agreement (FFA) for the Oak Ridge Reservation

- Federal Facilities Compliance Agreement-Toxic Substances Control Act (FFCA-TSCA) dated February 20, 1992

- Clean Air Act regulations under 40 CFR 60-65

- Clean Water Act regulations under 40 CFR 110-146 and 400-471

- DOE orders

Refer to the Regulatory Compliance Chapter of Vol. 1 applicable proposed and promulgated environmental laws, signed and pending agreements for the Oak Ridge Reservation, radiation protection standards, DOE orders, and nonregulatory guidance. As site- and waste-specific characteristics are provided for each technology, specific regulatory requirements will be specified.

\section{Subelement: Waste stabilization}

Alternatives: Solidification, macroencapsulation, microencapsulation, chemical fixation

Technology: Thermosetting plastics encapsulation. Vinyl Ester Styrene (VES) encapsulation is a form of thermosetting plastics encapsulation. This thermosetting encapsulation is accomplished by infiltration of, or mixing with, the monomer followed by polymerization, encapsulating the waste. Thermoplastic (polyethylene and bitumen) encapsulations require melting the polymer and mechanically mixing it with waste, requiring elevated temperatures and dried wastes. Thermoset encapsulation does not require elevated temperatures or dried wastes. VES encapsulation originally was developed by Dow Chemical, but it is now being developed and marketed by Diversified Technologies of Chestertown, Md. Primarily, it has been developed as a means of encapsulating ion-exchange resin without the matrix failure experienced by cementitious solidification/stabilization methods when the resin shrinks and swells with wetting and drying. The process developed is quite simple and does not require resin drying or mechanical mixing. The resin bed is drained of aqueous solution, the VES monomer mix is inflitrated into the bed of wet resin (displacing residual water clinging to the resin beads), and finally, the monomer is polymerized, forming a monolith of VES, with the wet resin encapsulated inside. VES encapsulation of a resin bed does not result in a net 
increase in bulk volume, an important economic advantage for ultimate disposal. Likewise, it is also possible to encapsulate beds of other large solids. Fine solids, such as ashes from incineration, may be encapsulated, but mechanical mixing likely will be required to get a homogenous mix. Ostensibly, no interaction occurs between the waste and VES, but some materials may interfere with polymerization of the monomers, especially organic materials. Liquid wastes cannot be handled presently, except by evaporation to solids. But conceptually, an emulsion could be formed, leading to liquid waste dispersed throughout the solid VES. Technically, this does not meet some definitions of "no liquid" for disposal purposes. Also, this is a physical encapsulation technique with no chemical immobilization of the waste species. It is questionable whether VES encapsulation would pass the required TCLP test.

Status: VES is an accepted technology for encapsulating spent ion-exchange resins; it is adjudged to be at the predemonstration scale for disposal of ashes and other fine solids and is considered conceptual for disposal of liquid wastes.

Science/Technology Needs: These include developing this technology to where it can be used to stabilize (in compliance with regulations) more of the expected residues likely to be generated at the site than just the spent ion-exchange resins. These needs include, for example, conducting development studies to determine the following: whether the VES waste form will meet the TCLP leachability test for RCRA metals; the need to develop means to incorporate ashes into the monomer mix and test the performance of the resulting product, the need to determine what materials will interfere with polymerization of VES or weaken the resulting waste form, and the need to quantify residual monomer content after polymerization and determine the potential release rate of this monomer to the environment.

Implementation Needs: Developing and demonstrating the efficacy of this stabilization technique are estimated to cost $\$ 2 \mathrm{M}-\$ 5 \mathrm{M}$ (1992 dollars). However, payback for the process could be significant, if it is demonstrated that the technique can successfully encapsulate and isolate residues other than the spent ion-exchange resins for the required period.

Author: R. D. Spence/515-574-6782

\section{References:}

1. J. R. Conner, Chemical Fixation and Solidification of Hazardous Wastes, Van Nostrand Reinhold, New York, 1990.

September 1994

Decontamination and Decommissioning 
EM Problem: Waste management

Y-12 Plant Problem: Building 9201-4 (Alpha-4). Contaminated solid wastes

Problem Area/Constituents: Stabilization of final contaminated residues that result from decontamination and decommissioning, environmental restoration, and waste management operations performed at the site. These radioactive and/or hazardous wastes must be prepared for final disposal in compliance regulations.

\section{Reference Requirements:}

- Resource Conservation and Recovery Act of 1976 (RCRA), as amended, Land Disposal Restriction (LDR) Federal Facilities Compliance Agreement (FFCA) dated June 12, 1992

- Federal Facilities Agreement (FFA) for the Oak Ridge Reservation

- Federal Facilities Compliance Agreement-Toxic Substances Control Act (FFCA-TSCA) dated February 20, 1992

- Clean Air Act regulations under 40 CFR 60-65

- Clean Water Act regulations under 40 CFR 110-146 and 400-471

- DOE orders

Refer to the Regulatory Compliance Chapter of Vol. 1 applicable proposed and promulgated environmental laws, signed and pending agreements for the Oak Ridge Reservation, radiation protection standards, DOE orders, and nonregulatory guidance. As site- and waste-specific characteristics are provided for each technology, specific regulatory requirements will be specified.

\section{Subelement: Waste stabilization}

Alternatives: Solidification, macroencapsulation, microencapsulation, chemical fixation

Technology: High-temperature crystalline materials (ceramic). Ideally, mineral phases can be identified and generated for each waste specie, but this may not prove feasible, and some species may be encapsulated or limited to grain boundaries. One advantage of this waste form is its thermodynamic stability. The amorphous calcium-silicate-hydrate matrix of cementitious waste forms is thermodynamically unstable, although it may last for millennium in dry environments. Glass is a supercooled liquid. Ceramics are dense mineral phases, thermodynamically stable. As with glass, ceramics can be made with low porosity, making them less leachable than cementitious waste forms. As with cementitious waste forms, the chemical phases have low solubility, potentially making them less extractable than glass waste forms. Species relegated to grain boundaries, or physically encapsulated, may be just as extractable as before treatment. TCLP testing of specific waste forms is needed to verify this chemical stability. This technique is in its infancy. Some wastes are ready-made for conversion into ceramics, whereas others may require significant chemical conversion. The waste must not only be characterized as to elemental species, but also as to chemical species. This is a high-temperature technique with thermal destruction of organics, but may not be 
amenable to some volatile and semivolatile species, such as mercury, tritium, iodine, cesium, and technetium.

Status: Evolving technology/conceptual. Some laboratory work has been conducted on the technology.

Science/Technology Needs: These include, for example, the following: laboratory development to prove the concept for encapsulating certain wastes; development of the required ceramic wasteform; identification of the hazardous species that are incorporated into ceramic phases and those that are not; and demonstration of the required destruction and disposal of hazardous organics and nondestructible volatilized species that may be present in the waste. Also, the technology will need to be successfully demonstrated on a large pilotplant scale after laboratory development has been completed.

Implementation Needs: Development and demonstration of the efficacy of this stabilization method are estimated to cost $\$ 5 \mathrm{M}-\$ 10 \mathrm{M}$ (1992 dollars). However, payback for the process could be significant, if it is demonstrated that the technique can successfully encapsulate or isolate radioactive and/or hazardous compounds from the environment for the required period.

Author: R. D. Spence/615-574-6782

References: None 


\section{EM Problem: Waste management}

Y-12 Plant Problem: Building 9201-4 (Alpha-4). Contaminated solid wastes

Problem Area/Constituents: Stabilization of final contaminated residues that result from decontamination and decommissioning, environmental restoration, and waste management operations performed at the site. These radioactive and/or hazardous wastes must be prepared for final disposal in compliance with regulations.

\section{Reference Requirements:}

- Resource Conservation and Recovery Act of 1976 (RCRA), as amended, Land Disposal Restriction (LDR) Federal Facilities Compliance Agreement (FFCA) dated June 12, 1992

- Federal Facilities Agreement (FFA) for the Oak Ridge Reservation

- Federal Facilities Compliance Agreement-Toxic Substances Control Act (FFCA-TSCA) dated February 20, 1992

- Clean Air Act regulations under 40 CFR 60-65

- Clean Water Act regulations under 40 CFR 110-146 and 400-471

- DOE orders

Refer to the Regulatory Compliance Chapter of Vol. 1 applicable proposed and promulgated environmental laws, signed and pending agreements for the Oak Ridge Reservation, radiation protection standards, DOE orders, and nonregulatory guidance. As site- and waste-specific characteristics are provided for each technology, specific regulatory requirements will be specified.

\section{Subelement: Waste stabilization}

Alternatives: Solidification, macroencapsulation, microencapsulation, chemical fixation

Technology: High-temperature noncrystalline materials (vitrification). Vitrification is a physical encapsulation technique that has been accepted for high-level waste. Vitrification results in a net volume reduction, even starting with a bed of solids and no liquids. This results in significant economic savings for ultimate disposal to compensate for the higher capital and production costs required to generate this waste form compared to cementitious waste forms. Ostensibly, no interaction occurs with the waste, but it is questionable whether vitrified waste will pass the required TCLP test. The waste form is nonporous and less leachable than cementitious waste forms, but the RCRA metal may be just as extractable after treatment as before, if truly allowed to come to equilibrium, as intended in the TCLP test. Laboratory testing would be required to identify compatible melt compositions. This technique is performed at temperatures higher than those for thermoplastic encapsulation. Thus, water is driven off, but the method is inappropriate for volatiles and may not be suitable for semivolatiles. Organics likely are destroyed, but other volatiles and semivolatiles that cannot be destroyed (such as mercury, tritium, cesium, and technetium) may not be 
encapsulated by vitrification. Such contaminants are likely to be found in residues to be processed at the Y-12 Plant.

Status: Vitrification is adjudged to be at the predemonstration scale of development for treatment of low-level and/or hazardous wastes. However, a large data base exists for treating high-level nuclear wastes but not for low-level and hazardous wastes.

Science/Technology Needs: These include, for example, the following: the need to demonstrate the effectiveness of the technology for those semivolatile species commonly found in low-level waste and, for hazardous wastes, incinerator efficiencies must be demonstrated for organics as well as for the ability to handle volatile and semivolatile metals. Typically, mercury must be removed before vitrification, and Cs- 137 has been found in off-gas from vitrification units. Claims are made that the vitrified waste form will pass the TCLP test, but this must be verified and the RCRA metal limits in the vitrified waste, quantified. Also; laboratory testing of a specific waste or waste stream is necessary to establish the required glass composition. Some laboratory testing of certain wastes has been done, but would need repeating for the K-25 Site residues, and pilot-scale testing and demonstrations would have to be done. The development likely would have to be repeated on a case-by-case basis, just as with development of the cementitious waste forms.

Implementation Needs: Development and demonstration of the efficacy of this stabilization technique are estimated to cost $\$ 5 \mathrm{M}-\$ 10 \mathrm{M}$ (1992 dollars). This is expected to cover the costs of meeting the above-mentioned science/technology needs.

Author: R. D. Spence/615-574-6782

References: None

September 1994

Decontamination and Decommissioning 


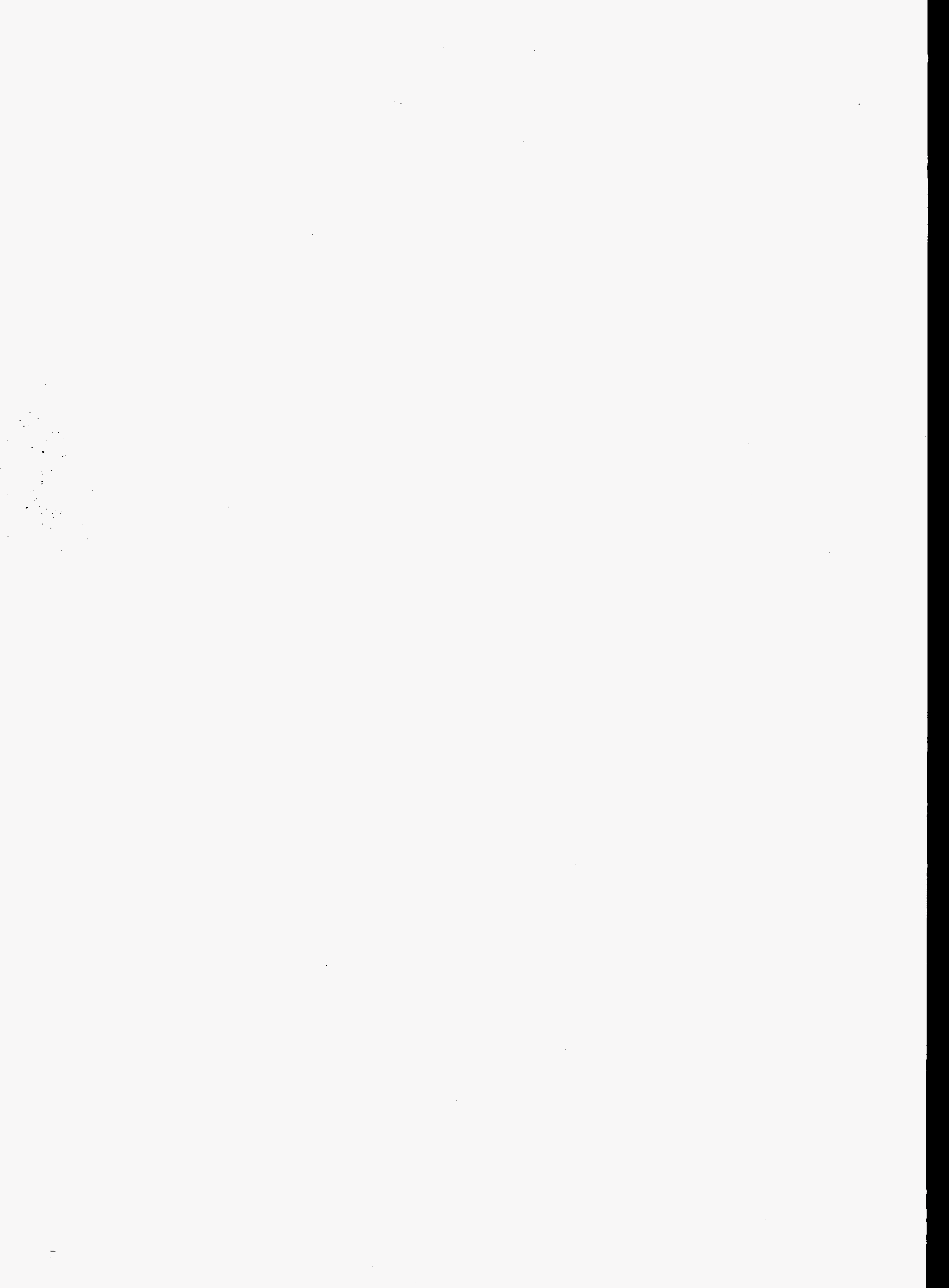




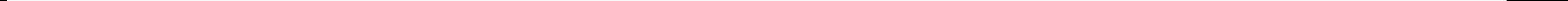

